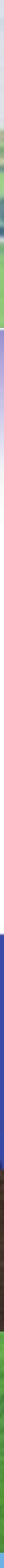

\title{
SWAP version 4
}

Theory description and user manual

J.G. Kroes, J.C. van Dam, R.P. Bartholomeus, P. Groenendijk, M. Heinen, R.F.A. Hendriks, H.M. Mulder,

I. Supit, P.E.V. van Walsum

WAGENINGEN

UNIVERSITY \& RESEARCH 



\section{SWAP version 4}

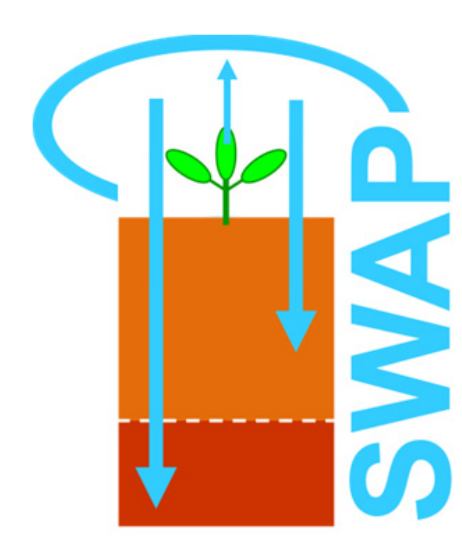

Theory description and user manual

J.G. Kroes ${ }^{1}$, J.C. van Dam ${ }^{1}$, R.P. Bartholomeus ${ }^{2}$, P. Groenendijk ${ }^{1}$, M. Heinen ${ }^{1}$, R.F.A. Hendriks ${ }^{1}$, H.M. Mulder ${ }^{1}$, I. Supit ${ }^{1}$, P.E.V. van Walsum ${ }^{1}$

${ }^{1}$ Wageningen UR - Wageningen Environmental Research (Alterra)

${ }^{2}$ KWR Watercycle Research Institute

This research is funded by a large group of financers: STOWA (Applied Research of the Water Boards), Ministry of Infrastructure and Environment, ACSG (Advisory Commission for Damage related to Groundwater), provinces Utrecht and Zuid-Holland, ZON (Zoetwatervoorziening Oost- Nederland), Water companies Vitens and Brabant Water, VEWIN, LTO and the Ministry of Economic Affairs (project KB-14-001-046).

Wageningen Environmental Research

Wageningen, May 2017

Report 2780

ISSN 1566-7197 
Kroes, J.G., J.C. van Dam, R.P. Bartholomeus, P. Groenendijk, M. Heinen, R.F.A. Hendriks, H.M. Mulder, I. Supit, P.E.V. van Walsum, 2017. SWAP version 4; Theory description and user manual. Wageningen, Wageningen Environmental Research, Report 2780. 244 pp.; 57 fig.; 17 tab.; 312 ref.

SWAP 4 simulates transport of water, solutes and heat in the vadose zone. It describes a domain from the top of canopy into the groundwater which may be in interaction with a surface water system. The program has been developed by Wageningen Environmental Research and Wageningen University, and is designed to simulate transport processes at field scale and during entire growing seasons. This is a new release with recent developments on atmosphere, soil water and crop growth interactions. This manual describes the theoretical background, model use, input requirements and output tables.

Keywords: agrohydrology, drainage, evaporation, irrigation, salinization, simulation model, soil water, solute transport, surface water management, transpiration, vadose zone

The pdf file is free of charge and can be downloaded at http://dx.doi.org/10.18174/416321 or via the website www.wur.nl/environmental-research (scroll down to Publications - Wageningen Environmental Research reports). Wageningen Environmental Research does not deliver printed versions of the Wageningen Environmental Research reports.

(C) 2017 Wageningen Environmental Research (an institute under the auspices of the Stichting Wageningen Research), P.O. Box 47, 6700 AA Wageningen, The Netherlands, $\mathrm{T}+31$ (0)317 4807 00, E info.alterra@wur.nl, www.wur.nl/environmental-research. Wageningen Environmental Research is part of Wageningen University \& Research.

- Acquisition, duplication and transmission of this publication is permitted with clear acknowledgement of the source.

- Acquisition, duplication and transmission is not permitted for commercial purposes and/or monetary gain.

- Acquisition, duplication and transmission is not permitted of any parts of this publication for which the copyrights clearly rest with other parties and/or are reserved.

Wageningen Environmental Research assumes no liability for any losses resulting from the use of the research results or recommendations in this report.

Wageningen Environmental Research Report 2780 | ISSN 1566-7197

Photo cover: The picture on the front cover shows SWAP's core processes in the soil below a grass vegetation positioned in a rural area with different land uses. 


\section{Contents}

$\begin{array}{ll}\text { Preface } & 7\end{array}$

$\begin{array}{ll}\text { Summary } & 9\end{array}$

1

Model overview

11

1.1 Model domain and processes $\quad 11$

1.2 SWAP installation $\quad 12$

$\begin{array}{lll}1.3 & \text { Model input } & 12\end{array}$

$\begin{array}{lll}1.4 & \text { Model run } & 15\end{array}$

$\begin{array}{lll}1.5 & \text { Model output } & 17\end{array}$

$\begin{array}{lll}1.6 & \text { Case studies } & 17\end{array}$

$\begin{array}{lll}1.7 & \text { Reading guide } & 17\end{array}$

2

$\begin{array}{lr}\text { Soil water flow } & 19\end{array}$

2.1 Basic equations $\quad 19$

2.2 Soil hydraulic functions $r$

2.2.1 General 19

2.2.2 Modification near saturation $\quad 20$

2.2.3 Soil hydraulic functions as tables $\quad 21$

2.2.4 Hysteresis 21

$\begin{array}{ll}2.2 .5 \text { Frozen soil conditions } & 23\end{array}$

$\begin{array}{lll}2.3 & \text { Lower boundary condition } & 23\end{array}$

2.4 Numerical implementation $\quad 24$

$\begin{array}{ll}2.4 .1 \text { Richards equation } & 25\end{array}$

$\begin{array}{ll}2.4 .2 \text { Numerical solution } & 26\end{array}$

$\begin{array}{lll}2.5 & \text { User instructions } & 28\end{array}$

2.5.1 General $\quad 28$

2.5.2 Bottom boundary conditions 32

$3 \quad$ Evapotranspiration and rainfall interception $\quad 37$

$\begin{array}{lll}3.1 & \text { Introduction } & 37\end{array}$

$\begin{array}{lll}3.2 & \text { Rainfall interception } & 38\end{array}$

3.2.1 Method Hoyningen-Braden 38

3.2.2 Method Gash $\quad 39$

3.3 Potential evapotranspiration of uniform surfaces 39

3.3.1 Penman Monteith method $\quad 40$

3.3.2 Reference evapotranspiration and crop factors $\quad 42$

3.4 Potential transpiration and evaporation fluxes of partly covered soils $\quad 42$

3.4.1 Distribution based on crop and soil factors 42

3.4.2 Distribution based on direct application of Penman-Monteith 43

3.5 Actual plant transpiration $\quad 44$

3.5.1 General approach $\quad 44$

3.5.2 Feddes function for root water uptake $\quad 45$

3.5.3 Detailed oxygen stress $\quad 45$

$\begin{array}{ll}3.5 .4 & \text { Detailed drought stress }\end{array}$

$\begin{array}{lll}3.6 & \text { Actual soil evaporation } & 49\end{array}$

3.7 User instructions $\quad 51$

3.7.1 General information $\quad 51$

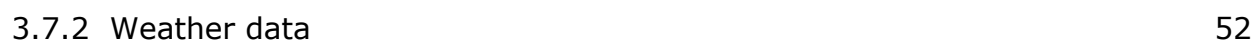

3.7.3 Soil data 53

$\begin{array}{ll}\text { 3.7.4 Plant data } & 54\end{array}$ 
4.1 Surface runoff $\quad 58$

4.2 Interflow $\quad 59$

4.3 Drain discharge $\quad 59$

4.3.1 Field scale drainage relation according to Hooghoudt and Ernst 61

4.3.2 Field scale drainage relation defined by a tabulated function 64

4.3.3 General aspects of regional scale drainage $\quad 65$

4.3.4 Regional scale drainage relation defined by a tabulated function 66

4.3.5 Multi-level drainage with fixed resistances and imposed drainage levels 67

4.3.6 Multi-level drainage with surface water dependent resistances and $\begin{array}{ll}\text { simulated drainage levels } & 68\end{array}$

4.4 Distribution with depth of drainage fluxes $\quad 69$

4.4.1 Implicit approach of travel times $\quad 69$

$\begin{array}{ll}4.4 .2 & \text { Discharge layers }\end{array}$

4.5 User instructions $\quad 72$

$\begin{array}{ll}4.5 .1 & \text { Surface runoff }\end{array}$

$\begin{array}{ll}4.5 .2 \text { Interflow } & 73\end{array}$

$\begin{array}{ll}4.5 .3 \text { Drainage } & 73\end{array}$

$\begin{array}{lll}5.1 & \text { Surface water balance } & 79\end{array}$

5.1.1 Multi-level drainage with imposed surface water levels $\quad 80$

5.1.2 Multi-level drainage with simulated surface water levels 80

$\begin{array}{lll}\text { 5.1.2.1 Fixed weir } & 80\end{array}$

5.1.2.2 Soil moisture controlled weir management 81

5.2 User instructions $\quad 82$

5.2.1 Input instructions $\quad 82$

$\begin{array}{lr}\text { Macropore flow } & 89\end{array}$

$\begin{array}{lll}6.1 & \text { Concept } & 89\end{array}$

6.1.1 Macropore geometry 90

$\begin{array}{lll}6.1 .1 .1 & \text { Continuity } & 90\end{array}$

$\begin{array}{lll}6.1 .1 .2 & \text { Persistency } & 93\end{array}$

$\begin{array}{ll}\text { 6.1.1.3 Horizontal distribution } & 97\end{array}$

6.1.2 Water flow and balance 98

6.2 Numerical implementation 103

$\begin{array}{ll}\text { 6.2.1 Macropore geometry } & 103\end{array}$

$\begin{array}{lll}6.2 .1 .1 & \text { Continuity } & 103\end{array}$

$\begin{array}{lll}6.2 .1 .2 & \text { Persistency } & 104\end{array}$

$\begin{array}{ll}\text { 6.2.1.3 Horizontal distribution } & 105\end{array}$

$\begin{array}{ll}\text { 6.2.2 Water flow and balance } & 105\end{array}$

6.3 User instructions $\quad 108$

$\begin{array}{ll}\text { 6.3.1 General input parameters } & 108\end{array}$

$\begin{array}{ll}\text { 6.3.2 Macropore input parameters } & 108\end{array}$

$\begin{array}{ll}\text { 6.3.2.1 Macropore geometry } & 109\end{array}$

$\begin{array}{ll}\text { 6.3.2.2 Water flow } & 114\end{array}$

$7 \quad$ Crop growth $r$

$\begin{array}{lll}7.1 & \text { Introduction } & 115\end{array}$

7.2 Simple module for static crop growth $\quad 116$

7.3 Detailed module for dynamic growth of arable crops $\quad 117$

$\begin{array}{ll}\text { 7.3.1 Phenological development stage } & 118\end{array}$

$\begin{array}{ll}\text { 7.3.2 Radiation fluxes above the canopy } & 120\end{array}$

$\begin{array}{ll}\text { 7.3.3 Radiation profiles within the canopy } & 121\end{array}$

7.3.4 Instantaneous assimilation rates per leaf layer $\quad 122$

$\begin{array}{ll}\text { 7.3.5 Daily gross assimilation rate of the canopy } & 123\end{array}$ 
7.3.7 Dry matter partitioning and growth respiration 125

7.3.8 Carbon balance check 126

$\begin{array}{lr}7.3 .9 \text { Senescence } & 126\end{array}$

$\begin{array}{ll}7.3 .10 \text { Net growth } & 128\end{array}$

7.3.11 Root growth $\quad 128$

7.4 Detailed module for dynamic growth of grass 129

7.5 Initialisation of crop growth (Germination) 130

7.5.1 Arable crops $\quad 130$

7.5.2 Grasland 131

$\begin{array}{lll}7.6 & \mathrm{CO}_{2} \text { changes } & 131\end{array}$

7.7 Nitrogen limited crop growth of arable crops 131

$\begin{array}{lll}7.8 & \text { User instructions } & 131\end{array}$

7.8.1 Crop rotation scheme 131

7.8.2 Simple crop module $\quad 132$

$\begin{array}{ll}\text { 7.8.3 Detailed crop module } & 136\end{array}$

$\begin{array}{ll}\text { 7.8.4 Detailed grass module } & 141\end{array}$

\section{Solute transport}

8.1 Introduction

8.2 Basic equations

8.2.1 Transport processes 146

8.2.2 Continuity and transport equation $\quad 147$

8.3 Boundary conditions 148

8.4 Crack solute transport $\quad 149$

8.5 Residence time in the saturated zone 149

$\begin{array}{lll}8.6 & \text { Salinity stress } & 150\end{array}$

8.7 Groundwater age 151

8.8 User instructions $\quad 152$

$9 \quad$ Soil temperature $r 55$

9.1 Temperature conductance equation $\quad 155$

9.2 Numerical solution $\quad 156$

9.3 Analytical solution $\quad 158$

9.4 User instructions $\quad 159$

$\begin{array}{llr}10 & \text { Snow and frost } & 161\end{array}$

$\begin{array}{llr}10.1 & \text { Snow } & 161\end{array}$

10.1.1Snowfall $\quad 161$

10.1.2Snowpack $\quad 161$

10.2 Frost $\quad 163$

10.3 User instructions $\quad 165$

11 Irrigation

$\begin{array}{lll}11.1 & \text { Fixed irrigation regime } & 167\end{array}$

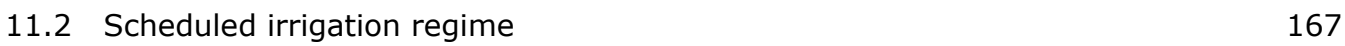

$\begin{array}{ll}\text { 11.2.1Timing criteria } & 167\end{array}$

$\begin{array}{ll}\text { 11.2.1.1 Allowable daily stress } & 167\end{array}$

11.2.1.2 Allowable depletion of readily available water 168

11.2.1.3 Allowable depletion of totally available water 168

11.2.1.4 Allowable depletion of field capacity water 168

$\begin{array}{ll}11.2 .1 .5 \text { Critical pressure head or moisture content } & 168\end{array}$

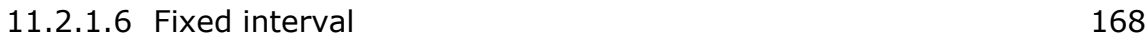

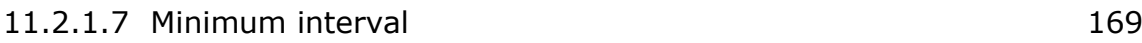


11.2.2Depth criteria 169

11.2.2.1 Back to Field Capacity 169

$\begin{array}{ll}11.2 .2 .2 \text { Fixed irrigation depth } & 169\end{array}$

$\begin{array}{ll}11.2 .2 .3 \text { Limited depth } & 169\end{array}$

$\begin{array}{lll}11.3 & \text { Flooding } & 169\end{array}$

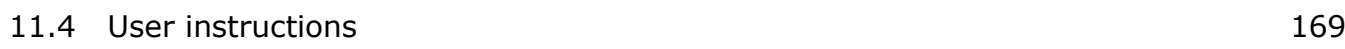

11.4.1Fixed irrigation regime 169

$\begin{array}{ll}\text { 11.4.2Scheduled irrigation } & 170\end{array}$

12 Case studies $r 3$

12.1 Hupsel Brook 173

$\begin{array}{lll}12.2 & \text { Grass growth } & 177\end{array}$

$\begin{array}{lr}12.3 \text { Macropore flow } & 179\end{array}$

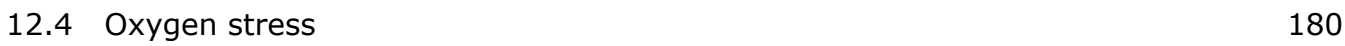

12.5 Salinity stress $\quad 181$

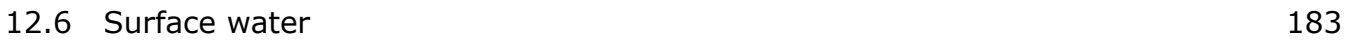

$\begin{array}{ll}\text { References } & 185\end{array}$

Appendix 1 Application Penman Monteith method 199

Appendix 2 Derivation of some macropore geometry equations 203

Appendix 3 Examples of description of macropore geometry 207

Appendix 4 Partial derivatives of $F_{i}$ to pressure heads 209

Appendix 5 Implicit linearization of hydraulic conductivities $\quad 211$

Appendix 6 Numerical solution heat flow equation 213

Appendix 7 Parameters of soil hydraulic functions: Staring series $\quad 217$

Appendix 8 Critical pressure head values of root water extraction 219

$\begin{array}{lll}\text { Appendix } 9 & \text { Salt tolerance data } & 221\end{array}$

$\begin{array}{lll}\text { Appendix } 10 & \text { Shrinkage characteristic data } & 223\end{array}$

Appendix 11 Examples of shrinkage characteristics of peat 225

$\begin{array}{lll}\text { Appendix } 12 & \text { List of input array lengths } & 227\end{array}$

$\begin{array}{lll}\text { Appendix } 13 & \text { List of main SWAP subroutines } & 229\end{array}$

Appendix 14 Description of output files *.afo and *.aun 231

Appendix 15 Description of output files *.bfo and *.bun 233

Appendix 16 Crop growth of grassland: acronyms 237

Appendix 17 Numerical scheme soil water boundary conditions 239

$\begin{array}{lll}\text { Appendix } 18 & \text { Explanation of header of crop output file } & 243\end{array}$ 


\section{Preface}

The ecohydrological model SWAP has a history of more than 40 years. The first version (called SWATR) was developed by Reinder Feddes and colleagues in The Netherlands and published in 1978. During it's history regularly updates were spread with derived acronyms SWATRE, SWACROP, SWAP93, and SWAP: Feddes et al. (1978); Belmans et al. (1983); Wesseling et al. (1991); Kabat et al. (1992); Van den Broek et al. (1994); Van Dam et al. (1997); Kroes et al. (2001; 2003; 2008). The last standard Internet version was published as SWAP3.2.36 by Kroes et al. (2009).

SWAP is used by researchers, engineers and students in the field of environmental science. Researchers employ the interactions between soil, water, plant and atmosphere as simulated by SWAP and the easy way in which the model can be adapted to specific research questions. Environmental engineers use the model as published and employ the practical boundary conditions of the model. In education SWAP is used to demonstrate typical transport situations in the biosphere. Students, ranging from Bachelor to PhD level, practice with the model. Some PhD students use SWAP as a research platform, to which they add innovative concepts.

At Wageningen University and Research centre fundamental and applied research are closely entangled. Scientists and students of various environmental disciplines and from all continents collaborate intensively. This creates much synergy, of which SWAP is one of the products.

Since the last release in 2009 a large number of new ideas have been tested with SWAP. Currently in environmental research much attention is paid to the food-water-energy nexus. In this research interaction between soil hydrology and crop growth plays a key role. In WaterVision Agriculture (Hack-ten Broeke et al., 2016; in Dutch: 'Waterwijzer Landbouw') we bring together the Dutch experience on how crop growth is affected by soil, climate and stresses due to lack of oxygen, lack of water or due to excess of salts. In the frame of WaterVision Agriculture we release this new SWAP version in which we implemented versatile improvements of recent years.

Our exciting journey does not end here. The future will bring new challenges. Therefore we supply SWAP with a well-structured source code and invite users to implement and test their own ideas. We very much appreciate feedback on the use of SWAP and suggestions for further improvements.

The SWAP team

Wageningen, May 2017 


\section{Summary}

SWAP (Soil-Water-Atmosphere-Plant) simulates transport of water, solutes and heat in the vadose zone in interaction with vegetation development. In the vertical direction the model domain reaches from a plane just above the canopy to a plane in the shallow groundwater. In this zone the transport processes are predominantly vertical, therefore SWAP is a one-dimensional, vertically directed model. In the horizontal direction, SWAP'S main focus is the field scale.

The SWAP model can be downloaded from site www.swap.alterra.nl. The model input may consist of files for main input, meteorological data, crop growth and drainage. SWAP employs the TTUTIL library to read the ASCII input files in easy format. Output is generated in ASCII and binary files. The internet site contains a large number of SWAP applications in scientific literature (Chapter 1 ).

Soil water flow is calculated with the Richards equation. The Mualem-Van Genuchten relations, with a modification near saturation, describe the soil hydraulic functions. Scaling of main drying and wetting curves is used to describe hysteresis in the retention function. The bottom boundary is controlled by head, flux or the relation between flux and head. SWAP solves the Richards equation numerically with an implicit, backward, finite difference scheme. The Newton-Raphson iterative procedure ensures mass conservation and rapid convergence (Chapter 2 ).

For agricultural crops and grassland, SWAP computes the interception following Von Hoyningen-Hüne and Braden. The interception concept of Gash is available for forests. The Penman-Monteith equation can be used to calculate the potential evapotranspiration of uniform surfaces (wet and dry vegetation, bare soil). An alternative is providing input of reference evapotranspiration in combination with crop factors. Next the potential transpiration and evaporation fluxes of partly covered soils are derived, taking into account interception and soil cover. Actual transpiration depends on the moisture and salinity conditions in the root zone, weighted by the root density. Actual evaporation depends on the capacity of the soil to transport water to the soil surface. SWAP uses the soil hydraulic functions and semi-empirical equations to determine this transport capacity (Chapter 3 ).

Surface runoff will be calculated when the height of water ponding on the soil surface exceeds a critical depth. The rate of surface runoff depends on a specified resistance. Interflow may occur when the groundwater level becomes higher than the interflow drainage level. Drainage can be calculated with the Hooghoudt or Ernst equations, with a table relating drainage flux and groundwater level, or with drainage resistances per drainage system. In order to calculate proper residence times of solutes, the drainage fluxes are vertically distributed according to so-called discharge layers (Chapter 4). The water balance of the surface water system can be calculated to analyse water management options. Surface water levels can be imposed, or derived by setting soil moisture criteria (groundwater level, pressure head, minimum storage) in combination with a weir (Chapter 5).

Macroporosity can be caused by shrinking and cracking of soil, by plant roots, by soil fauna, or by tillage operations. The macropore module in SWAP includes infiltration into macropores at the soil surface, rapid transport in macropores to deeper layers, lateral infiltration into and exfiltration out of the soil matrix, water storage in macropores, and rapid drainage to drainage systems. The macropores are divided in a main bypass domain (network of continuous, horizontal interconnected macropores) and an internal catchment domain (discontinuous macropores ending at different depths). The internal catchment domain causes infiltration of macropore water at different, relatively shallow depths. In addition, the macropores are divided in static and dynamic volumes. The dynamic volumes depend on shrinkage characteristics (Chapter 6).

The simple crop module prescribes crop development, independent of external stress factors. Its main function is to provide a proper upper boundary condition for soil water movement. In addition, SWAP includes the generic crop growth module WOFOST. In this module, the absorbed radiation is a function of solar radiation and crop leaf area. Next the produced carbohydrates $\left(\mathrm{CH}_{2} \mathrm{O}\right)$ are calculated, taking into account photosynthetic leaf characteristics and possible water and/or salinity stress. The carbohydrates provide energy for living biomass (maintenance respiration) and are converted into structural material during which weight is lost as growth respiration. The material produced is partitioned among roots, leaves, stems and storage organs, using partioning factors that depend on the crop development stage. The fraction partioned to the leaves, determines leaf area development 
and hence the dynamics of light interception. During crop development a part of the living biomass dies due to senescence (Chapter 7).

Grass growth is special: it is perennial, very sensitive to nitrogen, and grass is either grazed or mowed. Therefore SWAP includes a separate WOFOST module for grass, which simulates these special grass features (Chapter 7).

SWAP simulates transport of salts, pesticides and other solutes that can be described with basic physical relations: convection, diffusion, dispersion, root uptake, Freundlich adsorption and first order decomposition. In case of advanced pesticide transport, including volatilization and kinetic adsorption, SWAP can be used in combination with PEARL. In case of advanced transport of nitrogen and phosphorus, SWAP can be used in combination with ANIMO or Soil-N (Chapter 8).

SWAP may simulate soil temperature analytically, using an input sine function at the soil surface and the soil thermal diffusivity. In the numerical approach, SWAP takes into account the influence of soil moisture on soil heat capacity and soil thermal conductivity. The top boundary condition may include air temperatures or soil surface temperatures (Chapter 9).

The snow module calculates the accumulation and melting of a snowpack when the air temperature is below a threshold value. The water balance of the snow pack includes storage, incoming snow and rain and outgoing melting and sublimation. Melting may occur due to air temperature rise or heat release from rainfall. When a snowpack is present, the soil temperature top boundary condition is adjusted in order to account for the insulating effect of the snowpack. In case of frost, reduction factors can be calculated for the hydraulic conductivity, root water uptake, drainage fluxes and bottom flux (Chapter 10).

Irrigations with fixed date, depth and quality can be specified as input. In addition, SWAP can be used to schedule and optimize irrigation. Timing criteria include allowable daily stress, allowable depletion amount and critical pressure head or water content. Depth criteria include back to field capacity and fixed depth (Chapter 11).

The SWAP installation file includes cases which can be run directly after extraction. Chapter 12 describes the context and results of the following cases:

1. HupselBrook (typical Dutch field with subsurface drains and crop rotation),

2. GrassGrowth (growth and yield of a Dutch grassland field in Ruurlo),

3. MacroporeFlow (field experiment where macro pore flow occurs, Andelst),

4. OxygenStress (grassland field with shallow groundwater and oxygen stress),

5. SalinityStress (potato crop under saline conditions, saltFarm, Texel),

6. SurfaceWater (surface water management in a rural estate Wildenborch).

Most cases include one or more R-procedures $(R, 2013)$ to graphically display some model results. Observations are sometimes included to have reference points during visual comparisons with simulation results.

The appendices contain information on the parameters of the soil hydraulic functions, critical pressure heads for root water extraction, salt tolerance data, shrinkage characteristic data, numerical solution of water and heat flow, contents of binary output files and names of the main SWAP subroutines. 


\subsection{Model domain and processes}

SWAP simulates transport of water, solutes and heat in the vadose zone in interaction with vegetation development. The model employs the Richards equation including root water extraction to simulate soil moisture movement in variably saturated soils. In addition to ordinary matrix flow, SWAP considers flow through macropores as may occur in clay and peat soils. Solute transport includes the basic processes convection, dispersion, adsorption and decomposition. For more detailed solute transport studies, SWAP can be used in combination with specialized chemical transport models such as PEARL for pesticides and Soil-N or ANIMO for nitrogen. SWAP simulates soil heat flow taking into account actual heat capacities and thermal conductivities. The generic crop growth module WOFOST is incorporated to simulate leaf photosynthesis and plant growth. The soil moisture, heat and solute modules exchange status information each time step to account for their interactions. On a daily basis crop growth is affected by actual conditions of weather, soil moisture and salinity. An extensive test protocol ensures the numerical code quality of SWAP.

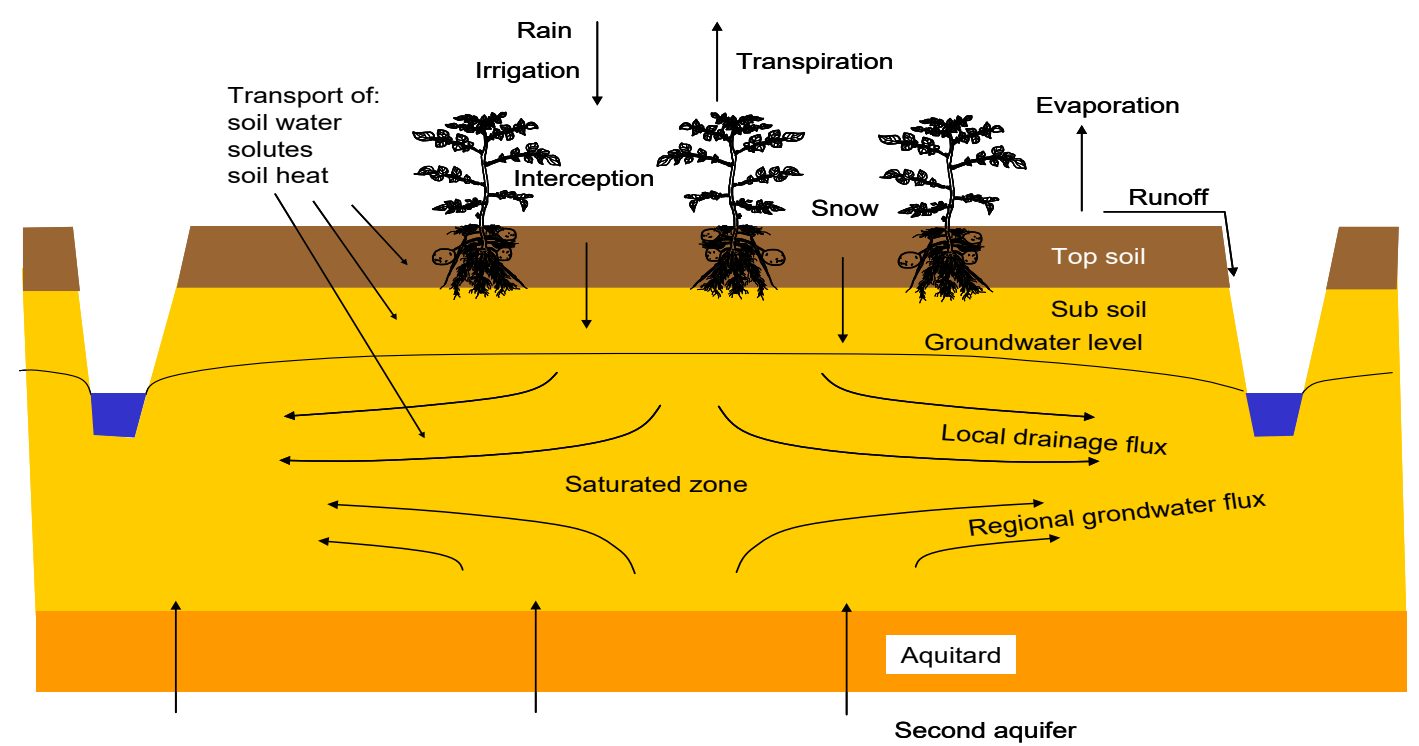

Figure 1.1 SWAP model domain and transport processes.

In the vertical direction the model domain reaches from a plane just above the canopy to a plane in the shallow groundwater (Figure 1.1). In this zone the transport processes are predominantly vertical, therefore SWAP is a one-dimensional, vertical directed model. The flow below the groundwater level may include lateral drainage fluxes, provided that these fluxes can be prescribed with analytical drainage formulas. The model is very flexible with respect to input data at the top and bottom of the soil column. At the top in general daily weather conditions will suffice. For Nordic conditions a simple snow storage module has been implemented. In case of more focussed studies (e.g. runoff or diurnal transpiration fluxes) evapotranspiration and rainfall data can be specified in more detail. At the bottom various forms of head and flux based conditions are used.

In the horizontal direction, SWAP's main focus is the field scale. At this scale most transport processes can be described in a deterministic way, as a field generally can be represented by one microclimate, one vegetation type, one soil type, and one drainage condition. Also many cultivation practices occur at field scale, which means that many management options apply to this scale. Upscaling from field to regional scale for broader management policy studies is possible with geographical information systems. 
The smallest time steps in SWAP are in the order of seconds for fast transport processes such as intensive rain showers with runoff or flow in macroporous clay soils. These time steps are automatically increased in periods with more slow flow conditions. Depending on simulation complexity, computation times for 50 year periods range from 30 to 500 seconds on ordinary personal computers.

\subsection{SWAP installation}

The SWAP model can be downloaded from Internet site www.swap.alterra.nl. This site contains general information on model features, applications and test reports. Various SWAP versions are available at the Internet site. This manual applies to SWAP 4. Only the most recent SWAP version is supported by the Swap team.

To allow installation on different operating system, we compiled a versatile set for distribution and packed all files into one zip-file. The zip-file can be unpacked on an arbitrary folder. Once unpacked a number of subfolders are created (Figure 1.2), which contain:

- Swap executable

- Swap source code

- User manual

- Several case studies

- Additional input data:

- Daily weather data of Wageningen meteorological station of the period 1971-2000.

- Simple crop input data for grass, fodder maize, potato, sugar beet and winter wheat.

- Detailed crop input data for winter wheat, grain maize, spring barley, rice, sugar beet, potato, field bean, soy bean, winter oilseed rape and sunflower.

SWAP can be automatically launched for the example Hupsel. Also runs can be made with the other 5 case studies described

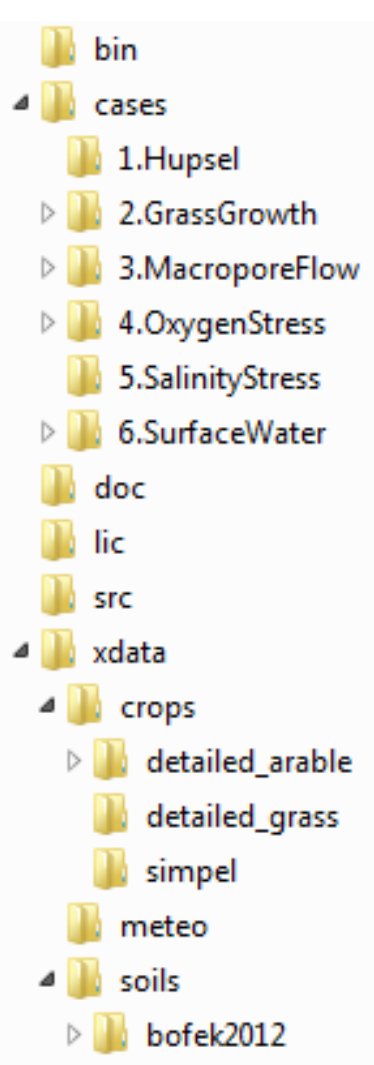

Figure 1.2 Installed folders by standard SWAP installation. in Chapter 12.

\subsection{Model input}

The input data of SWAP are divided over 4 different file types:

- Main input file (*.swp)

- Meteorological file (*.yyy)

- Crop growth file (*.crp)

- Drainage file (*.dra)

Box 1.1 provides an overview of the information in these input files. The main input file and the meteorological data file are always required. Input files of crop growth and drainage are optional. The extensions of the files are fixed. An exception is the meteorological file, which has an extension equal to the last 3 digits of the year (e.g. 2017 gives .017). The names of the input files are free to choose and are specified in the main input file. As listed in Box 1.1, the main input file contains general information with regard to the simulation, meteorology, crop rotation scheme, irrigation, soil water flow, heat flow and solute transport. For meteorological data, commonly a file with daily data is used. In Chapter 3 also more detailed input of evapotranspiration and rainfall fluxes will be discussed. The detailed crop growth input file is required to simulate crop development and biomass assimilation. As an alternative, the development of crop parameters as leaf area index or rooting depth can be 
prescribed in the simple crop growth input file. The drainage input file contains two sections. The first, basic drainage section provides input for drainage towards ditches and/or drains. The second, extended drainage section provides input for drainage including simulation of surface water levels.

Box 1.1 Summary of information in input files. Optional files are denoted with \#.

\section{Main input file (*.swp)}

- General section

- Environment

- Timing of simulation period

- Timing of boundary conditions

- Processes which should be simulated

- Optional output files

- Meteorology section

- Name of file with meteorological data

- Rainfall intensity

- Crop section

- Crop rotation scheme (calendar and files)

- Crop data input file

- Calculated irrigation input file

- Crop emergence and harvest

- Fixed irrigation parameters (Amount and quality of prescribed irrigation applications)

- Soil water section

- Initial moisture condition

- Ponding

- Soil evaporation

- Vertical discretization of soil profile

- Soil hydraulic functions

- Hysteresis of soil water retention function

- Maximum rooting depth

- Similar media scaling of soil hydraulic functions

- Preferential flow due to soil volumes with immobile water

- Preferential flow due to macro pores

- Snow and frost

- Numerical solution of Richards' equation

- Lateral drainage section

- (optional) name of file with drainage input data

- (optional) name of file with runon input data

- Bottom boundary section

- (optional) name of file with bottom boundary conditions

- selection out of 8 options

- Heat flow section

- calculation method

- Solute transport section

- Specify whether simulation includes solute transport or not

- Top boundary and initial condition

- Diffusion, dispersion, and solute uptake by roots

- Adsorption

- Decomposition

- Transfer between mobile and immobile water volumes (if present)

- Solute residence in the saturated zone

\section{File with daily meteorological data (*.yyy)}

- Radiation, temperature, vapour pressure, wind speed, rainfall and/or reference evapotranspiration,

- rainfall intensities

\section{File with Detailed crop growth (*.crp) \#}

- Crop section

- Crop height

- Crop development

- Initial values

- Green surface area

- Assimilation

- Assimilates conversion into biomass

- Maintenance respiration

- Dry matter partitioning

- Death rates

- Crop water use

- Salt stress

- Interception

- Root growth and density distribution

- Calculated Irrigation section

- General

- Irrigation time criteria

- Irrigation depth criteria

\section{File with Simple crop growth (*.crp) \#}

- Crop section

- Crop development

- Light extinction

- Leaf area index or soil cover fraction

- crop factor or crop height

- rooting depth

- yield response

- soil water extraction by plant roots

- salt stress

- interception

- Root density distribution and root growth

- Calculated Irrigation section

- General

- Irrigation time criteria

- Irrigation depth criteria

File with drainage data (*.dra) \#

- Basic drainage section

- Table of drainage flux - groundwater level

- Drainage formula of Hooghoudt or Ernst

- Drainage and infiltration resistances

- Extended drainage section

- Drainage characteristics

- Surface water level of primary and/or secondary system

- Simulation of surface water level

- Weir characteristics 
SWAP uses the TTUTIL library (Kraalingen \& Rappoldt, 2000) for reading input files. Box 1.2 gives an example of a part of the *.swp input file. General rules for the format of input files are:

- free format with the structure 'VariableName' = 'value' or, in case of arrays, in a table with variable names in the first line;

- order of variables is free;

- comment in lines is allowed starting with symbols '*' or '!';

- blank lines are allowed.

The input files list of each parameter their symbolic name, a description and an identification. The identification between square brackets provides information on:

- the range

- the unit

- the data type ( $\mathrm{I}=$ Integer, $\mathrm{R}=$ Real, $\mathrm{Ax}=$ character string of $\mathrm{x}$ positions)

For example: $[-5000 . .100 \mathrm{~cm}, \mathrm{R}]$ means: value between -5000 and +100 with a unit in $\mathrm{cm}$, given as a Real data type (which means that the value in the input file should contain a dot).

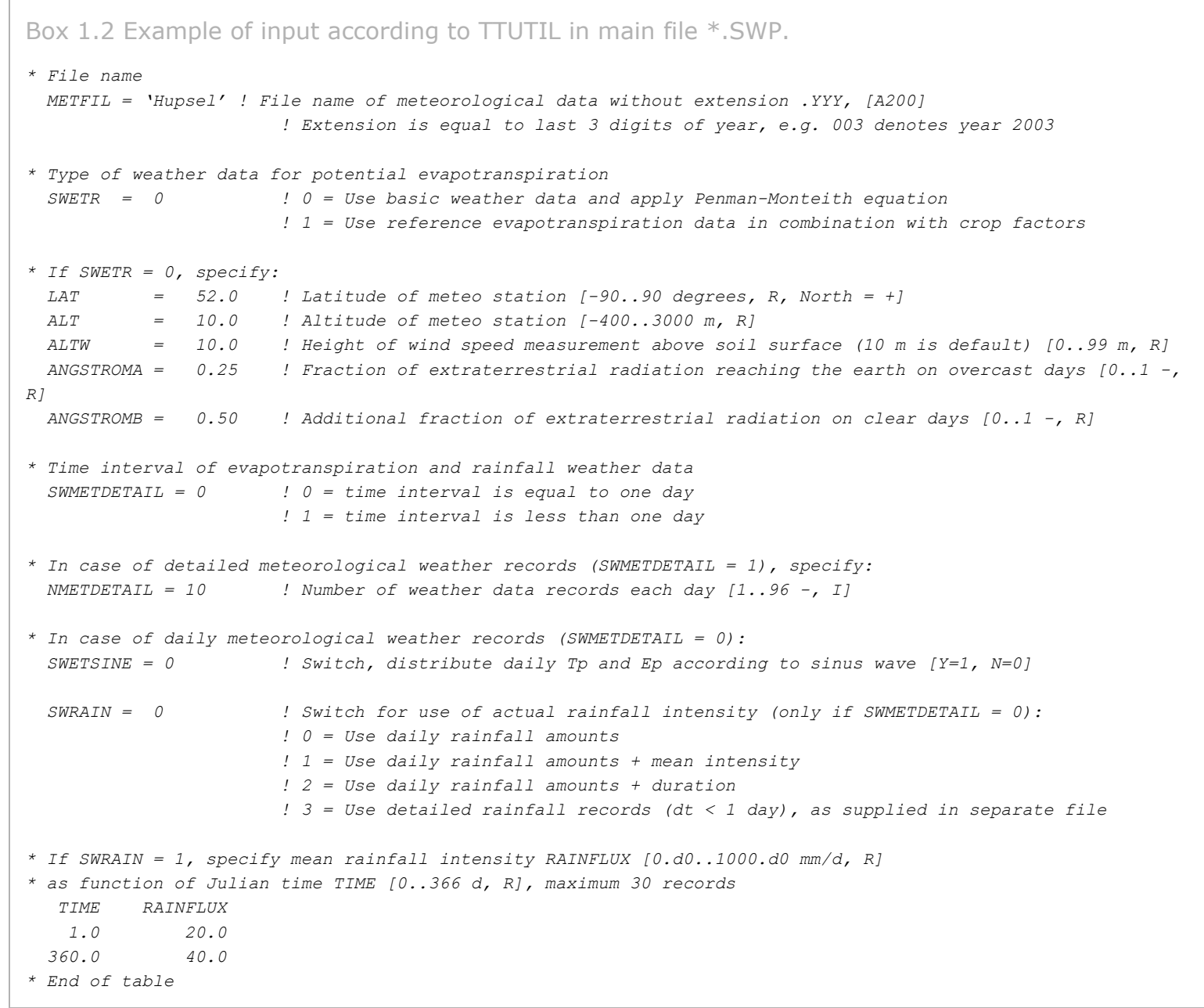

SWAP will read times according to the following format: 10-feb-2017_16:30:00.00 denotes February 10, 2017 at 4.30 PM. If in the input only dates and no times are specified, SWAP will assume time 0:00. In case of input data series as function of time (e.g. groundwater levels) or depth (e.g. initial pressure heads), SWAP will apply linear interpolation for times and depths in between. Outside the specified range, the closest value will be adopted. For instance in Box 1.2, the rainfall intensity (RAINFLUX) will gradually increase from 20 to $40 \mathrm{~mm} \mathrm{~d}^{-1}$ between $t=1.0$ and $t=360.0 \mathrm{~d}$. At $t>360.0 \mathrm{~d}$, the rainfall intensity will be $40 \mathrm{~mm} \mathrm{~d}^{-1}$. 


\section{$1.4 \quad$ Model run}

The most common way to run SWAP is by executing a batch file, which refers to the SWAP executable and the main input file *.swp. The batch file and the *.swp file need to be present in the same directory. The *.swp file contains the names and locations of other input files. In this way the meteorological, crop and drainage data can be specified on separate folders.

An example of the batch file is given in Box 1.3. In this case SWAP will use Hupsel.swp as main input file. If no name is specified behind the executable call, SWAP assumes 'Swap.swp' as main input file. The pause statement keeps the window box with screen messages open; this is convenient when runtime warnings or errors occur.

Box 1.3 Example of batch file to run SWAP with input file Hupsel.swp.

c: $\backslash$ Program Files $\backslash S W A P \backslash S w a p$.exe Hupsel.swp pause

An alternative to run SWAP is by double clicking file Swap.exe. In that case the main input file should be called 'Swap.swp', and should be located in the same folder as file Swap.exe.

Three types of messages may occur during a model run:

- error messages with respect to the input data

- warnings with the advise to adapt the combination of selected options because the specified combination is not feasible

- fatal calculation errors which stop the simulation

Output files will be generated in the same directory as the main input file. Here also the log file named '*_Swap.log' can be found. This log file contains a copy of the *.swp file, possible errors and warnings, and in case of a successful simulation run the statement: 'Swap simulation okay!' 
Short water and solute balance (*.bal)

Final and initial water and solute storage

Water balance components

Solute balance components

\section{Extended water balance (*.blc) \#}

Final and initial water storage

Water balance components of sub systems

Incremental water balance $(*$.inc)

Gross rainfall and irrigation

Interception

Runon and runoff

Potential and actual transpiration

Potential and actual evaporation

Net drainage and bottom flux

\section{Cumulative water balance (*.wba)}

Gross and net rainfall

Runon and runoff

Potential and actual transpiration

Potential and actual evaporation

Net lateral flux (drainage)

Net bottom flux

Change water storage in profile

Groundwater level

Water balance error

\section{Log file (SWAP.log)}

Echo of input (*.swp-file)

Errors and warnings

\section{Cumulative solute balance (*.sba) \#}

Flux at soil surface

Amount decomposed

Amount taken up by plant roots

Amount in soil profile

Amount in cracks

Flux at soil profile bottom

Drainage flux

Bypass flux from cracks

Amount in defined saturated aquifer

Flux from defined saturated aquifer

Soil temperatures (*.tem) \#

Soil temperature of selected nodes

\section{Soil profiles (*.vap) \#}

Profiles of water content, pressure head, solute concentration, temperature, water flux, root extraction and solute flux

\section{Irrigation (*.irg) \#}

Calculated irrigation applications

\section{Crop growth (*.crp)}

Development stage

Temperature sum

Leaf area index (potential and actual)

Crop height and crop factor

Potential and actual rooting depth

Cumulative relative transpiration during $0-2$ and

1-2 DVS

Cumulative potential and actual dry weight of leaves

Cumulative potential and actual dry weight of stems
Cumulative potential and actual dry weight of roots Cum. pot. and actual weight of crop dry matter above ground

Cumulative potential and actual dry weight of storage organ

Cum. pot. and actual weight of grass dry matter above ground

Cumulative potential and actual dry weight of mowed grass

Cumulative potential and actual dry weight of grazed grass

\section{Transpiration stress (*.str)}

Potential and actual transpiration

Transpiration reduction due to wet, dry, saline and frost conditions

Extended drainage components (*.drf) \#

Drainage fluxes of each level

Total drainage flux

Net runoff

Rapid drainage

\section{Surface water management 1 (*.swb) \#}

Groundwater level

Weir target level

Surface water level

Storage in surface water reservoir

Sum of drainage, runoff and rapid drainage

External supply to surface water reservoir

Outflow from surface water reservoir

Surface water management 2 (*.man) \# Weir type

Groundwater level

Pressure head for target level

Total air volume in soil profile

Weir target level

Surface water level and outflow

Number of target level adjustments

Indicator weir overflow

Weir crest level

\section{Snowpack water balance (*.snw) \# \\ Final and initial water storage \\ Water balance components}

Detailed waterbalance macropores (*.bma) \# Final and initial water storage

Water balance components

Soil physical parameters (soilphysparam.csv) For each soil layer the relation between: soil water pressure head $\mathrm{h}(\mathrm{cm})$, theta $\theta\left(\mathrm{cm}^{3} . \mathrm{cm}^{-3}\right)$, differential capacity $\mathrm{C}\left(\mathrm{cm}^{-1}\right)$, RelSat $\mathrm{S}_{\mathrm{e}}(-)$ and hydraulic conductivity $\mathrm{K}\left(\mathrm{cm} \cdot \mathrm{d}^{-1}\right)$

\section{Soil heat conductivity and capacity} (heatparam.csv) \#

For each soil layer the relation between theta heatcapacity and heat conducitivity

Final values of state variables (*.end)

Snow and ponding layer

Soil water pressure heads

Solute concentrations

Soil temperatures 


\section{$1.5 \quad$ Model output}

Output from SWAP is stored in general ASCII files, which can be read with any editor or word processor. You may open the output file directly in Excel by adding .CSV to the file extension. Some output files are always generated, other files are optional. Box 1.4 provides an overview of the variables that are printed in each output file. All output files have the same header with the project name, file content, file name, model version, generation time, calculation period and soil profile depth. The output interval may range from 0.001 day to 1.0 year. If the output time only consists of a date, the output represents the situation at the end of the particular day. We may distinguish output of state variables, incremental fluxes since the last output time, and cumulative fluxes since a specified date. The output file with final values of state variables (OUTFIL.END) can be used as input for a subsequent simulation period. This might be useful to derive suitable initial conditions.

In addition to the ASCII files, formatted and unformatted (binary) export files can be generated with data that cover the entire simulation period. These output files can be used as input for advanced solute transport models, such as PEARL (Leistra et al., 2001) for pesticides and ANIMO (Groenendijk et al., 2005) for nutrients. A description of these export files is given in Appendix 14 and 15.

\subsection{Case studies}

Chapter 12 contains 6 case studies with SWAP. The input files of these cases come with the installation of SWAP. The case studies are:

- Hupsel Brook

- Grass growth

- Macropore flow

- Oxygen stress

- Salinity stress

- Surface water

The first case of a field in Hupsel Brook catchment includes a simulation with 3 different crop growth modules. This case is used to illustrate the input files in this SWAP manual.

\subsection{Reading guide}

In the next chapters we discuss subsequently:

Chapter 2. Soil water flow

Chapter 3. Evapotranspiration and rainfall interception

Chapter 4. Surface runoff, interflow and drainage

Chapter 5. Surface water system

Chapter 6. Macropore flow

Chapter 7. Crop growth

Chapter 8. Solute transport

Chapter 9. Soil heat flow

Chapter 10. Snow and frost

Chapter 11. Irrigation

Chapter 12. Case studies

The first part of chapters 2-11 describe the physical relations incorporated in SWAP. This part also describes implemented numerical procedures, if required to use SWAP in a proper way. The second part of each chapter describes the model input. If relevant, suggestions for input are included.

The appendices contain information on:

- Application of the Penman Monteith method

- Derivation and examples of macropore equations

- Equations for the partial derivatives of $F_{i}$ to pressure heads 
- Equations for the implicit linearization of hydraulic conductivities

- Equations for the numerical solution of heat flow

- Tables with soil hydraulic functions: Staring Series

- Tables with critical pressure heads for root water extraction

- Tables with salt tolerance data

- Tables with shrinkage characteristic data

- Tables with shrinkage characteristic data for peat soils

- List of subroutines

- List of fixed ranges of array lengths

- Listing of formatted and unformatted binary output files

- Acronyms in the WOFOST growth routine for grassland

- Numerical scheme of soil water boundary conditions 


\section{Soil water flow}

\section{$2.1 \quad$ Basic equations}

Gradients of the soil water potential cause soil water movement. Darcy's equation is commonly used to quantify this soil water movement. For one-dimensional vertical flow, Darcy's equation can be written as:

$$
q=-K(h) \frac{\partial(h+z)}{\partial z}
$$

where $q$ is soil water flux density (positive upward) $\left(\mathrm{cm}^{3} \mathrm{~cm}^{-2} \mathrm{~d}^{-1}=\mathrm{cm} \mathrm{d}^{-1}\right), K(h)$ is hydraulic conductivity $\left(\mathrm{cm} \mathrm{d}^{-1}\right), h$ is soil water pressure head $(\mathrm{cm})$ and $z$ is the vertical coordinate $(\mathrm{cm})$, taken positively upward.

Water balance considerations of an infinitely small soil volume result in the continuity equation for soil water:

$$
\frac{\partial \theta}{\partial t}=-\frac{\partial q}{\partial z}-S_{a}(h)-S_{d}(h)-S_{m}(h)
$$

where $\theta$ is volumetric water content $\left(\mathrm{cm}^{3} \mathrm{~cm}^{-3}\right), t$ is time $(\mathrm{d}), S_{a}(h)$ is soil water extraction rate by plant roots $\left(\mathrm{cm}^{3} \mathrm{~cm}^{-3} \mathrm{~d}^{-1}\right), S_{d}(h)$ is the extraction rate by drain discharge in the saturated zone $\left(\mathrm{cm}^{3} \mathrm{~cm}^{-3} \mathrm{~d}^{-1}\right)$ and $S_{m}(h)$ is the exchange rate with macro pores $\left(\mathrm{cm}^{3} \mathrm{~cm}^{-3} \mathrm{~d}^{-1}\right)$.

Combination of Eqs. (2.1) and (2.2) provides the general water flow equation in variably saturated soils, known as the Richards equation:

$$
\frac{\partial \theta}{\partial t}=\frac{\partial\left[K(h)\left(\frac{\partial h}{\partial z}+1\right)\right]}{\partial z}-S_{a}(h)-S_{d}(h)-S_{m}(h)
$$

SWAP applies Richards equation integrally for the unsaturated-saturated zone, including possible transient and perched groundwater levels. SWAP solves Eq. (2.3) numerically, using specified boundary conditions and relations between $\theta, h$ and $K$.

\subsection{Soil hydraulic functions}

\subsubsection{General}

SWAP uses the Mualem-Van Genuchten functions (Mualem, 1976; Van Genuchten, 1980) which have been used in numerous studies and form the base of several national and international data-bases (e.g. Carsel and Parrish, 1988; Yates et al., 1992; Leij et al., 1996; Wösten et al., 2001; Vereecken et al., 2010). The analytical $\theta(h)$ function proposed by Van Genuchten (1980) reads:

$$
\theta=\theta_{\text {res }}+\left(\theta_{\text {sat }}-\theta_{\text {res }}\right)\left(1+|\alpha h|^{n}\right)^{-m}
$$

where $\theta_{\text {sat }}$ is the saturated water content $\left(\mathrm{cm}^{3} \mathrm{~cm}^{-3}\right), \theta_{\text {res }}$ is the residual water content in the very dry range $\left(\mathrm{cm}^{3} \mathrm{~cm}^{-3}\right)$ and $\alpha\left(\mathrm{cm}^{-1}\right), n(-)$ and $m(-)$ are empirical shape factors. Without loosing much flexibility, $m$ can be taken equal to:

$$
m=1-\frac{1}{n}
$$


Using the above $\theta(h)$ relation, with $m=1-1 / n$, and applying the theory on unsaturated hydraulic conductivity by Mualem (1976), the following $K(\theta)$ function results:

$$
K=K_{\mathrm{sat}} S_{\mathrm{e}}^{\lambda}\left[1-\left(1-S_{\mathrm{e}}^{\frac{1}{m}}\right)^{m}\right]^{2}
$$

where $K_{\text {sat }}$ is the saturated conductivity $\left(\mathrm{cm} \mathrm{d}^{-1}\right), \lambda$ is a shape parameter $(-)$ depending on flow path tortuosity, and $S_{\mathrm{e}}$ is the relative degree of saturation defined as:

$$
S_{\mathrm{e}}=\frac{\theta-\theta_{\text {res }}}{\theta_{\text {sat }}-\theta_{\text {res }}}
$$

The numerical solution to the Richards equation requires an expression of the differential water capacity $C\left(\mathrm{~cm}^{-1}\right)$, which is defined as the derivative of $\theta$ to $h$ (Eq. (2.4):

$$
C=\frac{\partial \theta}{\partial h}=\alpha m n|\alpha h|^{n-1}\left(\theta_{s a t}-\theta_{r e s}\right)\left(1+|\alpha h|^{n}\right)^{-(m+1)}
$$

A numerical approach to Eq. (2.3) yielding a steady-state solution requires an implicit treatment of the hydraulic conductivity. This implies the use of the derivative of the hydraulic conductivity to the pressure head $\mathrm{d} K / \mathrm{d} h$. Expressions are given in Appendix 5.

\subsubsection{Modification near saturation}

Near saturation a modification to the Mualem-Van Genuchten function (Schaap and Van Genuchten, 2006) has been implemented in SWAP. The modification is based on the introduction of a small minimum capillary height $h_{\mathrm{e}}$ which can be regarded as an air-entry value, causing a minor shift in the retention curve (Vogel et al., 2001). We follow Ippisch et al. (2006) by defining the relative water content as:

$$
S_{\mathrm{e}}=\left\{\begin{array}{cl}
\frac{1}{S_{\mathrm{c}}}\left[1+|\alpha h|^{n}\right]^{-m} & h<h_{\mathrm{e}} \\
1 & h \geq h_{\mathrm{e}}
\end{array}\right.
$$

where $S_{\mathrm{e}}$ is the relative saturation at the cut-off point $h_{\mathrm{e}}$ in the original Van Genuchten model, given by:

$$
S_{c}=\left[1+\left|\alpha h_{e}\right|^{n}\right]^{-m}
$$

The hydraulic conductivity is then given by:

$$
K=\left\{\begin{array}{cc}
K_{\text {sat }}\left(S_{\mathrm{e}}\right)^{\lambda}\left[\frac{1-\left(1-\left(S_{\mathrm{e}} S_{\mathrm{c}}\right)^{1 / m}\right)^{m}}{1-\left(1-\left(S_{\mathrm{c}}\right)^{1 / m}\right)^{m}}\right]^{2} & S_{\mathrm{e}}<1 \\
K_{\text {sat }} & S_{\mathrm{e}} \geq 1
\end{array}\right.
$$

This model reduces to Eqs. [2.4] and [2.6] for $h_{\mathrm{e}}=0$. For a detailed discussion of above equations we refer to Vogel et al. (2001), Schaap and Van Genuchten (2006) and Ippisch et al. (2006). They showed that this modification affects the shape of the retention curve only minimally relative to the original function. However, the impacts on the unsaturated hydraulic conductivity of fine-textured soils can be large.

To avoid numerical instabilities of the solution scheme, the soil moisture retention curve in the range $0.01>h>1.05 h_{e} \mathrm{~cm}$ is approached by a cubic spline of which the parameters preserve the continuity of the soil moisture retention curve and the differential moisture capacity function. 
A second modification concerns the saturated hydraulic conductivity itself. The parameter $K_{\text {sat }}$ of the $K\left(S_{e}\right)$ relation is usually derived from experiments with unsaturated flow. These experiments may yield a poor estimate of the hydraulic conductivity at saturated conditions where soil structure usually dominates soil texture. However, to simulate accurately runoff conditions and drainage, a correct value of the saturated hydraulic conductivity is essential. Therefore SWAP users may specify in addition to the parameter $K_{\text {sat }}$ the experimentally determined value of the saturated hydraulic

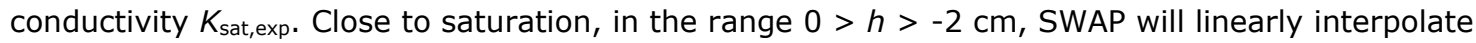
between $K_{\text {sat,exp }}$ and $K\left(S_{e}\right)$ at $h=-2 \mathrm{~cm}$.

\subsubsection{Soil hydraulic functions as tables}

The (modified) uni-modal Mualem - van Genuchten representation of the $\theta(h)$ and $K(\theta)$ or $K(h)$ relationships is just one of the many alternatives that are available in literature. Leij et al. (1997) listed 14 such relationships commonly used in soil physics, Leong and Rahardjo (1997) presented eight relationships often used in geotechnical science, and Kosugi et al. (2002) described the most widely used expressions, including those of Brooks and Corey $(1964,1966)$ and van Genuchten (1980). Currently in SWAP it has been decided not to implement many alternatives in the source code. Instead, the user is given the possibility to supply $\theta(h)$ and $K(h)$ relationships as tabulated input. In that way any relationship can be considered. During the computations interpolation is used to obtain $\theta$, $K, C(=\mathrm{d} \theta / \mathrm{d} h)$ and $\mathrm{d} K / \mathrm{d} h$ for any $h$. Interpolation is done as piecewise cubic Hermite interpolation, preserving the monotonic character of the relationships (using PCHIP routines from the public domain software library SLATEC, http://www.netlib.org/slatec/). Due to the interpolation method, the outcome does depend on the user-supplied information in the input tables. Best results are obtained when the $h-\theta-K$ tables contain many data points nicely distributed over all variables, with special attention to data close to saturation and data close to wilting point and beyond. For example, in case the user has data available measured in the laboratory it is advised to first describe these data by some kind of analytical expression, and then derive tables based on this analytical expression. Experience has shown that supplying a coarse (or irregular) grid of $h-\theta-K$ data points may cause unrealistic behaviour of the interpolated results, e.g. in the derivation of $C$.

\subsubsection{Hysteresis}

Hysteresis refers to non-uniqueness of the $\theta(h)$ relation and is caused by variations of the pore diameter (inkbottle effect), differences in radii of advancing and receding meniscus, entrapped air, thermal gradients and swelling/shrinking processes (Hillel, 1980; Feddes et al., 1988). Gradual desorption of an initially saturated soil sample gives the main drying curve, while slow absorption of an initially dry sample results in the main wetting curve. In the field partly wetting and drying occurs in numerous cycles, resulting in so-called drying and wetting scanning curves lying between the main drying and the main wetting curves (Figure 2.1).

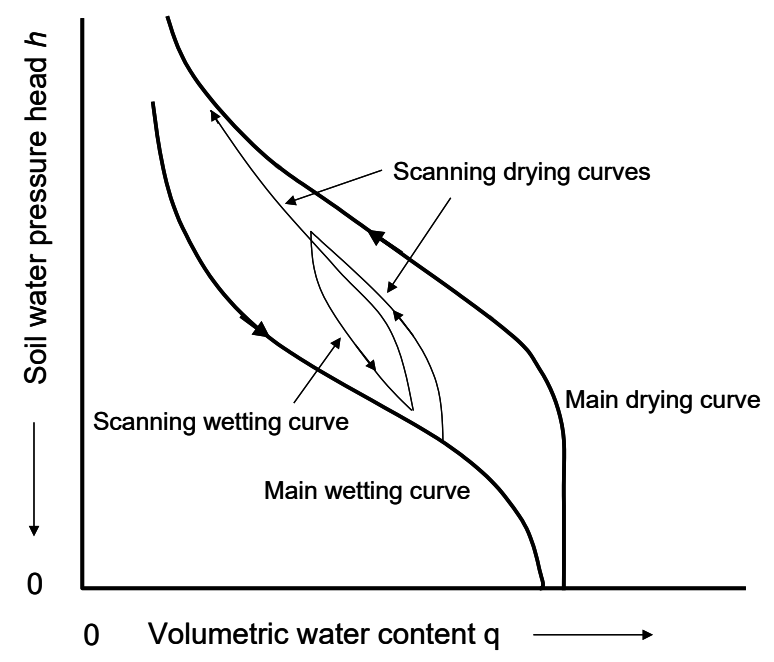

Figure 2.1 Water retention function with hysteresis, showing the main wetting, main drying and scanning curves. 
In simulation practice, often only the main drying curve is used to describe the $\theta(h)$ relation. This is mainly due to the time and costs involved in measurement of the complete $\theta(h)$ relationship, including the main wetting, the main drying and the scanning curves, especially in the dry range. For instance, a generally applied soil hydraulic data base in The Netherlands, known as the Staring series (Wösten et al., 1994, 2001), contains only $\theta(h)$ data of the main drying curve. Nevertheless, it is obvious that the simulation of infiltration events with the main drying curve can be inaccurate. Therefore the scaling method of Scott et al. (1983), who derived scanning curves by rescaling the main wetting or the main drying curve to the actual water content, has been implemented in SWAP.

The main drying and main wetting curve are described analytically with the Mualem-van Genuchten parameters $\left(\alpha, n, \theta_{\text {res }}, \theta_{\text {sat, }}, K_{\text {sat, }}\right.$ and $\left.\lambda\right)$. Some of the parameters describing the main wetting and main drying curve are related. We assume $\theta_{\text {res }}$ and $\theta_{\text {sat }}$ to be equal for both curves. Usually the $K(\theta)$ function shows only minor hysteresis effects, which can be achieved by choosing for the main wetting and main drying curve a common value for $n$. Ultimately the two curves only differ in the parameter $\alpha$, as depicted in Figure 2.2. The scanning curves are derived by linear scaling of either the main wetting or main drying curve, such that the scanning curve includes the current $\theta-h$ combination and approaches the main wetting curve in case of a wetting scanning curve and the main drying curve in case of a drying scanning curve.

The scaling principle in case of a drying scanning curve is depicted in Figure 2.2A. Based on its wetting and drying history, at a certain time and depth the soil shows an actual water content $\theta_{\text {act }}$ at the soil water pressure head $h_{\text {act. }}$. The valid drying scanning curve should pass through the point ( $\theta_{\text {act, }} h_{\text {act }}$ ), and approach the main drying curve at smaller water contents.
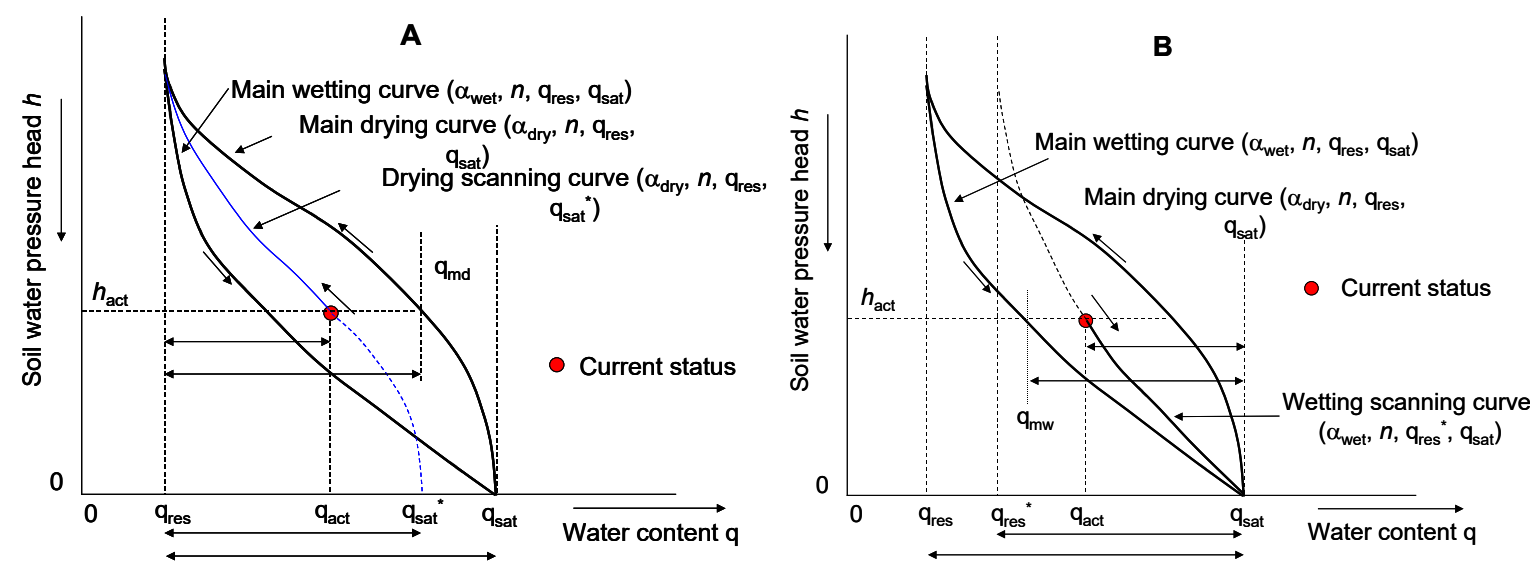

Figure 2.2 (A) Linear scaling of the main drying water retention curve in order to derive a drying scanning curve; $(B)$ Linear scaling of the main wetting water retention curve in order to derive a drying wetting curve.

We may define $\theta_{m d}$ as the water content of the main drying curve at $h_{\text {act }}$, and $\theta_{\text {sat }}{ }^{*}$ as the saturated water content of the drying scanning curve. Linear scaling of the main drying curve with respect to the vertical axis $\theta=\theta_{\text {res }}$ gives (Figure 2.2A):

$$
\frac{\theta_{\text {sat }}^{*}-\theta_{\text {res }}}{\theta_{\text {sat }}-\theta_{\text {res }}}=\frac{\theta_{\text {act }}-\theta_{\text {res }}}{\theta_{\text {md }}-\theta_{\text {res }}} \Rightarrow \theta_{\text {sat }}^{*}=\theta_{\text {res }}+\left(\theta_{\text {sat }}-\theta_{\text {res }}\right) \frac{\theta_{\text {act }}-\theta_{\text {res }}}{\theta_{\text {md }}-\theta_{\text {res }}}
$$

The only unknown in Eq. (2.12) is $\theta_{\text {sat }}{ }^{*}$, which can be solved directly. The drying scanning curve is described accordingly with the parameters $\left(\alpha_{\text {dry }}, n, \theta_{\text {res }}, \theta_{\text {sat }}{ }^{*}\right)$. As long as the soil keeps drying, this drying scanning curve is valid.

The opposite occurs when the soil gets wetter. Again we start from the arbitrary actual water content $\theta_{\text {act }}$ at the soil water pressure head $h_{\text {act}}$, and now define $\theta_{\mathrm{mw}}$ as the water content of the main wetting 
curve at $h_{\text {act, }}$ and $\theta_{\text {res }}{ }^{*}$ as the residual water content of the wetting scanning curve. Linear scaling of the main wetting curve with respect to the vertical axis $\theta=\theta_{\text {sat }}$ gives (Figure 2.2B):

$$
\frac{\theta_{\text {sat }}-\theta_{\text {res }}^{*}}{\theta_{\text {sat }}-\theta_{\text {res }}}=\frac{\theta_{\text {sat }}-\theta_{\text {act }}}{\theta_{\text {sat }}-\theta_{\text {mw }}} \Rightarrow \theta_{\text {res }}^{*}=\theta_{\text {sat }}-\left(\theta_{\text {sat }}-\theta_{\text {res }}\right) \frac{\theta_{\text {sat }}-\theta_{\text {act }}}{\theta_{\text {sat }}-\theta_{\text {mw }}}
$$

From Eq. (2.13), $\theta_{\text {res }}{ }^{*}$ can be directly solved. The wetting scanning curve is accordingly described with the parameters ( $\alpha_{\text {wet }}, n, \theta_{\text {res }}{ }^{*}, \theta_{\text {sat }}$ ), and is valid as long as the soil keeps wetting. As the wetting-drying history is different at each soil depth, each node may show a different scanning curve. The unique $K(\theta)$ relation of a soil layer always follows from the parameter set $\left(n, \theta_{\text {res }}, \theta_{\text {sat }}, K_{\text {sat }}, \lambda\right)$ according to Eq. (2.6).

\subsubsection{Frozen soil conditions}

The effect of frozen soil moisture on the hydraulic conductivity is described by:

$$
K^{*}=K_{\min }+\left(K-K_{\min }\right) \cdot \max \left(0, \min \left(1, \frac{T-T_{2}}{T_{1}-T_{2}}\right)\right)
$$

where $K^{*}$ is the adjusted hydraulic conductivity $\left(\mathrm{cm} \mathrm{d}^{-1}\right), T$ is the soil temperature $\left({ }^{\circ} \mathrm{C}\right), T_{1}$ and $T_{2}\left({ }^{\circ} \mathrm{C}\right)$ denote the temperature range over which the hydraulic conductivity linearly declines, and $K_{\min }$ is a minimum value of the hydraulic conductivity $\left(\mathrm{cm} \mathrm{d}^{-1}\right)$ which is valid for temperatures less than $T_{2}$.

\subsection{Lower boundary condition}

The bottom boundary of the one-dimensional SWAP is either in the unsaturated zone or in the upper part of the saturated zone where the transition takes place to three-dimensional groundwater flow. The lower boundary conditions in SWAP can be specified, depending on the application and the relevant spatial scale.

Three general types and some special cases of lower boundary conditions are distinguished:

1. The Dirichlet condition

The head controlled boundary is often referred as to the Dirichlet condition and involves the imposing of a pressure head $h_{\text {bot }}$ at the lower boundary. A special case involves the use of a recorded groundwater elevation. The pressure head at the groundwater elevation $\phi_{\text {avg }}$ is defined as $h=0$. This yields a linear relation between the pressure heads at the grid points above and below $\phi_{\text {avg: }}$

$$
h_{i+1}=-h_{i} \frac{\phi_{\mathrm{avg}}-z_{i+1}}{z_{i}-\phi_{\mathrm{avg}}}
$$

2. The Neumann condition

The flux boundary condition is often referred as to the Neumann condition and involves prescribing a flux $q_{\text {bot }}$ at the bottom. Since the model employs an explicit linearization scheme, the flux groundwater level relations are treated as a Neumann condition, where the actual flux is calculated from the groundwater level of the previous time step. The relation between flux and groundwater level can be obtained from regional groundwater flow models (e.g. Van Bakel, 1986). Some special options are available to define $q_{\text {bot }}$ :

- A zero bottom flux may be applied when an impermeable layer exists at the bottom of the profile.

- Impose a time series of $q_{\text {bot }}$

- Calculate $q_{\text {bot }}$ at the start of a time step as a function of the groundwater level $\phi_{\text {avg }}$ of the previous timestep, either by interpolation in a tabulated function or by using an exponential function, defined as:

$$
q_{\mathrm{bot}}=a_{\mathrm{bot}} \exp \left(b_{\mathrm{bot}}\left|\phi_{\mathrm{avg}}\right|\right)+c_{\mathrm{bot}}
$$

where $a_{\text {bot }}\left(\mathrm{cm} \mathrm{d}^{-1}\right), b_{\text {bot }}\left(\mathrm{cm}^{-1}\right)$ and $c_{\text {bot }}\left(\mathrm{cm} \mathrm{d}^{-1}\right)$ are empirical coefficients. 
- Calculate $q_{\text {bot }}$ at the start of a time step as a function of the groundwater level $\phi_{\text {avg }}$ of the previous timestep, the hydraulic head in a semi-confined aquifer $\phi_{\text {aquif }}(\mathrm{cm})$, and the resistance of the semi-confining layer $c_{1}(d)$, according to:

$$
q_{\text {bot }}=\frac{\phi_{\text {aquif }}-\phi_{\text {avg }}}{c_{1}+\sum_{\mathrm{i}=\mathrm{i}_{\mathrm{gwl}}}^{\mathrm{n}} \frac{\Delta \mathrm{z}_{\mathrm{i}}}{K_{\mathrm{sat}, \mathrm{i}}}}
$$

where the subscript $i_{\text {gwl }}$ points to the compartment number in which the groundwater level is located. The flow resistance in the saturated zone between the groundwater level and the lower boundary has been accounted for by summation of the flow resistances $\frac{\Delta z_{i}}{K_{\text {sat,i }}}$ in this zone. Different options for defining $\phi_{\text {aquif }}$ are available.

3. The Cauchy condition

In this case the flux $q$ at the lower boundary is defined as a function of the prevailing pressure head. This condition can be used when unsaturated flow models are combined with models for regional groundwater flow and when an implicit handling of $q_{\text {bot }}$ in the iterative computation scheme is required. The flux through the bottom boundary is defined by the difference of the total hydraulic head $(h+z)$ and the hydraulic head $\phi(\mathrm{cm})$ of the regional groundwater outside the flow domain described by the model, divided by a flow resistance $c(d)$.

$$
q_{\mathrm{bot}}=\frac{[h+z]_{z=\mathrm{bot}}-\phi}{c}
$$

4. Special cases

Two special cases involve the option to define a seepage face at the lower boundary and to define free drainage. The seepage face is meant to simulate moisture flow in a lysimeter and combines a head controlled and zero flux controlled condition in the following way:

$$
\begin{aligned}
& h_{z=\mathrm{bot}}<0 \quad \rightarrow \quad q_{\mathrm{bot}}=0 \\
& h_{z=\mathrm{bot}} \geq 0 \quad \rightarrow \quad q_{\mathrm{bot}}=-K_{\mathrm{n}}\left[\frac{\partial(h+z)}{\partial z}\right]_{z=\mathrm{bot}} \quad \operatorname{setting} h_{z=\mathrm{bot}}=0
\end{aligned}
$$

The free drainage option results from the assumption that the pressure head gradient equals zero, implying that $q_{\text {bot }}$ equals the hydraulic conductivity of the lowest compartment $n$ :

$$
\left[\frac{\partial(h+z)}{\partial z}\right]_{z=\mathrm{bot}}=1 \quad \rightarrow \quad q_{\mathrm{bot}}=-K_{\mathrm{n}}
$$

During frost conditions, $q_{\text {bot }}$ will be reduced according to:

$$
q_{\mathrm{bot}}=f_{\mathrm{T}}(z) q_{\mathrm{bot}}
$$

where the reduction factor $f_{\mathrm{T}}$ is based on Eq. (2.14). The bottom flux can be reduced even further in case of the presence of frozen layers (see Chapter 10).

\subsection{Numerical implementation}

Accurate numerical solution of the Richards partial differential equation is difficult due to its hyperbolic nature, the strong non-linearity of the soil hydraulic functions and the rapid changing boundary conditions near the soil surface. Calculated soil water fluxes can be significantly affected by the structure of the numerical scheme, the applied time and space discretizations, and the procedure for the top boundary condition (Van Genuchten, 1982; Milly, 1985; Celia et al., 1990; Warrick, 1991; Zaidel and Russo, 1992). The numerical scheme chosen in SWAP solves the one-dimensional Richards equation with an accurate mass balance and converges rapidly. This scheme in combination with the top boundary procedure has been shown to handle rapid soil water movement during infiltration in dry soils accurately. At the same time the scheme is computationally efficient (Van Dam and Feddes, 2000). 


\subsubsection{Richards equation}

The current numerical scheme of SWAP to solve Richards equation is the implicit, backward, finite difference scheme with explicit linearization of hydraulic conductivities as described by Haverkamp et al. (1977) and Belmans et al. (1983), but with the following adaptations:

- The numerical scheme applies to both the unsaturated and saturated zone and the flow equations are solved in both zones simultaneously;

- The water storage term $\frac{\partial \theta}{\partial t}$ is evaluated instead of using an approximation for the term $C \frac{\partial h}{\partial t}$, where $C$ is the water capacity $\left(\mathrm{cm}^{-1}\right)$;

- There are several options for calculating the internodal conductivity.

The implicit, backward, finite difference scheme of Eq. (2.3) with explicit linearization, yields the following discretization of Richards equation:

$$
\begin{aligned}
\frac{\theta_{i}^{j+1}-\theta_{i}^{j}}{\Delta t^{j}}= & \frac{1}{\Delta z_{i}}\left[K_{i-1 / 2}^{j+\kappa} \frac{h_{i-1}^{j+1}-h_{i}^{j+1}}{1 / 2\left(\Delta z_{i-1}+\Delta z_{i}\right)}+K_{i-1 / 2}^{j+\kappa}-K_{i+1 / 2}^{j+\kappa} \frac{h_{i}^{j+1}-h_{i+1}^{j+1}}{1 / 2\left(\Delta z_{i}+\Delta z_{i+1}\right)}-K_{i+1 / 2}^{j+\kappa}\right] \\
& -S_{a, i}^{j}-S_{d, i}^{j}-S_{m, i}^{j+1}
\end{aligned}
$$

where $\Delta t^{j}=t^{j+1}-t^{j}$ and $\Delta z_{i}$ is the compartment thickness. Handling of the water storage term is further elaborated in the next section. The sink terms representing the root extraction $S_{a}$ and the flow to drains $S_{d}$ are evaluated at the old time level $j$ (explicit linearization). The macro pore exchange rate $S_{m}$ is evaluated at the new time level $j+1$ and the internodal conductivity $K_{i-1 / 2}^{j+\kappa}$ can be evaluated at the old time level $j(\kappa=0)$ or at the new time level $j+1(\kappa=1)$. The internodal conductivity $K_{i-1 / 2}^{j+\kappa}$ can be calculated as:

Arithmic mean:

$$
K_{i-1 / 2}^{j+\kappa}=1 / 2\left(K_{i-1}^{j+\kappa}+K_{i}^{j+\kappa}\right)
$$

Weighted arithmic mean:

$$
K_{i-1 / 2}^{j+\kappa}=\frac{\Delta z_{i-1} K_{i-1}^{j+\kappa}+\Delta z_{i} K_{i}^{j+\kappa}}{\Delta z_{i-1}+\Delta z_{i}}
$$

Geometric mean:

$$
K_{i-1 / 2}^{j+\kappa}=\left(K_{i-1}^{j+\kappa}\right)^{1 / 2}\left(K_{i}^{j+\kappa}\right)^{1 / 2}
$$

Weighted geometric mean:

$$
K_{i-1 / 2}^{j+\kappa}=\left(K_{i-1}^{j+\kappa}\right)^{\frac{\Delta z_{i-1}}{\Delta z_{i-1}+\Delta z_{i}}}\left(K_{i}^{j+\kappa}\right)^{\frac{\Delta z_{i}}{\Delta z_{i-1}+\Delta z_{i}}}
$$

Haverkamp and Vauclin (1979), Belmans et al. (1983) and Hornung and Messing (1983) proposed to use the geometric mean. In their simulations the geometric mean increased the accuracy of calculated fluxes and caused the fluxes to be less sensitive to changes in nodal distance. However, the geometric mean has serious disadvantages too (Warrick, 1991). When simulating infiltration in dry soils or high evaporation from wet soils, the geometric mean severely underestimates the water fluxes. Other researchers proposed to use the harmonic mean of $K$ or various kind of weighted averages (Ross, 1990; Warrick, 1991; Zaidel and Russo, 1992; Desbarats, 1995). Van Dam and Feddes (2000) showed that, although arithmetic averages at larger nodal distances overestimate the soil water fluxes in case of infiltration and evaporation events, at nodal distances in the order of $1 \mathrm{~cm}$ non-weighted arithmetic averages are more close to the theoretically correct solution than geometric averages. Also they show that the remaining inaccuracy between calculated and theoretically correct fluxes, is relatively small compared to expected effects of soil spatial variability and hysteresis. Therefore the SWAP development team has a preference for applying weighted arithmetic averages of $K$, which is in line with commonly applied finite element models (Kool and Van Genuchten, 1991; Šimůnek et al., 1992). Therefore default choices are weighted arithmetic mean and no update of hydraulic conductivity.

Starting in the saturated zone, the groundwater table is simply found at $h=0$. Also perched water tables may occur above dense layers in the soil profile. Since SWAP is designed to describe a wide range of layered soil profiles combined with different types of boundary conditions, the nodal distance is made variable and should be specified by the user. 


\subsubsection{Numerical solution}

The discrete form of the Richards equation is solved iteratively using the pressure heads as state variables. Taylor-expansion of the new moisture fraction at a new iteration level with respect to the moisture fraction at the preceding iteration step is defined by:

$$
\theta_{i}^{j+1, p+1}\left(h_{i}^{j+1}\right) \approx \theta_{i}^{j+1, p}\left(h_{i}^{j+1, p}\right)+\left(h_{i}^{j+1, p+1}-h_{i}^{j+1, p}\right) \frac{\partial \theta_{i}^{j+1, p}}{\partial h_{i}^{j+1, p}}+\cdots+\cdots
$$

Ignoring the second and higher order terms of the Taylor series yields an expression which can substitute the moisture fraction variable at the new time-level. The first order derivate of the moisture fraction to the pressure head is identical to the water capacity $C^{j+1, p}$. In fact the numerical method proposed by Celia et al. (1990) complies with the assumptions made in the Newton-Raphson iteration procedure. An improvement to this method is made by defining $F_{i}$ based on the closure term of the water balance as a function of $h_{i}^{j+1}$ :

$$
\begin{aligned}
F_{i} & =\frac{\Delta z_{i}}{\Delta t^{j}}\left(\theta_{i}^{j+1, p}-\theta_{i}^{j}\right)-K_{i-1 / 2}^{j+\kappa, \kappa p} \frac{h_{i-1}^{j+1, p}-h_{i}^{j+1, p}}{1 / 2\left(\Delta z_{i-1}+\Delta z_{i}\right)}-K_{i-1 / 2}^{j+\kappa, \kappa p} \\
& +K_{i+1 / 2}^{j+\kappa, \kappa p} \frac{h_{i}^{j+1, p}-h_{i+1}^{j+1, p}}{1 / 2\left(\Delta z_{i}+\Delta z_{i+1}\right)}+K_{i+1 / 2}^{j+\kappa, \kappa p}+\Delta z_{i} S_{a, i}^{j+\kappa, p}+\Delta z_{i} S_{d, i}^{j}+\Delta z_{i} S_{m, i}^{j+1, p}
\end{aligned}
$$

where the superscript $p+1$ points to the solution of iteration round $p$. This discrete form of the Richards equation allows for a straightforward evaluation of the storage term and is flexible with the respect to adding of $h_{i}^{j+1}$-dependent source and sink-terms. Solving the set of non-linear equations numerically implies root finding of the function $F_{i} \approx 0$ for $i=1 . . n$, with $n$ the number of compartments. The Newton Raphson-iteration scheme for the set of $n$ equations is written as follows:

$$
\left(\begin{array}{c}
h_{1}^{j+1, p+1} \\
\vdots \\
h_{i}^{j+1, p+1} \\
\vdots \\
h_{n}^{j+1, p+1}
\end{array}\right)=\left(\begin{array}{c}
h_{1}^{j+1, p} \\
\vdots \\
h_{i}^{j+1, p} \\
\vdots \\
h_{n}^{j+1, p}
\end{array}\right)-\left(\begin{array}{ccccc}
\frac{\partial F_{1}}{\partial h_{1}^{j+1, p}} & \frac{\partial F_{1}}{\partial h_{2}^{j+1, p}} & 0 & 0 & 0 \\
\frac{\partial F_{2}}{\partial h_{1}^{j+1, p}} & \frac{\partial F_{2}}{\partial h_{2}^{j+1, p}} & \ldots & 0 & 0 \\
0 & \frac{\partial F_{i}}{\partial h_{i-1}^{j+1, p}} & \frac{\partial F_{i}}{\partial h_{i}^{j+1, p}} & \frac{\partial F_{i}}{\partial h_{i+1}^{j+1, p}} & 0 \\
0 & 0 & \ldots & \frac{\partial F_{n-1}}{\partial h_{n-1}^{j+1, p}} & \frac{\partial F_{n-1}}{\partial h_{n}^{j+1, p}} \\
0 & 0 & 0 & \frac{\partial F_{n}}{\partial h_{n-1}^{j+1, p}} & \frac{\partial F_{n}}{\partial h_{n}^{j+1, p}}
\end{array}\right)^{-1}\left(\begin{array}{c}
F_{1} \\
\vdots \\
F_{i} \\
\vdots \\
F_{n}
\end{array}\right)
$$

The starting values are the results of the previous iteration round, indicated by the superscript $p$. The solution of the second part of the right hand side is found by solving efficiently a tri-diagonal system of equations (Press et al., 1989). The coefficients of the Jacobian are listed in Appendix 4. The

contribution of the partial derivative of the macro-pore exchange to the pressure head $\left(\frac{\partial S_{m, i}^{j+1, p}}{\partial h_{i}^{j+1, p}}\right)$ is discussed in Chapter 6. If the option to treat the hydraulic conductivities implicitly is used $(k=1)$, the contribution of the partial derivates of the internodal conductivity relation to the pressure head should also accounted. Expressions for these terms are given in Appendix 5.

Newton's method for solving nonlinear equations might wander off into the wild blue yonder if the initial guess is not sufficiently close to the root. 


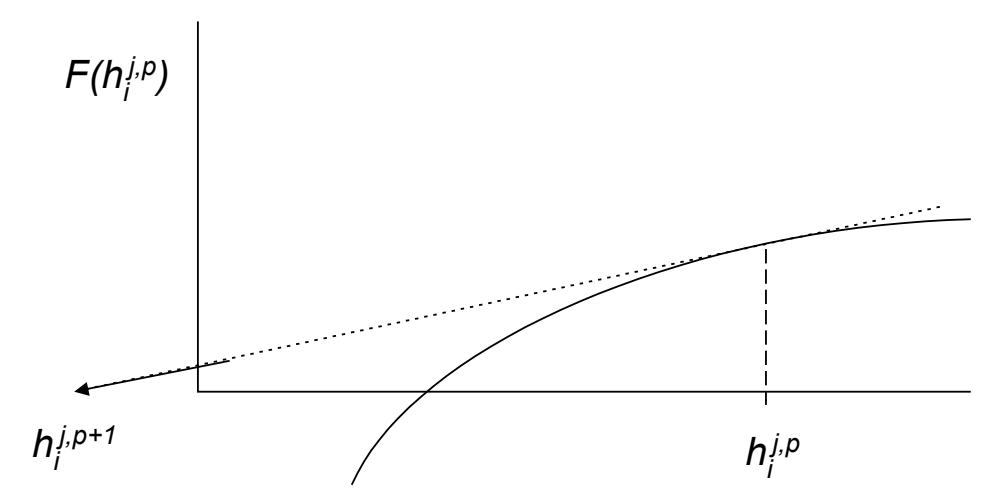

Figure 2.3 Influence of initial estimate on an intermediate solution of a first order approximation based root finding procedure.

The solution to the second part of the right hand side of Eq. (2.29) is referred to as the Newton-step $\Delta h_{i}^{j+1, p}$ :

$$
\left(\begin{array}{c}
h_{1}^{j+1, p+1} \\
\vdots \\
h_{i}^{j+1, p+1} \\
\vdots \\
h_{n}^{j+1, p+1}
\end{array}\right)=\left(\begin{array}{c}
h_{1}^{j+1, p} \\
\vdots \\
h_{i}^{j+1, p} \\
\vdots \\
h_{n}^{j+1, p}
\end{array}\right)+\lambda\left(\begin{array}{c}
\Delta h_{1}^{j+1, p} \\
\vdots \\
\Delta h_{i}^{j+1, p} \\
\vdots \\
\Delta h_{n}^{j+1, p}
\end{array}\right)
$$

We always first try the full Newton step and we check at each iteration that the proposed step reduces $1 / 2 \sum_{i=1}^{n} F_{i}^{2}$. If not, we backtrack along the Newton direction until we have an acceptable step. The aim is to find $\lambda$ which results in a decrease of $1 / 2 \sum_{i=1}^{n}\left[F_{i}\left(h_{i}^{j+1, p}+\lambda \Delta h_{i}^{j+1, p}\right)\right]^{2}$. The first estimate of $\lambda$ amounts to 1 . If it is decided that a second estimate is needed, $\lambda$ is set to $1 / 3$. The third estimate amounts to $1 / 9$. Thereafter, no further reduction of $\lambda$ is applied but a new Newton-iteration step is performed.

In SWAP the main convergence criterium in the unsaturated zone is based on the water closure term of the water balance $F$. If $\left|F_{i}\right|$ is less than a user defined criterion for all compartments, it is decided that the iteration cycle has resulted into a sufficiently accurate solution.

Numerical implementation of the top and bottom boundary conditions is described in Appendix 17. 


\subsection{User instructions}

\subsubsection{General}

Box 2.1 shows the general input of soil water flow. The initial soil moisture condition (Part $\mathbf{1}$ ) is defined by the soil water pressure head. Initial values can by specified as function of soil depth with linear interpolation between depths or can be calculated assuming hydrostatic equilibrium with a groundwater level. A third option is to use the output of an earlier SWAP simulation. This option is very useful when no data are available of the initial soil moisture condition.

Part 4 describes the vertical discretization of the soil profile. In addition to the natural soil layers with different hydraulic functions, the thicknesses of the calculation compartments should be defined. For correct simulation of infiltration and evaporation fluxes near the soil surface, the compartment thickness near the soil surface should be $\leq 1 \mathrm{~cm}$. Deeper in the soil profile, where the soil water flow is less dynamic, the compartment thicknesses may increase (to 10 or more $\mathrm{cm}$ ). In Part $\mathbf{5}$ the hydraulic parameters of each distinct soil layer are defined, which describe the water retention and hydraulic conductivity functions. The use of the air-entry-value concept $h_{\mathrm{e}}$ requires special care. Even a small value of $\left|h_{e}\right|$ can cause a large change in $K(\theta)$ of fine-textured soils (see Section 2.2.2). In fact, the use of the air-entry-value concept requires the re-fitting of the other parameters of the classical Mualem-Van Genuchten model on the original experimental data. In case the soil hydraulic functions are provided as tables, for each soil layer a separate file should be provided. Each file should provide the following column-oriented data: headtab (for $h$ ), thetatab (for $\theta$ ) and conductab (for $K$ ). Due to the interpolation method, the outcome does depend on the user-supplied information in the input tables. Best results are obtained when the $h-\theta-K$ tables contain many data points nicely distributed over all variables (e.g., based on equal log-transformed $h$ interval (e.g., 0.1), or a dense grid of effective degree of saturation), with special attention to data close to saturation and data close to wilting point and beyond (see example in Table 2.2). For example, in case the user has data available measured in the laboratory it is advised to first describe these data by some kind of analytical expression, and then derive tables based on this analytical expression. Experience has shown that supplying a coarse (or irregular) grid of $h-\theta-K$ data points may cause unrealistic behaviour of the interpolated results, e.g. in the derivation of $C$.

The piecewise cubic Hermite interpolation requires information regarding the behaviour at the endpoints of the tabulated data. Currently, this is internally defined as provided in Table 2.1. The current pre-defined behaviour at the end-points is in agreement with the analytical behaviour of the Mualemvan Genuchten relationships. In case other relationships are used, this behaviour might be in conflict with these pre-defined characteristics. In a future release this might be solved by giving the user the opportunity to supply endpoint behaviour as input.

Table 2.1 Currently the behaviour of the interpolation technique at the end-points of the tabulated water retention and tabulated hydraulic conductivity relationships are fixed according to the first or second derivatives as provided here.

\begin{tabular}{|c|c|c|c|}
\hline End point in $h$ & Retention & Conductivity & Comment \\
\hline$h \rightarrow-\infty$ & $\mathrm{d}^{2} \theta / \mathrm{d} h^{2}=0$ & $\mathrm{~d}^{2} K / \mathrm{d} h^{2}=0$ & $\begin{array}{l}\text { Thus data for a very large negative value should be } \\
\text { provided }\end{array}$ \\
\hline
\end{tabular}


Table 2.2 Example of tabulated soil physical input data for a soil layer. In this case headtab, thetatab and conductab were constructed from the Mualem-van Genuchten relationship for effective degrees in satuarion of $S_{e}=0.001,0.01,0.02,0.03, \ldots, 0.99,0.991,0.992, \ldots, 0.999,0.9991$, $0.9992, \ldots, 0.9999,1$.

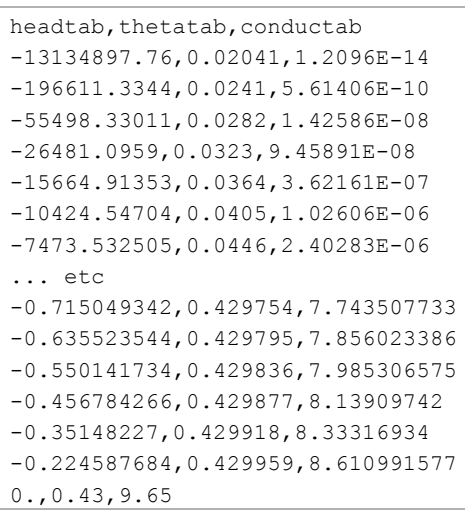

In Part $\mathbf{6}$ the inclusion of hysteresis in the water retention function can be selected. In case of hysteresis, the parameter ALFAW of the wetting curve (Part 5) should be properly defined. Whether the initial condition is wetting or drying, may have a large impact on the water balance. In general the simulations are not sensitive to the minimum head difference to change from wetting to drying scanning curves and vice versa (TAU). The parameter TAU is usually set equal to $0.2 \mathrm{~cm}$.

In Part 10 various parameters are defined that may affect the numerical solution of the Richards equation. In general, the default values will garantuee an accurate numerical solution of the Richards equation for common soil profiles. In extreme hydrologic or textural cases adjustment of the default values might be required. The user should specify a minimum and a maximum time step, $\Delta t_{\min }$ and $\Delta t_{\max }(\mathrm{d})$. SWAP will determine the optimal time step which minimizes the computational effort of a simulation while the numerical solution still meets the convergence criteria. For this purpose, SWAP employes the number of iterations needed to reach convergence, $N_{\text {it, }}$ in the following way (Kool and Van Genuchten, 1991):

- $N_{\text {it }}<3 \quad$ : multiply time step with a factor 2

- $3 \leq N_{\text {it }} \leq N_{\max }$ : keep time step the same

- $N_{\text {it }}>N_{\max } \quad$ : divide time step by a factor 2

where $N_{\max }$ is the defined maximum number of iterations (default 30 ). Also the maximum number of back-track cycles should be specified by the user. A common value for this maximum is 3 .

Routinely, SWAP uses 4 convergence criteria:

- the water balance error of each soil compartment: should be less than $10^{-6} \mathrm{~cm}$

- between iterations the relative difference in pressure head (-) per compartment should be less than a user specified criterion (CritDevH1CP, e.g. $10^{-2}$ )

- between iterations the absolute difference in pressure head $(\mathrm{cm})$ per compartment should be less than a user specified criterion (CritDevH2CP, e.g. $10^{-1} \mathrm{~cm}$ )

- the water balance error of a possible ponding layer should be less than a user specified criterion (CritDevPondDt, e.g. $10^{-4} \mathrm{~cm}$ )

In addition, SWAP will give a warning when groundwater levels between time steps fluctuate more than a user specified criterion (GWLCONV).

For the initial time step, SWAP will take $\Delta t=\sqrt{\Delta t_{\min } \Delta t_{\max }}$. Depending on $N_{\text {it, }}$ the time step will be decreased, maintained or increased for the following time steps as described above. The time step is always confined to the range $\Delta t_{\min } \leq \Delta t \leq \Delta t_{\max }$. When the actual time step during simulation reaches its minimum value ( $\Delta t=\Delta t_{\min }$ ), the maximum number of iterations is expanded to $2 * N_{\max }$. If still the numerical solution shows no convergence, SWAP prints a warning in the log file, and continues the simulation at the next time step with the pressure heads of the last iteration. 
In Part 10 also a choice can be made with respect to spatial averaging of hydraulic conductivity and updating hydraulic conductivity in the numerical solution. Default choices are weighted arithmetic mean (SWKMEAN $=2$ ) and no update of hydraulic conductivity (SWKIMPL $=0$ ) (see also paragraph 2.4.1).

\section{Box 2.1 Information on soil water flow in main file *. swp}

* Part 1: Initial soil moisture condition

SWINCO = 2 ! Switch, type of initial soil moisture condition:

$! 1$ = pressure head as function of soil depth

! 2 = pressure head of each compartment is in hydrostatic equilibrium with initial groundwater level

! 3 = read final pressure heads from output file of previous Swap simulation

* If SWINCO $=1$, specify soil depth ZI $[-1 . d 5.0 \mathrm{~cm}, \mathrm{R}]$ and initial

* soil water pressure head H $[-1 . \mathrm{d} 10 \ldots 1 . \mathrm{d} 4 \mathrm{~cm}, \mathrm{R}]$ (maximum MACP):

$$
\begin{array}{rr}
\text { ZI } & \text { H } \\
-0.5 & -93.0 \\
-195.0 & 120.0
\end{array}
$$

* End of table

* If SWINCO $=2$, specify initial groundwater level:

GWLI $=-75.0$ ! Initial groundwater level $[-10000 \ldots 1000 \mathrm{~cm}, \mathrm{R}]$

* If SWINCO $=3$, specify output file with initial values for current run:

INIFIL $=$ 'result.end' ! name of output file *.END which contains initial values [A200]

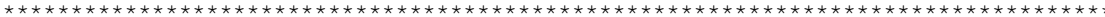

* Part 4: Vertical discretization of soil profile

* Specify the following data (maximum MACP lines):

* ISUBLAY = number of sub layer, start with 1 at soil surface $[1 \ldots$ MACP, I]

* ISOILLAY $=$ number of soil physical layer, start with 1 at soil surface [1..MAHO, I]

* HSUBLAY = height of sub layer $[0 \ldots 1 . \mathrm{d} 4 \mathrm{~cm}, \mathrm{R}]$

* HCOMP = height of compartments in the sub layer $[0.0 \ldots 1000.0 \mathrm{~cm}, \mathrm{R}]$

$\star \operatorname{NCOMP}=$ number of compartments in the sub layer (Mind NCOMP $=$ HSUBLAY/HCOMP) $[1 \ldots \mathrm{MACP}, \mathrm{I}]$

$\begin{array}{ccccr}\text { ISUBLAY } & \text { ISOILLAY } & \text { HSUBLAY } & \text { HCOMP } & \text { NCOMP } \\ 1 & 1 & 10.0 & 1.0 & 10 \\ 2 & 1 & 20.0 & 5.0 & 4 \\ 3 & 2 & 30.0 & 5.0 & 6 \\ 4 & 2 & 140.0 & 10.0 & 14\end{array}$

* end of table

* Part 5: Soil hydraulic functions

* Switch for analytical functions or tabular input:

SWSOPHY $=0 \quad ! \quad 0=$ Analytical functions with input of Mualem - van Genuchten parameters $! 1$ = Soil physical tables

* If SWSOPHY $=0$, specify MvG parameters for each soil physical layer (maximum MAHO):

* ISOILLAY1 = number of soil physical layer, as defined in part 4 [1..MAHO, I]

$\star$ ORES = Residual water content $[0.1 \mathrm{~cm} 3 / \mathrm{cm} 3, \mathrm{R}]$

* OSAT = Saturated water content $[0 \ldots 1 \mathrm{~cm} 3 / \mathrm{cm} 3, \mathrm{R}]$

* ALFA = Parameter alfa of main drying curve $[0.0001 \ldots 100 / \mathrm{cm}, \mathrm{R}]$

* NPAR = Parameter $\mathrm{n}[1.001 \ldots 9-, \mathrm{R}]$

* KSATFIT = Fitting parameter Ksat of hydraulic conductivity function [1.d-5..1d5 cm/d, R]

* LEXP = Exponent in hydraulic conductivity function $[-25 \ldots 25-$, R]

* ALFAW = Alfa parameter of main wetting curve in case of hysteresis $[0.0001 \ldots 100 / \mathrm{cm}, \mathrm{R}]$

* $\mathrm{H}$ ENPR = Air entry pressure head $[-40.0 \ldots 0.0 \mathrm{~cm}, \mathrm{R}]$

* $\overline{\text { KSATEXM }}=$ Measured hydraulic conductivity at saturated conditions $[1 . \mathrm{d}-5 \ldots 1 \mathrm{~d} 5 \mathrm{~cm} / \mathrm{d}, \mathrm{R}]$

\begin{tabular}{|c|c|c|c|c|c|c|c|c|c|c|}
\hline ISOILLAY1 & ORES & OSAT & ALFA & NPAR & KSATFIT & LEXP & ALFAW & H_ENPR & KSATEXM & BDENS \\
\hline 1 & 0.01 & 0.43 & 0.0227 & 1.548 & 9.65 & -0.983 & 0.0454 & $\begin{array}{ll}4 & 0.0\end{array}$ & 9.65 & 1315.0 \\
\hline 2 & 0.02 & 0.38 & 0.0214 & 2.075 & 15.56 & 0.039 & 0.0428 & 0.0 & 15.56 & 1315.0 \\
\hline
\end{tabular}

* BDENS = Dry soil bulk density $[100 \ldots 1 \mathrm{~d} 4 \mathrm{mg} / \mathrm{cm} 3, \mathrm{R}]$

* --- end of table

* If SWSOPHY $=1$, specify names of input files [A80] with soil hydraulic tables for each soil layer: FILENAMESOPHY = 'topsoil_sand_B2.cSV', 'subsoil_sand O2.cSV' 
* Part 6: Hysteresis of soil water retention function

* Switch for hysteresis:

SWHYST $=0 \quad ! \quad 0=$ no hysteresis

! 1 = hysteresis, initial condition wetting

! 2 hysteresis, initial condition drying

* If SWHYST $=1$ or 2 , specify:

TAU $=0.2$ ! Min. pressure head difference to change from wetting to drying and vice versa, [0..1 cm, R]

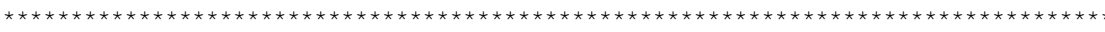

* Part 7: Maximum rooting depth

RDS $=200.0$ ! Maximum rooting depth allowed by the soil profile $[1.5000 \mathrm{~cm}, \mathrm{R}]$ $* * * * * * * * * * * * * * * * * * * * * * * * * * * * * * * * * * * * * * * * * * * * * * * * * * * * * * * * * * * * * * * * * * * * * * * * * * * * * * * * * *$

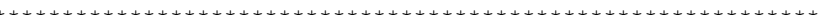

* Part 8: Preferential flow due to macropores

SWMACRO $=0 \quad$ ! Switch for macropore flow $[0 . .2$, I $]$ :

! 0 = no macropore flow

! 1 = macropore flow

* Part 9: Snow and frost

* Snow

SWSNOW $=0 \quad$ ! Switch, calculate snow accumulation and melt $[Y=1, N=0]$

* If SWSNOW $=1$, specify:

SNOWINCO $=22.0 \quad$ ! Initial snow water equivalent $[0.1000 \mathrm{~cm}, \mathrm{R}]$

TEPRRAIN $=2.0 \quad$ ! Temperature above which all precipitation is rain $\left[0 \ldots 10{ }^{\circ} \mathrm{C}, \mathrm{R}\right]$

TEPRSNOW $=-2.0 \quad$ ! Temperature below which all precipitation is snow $\left[-10 \ldots 0^{\circ} \mathrm{C}, \mathrm{R}\right]$

SNOWCOEF $=0.3 \quad$ ! Snowmelt calibration factor $[0.0 \ldots 10.0-, \mathrm{R}]$

* Frost

SWFROST $=0$ ! Switch, in case of frost reduce soil water flow [Y=1, N=0]

* If SWFROST $=1$, specify soil temperature range in which soil water flow is reduced

TFROSTSTA $=0.0$ ! Soil temperature $\left({ }^{\circ} \mathrm{C}\right)$ at which reduction of water fluxes starts $\left[-10.5{ }^{\circ} \mathrm{C}, \mathrm{R}\right]$

TFROSTEND $=-1.0$ ! Soil temperature $\left({ }^{\circ} \mathrm{C}\right)$ at which reduction of water fluxes ends $\left[-10.5{ }^{\circ} \mathrm{C}, \mathrm{R}\right]$

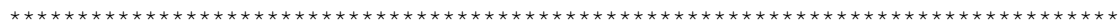

* Part 10 Numerical solution of Richards' equation for soil water flow

DTMIN $=1.0 \mathrm{~d}-6 \quad$ ! Minimum timestep $[1 . \mathrm{d}-7 \ldots 0.1 \mathrm{~d}, \mathrm{R}]$

DTMAX $=0.2 \quad$ ! Maximum timestep [dtmin..1 d, R]

GWLCONV $=100.0 \quad$ ! Max. difference of groundwater level between iterations $[1 . \mathrm{d}-5 \ldots 1000 \mathrm{~cm}, \mathrm{R}]$

CRITDEVH1CP $=1.0 \mathrm{~d}-2 \quad !$ Max. rel. difference in pressure heads per compartment [1.0d-10..1.d3 -, R]

CRITDEVH2CP $=1.0 \mathrm{~d}-1 \quad$ ! Max. abs. difference in pressure heads per compartment $[1.0 \mathrm{~d}-10.1 . \mathrm{d} 3 \mathrm{~cm}, \mathrm{R}]$

CRITDEVPONDDT $=1.0 \mathrm{~d}-4 \quad$ ! Maximum water balance error of ponding layer $[1.0 \mathrm{~d}-6.0 .1 \mathrm{~cm}, \mathrm{R}]$

MAXIT $=30 \quad$ ! Maximum number of iteration cycles [5..100 -, I]

MAXBACKTR $=3$ ! Maximum number of back track cycles within an iteration cycle [1..10 -, I]

* Switch for averaging method of hydraulic conductivity [1..4 -, I]:

SWKMEAN = $1 \quad ! 1$ = unweighted arithmic mean

! 2 = weighted arithmic mean

! 3 = unweighted geometric mean

! 4 = weighted geometric mean

* Switch for updating hydraulic conductivity during iteration [0..1 -, I]

SWKIMPL $=0 \quad ! \quad 0=$ no update

! 1 = update

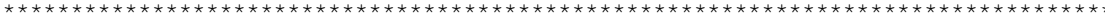




\subsubsection{Bottom boundary conditions}

SWAP offers various options to prescribe the lower boundary condition, each having their typical scale of application (Table 2.3, Box 2.2).

Table 2.3 Options for the lower boundary condition.

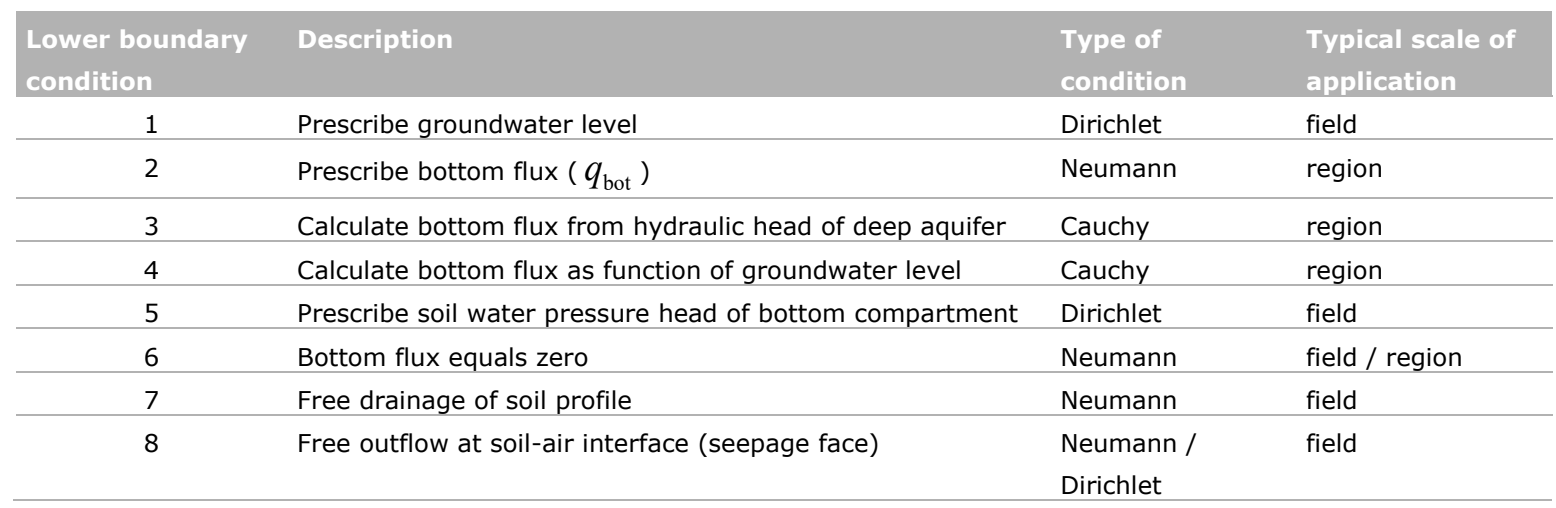

In case of options $1,2,3,5$ and 6 , in addition to the flux across the bottom of the modelled soil profile $\left(q_{\text {bot }}\right)$, a drainage flux $\left(q_{\text {drain }}\right)$ can be defined (Chapter 4$)$. In case of option 4 , the lower boundary includes drainage to local ditches or drains so $q_{\text {drain }}$ should not be defined separately. In case of options 7 and 8 , the simulated soil profile is unsaturated, so lateral drainage will not occur. We will discuss the 8 available bottom boundary conditions sequentially.

\section{Prescribed groundwater levels}

In this case a field-averaged groundwater level ( $\phi_{\text {avg }}$ ) is given as a function of time (Box 2.2). SWAP will linearly interpolate between the dates and times at which the groundwater levels are specified. SWAP will read times according to the following format: 05-jan-2005_14:30:00.00 denotes January 5, 2005 at 2.30 PM. If only dates and no times are specified (as in Box 2.2), SWAP will assume time $0: 00$.

The main advantage of this boundary condition is the easy recording of the phreatic surface in case of a present groundwater table. A drawback is that at shallow groundwater tables the simulated phreatic surface fluctuations are very sensitive to the soil hydraulic functions and the top boundary condition. If the top and bottom boundary condition not match accurately, or the soil hydraulic functions deviate from reality, strong fluctuations of simulated water fluxes across the lower boundary may result. Especially when the output of SWAP is used as input in water quality calculations, it is recommended to use another type of lower boundary condition. The option of prescribed groundwater levels is disabled for macropore flow simulations.

Experience has shown that SWAP cannot correctly handle this boundary condition in case the (interpolated) prescribed groundwater level is inside frozen soil. An alternative boundary condition to achieve approximately the desired groundwater levels is to impose pressure heads at the bottom of the simulated soil profile which are in hydrostatic equilibrium with the desired phreatic surface (Option 5).

\section{Prescribed bottom flux}

In this case the bottom flux $\left(q_{\mathrm{bot}}\right)$ might be given as function of time with linear interpolation between the data pairs, or as a sine function (Box 2.2). This option has a similar disadvantage as the previously described option with the prescribed groundwater level at the field scale. When a mismatch occurs between boundary conditions (e.g. drainage + leakage to deep aquifer exceeds net precipitation excess) the result may be a continuously declining or increasing groundwater level. In particular in cases where the output of SWAP is used as input in water quality calculations, it is recommended to use another type of lower boundary condition. 
3. Calculate the bottom flux from the hydraulic head of a deep aquifer

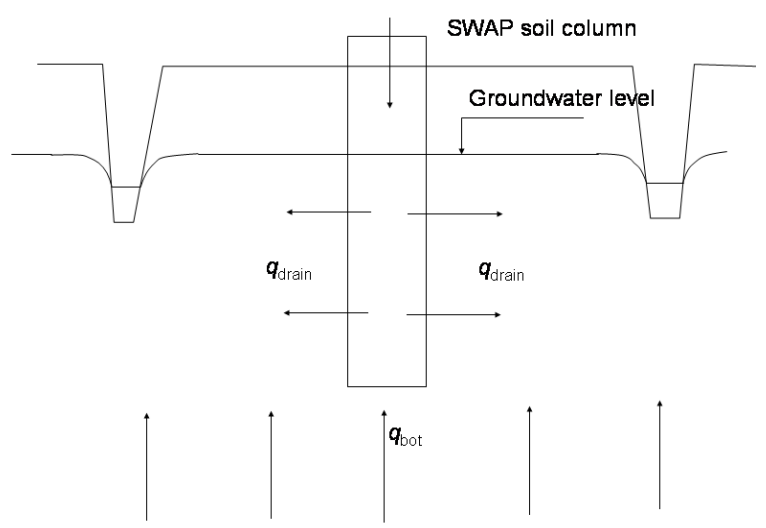

Figure 2.4 Pseudo two-dimensional Cauchy lower boundary conditions, in case of drainage to ditches and seepage from a deep aquifer.

Figure 2.4 shows a soil profile which is drained by ditches and which receives a seepage flux from a semi-confined aquifer. SWAP makes a distinction between $q_{\text {drain, }}$ the local drainage flux to ditches and drains (see Chapter 4), and $q_{\text {bot, }}$ the bottom flux due to regional groundwater flow.

The bottom flux $q_{\text {bot }}$ depends on the average groundwater level $\phi_{\text {avg }}(\mathrm{cm})$, the hydraulic head in the semi-confined aquifer $\phi_{\text {aquif }}(\mathrm{cm})$, and the resistance of the semi-confining layer $c_{1}(\mathrm{~d})$ :

$$
q_{\mathrm{bot}}=\frac{\phi_{\mathrm{aquif}}-\phi_{\mathrm{avg}}}{c_{1}+\sum_{i=i_{\mathrm{gwl}}}^{n} \frac{\Delta z_{i}}{K_{\mathrm{sat}, i}}}
$$

where the subscript $i_{\text {gwl }}$ points to the compartment number in which the groundwater level is located. The vertical resistance between the bottom of the model and the groundwater level may be taken into account by adding it to the aquitard resistance $c_{1}$. The hydraulic head $\phi_{\text {aquif }}$ is prescribed using a sinusoidal wave:

$$
\phi_{\text {aquif }}=\phi_{\text {aquif,m }}+\phi_{\text {aquif,a }} \cos \left(\frac{2 \pi}{\phi_{\text {aquif,p }}}\left(t-t_{\text {max }}\right)\right)
$$

where $\phi_{\text {aquif,m }}, \phi_{\text {aquif,a }}$, and $\phi_{\text {aquif,p }}$ are the mean $(\mathrm{cm})$, amplitude $(\mathrm{cm})$ and period (d) of the hydraulic head sinus wave in the semi-confined aquifer, and $t_{\max }$ is the time (d) at which $\phi_{\text {aquif }}$ reaches its maximum.

SWAP includes the option for the implicit treatment of pressure head in lowest compartment by substitution of $\phi_{\text {avg }}$ by $h_{n}^{j+1}+z_{n}$ and considering the vertical resistance within the model domain only between the lowest node and the lower boundary. Another option involves the possibility to specify a groundwater flux additional to $q_{\text {bot }}$ to facilitate the coupling of the SWAP model to a regional groundwater model.

4. Calculate bottom flux as a function of groundwater level The relation between $q_{\text {bot }}$ and $\phi_{\text {avg }}$ can be given as an exponential relation or as a table (Box 2.2). The exponential relationship is formulated as:

$$
q_{\mathrm{bot}}=a_{\mathrm{qbot}} \exp \left(b_{\mathrm{qbot}}\left|\phi_{\mathrm{avg}}\right|\right)+c_{\mathrm{qbot}}
$$

where $a_{\mathrm{qbot}}\left(\mathrm{cm} \mathrm{d}^{-1}\right), b_{\mathrm{qbot}}\left(\mathrm{cm}^{-1}\right)$ and $c_{\mathrm{qbot}}\left(\mathrm{cm} \mathrm{d}^{-1}\right)$ are empirical coefficients. This type of exponential relationship was derived for deep sandy areas in the eastern part of The Netherlands (Massop and De Wit, 1994).

Special care should be taken with respect to the distinction between drainage and bottom boundary flux. The relationship that may be used to compute drainage (Chapter 4) can conflict with the relation for $q_{\text {bot. }}$ It may then be appropriate to apply another type of boundary condition.

When the relation between $q_{\text {bot }}$ and $\phi_{\text {avg }}$ is given as a table, $q_{\text {bot }}$ results from an interpolation between groundwater level and bottom flux listed in the table, using the simulated groundwater level $\left(\phi_{\text {gwl }}\right)$.

\section{Prescribed soil water pressure heads at the bottom of the model}

In this case values of $h_{\text {bot }}$ should be specified as function of time. For times between observations, SWAP will interpolate linearly. 


\section{Zero flux at the bottom of the model domain}

A bottom flux ( $\left.q_{\mathrm{bot}}\right)$ of zero may be applied when an impervious layer exists at the profile bottom or when groundwater flow below the profile bottom can be neglected. This option is implemented with a simple switch, which forces $q_{\text {bot }}$ to zero.

\section{Free drainage}

In case of free drainage, the total hydraulic head $H$ gradient is assumed to be equal to one at the bottom boundary, which sets $q_{\text {bot }}$ equal to the hydraulic conductivity of the lowest compartment:

$$
\frac{\partial H}{\partial z}=1 \quad \text { thus: } \quad q_{\text {bot }}=-K_{n}
$$

\section{Free outflow (seepage face)}

In this case, drainage will only occur if the pressure head in the bottom compartment $\left(h_{n}\right)$ becomes larger than zero. During drainage and after a drainage event, $h_{n}$ is set equal to zero and $q_{\mathrm{bot}}$ is calculated by solving the Richards equation. This option is commonly applied for lysimeters, where outflow only occurs when the lowest part of the lysimeter becomes saturated. In the field this condition is appropriate when the soil profile is drained by a coarse gravel layer. Lysimeters with groundwater table controlling provisions can also be simulated imposing a zero bottom flux condition $($ SWBOTB $=6)$, combined with a single drainage system, where the drainage resistance is low.

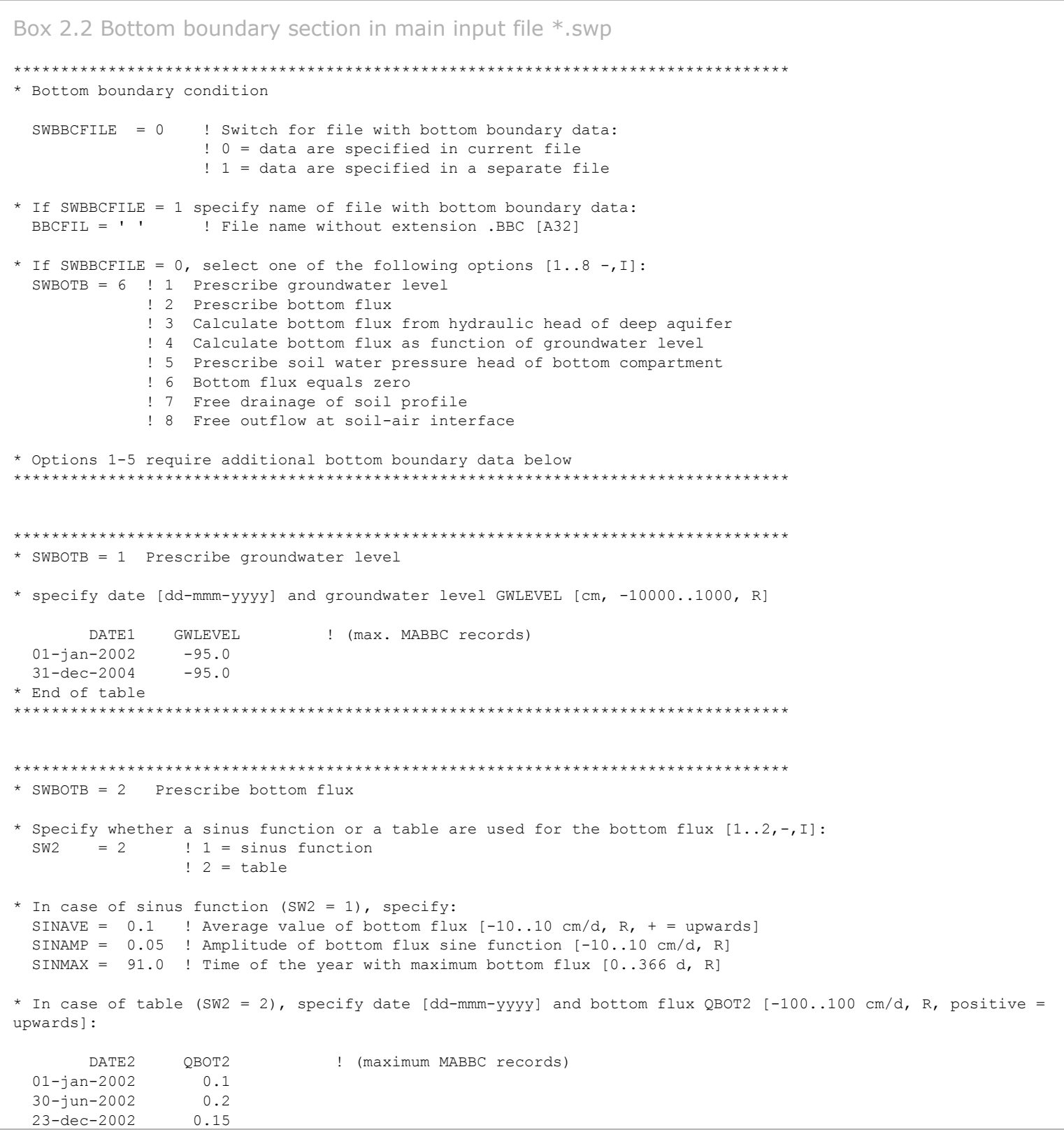




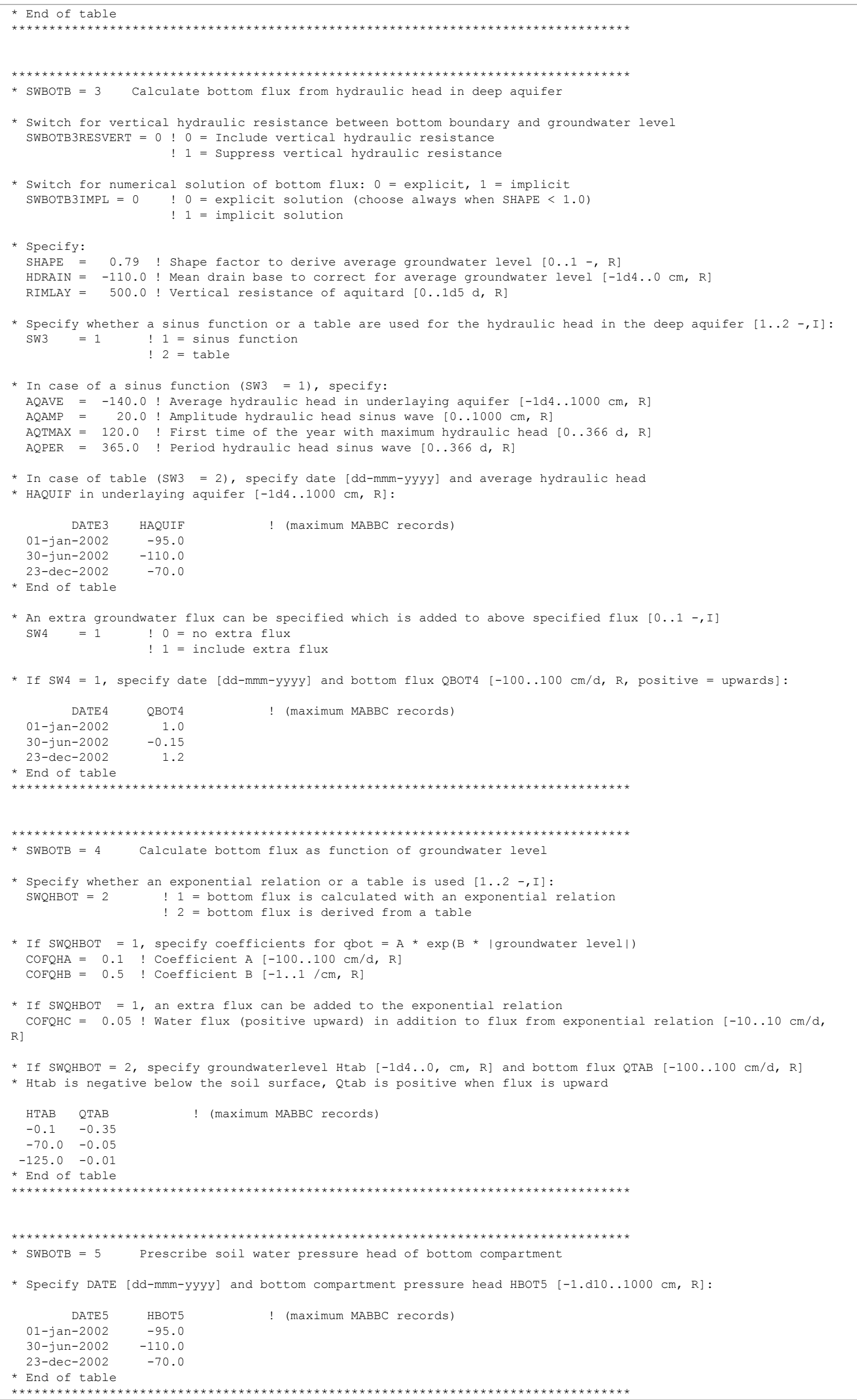




\section{Evapotranspiration and rainfall interception}

\subsection{Introduction}

In contrast to rainfall, accurate measurement of evapotranspiration fluxes is far from trivial. Evapotranspiration fluxes strongly vary with the local hydrological conditions. Therefore SWAP simulates evapotranspiration fluxes employing basic weather data or reference crop evapotranspiration data, as discussed in this chapter.

Rainfall and irrigation minus the sum of transpiration, evaporation and interception determine the amount of infiltration in the soil (Figure 3.1) and groundwater fluxes. In general the sums of rainfall+irrigation and transpiration+evaporation+interception are large compared to their difference, which equals the infiltration. This means that relative errors in these sums will magnify in relative errors of infiltration and groundwater fluxes. Therefore, reliable soil water and groundwater fluxes require accurate simulation of evapotranspiration and interception fluxes.

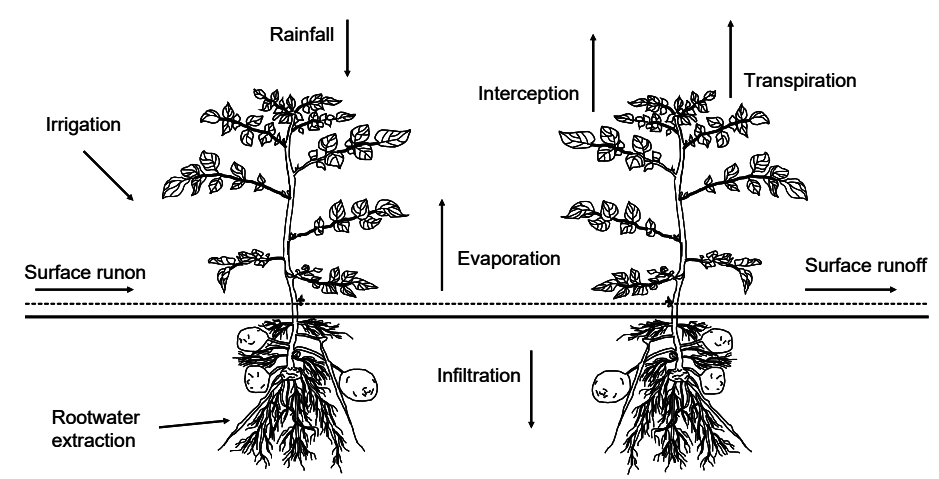

Figure 3.1 Water fluxes near the soil surface.
In general the daily water fluxes passing through a canopy are large compared to the amounts of water stored in the canopy itself.

Therefore we will assume that root water extraction in the soil is equal to plant transpiration. While root water extraction may occur throughout the root zone, soil evaporation occurs at the interface soil-atmosphere. The consequence is that during drying conditions, evaporation fluxes decline much

more rapidly than transpiration fluxes. Water harvesting, by leaving fields fallow during one or several seasons, uses this phenomenon. Because of the different physical behaviour of transpiration and evaporation, SWAP will consider evaporation and transpiration separately.

In this chapter we will first discuss the rainfall interception as used for low vegetation and forests. Next we describe the simulation of potential evapotranspiration and its distribution into potential transpiration and evaporation for partly covered soils. Then we will discuss the reduction of transpiration for wet, dry and saline soil conditions, and the reduction of evaporation for dry top soils. In the last part the related model input is described. 


\subsection{Rainfall interception}

Two methods are available in SWAP to simulate rainfall interception: method Hoyningen-Braden for agricultural crops and method Gash for forests.

\subsubsection{Method Hoyningen-Braden}

For agricultural crops and for pasture, SWAP computes the interception following Von Hoyningen-Hüne (1983) and Braden (1985). They proposed the following general formula for canopy interception (Figure 3.2):

$$
P_{\mathrm{i}}=a \cdot L A I\left(1-\frac{1}{1+\frac{b \cdot P_{\mathrm{gross}}}{a \cdot L A I}}\right)
$$

where $P_{i}$ is intercepted precipitation $\left(\mathrm{cm} \mathrm{d}^{-1}\right), L A I$ is leaf area index, $P_{\text {gross }}$ is gross precipitation ( $\mathrm{cm}$ $\left.\mathrm{d}^{-1}\right), a$ is an empirical coefficient $\left(\mathrm{cm} \mathrm{d}^{-1}\right)$ and $b$ represents the soil cover fraction (-). For increasing amounts of precipitation, the amount of intercepted precipitation asymptotically reaches the saturation amount $a L A I$. In principle a must be determined experimentally and should be specified in the input file. In case of ordinary agricultural crops we may assume $a=0.025 \mathrm{~cm} \mathrm{~d}^{-1}$. When LAI is given, SWAP estimates the soil cover fraction $b$ as:

$$
b=1-e^{-\kappa_{g r} L A I}
$$

where $\kappa_{g r}(-)$ is the extinction coefficient for solar radiation (see Par. 3.4).

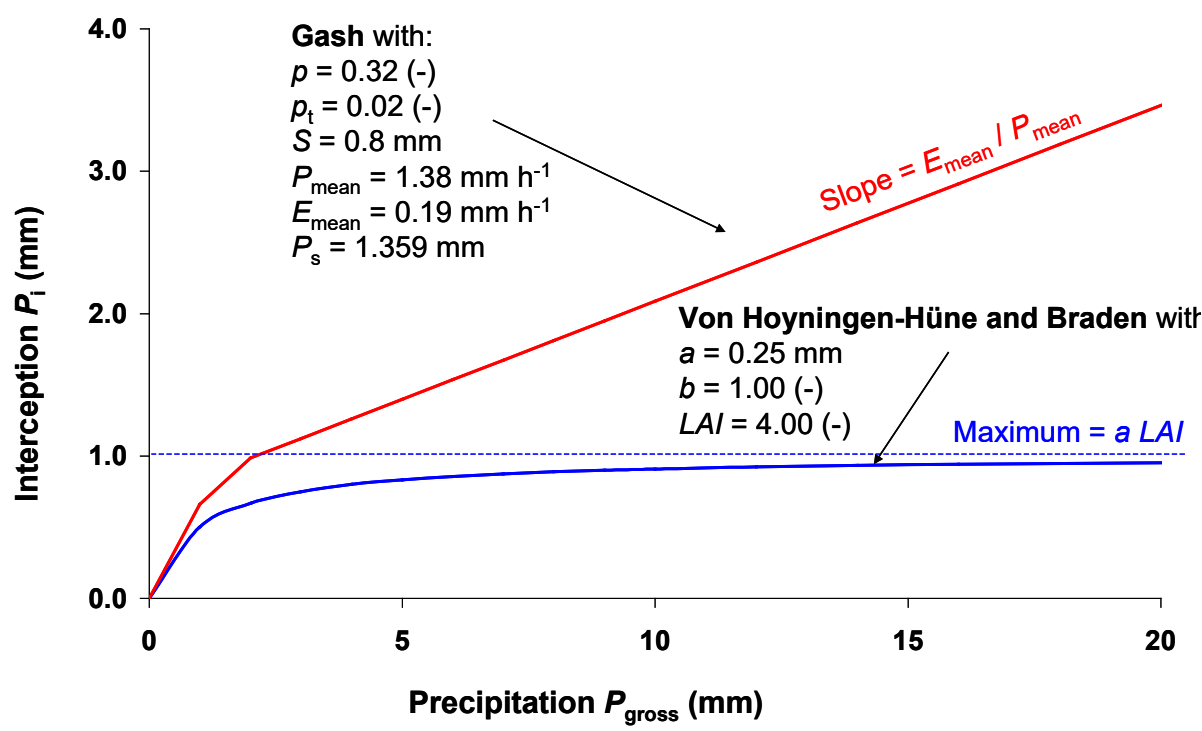

Figure 3.2 Interception for agricultural crops (Von Hoyningen-Hüne, 1983; Braden, 1985) and forests (Gash, 1979; 1985).

In case irrigation water is applied with sprinklers, SWAP will simulate separately interception of rainfall and irrigation. This is required because the solute concentration of both water sources may be different. Observed rainfall $P_{\text {gross }}$ minus intercepted rainfall $P_{\mathrm{i}}$ is called net rainfall $P_{\text {net. }}$. Likewise, applied irrigation depth $I_{\text {gross }}$ minus intercepted irrigation water is called net irrigation depth $I_{\text {net }}$.

The method of Von Hoyningen-Hüne and Braden is based on daily precipitation values. Although rainfall amounts may be specified in smaller time steps, the interception will be based on daily amounts. 


\subsubsection{Method Gash}

A main drawback of the method of Von Hoyningen-Hüne and Braden is that the effect of rain duration and evaporation during the rain event is not accounted for. In case of trees and forests, evaporation of intercepted water during rainfall can not be neglected. Gash $(1979,1985)$ formulated a physically based and widely used interception formula for forests. He considered rainfall to occur as a series of discrete events, each comprising a period of wetting up, a period of saturation and a period of drying out after the rainfall. The canopy is assumed to have sufficient time to dry out between storms. During wetting up, the increase of intercepted amount is described by:

$$
\frac{\partial P_{\mathrm{i}}}{\partial t}=\left(1-p-p_{\mathrm{t}}\right) P_{\text {mean }}-\frac{P_{\mathrm{i}}}{S} E_{\text {mean }}
$$

where $p$ is a free throughfall coefficient $(-), p_{\mathrm{t}}$ is the proportion of rainfall diverted to stemflow $(-)$, $P_{\text {mean }}$ is the mean rainfall rate $\left(\mathrm{mm} \mathrm{h}^{-1}\right), E_{\text {mean }}$ is the mean evaporation rate of intercepted water when the canopy is saturated $\left(\mathrm{mm} \mathrm{h}^{-1}\right)$ and $S$ is the maximum storage of intercepted water in the canopy $(\mathrm{mm})$. Integration of Eq. (3.3) yields the amount of rainfall which saturates the canopy, $P_{\mathrm{s}}(\mathrm{mm})$ :

$$
P_{\mathrm{s}}=-\frac{P_{\text {mean }} S}{E_{\text {mean }}} \ln \left(1-\frac{E_{\text {mean }}}{P_{\text {mean }}\left(1-p-p_{\mathrm{t}}\right)}\right) \quad \text { with } \quad 1-\frac{E_{\text {mean }}}{P_{\text {mean }}\left(1-p-p_{\mathrm{t}}\right)} \geq 0
$$

For small storms $\left(P_{\text {gross }}<P_{\mathrm{s}}\right)$ the interception can be calculated from:

$$
P_{\mathrm{i}}=\left(1-p-p_{\mathrm{t}}\right) P_{\text {gross }}
$$

For large storms $\left(P_{\text {gross }}>P_{\mathrm{s}}\right)$ the interception according to Gash (1979) follows from:

$$
P_{\mathrm{i}}=\left(1-p-p_{\mathrm{t}}\right) P_{\mathrm{s}}+\frac{E_{\text {mean }}}{P_{\text {mean }}}\left(P_{\text {gross }}-P_{\mathrm{s}}\right)
$$

Figure 3.2 shows the relation of Gash for typical values of a pine forest as function of rainfall amounts. The slope $\partial P_{\mathrm{i}} / \partial P_{\text {gross }}$ before saturation of the canopy equals $\left(1-p-p_{t}\right)$, after saturation of the canopy this slope equals $E_{\text {mean }} / P_{\text {mean }}$.

SWAP uses mean intensities of rainfall and evaporation rate to calculate the amount of rainfall which saturates the canopy, according to Eq. (3.4). Next, depending on the total amount of rainfall during a day, the amount of interception is calculated according to either Eq. (3.5) or Eq. (3.6).

\subsection{Potential evapotranspiration of uniform surfaces}

Evapotranspiration refers to both transpiration of the plants and evaporation of the soil or of water intercepted by vegetation or ponding on the soil surface. The addition 'potential' refers to non-limiting soil conditions. The potential evapotranspiration flux is therefore only determined by atmospheric conditions and plant characteristics. In SWAP we assume the atmospheric conditions to be external conditions, which are representative for the area for which the simulations are performed. Starting point in the calculations is the determination of the potential evapotranspiration of different uniform surfaces. The model offers two methods to calculate this potential evapotranspiration (see Figure 3.3): the Penman-Monteith method and the reference evapotranspiration method. Both methods can be combined with crop factors; Penman-Monteith can also be applied without crop factors. 


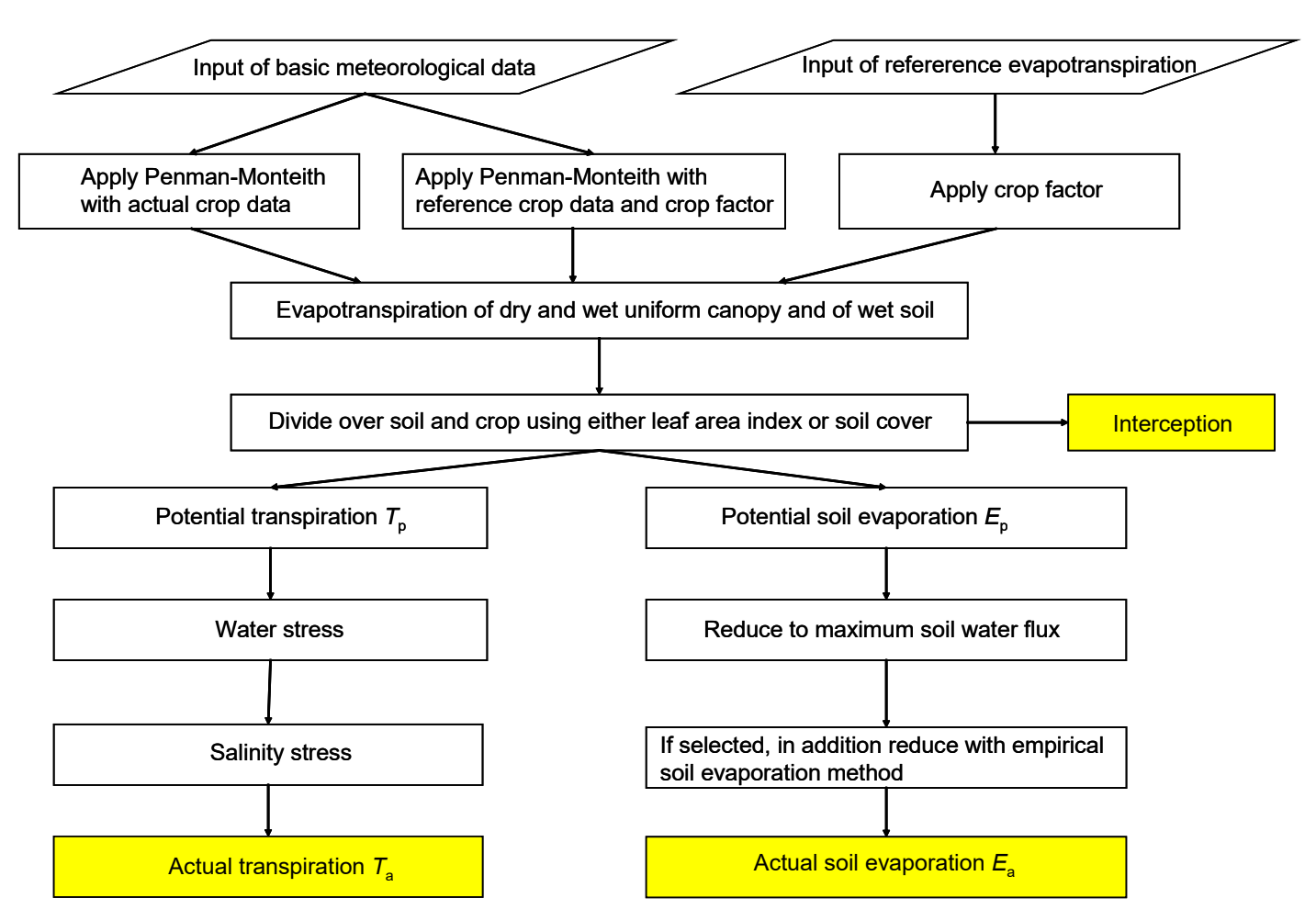

Figure 3.3 Method used in SWAP to derive actual transpiration and soil evaporation of partly covered soils from basic input data.

\subsubsection{Penman Monteith method}

Using similar physics as Penman (1948), Monteith (1965) derived an equation that describes the evapotranspiration from a dry, uniform, vegetated surface, which is optimally supplied with water. This equation is known as the Penman-Monteith equation. Jensen et al. (1990) analyzed the performance of 20 different evapotranspiration formula using lysimeter data of 11 stations around the world under different climatic conditions. The Penman-Monteith formula ranked as the best for all climatic conditions. Therefore this equation has become an international standard for calculation of potential evapotranspiration (Allen et al., 1998), and is applied by SWAP.

For a closed canopy with insignificant evaporation from the soil the Penman-Monteith equation can be written as (Monteith, 1965, 1981):

$$
E T_{\mathrm{p}}=\frac{\frac{\Delta_{\mathrm{v}}}{\lambda_{\mathrm{w}}}\left(R_{\mathrm{n}}-G\right)+\frac{p_{1} \rho_{\text {air }} C_{\text {air }}}{\lambda_{w}} \frac{e_{\text {sat }}-e_{\mathrm{a}}}{r_{\text {air }}}}{\Delta_{\mathrm{v}}+\gamma_{\text {air }}\left(1+\frac{r_{\text {crop }}}{r_{\text {air }}}\right)}
$$

where $E T$ is the transpiration rate of the canopy $\left(\mathrm{mm} \mathrm{d}^{-1}\right), \Delta_{\mathrm{V}}$ is the slope of the vapour pressure curve $\left(\mathrm{kPa}{ }^{\circ} \mathrm{C}^{-1}\right), \lambda_{w}$ is the latent heat of vaporization $\left(\mathrm{J} \mathrm{kg}^{-1}\right), R_{\mathrm{n}}$ is the net radiation flux at the canopy surface $\left(\mathrm{J} \mathrm{m}^{-2} \mathrm{~d}^{-1}\right), G$ is the soil heat flux $\left(\mathrm{J} \mathrm{m}^{-2} \mathrm{~d}^{-1}\right), p_{1}$ accounts for unit conversion $\left(=86400 \mathrm{~s} \mathrm{~d}^{-1}\right)$, $\rho_{a i r}$ is the air density $\left(\mathrm{kg} \mathrm{m}^{-3}\right), C_{\text {air }}$ is the heat capacity of moist air $\left(\mathrm{J} \mathrm{kg}^{-1}{ }^{\circ} \mathrm{C}^{-1}\right), e_{\text {sat }}$ is the saturation vapour pressure $(\mathrm{kPa}), e_{\mathrm{a}}$ is the actual vapour pressure $(\mathrm{kPa}), \gamma_{a i r}$ is the psychrometric constant ( $\mathrm{kPa}$ $\left.{ }^{\circ} \mathrm{C}^{-1}\right), r_{\text {crop }}$ is the crop resistance $\left(\mathrm{s} \mathrm{m}^{-1}\right)$ and $r_{\text {air }}$ is the aerodynamic resistance $\left(\mathrm{s} \mathrm{m}^{-1}\right)$.

The FAO has proposed a clearly defined and well established methodology to apply the PenmanMonteith equation for evapotranspiration estimates at a daily time scale using routinely measured weather data (Allen et al., 1998). The required weather data include daily values of air temperature (preferably the minimum as well as the maximum value), global radiation, wind speed and relative humidity. The FAO methodology is applied in SWAP and the basic equations are listed in Appendix 1. 
In general the parameter $r_{\text {crop }}$ is used to calculate $E T_{\mathrm{p}}$ from a mixture of vegetation and bare soil, in which case this parameter is called the surface resistance $r_{\mathrm{s}}$. When crop factors are used, SWAP applies the Penman-Monteith method to either vegetations fully covering the soil or bare soils. Therefore we replace $r_{\mathrm{s}}$ by $r_{\text {crop }}$ (at bare soils $r_{\text {crop }}$ is absent and equals zero). SWAP calculates fluxes for three uniform surfaces:

- $E T_{\text {wo }}\left(\mathrm{cm} \mathrm{d}^{-1}\right)$, evapotranspiration rate of a closed wet canopy;

- $E T_{\mathrm{po}}\left(\mathrm{cm} \mathrm{d}^{-1}\right)$, evapotranspiration rate of a closed dry canopy;

- $E_{\mathrm{p} 0}\left(\mathrm{~cm} \mathrm{~d}^{-1}\right)$, evaporation rate from a wet, bare soil.

These ET-quantities are obtained by varying the values for crop resistance $\left(r_{\text {crop }}\right)$, crop height $\left(h_{\text {crop }}\right)$ and reflection coefficient $\left(\alpha_{r}\right)$ for the three uniform surfaces as listed in Table 3.1. For a dry crop completely covering the soil with optimal water supply in the soil $r_{\text {crop }}$ is minimal and varies between $30 \mathrm{~s} \mathrm{~m}^{-1}$ for arable crop to $150 \mathrm{~s} \mathrm{~m}^{-1}$ for trees in a forest (Allen et al., 1986, 1989). This value is input, as is the crop height. For a wet, bare soil SWAP will assume $r_{\text {crop }}=0$ and 'crop height' $h_{\text {crop }}=0.1 \mathrm{~cm}$.

As Figure 3.3 shows, the Penman-Monteith method can be applied for the reference grass, in combination with crop factors. This method has been extensively discussed by Allen et al., (1998). In that case SWAP will set $r_{\text {crop }}=70 \mathrm{~s} \mathrm{~m}^{-1}, h_{\text {crop }}=12 \mathrm{~cm}$ and $\alpha_{\mathrm{r}}=0.23$, as generally defined for the reference grass. Table 3.2 shows how the crop factors relate $E T_{\text {wo }}$ and $E T_{\mathrm{p} 0}$ to the corresponding values for grass. The crop factors belong to a certain crop and depend on its development stage. In case of bare soils the 'crop factor' has just one value, and is called soil factor. The use of a soil factor is optional. Without soil factor SWAP will directly calculate $E_{\mathrm{po}}$ with the Penman-Monteith method. With soil factor SWAP wil relate $E_{\mathrm{p} 0}$ to the reference evapotranspiration rate calculated with PenmanMonteith for grass, $E T_{\mathrm{p} 0 \text {, grass. }}$. For ponding conditions potential evaporation is related to the potential evaporation of intercepted water (see Table 3.2).

Table 3.1 Three uniform surfaces and its corresponding potential evapotranspiration $E T_{p}$ : parameter settings for the method Penman-Monteith.

\begin{tabular}{lllll} 
Description of uniform surface & $E T_{\mathrm{p}}$ & $r_{\text {crop }}$ & $\begin{array}{l}h_{\text {crop }} \\
(\mathrm{sm})\end{array}$ & $\begin{array}{c}a_{r} \\
(-)\end{array}$ \\
wet canopy, completely covering the soil & $E T_{\text {wo }}$ & input & input & input \\
\hline dry canopy completely covering the soil & $E T_{p 0}$ & input & input & input \\
wet, bare soil & $E_{p 0}$ & 0.0 & 0.1 & 0.15 \\
\hline
\end{tabular}

Table 3.2 Options in SWAP to derive potential evapotranspiration rates for uniform surfaces.

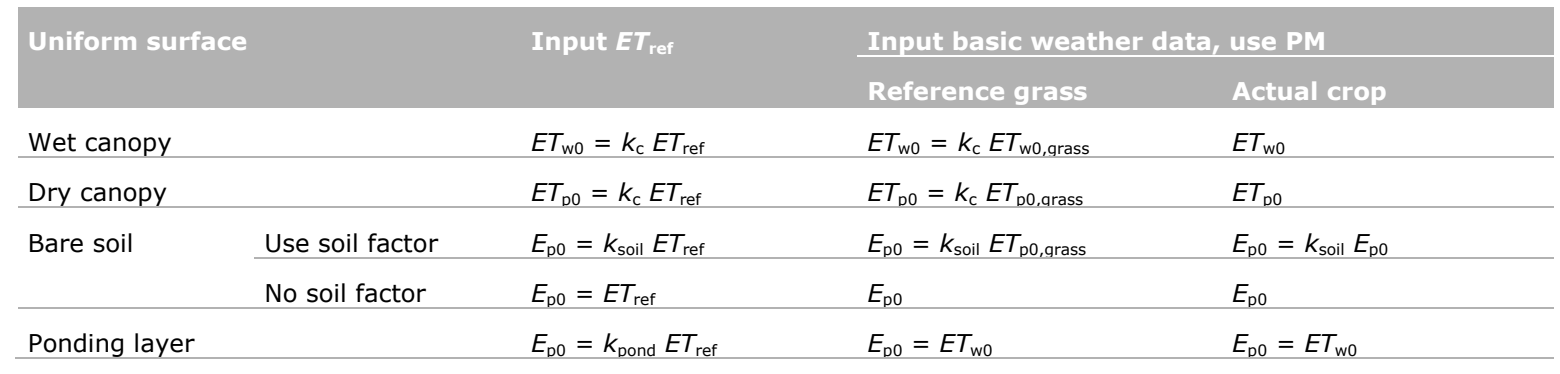




\subsubsection{Reference evapotranspiration and crop factors}

Application of the Penman-Monteith equation requires daily values of air temperature, net radiation, wind speed and air humidity, which data might not be available. Also in some studies other methods than Penman-Monteith might be more appropriate. For instance in The Netherlands the Makkink equation is widely used (Makkink, 1957; Feddes, 1987). Therefore SWAP allows the use of a reference evapotranspiration rate $E T_{\text {ref }}\left(\mathrm{cm} \mathrm{d}^{-1}\right)$ (Figure 3.3). In that case the potential evapotranspiration rate for the dry canopy $E T_{\mathrm{po}}$ is calculated by:

$$
E T_{\mathrm{p} 0}=k_{c} E T_{\text {ref }}
$$

where $k_{\mathrm{c}}$ is the so called crop factor, which depends on the crop type and the method employed to obtain $E T_{\text {ref. }}$ In a similar way the potential evapotranspiration rate for the wet canopy $E T_{\text {wo }}$ is derived:

$$
E T_{\mathrm{w} 0}=k_{c} E T_{\text {ref }}
$$

The evaporation rate of a wet, bare soil, can be derived with a soil factor $k_{\text {soil }}$ :

$$
E_{\mathrm{p} 0}=k_{\text {soil }} E T_{\text {ref }}
$$

Without soil factor, $E_{\mathrm{p} 0}$ is set equal to $E T_{\text {ref. }}$ For ponding conditions a separate factor $k_{\text {pond }}$ is used.

Table 3.2 provides an overview of the SWAP options when $E T_{\text {ref }}$ and crop, soil and pond factors are used.

The reference evapotranspiration rate can be determined in several ways, such as pan evaporation, the Penman open water evaporation (Penman, 1948), the FAO modified Penman equation (Doorenbos and Pruitt, 1977), the Penman-Monteith equation applied for a reference crop (Allen et al., 1998), Priestly-Taylor (1972), Makkink (Makkink, 1957; Feddes, 1987) or Hargreaves et al. (1985). In case of Priestly-Taylor and Makkink, only air temperature and solar radiation data are required. Hargreaves requires solely air temperature data.

In SWAP the crop factors are used to convert the evapotranspiration rate of a reference crop fully covering the soil to the potential evapotranspiration rate of the actual crop fully covering the soil (Figure 3.3). This is different from programs like CROPWAT (Smith, 1992) and CRIWAR (Bos et al., 1996), which use crop factors that depend on the crop development stage and soil cover. Because the soil has generally a dry top layer, soil evaporation is usually below the potential evaporation rate. Hence, these crop factors semi-empirically combine the effect of an incomplete soil cover and reduced soil evaporation. Instead SWAP uses the crop factor to relate uniform, cropped surfaces. Therefore crop factors in SWAP can be larger than those in CROPWAT and CRIWAR.

\subsection{Potential transpiration and evaporation fluxes of partly covered soils}

For accurate agrohydrological analysis we need the distribution of the atmospheric demand over potential transpiration and potential evaporation. This is important for proper simulation of crop growth and root uptake of nutrients and salts, which depend on the reduction of transpiration only, and for proper simulation of actual evaporation and transpiration fluxes. SWAP offers two methods for this distribution:

1. Distribution based on crop and soil factors

2. Distribution based on direct application of Penman-Monteith

\subsubsection{Distribution based on crop and soil factors}

The application of crop and soil factors as described in Par. 3.3 yields fluxes of uniform surfaces: either a wet $\left(E T_{\mathrm{w} 0}\right)$ or dry $\left(E T_{\mathrm{p} 0}\right)$ canopy and a wet soil $\left(E_{\mathrm{p} 0}\right)$. These uniform fluxes are used to derive the potential transpiration rate $T_{\mathrm{p}}$ and potential soil evaporation rate $E_{\mathrm{p}}$ for partly covered soils.

When the crop is wet due to interception, SWAP assumes that the energy available for evapotranspiration is entirely used to evaporate the intercepted water, independent of the soil cover fraction. This is at least 
valid for higher soil cover fractions. At small soil cover fractions, this assumption might overestimate the actual evaporation rate of intercepted water. However, as at small soil fractions the interception amounts are minor, this seems not important. The fraction of the day that the crop is wet, $W_{\text {frac }}(-)$, therefore follows from the ratio of the daily amount of intercepted precipitation $P_{\mathrm{i}}$ (Par. 3.2) and $E T_{\text {wo }}$ :

$$
W_{\text {frac }}=\frac{P_{\mathrm{i}}}{E T_{\mathrm{w} 0}} \quad \text { with } \quad \mathrm{W}_{\text {frac }} \leq 1.0
$$

Next the potential soil evaporation rate can be derived from $E_{\mathrm{p} 0}$, taking into account reductions due to shadow by crops and periods without evaporation when the crop is wet. The most common method assumes that the net radiation inside the canopy decreases according to an exponential function (Goudriaan, 1977; Belmans, 1983). In that case, the potential soil evaporation rate $E_{\mathrm{p}}$ is equal to:

$$
E_{\mathrm{p}}=E_{\mathrm{p} 0}\left(1-W_{\text {frac }}\right) \mathrm{e}^{-\kappa_{\mathrm{gr}} L A I}
$$

where $\kappa_{\mathrm{gr}}(-)$ is the extinction coefficient for solar radiation. Ritchie (1972) and Feddes (1978) used $\kappa_{\mathrm{gr}}$ $=0.39$ for common crops. More recent approaches estimate $\kappa_{\mathrm{gr}}$ as the product of the extinction coefficient for diffuse visible light, $\kappa_{\mathrm{df}}(-)$, which varies with crop type from 0.4 to 1.1 , and the extinction coefficient for direct visible light, $\kappa_{\text {dir }}(-)$ :

$$
\kappa_{\mathrm{gr}}=\kappa_{\mathrm{df}} \kappa_{\mathrm{dir}}
$$

Sometimes reliable figures of LAI as function of crop development are lacking. For such cases, the soil cover fraction is a practical alternative to account for the reduction of solar radiation due to crop cover:

$$
E_{\mathrm{p}}=E_{\mathrm{p} 0}\left(1-W_{\text {frac }}\right)(1.0-S C)
$$

Next the most important evapotranspiration term should be calculated: the potential transpiration rate $T_{\mathrm{p}}$ of the dry crop which partly covers the soil. During transpiration of dry crops, the total energy for evapotranspiration will be distributed over $E_{\mathrm{p}}$ and $T_{\mathrm{p}}$. SWAP assumes that the total evapotranspiration rate in periods with dry canopy corresponds to $E T_{\mathrm{p} 0}$, as this rate is best defined. Therefore $T_{\mathrm{p}}$, taking into account rainfall interception and partly soil cover, follows from:

$$
T_{\mathrm{p}}=E T_{\mathrm{p} 0}\left(1.0-W_{\text {frac }}\right)-E_{\mathrm{p}}
$$

\subsubsection{Distribution based on direct application of Penman-Monteith}

In the PM-direct method, crop or soil factors to translate reference ET to crop ET are no longer needed. In this way the cumbersome calibration of crop and soil factors during the growing season is avoided, and the method can be made climate proof if used in combination with an adequate crop growth model. The method requires biophysical data on leaf area index, albedo, crop height, minimal stomatal resistance and minimal soil resistance.

In the PM-direct method as implemented in SWAP we assume that during the fraction of time that the crop is wet, $W_{\text {frac }}$, the transpiration is suppressed due to the fact that the interception evaporation has a very low resistance, creating a near-saturated atmosphere around the leaves. The potential transpiration of a dry canopy $T_{\mathrm{p}}\left(\mathrm{mm} \mathrm{d}^{-1}\right)$ and potential evaporation $E_{\mathrm{p}}\left(\mathrm{mm} \mathrm{d}^{-1}\right)$ of the actual crop are thus calculated directly with:

$$
\begin{gathered}
T_{p}=\frac{\left(1-W_{\text {frac }}\right)\left(V_{\mathrm{c}} \frac{\Delta_{\mathrm{v}}}{\lambda_{\mathrm{w}}}\left(R_{\mathrm{n}}-G\right)+\frac{p_{1} \rho_{\mathrm{a}} C_{\mathrm{a}}}{\lambda_{\mathrm{w}}}\left(\frac{e_{\mathrm{sat}}-e_{\mathrm{a}}}{r_{\mathrm{a}, \text { can }}}\right)\right)}{\Delta_{\mathrm{v}}+\gamma_{\mathrm{a}}\left(1+\frac{r_{\mathrm{s}, \text { min }}}{r_{\mathrm{a}, \text { can }} L A I_{\text {eff }}}\right)} \\
E_{\mathrm{p}}=\frac{\left(1.0-V_{\mathrm{c}}\right) \frac{\Delta_{\mathrm{v}}}{\lambda_{\mathrm{w}}}\left(R_{\mathrm{n}}-G\right)+\frac{p_{1} \rho_{\mathrm{a}} C_{\mathrm{a}}}{\lambda_{\mathrm{w}}}\left(\frac{e_{\text {sat }}-e_{\mathrm{a}}}{r_{\mathrm{a}, \text { soil }}}\right)}{\Delta_{\mathrm{v}}+\gamma_{\mathrm{a}}\left(1.0+\frac{r_{\text {soil }}}{r_{\mathrm{a}, \text { soil }}}\right)}
\end{gathered}
$$


where $W_{\text {frac }}$ is the fraction of the day in which the canopy is wet $(-), V_{\mathrm{c}}$ is vegetation cover $(-), r_{\mathrm{s}, \min }$ is the minimal stomatal resistance $\left(\mathrm{s} \mathrm{m}^{-1}\right), L A I_{\text {eff }}$ is the effective leaf area index $(-)$ and $r_{\text {soil }}$ is the soil resistance of a wet soil $\left(\mathrm{s} \mathrm{m}^{-1}\right)$. Note that instead of the overall crop resistance, Eq. (3.16) uses the minimal stomatal resistance which is corrected with $L A I_{\text {eff. }}$

The vegetation cover is based on solar light extinction within a canopy:

$$
V_{\mathrm{c}}=1-\mathrm{e}^{-\kappa_{\mathrm{dir}} \kappa_{\mathrm{dif}} L A I}
$$

where $\kappa_{\text {dir }}$ and $\kappa_{\text {dif }}$ are the extinction coefficients for direct and diffuse solar light, respectively (-) and $L A I$ is the actual leaf area index (-).

$L A I_{\text {eff }}$ is related to the stomata that participate in transpiration and is derived from the actual leaf area index $L A I$ with (Bastiaanssen et al., 2012):

$$
L A I_{\text {eff }}=\frac{L A I}{0.3 L A I+1.2}
$$

The aerodynamic resistances are first calculated for uniform crop and soil surfaces according to Allen et al. (1998). Next these resistances are corrected for the vegetation cover:

$$
\begin{aligned}
& r_{\mathrm{a}, \text { can }}=\frac{r_{\mathrm{a}, \mathrm{can}, 0}}{V_{\mathrm{c}}} \\
& r_{\mathrm{a}, \text { soil }}=\frac{r_{\mathrm{a}, \text { soil, } 0}}{1-V_{\mathrm{c}}}
\end{aligned}
$$

where $r_{\mathrm{a}}$ can,0 and $r_{\mathrm{a}, \text { soil, } 0}$ are the aerodynamic resistances of uniform crop and soil surfaces.

For the interception evaporation of a wet crop, $E_{\mathrm{i}}$, we apply Eq. (3.16) with a lower value of the minimal stomatal resistance than for a dry crop, with the default value set to zero. The fraction of a day that the canopy is wet is then calculated as:

$$
W_{\text {frac }}=\frac{P_{\mathrm{i}}}{E_{\mathrm{i}}}
$$

where $P_{\mathrm{i}}$ is the rainfall interception $\left(\mathrm{mm} \mathrm{d}^{-1}\right)$ and $E_{\mathrm{i}}$ is the evaporation flux of a wet canopy $\left(\mathrm{mm} \mathrm{d}^{-1}\right)$.

On a daily base, the soil heat flux $G$ is assumed to be negligible.

\subsection{Actual plant transpiration}

\subsubsection{General approach}

The potential transpiration and evaporation fluxes $\left(T_{\mathrm{p}}\right.$ and $\left.E_{\mathrm{p}}\right)$ as described in Par. 3.4 are based on atmospheric conditions and vegetation properties, assuming favourable soil moisture conditions. In this paragraph we describe the reduction of root water uptake and thus transpiration due to oxygen stress and drought stress. In Par. 3.6 the reduction of soil evaporation due to drying of the top soil is described. Paragraph 8.6 considers the effect of high salt concentrations on root water uptake and Par. 10.2 the effect of frozen soils. Ultimately the calculations yield the actual transpiration and evaporation fluxes $\left(T_{\mathrm{a}}\right.$ and $\left.E_{\mathrm{a}}\right)$ as depicted in Figure 3.3.

The maximum possible root water extraction rate, integrated over the rooting depth, should equal $T_{\mathrm{p}}$ $\left(\mathrm{cm} \mathrm{d}^{-1}\right)$. Taking into account the root length density distribution (Bouten, 1992), the potential root water extraction rate at a certain depth, $S_{p}(z)\left(d^{-1}\right)$ can be calculated by:

$$
S_{\mathrm{p}}(z)=\frac{\ell_{\text {root }}(z)}{\int_{-D_{\text {root }}}^{0} \ell_{\text {root }}(z) \mathrm{d} z} T_{\mathrm{p}}
$$

where $D_{\text {root }}$ is the root layer thickness $(\mathrm{cm})$. 
These potential fluxes to the roots are reduced due to suboptimal soil conditions: too wet, too dry, too saline or too cold. SWAP determines stress factors for these factors at each soil compartment. In case of combined water and salt stress, Skaggs et al. (2006) argue that the stress factors for water and salt stress can be multiplied. In SWAP we follow this multiplication approach for all stresses and calculate the actual root water flux, $S_{\mathrm{a}}(z)\left(\mathrm{d}^{-1}\right)$, as:

$$
S_{\mathrm{a}}(z)=\alpha_{\mathrm{rw}} \alpha_{\mathrm{rd}} \alpha_{\mathrm{rs}} \alpha_{\mathrm{rf}} S_{\mathrm{p}}(z)
$$

where $\alpha_{\text {rd }}(-), \alpha_{r w}(-), \alpha_{r s}(-)$ and $\alpha_{r f}(-)$ are the reduction factors for conditions which are too wet, too dry, too saline, or too cold, respectively.

Integration of $S_{\mathrm{a}}(z)$ over the root layer yields the actual transpiration rate $T_{a}\left(\mathrm{~cm} \mathrm{~d}^{-1}\right)$ :

$$
T_{\mathrm{a}}=\int_{-D_{\text {root }}}^{0} S_{\mathrm{a}}(z) \partial z
$$

In many applications the proportion of occurring stress factors is relevant information. Therefore SWAP will split the total transpiration reduction into individual contributions of the occurring stress factors by multiplying $\left(S_{p}(z)-S_{a}(z)\right)$ with the proportion of the logarithmic value of each of the reduction factors $\log \left(\alpha_{\mathrm{j}}\right) / \sum_{\mathrm{i}=1} \log \left(\alpha_{\mathrm{i}}\right)$.

\subsubsection{Feddes function for root water uptake}

Stresses due to dry or wet conditions and/or high salinity concentrations may reduce $S_{p}(z)$. The water stress in SWAP is described by the function proposed by Feddes et al. (1978), which is depicted in Figure 3.4. In the range $h_{3}<h<h_{2}$ root water uptake is optimal. Below $h_{3}$ root water uptake linearly declines due to drought until zero at $h_{4}$ (wilting point). The critical pressure head $h_{3}$ increases for higher potential transpiration rates $T_{\mathrm{p}}$. Above $h_{2}$ root water uptake linearly declines due to insufficient aeration until zero at $h_{1}$, i.e. representing threshold values for minimum and maximum oxygen deficiency.

Sometimes only some parts of the root zone are

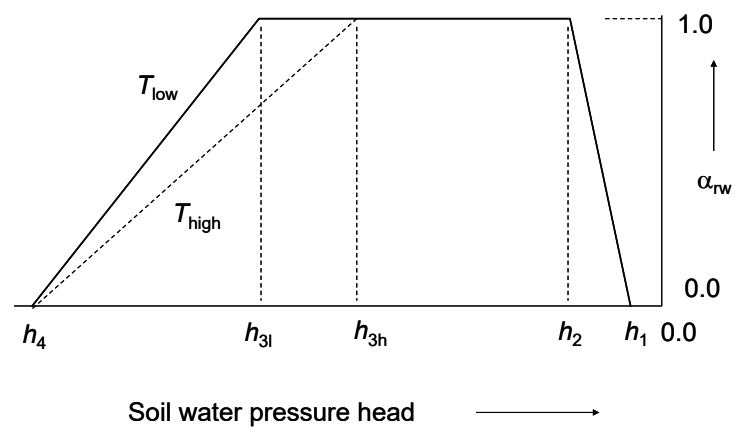

Figure 3.4 Reduction coefficient for root water uptake, $\alpha_{r w}$ as function of soil water pressure head $h$ and potential transpiration rate $T_{p}$ (after Feddes et al., 1978). stressed and show reduction of root water uptake, while other parts have favourable conditions for root water uptake. In these conditions the reduction in the stressed parts might be compensated by extra root water uptake in the parts with favourable conditions. This compensation was not part of the original Feddes reduction function. In SWAP the user might add this compensation to the Feddes function. The compensation concept implemented has been described by Jarvis $(1989 ; 2011)$.

Constant critical pressure heads for oxygen stress seem not realistic, as oxygen stress depends on various abiotic and biotic factors. Therefore, as alternative the process-based method of Bartholomeus et al. (2008) has been implemented (Par. 3.5.3). At the dry side, the input values for $h_{31}$ and $h_{3 \mathrm{~h}}$ depend on plant type, soil texture, root density and atmospheric demand, input values which are not commonly available. Also the linear decline of the reduction factor in the range $h_{4}<h<h_{3}$ may deviate from reality. Therefore also a detailed, microscopic root water uptake module for drought has been added to SWAP (Par. 3.5.4).

\subsubsection{Detailed oxygen stress}

When the availability of oxygen or water is insufficient to meet the metabolic demands of plants for these resources, the plant suffers from oxygen and drought stress, respectively. The first physiological process inhibited at oxygen stress (resulting from high soil moisture contents) is plant root respiration, i.e. oxygen consumption in the roots, a process that increases with rising temperatures. High soil 
moisture contents hamper oxygen transport from the atmosphere, through the soil - where part of the oxygen is additionally consumed by soil microorganisms - and to the root cells. Reduced respiration negatively affects the energy supply to plant metabolism (Bartholomeus et al. 2011).

Root respiration is determined by interacting respiratory (i.e. oxygen consuming) and diffusive (i.e. oxygen providing) processes in and to the soil. Plant roots respire at a potential rate under optimal soil aeration and thus non-limiting oxygen availability. This potential root respiration is in equilibrium with the oxygen demand of plant roots, which is determined by plant characteristics and soil temperature (as simulated with SWAP). Upon increasingly wetter conditions, however, the gas-filled porosity of the soil decreases and oxygen availability becomes insufficient for potential root respiration. Daily respiration reduction (i.e. potential minus actual respiration) is calculated with a model for oxygen transport and consumption, which uses generally applied physiological and physical relationships to calculate both the oxygen demand of, and the oxygen supply to plant roots (Figure 3.5). For details and equations of the implemented method we refer to Bartholomeus et al. (2008).

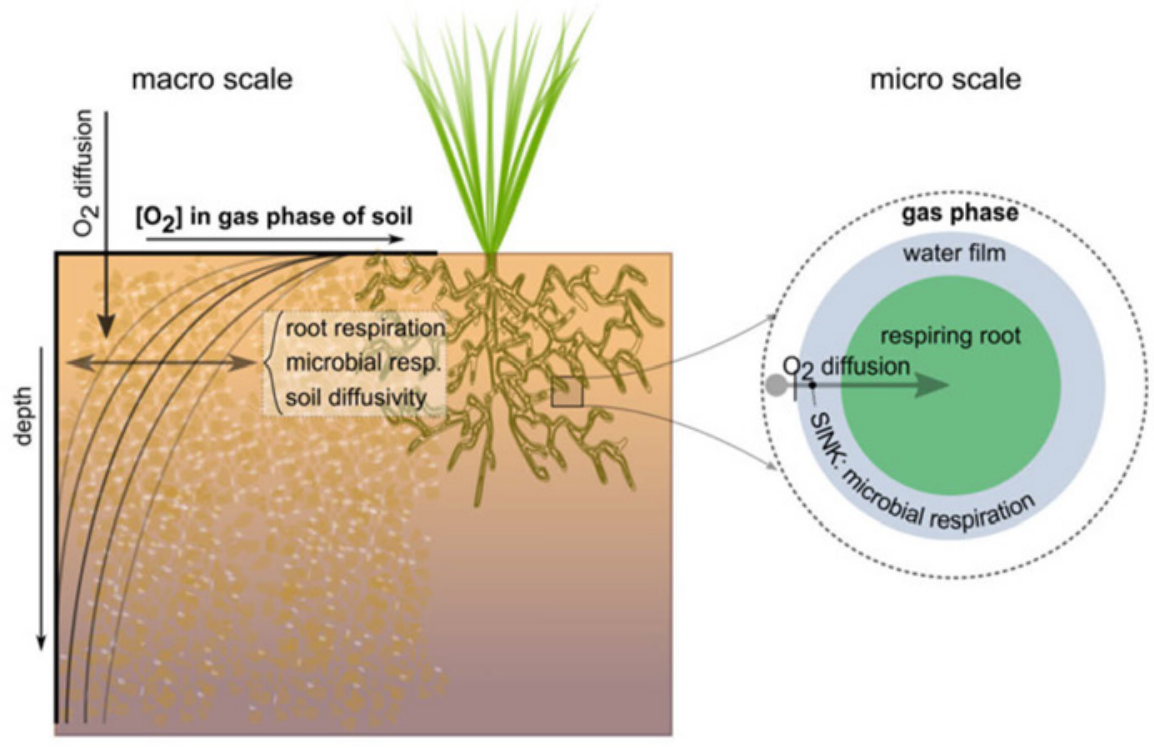

Figure 3.5 Schematization of the oxygen module used to simulate daily respiration reduction. The model combines interacting physiological processes (i.e. root respiration and microbial respiration) and physical processes (i.e. macro-scale and micro-scale oxygen diffusion) (figure from Bartholomeus et al. 2011). Details of equations involved are given in Bartholomeus et al. (2008).

Simulation of the actual root respiration for a crop requires actual data on soil type, daily soil temperature and daily gas-filled porosity in each soil layer. The latter two variables are simulated within SWAP. Simulation of soil temperature should thus be switched on. The model of Bartholomeus et al. (2008) is applied to all soil layers of SWAP, to account for layer-specific soil physical properties, moisture contents and temperatures. The difference between potential and actual root respiration is calculated for each soil layer separately. Subsequently, the reduction coefficient $a_{r w}$ due to oxygen stress is calculated for each soil layer, based on the assumption that root water uptake is proportional to growth respiration.

The latter assumption is supported by several arguments (Bartholomeus et al. 2008). If stomata are fully opened, root water uptake is optimal $\left(a_{r w}=1\right)$ and dominated by hydraulic forces. At fully open stomata, potential photosynthesis is achieved and oxygen is needed for both maintenance and growth respiration. Potential root water uptake is thus only possible as long as there is no oxygen stress. Oxygen stress induces stomatal closure (e.g. Glínski and Stępniewksi, 1985; Kramer, 1951). At complete stomatal closure, the actual transpiration and photosynthesis approach zero $\left(a_{\mathrm{rw}}=0\right)$. In that case, oxygen is solely used for maintenance respiration.

Between the limits $a_{r w}=1$ and $a_{r w}=0$, it is assumed that the reduction of root water uptake is directly proportional to the reduction in root respiration. Kamaluddin and Zwiazek (2001) 
demonstrated, by inhibition of the metabolism of the roots, that a positive correlation between root respiration and water uptake exists. This implies a gradual decrease of root water uptake with increased oxygen stress (Bartholomeus et al. 2008).

\subsubsection{Detailed drought stress}

In contrast to the macroscopic Feddes model as described in Par. 3.5.2, microscopic models describe the radial flow of soil water towards individual roots. The roots are considered as linear tubes. The root system as a whole is considered as a set of such individual tubes, regularly spaced in the soil at definable distances (Figure 3.6). The density of the tubes with depth may vary, similar to root density in a root zone.

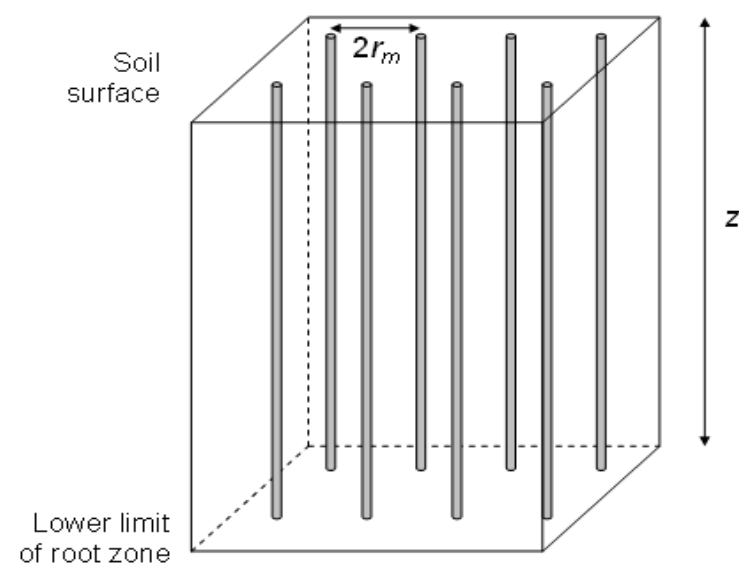

Figure 3.6 Schematization of root system by equally spaced tubes. Density in the vertical may differ.

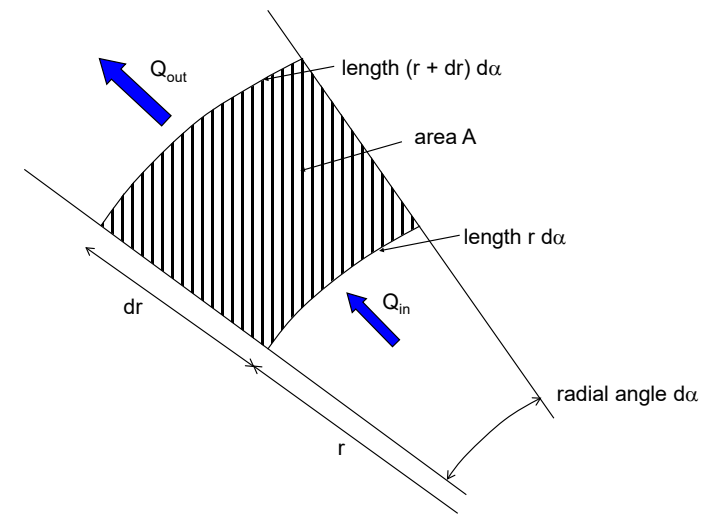

Figure 3.7 Water balance of a radial segment.

In such a geometry, a radial flow pattern towards the roots exists. Figure 3.7 depicts this flow pattern for a segment with angle da (rad). The inflow $Q_{\text {in }}\left(\mathrm{m}^{2} \mathrm{~d}^{-1}\right)$ can be written as:

$$
Q_{\text {in }}=q r \mathrm{~d} \alpha
$$

and the outflow $Q_{\text {out }}\left(\mathrm{m}^{2} \mathrm{~d}^{-1}\right)$ equals:

$$
Q_{\text {out }}=\left(q+\frac{\partial q}{\partial r} \mathrm{~d} r\right)(r+\mathrm{d} r) \mathrm{d} \alpha
$$

where $q\left(\mathrm{~m} \mathrm{~d}^{-1}\right)$ is the soil water flux density and $r(\mathrm{~m})$ is the radial distance from the root centre. Calculation of the terms of Eq. (3.27) and subsequently the difference $Q_{\text {in }}-Q_{\text {out }}$ yields:

$$
Q_{\text {in }}-Q_{\text {out }}=-\mathrm{d} \alpha\left[q \mathrm{~d} r+r \frac{\partial q}{\partial r} \mathrm{~d} r+\frac{\partial q}{\partial r}(\mathrm{~d} r)^{2}\right]
$$


The segment area $A\left(\mathrm{~m}^{2}\right)$ between radial distances $r$ and $r+\mathrm{d} r(\mathrm{~m})$ from the root centre is equal to:

$$
A=\pi(r+\mathrm{d} r)^{2} \frac{\mathrm{d} \alpha}{2 \pi}-\pi r^{2} \frac{\mathrm{d} \alpha}{2 \pi}=\left(r d r+\frac{\mathrm{d} r^{2}}{2}\right) \mathrm{d} \alpha
$$

As we are dealing with infinite small differences, we may omit higher order terms with respect to first order terms: the third term in Eq. (3.28), and the second term in Eq. (3.29). The water balance of segment $A$ can be written with the simplified equations (3.28) and (3.29) as:

$$
\frac{\partial \theta}{\partial t} A=Q_{\text {in }}-Q_{\text {out }} \quad \rightarrow \quad \frac{\partial \theta}{\partial t} r \mathrm{~d} r \mathrm{~d} \alpha=-\mathrm{d} \alpha\left[q \mathrm{~d} r+r \mathrm{~d} r \frac{\partial q}{\partial r}\right]
$$

where $\theta$ is the volumetric water content $\left(\mathrm{m}^{3} \mathrm{~m}^{-3}\right)$ and $t$ is the time $(\mathrm{d})$. Therefore we may write the water balance of the radial flow pattern towards root as:

$$
\frac{\partial \theta}{\partial t}=-\frac{q}{r}-\frac{\partial q}{\partial r}
$$

The soil water flux itself can be described by the Darcy equation without the gravity component:

$$
q=-k \frac{\partial h}{\partial r}
$$

where $k$ is hydraulic conductivity and $h$ is soil water pressure head $(\mathrm{cm})$.

When we solve Eq. (3.32) using realistic values for extraction rates and soil hydraulic properties, we get very strong gradients $\partial h / \partial r$ near the root surface. This is caused by the rapid decline of the hydraulic conductivity at lower $h$ values and by the increasing flux density due to converging flow lines near the roots. In order to solve Eq. (3.31) numerically, in the past gross simplifications were required. However, Eq. (3.31) can be solved more accurately when we use the matric flux potential as driving variable, instead of the soil water pressure head. The matric flux potential $M\left(\mathrm{~m}^{2} \mathrm{~d}^{-1}\right)$ is defined as:

$$
M=\int_{h_{\mathrm{w}}}^{h} k(h) \mathrm{d} h
$$

where $h_{\mathrm{w}}$ is the pressure head corresponding to plant wilting point. With the matric flux potential we can simplify the Darcy equation:

$$
q=-k \frac{\partial h}{\partial r}=-\frac{\partial M}{\partial r}
$$

When we numerically solve Eq. (3.31) with the matric flux potential, resulting $M(r)$ profiles are much more linear than $h(r)$ profiles, which is a consequence of the linear character of Eq. (3.34). Use of the matric flux potential yields even analytical solutions for microscopic root water extraction, which was a prerequisite for use in ecohydrological models as SWAP. De Jong van Lier et al. (2008) describe the analytical solution in detail, and did upscale the microscopic approach to entire root zones with variable root densities and soil moisture conditions. In a later paper (De Jong van Lier et al., 2013) the authors extended the microscopic concept in the soil with radial and axial hydraulic resistances within the plant system. The approach of De Jong van Lier et al. (2013) been implemented in SWAP. For detailed theoretical background and applications we refer to both papers. 


\subsection{Actual soil evaporation}

At a wet soil, soil evaporation equals its potential rate $E_{\mathrm{p}}$. This is also the case at ponded conditions, during which SWAP will increase $E_{\mathrm{p}}$ to the evaporation rate of intercepted water with or without a ponding factor (Table 3.2). When the soil becomes drier, the soil hydraulic conductivity decreases, which may reduce $E_{\mathrm{p}}$ to evaporation rate, $E_{\mathrm{a}}\left(\mathrm{cm} \mathrm{d}^{-1}\right)$. In SWAP the maximum evaporation rate that the top soil can sustain, $E_{\max }\left(\mathrm{cm} \mathrm{d}^{-1}\right)$, is calculated according to Darcy's law:

$$
E_{\max }=K_{1 / 2}\left(\frac{h_{\mathrm{atm}}-h_{1}-z_{1}}{z_{1}}\right)
$$

where $K_{1 / 2}$ is the average hydraulic conductivity $\left(\mathrm{cm} \mathrm{d}^{-1}\right)$ between the soil surface and the first node, $h_{\text {atm }}$ is the soil water pressure head $(\mathrm{cm})$ in equilibrium with the air relative humidity, $h_{1}$ is the soil water pressure head $(\mathrm{cm})$ of the first node, and $z_{1}$ is the soil depth $(\mathrm{cm})$ at the first node. Equation (3.35) excludes water flow due to thermal differences in the top soil and due to vapour flow, as on daily basis the concerned flow amounts are probably negligible compared to isothermal, liquid water flow (Koorevaar et al., 1983; Ten Berge, 1986; Jury et al., 1991). Note that the value of $E_{\max }$ in Eq. (3.35) depends on the thickness of the top soil compartments. Increase of compartment thickness, generally results in smaller values for $E_{\max }$ due to smaller hydraulic head gradients. For accurate simulations at extreme hydrological conditions, the thickness of the top compartments should not be more than $1 \mathrm{~cm}$ (Van Dam and Feddes, 2000).

There is one serious limitation of the $E_{\max }$ procedure as described above. $E_{\max }$ is governed by the soil hydraulic functions $\theta(h)$ and $K(\theta)$. It is still not clear to which extent the soil hydraulic functions, that usually represent a top layer of a few decimeters, are valid for the top few centimeters of a soil, which are subject to splashing rain, dry crust formation, root extension and various cultivation practices, including mulch additions. Therefore also empirical evaporation functions may be used, which require calibration of their parameters for the local climate, soil, cultivation and drainage situation. SWAP has the option to choose the empirical evaporation functions of Black (1969) or Boesten and Stroosnijder (1986).

Reduction of soil evaporation according to Black

Black (1969) calculated the cumulative actual evaporation during a drying cycle, $\Sigma E_{a}(\mathrm{~cm})$ as:

$$
\sum E_{\mathrm{a}}=\beta_{1} t_{\mathrm{dry}}^{1 / 2}
$$

where $\beta_{1}$ is a soil specific parameter $\left(\mathrm{cm} \mathrm{d}^{-1 / 2}\right)$, characterizing the evaporation process and $t_{\mathrm{dry}}$ is the time (d) after a significant amount of rainfall, $P_{\mathrm{min}}$. SWAP resets $t_{\mathrm{dry}}$ to zero if the net precipitation $P_{\text {net }}$ exceeds $P_{\min }$.

\section{Reduction of soil evaporation according to Boesten and Stroosnijder}

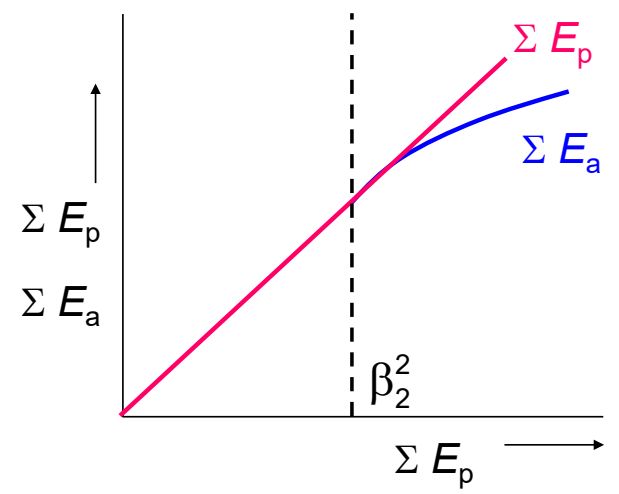

Figure 3.8 Evaporation fluxes according to Boesten and Stroosnijder (1986). 
The Black-parameter $\beta_{1}$ will be affected by the potential evaporation rates in the drying period. Therefore Boesten and Stroosnijder (1986) proposed to replace time by the sum of potential evaporation, $\Sigma E_{\mathrm{p}}(\mathrm{cm})$, as time variable (Figure 3.8):

$$
\begin{array}{llll}
\sum E_{\mathrm{a}}=\sum E_{\mathrm{p}} & \text { for } & \sum E_{\mathrm{p}} \leq \beta_{2}^{2} \\
\sum E_{\mathrm{a}}=\beta_{2}\left(\sum E_{\mathrm{p}}\right)^{1 / 2} & \text { for } & \sum E_{\mathrm{p}}>\beta_{2}^{2}
\end{array}
$$

where $\beta_{2}$ is a soil parameter $\left(\mathrm{cm}^{1 / 2}\right)$, which should be determined experimentally. The parameter $\beta_{2}$ determines the length of the potential evaporation period, as well as the slope of the $\Sigma E_{\mathrm{a}}$ versus $\left(\Sigma E_{\mathrm{p}}\right)^{1 / 2}$ relationship in the soil limiting stage.

Boesten and Stroosnijder suggest the following procedure with respect to updates of $\Sigma E_{\mathrm{p}}$. On days with no excess in rainfall $\left(P_{\text {net }}<E_{\mathrm{p}}\right), \Sigma E_{\mathrm{p}}$ follows from Eq. (3.37):

$$
\left(\sum E_{\mathrm{p}}\right)^{j}=\left(\sum E_{\mathrm{p}}\right)^{j-1}+\left(E_{\mathrm{p}}-P_{\text {net }}\right)^{j}
$$

in which superscript $j$ is the day number. $\left(\Sigma E_{\mathrm{a}}\right)^{\mathrm{j}}$ is calculated from $\left(\Sigma E_{\mathrm{p}}\right)^{\mathrm{j}}$ with $\mathrm{Eq} .(3.37)$ and $E_{\mathrm{a}}$ is calculated with

$$
E_{\mathrm{a}}^{j}=P_{\text {net }}^{j}+\left(\sum E_{\mathrm{a}}\right)^{j}-\left(\sum E_{\mathrm{a}}\right)^{j-1}
$$

On days of excess in rainfall $\left(P_{\text {net }}>E_{\mathrm{p}}\right)$

$$
E_{\mathrm{a}}^{j}=E_{\mathrm{p}}^{j}
$$

and the excess rainfall is subtracted from $\Sigma E_{\mathrm{a}}$

$$
\left(\sum E_{\mathrm{a}}\right)^{j}=\left(\sum E_{\mathrm{a}}\right)^{j-1}-\left(P_{\text {net }}-E_{\mathrm{p}}\right)^{j}
$$

Next $\left(\Sigma E_{\mathrm{p}}\right)^{j}$ is calculated from $\left(\Sigma E_{\mathrm{a}}\right)^{j}$ with Eq. (3.37). If the daily rainfall excess is larger than $\left(\Sigma E_{\mathrm{p}}\right)^{j-1}$, then both $\left(\Sigma E_{\mathrm{a}}\right)^{j}$ and $\left(\Sigma E_{\mathrm{p}}\right)^{j}$ are set to zero.

SWAP will determine $E_{\mathrm{a}}$ by taking the minimum value of $E_{\mathrm{p}}, E_{\max }$ and, if selected by the user, one of the empirical functions. 


\subsection{User instructions}

\subsubsection{General information}

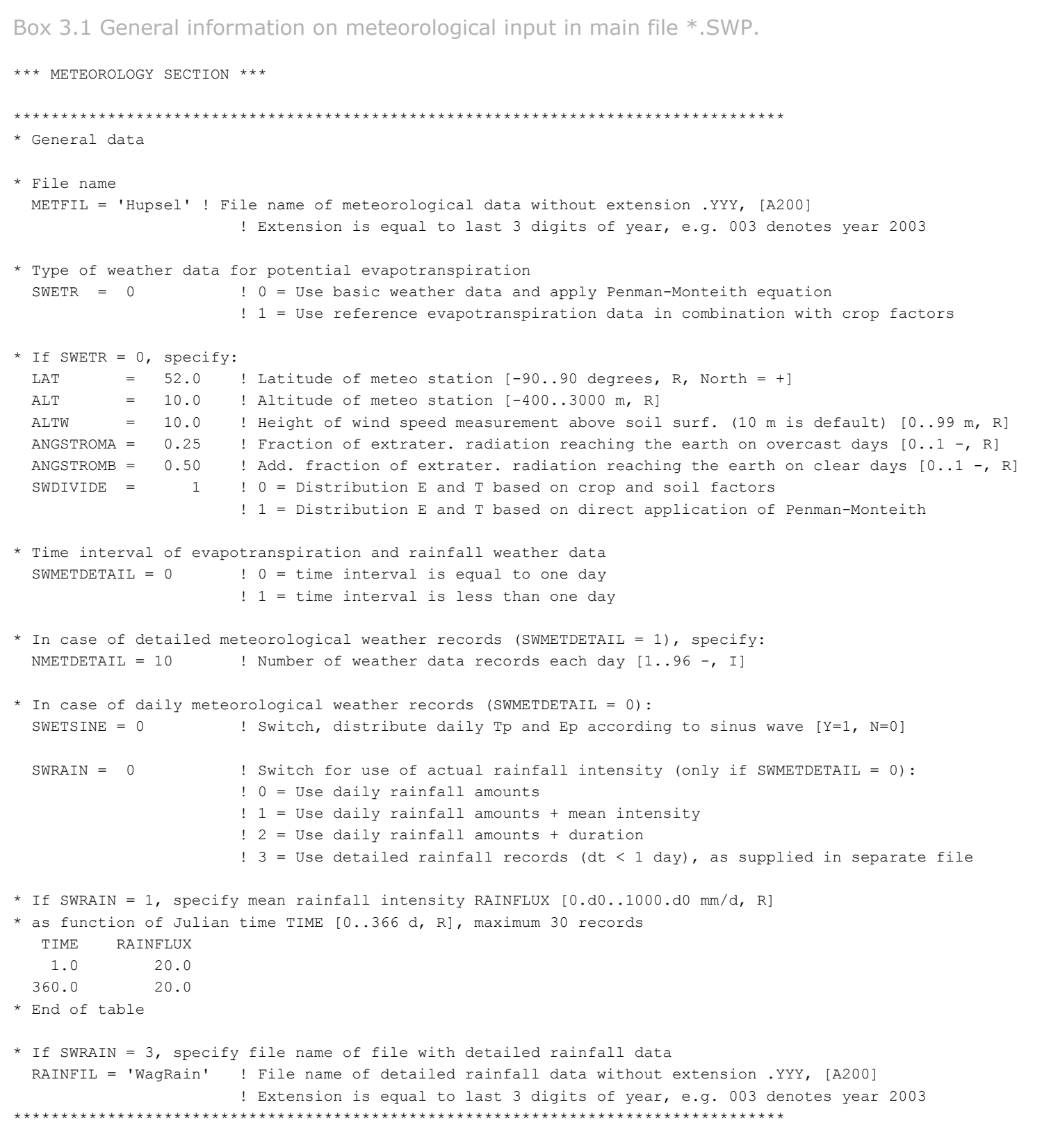

Box 3.1 lists the general input data with respect to evapotranspiration. The name of the meteorological files is generic, and the extension denotes the year. A main choice is whether precalculated $E T_{\text {ref }}$ are used $($ SWETR $=1$ ) or basic data on solar radiation, air temperature, air humidity and wind speed. These basic weather data may be specified daily or with shorter, constant time intervals (SWMETDETAIL = 1). In case of daily meteorological weather records, SWAP may distribute the evapotranspiration fluxes uniform over the day (default) or sinusoïdal during daylight (SWETSINE $=1$ ). As listed in Box 3.1, the rainfall input may range from daily amounts to short time rainfall amounts.

As described in Par. 3.4, the partitioning of potential transpiration and evaporation fluxes can be based on crop and soil factors, or on direct application of the Penman-Monteith equation. With the switch SWDIVIDE the user selects the preferred method. For many applications daily input of solar radiation, air temperature, air humidity and wind speed is preferred. In that case SWAP may apply the 
Penman-Monteith method to determine $E T_{\mathrm{p}}$. If some of these data are missing or unreliable, alternative methods to determine $E T_{\text {ref }}$ in combination with crop factors are advised.

Input of daily rainfall amounts will suffice for most applications. However when surface runoff is expected, daily rainfall amounts may underestimate the amount of surface runoff. To calculate surface runoff, actual rainfall intensities should be used.

\subsubsection{Weather data}

In case of daily weather records, the data should be specified as listed in Box 3.2. Missing data are given the number -99.9. When SWAP should use Penman Monteith (SWETR = 0, Box 3.1), data on solar radiation, air temperature ( $\min$ and $\max$ ), air humidity and wind speed are required. When SWAP should simulate detailed crop growth (Chapter 7), data on solar radiation and air temperature ( $\min$ and max) are required. For rainfall, either daily amounts (SWRAIN $=0$, Box 3.1 ) or daily amounts plus duration (SWRAIN $=2$, Box 3.1) should be specified.

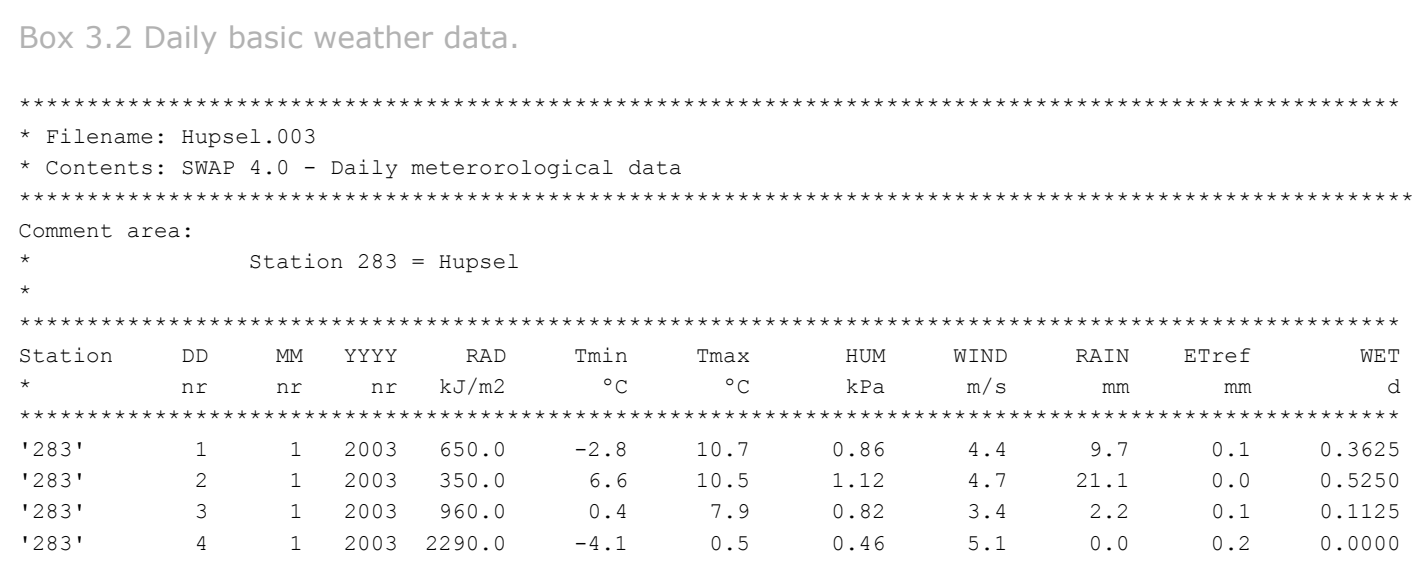

Alternatively weather records can be specified with short, constant time intervals ( $>15$ minutes) according to the format listed in Box 3.3. Radiation and rainfall denote total amounts during the time interval. Air temperature, humidity and wind speed denote average values during the time interval.

A third combination is daily evapotranspiration data and detailed rainfall data. In this case evapotranspiration data are input according to Box 3.2, while the rainfall data are input according to Box 3.4. The rainfall data follow the format of a tipping bucket measurement device: the rainfall amount corresponds to the total amount in the previous period. 


\begin{tabular}{|c|c|c|c|c|c|c|}
\hline \multicolumn{7}{|c|}{ 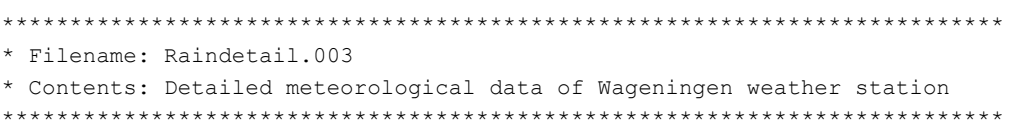 } \\
\hline \multicolumn{7}{|c|}{$\begin{array}{l}\star \\
\star\end{array}$} \\
\hline \multicolumn{7}{|c|}{ 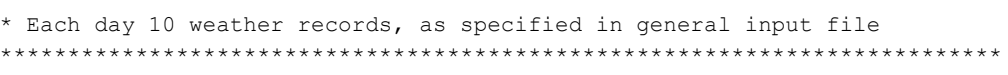 } \\
\hline * Date & $\begin{array}{r}\text { Record } \\
n r\end{array}$ & $\begin{array}{r}\mathrm{Rad} \\
\mathrm{kJ} / \mathrm{m} 2\end{array}$ & $\begin{array}{r}\text { Temp } \\
\text { 'C }\end{array}$ & $\begin{array}{l}\mathrm{Hum} \\
\mathrm{kPa}\end{array}$ & $\begin{array}{l}\text { Wind } \\
\mathrm{m} / \mathrm{s}\end{array}$ & $\begin{array}{r}\text { Rain } \\
\mathrm{mm}\end{array}$ \\
\hline \multicolumn{7}{|c|}{ 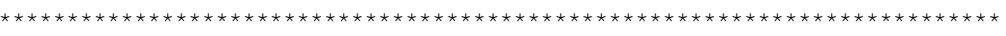 } \\
\hline $01-m a y-2003$ & 1 & 0.0 & 10.0 & 0.75 & 0.5 & 0.0 \\
\hline $01-m a y-2003$ & 2 & 3.0 & 10.0 & 0.76 & 0.4 & 0.1 \\
\hline $01-m a y-2003$ & 3 & 1347.0 & 9.0 & 0.76 & 0.6 & 1.2 \\
\hline $01-m a y-2003$ & 4 & 3622.0 & 8.5 & 0.74 & 1.2 & 4.7 \\
\hline $01-m a y-2003$ & 5 & 5029.0 & 10.5 & 0.78 & 2.4 & 0.0 \\
\hline $01-m a y-2003$ & 6 & 5029.0 & 15.3 & 0.85 & 4.0 & 0.0 \\
\hline $01-m a y-2003$ & 7 & 3622.0 & 14.0 & 0.84 & 3.5 & 0.0 \\
\hline $01-m a y-2003$ & 8 & 1347.0 & 13.5 & 0.82 & 2.0 & 0.0 \\
\hline $01-m a y-2003$ & 9 & 3.0 & 11.8 & 0.75 & 1.1 & 0.0 \\
\hline $01-m a y-2003$ & 10 & 0.0 & 10.5 & 0.73 & 0.7 & 0.0 \\
\hline $02-m a y-2003$ & 1 & 0.0 & 9.8 & 0.71 & 0.4 & 0.0 \\
\hline $02-m a y-2003$ & 2 & 8.0 & 9.0 & 0.69 & 0.3 & 0.0 \\
\hline $02-m a y-2003$ & 3 & 2046.0 & 8.5 & 0.68 & 0.5 & 0.0 \\
\hline $02-m a y-2003$ & 4 & 5429.0 & 12.0 & 0.76 & 1.2 & 0.0 \\
\hline $02-m a y-2003$ & 5 & 7520.0 & 14.5 & 0.82 & 2.5 & 0.0 \\
\hline
\end{tabular}

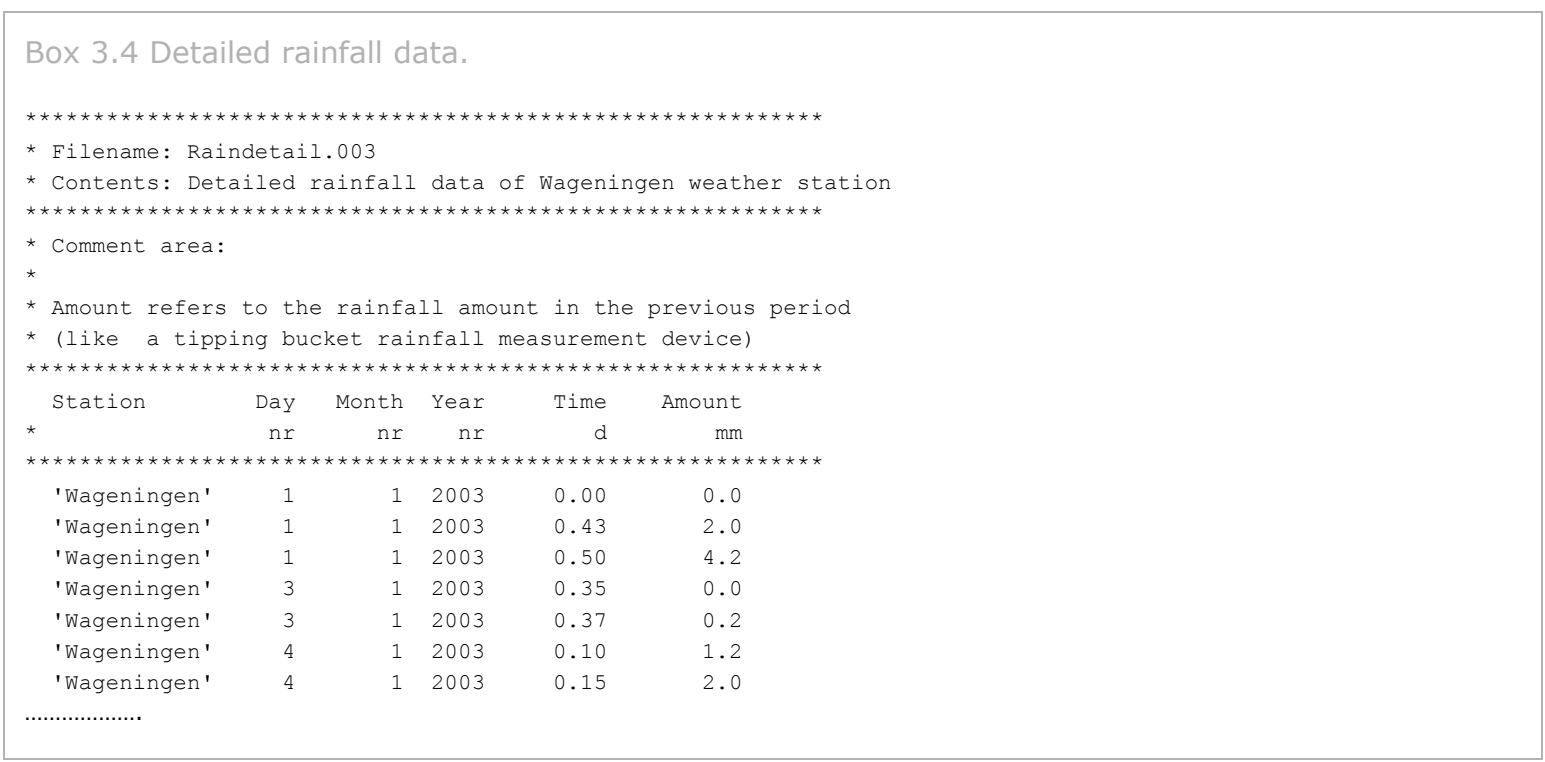

\subsubsection{Soil data}

Box 3.5 lists the soil data which are required to determine the actual evaporation at the soil surface. The soil factor CFBS can be used to transform reference crop evapotranspiration into potential soil evaporation (see Section 3.3.2 and Table 3.2). Commonly a soil factor CFBS $=0.5$ is used.

Three options are offered to reduce soil evaporation according to the maximum water flux which can be delivered by the soil. Applying straight soil physical theory, the maximum soil water flux according to Darcy would suffice. However, as discussed in Section 3.6, this method in general overestimates actual soil evaporation. Therefore we recommend to use in addition to the Darcy flux, reduction with either the Black or Boesten/Stroosnijder method (SWREDU $=1$ or 2).

Default soil evaporation coefficient for Black equals $0.35 \mathrm{~cm} \mathrm{~d}^{-0.5}$, and for Boesten/Stroosnijder $0.54 \mathrm{~cm}^{-0.5}$. 


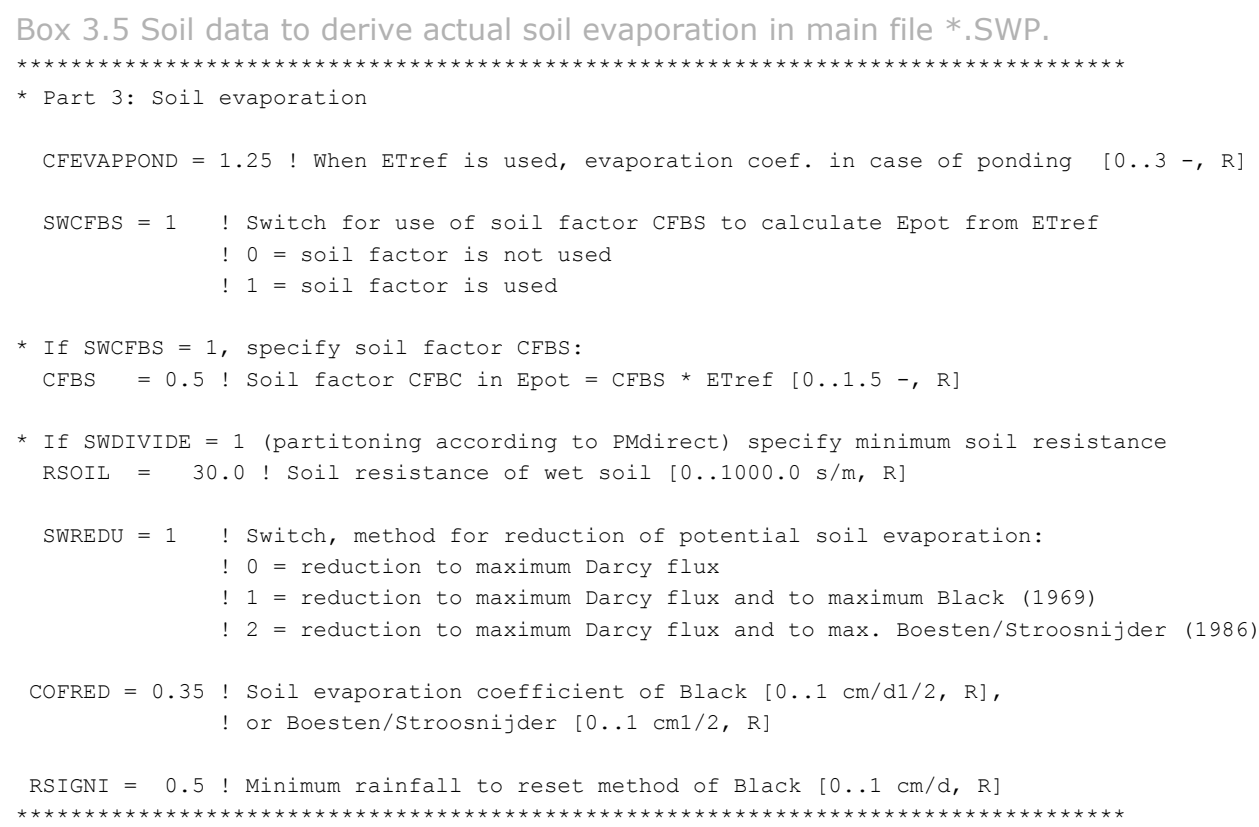

\subsubsection{Plant data}

The required plant input data to calculate evapotranspiration and rainfall interception will be listed at the end of Chapter 7. The theory in this chapter and the description in the input file should be sufficient to provide the proper input values for most variables. In this section we provide more information on the input for the detailed oxygen module (Par. 3.5.3). This module calculates the root uptake reduction factor $a_{\mathrm{rw}}$ as function of plant characteristics and plant physiological processes, rooting depth, gas filled porosity, soil temperature, soil physical properties and soil microbial activity.

Most input parameters required for the oxygen module (Table 3.3) are already standard input to SWAP simulations. When SWAP is used with the simple, static crop module, crop development is input and plant physiological processes are not simulated. Consequently, for the simulation of oxygen stress some additional crop parameters are required of i) characteristics of the plant roots to simulate the root oxygen demand and ii) soil characteristics to simulate the oxygen consumption by soil microbial activity (Table 3.3, column 'Stat'). Using dynamic crop development decreases the number of extra input parameters from nine to four (Table 3.3, column 'Dyn').

Using dynamic crop development (WOFOST) allows to describe the plant physiological processes, that determine the oxygen demand of plant roots, in more detail than with the static crop module. The following variables are relevant:

- rooting depth

- root biomass

- ratio total root respiration : maintenance respiration

WOFOST simulates rooting depth and root biomass dynamically (daily). Root biomass is used to simulate the root maintenance respiration. The oxygen module calculates total root respiration from the maintenance respiration by multiplication with a factor $\eta$ : the ratio of total respiration and maintenance respiration (Bartholomeus et al., 2008). The factor $\eta$ is derived on a daily basis from root growth respiration and maintenance respiration as simulated with WOFOST. For simulations with the simple crop module, $\eta$ is an input variable.

It should be noted that WOFOST uses air temperature to simulate the temperature dependence of the plants' maintenance respiration. This also holds for the simulation of root respiration. Within the oxygen module however, maintenance respiration is simulated with soil temperature as input.

It is optional to either calculate or simulate the root radius (Table 3.3, SwRootRadius). The latter option requires four extra input parameters. 
Table 3.4 provides oxygen stress parameter values for a static grass crop; Table 3.5 provides parameter values for dynamic crop growth simulation (WOFOST, see Chapter 7) for the crops grass, potato and maize.

Tabel 3.3 Overview of input parameters required for the simulation of oxygen stress for both a static (Stat) and dynamic (Dyn) crop. $X$ indicates if a parameter is input to Stat and/or Dyn.

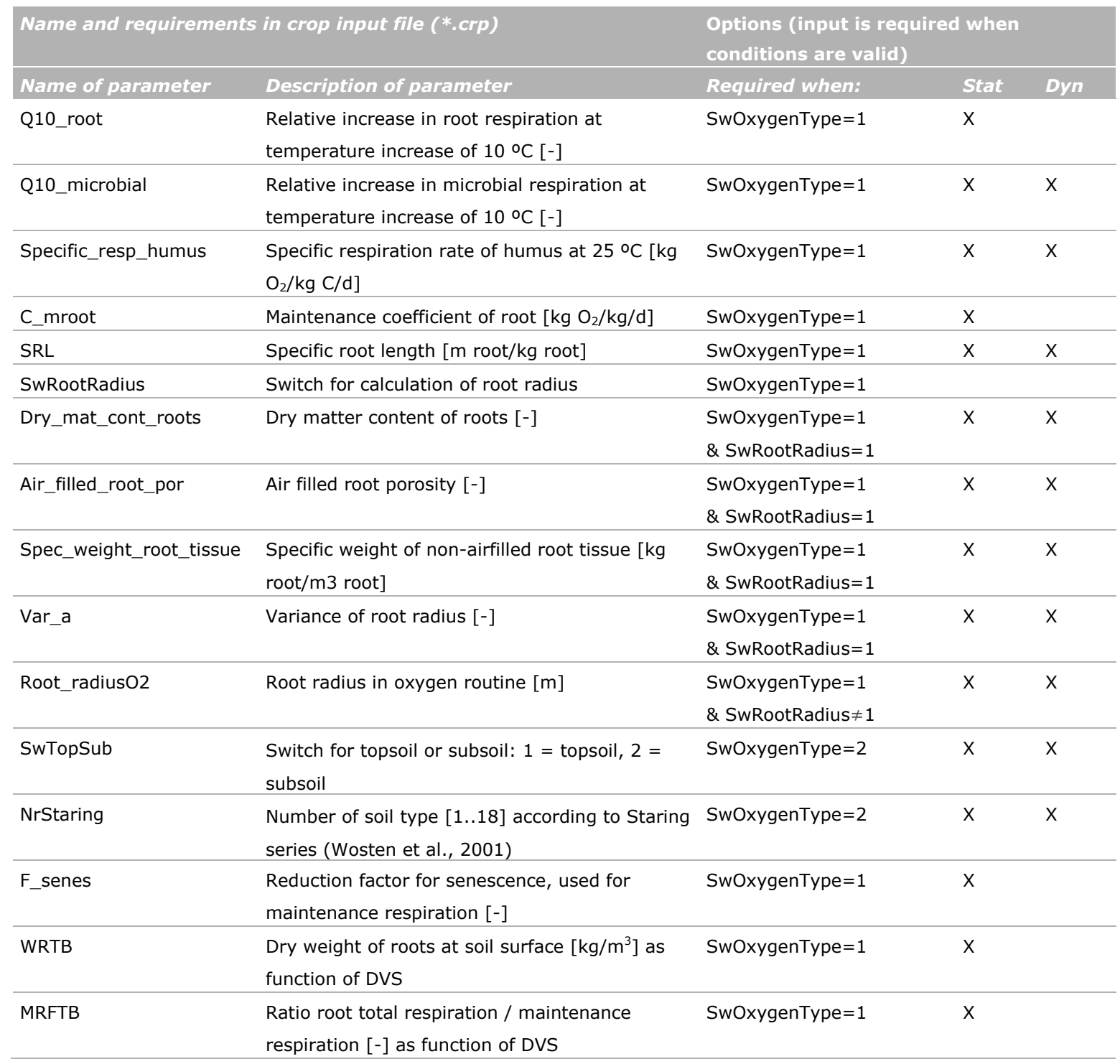


Table 3.4 Parameter values for grass for the simulation of oxygen stress with SWAP and a static crop.

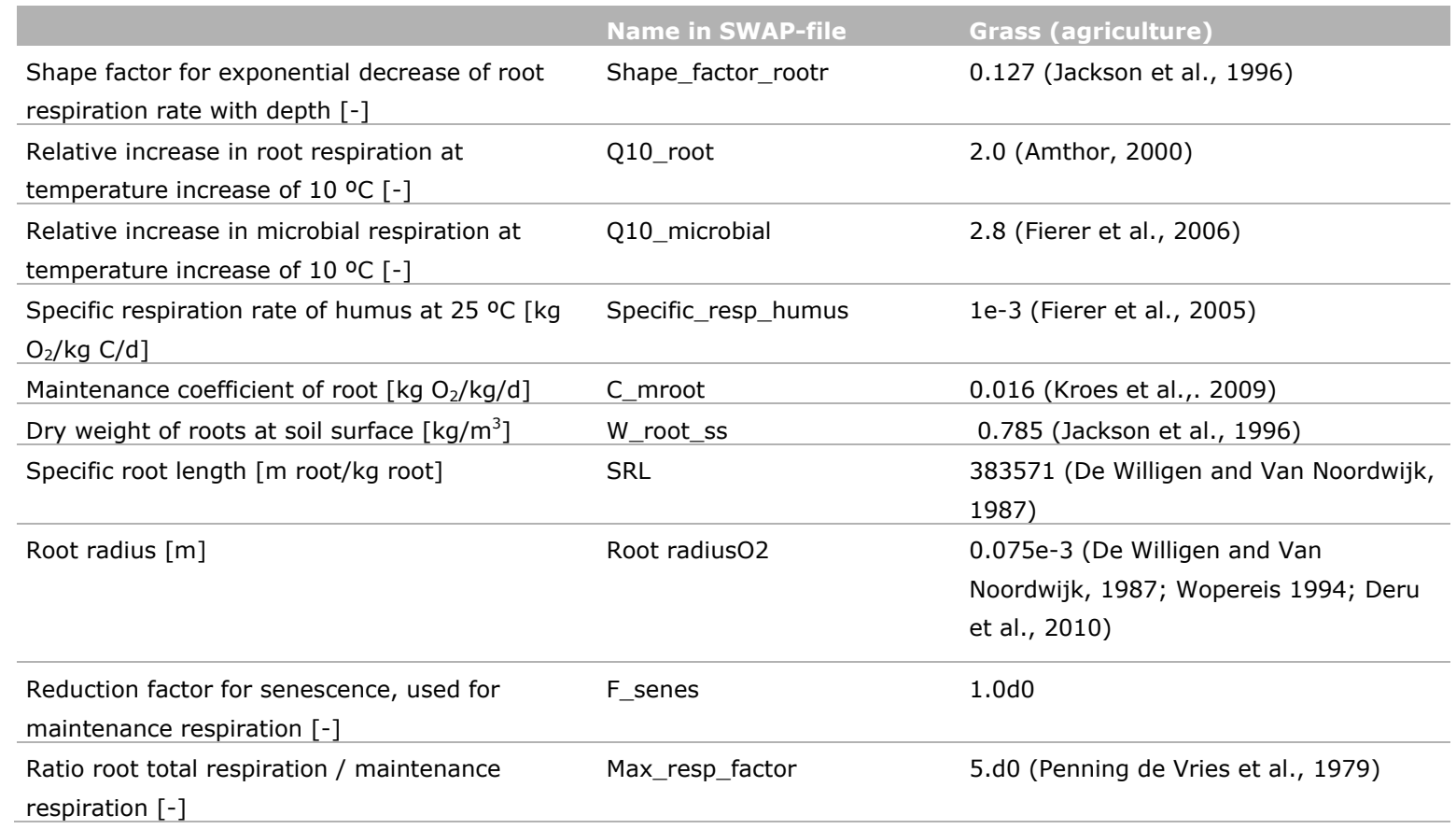

Table 3.5 Parameter values for the simulation of oxygen stress with SWAP-WOFOST for grass, potato and maize.

\begin{tabular}{|c|c|c|c|c|}
\hline & Name in SWAP-file & Grass & Potato & Maize \\
\hline $\begin{array}{l}\text { Specific respiration rate of } \\
\text { humus at } 25^{\circ} \mathrm{C}\left[\mathrm{kg} \mathrm{O}_{2} / \mathrm{kg}\right. \\
\mathrm{C} / \mathrm{d}]\end{array}$ & Specific_resp_humus & $\begin{array}{l}1.6 e-3 \text { (Fierer et al., } \\
2005 \text { ) - 'medium litter } \\
\text { quality' }\end{array}$ & Idem & Idem \\
\hline $\begin{array}{l}\text { Specific root length [m } \\
\text { root/kg root] }\end{array}$ & SRL & $\begin{array}{l}383571 \text { (De Willigen and } \\
\text { Van Noordwijk, 1987) }\end{array}$ & $\begin{array}{l}234000 \text { (De Willigen and } \\
\text { Van Noordwijk, 1987) }\end{array}$ & $\begin{array}{l}151375 \text { (De } \\
\text { Willigen and Van } \\
\text { Noordwijk, 1987) }\end{array}$ \\
\hline Root radius [m] & Root radius $\mathrm{O} 2$ & $\begin{array}{l}0.075 \text {-3 (De Willigen } \\
\text { and Van Noordwijk, } \\
\text { 1987; Wopereis 1994; } \\
\text { Deru et al., 2010) }\end{array}$ & $\begin{array}{l}0.14 \text {-3 (Rawsthorne } \\
\text { and Brodie, 1986; Vos } \\
\text { and Groenwold, 1986; } \\
\text { De Willigen and Van } \\
\text { Noordwijk, 1987; Iwama } \\
\text { 1998; Ehlert et al., } \\
\text { 2004) }\end{array}$ & $\begin{array}{l}0.15 e-3 \text { (De } \\
\text { Willigen and Van } \\
\text { Noordwijk, 1987; } \\
\text { Qin et al., 2006) }\end{array}$ \\
\hline
\end{tabular}




\section{Surface runoff, interflow and drainage}

The interaction between soil water and surface water is of importance in lowland areas. Dependent on the specific setting in the landscape of the field studied, different types of pathways and interconnections may play a role.

Surface runoff that occurs when the rainfall rate exceeds the infiltration rate is called Horton overland flow. A second form of runoff occurs after the water storage volume of a soil has been exceeded, which means that the groundwater table has reached the soil surface. This runoff is commonly called the Dunne overland flow. It occurs in areas with a shallow groundwater table and moderate rainfall of long duration.

Interflow can be defined as the near-surface flow of water within the soil profile resulting in seepage to a stream channel within the time frame of a storm hydrograph. Interflow involves both unsaturated and saturated flows, the latter being in zones of limited vertical extent caused by soil horizons impeding vertical percolation. The mechanisms by which subsurface flow enters streams quickly enough to contribute to streamflow responses to individual rainstorms are summarized in various publications (Beven 1989).

Infiltration excess moves slowly downwards and once it has reached the saturated zone, it is called ground water. Ground water moves downward and laterally through the subsurface and eventually discharges through tile drains, field ditches or other open conduits. A tile drain is a perforated conduit, such as tile, pipe or tubing, installed below the ground surface to intercept and convey drainage water.

The SWAP model can take account for the different types of interconnections between soil moisture/groundwater and surface water by offering options for describing surface runoff as a nonlinear function of water storage on the field, interflow as a non-linear function of the groundwater elevation when it has reached the near-surface zone and the discharge to a series of drainage systems. Options to simulate dynamically the levels in surface water systems provide the possibility to describe the feedback and the close interconnection between groundwater and surface water in stream valleys and polders. 


\subsection{Surface runoff}

Surface runoff is one of the terms in the water balance of the ponding reservoir. The ponding reservoir stores a certain amount of excess water on top of the soil surface (Fig.4.1).

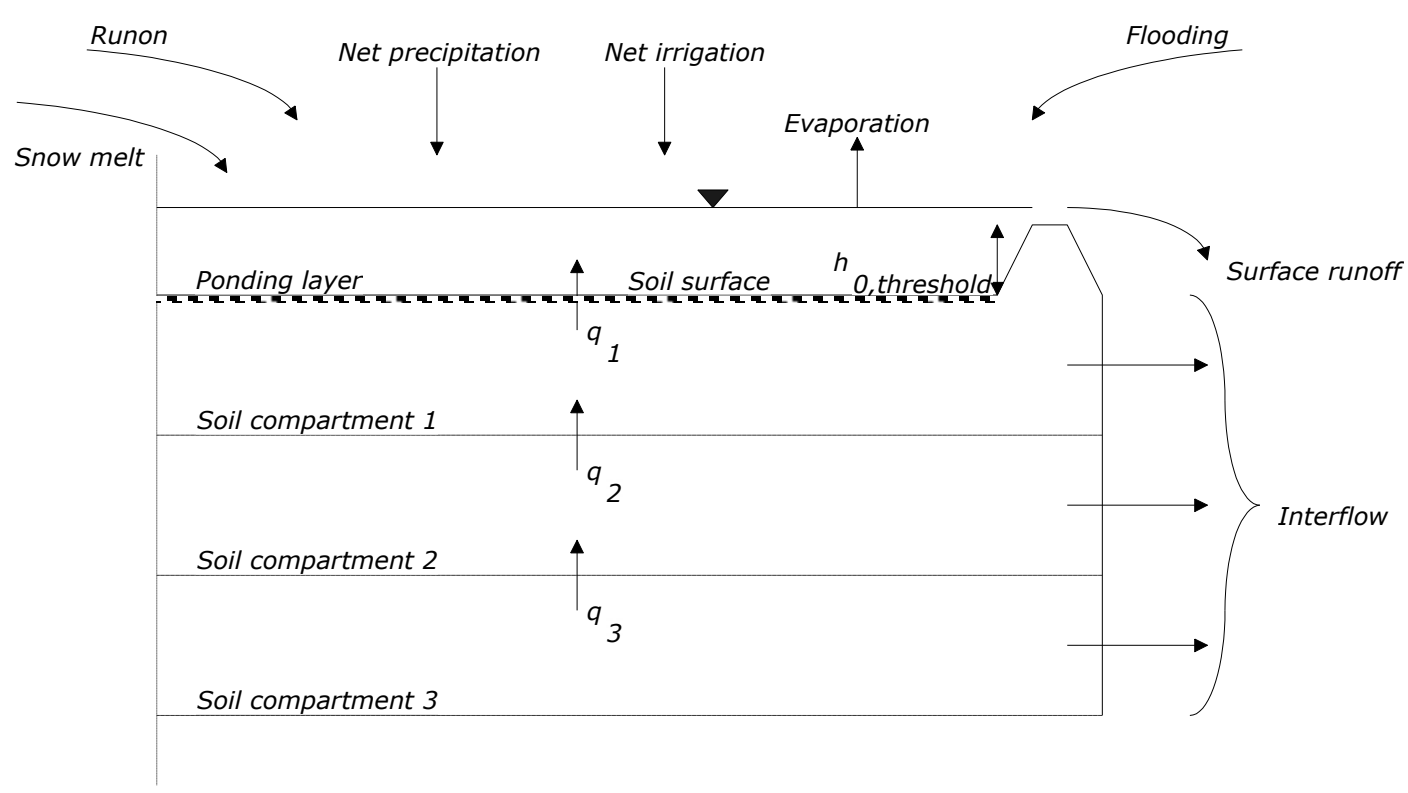

Figure 4.1 Schematic representation of the near surface flux to a surface water system and the water balance of the ponding layer.

The water balance of the ponding reservoir is governed by:

$$
\frac{\Delta h_{0}}{\Delta t}=q_{\text {prec }}+q_{\text {irri }}+q_{\text {melt }}+q_{\text {runon }}+q_{\text {inun }}+q_{1}-q_{\text {e,pond }}-q_{\text {runoff }}-I_{r u}
$$

Where $\Delta h_{0}$ is the storage change of the ponding reservoir $\left(\mathrm{cm} \mathrm{d}^{-1}\right), q_{\text {prec }}$ is the precipitation flux subtracted with interception, $\left(\mathrm{cm} \mathrm{d}^{-1}\right), q_{\text {irri }}$ is the irrigation flux subtracted with interception $\left(\mathrm{cm} \mathrm{d}^{-1}\right)$, $q_{1}$ is the flux from the first model compartment to the ponding layer ( $\left.\mathrm{cm} \mathrm{d}^{-1}\right), q_{\text {melt }}$ is snowmelt $\left(\mathrm{cm} \mathrm{d}^{-1}\right), q_{\text {runon }}$ is the runon flux of water which enters the field from an upstream adjacent field $\left(\mathrm{cm} \mathrm{d}^{-1}\right), q_{\text {inun }}$ is the inundation or flooding from surface water to the field $\left(\mathrm{cm} \mathrm{d}^{-1}\right), q_{\text {runoff }}$ is surface runoff flux $\left(\mathrm{cm} \mathrm{d}^{-1}\right)$ and $q_{e, p o n d}$ is the evaporation flux of the open water stored on the soil surface $\left(\mathrm{cm} \mathrm{d}^{-1}\right)$ and $I_{r u}$ is the runoff into the macropores $\left(\mathrm{cm} \mathrm{d}^{-1}\right.$, see Section 6.1.2)

Surface runoff occurs when the water storage in the ponding layer exceeds the critical depth of $h_{0, \text { threshold }}(\mathrm{cm})$ :

$$
q_{\text {runoff }}=\frac{1}{\gamma}\left(\max \left(0,\left(h_{0}-h_{0, \text { threshold }}\right)\right)^{\beta}\right.
$$

where $h_{0}$ is the ponding depth of water $(\mathrm{cm})$ on the soil surface, $\mathrm{y}$ is a resistance parameter $\left(\mathrm{cm}^{\beta-1} \mathrm{~d}\right)$ and $\beta$ is an exponent (-) in the empirical relation. Inundation of the field from an adjacent water

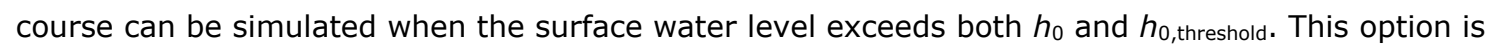
only available when the so-called extended drainage option is chosen (see Chapter 5). 


\subsection{Interflow}

In some applications one may wish to describe an interflow system, which has a rapid discharge with short residence times of the water in the soil system. If the groundwater level $\phi_{g w l}$ is higher than the hydraulic head of the drain $\phi_{d r a i n, n}$ the interflow flux is optionally calculated as:

$$
q_{\mathrm{drain}, \mathrm{n}}=\frac{\phi_{\mathrm{gwl}}-\phi_{\mathrm{drain}, \mathrm{n}}}{\max \left(\gamma_{\mathrm{min}}, 1 \cdot \phi_{\mathrm{gwl}}-\gamma_{\mathrm{ref}}\right)}
$$

or as:

$$
q_{\text {drain,n }}=A_{\text {interflow }}\left(\phi_{\mathrm{gwl}}-\phi_{\text {drain,n }}\right)^{B_{\text {interlow }}}
$$

where $q_{\mathrm{drain}, \mathrm{n}}$ is the interflow flux $\left(\mathrm{cm} \mathrm{d}^{-1}\right), \gamma_{\min }$ and $\gamma_{\text {ref }}$ are the minimum and the reference resistance related to the interflow process, ' 1 ' is a factor that expresses the unit conversion and is equal to one $\left(\mathrm{d} \mathrm{cm}^{-1}\right)$ in this case. $A_{\text {interflow }}$ is a conductance parameter $\left(\mathrm{cm}^{1-B} \mathrm{~d}^{-1}\right)$ and $B_{\text {interflow }}$ is an exponent (-) in the empirical relation. The subscript $n$ points to the rule that in the SWAP model interflow is always assigned to the highest order of distinguished drainage systems.

\subsection{Drain discharge}

Although the entity for which the SWAP model operates is at field scale, the model is used both for field studies and for regional studies. The different spatial scales of operation are expressed among other things by the type of drainage relation and its associated parameters chosen. For the purpose of a drainage system at field scale, one may use one of the classical drainage equations, but for simulation of water discharge in the spatial entity of a sub-catchment, the use of a multiple drainage system formulation is more convenient. Table 4.1 provides a brief overview of the drainage options available in the SWAP model. Additionally, options are available to take account for the influence of surface water management strategies on soil water flow and drain discharge. The background and the implementation of this option is presented in Chapter 5.

\begin{tabular}{|c|c|c|c|}
\hline $\begin{array}{l}\text { Scale of } \\
\text { application }\end{array}$ & No of systems & Drainage flux relation & Drainage level \\
\hline Field & $\begin{array}{l}\text { Single drainage } \\
\text { system }\end{array}$ & Hooghoudt or Ernst equation & Specified in model input \\
\hline \multirow[t]{3}{*}{ Regional } & $\begin{array}{l}\text { Single drainage } \\
\text { system }\end{array}$ & Tabulated input & Implicitly included in tabulated input \\
\hline & \multirow{2}{*}{$\begin{array}{l}\text { Multiple drainage } \\
\text { system }\end{array}$} & \multirow[t]{2}{*}{$\begin{array}{l}\text { Drainage resistance per sub-system dependent } \\
\text { on wetted perimeter of drains }\end{array}$} & $\begin{array}{l}\text { Specified in model input per } \\
\text { drainage system }\end{array}$ \\
\hline & & & Simulated (see Chapter 5) \\
\hline
\end{tabular}

Table 4.1 Options to simulate drain discharge at field scale and at regional scale.

The options provided by the SWAP model are limited to lowland conditions. Subsurface groundwater flow and drainage response of sloping fields can better be described by 2D or 3D models or Boussinesq-equation based models. Another limitation of the drainage equations involves the steadystate assumption. Hysteresis phenomena in the groundwater - discharge relation as they can be observed in experimental data are attributed to the different possible shapes of the groundwater elevation surface pertaining to one groundwater level value. Although there are possibilities to conceptualize the 2D groundwater depth discharge relation for nonsteady-state conditions (Kraijenhoff van de Leur, 1957, Wesseling and Wesseling, 1984), such relation is not implemented. These relations consider only one over-all value for the storage coefficient and neglect the influence of the pressure 
head variations in space and time on the storativity. If such phenomena are of interest for drainage flow simulations, the reader is referred to 2D and 3D models as MODFLOW-VSF (Thoms et al., 2006), SUTRA (Voss and Provost, 2002), FEMWATER (Lin et al., 1997), and HYDRUS (Šimůnek et al., 2007).

The drainage flux are incorporated in the numerical solution to the Richards equation (see Chapter 2) by specifying it as a sink term. The drainage relations presented in Section 4.3 are conceptualizations of $2 \mathrm{D}$ and $3 \mathrm{D}$ saturated groundwater flow to surface water systems and are based on the head difference between groundwater elevation and drainage level. Assignment of drainage sink term values in the Richards equation involves a conceptualization of the 2D and 3D flow field, which is briefly explained in Par. 4.4. Optionally, to provide possibilities to compare the SWAP model with other 1D soil moisture models as HYDRUS1D (Šimůnek et al., 1998), the drain flux can be described as a vertical flow in the model, which leaves the flow domain at the bottom.

It should be noticed that the calculation of drainage resistance should be attuned to the definition of the groundwater elevation as one of the driving forces of groundwater discharge and to the lower boundary condition one wants to impose. The general formulation of the drainage equation:

$$
q_{\text {drain }}=\frac{\left(\phi_{\mathrm{gwl}} \text { or } \phi_{\mathrm{avg}}\right)-\phi_{\mathrm{drain}}}{\gamma_{\text {drain }}}
$$

where $\phi_{\mathrm{gwl}}$ is the phreatic groundwater level midway between the drains or ditches $(\mathrm{cm}), \phi_{\mathrm{avg}}$ is the averaged phreatic groundwater level midway between the drains or ditches $(\mathrm{cm}), \phi_{\text {drain }}$ is the drainage level $(\mathrm{cm})$ and $\Upsilon_{\text {drain }}$ is the drainage resistance $(\mathrm{d})$.

Drainage relations are generally derived from the groundwater elevation as a function of distance. An example is given in Figure 4.2.

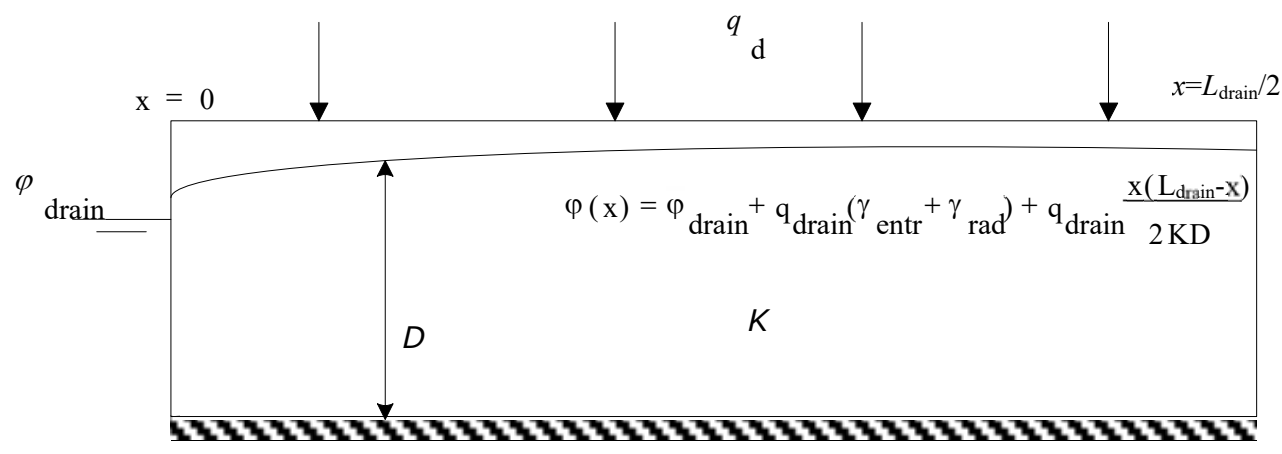

Figure 4.2 Groundwater elevation as a function of distance as the basis for drainage equations.

For drainage design purposes, one may be interested in the maximum groundwater elevation $\left(\phi_{\mathrm{gwl}}\right)$, but for the analysis of regional water management, the average groundwater elevation ( $\left.\phi_{\text {avg }}\right)$ is often a key variable to be studied. The different backgrounds reveals itself in the manner the drainage flux is calculated. For field applications, the relation between the drainage flux and the groundwater elevation can be expressed by the Ernst equation, modified with respect to the introduction of an additional entrance resistance :

$$
\phi_{\mathrm{gwl}}=\phi_{\text {drain }}+q_{\text {drain }}\left(\gamma_{\mathrm{entr}}+\gamma_{\mathrm{rad}}+\frac{L_{\mathrm{drain}}^{2}}{8 K D}\right) \rightarrow q_{\mathrm{drain}}=\frac{\phi_{\mathrm{gwl}}-\phi_{\mathrm{drain}}}{\gamma_{\mathrm{entr}}+\gamma_{\mathrm{rad}}+\frac{L_{\text {drain }}^{2}}{8 K D}}
$$


and for regional applications:

$$
\phi_{\text {avg }}=\phi_{\text {drain }}+q_{\text {drain }}\left(\gamma_{\text {entr }}+\gamma_{\text {rad }}+\frac{L_{\text {drain }}^{2}}{12 K D}\right) \rightarrow q_{\text {drain }}=\frac{\phi_{\text {avg }}-\phi_{\text {drain }}}{\gamma_{\text {entr }}+\gamma_{\text {rad }}+\frac{L_{\text {drain }}^{2}}{12 K D}}
$$

By comparing Eq. (4.6) with Eq. (4.7) it can be seen that the two definitions of $\gamma_{\text {drain }}$ in the equations differ by the so-called shape factor. The shape factor $a$ is the ratio between the mean and the maximum groundwater level elevation above the drainage base:

$$
\alpha=\frac{\phi_{\text {avg }}-\phi_{\text {drain }}}{\phi_{\mathrm{gwl}}-\phi_{\text {drain }}}
$$

The shape factor depends on the vertical, horizontal, radial and entrance resistances of the drainage system (Ernst, 1978). For regional situations, where the 'horizontal' resistance to flow plays an important role, the shape factor is relatively small $(\approx 0.7)$. The smaller the horizontal resistance becomes, the more 'rectangular' shaped the water table: in the most extreme case with all the resistance concentrated in the direct vicinity of the channel, the water table is level, except for the abrupt decrease towards the drainage base. In that case the shape factor approaches to unity.

It should be noted that the parameters chosen to describe the relation between discharge and groundwater elevation should be attuned to the hydrological schematization. The combination of a Cauchy condition for the bottom boundary with a drainage relation for the lateral boundary may require an other formula (De Lange, 1999) than the one usually applied for drainage combined with a flux bottom boundary condition. Also the coupling of the SWAP model to a regional groundwater model by exchanging information concerning fluxes and hydraulic heads at the bottom of the schematization may require alternative formulations for the drainage equation to be used.

The influence of frost at a certain depth can optionally be accounted for by the reduction of prevailing drainage fluxes at that depth similar to the reduction of hydraulic conductivities (see Chapter 2).

\subsubsection{Field scale drainage relation according to Hooghoudt and Ernst}

The drainage equations of Hooghoudt and Ernst allow the evaluation of drainage design and are based on the drainage flux as a function of the head difference the maximum groundwater elevation midway between the drains and the drainage level. Depending on position of the groundwater level, the drainage level and the possibility for water supply in the surface water system, the channels will act as either drainage or sub-irrigation media. The theory behind the field drainage equations used for drainage design purposes is summarized by Ritzema (1994). Five typical drainage situations are distinguished (Table 4.2). For each of these situations the drainage resistance $\gamma_{\text {drain }}$ (d) can be defined. 
Table 4.2 Five field drainage situations considered in SWAP (after Ritzema, 1994).

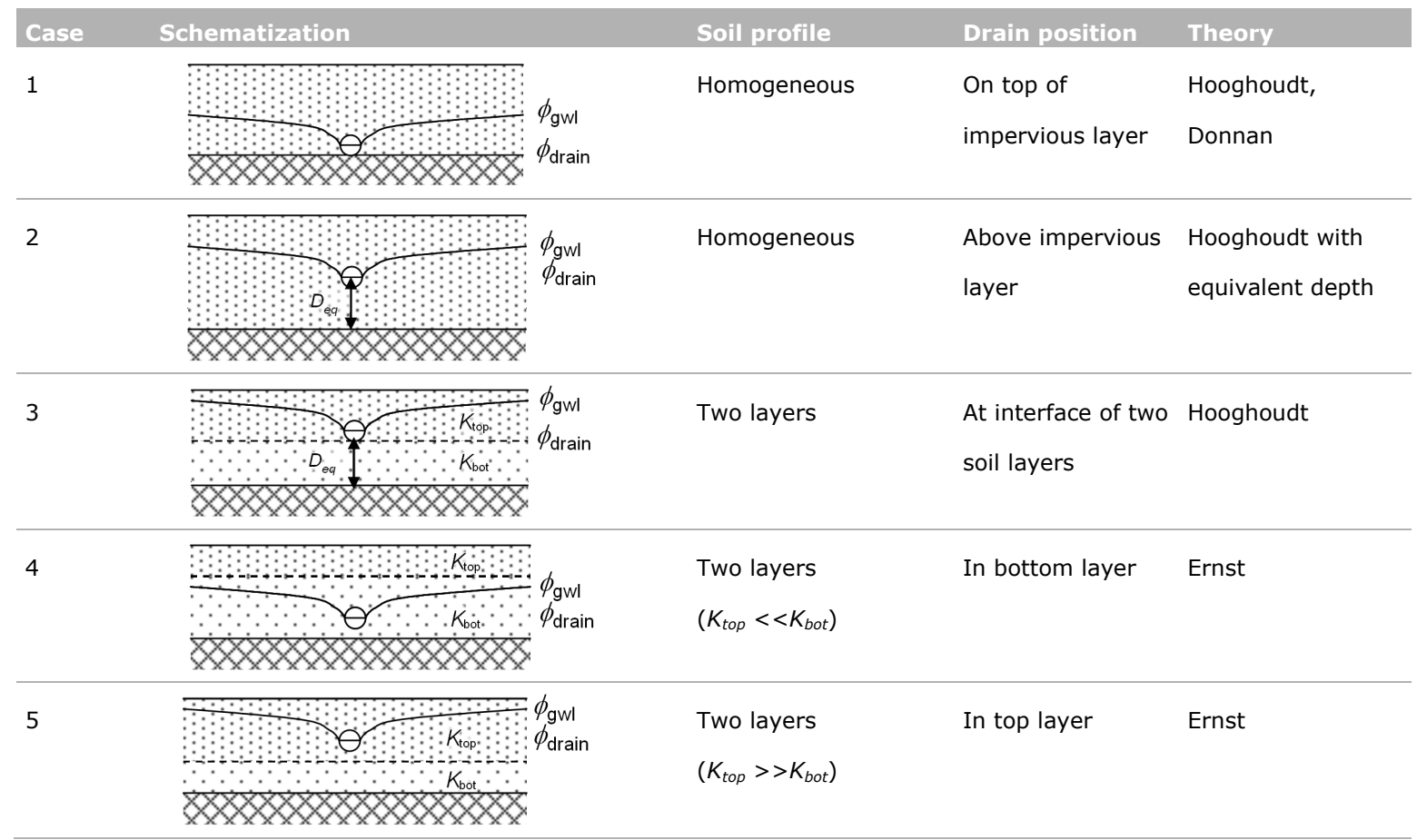

Case 1: Homogeneous profile, drain on top of impervious layer

The drainage resistance is calculated as:

$$
\gamma_{\text {drain }}=\frac{L_{\text {drain }}^{2}}{4 K_{\text {hprof }}\left(\phi_{\text {gwl }}-\phi_{\text {drain }}\right)}+\gamma_{\text {entr }}
$$

with $K_{\text {hprof }}$ the horizontal saturated hydraulic conductivity above the drainage basis ( $\mathrm{cm} \mathrm{d}^{-1}$ ), $L_{\text {drain }}$ the drain spacing $(\mathrm{cm})$ and $\gamma_{\text {entr }}$ the entrance resistance into the drains and/or ditches (d). The value for $\gamma_{\text {entr }}$ can be obtained, analogous to the resistance value of an aquitard, by dividing the 'thickness' of the channel walls with the permeability. If this permeability does not differ substantially from the conductivity in the surrounding subsoil, the numerical value of the entry resistance will become relatively minor.

\section{Case 2: Homogeneous profile, drain above impervious layer}

This drainage situation has been originally described by Hooghoudt (1940). An extension for the entrance resistance has been added later on. The drainage resistance follows from:

$$
\gamma_{\text {drain }}=\frac{L_{\text {drain }}^{2}}{8 K_{\text {hprof }} D_{\text {eq }}+4 K_{\text {hprof }}\left(\phi_{\mathrm{gwl}}-\phi_{\text {drain }}\right)}+\gamma_{\text {entr }}
$$

where $D_{e q}$ is the equivalent depth $(\mathrm{cm})$ that accounts for the extra head loss near the drains caused by converging flow lines (Hooghoudt, 1940). The numerical solution of Van der Molen and Wesseling (1991) is employed to obtain an estimate for $D_{\text {eq. }}$. A characteristic dimensionless length scale $x$ is used:

$$
x=2 \pi \frac{\phi_{\text {drain }}-z_{\text {imp }}}{L_{\text {drain }}}
$$

where $z_{\text {imp }}$ is the level of the impervious layer. The equivalent depth $D_{\text {eq }}$ is approximated for three ranges of $x$ as:

If $x<10^{-6}: \quad D_{\text {eq }}=\phi_{\text {drain }}-z_{\text {imp }}$ 
If $10^{-6}<x<0.5$ :

$$
\begin{aligned}
& D_{\text {eq }}=\frac{L_{\text {drain }}}{\frac{8}{\pi} \ln \left(\frac{\phi_{\text {drain }}-z_{\text {imp }}}{\pi r_{\text {drain }}}\right)+\frac{L_{\text {drain }}}{\phi_{\text {drain }}-z_{\text {imp }}}} \\
& D_{\text {eq }}=\frac{L_{\text {drain }}}{\frac{8}{\pi}\left(\ln \left(\frac{L_{\text {drain }}}{\pi r_{\text {drain }}}\right)+\sum_{k=1,3,5, . .}^{\infty} \frac{4 e^{-2 k x}}{k\left(1-e^{-2 k x}\right)}\right)}
\end{aligned}
$$

\section{Case 3: Heterogeneous soil profile, drain at interface between both soil layers}

The drainage resistance follows from:

$$
\gamma_{\text {drain }}=\frac{L_{\text {drain }}^{2}}{8 K_{\text {hbot }} D_{\text {eq }}+4 K_{\text {htop }}\left(\phi_{\mathrm{gwl}}-\phi_{\text {drain }}\right)}+\gamma_{\text {entr }}
$$

with $K_{\text {htop }}$ and $K_{\text {hbot }}$ the horizontal saturated hydraulic conductivity $\left(\mathrm{cm} \mathrm{d}^{-1}\right)$ of upper and lower soil layer, respectively. The equivalent depth $D_{\text {eq }}$ is calculated using Eq. (4.11) to Eq. (4.14).

\section{Case 4: Heterogeneous soil profile, drain in bottom layer}

The drainage resistance is calculated according to Ernst (1956) with later extensions for the entrance resistance as:

$$
\gamma_{\text {drain }}=\gamma_{\text {ver }}+\gamma_{\text {hor }}+\gamma_{\text {rad }}+\gamma_{\text {entr }}
$$

where $\gamma_{\text {ver, }} \gamma_{\text {hor, }} \gamma_{\text {rad }}$ and $\gamma_{\text {entr }}$ are the vertical, horizontal, radial and entrance resistance $\left(d^{-1}\right)$, respectively. The vertical resistance is calculated by:

$$
\gamma_{\mathrm{ver}}=\frac{\phi_{\mathrm{gwl}}-z_{\text {int }}}{K_{\mathrm{vtop}}}+\frac{z_{\text {int }}-\phi_{\mathrm{drain}}}{K_{\mathrm{vbot}}}
$$

with $z_{\text {int }}$ the level of the transition $(\mathrm{cm})$ between the upper and lower soil layer, and $K_{\mathrm{vtop}}$ and $K_{\mathrm{vbot}}$ the vertical saturated hydraulic conductivity $\left(\mathrm{cm} \mathrm{d}^{-1}\right)$ of the upper and lower soil layer, respectively. The horizontal resistance is calculated as:

$$
\gamma_{\text {hor }}=\frac{L_{\text {drain }}^{2}}{8 K_{\mathrm{hbot}} D_{\text {bot }}}
$$

with $D_{\text {bot }}$ the depth of the contributing layer below the drain level $(\mathrm{cm})$, which is calculated as the minimum of ( $\left.\phi_{\text {drain }}-z_{\text {imp }}\right)$ and $1 / 4 L_{\text {drain }}$. The radial resistance is calculated using:

$$
\gamma_{\text {rad }}=\frac{L_{\text {drain }}}{\pi \sqrt{K_{\text {hbot }} K_{\text {vbot }}}} \ln \left(\frac{D_{\text {bot }}}{u_{\text {drain }}}\right)
$$

with $u_{\text {drain }}$ the wet perimeter $(\mathrm{cm})$ of the drain.

\section{Case 5: Heterogeneous soil profile, drain in top layer}

Again the approach of Ernst (1956), with later extensions for the entrance resistance, is applied. The resistances are calculated as:

$$
\begin{aligned}
& \gamma_{\text {ver }}=\frac{\phi_{\mathrm{gwl}}-\phi_{\text {drain }}}{K_{\mathrm{vtop}}} \\
& \gamma_{\text {hor }}=\frac{L_{\text {drain }}^{2}}{8 K_{\mathrm{htop}} D_{\text {top }}+8 K_{\mathrm{hbot}} D_{\text {bot }}} \\
& \gamma_{\text {rad }}=\frac{L_{\text {drain }}}{\pi \sqrt{K_{\text {hbot }} K_{\text {vbot }}}} \ln \left(g_{\text {drain }} \frac{\phi_{\text {drain }}-z_{\text {int }}}{u_{\text {drain }}}\right)
\end{aligned}
$$


with $D_{\text {top }}$ equal to ( $\phi_{\text {drain }}-z_{\text {int }}$ ) and $g_{\text {drain }}$ is the drain geometry factor, to be specified in the input. The value of $g_{\text {drain }}$ in Eq. (4.22) depends on the ratio of the hydraulic conductivity of the bottom $\left(K_{\text {hbot }}\right)$ and the top $\left(K_{\text {htot }}\right)$ layer. Ernst (1962) distinguished the following situations:

$K_{\mathrm{hbot}} / K_{\mathrm{htop}}<0.1: \quad$ the bottom layer can be considered impervious and the case is reduced to

$0.1<K_{\text {hbot }} / K_{\text {htop }}<50: \quad g_{\text {drain }}$ depends on the ratios $K_{\text {hbot }} / K_{\text {htop }}$ and $D_{\text {bot }} / D_{\text {top }}$ as given in Table 4.3

$K_{\text {hbot }} / K_{\text {htop }}>50 \quad g_{\text {drain }}=4$

Table 4.3 The geometry factor $g_{d r a i n}(-)$, as obtained by the relaxation method (after Ernst, 1962).

\begin{tabular}{|c|c|c|c|c|c|c|c|}
\hline $\boldsymbol{K}_{\text {hbot }} / \boldsymbol{K}_{\text {htop }}$ & $D_{\text {bot }} / D_{\text {top }}$ & & & & & & \\
\hline & 1 & 2 & 4 & 8 & 16 & 32 & 1 \\
\hline 1 & 2.0 & 3.0 & 5.0 & 9.0 & 15.0 & 30.0 & 2.0 \\
\hline 3 & 2.6 & 3.3 & 4.5 & 5.5 & 6.8 & 8.0 & 2.6 \\
\hline 5 & 2.8 & 3.5 & 4.4 & 4.8 & 5.6 & 6.2 & 2.8 \\
\hline 20 & 3.6 & 3.7 & 4.0 & 4.2 & 4.4 & 4.6 & 3.6 \\
\hline 50 & 3.8 & 4.0 & 4.0 & 4.0 & 4.2 & 4.6 & 3.8 \\
\hline
\end{tabular}

\subsubsection{Field scale drainage relation defined by a tabulated function}

The SWAP model provides an option to specify a tabulated drainage flux relationship as a function of the groundwater level. When this option is chosen, one should specify a number of $\left(\varphi_{\mathrm{gwl}}, q_{\mathrm{drain}}\right)$ datapairs. For a linear relation only two data-pairs suffices, but a non-linear relation requires more datapairs. A non-linear relation can be either the result of:

- describing the drainage flux by the Hooghoudt equation;

- an analysis of the flux relation in a stratified profile by means of a numerical model

- an analysis of measured field data.

The general shape of such a relation is given in Figure 4.3. Specifying a non-linear relation by means of a tabulated function involves a linearization, since flux values are derived by linear interpolation. Specifying more data pairs can reduce the inaccuracy which results from this type of linearization.

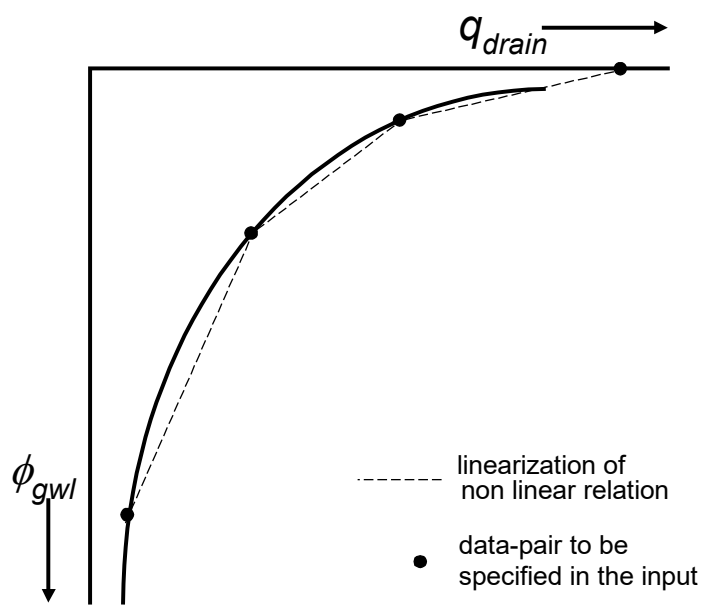

Figure 4.3 Linearization of a non-linear drainage flux relation. 


\subsubsection{General aspects of regional scale drainage}

\section{Schematization}

The groundwater-surface water system is described at the scale of a horizontal sub-region. A network of drainage devices consists of a hierarchical system of different order and incision depth, but only a single representative groundwater level is simulated for a sub-region, which is 'stretched' over a scale that in reality involves a variety of groundwater levels. In the following, due consideration will be given to the schematization of the surface water system, the simulation of drainage/sub-irrigation fluxes, and the handling of an open surface water level.

The regional surface water system consists of a hierarchical system of different order drainage devices (Figure 4.4), each with its own with bed level, bed width, side-slope, and spacing conveyance capacity. The drainage devices can be connected to each other in different ways. In the man-made the ditches of the network systems act as perennial streams, connected to larger canals with a nearly equal surface water level. In the alluvial sandy areas of the Netherlands, the smaller streams may have intermittent character which only discharge water in periods with rainwater excess.

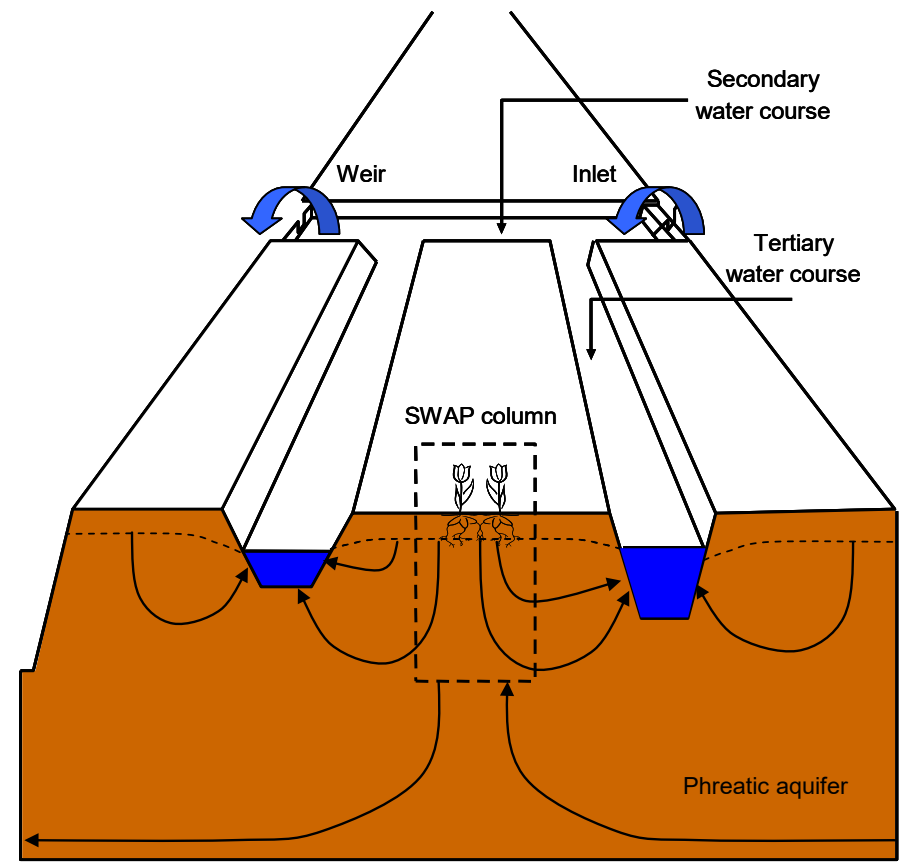

It should be noted that contrary to the classification notation used in geomorphological sciences, in this report the stream and canal order is dictated by the level of the stream bed or water level (drainage level) compared to the land surface level: the deeper the drainage level, the lower classification index.

The representative distance between drain devices $L_{\text {drain, }}(m)$ is derived by dividing the area of the subregion $A_{\text {reg }}$ $\left(\mathrm{m}^{2}\right)$ by the total length of the $i^{\text {th }}$ order channels, $I_{\text {drain,i }}(\mathrm{m})$ :

$$
L_{\text {drain,i }}=\frac{A_{\text {reg }}}{l_{\text {drain, } \mathrm{i}}}
$$

Figure 4.4 Schematization of surface water system in a control unit.

In the surface water model, we assume that the different channels orders are connected in a dendritic manner. Together they form a surface water 'control unit' with a single outlet (indicated by the weir in Figure 4.4) and, if present, a single inlet. The surface water level at the outlet is assumed to be omnipresent in the subregion. Friction losses are neglected and thus the slope of the surface water level is assumed to be zero. This means that in all parts of the subregion the surface water level has the same depth below soil surface.

In the so-called 'multi-level' drainage or sub-irrigation approach employed by the SWAP model, it is possible that more than one type of surface water channel become active simultaneously. In the following, we will refer to channels in terms of their 'order' if their role as part of the surface water system is being considered. When considering their drainage characteristics we will refer to them in terms of their 'level'.

The SWAP model has the option for specifying resistances for calculating the sub-irrigation flux that differ from the resistance values used for drainage. An additional model option involves the limitation of the sub-irrigation rate by defining the groundwater level $\phi_{\mathrm{avg}}^{\mathrm{min}}$ at which the maximum sub-irrigation 
rate is reached. Such a limitation is needed because the sub-irrigation rate does not increase infinitely when the groundwater level lowers.

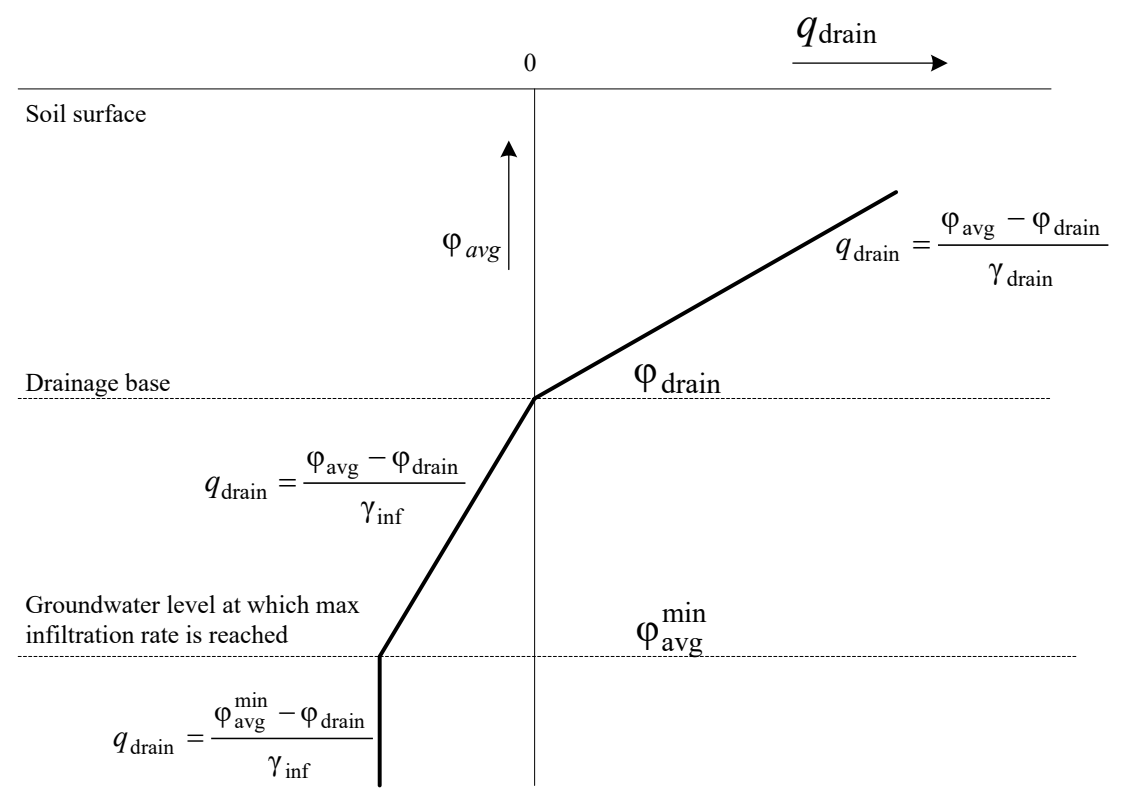

Figure 4.5 Lineair relationships between drainage $\left(q_{d r a i n}>0\right)$ and infiltration $\left(q_{d r a i n}<0\right)$ flux and mean groundwater level $\phi_{a v g}$.

\subsubsection{Regional scale drainage relation defined by a tabulated function}

An example of a non-linear relation between discharge and groundwater elevation resulting from an analysis of observed field data is presented in Figure 4.6 (Massop and De Wit, 1994). It can be seen from this figure that the non-linear relation may be linearized to a piece-wise linear relation in which each part of this relation corresponds to a certain type of drainage system. From Figure 4.6 one can infer that the drainage base of the larger channels is roughly at $z=-120 \mathrm{~cm}$, as no discharges were measured below that level. The schematized $q_{\text {drain }}\left(\phi_{\text {avg }}\right)$-relationship has transition points at mean groundwater levels of 80 and $55 \mathrm{~cm}$ below soil surface. These transition points correspond to the 'representative' bed levels of the second and third order channels. These levels could be imposed to the SWAP model as drainage levels. 


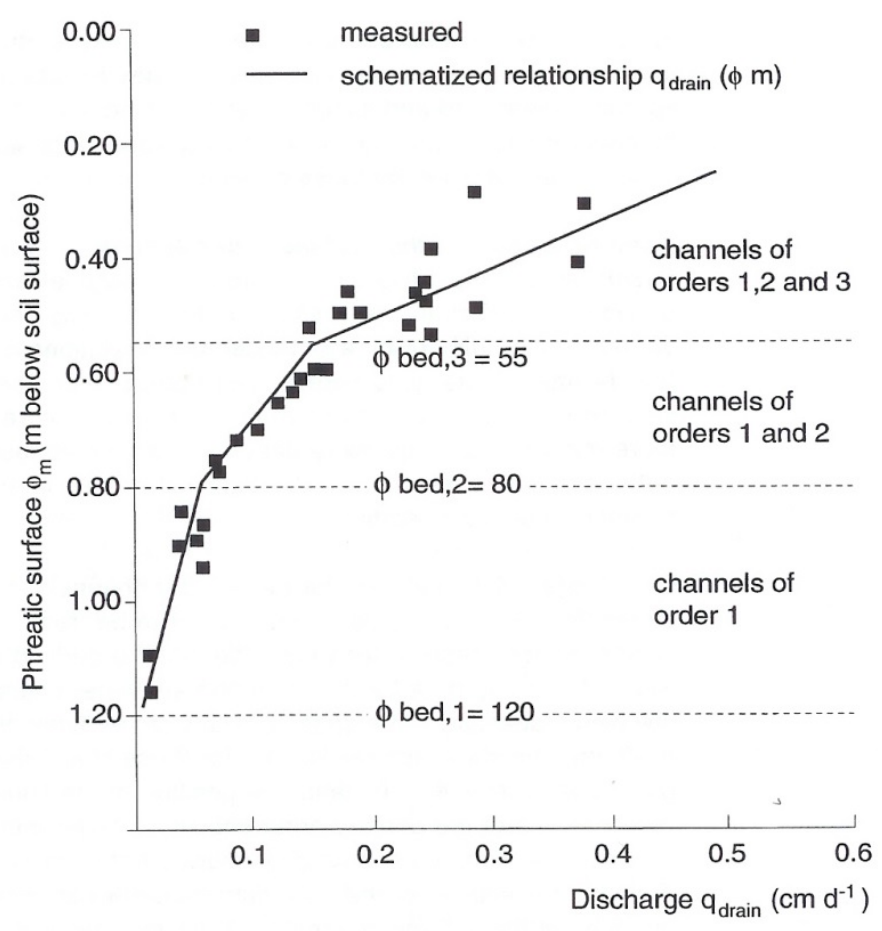

Figure 4.6 Discharge $q_{d r a i n}$ as function of mean phreatic surface $\phi_{a v g}$ in the Beltrum area (Massop and de Wit, 1994).

\subsubsection{Multi-level drainage with fixed resistances and imposed drainage levels}

Prior to any calculation of the drainage/sub-irrigation rate, we determine whether the flow situation involves drainage, sub-irrigation, or neither. No drainage or sub-irrigation will occur if both the groundwater level and surface water level are below the drainage base.

Drainage will only occur if the following two conditions are met:

- the groundwater level is higher than the channel bed level;

- the groundwater level is higher than the surface water level.

Sub-irrigation can only occur if the following two conditions are met:

- the surface water level is higher than the channel bed level;

- the surface water level is higher than the groundwater level.

In both cases we take for the drainage base, $\phi_{\text {drain }}(\mathrm{cm})$, either the surface water level, $\phi_{\text {sur }}(\mathrm{cm})$, or the channel bed level, $z_{\text {bed }}(\mathrm{cm})$, whichever is higher:

$$
\phi_{\text {drain }}=\max \left(\phi_{\text {sur }}, z_{\text {bed }}\right)
$$

The hydraulic head $\phi$ is defined positive upward, with zero at the soil surface.

The drainage/infiltration flux $q_{\mathrm{drain}, \mathrm{i}}\left(\mathrm{cm} \mathrm{d}^{-1}\right)$ to/from each surface water system $i$ is calculated from the linear relation:

$$
q_{\text {drain,i }}=\frac{\phi_{\text {avg }}-\phi_{\text {drain,i }}}{\gamma_{\text {drain,i }}}
$$

where $\phi_{\text {drain, } i}$ is the drainage base is equal to the surface water level of system $i$ (cm below the soil surface) and $\gamma_{\text {drain, } i}$ is the drainage resistance of system $i$ (d). Similar to the case of single-level drainage, a drainage level is only 'active' if either the groundwater level or the surface water level is higher than the channel bed level. The drainage base is determined separately for each of the 
drainage levels. In computing the total flux $q_{\text {drain }}^{\text {tot }}$ to/from surface water, the contributions of the different channel orders are simply added:

$$
q_{\mathrm{drain}}^{\mathrm{tot}}=\sum_{i=1}^{n} q_{\mathrm{drain}, \mathrm{i}}
$$

\subsubsection{Multi-level drainage with surface water dependent resistances and simulated drainage levels}

In most applications, the control unit involves the primary watercourse; the largest canals with the deepest channels beds. An option is available to specify that the primary watercourse, e.g., a large river, functions separately from the other watercourses within the sub-regional surface water system. In that case the primary water courses have their own surface water level which should be specified in the input. In the real situation there may be some interaction between the primary water course and the control unit: for instance a pumping station for removal of drainage water, and/or an inlet for letting in external surface water supply (Figure 4.4). The hydraulics of such structures are not included in the model. Conveyance processes within the surface water devices are not described.

Contrary to the model option described in Par 4.3.5, the influence of the surface water level on drainage resistances can be accounted for by distinguishing two parts of the resistance: 1) a part independent of the surface water level and 2) a part that is adjusted by the level. For the drainage case:

$$
\gamma_{\text {drain,i }}=\gamma_{\text {drain,i }}^{0}+\gamma_{\text {entr,i }}^{*} \frac{L_{\text {drain,i }}}{u_{\text {drain,i }}}
$$

And for the sub-infiltration case:

$$
\gamma_{\text {infil,i }}=\gamma_{\text {infil,i }}^{0}+\gamma_{\text {exit,i }}^{*} \frac{L_{\text {drain,i }}}{u_{\text {drain,i }}}
$$

where $\gamma_{\text {drain,i }}^{0}$ and $\gamma_{\text {infili, }}^{0}$ are the level independent parts of the drainage and infiltration resistance and resp. $\gamma_{\text {entr,i }}^{*}$ and $\gamma_{\text {exit,i }}^{*}$ are the entrance and exit resistance factor per unit drain distance $\left(L_{\text {drain,i }}\right)$ and divided by the wetted perimeter $\left(u_{\text {drain,i }}\right)$. The radial resistance has been lumped with the entrance or exit resistance. By assuming a trapezoidal cross section of the water courses, the wetted perimeter can be calculated as:

$$
u_{\text {drain }, i}=w_{\text {bed }, i}+\max \left(0,2 \cdot\left(\varphi_{\text {sur }}-z_{\mathrm{bed}, \mathrm{i}}\right) \sqrt{1+1 / \alpha_{\mathrm{sl}, \mathrm{i}}^{2}}\right)
$$

where $w_{\text {bed,i }}$ is the channel bed width $(\mathrm{cm})$ and $\alpha_{\mathrm{sl}, \mathrm{i}}(-)$ is the slope of the channel bank.

Another feature of this model option includes the ability to simulate the flooding of the field when the surface water level higher appears to be higher than both the ponding sill and the ponding level or groundwater level (Figure 4.7). 


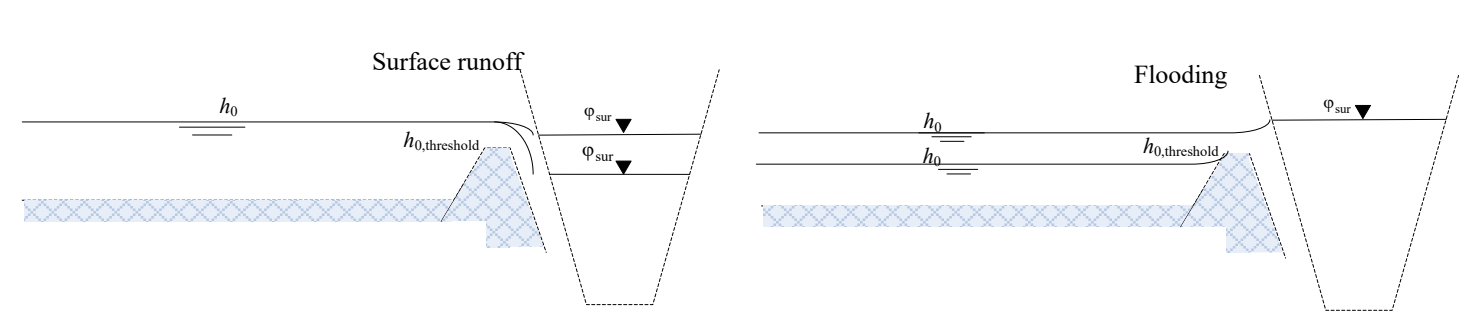

Figure 4.7 In-between position of groundwater/ponding level $h_{0}$ and surface waterlevel $\phi_{\text {sur }}$ in case of surface runoff (left) and flooding (right).

The model concept for flooding does not take account for the resistance of water flowing on the field surface and an immediate equilibrium between the ponding level and the surface water level is assumed when flooding occurs.

\subsection{Distribution with depth of drainage fluxes}

\subsubsection{Implicit approach of travel times}

In this section, the concept for the distribution drainage fluxes with depth as one of the sink terms in the SWAP model is described. Although the concept discussed here is valid for a region having any number of drainage levels, only three drainage systems are considered for reasons of convenience.

One-dimensional leaching models generally represent a vertical soil column. Within the unsaturated zone, solutes are transported by vertical water flows, whereas in the saturated zone the drainage discharge can have a three dimensional flow pattern. Van Ommen (1986) has shown that for a simple single-level drainage system, the travel time distribution is independent from the size and the shape of the recharge area. Under these assumptions, the average concentration in drainage water can mathematically be described by the linear behaviour of a single reservoir. But, the non-homogeneous distribution of exfiltration points, the variety of hydraulic properties and the influence of stratified soil chemical characteristics necessitates to distinguish between the different soil layers.

The distribution of drainage fluxes with depth is used to describe the travel time distribution of drainage water in an implicit manner. Drainage fluxes are treated as lateral sink terms of the water balance in the SWAP model. The vertical flux qy in the saturated zone of the SWAP model relates to the distribution of lateral drainage rate sink terms according to:

$$
\frac{\mathrm{d} q_{\mathrm{y}}}{\mathrm{d} y}=-\frac{q_{\mathrm{drain}}}{D}
$$

where $D$ is the depth of the zone for which $q_{\text {drain }}$ has a certain value. Assume that a fluid particle is at the depth of $y_{0}$ at time $t_{0}$. The time it takes for this particle to reach a depth $y$ is given by:

$$
t-t_{0}=\int_{y_{0}}^{y} \frac{\varepsilon \mathrm{d} y}{q_{y}(y)}
$$

The travel time relation is governed by the vertical flux as a function of depth and the porosity. It can be seen from Eq. (4.30) that the vertical flux coheres with the drainage flux.

\subsubsection{Discharge layers}

The concept of the distribution of the drainage flux with depth for a single level drainage system can be very simple. The Dupuit-Forcheimer assumption involves disregarding the head loss due to radial flow and vertical flow in the largest part of the flow domain. Based on this rule, the groundwater movement towards drains in a non-stratified aquifer is considered as an uni-directional flow and the drainage flux is distributed uniformly with depth. 
The distribution with depth for a multi-level drainage system should ideally be based on the 3-D flow paths of water parcels migrating to drains. But since such type of information is not available in the 1-D vertical model, additional assumptions have to be made. The concept of discharge layers has been introduced, representing the flow systems associated with each of the drains. Although the verification of the concept by comparing the depth of discharge layers with the streamline patterns generated by 2D models do not always agree, the concept enables the accounting of the different types of water courses and the stratification of hydraulic properties in the implicit travel time approach.

The discharge layers are considered as horizontal layers. Each layer occupies a certain part of the groundwater volume. The ratio between the occupied flow volumes $V_{i}$ is derived from the proportionality between flow volumes and volumetric discharge rates:

$$
\frac{V_{\mathrm{i}}}{V_{\mathrm{i}-1}}=\frac{Q_{\text {drain,i }}}{Q_{\text {drain,i-1 }}}
$$

The volumetric flux $\mathrm{Q}_{\mathrm{drain}, \mathrm{i}}$ to drainage system $\mathrm{i}$, is calculated as:

$$
Q_{\text {drain,i }}=L_{\text {drain,i }} q_{\text {drain,i }}
$$
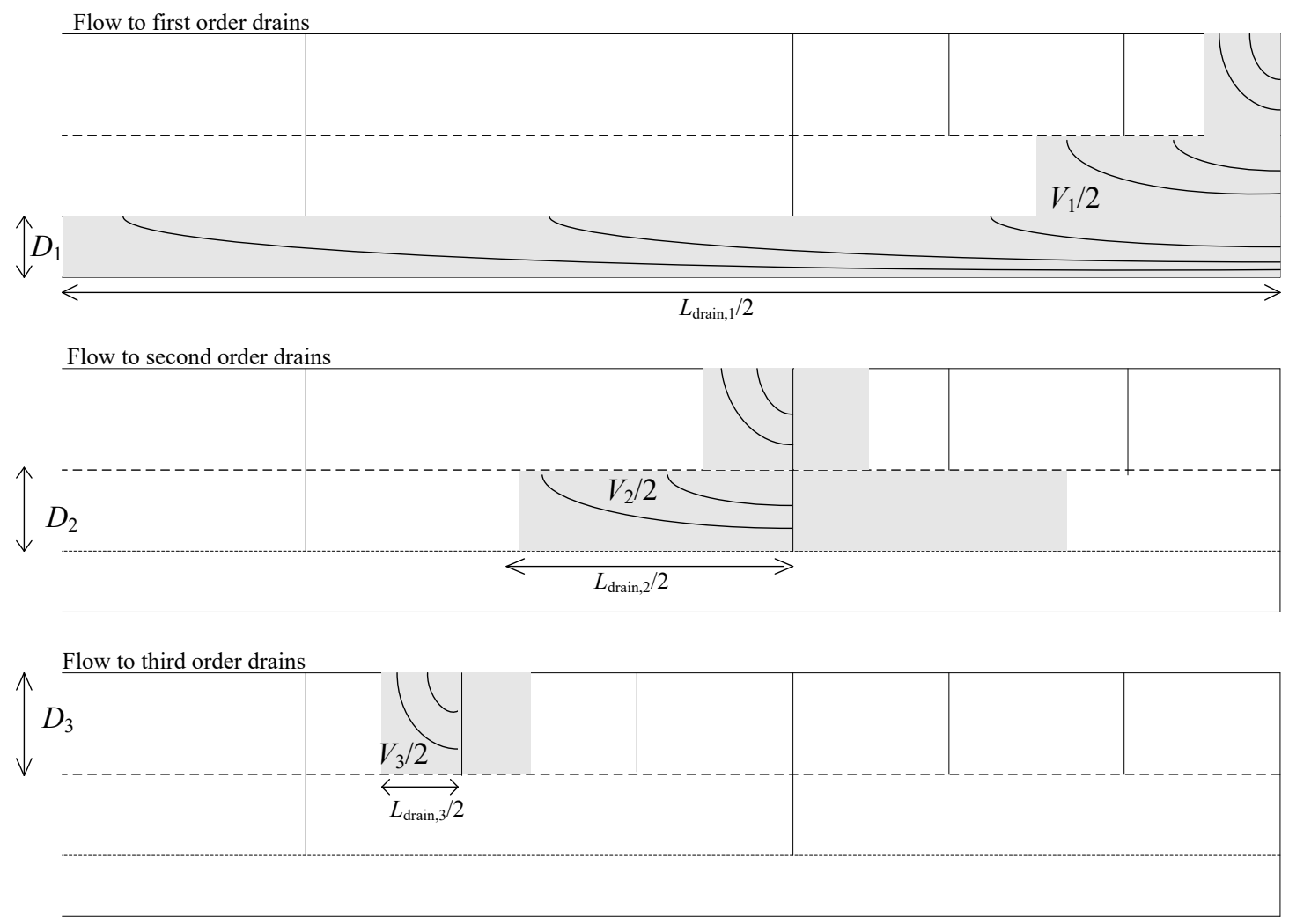

Figure 4.8 Schematization of regional groundwater flow to drains of three different orders.

First order drains act also as field ditches and trenches and next higher drains act partly as third order drains. In the SWAP-model the lumped discharge flux per drainage system is computed from the relation between groundwater elevation and drainage resistance. Figure 4.8 shows the schematization of the regional groundwater flow, including the occupied flow volumes for the nested drain systems. The volume $V_{\mathrm{i}}$ consists of summed rectangles $L_{\mathrm{i}} D_{\mathrm{i}}$ of superposed drains, where $D_{\mathrm{i}}$ is the thickness $(\mathrm{cm})$ of discharge layer $i$. 
The flow volume $V_{\mathrm{i}}$ assigned to drains of order 1,2 and 3 is related to drain distances $L_{\mathrm{i}}$ and thickness $D_{\mathrm{i}}$ of discharge layers as follows:

$$
\begin{aligned}
& V_{1}=L_{1} D_{1}+L_{2} D_{2}+L_{3} D_{3} \\
& V_{2}=L_{2} D_{2}+L_{3} D_{3} \\
& V_{3}=L_{3} D_{3}
\end{aligned}
$$

Rewriting Eq. (4.34) to (4.36) and substituting Eq. (4.32) and Eq. (4.33) yields an expression which relates the proportions of the discharge layer to the discharge flow rates:

$$
\begin{aligned}
& L_{1} D_{1}: L_{2} D_{2}: L_{3} D_{3}= \\
& \left(q_{\text {drain }, 1} L_{\text {drain }, 1}-q_{\text {drain }, 2} L_{\text {drain }, 2}\right):\left(q_{\text {drain }, 2} L_{\text {drain }, 2}-q_{\text {drain, }, 3} L_{\text {drain, }, 3}\right):\left(q_{\text {drain }, 3} L_{\text {drain }, 3}\right)
\end{aligned}
$$

In theory, the terms $\left(q_{\text {drain,1 }} L_{\text {drain,1 }}-q_{\text {drain, } 2} L_{\text {drain, } 2}\right)$ and $\left(q_{\text {drain,2 }} L_{\text {drain,2 }}-q_{\text {drain,3 }} L_{\text {drain,3 }}\right)$ can take negative values for specific combinations of $q_{\text {drain,1 }} L_{\text {drain,1 }}, q_{\text {drain, } 2} L_{\text {drain, } 2}$ and $q_{\text {drain,3 }} L_{\text {drain,3 }}$. When $\left(q_{\text {drain, } 1} L_{\text {drain,1 }}-q_{\text {drain,2 }} L_{\text {drain,2 }}\right)<0$ it is assumed that $D_{1}$ will be zero and the nesting of superposed flows systems on top of the flow region assigned to drainage class 1 will not occur. Likewise, a separate nested flow region related to a drainage class will not show up when $\left(q_{\text {drain,2 }} L_{\text {drain,2 }}-q_{\text {drain,3}} L_{\text {drain,3 }}\right)<0$. These cases are depicted schematically in Figure 4.9.
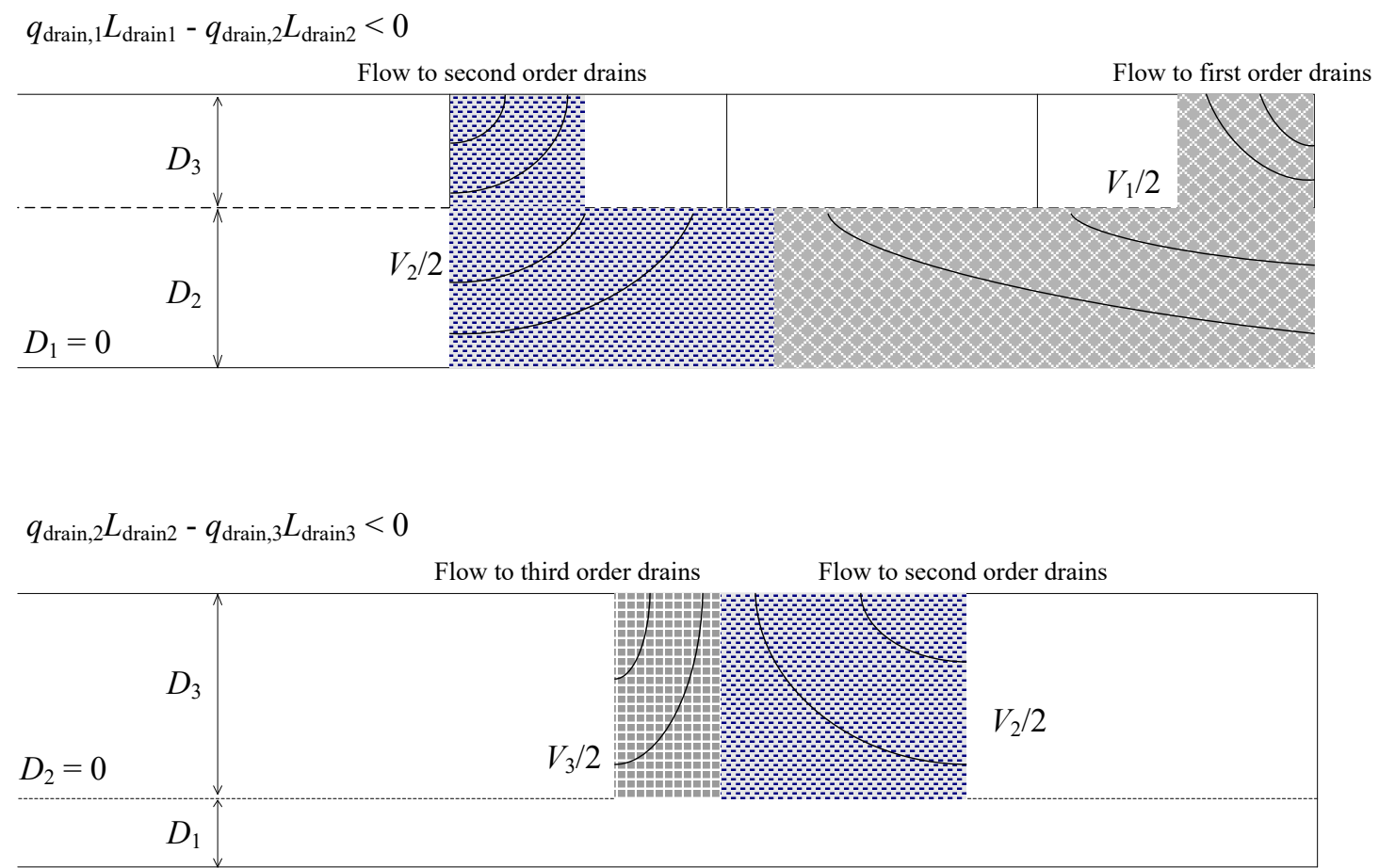

Figure 4.9 Schematization of regional groundwater flow to drains of three orders when either $q_{d r a i n, 1} L_{d r a i n, 1}-q_{d r a i n, 2} L_{d r a i n, 2}<0$ or $q_{d r a i n, 2} L_{d r a i n, 2}-q_{d r a i n, 3} L_{d r a i n, 3}<0$.

If the soil profile is stratified with respect to horizontal conductivities, the heterogeneity can be taken into account by substituting transmissivities $k D$ for layer thicknesses in Eq. (4.37):

$$
K D_{1}: K D_{2}: K D_{3}=\frac{q_{\text {drain }, 1} L_{\text {drain }, 1}-q_{\text {drain }, 2} L_{\text {drain }, 2}}{L_{\text {drain }, 1}}: \frac{q_{\text {drain }, 2} L_{\text {drain }, 2}-q_{\text {drain }, 3} L_{\text {drain }, 3}}{L_{\text {drain }, 2}}: q_{\text {drain }, 3}
$$

Lateral drainage fluxes to a certain drainage system per nodal point are calculated by multiplying the flux and the transmissivity proportion of that nodal point in the total transmissivity of the discharge layer. 
In deep aquifers, the thickness of a model discharge layer is limited by:

$$
D \leq \frac{L_{\text {drain }}}{4} \sqrt{\frac{K_{\mathrm{v}}}{K_{\mathrm{h}}}}
$$

where $K_{\mathrm{v}}$ is the vertical conductivity and $K_{\mathrm{h}}$ is the horizontal conductivity. In stratified aquifers, the weighted arithmetic mean is used for the horizontal conductivity and the weighted harmonic mean is used for the vertical conductivity.

The top of any of the discharge layers is situated at the average groundwater level. This implies that solute transport to drains is calculated for the soil compartments between the simulated groundwater level and the bottom of the discharge layer. The groundwater level as the defined top of the zone that contributes to surface water loading may be inaccurate in case of concentration profiles with steep gradients. In reality the surface water load is determined by the present concentrations and water fluxes at the exfiltration zone in the drain. From Figure 4.10 (left) it can be seen that the concentrations at the drain bottom and at the depth of the surface water level are lower than the ones at the groundwater level. In such case the concept will lead to an over estimation to the surface water load relative to the results of 2D models. The SWAP model provides an option to specify the top of the zone that contributes to surface water loading as a function of the average groundwater level and the drainage level ( $z_{\text {top }}$, Figure 4.10 ; right) according to:

$$
z_{\text {top }}=f_{\text {ztop }} \varphi_{\text {avg }}+\left(1-f_{\text {ztop }}\right) \varphi_{\text {drain }}
$$
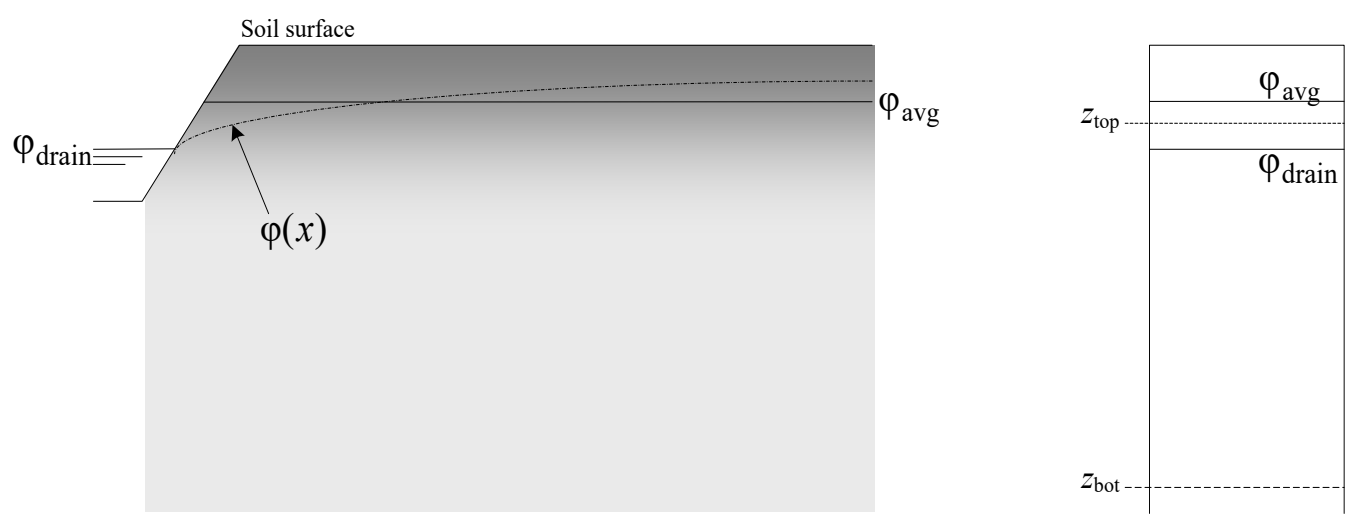

Figure 4.10 2D-schematization of the saturated flow domain with a hypothetical concentration profile indicated by gray shading (left) and the schematization of the top of the zone that contributes to surface water loading (right).

\subsection{User instructions}

\subsubsection{Surface runoff}

The maximum height of ponded water stored $h_{0 \text {,threshold }}$ on the field surface is determined by the irregularities and the slope of the soil surface. Also the extend to which local field depressions are connected to each other and are connected to the surface water affects the value of $h_{0, \text { threshold. Typical }}$ values range from 0.5 to $2 \mathrm{~cm}$ for well maintained agricultural fields. Since it is impossible to measure

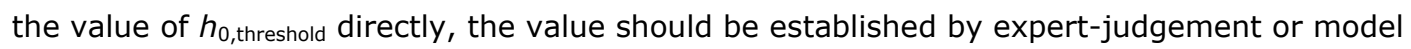
calibration. The value can be specified as a constant, or may fluctuate with time.

As surface runoff is a rapid process, the resistance $r$ will typically take values of less than $1 \mathrm{~d}$. When the dynamics of surface runoff are relevant, the values of resistance $\gamma$ (RSRO) and exponent $\beta$ (RSROEXP) might be derived from experimental data or from a hydraulic model of soil surface flow. 


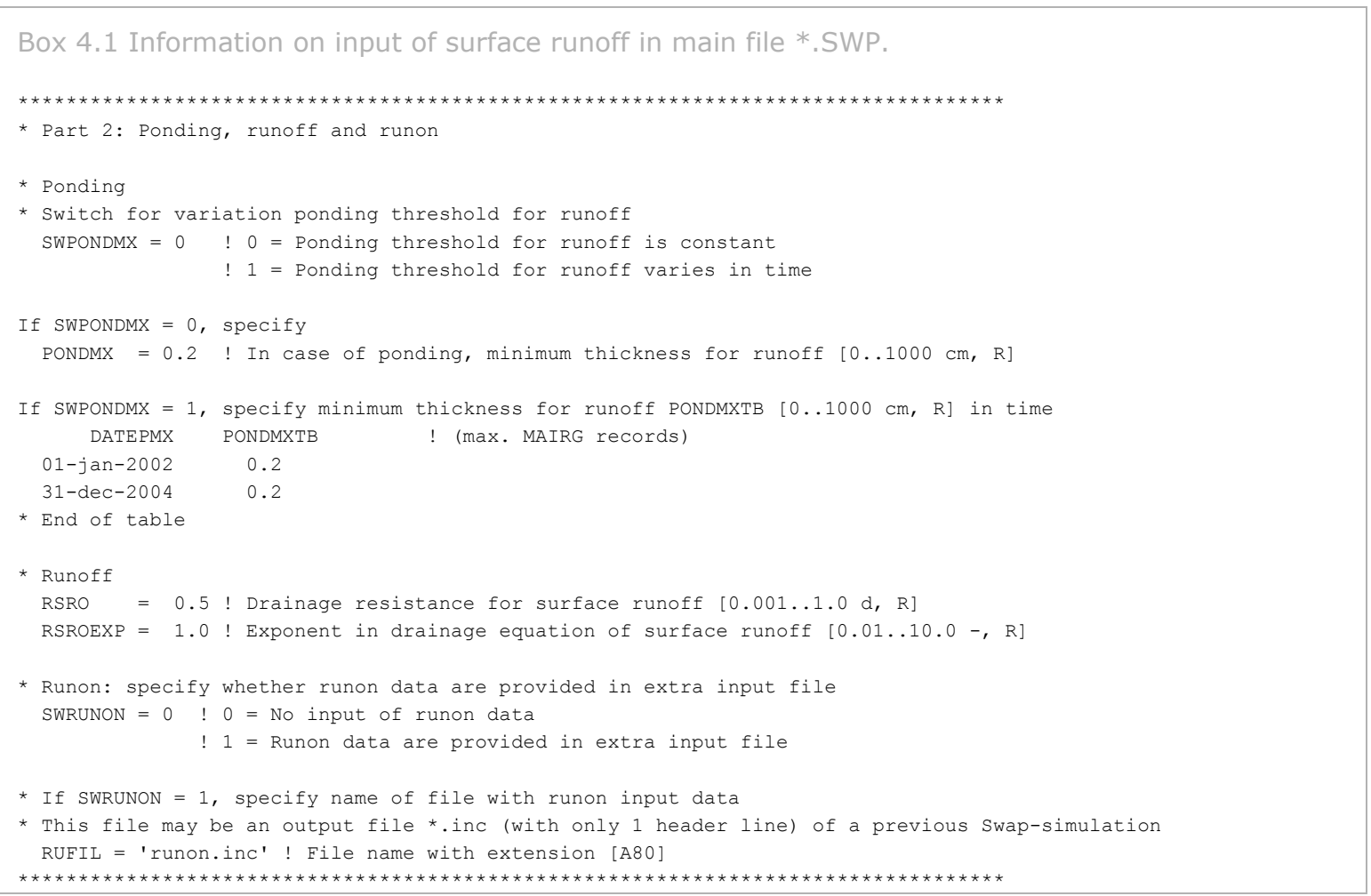

\subsubsection{Interflow}

For describing the interflow process, a non-linear relation can be used. Such relation may useful for taking account for the horizontal flow in the saturated zone above drainage level may yield a nonlinear relation contrary to relation based on the assumption made in the derivation of the horizontal resistance in the Ernst-equation. Another reason to introduce a non-linear relation for interflow may be the occurrence of hillslope. Sometimes it is possible to relate the parameters $A_{\text {interflow }}$ and $B_{\text {interflow }}$ to a specific flow concept, but most of the model user has to rely on expert judgement of model calibration.

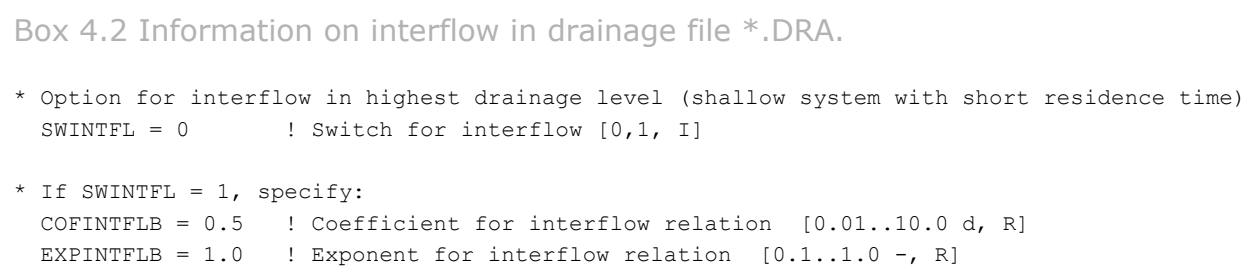

\subsubsection{Drainage}

The input requirements for the simulation of a field scale drainage relation according to Hooghoudt and Ernst is given in Box 4.3. 


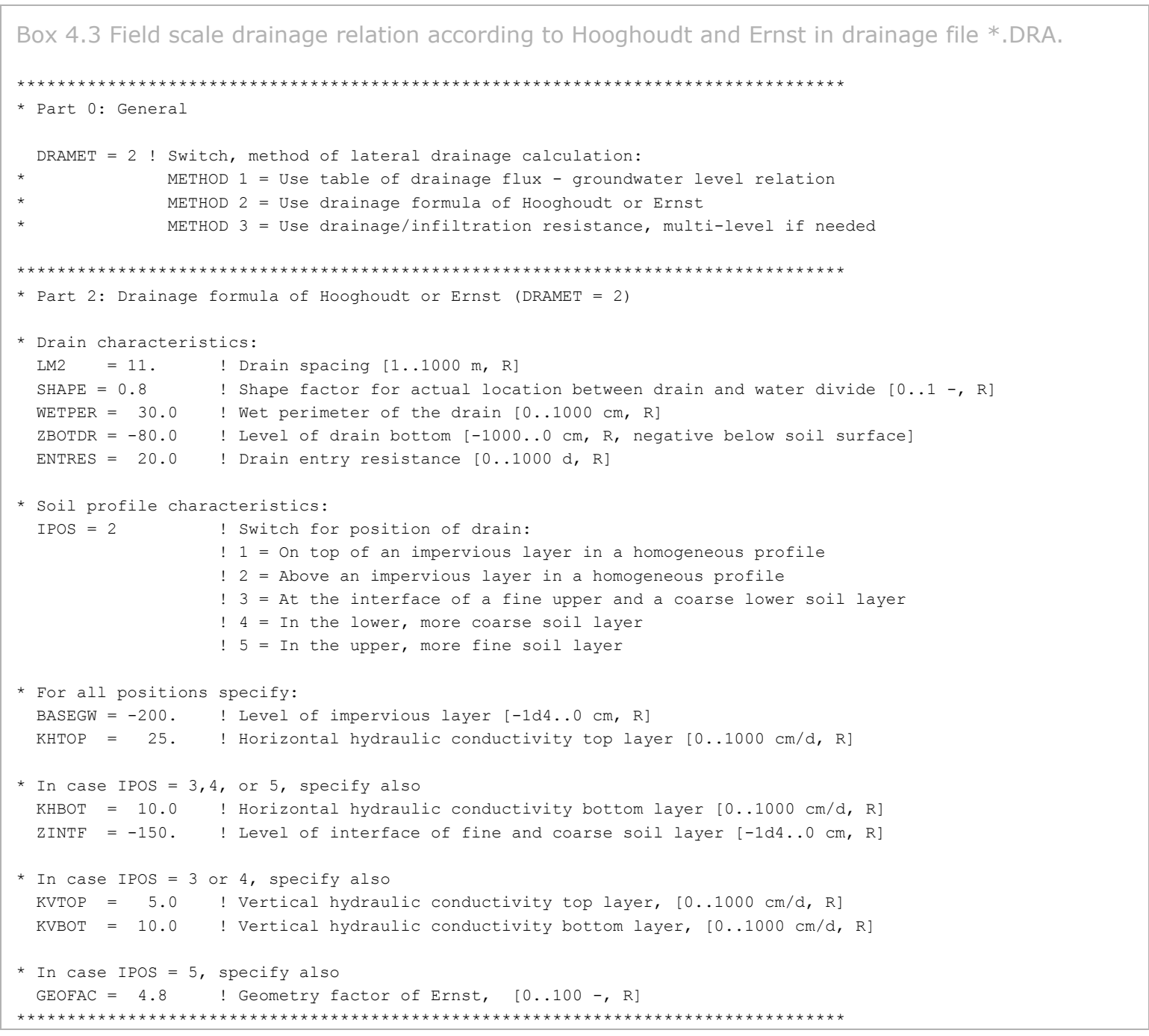

The input requirements for the simulation of a field scale drainage relation defined by a tabulated function is given in Box 4.4 .

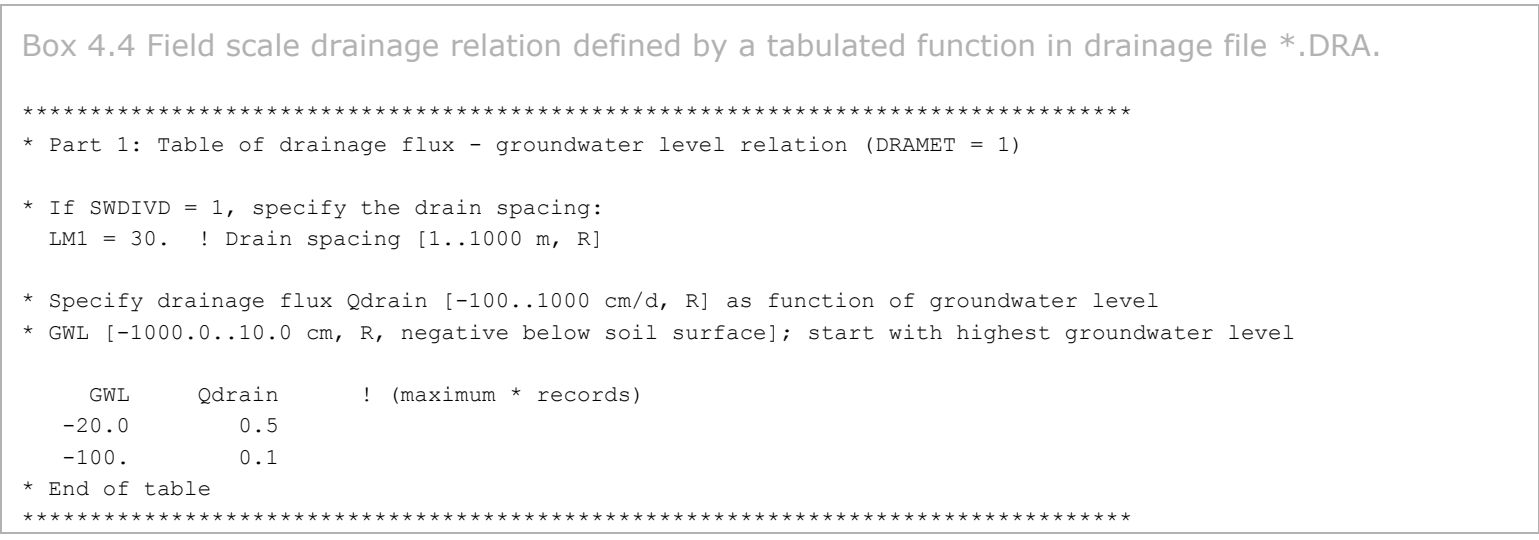

The input requirements for the simulation of multi-level drainage given in Box 4.5 for a basic system with fixed resistances and imposed levels. In Box 4.6 the requirements are given for an extended system with surface water dependent resistances and simulated drainage levels.

Up to five different drainage levels can be specified. For each level the user can specify whether drainage or infiltration or both are allowed. Both the drainage and infiltration resistance needs to be specified by the user. In case of sub-irrigation, the entrance resistance (then denoted as $\gamma_{\text {inf }}$ ) can be 
either higher or lower than that for drainage $\left(\gamma_{\text {drain }}\right)$, depending on local conditions. A substantial raising of the surface water level can for instance result in infiltration through a more conductive 'bioactive' zone which will reduce the entrance resistance. In most situations with sub-irrigation the radial resistance will be higher than with drainage, because the wetted section of the subsoil is less than in the situation with drainage (the groundwater table becomes concave instead of convex). Especially if the conductivity is larger in the subsoil above the drainage base than in the deeper subsoil, $\gamma_{\text {inf }}$ will be substantially higher than $\gamma_{\text {drain }}$. To deal with such cases, the model has the option for using subirrigation resistances that differ from the ones for drainage (e.g. $\gamma_{\text {inf }} \approx 3 / 2 \gamma_{\text {drain }}$ in Figure 4.5).

When calibrating SWAP against measured groundwater levels it should be realised that SWAP calculates a field-average groundwater level. Measured groundwater levels represent a point of the convex or concave shaped groundwater table, depending on the position of the piezometer in the field in relation to the drains. Piezometers in the middle of two parallel drains will display stronger fluctuations of the groundwater level than the field-average level. Calibrating SWAP against these strong fluctuating groundwater levels will result in a calibrated model that deviates strongly from groundwater behavior at the field scale. To overcome this inaccuracy, it is advised to measure groundwater level movement in a row of piezometers perpendicular to the drain starting at a close $(0.5 \mathrm{~m})$ distance of the drain, including the drain level. From these measurements a shape-factor can be calculated that represents the relationship between the measured convex or concave groundwater table and the field average. This shape-factor can be used to convert measured levels into levels that are suitable for calibration of SWAP. 
Box 4.5 Multi-level drainage with fixed resistances and imposed drainage levels in drainage file $*$.DRA.

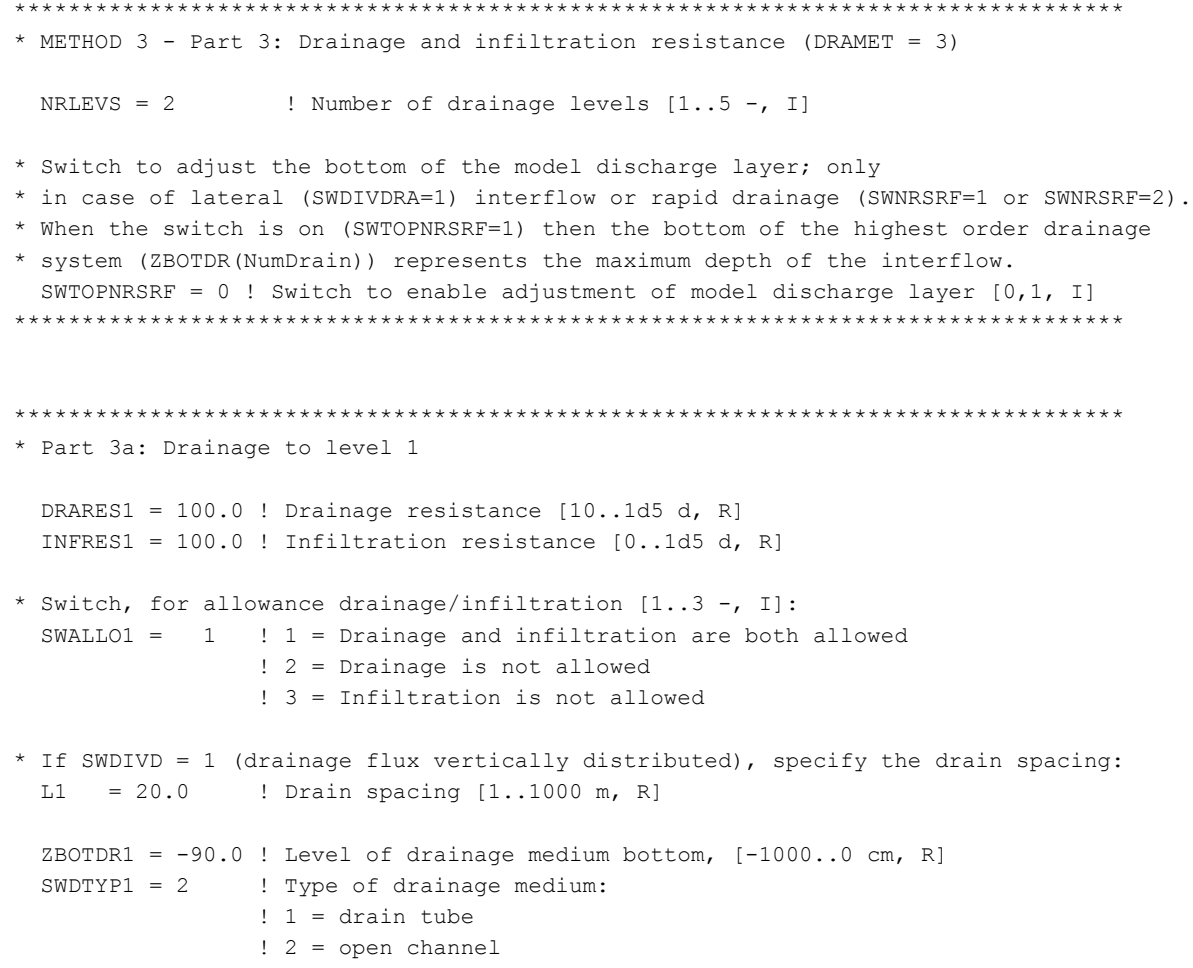

* In case of open channel (SWDTYP1 = 2), specify date DATOWL1 [dd-mmm-yyyy] and channel

* water level LEVEL1 [cm, negative if below soil surface]:

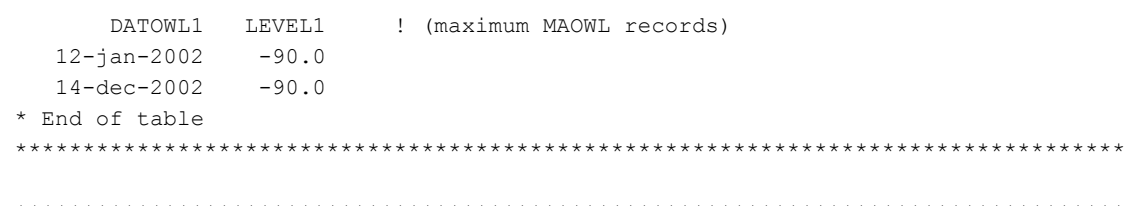

* Part 3b: Drainage to level 2

DRARES2 $=100.0$ ! Drainage resistance $[10 \ldots 1 \mathrm{~d} 5 \mathrm{~d}, \mathrm{R}]$

INFRES2 $=100.0$ ! Infiltration resistance $[0 \ldots 1 \mathrm{~d} 5 \mathrm{~d}, \mathrm{R}]$

* Switch, for allowance drainage/infiltration [1..3 -, I] :

SWALLO2 = 1 ! 1 = Drainage and infiltration are both allowed

! 2 = Drainage is not allowed

! 3 = Infiltration is not allowed

* If SWDIVD $=1$ (drainage flux vertically distributed), specify the drain spacing: $\mathrm{L} 2=20$. $\quad$ Drain spacing $[1 \ldots 1000 \mathrm{~m}, \mathrm{R}]$

ZBOTDR2 $=-90.0$ ! Level of drainage medium bottom, $[-1000 \ldots 0 \mathrm{~cm}, \mathrm{R}]$ SWDTYP2 $=2$ ! Type of drainage medium:

! 1 = drain tube

! 2 = open channel

* In case of open channel (SWDTYP2 $=2$ ), specify date DATOWL2 [dd-mmm-yyyy] and channel * water level LEVEL2 [cm, negative if below soil surface]:

$$
\begin{aligned}
& \text { DATOWL2 LEVEL2 ! (maximum MAOWL records) } \\
& \text { 12-jan-2002 }-90.0 \\
& 14-\text { dec-2002 }-90.0 \\
& \text { * End of table }
\end{aligned}
$$

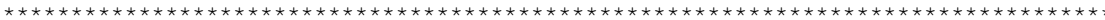

If the Number of drainage levels (NRLEVS) is larger than 2, then

similar input is required for levels $3-5$ 
Box 4.6 Multi-level drainage with surface water dependent resistances and simulated drainage levels in drainage file $*$. DRA.

$\star \star \star$ EXTENDED DRAINAGE SECTION $\star * \star$

* Part 0: Reference level

ALTCU $=0.0$ ! ALTitude of the Control Unit relative to reference level

* $\quad \mathrm{AltCu}=0.0$ means reference level coincides with

surface level $[-300000 \ldots 300000 \mathrm{~cm}, \mathrm{R}]$

* Part 1a: drainage characteristics

*

NRSRF $=2$ ! number of subsurface drainage levels $[1 \ldots 5$, I $]$

*** Table with physical characteristics of each subsurface drainage level:

* LEVEL ! drainage level number [1..NRSRF, I]

* SWDTYP ! type of drainage medium [open=0, closed=1]

* L ! spacing between channels/drains [1.1000 m, R]

* ZBOTDRE ! altitude of bottom of channel or drain [ALTCU-1000..ALTCU-0.01 cm, R]

* GWLINF ! groundw. level for max. infiltr. [-1000..0 cm rel. to soil surf., R]

* RDRAIN ! drainage resistance $[1 \ldots 100000 \mathrm{~d}, \mathrm{R}]$

* RINFI ! infiltration resistance $[1 \ldots 100000 \mathrm{~d}, \mathrm{R}]$

* Variables RENTRY, REXIT, WIDTHR and TALUDR must have realistic values when the

* type of drainage medium is open (second column of this table:SWDTYP=0)

For closed pipe drains (SWDTYP=1) dummy values may be entered

* RENTRY ! entry resistance [1.100 d, R]

* REXIT ! exit resistance [1.100 d, R]

* WIDTHR ! bottom width of channel $[0 \ldots 100 \mathrm{~cm}, \mathrm{R}]$

* TALUdR ! side-slope $(\mathrm{dh} / \mathrm{dw})$ of channel $[0.01 \ldots 5, \mathrm{R}]$

LEV SWDTYP L ZBOTDRE GWLINF RDRAIN RINFI RENTRY REXIT WIDTHR TALUDR

$\begin{array}{lllllllllll}1 & 0 & 250.0 & 1093.0 & -350.0 & 150.0 & 4000.0 & 0.8 & 0.8 & 100.0 & 0.66\end{array}$

$\begin{array}{lllllllllll}2 & 0 & 200.0 & 1150.0 & -300.0 & 150.0 & 1500.0 & 0.8 & 0.8 & 100.0 & 0.66\end{array}$

* End_of_table

* Part 1b: Separate criteria for highest (shallow) drainage system

SWNRSRF $=0 \quad$ ! Switch to introduce rapid subsurface drainage $[0 \ldots 2$, I]

* $\quad 0=$ no rapid drainage

* $\quad 1$ = rapid drainage in the highest drainage system (=NRSRF)

(implies adjustment of RDRAIN of highest drainage system)

2 = rapid drainage as interflow according to a power relation (implies adjustment of RDRAIN of highest drainage system)

When SWRNSRF $=1$, then enter realistic values for rapid drainage

RSURFDEEP $=30.0$ ! maximum resistance of rapid subsurface Drainage $[0.001 \ldots 1000.0 \mathrm{~d}, \mathrm{R}]$

RSURFSHALLOW $=10.0 \quad !$ minimum resistance of Rapid subsurface Drainage $[0.001 \ldots 1000.0$ d, R]

* When SWRNSRF $=2$, then enter coefficients of power function

COFINTFL $=0.1 \quad !$ coefficient of interflow relation $[0.01 \ldots 10.0 \mathrm{~d}-1, \mathrm{R}]$

EXPINTFL $=0.5 \quad$ ! exponent of interflow relation $[0.1 \ldots 1.0-, \mathrm{R}]$

* Switch to adjust the bottom of the model discharge layer in case of lateral (swdivdra=1)

* interflow or rapid drainage (Swnrsrf=1 or Swnrsrf=2).

* When the switch is on (SwTopnrsrf=1) then the bottom of the highest order drainage

* system (Zbotdr(NumDrain)) represents the max depth of the interflow.

SwTopnrsrf $=0$ ! Switch to enable adjustment of model discharge layer [0,1, I]

The input requirements to influence the distribution of drainage fluxes with depth are given in Box 4.7 and 4.8 .

An implicit approach for travel times distribution (Section 4.4.1) requires specification of an anisotropy factor (COFANI) for each soil layer. This factor represents the division of horizontal over vertical saturated hydraulic conductivity and will generally increase with depth.

The exact location of the so-called discharge layers may be influenced by an adjustment of the upper boundary of the discharge layer (Box 4.8 and Section 4.4.2). This adjustment requires expert judgement and it is generally not recommended to apply/adjust it without thorough knowledge of the underlying processes. 
Box 4.7 Implicit approach of travel times in drainage file *.DRA.

* Part 0: General

SWDIVD = 1 ! Calculate vertical distribution of drainage flux in groundwater $[\mathrm{Y}=1, \mathrm{~N}=0$ ]

* If SWDIVD $=1$, specify anisotropy factor COFANI (horizontal/vertical Ksat) [0..1000 -, R]

* for each physical soil layer (maximum MAHO):

COFANI $=1.0 \quad 1.0$

Box 4.8 Discharge layers in drainage file *.DRA.

* Switch to adjust upper boundary of model discharge layer

SWDISLAY $=0 \quad$ ! switch to adjust discharge layer $[0 \ldots 2-$, I $]$

* If SWDISLAY $=1$, specify for the drainage systems 1 - NRLEVS or NRSRF:

* SwTopDislay = switch, for each drainage level, to distribute drainage flux vertically

* With a given position of the top of the model discharge layers [Y=1, N=0]

* ZTopDislay = array with depth of top of model discharge layer for each drain level [*..* cm, R]

* If SWDISLAY $=2$, specify FTopDislay instead of ZTopDislay:

* FTopDislay = array with factor of top of model discharge layer for each drain level

Level SwTopDislay ZTopDisLay FTopDisLay ! (maximum MADR records)

$\begin{array}{llll}1 & 1 & -200.0 & 0.5 \\ 2 & 0 & -0.01 & 0.0\end{array}$

* end of tabel

* 


\section{$5 \quad$ Surface water management}

\subsection{Surface water balance}

Surface water management options have been implemented in the SWAP model by taking account of the water balance of the surface water. The groundwater-surface water system is described at the scale of a horizontal subregion. The subregion has a single representative groundwater level and it is assumed that the soil profile occupies the whole surface area. This results in water balance terms of the soil profile that are computed per unit area $\left(\mathrm{cm}^{3} \mathrm{~cm}^{-2}\right)$ and have the same numerical value for the sub-region as a whole. The surface water system is simplified to a control unit for which the following surface water balance equation is formulated:

$$
\frac{d V_{\text {sur }}}{d t}=q_{\text {sup }}-q_{\text {dis }}+q_{\text {drain }}+q_{\text {crackfl }}+q_{\text {runoff }}
$$

where $V_{\text {sur }}$ is the regional surface water storage $\left(\mathrm{cm}^{3} \mathrm{~cm}^{-2}\right), q_{\text {sup }}$ is external supply to the control unit $\left(\mathrm{cm}^{3} \mathrm{~cm}^{-2} \mathrm{~d}^{-1}\right), q_{\text {dis }}$ is discharge from control unit $\left(\mathrm{cm}^{3} \mathrm{~cm}^{-2} \mathrm{~d}^{-1}\right), q_{\text {drain }}$ is regional drainage flow $\left(\mathrm{cm}^{3}\right.$ $\left.\mathrm{cm}^{-2} \mathrm{~d}^{-1}\right), q_{\text {crackfl }}$ is bypass flow through cracks of a clay soil to drains or ditches $\left(\mathrm{cm}^{3} \mathrm{~cm}^{-2} \mathrm{~d}^{-1}\right)$, and $q_{\text {runoff }}$ is either surface runoff or surface runon $\left(\mathrm{cm}^{3} \mathrm{~cm}^{-2} \mathrm{~d}^{-1}\right)$.

The regional surface water storage $V_{\text {sur }}\left(\mathrm{cm}^{3} \mathrm{~cm}^{-2}\right)$ is the sum of the surface water storage in each order of the surface water system:

$$
V_{\text {sur }}=\frac{1}{A_{\text {reg }}} \sum_{i=1}^{n} l_{\mathrm{i}} A_{\mathrm{d}, \mathrm{i}}
$$

in which $A_{\text {reg }}$ is the total area of the sub-region $\left(\mathrm{cm}^{2}\right), l_{i}$ the total length of channels/drains of order $i$ in the sub-region $(\mathrm{cm})$, and $A_{\mathrm{d}, i}$ is the wetted area of a channel vertical cross-section $\left(\mathrm{cm}^{2}\right)$. The wetted area $A_{\mathrm{d}, i}$ is calculated from the surface water level $\phi_{\text {sur, }}$ the channel bed level, the bottom width, and the side-slope. Substitution of Eq. (4.23) in Eq. (5.2) yields the expression:

$$
V_{\text {sur }}=\sum_{i=1}^{n} \frac{A_{\mathrm{d}, \mathrm{i}}}{L_{\mathrm{i}}}
$$

Channels of order $\mathrm{i}$ only contribute to the storage if $\phi_{\text {sur }}>z_{\text {bed,i. }}$. The storage in tile drains is neglected. SWAP calculates the net discharge $\mathrm{q}_{\text {dis }}-\mathrm{q}_{\text {sup }}$ for a given timestep and for specified surface water levels $\phi_{\text {sur }}^{j}$ and $\phi_{\text {sur }}^{j+1}$ :

$$
q_{\mathrm{dis}}-q_{\mathrm{sup}}=\frac{V_{\mathrm{sur}}^{j}\left(\phi_{\mathrm{sur}}^{j}\right)-V_{\mathrm{sur}}^{j+1}\left(\phi_{\mathrm{sur}}^{j+1}\right)}{\Delta t^{j}}+q_{\mathrm{drain}}+q_{\text {crackfl }}+q_{\text {runoff }}
$$

If the sum of the terms on the right hand side is positive, discharge has taken place and the supply is equal to zero. If the sum is negative, supply has taken place and the discharge is equal to zero.

The drainage flux is calculated by:

$$
q_{\text {drain }}=\sum_{i=1}^{n} \frac{\phi_{\text {avg }}-\phi_{\text {drain,i }}}{\gamma_{\text {drain,i }}}
$$

where the drainage level $\phi_{\text {drain,i }}$ is in this case equal to the channel bed level, $z_{\text {bed, } i}$. When the groundwater level is situated above the highest bed level and with the surface water level is below the lowest one the total drainage flux. If the surface water level tends to rise to levels higher than the channel bed level $z_{\text {bed,i, }}$ the latter is replaced by the surface water level. 
Calculation of the discharge rate $q_{\text {dis }}$ is the last step in solving Eq. (5.4):

1. If the supply rate $q_{\text {sup }}$ takes a positive value, the discharge is set to zero. The calculation of the supply rate is based on the comparison the target level and that actual surface water level. After establishing a target level it is examined whether the surface water level can take the target value. If necessary, surface water supply is used to attain the target level. The water supply should meet to the following criteria:

a. The supply rate should not exceed a user defined value of the maximum supply rate $q_{\text {sup,max }}$

b. Water supply only occurs when the surface water level takes a value below the water supply level. This water supply level is defined as a tolerance value in relation to the target level.

2. For the fixed weir, the discharge follows from the iterative procedure to establish a target level from the stage - discharge relationship. This relationship can either be expressed in tabulated form or as a power function for "weir flow" (see Par 5.1.2.1).

3. For a soil moisture controlled weir, the discharge follows simply from the water balance equation as given by Eq. (5.4), with $q_{\text {sup }}$ set to zero and the storage $V_{\text {sur }}^{j+1}$ set equal to the storage for the target level. The discharge $q_{\text {dis }}$ is then the only unknown left, and can be solved directly.

\subsubsection{Multi-level drainage with imposed surface water levels}

SWAP comprises an option for imposing a surface water level time series to be used as drainage level time series. When using this option it is assumed that the surface water level is equal for all drainage systems. Surface waters associated to the different drainage systems have open connections to each other and conduit resistances are neglected. This results to only one overall surface water level. The number of drainage systems to account for depends on the position of the groundwater level and the surface water level relative to the channel bed level.

\subsubsection{Multi-level drainage with simulated surface water levels}

Another option in the SWAP model is to simulate the surface water level on the basis of the control unit surface water balance. Then, the discharge is governed by a either a fixed weir or an automated weir. The user can specify different water management periods for which the settings of the weirs can be different. During each time step, SWAP determines in subsequent order:

1. the target level;

2. whether the target level is reached, and the amount of external supply that is needed (if any);

3. the discharge that takes place (if any) and the surface water level at the end of the time step.

Two options for describing the functioning of a weir are available: 1) the target level of a fixed weir coincides with the crest level, which has a constant value within a certain management period, or 2) the target level of soil moisture controlled weir is a function of a soil moisture state variable and is defined by a water management scheme.

\subsubsection{Fixed weir}

The fixed weir-option employs a power function based 'stage-discharge' relationship $q_{\text {dis }}\left(\phi_{\text {sur }}\right)$ for which the parameters in the input are specified in SI-units ${ }^{1}$ or a tabulated relationship. The power function based 'stage-discharge' relationship reads as:

$$
q_{\text {dis }}=\frac{Q_{\text {dis }}}{A_{\text {cu }}}=\frac{\alpha_{\text {weir }}\left(\phi_{\text {sur }}-z_{\text {weir }}\right)^{\beta_{\text {weir }}}}{A_{\text {cu }}}
$$

in which $Q_{\text {dis }}$ is the volumetric discharge $\left(\mathrm{m}^{3} \mathrm{~s}^{-1}\right), A_{\mathrm{cu}}$ is the area of the control unit $\left(\mathrm{m}^{2}\right), z_{\text {weir }}$ is the weir crest level $(m), a_{\text {weir }}$ is the discharge coefficient $\left(m^{3-\beta} d^{-1}\right)$ and $\beta_{\text {weir }}$ is the discharge exponent (-). For a broad-crested rectangular weir, $\alpha_{\text {weir }}$ is (approximately) given by $\alpha_{\text {weir }}=1.7 \mathrm{x}$ "weir width" The stage-discharge relationship can optionally be specified by a table. In that case the relationship should be defined for each management period.

\footnotetext{
1 Literature values are often given in these units. The conversion to internal units is handled by SWAP.
} 


\subsubsection{Soil moisture controlled weir management}

This model option assumes the target level of a weir to be controlled by one or more soil moisture state variables. The so called water management scheme defines the setting of the water level $\phi_{\text {sur,tar }}$ aimed for in relation to a soil moisture state variable. The target level is defined as a combinational function of three state variables related to soil moisture:

1. the average groundwater level $\phi_{\text {avg }}(\mathrm{cm})$. Lower target levels a higher groundwater levels may prevent waterlogging and can contribute to minimize crop yield reduction.

2. the soil water pressure head $h(\mathrm{~cm})$ at a certain depth. A soil water pressure value appears to be a better indicator for water logging in nature reserves than a groundwater level criteriumPar.

3. the capacity of the unsaturated soil profile to store water $V_{\text {uns }}(\mathrm{cm})$. This state variable is an indicator for the possibility of buffering extreme rainfall events. Maintaining a certain minimum amount of storage, reduces the risk of flooding and subsequent discharge peaks.

During the simulation, the SWAP model selects the target level for which all three criteria are met. A scheme maintained by a soil moisture controlled weir is illustrated in Table 5.1. The minimum target level is specified in the first column. The second, third and fourth column represent values for the corresponding groundwater level, soil water pressure head and soil water storage capacity. The first row contain zeros, indicating that irrespective the conditions, the minimum target level should never drop below that level.

Table 5.1 Example of a water management scheme, with $\phi_{s u r, t a r}$ as the target level, the groundwater level criterium $\phi_{a v g, m a x}$ the pressure head criterium $h_{\max }$ and the soil water storage criterium $V_{u n s, m i n}$.

\begin{tabular}{|c|c|c|c|}
\hline$\phi_{\text {sur,tar }}(\mathrm{cm})$ & $\phi_{\text {avg,max }}(\mathrm{cm})$ & $h_{\max }(\mathrm{cm})$ & $V_{\text {uns, } \min }(\mathrm{cm})$ \\
\hline-180 & 0 & 0 & 0 \\
\hline-160 & -80 & -100 & 1.5 \\
\hline-120 & -100 & -200 & 2.5 \\
\hline-100 & -120 & -250 & 3.0 \\
\hline
\end{tabular}

To avoid the target level reacting too fast on the prevailing groundwater level, a maximum drop rate parameter has been introduced specifying the maximum permitted change of the target level per time unit $\left(\mathrm{cm} \mathrm{d}^{-1}\right)$. The limitation of the target level change can become effective in periods with surface water supply combined with a rising groundwater level.

In periods with heavy rainfall and high discharge, the maximum capacity of a soil moisture controlled weir can be reached and the crest level will drop to its minimum level. Then, the surface water level is not controlled by any of the criteria mentioned before any longer, but will be a function of the discharge characteristics of the surface water infrastructure. Therefore, the management scheme of a soil moisture controlled weir should always be combined with a table defining a stage discharge relationship. This tabulated relationship should be defined for every management period. The minimum level of management scheme should identical to the minimum level of the discharge relationship. 


\subsection{User instructions}

\subsubsection{Input instructions}

The user first has to select the option for extended drainage (Box 5.1).

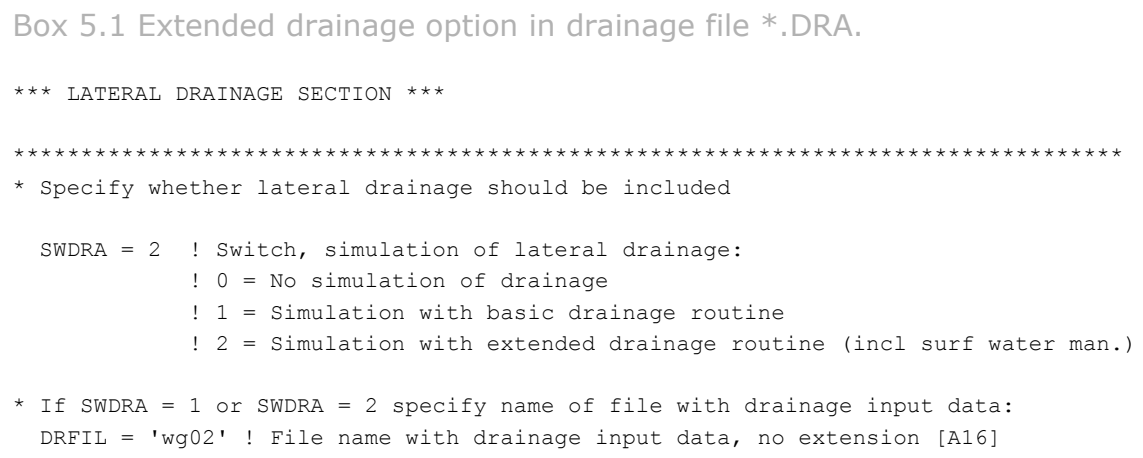

Parameters and input variables are specified in a separate file called DRFIL. The input data are given in 2 sections:

- Section 1: drainage characteristics (described in Chapter 4)

- Section 2: surface water system (described in Chapter 5)

In Section 1, the user should specify the altitude of the control unit (= soil surface), with respect to a certain reference level (ALTCU). In Section 2, water management levels are given with respect to the same reference. The user may choose to define the soil as surface reference level by specifying zero for the altitude.

A flow chart of the input for the surface water module (Section 2 in the input file) is given in Figure 5.1. The user should make selections for the kind of surface water system (SWSRF) and the kind of control (SWSEC). The different parts of Section 2 are described hereafter. 


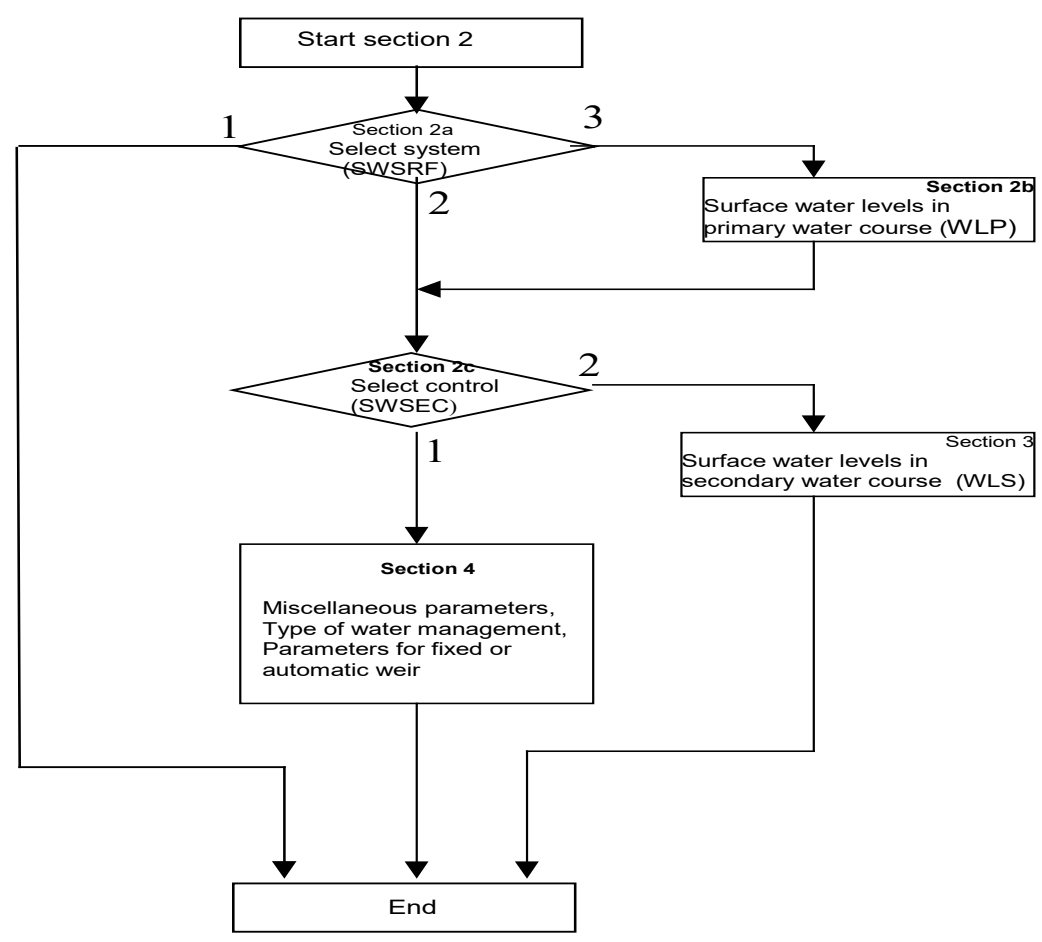

Figure 5.1 Flow chart for input data of surface water system

Section 2 starts with a switch (Section 2a, variable SWSRF) for three options:

1. no surface water system is simulated;

2. surface water system is simulated with no separate primary water course;

3. surface water system is simulated with a primary water course (level 1) separate from the control unit

If the first option ( $S W S R F=1$ ) has been chosen, the user may skip the rest of Section 2 . For the second or third option ( $S W S R F=2$ or 3 ) the user has also to specify how the surface water level in the control unit is determined (Section 2c, variable SWSEC):

1. the surface water level is simulated;

2. the surface water level is obtained from input data.

If the third option (SWSRF=3) has been chosen, the user should also specify (Section $2 b$ ) the time variation of the surface water level in the primary water course. The specification is done in terms of data pairs (date, water level). To obtain levels at intermediate dates, the model performs a linear interpolation. 


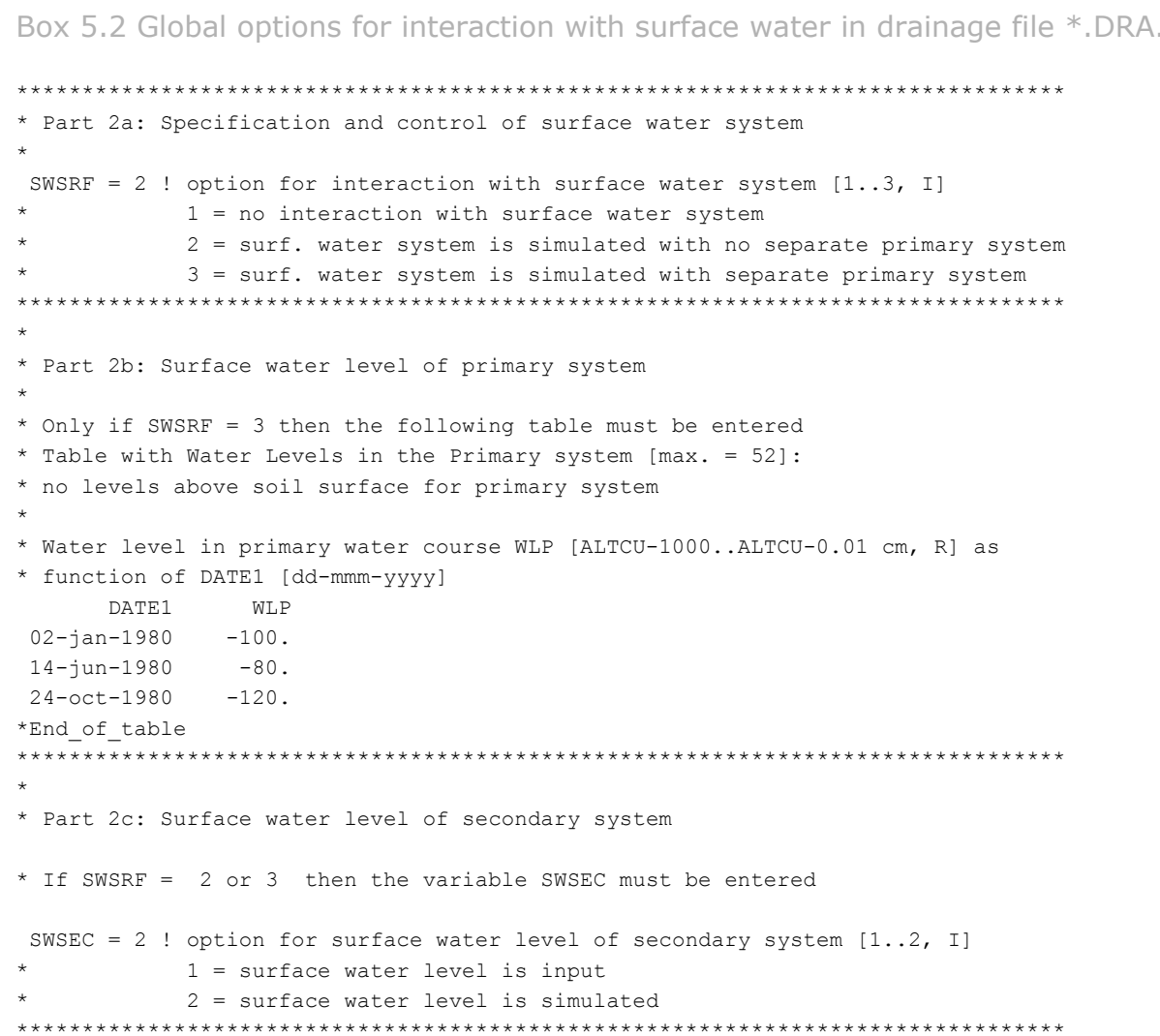

If the option is chosen to obtain surface water levels from input data (SWSEC=1), the surface water level of the secondary watercourse has to be specified in the form of data pairs (Section 3).

If the option is chosen to simulate surface water levels (SWSEC $=2$ ), the user has to specify how the surface water system in the control unit functions and how it is managed (Section 4).

Section 4 starts with some miscellaneous parameters (Section $4 a$ ):

- the initial surface water level in the control unit;

- the criterium for detecting oscillation of the surface water level;

- the number of water management periods.

In the next Section $4 \mathrm{~b}$ the management period are defined as well as the type of watermanagent (1- fixed weir crest; 2 - automatic weir), the water supply capacity and a tolerance value (WLDIP). The tolerance value WLDIP relates the water supply level to the target level preventing oscillations and too fast unrealistic responses of surface water management to the prevailing conditions. This tolerance can be seen as the allowed dip of the surface water level and can take a value of e.g. $10 \mathrm{~cm}$. An appropriate setting of this parameter can save a substantial amount of water.

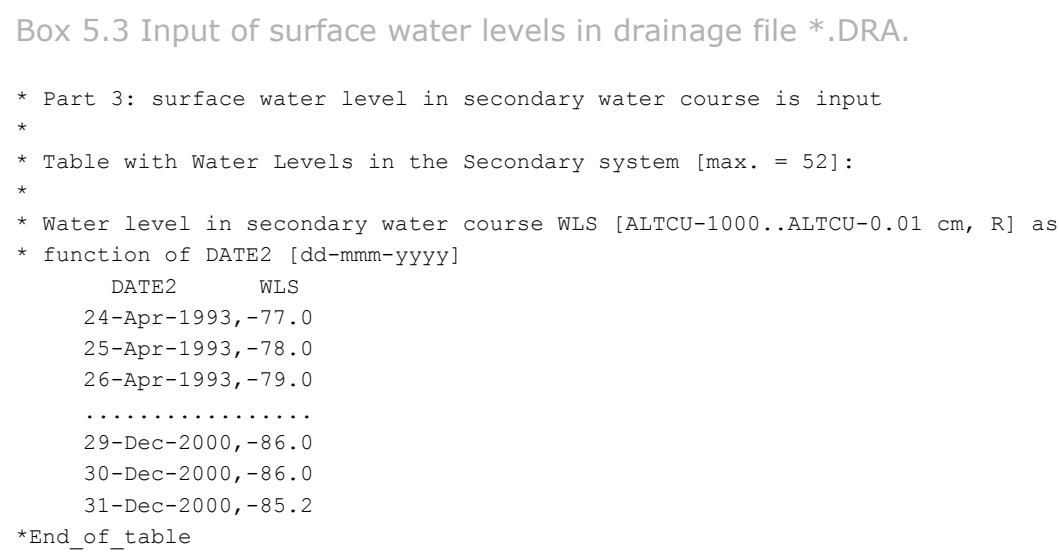




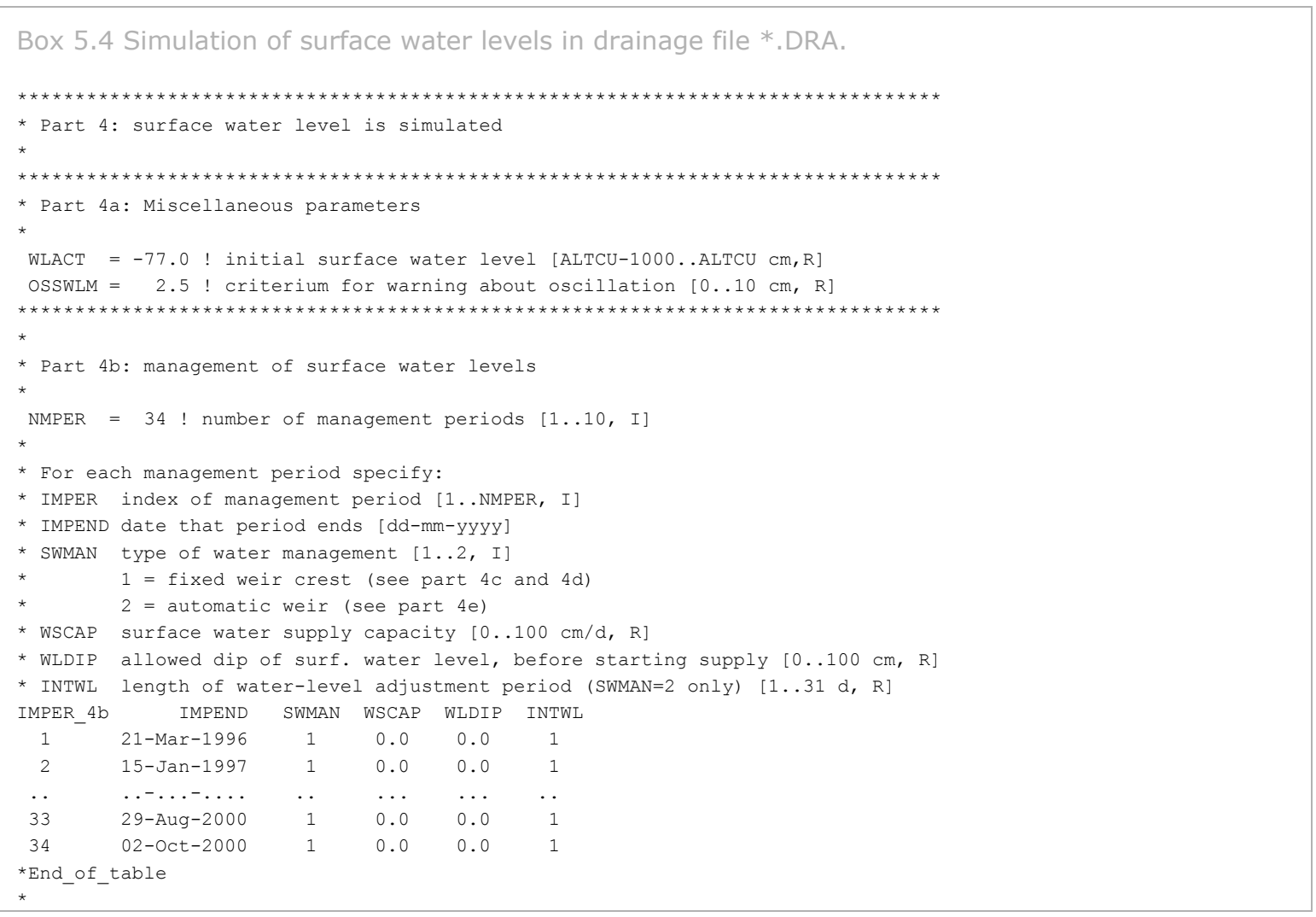

Dependent on the discharge relationship for the weir, the user has to specify:

- either Section $4 c$ (SWQHR=1, exponential relation)

- or Section $4 d(S W Q H R=2$, relation given as table)

If an exponential relations is chosen then, for each water management period with a fixed weir crest using weir characteristics, the user should specify (Section 4c):

- Size of the control unit (catchment) (ha);

- A table with weir characteristics for each management period:

- Index for management period (-);

- Elevation $(\mathrm{H})$ of the weir crest $(\mathrm{cm})$;

- dischargecoefficient $\alpha_{\text {input }}\left(\mathrm{m}^{3-\beta} \mathrm{s}^{-1}\right)$;

- discharge exponent $\beta(-)$

Head-discharge relationships are given in SI-units, i.e. $\mathrm{m}$ for length and $\mathrm{s}$ for time and the discharge is computed as a volume rate $\left(\mathrm{m}^{3} \mathrm{~s}^{-1}\right)$. To facilitate the input for the user we conformed to hydraulic literature. This implies that the user has to specify the weir characteristics that define a relationship of the following form:

$$
Q=\alpha_{\text {input }} H^{\beta}
$$

where $Q$ is the discharge $\left(\mathrm{m}^{3} \mathrm{~s}^{-1}\right), H$ is the head above the crest $(\mathrm{m})$ and $\alpha_{\text {input }}$ is a weir coefficient $\left(\mathrm{m}^{3-}\right.$ $\left.{ }^{\beta} \mathrm{s}^{-1}\right), \beta$ is a weir exponent (-).

The preparatory work that the user has to do is to compute the value of $\alpha_{\text {input }}$ from the various coefficients preceding the upstream head above the crest. For instance, for a broad-crested rectangular weir, $\alpha_{\text {input }}$ is (approximately) given by:

$$
\alpha_{\text {input }}=1.7 \mathrm{~b}
$$

where 1,7 is the discharge coefficient of the weir (based on SI-units), $b$ is the width of the weir (m). To correct for units, SWAP carries out the following conversion:

$$
\alpha_{\text {weir }}=\frac{8.65 * 100^{(1-\beta)}}{A_{c u}}
$$

where $A_{c u}$ is the size of the control unit (ha) 
The model requires input of the size of the control unit $\left(A_{c u}\right)$, which in simple cases will be identical to the size of the simulation unit.

If the discharge relation is described using a table (SWQHR=2) then, for each water management period with a fixed weir crest using weir characteristics, the user should specify a table in Section 4d.

In Section $4 \mathrm{e}$ of the input file the required parameters should be given to introduce an automatic weir (SWMAN=2) controlled by soil moisture characteristics (see also Par. 5.1.2.2). For each management period with an automatic weir the user should specify in Section 4e:

- the maximum allowed drop rate of the water level setting

- the depth (HDEPTH) in the soil profile for a comparison between simulated and required soil moisture criterium (HCRIT)

The three state variables (GWLCRIT, HCRIT, VCRIT) that define the target weir level are given in a separate table.

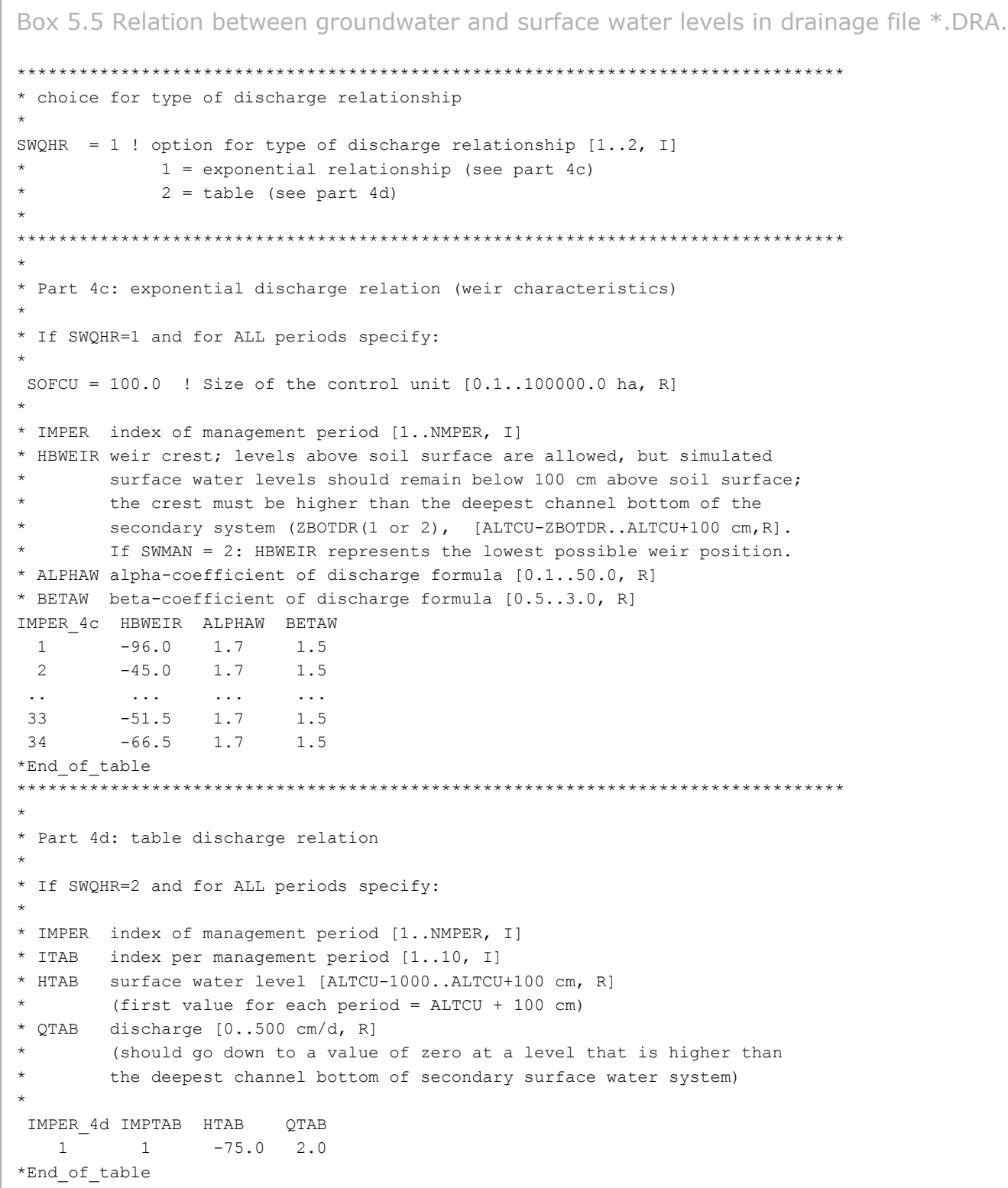


Box 5.6 Automatic and soil moisture controlled Weir management - in drainage file *.DRA.

* Part 4e: automatic weir control

*

* For the periods when SWMAN=2 specify next two tables:

$\star * \star$ Table \#1

$\star$

* IMPer index of management period [1..nMPER, I]

* DROPR maximum drop rate of surface water level [0..100 cm/d, positive, R]

* if the value is set to zero, the parameter does not play any role at all

* HDEPTH depth in soil profile for comparing with HCRIT

* $\quad[-100 \ldots 0 \mathrm{~cm}$ below soil surface, R]

IMPER_4E1 DROPR HDEPTH

$\begin{array}{ccc}-1 & 0.0 & -15.0\end{array}$

*End_of_table

*

$\star \star \star$ Table \#2

* IMPER index of management period [1..NMPER, I]

* IPHASE index per management period [1.10, I]

* WLSMAN surface water level of phase IPHASE [ALTCU-500.0.ALTCU cm,R]

* GWLCRIT groundwater level of phase IPHASE, max. value

* [-500.0 cm below soil surface, R]

* HCRIT critical pressure head, max. value, (at HDEPTH, see above)

* for allowing surface water level $[-1000 \ldots 0 \mathrm{~cm}$, neg., R]

* VCRIT critical unsaturated volume (min. value) for all

* surface water level $[0.20 \mathrm{~cm}, \mathrm{R}]$

* Notes: 1) The zero's for the criteria on the first record are in fact dummy's, because under all circumstances the scheme will set the surface water level at least to wlsman(imper, 1)

2) The lowest level of the scheme must still be above the

deepest channel bottom of the secondary surface water system

IMPER_4E2 IMPPHASE WLSMAN GWLCRIT HCRIT VCRIT

$\begin{array}{rrrrrr}2 & 1 & 1114.0 & 0.0 & 0.0 & 0.0 \\ 2 & 2 & 1124.0 & -80.0 & 0.0 & 0.0 \\ 2 & 3 & 1124.0 & -90.0 & 0.0 & 0.0 \\ 2 & 4 & 1154.0 & -100.0 & 0.0 & 0.0 \\ 3 & 1 & 1114.0 & 0.0 & 0.0 & 0.0 \\ 3 & 2 & 1124.0 & -80.0 & 0.0 & 0.0 \\ 3 & 3 & 1124.0 & -90.0 & 0.0 & 0.0 \\ 3 & 4 & 1154.0 & -100.0 & 0.0 & 0.0\end{array}$




\section{Macropore flow}

In structured soils such as clay and peat, preferential flow occurs through large pores or macropores in the (un)saturated soil matrix. Macropores are defined as pores with a diameter or width equal to or larger than $100 \mu \mathrm{m}$. Macroporosity can be caused by shrinking and cracking of soil, by plant roots, by soil fauna, or by tillage operations.

Due to the very rapid flow through macropores, water and solutes can reach large depths almost immediately after the start of a shower, bypassing the capacity of the soil matrix for storage, adsorption and transformation of potential pollutants. This process is known as 'bypass flow' or 'shortcircuiting' (Hoogmoed and Bouma, 1980). Because of the great impact of macropores on water flow and solute transport through the vadose zone, a concept has been implemented in SWAP for simulating preferential flow at the field scale.

Cracked clay soils reveal a large spatial variability of water contents and solute concentrations at given depth (Beven and Germann, 1982; Bronswijk et al., 1995). Therefore, instead of trying to describe water flow and solute transport at each location, the field-average behaviour may be easier to catch in a model. In order to make the model suitable for process and scenario analysis, concepts should be used that are generally applicable, thus physically based. Furthermore, model calibration requires a limited number of parameters, and preferably parameters that can be measured directly in the field. The description of macropore flow in SWAP has been developed taking into account these requirements.

The macropore flow concept is described by Hendriks et al. (in prep). It is new in the present SWAP and therefore, and to promote a well-considered use of this option its description is rather extensive and detailed. The concept of macropore flow is described in Section 6.1. The numerical implementation in the SWAP model is discussed in Section 6.2. User instructions are given in Section 6.3.

\subsection{Concept}

In the SWAP model macropore water flow includes the following processes:

- uptake of water by macropores at the soil surface;

- vertical transport to deeper layers or the groundwater bypassing the soil matrix;

- lateral infiltration into and exfiltration out of the soil matrix;

- rapid drainage to drainage systems;

- water storage in the macropores.

The simulation of these processes is based on the description of the macropore geometry as proposed by Hendriks et al. (1999). The description of this geometry that SWAP uses, is discussed in Section 6.1.1. Water flowing into macropores is instantaneously added to the water storage at the bottom of the macropores. Lateral infiltration into the soil matrix of macropore water running rapidly downwards is neglected. Because such downward flow occurs in a low number of contracted films, contact areas with the matrix are small, and consequently this infiltration is negligible (Hoogmoed and Bouma, 1980; Booltink, 1993). Some of the macropore inflow is trapped in discontinuous macropores and is therefore forced to infiltrate into the unsaturated matrix at different depths. This process is called 'internal catchment' (Bouma and Dekker, 1978; Van Stiphout et al., 1987). The water flow and balance are described in more detail in Section 6.1.2. 


\subsubsection{Macropore geometry}

In SWAP the geometry of macropore structure is described by characterising the macropore volume according to three main properties:

1. Continuity: vertical continuity controls flow of water that is taken up at the soil surface to different depths in the profile and horizontal continuity controls exchange of water between macropores (Section 6.1.1.1);

2. Persistency: static macropore volume is permanent, while dynamic macropore volume (shrinkage cracks) depends on soil moisture status (Section 6.1.1.2);

3. Horizontal distribution: in the horizontal plane, macropore volume is distributed over cracks and holes. The shape of the horizontal cross-section of the macropore volume has a large impact on the water exchange between macropore volume and soil matrix, and on rapid drainage (Section 6.1.1.3).

The concept provides a functional rather than a meticulous description of these macropore geometry properties. With a limited number of input parameters it determines a functional macropore bottom depth distribution, and magnitude and horizontal shape of the macropore volume as a function of depth.

A basic assumption in the concept is that property 'persistency' is not correlated with both other properties. 'Persistency' refers to volume and not to pore structure: static macropore volume and dynamic macropore volume form together the total macropore volume. Characterisation according to the other two properties applies to the total volume. The properties 'continuity' and 'horizontal distribution' are correlated: the horizontal macropore volume distribution as a function of depth depends on the macropore bottom depth distribution.

\subsubsection{Continuity}

With respect to vertical and horizontal continuity the macropores are divided into two classes that are integrated in two domains:

1. Main Bypass (MB) flow domain: the network of continuous, horizontal interconnected macropores;

2. Internal Catchment (IC) domain: discontinuous non-interconnected macropores, ending at different depths.

The MB domain represents the main system of continuous structural and shrinkage cracks, as well as root and worm holes. These macropores penetrate relatively deep into the soil profile and are assumed to be horizontal interconnected, for example in a network of structural and shrinkage cracks. In the MB domain water is transported relatively fast and deep into the profile bypassing the soil matrix. This may lead to short-circuiting between soil surface and groundwater, and rapid drainage to drains.

The IC domain represents macropores - cracks and holes - that are not interconnected and not connected to the MB domain, and that end at different depths in the profile. In this domain macropore inflow is entrapped at the bottom of individual macropores, resulting in forced infiltration of macropore water into the (mainly) unsaturated soil matrix at different, relatively shallow depths. 
A

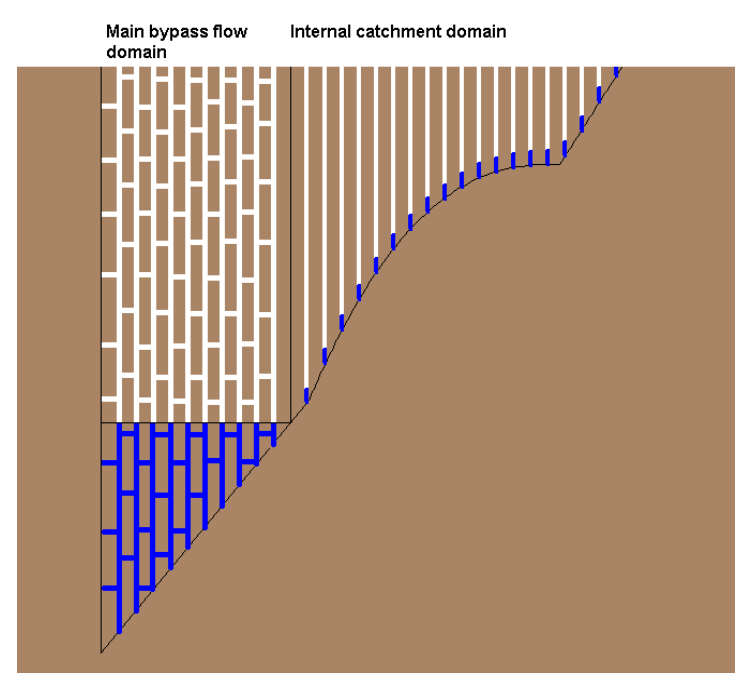

\section{B}

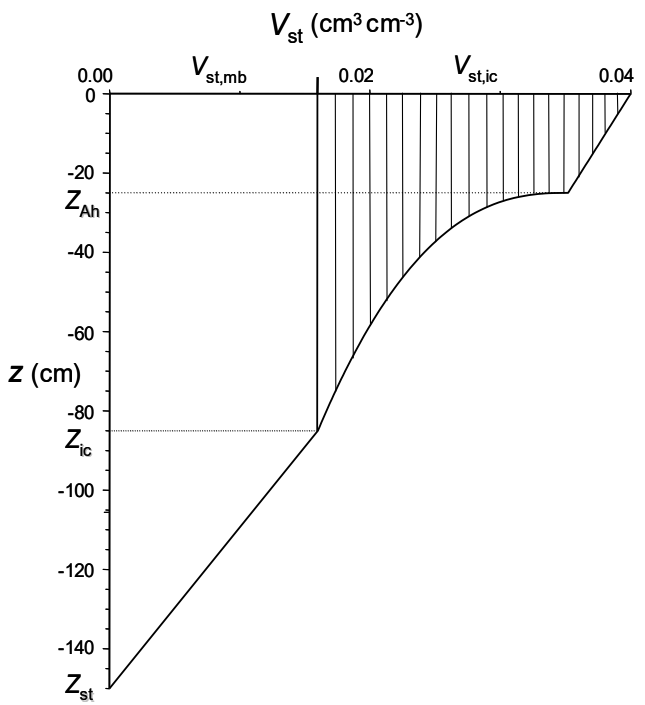

Figure 6.1 Aschematic representation of the two main domains: 1) Main bypass flow domain (MB): transporting water deep into the profile and possibly leading to rapid drainage, 2) Internal catchment domain (IC): infiltration of trapped water into the (mainly) unsaturated matrix at different depths; $B$ : mathematical description of the two domains, as static macropore volume fraction $V_{s t}$ as a function of depth for the MB $\left(V_{s t, m b}\right)$ and IC $\left(V_{s t, i c}\right)$ domain, with the IC domain divided in several sub-domains.

Figure 6.1.A presents a conceptual visualisation of the two classes of macropores; Figure 6.1.B depicts a mathematical representation of the conceptual figure. It describes the static macropore volume fraction $V_{\text {st }}\left(\mathrm{cm}^{3} \mathrm{~cm}^{-3}\right)$ of the two domains, $V_{\text {st, mb }}$ and $V_{\text {st,ic, }}$ as a function of depth. From these two volume fractions the partition of the static macropore volume over the two domains at any depth can be calculated. This partition as a function of depth is a crucial property of macropore geometry in the concept. It determines the distribution of macropore volume and water flow over both domains. It is expressed in the volumetric proportion $P_{\mathrm{dm}}(-)$ of each domain $d m$ :

$$
P_{\mathrm{mb}}=\frac{V_{\mathrm{st}, \mathrm{mb}}}{V_{\mathrm{st}, \mathrm{mb}}+V_{\mathrm{st}, \mathrm{ic}}} \text { and } P_{\mathrm{ic}}=\frac{V_{\mathrm{st}, \mathrm{ic}}}{V_{\mathrm{st}, \mathrm{mb}}+V_{\mathrm{st}, \mathrm{ic}}}
$$

and

$$
P_{\mathrm{mb}}=1-P_{\mathrm{ic}}
$$

where subscripts ' $m b^{\prime}$ ' and 'ic' refer to MB and IC domains, respectively.

In the concept, static macropore volume is not necessary always present. But even in the absence of this volume the volumetric proportions are required to partition the dynamic volume over the domains. Therefore, the calculation of the volumetric proportions as a function of depth is independent of the magnitude of the dynamic macropore volume. This calculation provides the 'blueprint' of the macropore domains. In order to visualize this 'blueprint' it is more illustrative to show only the static macropore volume as is done in Figure 6.1.B. Since the dynamic volume changes with time and depth, depending on shrinkage characteristic and soil moisture status that may differ at each depth, it would distort the image of the 'blueprint'.

The volumetric proportion of each domain as a function of depth is described by analytical equations with four basic input parameters: two depths $(\mathrm{cm}), Z_{\mathrm{AH}}$ representing the bottom of the A-horizon and $Z_{\text {ic }}$ representing the bottom of the IC domain, proportion $P_{\mathrm{ic}, 0}(-)$ of the IC domain at soil surface and power $m(-)$, a shape factor. In order to describe the individual IC macropores, the IC domain is divided into sub-domains. Strictly speaking, this subdivision of the IC macropore volume is an aspect of the numerical implementation and therefore is discussed in Section 6.2.1.1. 
The IC volumetric proportion at the soil surface $P_{\mathrm{ic}, 0}$ is an essential parameter of the concept. It determines the distribution over the two main domains of the precipitation water routed into the macropores at the soil surface, the major source of macropore water. It is assumed that the IC macropore volume consists of many individual small macropores that originates at the soil surface and functional end at different depths. In this sense 'functional' implies that flow is blocked at the depth of ending of the macropores. Thus, the functional volume of IC macropores gradually declines to zero at depth $Z_{\text {ic. }}$.

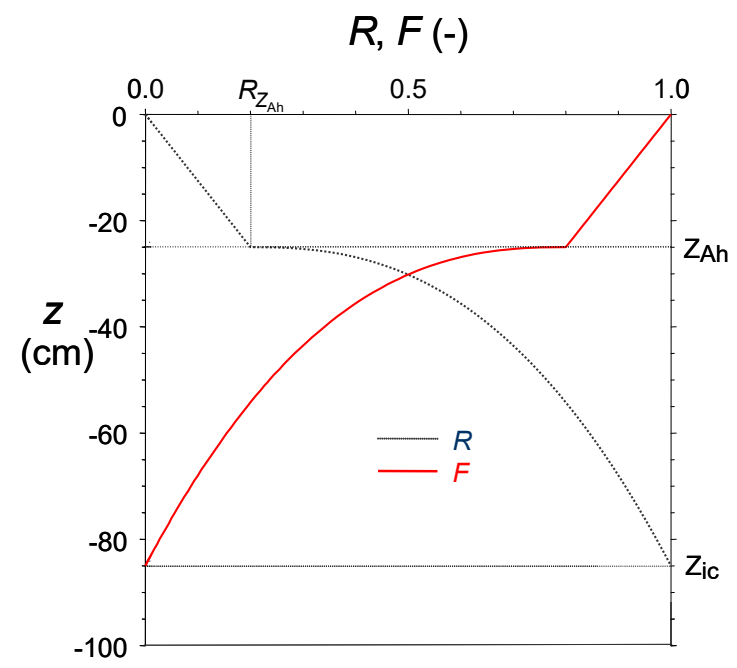

Figure 6.2 Cumulative frequency distribution $R$ of the depth $z$ at which the functional IC macropores end and the fraction F of IC macropores that is functional at depth $z$.

The cumulative frequency distribution of the depth $z$ at which the functional IC macropores end in the concept is described with a power law function (Figure 6.2):

$$
\begin{array}{ll}
R=R_{Z_{\mathrm{Ah}}} \frac{z}{Z_{\mathrm{Ah}}} & \text { for } 0 \geq z>Z_{\mathrm{Ah}} \\
R=R_{Z_{\mathrm{Ah}}}+\left(1-R_{Z_{\mathrm{Ah}}}\right)\left(\frac{Z_{\mathrm{Ah}}-z}{Z_{\mathrm{Ah}}-Z_{\mathrm{ic}}}\right)^{m} & \text { for } Z_{\mathrm{Ah}} \geq z \geq Z_{\mathrm{ic}}
\end{array}
$$

where the depths $z, Z_{\text {Ah }}$ and $Z_{\text {ic }}(\mathrm{cm})$ are defined negative downwards and the power $m(-)$ is a shapefactor. Power $m<1$ describes shallow IC systems, while $m>1$ describes deep IC systems; $m=1$ describes an intermediate system with a linear decline of functional IC macropores with depth. $R_{\text {ZAH }}(-)$ is an optional parameter with which a linear increase of the $R$-curve over the thickness of the Ahorizon can be described. Its default value is zero.

Curve $F$ in Figure 6.2 depicts the complement of $R$, the cumulative frequency distribution of the depth at which IC macropores are not ended in the concept, i.e. the fraction of IC macropores that is functional at that depth $z$. Functional in the sense of downward transport and storage of water, and lateral infiltration of macropore water into the soil matrix:

$$
F=1-R
$$

The volumetric proportion of IC macropore volume as a function of depth can be written in terms of the constant $P_{\mathrm{ic}, 0}$ and the function $F$ :

$$
\begin{aligned}
& P_{\mathrm{ic}}=\frac{F}{\frac{1}{P_{\mathrm{ic}, 0}}+F-1} \quad \text { for } 0 \geq z>Z_{\mathrm{ic}} \quad \text { and } \quad 0<P_{\mathrm{ic}, 0} \leq 1 \\
& P_{\mathrm{ic}}=0 \quad \text { for } z \leq Z_{\mathrm{ic}} \quad \text { and } / \text { or for } \quad P_{\mathrm{ic}, 0}=0
\end{aligned}
$$


The volumetric proportion of MB macropore volume as a function of depth is calculated from function $P_{\text {ic }}$ with Eq. (6.2). This results in a proportion $P_{\mathrm{mb}}$ of 1 for depths below $Z_{\text {ic }}$ where IC macropore volume is absent and all macropore volume is MB volume.

\subsubsection{Persistency}

With respect to persistency the macropore volume of each of the domains consists of:

1. Static macropore volume, expressed as volume fraction $V_{\mathrm{st}}\left(\mathrm{cm}^{3} \mathrm{~cm}^{-3}\right)$ : macropores that are permanent present. The static volume as a function of depth is constant in time;

2. Dynamic macropore volume, expressed as volume fraction $V_{\mathrm{dy}}\left(\mathrm{cm}^{3} \mathrm{~cm}^{-3}\right)$ i.e. shrinkage cracks. The dynamic volume as a function of depth is not constant in time.

The dynamic shrinkage volume is added up to the static volume, if present, and in this way enlarges the total macropore volume (Figure 6.3). The total macropore volume fraction $V_{\mathrm{mp}}\left(\mathrm{cm}^{3} \mathrm{~cm}^{-3}\right)$ is distributed over the two domains according to their volumetric proportion:

$$
\begin{aligned}
& V_{\mathrm{mp}}=V_{\mathrm{st}}+V_{\mathrm{dy}} \\
& V_{\mathrm{mb}}=P_{\mathrm{mb}} V_{\mathrm{mp}}=P_{\mathrm{mb}}\left(V_{\mathrm{st}}+V_{\mathrm{dy}}\right)=V_{\mathrm{st}, \mathrm{mb}}+P_{\mathrm{mb}} V_{\mathrm{dy}} \\
& V_{\mathrm{ic}}=P_{\mathrm{ic}} V_{\mathrm{mp}}=P_{\mathrm{ic}}\left(V_{\mathrm{st}}+V_{\mathrm{dy}}\right)=V_{\mathrm{st}, \mathrm{ic}}+P_{\mathrm{ic}} V_{\mathrm{dy}}
\end{aligned}
$$

This implies that below depth $Z_{\text {ic }}$ all dynamic volume is part of the MB domain. And below depth $Z_{\text {st }}$ only dynamic volume may occur.

\section{Static macropore volume}

The static macropore volume consists of structural shrinkage cracks, bio-pores (e.g., worm and root holes) and macropores that originate from tillage operations. Contrary to dynamic macropore volume, it is independent of the soil moisture status. The volume fraction of static macropores $V_{\mathrm{st}}\left(\mathrm{cm}^{3} \mathrm{~cm}^{-3}\right)$ as a function of depth $z$ is described with the constant $P_{\mathrm{ic}, 0}$, the function $F$ (Eq. (6.4)) and the two additional input parameters $V_{\text {st }, 0}(-)$, describing the volume fraction of static macropores at the soil surface, and $Z_{\text {st }}(\mathrm{cm})$, signifying the depth of static macropores.

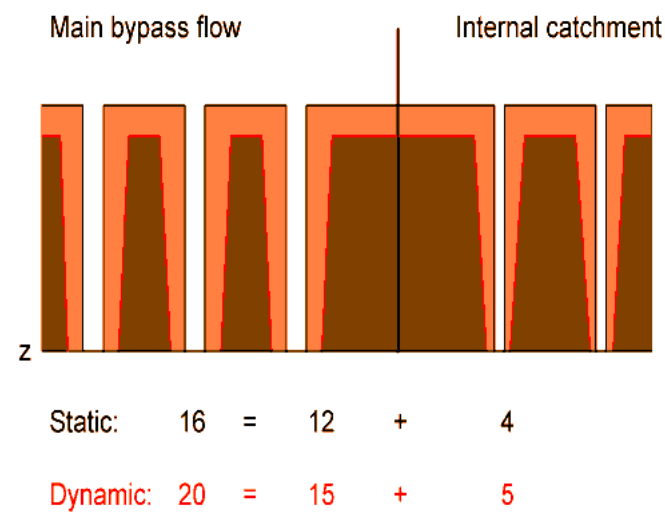

Figure 6.3 Partitioning of static and dynamic macropore volume over the two macropore domains according to the volumetric proportions of the domains $\left(P_{i c}=0.25\right)$ : ratio between MB and IC domains is equal for static and dynamic macropore volume. White areas represent static and light areas dynamic macropore volume. Dark colour is the soil matrix. Numbers are imaginary macropore volumes meaning: Total = Main Bypass + Internal Catchment. 
In general (all $V$ in $\mathrm{cm}^{3} \mathrm{~cm}^{-3}$ ):

$$
V_{\mathrm{st}}=V_{\mathrm{st}, \mathrm{mb}}+V_{\mathrm{st}, \mathrm{ic}} \quad \text { and thus } \quad V_{\mathrm{st}, 0}=V_{\mathrm{st}, \mathrm{mb}, 0}+V_{\mathrm{st}, \mathrm{cc}, 0}
$$

where:

$$
V_{\mathrm{st}, \mathrm{c}, 0}=P_{\mathrm{ic}, 0} V_{\mathrm{st}, 0} \quad \text { and } \quad V_{\mathrm{st}, \mathrm{mb}, 0}=\left(1-P_{\mathrm{ic}, 0}\right) V_{\mathrm{st}, 0}
$$

The static macropore volume fraction of the MB domain as a function of depth is calculated as:

$$
\begin{array}{ll}
V_{\mathrm{st}, \mathrm{mb}}=V_{\mathrm{st}, \mathrm{mb}, 0} & \text { for } 0 \geq z>Z_{\mathrm{ic}} \\
V_{\mathrm{st}, \mathrm{mb}}=V_{\mathrm{st}, \mathrm{mb}, 0} \frac{z-Z_{\mathrm{st}}}{Z_{\mathrm{ic}}-Z_{\mathrm{st}}} & \text { for } Z_{\mathrm{ic}} \geq z>Z_{\mathrm{st}} \\
V_{\mathrm{st}, \mathrm{mb}}=0 & \text { for } z \leq Z_{\mathrm{st}}
\end{array}
$$

And the static macropore volume fraction of the IC domain as a function of depth:

$$
\begin{array}{ll}
V_{\mathrm{st}, \mathrm{ic}}=F V_{\mathrm{st}, \mathrm{i}, 0} & \text { for } 0 \geq z>Z_{\mathrm{ic}} \\
V_{\mathrm{st}, \mathrm{ic}}=0 & \text { for } z \leq Z_{\mathrm{ic}}
\end{array}
$$

\section{Dynamic macropore volume}

The dynamic macropore volume originates from the shrinking of the soil matrix due to soil moisture loss. In general, this process is restricted to soils that contain substantial amounts (>10-15 mass-\%) of clay minerals (except kaolonite) and/or organic matter. Mostly, the shrinkage volume occurs as shrinkage cracks. But shrinkage of the matrix may also enlarge cylinder-shaped macropores. In SWAP it is assumed that shrinkage enlarges the present permanent macropore volume and consequently the shrinkage volume is added up to the static volume (Eq. (6.3)).

Soil matrix shrinkage occurs in vertical and horizontal direction. Vertical shrinkage leads to soil surface subsidence, horizontal shrinkage to dynamic macropore volume. The dynamic volume is calculated from overall and vertical shrinkage as:

$$
V_{\text {dy }}=V_{\text {sh }}-V_{\text {su }}
$$

where $V_{\mathrm{sh}}, V_{\mathrm{dy}}$ and $V_{\mathrm{su}}$ (all in $\mathrm{cm}^{3} \mathrm{~cm}^{-3}$ ) are the volume fraction of overall matrix shrinkage, the dynamic macropore volume fraction and the subsidence volume fraction, respectively.

In the present version of SWAP the vertical shrinkage does not affect the vertical coordinate system of SWAP. This approach avoids numerical problems that may result from solving Richards equation for a dynamical vertical coordinate. We assume that at the field scale the effects of ignoring changes of the soil matrix in vertical direction are small as compared to effects of uncertainties in other processes and input parameters.

However, the approach of ignoring vertical changes in soil matrix does affect the calculation of the dynamic macropore volume. This volume is corrected for the vertical shrinkage according to:

$$
V_{\mathrm{dy}}=\frac{V_{\mathrm{sh}}-V_{\mathrm{su}}}{1-V_{\mathrm{su}}}
$$

In this way the ratio between $V_{\mathrm{dy}}$ on one hand, and the matrix volume fraction and the static macropore volume fraction $V_{\text {st }}$ on the other hand is consistent.

If static macropore volume is present, the horizontal area fraction of the matrix equals $1-V_{\mathrm{st}} \mathrm{cm}^{2}$ $\mathrm{cm}^{-2}$ and consequently the dynamic macropore volume fraction is calculated as:

$$
V_{\mathrm{dy}}=\left(1-V_{\mathrm{st}}\right) \frac{V_{\mathrm{sh}}-V_{\mathrm{su}}}{1-V_{\mathrm{su}}}
$$


The vertical shrinkage component is determined from the overall matrix shrinkage as (Bronswijk, 1988):

$$
V_{\mathrm{su}}=1-\left(1-V_{\mathrm{sh}}\right)^{\frac{1}{r_{\mathrm{s}}}}
$$

where exponent $r_{\mathrm{s}}(-)$ is the geometry factor (Rijniersce, 1983). In case of three-dimensional isotropic shrinkage, $r_{\mathrm{s}}=3$. When cracking dominates subsidence $r_{\mathrm{s}}>3$, when subsidence dominates cracking $1<r_{\mathrm{s}}<3$. In case of subsidence only, $r_{\mathrm{s}}=1$. The geometry factor is an input parameter.

The matrix shrinkage volume fraction $V_{\text {sh }}$ is a function of volumetric moisture content and shrinkage characteristic. A shrinkage characteristic describes the relationship between soil volume and soil moisture content. Many forms of shrinkage characteristics exist. A very convenient one is the characteristic that takes the constant volume fraction of the solid soil fraction $V_{\text {sol }}$ as reference for all variable volume fractions and expresses the soil matrix volume fraction $V_{\mathrm{m}}$ in terms of pore volume fraction $V_{\mathrm{p}}$ relative to $V_{\text {sol }}$ (Bronswijk, 1988) (all $V$ in $\mathrm{cm}^{3} \mathrm{~cm}^{-3}$ ):

$$
V_{\mathrm{m}}=V_{\mathrm{sol}}+V_{\mathrm{p}}=(1+e) V_{\mathrm{sol}}
$$

where $e\left(\mathrm{~cm}^{3} \mathrm{~cm}^{-3}\right)$ is the void ratio:

$$
e=\frac{V_{\mathrm{p}}}{V_{\mathrm{sol}}}
$$

The shrinkage volume fraction $V_{s h}$ is equal to the fraction of volume loss of the matrix that in its turn equals the fraction loss of pore volume. The latter is expressed in terms of $e$ and $V_{\text {sol }}$ :

$$
V_{\mathrm{sh}}=-\Delta V_{\mathrm{m}}=-\Delta V_{\mathrm{p}}=-\Delta e V_{\mathrm{sol}}=-\left(e-e_{\mathrm{s}}\right) V_{\mathrm{sol}}
$$

where $e_{\mathrm{s}}$ is the void ratio at saturation.

The shrinkage characteristic expresses the variable $e$ as a function of the variable $\vartheta\left(\mathrm{cm}^{3} \mathrm{~cm}^{-3}\right)$, which represents the moisture ratio:

$$
e=\mathrm{f}(\vartheta)
$$

where the moisture ratio is defined as:

$$
\vartheta=\frac{V_{\mathrm{w}}}{V_{\mathrm{sol}}}=\frac{\theta}{1-\theta_{\mathrm{s}}}
$$

with $V_{\mathrm{w}}\left(\mathrm{cm}^{3} \mathrm{~cm}^{-3}\right)$ the actual water volume fraction that equals $\theta$, the volumetric moisture content of the matrix. Volume fraction of solids $V_{\text {sol }}\left(\mathrm{cm}^{3} \mathrm{~cm}^{-3}\right)$ equals $1-\theta_{\mathrm{s}}\left(\theta_{\mathrm{s}}=\theta\right.$ at saturation).

The exact form of the shrinkage characteristic depends on soil texture, in terms of content and nature of clay minerals, and organic matter. Shrinkage characteristics of clay and organic soils - peat and peaty soils - differ strongly.

Shrinkage characteristic of clay and clayey soils

Figure 6.4.A shows a typical shrinkage characteristic of a clay soil. Three stages can be distinguished (Stroosnijder, 1975; Bronswijk, 1988):

1. Normal shrinkage: volume decrease of clay aggregates is equal to moisture loss. The aggregates remain fully saturated;

2. Residual shrinkage: upon drying the volume of the aggregates still decreases, but moisture loss is greater than volume decrease. Air enters the pores of the aggregates;

3. Zero shrinkage: soil particles reach their most dense configuration. Upon further moisture extraction, the volume of the aggregates remains constant. Moisture loss equals air volume increase of the aggregates. Rigid soils, like sands, only show this stage.

A fourth shrinkage stage that precedes normal shrinkage may be recognized: structural shrinkage. When saturated soils dry, large water filled pores may be emptied. As a result, aggregates can get a 
somewhat denser packing. Overall, the volume changes in this stage are negligible, but water losses can be considerable. In SWAP, structural shrinkage is explicitly accounted for in the form of the static macropores, e.g. structural shrinkage cracks. The first three real shrinkage stages are computed as a function of moisture content with the equation of Kim (1992):

$$
e=\alpha_{\mathrm{K}} \exp \left(-\beta_{\mathrm{K}} \vartheta\right)+\gamma_{\mathrm{K}} \vartheta \text { for } 0<\vartheta<\vartheta_{\mathrm{s}} \quad \text { where } \quad \vartheta_{\mathrm{s}}=\frac{\theta_{\mathrm{s}}}{1-\theta_{\mathrm{s}}}
$$

with $\alpha_{\mathrm{K}}\left(\mathrm{cm}^{3} \mathrm{~cm}^{-3}\right)$ equals $e_{0}$ the void ratio at $\vartheta=0, \beta_{\mathrm{K}}$, and $\gamma_{\mathrm{K}}$ are dimensionless fitting parameters and $\vartheta_{\mathrm{s}}$ is void ratio at saturation. Using Eq. (6.18), e may become smaller than $e_{0}$, in which case the model sets $e$ to $e_{0}$ (zero shrinkage).

\section{Shrinkage characteristic of peat and peaty soils}

According to Hendriks (2004), for peat soils three shrinkage stages can be distinguished as well (Figure 6.4.B):

1. Near-normal shrinkage: volume reduction equals nearly moisture loss, little air enters the pores and the peat matrix remains close to saturation;

2. Subnormal shrinkage: upon drying moisture loss exceeds volume reduction, air enters the relatively large pores while the small pores in the organic fibres, that form the 'skeleton' around the larger pores, remain water-filled;

3. Supernormal shrinkage: volume reduction exceeds by far moisture loss, small pores are emptied and the skeleton collapses, so that air is driven out of the larger pores and the matrix reaches its final, smallest volume when the moisture content is zero.
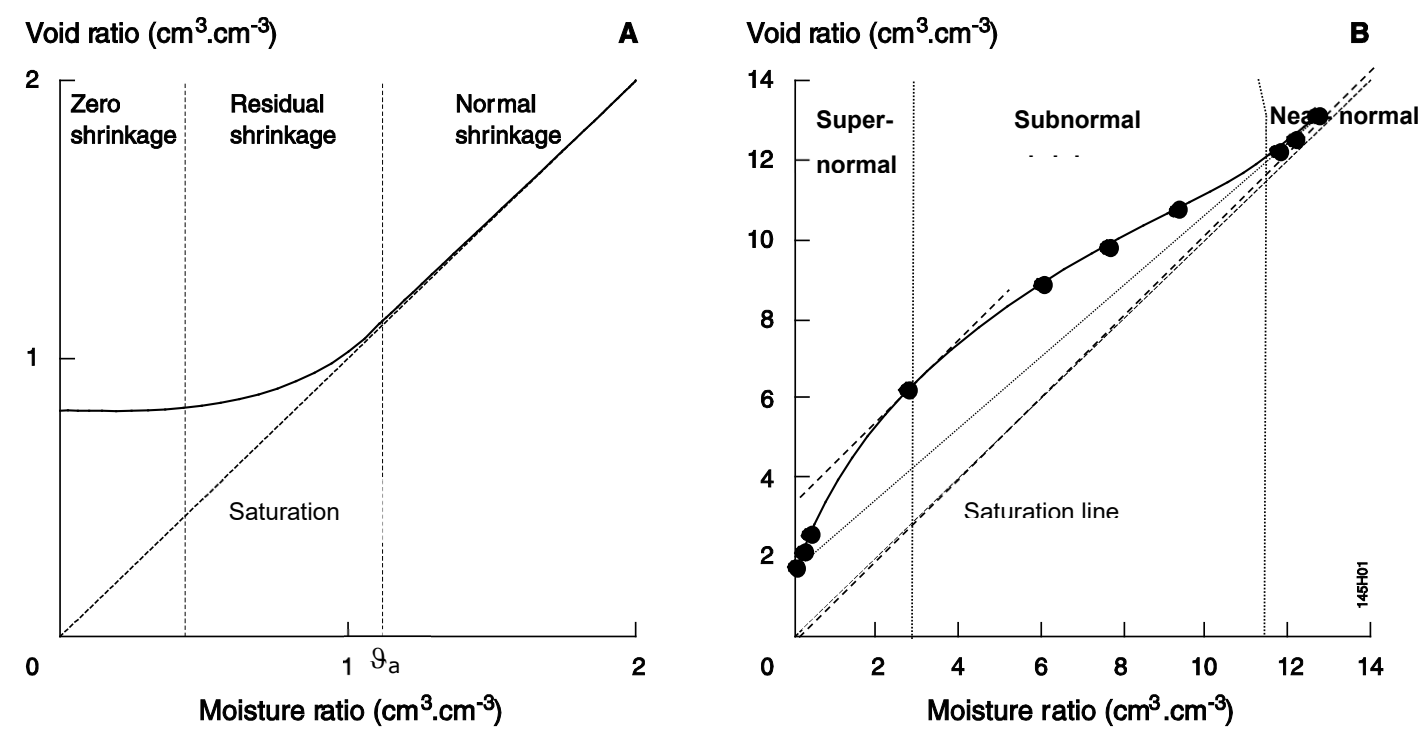

Figure 6.4 Typical shrinkage characteristic of A. clay (modified after Bronswijk, 1988) and B. peat (after Hendriks, 2004), expressed as void ratio e as a function of moisture ratio $\vartheta$, showing the three shrinkage stages. Black dots in B. are measurements while solid line is fit with Eq.(6.19).

Hendriks' (2004) equation for the shrinkage curve of peat and peaty soils reads:

$$
\begin{array}{ll}
e=e_{\mathrm{t}}\left(1+P_{\mathrm{H}} \frac{\vartheta_{\mathrm{t}}^{\alpha_{\mathrm{H}}}\left(\exp \left(-\beta_{\mathrm{H}} \vartheta_{\mathrm{t}}\right)-\exp \left(-\beta_{\mathrm{H}}\right)\right)}{\vartheta_{\mathrm{t}, \mathrm{P}}^{\alpha_{\mathrm{H}}}\left(\exp \left(-\alpha_{\mathrm{H}}\right)-\exp \left(-\beta_{\mathrm{H}}\right)\right)}\right) \text { for } & 0<\vartheta<\vartheta_{\mathrm{a}} \\
e=e_{\mathrm{t}} & \text { for } \vartheta_{\mathrm{a}} \leq \vartheta<\vartheta_{\mathrm{s}}
\end{array}
$$

with:

$$
e_{\mathrm{t}}=e_{0}+\left(e_{\mathrm{s}}-e_{0}\right) \frac{\vartheta}{\vartheta_{\mathrm{s}}} ; e_{\mathrm{s}}=\vartheta_{\mathrm{s}}=\frac{\theta_{\mathrm{s}}}{1-\theta_{\mathrm{s}}}
$$




$$
\vartheta_{\mathrm{t}}=\frac{\vartheta}{\vartheta_{\mathrm{a}}} ; \vartheta_{\mathrm{t}, \mathrm{P}}=\frac{\alpha_{\mathrm{H}}}{\beta_{\mathrm{H}}} \quad \text { for } 0<\alpha_{\mathrm{H}}<\beta_{\mathrm{H}}
$$

where $\vartheta_{a}$ is the moisture ratio at the transition of the near-normal shrinkage stage to the subnormal shrinkage stage, when air entry increases substantially. $a_{\mathrm{H}}, \beta_{\mathrm{H}}$ and $P_{\mathrm{H}}$ are dimensionless fitting parameters.

\section{Overburden pressure}

Shrinking and swelling behaviour in the field may deviate from that in the laboratory because of overburden pressure of overlaying soil layers in the field. This may result in delayed horizontal shrinkage in favour of vertical shrinkage. To account for this process, a threshold moisture content $\theta_{\text {crack }}$ is introduced. For moisture contents $\theta \geq \theta_{\text {crack }}$ all shrinkage is vertical and for $\theta<\theta_{\text {crack }}$ shrinkage is vertical and horizontal according to geometry factor $r_{\mathrm{s}}$. This concept does not apply to swelling: shrinkage cracks are not closed before saturation. $\theta_{\text {crack }}$ is an input parameter.

\subsubsection{Horizontal distribution}

In the horizontal plane in the field, macropore volume is distributed over different forms of macropores: from holes with a diameter of $100 \mu \mathrm{m}$ to several centimetres wide, several decimetres long cracks. This distribution determines the functional horizontal shape of the macropores, which forms the basis of the calculation of several important parameters:

1. two parameters that affect lateral water exchange between macropores and soil matrix:

a. the vertical area of macropore walls per unit of volume, and

b. the distance from macropore wall to centre of matrix polygons;

2. the lateral hydraulic conductivity of cracks in case of rapid drainage.

For simplicity and input parameter limitation, cracks and hole-shaped macropores are not explicitly distinguished. Instead, they are implicit in an effective functional horizontal macropore shape that is described by an effective matrix polygon diameter $d_{\text {pol }}(\mathrm{cm})$ as a function of depth.

Assuming effective regular soil matrix polygons, the effective vertical area of macropore walls per unit of volume $A^{*}$ wall $\left(\mathrm{cm}^{2} \mathrm{~cm}^{-3}\right)$ is equal to the quotient of the perimeter divided by the area of the polygons. For all even-sided regular polygons, from square to circle, this quotient equals (see Appendix 2 for derivation):

$$
A_{\mathrm{wall}}^{*}=\frac{4}{d_{\mathrm{pol}}}
$$

The effective horizontal distance $x_{\text {pol }}(\mathrm{cm})$ from macropore wall to matrix polygon centre is taken equal to:

$$
x_{\mathrm{pol}}=\frac{1}{2} d_{\mathrm{pol}}
$$

The effect of horizontal macropore shape on rapid drainage is expressed through the effect on the lateral hydraulic conductivity of cracks which depends on the effective crack width $w_{\mathrm{cr}}(\mathrm{cm})(\mathrm{see}$ Section 6.1.2.3). This width is calculated from the effective polygon diameter and the volume fraction $V_{\mathrm{mb}}$ of macropores in the MB domain (see 0 for derivation):

$$
w_{\mathrm{cr}}=d_{\mathrm{pol}}\left(1-\sqrt{1-V_{\mathrm{mb}}}\right)
$$

It is assumed that the effective diameter $d_{\text {pol }}$ of the soil polygons is a function of depth with its minimum value at the soil surface where macropore density is maximal and consequently distances between macropores are relatively small, and its maximum value deeper in the profile where macropore density is minimal. $d_{\text {pol }}$ as a function of depth is determined from a maximum $d_{p, \max }$ and minimum $d_{\mathrm{p} \text {, min }}$ polygon diameter, both input parameters, and the relative macropore density $M(-)$ as a function of depth according to:

$$
d_{\mathrm{pol}}=d_{\mathrm{p}, \min }+\left(d_{\mathrm{p}, \max }-d_{\mathrm{p}, \min }\right)(1-M)
$$


where $M$ depends on the static macropore volume if present:

$$
M=\frac{V_{\mathrm{st}}}{V_{\mathrm{st}, 0}} \quad \text { for } V_{\mathrm{st}, 0}>0
$$

If no static macropore volume is present $M$ depends on the volumetric proportion of the IC domain:

$$
M=\frac{P_{\mathrm{ic}}}{P_{\mathrm{ic}, 0}} \quad \text { for } V_{\mathrm{st}, 0}=0 \quad \text { and } \quad P_{\mathrm{ic}, 0}>0
$$

If no static macropore volume and no IC domain are present, $M$ can be defined as a function of depth with $Z_{\mathrm{dpmax}}$ as the depth below which $d_{\mathrm{pol}}$ equals $d_{\mathrm{p} \text {, max }}$ :

$$
M=\max \left(0,1-\frac{z}{Z_{\mathrm{dpmax}}}\right) \quad \text { for } V_{\mathrm{st}, 0}=0 \text { and } P_{\mathrm{ic}, 0}=0
$$

\subsubsection{Water flow and balance}

In SWAP macropore water flow and balance comprise (Figure 6.5):

1. storage of water in the macropore domains $S_{\mathrm{mp}}(\mathrm{cm})$;

2. infiltration of water into macropores at soil surface, by precipitation, irrigation and snowmelt water falling directly into macropores $I_{\mathrm{pr}}$ and by overland flow (runoff) into the macropores $I_{\mathrm{ru}}\left(\mathrm{cm} \mathrm{d}^{-1}\right)$;

3. lateral infiltration into the unsaturated soil matrix $q_{\mathrm{lu}}\left(\mathrm{cm} \mathrm{d}^{-1}\right)$;

4. lateral infiltration into and exfiltration out of the saturated soil matrix $q_{\mathrm{ls}}\left(\mathrm{cm} \mathrm{d}^{-1}\right)$;

5. lateral exfiltration out of the saturated soil matrix by interflow out of a zone with perched groundwater $q_{\mathrm{li}}\left(\mathrm{cm} \mathrm{d}^{-1}\right)$;

6. rapid drainage to drainage systems $q_{\mathrm{rd}}\left(\mathrm{cm} \mathrm{d}^{-1}\right)$.

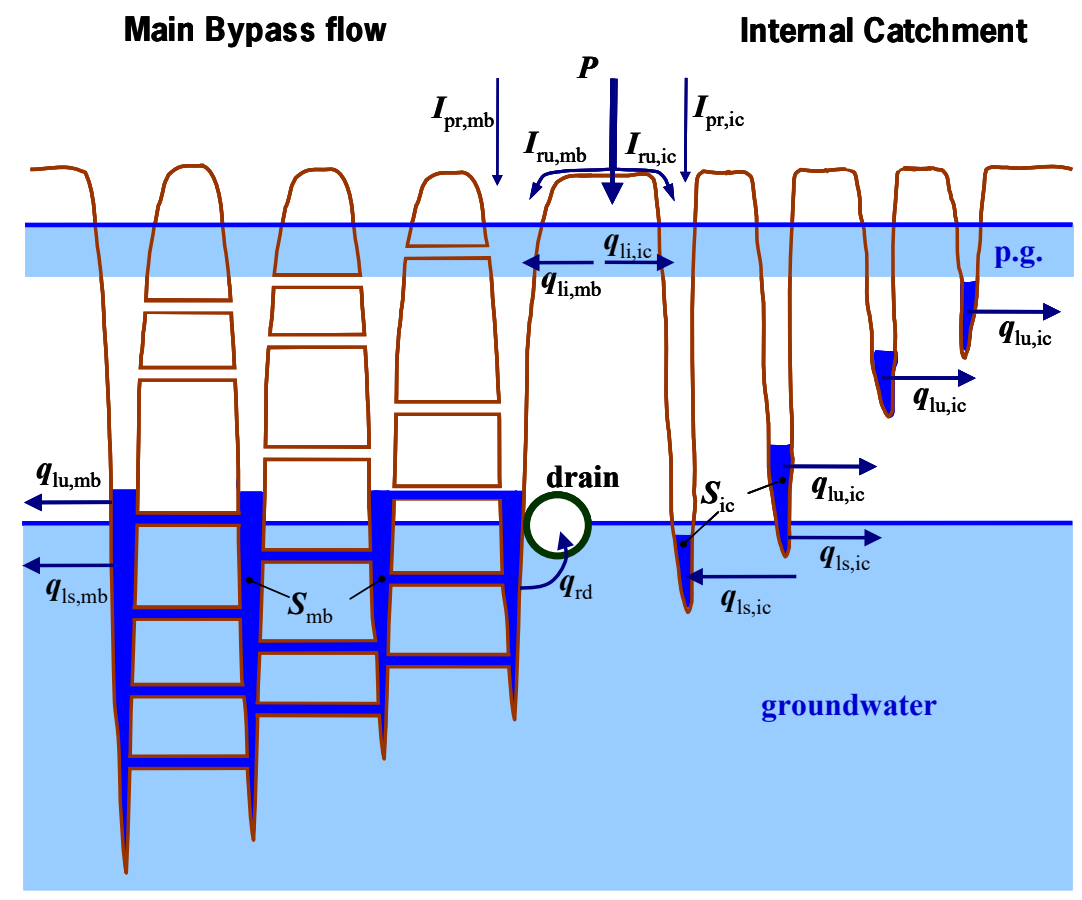

Figure 6.5 Schematic representation of the soil profile with soil matrix, drain, groundwater, perched groundwater (p.g.), macropores in MB and IC domains, and the various macropore water balance terms. Mark that the saturated lateral exchange flux $q_{\text {ls }}$ can occur in two directions. See text for explanation of terms. 


\section{Water balance}

The water balance of the MB domain for a given time interval $\mathrm{d} t=t_{0} \rightarrow t$ reads:

$$
S_{\mathrm{mb}}^{t}-S_{\mathrm{mb}}^{t_{0}}=\int_{t_{0}}^{t}\left(I_{\mathrm{pr}, \mathrm{mb}}+I_{\mathrm{ru}, \mathrm{mb}}+q_{\mathrm{li}, \mathrm{mb}}-q_{\mathrm{lu}, \mathrm{mb}}-q_{\mathrm{ls}, \mathrm{mb}}-q_{\mathrm{rd}}\right) \mathrm{d} t
$$

where:

$$
q_{\mathrm{li}, \mathrm{mb}}=\int_{z_{\mathrm{if}, \mathrm{bot}}}^{z_{\mathrm{ifftop}}} q_{\mathrm{li}, \mathrm{mb}}^{*} \mathrm{~d} z ; \quad q_{\mathrm{lu}, \mathrm{mb}}=\int_{z_{\text {uns,bot }}}^{z=0} q_{\mathrm{lu}, \mathrm{mb}}^{*} \mathrm{~d} z ; q_{\mathrm{ls}, \mathrm{mb}}=\int_{z_{\text {prof,bot }}}^{z_{\text {uns,bot }}} q_{\mathrm{ls}, \mathrm{mb}}^{*} \mathrm{~d} z
$$

All balance terms are positive except $q_{\mathrm{Is}}$ which is positive in case of infiltration into the matrix and negative in case of exfiltration out of the matrix. The storage term is always less than or equal to the actual macropore volume. All flux densities $q^{*}$ are defined per unit of depth $\left(\mathrm{cm} \mathrm{cm}^{-1} \mathrm{~d}^{-1}\right)$. Depths $z_{\text {if,top }}, z_{\text {if, bot }}, z_{\text {uns,bot }}$ and $z_{\text {prof,bot }}(\mathrm{cm})$ refer to top and bottom of interflow zone, and bottom of unsaturated zone and soil profile, respectively.

The water balance calculation of the IC domain is equal to that of the MB domain with the exception of the rapid drainage term. Per definition, rapid drainage occurs exclusively in the MB domain.

Inflow at soil surface $\left(I_{p r}\right.$ and $\left.I_{r u}\right)$

The rate $I_{\mathrm{pr}}$ of precipitation, irrigation and snowmelt water routed directly into the macropores at the soil surface at a given precipitation/irrigation/snowmelt intensity $P\left(\mathrm{~cm} \mathrm{~d}^{-1}\right)$ is calculated as:

$$
I_{\mathrm{pr}}=A_{\mathrm{mp}} P
$$

where $A_{\mathrm{mp}}\left(\mathrm{cm}^{2} \mathrm{~cm}^{-2}\right)$ is the horizontal macropore area fraction at the soil surface which equals $V_{\mathrm{mp}, 0}$ $\left(\mathrm{cm}^{3} \mathrm{~cm}^{-3}\right)$ the total macropore volume fraction at soil surface. For macropore domain $d m$ (subscript $d m=\mathrm{mb}$ or $\mathrm{ic})$ this direct infiltration reads:

$$
I_{\mathrm{pr}, d m}=A_{d m} P \quad \text { with } \quad A_{d m}=P_{d m, 0} V_{\mathrm{mp}, 0}
$$

where $P_{d m, 0}(-)$ is the volumetric proportion of domain $d m$ at the soil surface. In case of a snowpack on top of the soil surface, it is assumed that the macropores are sealed off from the atmosphere and consequently $I_{\mathrm{pr}}=0$, except when snowmelt occurs.

Infiltration rate term $I_{\mathrm{ru}}$ occurs when the head boundary condition holds for the top boundary of the soil matrix (see Section 2.7.3.1). In that case the water balance of the ponding layer is calculated, which includes $I_{\mathrm{ru}}$. Ponding occurs when the total of precipitation, irrigation, snow melt, runon and inundation intensity exceeds soil matrix infiltration capacity. Runoff into the macropores is described in the same form as used for regular runoff to surface water or adjacent fields (Section 4.1) to allow for similar incorporation into the numerical solution of the top boundary (Appendix 17):

$$
I_{\mathrm{ru}}=\frac{h_{0}}{\gamma_{\text {Iru }}}
$$

where $h_{0}(\mathrm{~cm})$ is the pressure head at the soil surface that equals the ponding height and $\gamma_{\text {Iru }}(\mathrm{d})$ is the resistance for macropore inflow at the soil surface. The macropore inflow resistance is estimated from the maximum ponding height $h_{0, \max }$ assuming no runoff, either into macropores or regular, and the vertical hydraulic conductivity of the macropores at soil surface $K_{\mathrm{ver}, \mathrm{mp}}\left(\mathrm{cm} \mathrm{d}^{-1}\right)$. The latter is derived as a function of effective macropore width at soil surface from a theoretical slit model presented by Bouma and Anderson (1973):

$$
\gamma_{\text {Iru }}=\frac{h_{0, \text { max }}}{k_{\text {ver,mp }}} \quad \text { with } \quad K_{\text {ver,mp }}=14.4 \cdot 10^{8} \frac{w_{\mathrm{mp}, 0}^{3}}{d_{\mathrm{pol}, 0}}
$$

It can be seen that even the lower limit of macropore width $(100 \mu \mathrm{m})$ yields large conductivities in the order of 100-1000 ( $\left.\mathrm{cm} \mathrm{d}^{-1}\right)$ and consequently very low inflow resistances of 0.001-0.01 d. This implies that ponding water is routed preferentially into macropores. To account for the micro relief at the soil surface, mostly a threshold value for ponding height is used that must be exceeded before regular 
runoff starts. It is assumed that this does not apply to runoff into macropores, because it is very likely that micro depressions at the soil surface are connected to macropores. As a consequence, runoff into macropores is favoured over regular runoff and thus $\gamma_{\text {Iru }}$ is not a very sensitive variable.

Distribution of $I_{\mathrm{ru}}$ over MB and IC domains is according to their proportions at the soil surface $P_{\mathrm{mb}, 0}$ and $P_{\text {ic }, 0 \text {. }}$

Lateral infiltration into the unsaturated matrix $q_{l u}$

Lateral infiltration of macropore water into the unsaturated soil matrix takes place strictly over the depth where stored macropore water is in contact with the unsaturated matrix. Two lateral infiltration mechanisms are relevant: absorption of macropore water when capillary forces dominate and Darcy flow due to a pressure head gradient from macropore wall to centre of the effective matrix polygon. Absorption is the dominant mechanism at low soil moisture contents. It will be negligible under wet conditions even when there is a large pressure head gradient. In the latter case Darcy flow will be dominant. Darcy flow is very small under dry conditions because of very low hydraulic conductivities. Therefore, for each situation the flow rates of both infiltration mechanisms are calculated and the unsaturated infiltration flux is set equal to the largest of these two rates.

Lateral absorption is described with Philip's sorptivity (Philip, 1957):

$$
I_{\mathrm{ab}, t}^{*}=A_{\mathrm{wall}, \mathrm{mtx}}^{*} S_{\mathrm{P}} \sqrt{t-t_{0}}=\frac{4 S_{\mathrm{P}} \sqrt{t-t_{0}}}{d_{\mathrm{pol}} \sqrt{1-V_{\mathrm{mp}}}}
$$

where $I^{*}$ ab,t is the lateral absorption per unit of depth $\left(\mathrm{cm} \mathrm{cm}^{-1}\right)$ over time interval $t_{0} \rightarrow t(\mathrm{~d})$ and $S_{\mathrm{p}}$ is Philip's sorptivity $\left(\mathrm{cm} \mathrm{d}^{-0.5}\right)$. The meaning of $A^{*}{ }_{\text {wall, mtx }}$ is explained in Appendix 2 (Eq.(A2.12)). $S_{\mathrm{p}}$ is a function of initial volumetric moisture content $\theta_{0}\left(\mathrm{~cm}^{3} \mathrm{~cm}^{-3}\right)$ at $t=t_{0}$, the time of first contact of macropore water with the matrix. It is empirically described as (adapted from Greco et al., 1996):

$$
S_{\mathrm{P}}=S_{\mathrm{P}, \max }\left(1-\frac{\theta_{0}-\theta_{\mathrm{r}}}{\theta_{\mathrm{s}}-\theta_{\mathrm{r}}}\right)^{\alpha_{\mathrm{s}}}=S_{\mathrm{P}, \max }\left(\frac{\theta_{\mathrm{s}}-\theta_{0}}{\theta_{\mathrm{s}}-\theta_{\mathrm{r}}}\right)^{\alpha_{\mathrm{s}}}
$$

where $S_{P, \max }$ is the maximum sorptivity when $\theta_{0}=\theta_{\mathrm{r}}$ (residual moisture content) and $\alpha_{S}$ is a fitting parameter (-).

Average, constant absorption rate $q_{\text {lu,ab }}^{*}$ per unit of depth $\left(\mathrm{cm} \mathrm{cm}^{-1} \mathrm{~d}^{-1}\right)$ for time interval $t_{1} \rightarrow t_{2}$ is obtained from:

$$
q_{\mathrm{lu}, \mathrm{ab}}^{*}=\frac{I_{\mathrm{ab}, t_{2}}^{*}-I_{\mathrm{ab}, t_{1}}^{*}}{t_{2}-t_{1}}
$$

Infiltration rate by Darcy flow per unit of depth $q_{\mathrm{lu}, \mathrm{D}}^{*}\left(\mathrm{~cm} \mathrm{~cm}^{-1} \mathrm{~d}^{-1}\right)$ reads:

$$
q_{\mathrm{lu}, \mathrm{D}}^{*}=f_{\text {shp }} A_{\mathrm{wall}}^{*} K_{\mathrm{h}} \frac{\left(h_{\mathrm{mp}}-h_{\mathrm{mt}}\right)}{x_{\mathrm{pol}}}=\frac{f_{s h p} 8 K_{\mathrm{h}}\left(h_{\mathrm{mp}}-h_{\mathrm{mt}}\right)}{d_{\mathrm{pol}}^{2}}
$$

where $K_{\mathrm{h}}\left(\mathrm{cm} \mathrm{d}^{-1}\right)$ is the hydraulic conductivity as a function of pressure head in the unsaturated matrix $h_{\mathrm{mt}}(\mathrm{cm})$ and term $\left(h_{\mathrm{mp}}-h_{\mathrm{mt}}\right) / x_{\mathrm{pol}}$ is the lateral pressure head gradient between macropore and centre of the matrix polygon. Parameter $f_{\mathrm{shp}}(-)$ is a shape factor to account for the uncertainties in the theoretical description of lateral infiltration by Darcy flow originating from uncertainties in the exact shape of the soil matrix polygons. Depending on whether the polygons are more plate or cylinder shaped, the figure in Eq. (6.32) should be somewhere between 8 and 16. Thus, theoretically, the value of $f_{\mathrm{shp}}$ lies between 1 and 2 . Pressure head $h_{\mathrm{mp}}$ as a function of depth is obtained from the macropore water level elevation $\phi_{\mathrm{mp}}$ and depth $z$ as:

$$
h_{\mathrm{mp}}=\phi_{\mathrm{mp}}-z
$$


Finally, the lateral infiltration fluxdensity into the unsaturated matrix per unit of depth $q_{\text {lu }}^{*}\left(\mathrm{~cm} \mathrm{~cm}^{-1}\right.$ $\mathrm{d}^{-1}$ ) is obtained by taking the largest flow rate:

$$
q_{\mathrm{lu}}^{*}=\max \left(q_{\mathrm{lu}, \mathrm{ab}}^{*}, q_{\mathrm{lu}, \mathrm{D}}^{*}\right)
$$

Distribution of $q_{\text {lu }}^{*}$ over MB and IC domains is according to their proportions $P_{\mathrm{mb}}$ and $P_{\mathrm{ic}}$ at the specific depth $z$.

Lateral infiltration into and exfiltration out of the saturated matrix $q_{\text {ls }}$ Lateral infiltration of macropore water into the saturated soil matrix and lateral exfiltration of soil matrix water into the macropores takes place strictly over the depth where stored macropore water is in contact with the saturated matrix. This only concerns static macropores below the groundwater table, since in the present concept in saturated condition the soil is assumed swollen to its maximum volume, without dynamic macropore volume.

The lateral in- and exfiltration rate per unit of depth $q_{\mathrm{Is,D}}^{*}\left(\mathrm{~cm} \mathrm{~cm}^{-1} \mathrm{~d}^{-1}\right)$ in case of water filled macropores $\left(h_{\mathrm{mp}}>0\right)$ is described by Darcy flow similar to Eq. (6.32):

$$
q_{\mathrm{ls}, \mathrm{D}}^{*}=f_{\mathrm{shp}} A_{\mathrm{wall}}^{*} K_{\mathrm{sat}} \frac{\left(h_{\mathrm{mp}}-h_{\mathrm{mt}}\right)}{x_{\mathrm{pol}}}=\frac{f_{\text {shp }} 8 K_{\mathrm{sat}}\left(h_{\mathrm{mp}}-h_{\mathrm{mt}}\right)}{d_{\mathrm{pol}}^{2}}
$$

The same shape factor $f_{\text {shp }}$ as in Eq. (6.32) is adopted since the same considerations about uncertainties in the exact shape of the soil matrix polygons apply. Infiltration occurs if $h_{\mathrm{mp}}>h_{\mathrm{mt}}$ and exfiltration if $h_{\mathrm{mp}}<h_{\mathrm{mt}}$.

Exfiltration rate out of the matrix into empty macropores $\left(h_{\mathrm{mp}}=0\right)$ is described as a seepage face: $q^{*}{ }_{\text {Is,seep }}\left(\mathrm{cm} \mathrm{cm}^{-1} \mathrm{~d}^{-1}\right)$. It is approached with a seepage resistance $\mathrm{Y}_{\text {seep }}(\mathrm{d})$ that is based on Ernst's drainage resistance equation Eq. (4.16) without term Yentr:

$$
\begin{aligned}
& q_{\mathrm{ls} \text {, seep }}^{*}=-\frac{h_{\mathrm{mt}}}{\gamma_{\text {seep }}}=-\frac{h_{\mathrm{mt}}}{\gamma_{\mathrm{ver}}+\gamma_{\mathrm{hor}}+\gamma_{\mathrm{rad}}} \\
& \gamma_{\text {ver }}=\frac{D_{\text {seep }}}{K_{\mathrm{sat}}} \quad \text { with } D_{\text {seep }}=\phi_{\mathrm{gwl}}-z_{\text {seep }} \\
& \gamma_{\text {hor }}=\frac{d_{\mathrm{pol}}^{2}}{8 K_{\mathrm{sat}} D_{\text {seep }}} \\
& \gamma_{\mathrm{rad}}=\frac{d_{\mathrm{pol}} \ln \left(\frac{D_{\text {seep }}}{u_{\text {seep }}}\right)}{\pi K_{\mathrm{sat}}}
\end{aligned}
$$

where $\phi_{\mathrm{gwl}}(\mathrm{cm})$ is the groundwater elevation, $z_{\text {seep }}(\mathrm{cm})$ is bottom of seepage layer which equals depth of either bottom of macropores or macropore water level, $D_{\text {seep }}(\mathrm{cm})$ is thickness of seepage zone and $u_{\text {seep }}(\mathrm{cm})$ is thickness of seepage face which is set to $10 \%$ of $D_{\text {seep. }}$. Eq. (6.36.b)-Eq. (6.36.d) are derived from Eq. (4.20)-Eq. (4.22).

Distribution of $q^{*}{ }_{\mathrm{li}}$ over MB and IC domains is according to their proportions $P_{\mathrm{mb}}$ and $P_{\mathrm{ic}}$ at the specific depth $z$.

Lateral exfiltration out of the saturated matrix as interflow $q_{l i}$ Lateral exfiltration $q^{*}{ }_{\mathrm{li}}\left(\mathrm{cm} \mathrm{cm}^{-1} \mathrm{~d}^{-1}\right)$ out of the saturated soil matrix into macropores by interflow (Section 4.2) out of a zone with perched groundwater occurs over the depth of perched groundwater. This process is a special case of exfiltration of soil water from the saturated zone into the macropores and is described in a similar way using Eq. (6.35) and Eq. (6.36), but with an opposite sign due to its definition in Eq. (6.24). If $h_{\mathrm{mp}}>h_{\mathrm{mt}}$, infiltration into the saturated matrix in the perched groundwater zone occurs according to Eq. (6.35). A perched groundwater zone is here defined as a saturated zone 
above groundwater level that is separated from the saturated zone below groundwater level by an unsaturated zone which contains at least a critical value $V_{\text {undsat,crit }}$ (default: $0.1 \mathrm{~cm}$ ) of under-saturated volume $V_{\text {undsat }}\left(=\int\left[\theta_{\mathrm{s}}-\theta\right] \mathrm{d} z \mathrm{~cm}\right)$.

Distribution of $q_{\text {li }}^{*}$ over the MB and IC domains is according to their proportions $P_{\mathrm{mb}}$ and $P_{\mathrm{ic}}$ at the specific depth $z$.

\section{Rapid drainage $q_{r d}$}

Rapid drainage to drainage systems can occur via a network of lateral interconnected cracks or via otherwise (nearly) interconnected macropores. Also when macropores are separated by just thin walls of soil matrix they can enhance drainage considerably. Fed by macropore water, the small matrix barriers will become saturated even when the soil matrix as a whole remains unsaturated. They then form part of a saturated macropore-soil-matrix-system that conducts water better in vertical and horizontal direction than the bulk of the soil matrix (Nieber et al., 2006). Sidle et al. (2001) proposed the concept of a self-organising network of preferential flow pathways, where the connections in the network are controlled by moisture level.

In SWAP, the complex process of rapid drainage is described with a drainage resistance. This resistance may depend on the width of macropores and of shrinkage cracks in particular: wider cracks have higher hydraulic conductivities. It may also depend on macropore water level: the higher this level, the more macropore volume involved, the higher the hydraulic conductivity and the lower the resistance. Therefore, the functional input parameter 'drainage resistance' is considered as a reference drainage resistance: it is valid for a defined reference situation. The actual drainage resistance is derived from the reference resistance according to the deviations from the actual situation to the reference situation.

The reference situation is preferably an average situation at the field scale and should be based on a relevant reference level. In this SWAP version it is fixed. The reference level is chosen to be the drainage basis: drain depth or surface water level. The definition of the reference situation is: soil moisture is in hydrostatic equilibrium with groundwater level at depth of drainage basis and with the water level in the MB macropores at a depth of three-quarters of the drainage basis depth.

The actual drainage resistance $Y_{\text {act }}(\mathrm{d})$ is calculated from the reference drainage resistance $Y_{\text {ref }}(\mathrm{d})$ according to the ratio between actual and reference transmissivity $K D$ of the MB macropores $\left(\mathrm{cm}^{2} \mathrm{~d}^{-1}\right)$ :

$$
\gamma_{\text {act }}=\frac{[K D]_{\text {act }}}{[K D]_{\text {ref }}} \gamma_{\text {ref }}
$$

where:

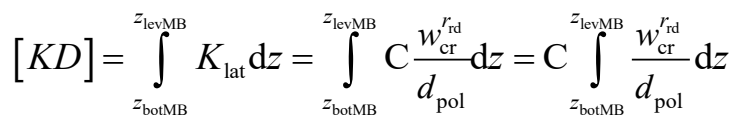

with $K_{\text {lat }}\left(\mathrm{cm} \mathrm{d}^{-1}\right)$ the lateral hydraulic conductivity of the macropores, $z_{\text {botmB }}$ and $z_{\text {levmB }}(\mathrm{cm})$ the depths of the bottom of and the water level in the MB macropores. $C$ is a constant that follows from the slit model for conductivity (Eq. (6.28)). Its value is irrelevant because it is eliminated in Eq. (6.37.a). The exponent $r_{\mathrm{rd}}(-)$ is a reaction coefficient that determines the effect of width $w_{\mathrm{cr}}(\mathrm{cm})$ on Yact. It varies between 0 and 3. When $r_{\text {rd }}=0$, Yact becomes independent of $w_{\text {cr. }}$.

Rapid drainage flux $q_{\mathrm{rd}}\left(\mathrm{cm} \mathrm{d}^{-1}\right)$ is calculated from MB domain water level elevation $\phi_{M B}(\mathrm{~cm})$ above drainage level $\phi_{\mathrm{db}}(\mathrm{cm})$ and $Y_{\mathrm{act}}\left(\mathrm{d}^{-1}\right)$ at actual moisture content:

$$
q_{\mathrm{rd}}=\frac{\phi_{\mathrm{MB}}-\phi_{\mathrm{db}}}{\gamma_{\mathrm{act}}}
$$




\subsection{Numerical implementation}

SWAP applies the same vertical spatial $(\Delta z)$ and temporal $(\Delta t)$ discretisation for macropore flow as is used for matrix flow. Besides, SWAP uses a horizontal discretisation in the form of macropore domains for macropore flow. In this section the horizontal discretisation, its relationship with vertical and temporal discretisation, and the numerical implementation of water balance and flow equations are described.

\subsubsection{Macropore geometry}

\subsubsection{Continuity}

To obtain the required resolution in IC macropores, the IC domain is divided into $n_{\mathrm{sd}}$ sub-domains. This partition represents the horizontal discretisation of the macropore system. The IC volume at soil surface, minus the $R_{\mathrm{ZAH}}$-volume, is equally distributed over the $n_{\mathrm{sd}}$ sub-domains. Thus, all $n_{\mathrm{sd}}$ subdomains take up an equal amount of infiltrating water at the soil surface. The volumetric proportion $P_{\mathrm{sd}, j}$ of sub-domain $j$ as a function of $z$ is calculated according to:

$$
\begin{array}{ll}
P_{\mathrm{sd}, j}=\frac{1}{\frac{1}{P_{\mathrm{ic}, 0}}+F-1} \frac{1-R_{Z_{\mathrm{Ah}}}}{n_{\mathrm{sd}}} & \text { for } 0 \geq z>z_{\mathrm{sd}, j+1} \\
P_{\mathrm{sd}, j}=\frac{\left(F-\frac{j-1}{n_{\mathrm{sd}}}\left(1-R_{Z_{\mathrm{Ah}}}\right)\right)}{\frac{1}{P_{\mathrm{ic}, 0}}+F-1} & \text { for } z_{\mathrm{sd}, j+1} \geq z>z_{\mathrm{sd}, j} \\
P_{\mathrm{sd}, j}=0 & \text { for } z \leq z_{\mathrm{sd}, j}
\end{array}
$$

where $j=1$ is the deepest and $j=n_{\text {sd }}$ the shallowest sub-domain (respectively left and right in Figure 6.1.B), and $z_{\mathrm{sd}, j}$ is the depth at which sub-domain $j$ ends:

$$
z_{\mathrm{sd}, j}=Z_{\mathrm{Ah}}-\left(Z_{\mathrm{Ah}}-Z_{\mathrm{ic}}\right)\left(1-\frac{j-1}{n_{\mathrm{sd}}}\right)^{\frac{1}{m}}
$$

If option $R_{\mathrm{ZAH}}>0$ is chosen, an extra $A h$-sub-domain $j=n_{\mathrm{sd}}+1$ is created with proportion:

$$
\begin{array}{ll}
P_{\mathrm{sd}, n_{\mathrm{sd}}+1}=\frac{R_{Z_{\mathrm{Ah}}}+F-1}{\frac{1}{P_{\mathrm{ic}, 0}}+F-1} & \text { for } 0 \geq z>Z_{\mathrm{Ah}} \\
P_{\mathrm{sd}, n_{\mathrm{sd}}+1}=0 & \text { for } z \leq Z_{\mathrm{Ah}}
\end{array}
$$

Because the MB domain is always present, though sometimes with very low proportion, the total number of domains $n_{\mathrm{dm}}=n_{\mathrm{sd}}+1$. In case of $R_{\mathrm{ZAH}}>0, n_{\mathrm{dm}}=n_{\mathrm{sd}}+2$. All domains are numbered from $j=1$ to $n_{\mathrm{dm}}$ with the MB domain being the first domain $j=1$ and the deepest IC domain the second $j=2$.

In the model, the vertical coordinate $z$ is partitioned into discrete model compartments $i$ with thickness $\Delta \mathrm{z}_{, i}(\mathrm{~cm})$ between $z_{\mathrm{b}, i}$ and $z_{\mathrm{t}, i}$ at the bottom and top of the compartment, respectively. For each compartment a discrete macropore volume per domain is required. Volumetric proportion $P_{j, i}$ for each combination of domain $j$ and compartment $i$ is obtained by integration of $P_{\mathrm{MB}}$ and $P_{\mathrm{sd}, j}$ as a function of $z$ over the compartment thickness and dividing by $\Delta z_{i}$ : 


$$
P_{1, i}=\frac{\int_{z_{\mathrm{b}, i}}^{z_{\mathrm{t}, i}} P_{\mathrm{MB}} \mathrm{d} z}{\Delta z_{i}} \quad \text { and } \quad P_{j, i}=\frac{\int_{z_{\mathrm{b}, i}}^{z_{\mathrm{t}, i}} P_{\mathrm{sd}, j-1} \mathrm{~d} z}{\Delta z_{i}} \text { for } 2 \leq j \leq n_{\mathrm{dm}}
$$

Per domain the macropore volume is vertically interconnected over the soil compartments. Domains $j>1$ to $j+n_{\mathrm{dl}, j}$ inclusive, that end in the same model compartment, are functionally equal and therefore are lumped for all compartments $i=1$ to $n d b_{j}$ (compartment number that contains bottom of domain $j$ ):

$$
P_{j, i}=P_{j, i}+\sum_{l=1}^{n_{\mathrm{dl}, j}} P_{j+l, i} \text { and } \quad P_{l, i}=P_{l+n_{\mathrm{d} l, j}, i} \quad \text { for all } l: j+1 \leq l \leq n_{\mathrm{dm}}-n_{\mathrm{dl}, j}
$$

For each lumped domain $n_{\mathrm{dm}}$ is reduced with 1 . In this way, the resolution of the horizontal discretisation in terms of $n_{\mathrm{dm}}$ is determined by $n_{\mathrm{sd}}$, the thickness of compartments and the shape of curve $F$ : the combination of large $n_{\text {sd, }}$ small $\Delta z^{\prime}$ s in the IC domain and a linear $F$-curve $(m=1)$ yields the largest $n_{\mathrm{dm}}$.

\subsubsection{Persistency}

The volume of macropores $V_{\mathrm{mp}, j, i}\left(\mathrm{~cm}^{3} \mathrm{~cm}^{-2}\right.$ unit of area) for domain $j$ in compartment $i$ is calculated for each time-step $\Delta t$ as:

$$
V_{\mathrm{mp}, j, i}=P_{j, i}\left(V_{\mathrm{st}, i}+V_{d y, i}\right)
$$

Static $V_{\mathrm{st}, i}$ and dynamic $V_{\mathrm{dy}, i}$ macropore volume $\left(\mathrm{cm}^{3} \mathrm{~cm}^{-2}\right)$ in each compartment $i$ are obtained as explained below. Dynamic volume is changing in time, static volume is not. Consequently, if dynamic volume is present in compartment $i$, the total macropore volume in this compartment is changing in time as well.

The total volume $V_{\mathrm{dm}, j}\left(\mathrm{~cm}^{3} \mathrm{~cm}^{-2}\right)$ of macropore domain $j$ equals:

$$
V_{\mathrm{dm}, j}=\sum_{i=1}^{n d b_{j}} V_{\mathrm{mp}, j, i}
$$

where $n d b_{j}$ is the number of the compartment that contains the bottom of domain $j$.

\section{Static macropore volume}

The volumes of static macropores per compartment $i$ for the MB and IC domain, $V_{\mathrm{st}, \mathrm{mb}, i}$ and $V_{\mathrm{st}, \mathrm{ic}, i}\left(\mathrm{~cm}^{3}\right.$ $\mathrm{cm}^{-2}$ ), are obtained by integration over $\Delta z_{i}$ :

$$
V_{\mathrm{s}, \mathrm{mb}, i}=\int_{z_{\mathrm{b}, i}}^{z_{\mathrm{t}, i}} V_{\mathrm{st}, \mathrm{mb}} \mathrm{d} z \quad \text { and } \quad V_{\mathrm{st}, \mathrm{ic}, i}=\int_{z_{\mathrm{b}, i}}^{z_{\mathrm{t}, i}} V_{\mathrm{st}, \mathrm{ic}} \mathrm{d} z
$$

The total volume of static macropores $V_{\mathrm{st}, i}$ in compartment $i$ equals:

$$
V_{\mathrm{st}, i}=V_{\mathrm{st}, \mathrm{mb}, i}+V_{\mathrm{st}, \mathrm{ic}, i}
$$

\section{Dynamic macropore volume}

Dynamic macropore volume $V_{\mathrm{dy}, i}\left(\mathrm{~cm}^{3} \mathrm{~cm}^{-2}\right)$ in compartment $i$ is computed for each time-step $\Delta t$ by substituting Eq. (6.13) in Eq. (6.12) and multiplying with compartment thickness $\Delta z_{i}$ :

$$
V_{\mathrm{dy}, i}=\left(1-\frac{V_{\mathrm{st}, i}}{\Delta z_{i}}\right) \frac{V_{\mathrm{sh}, i}+\left(1-V_{\mathrm{sh}, i}\right)^{\frac{1}{r_{\mathrm{s}, i}}}-1}{\left(1-V_{\mathrm{sh}, i}\right)^{\frac{1}{r_{\mathrm{s}, i}}}} \Delta z_{i}
$$

The shrinkage volume fraction $V_{\mathrm{sh}, i}\left(\mathrm{~cm}^{3} \mathrm{~cm}^{-3}\right)$ is calculated from actual moisture content and shrinkage characteristic of compartment $i$ at the beginning of $\Delta t$ : 


$$
V_{\mathrm{sh}, i}=\left(e_{\mathrm{s}, i}-e_{i}\right) V_{\mathrm{sol}, i}
$$

where:

$$
e_{i}=\mathrm{f}\left(\vartheta_{i}\right)_{i}, \vartheta_{i}=\frac{\theta_{i}}{V_{\mathrm{sol}, i}}, e_{\mathrm{s}, i}=\vartheta_{\mathrm{s}, i}=\frac{\theta_{\mathrm{s}, i}}{V_{\mathrm{sol}, i}} \text { and } \quad V_{\mathrm{sol}, i}=1-\theta_{\mathrm{s}, i}
$$

with $\theta_{i}$ is the actual and $\theta_{\mathrm{s}, i}$ the saturated volumetric moisture content $\left(\mathrm{cm}^{3} \mathrm{~cm}^{-3}\right)$ of compartment $i$.

In order to correctly model infiltration into the soil matrix at soil surface, thin model compartments in the order of $1 \mathrm{~cm}$ thick are advised for the top of the soil profile (Van Dam and Feddes, 2000). Because of the dynamical conditions at soil surface and the small storage capacity of the thin compartments, moisture contents may change rapidly. As a result shrinkage volume at soil surface may appear and disappear faster than in reality. Because the quantity of shrinkage volume at the soil surface is crucial for determining the amount of precipitation water infiltrating into the macropores, shrinkage volume of the first compartments is calculated on the basis of moisture content of the compartment that contains a reference depth $z_{\text {crack, }}$ where moisture conditions are less dynamical. $z_{\text {crack }}$ is an input parameter.

\subsubsection{Horizontal distribution}

Effective diameter of soil polygons $d_{\mathrm{pol}, i}(\mathrm{~cm})$ for compartment $i$ is calculated with Eq. (6.23) by substituting $V_{\mathrm{st}, i}, 1-P_{1, i}$ and $z_{i}$ for $V_{\mathrm{st}}, P_{\mathrm{ic}}$ and $z$, respectively. $z_{i}(\mathrm{~cm})$ is the depth of node $i$ which is in the middle of compartment $i$.

The effective vertical area of macropore walls per unit of horizontal area $A_{\text {wall,i }}\left(\mathrm{cm}^{2} \mathrm{~cm}^{-2}\right)$ is obtained by multiplying the vertical area of macropore walls per unit of volume $A^{*}{ }_{\text {wall, } i}$ with compartment thickness $\Delta z_{i}$ :

$$
A_{\mathrm{wall}, i}=A_{\mathrm{wall}}^{*} \Delta z_{i}=\frac{4}{d_{\mathrm{pol}, i}} \Delta z_{i}
$$

The effective horizontal distance $x_{\mathrm{pol}, i}(\mathrm{~cm})$ is calculated with Eq. (6.21) by substituting $d_{\mathrm{pol}, i}$ for $d_{\mathrm{pol}}$. The effective crack width $w_{\mathrm{cr}, i}(\mathrm{~cm})$ in the MB domain for compartment $i$ is calculated from Eq. (6.22) as:

$$
w_{\mathrm{cr}, i}=d_{\mathrm{pol}, i}\left(1-\sqrt{1-\frac{V_{\mathrm{mp}, 1, i}}{\Delta z_{i}}}\right)
$$

\subsubsection{Water flow and balance}

The water balance of macropore domain $j=1$ (MB domain) for time-step $\Delta t$ (d) reads in accordance with Eq. (6.24):

$$
S_{j}^{t}-S_{j}^{t-\Delta t}=\left(I_{\mathrm{pr}, j}+I_{\mathrm{ru}, j}+\sum_{i=n i t}^{n i b} q_{\mathrm{li}, j, i} \Delta z_{i}-\sum_{i=1}^{n u b} q_{\mathrm{lu}, j, i} \Delta z_{i}-\sum_{i=n u b+1}^{n d b_{j}} q_{\mathrm{ls}, j, i} \Delta z_{i}-q_{\mathrm{rd}}\right) \Delta t
$$

Water balance equations of all other domains $j>1$ are equal to Eq. (6.52) but with exclusion of the rapid drainage term $q_{\mathrm{rd}}$ that only applies to domain 1, the MB domain. The compartment numbers nit, $n i b$, nub and $n d b_{j}$ refer to the top and bottom compartment with interflow from perched groundwater, the bottom (deepest) unsaturated compartment and the compartment with bottom of domain $j$, respectively. Storage $S_{j}$ is always limited to $0 \leq S_{j} \leq V_{\mathrm{dm}, j}$. In case of water deficit $\left(S_{j}<0\right)$ all outgoing fluxes are decreased with a part of the deficit according to their relative rate. In case of water excess $\left(S_{j}>V_{\mathrm{dm}, j}\right)$ all incoming fluxes are decreased with a part of the excess according to their relative rate. The excess of the inflow at soil surface is distributed over macropore domains that are not fully filled up, that is, if $S<V_{\mathrm{dm}}$ for a particular domain. The remaining excess is added to the ponding layer. 
Inflow at soil surface $\left(I_{p r}\right.$ and $\left.I_{r u}\right)$

The inflow at the soil surface fluxes Ipr and Iru (cm d-1) are calculated according to Eq. (6.25) to (6.28) with the relevant properties of the first compartment. When these fluxes exceed storage capacity of total macropore volume, the inflow excess is added to the ponding layer before calculation of regular runoff takes place.

Distribution of $I_{\mathrm{pr}}$ and $I_{\mathrm{ru}}$ over all macropore domains $j$ is according to the domains proportions at soil surface, that is, in model compartment 1 :

$$
I_{\mathrm{pr}, j}=P_{j, 1} I_{\mathrm{pr}} \quad \text { and } \quad I_{\mathrm{ru}, j}=P_{j, 1} I_{\mathrm{ru}}
$$

Lateral infiltration into the unsaturated matrix $q_{/ u}$

Cumulative lateral absorption $I_{\mathrm{ab}, j, i}(\mathrm{~cm})$ for all compartments $i$ of the unsaturated matrix that are in contact with water in macropore domain $j$ is computed according to Eq. (6.29):

$$
I_{\mathrm{ab}, j, i}=P_{j, i} A_{\mathrm{wall}, \mathrm{mtx}, i} S_{\mathrm{P}, j, i} \sqrt{t_{\mathrm{cum}, j, i}}=P_{j, i} \frac{4 \Delta z_{i} S_{\mathrm{P}, j, i} \sqrt{t_{\mathrm{cum}, j, i}}}{d_{\mathrm{pol}, i} \sqrt{1-\frac{\left(V_{\mathrm{dy}, i}+V_{\mathrm{st}, i}\right)}{\Delta z_{i}}}}
$$

where $t_{\mathrm{cum}, j, i}(\mathrm{~d})$ is the cumulative time since first contact of compartment $i$ with water in macropore domain $j$. At each new contact event, $S_{p, j, i}$ and $t_{\mathrm{cum}, j, i}$ are updated.

The sorptivity approach assumes that the moisture content is not influenced by another process then sorption. In the model, moisture content $\theta_{i}$ is also affected by vertical matrix flow and uptake by roots. To account for this inadequacy, for each time-step sorptivity $S_{P_{1}, j, i}$ is corrected according to the deviation $\Delta \theta_{i}$ between actual $\theta_{i}$ and theoretical $\theta_{\text {the }, j, i}$ moisture content at the beginning of the timestep:

$$
S_{\mathrm{P}, j, i}=S_{\mathrm{P}, \max , i}\left(\frac{\theta_{\mathrm{s}, i}-\theta_{0, j, i}-\Delta \theta_{j, i}}{\theta_{\mathrm{s}, i}-\theta_{\mathrm{r}, i}}\right)^{\alpha_{\mathrm{s}, i}}
$$

where $\Delta \theta_{j, i}=\theta_{i}-\theta_{\text {the }, j, i}$. For further explanation, see Eq. (6.30). $\theta_{\text {the }, j, i}$ is computed with Eq. (6.54) on the basis of initial sorptivity $S_{p_{, j}, i}$ that is obtained by Eq. (6.55) without term $\Delta \theta_{j, i}$.

The lateral absorption rate during time step $\Delta t$ is linearised to obtain an average, constant rate $q_{\mid \mathrm{lu}, \mathrm{ab}, j, \mathrm{i}}$ $\left(\mathrm{cm} \mathrm{d}^{-1}\right)$ :

$$
q_{\mathrm{lu}, \mathrm{ab}, j, i}=\frac{I_{\mathrm{ab}, j, i}^{t}-I_{\mathrm{ab}, j, i}^{t-\Delta t}}{\Delta t}
$$

Infiltration rate $q_{\mathrm{lu}, \mathrm{D}, j, i}\left(\mathrm{~cm} \mathrm{~d}^{-1}\right)$ by lateral Darcy flow from domain $j$ into compartment $i$ reads in accordance to Eq. (6.32):

$$
q_{\mathrm{lu}, \mathrm{D}, j, i}=P_{j, i} f_{\mathrm{shp}} A_{\mathrm{wall}, i} K_{\mathrm{h}, i} \frac{\left(h_{\mathrm{mp}, j, i}-h_{\mathrm{mt}, i}\right)}{x_{\mathrm{pol}, i}}=P_{j, i} \frac{f_{\mathrm{shp}} 8 K_{\mathrm{h}, i}\left(h_{\mathrm{mp}, j, i}-h_{\mathrm{mt}, i}\right)}{d_{\mathrm{pol}, i}^{2}}
$$

where $h_{\mathrm{mp}, j, i}$ is calculated with Eq. (6.33) by using $\phi_{\mathrm{mp}, j}(\mathrm{~cm})$, the water level in domain $j$, and $z_{i}$.

The resulting lateral infiltration rate $q_{\mathrm{lu}, j, i}\left(\mathrm{~cm} \mathrm{~d}^{-1}\right)$ from macropore domain $j$ into unsaturated compartment $i$ is derived from Eq. (6.34).

Lateral infiltration into and exfiltration out of the saturated matrix $q_{\text {ls }}$ Rate of lateral water exchange $q_{\mathrm{ls}, j, i}\left(\mathrm{~cm} \mathrm{~d}^{-1}\right)$ between macropore domain $j$ and saturated compartment $i$ by Darcy flow is computed in accordance to Eq. (6.35):

$$
q_{\mathrm{ls}, \mathrm{D}, j, i}=P_{j, i} f_{\mathrm{shp}} A_{\mathrm{wall}, i} K_{\mathrm{sat}, i} \frac{\left(h_{\mathrm{mp}, j, i}-h_{\mathrm{mt}, i}\right)}{x_{\mathrm{pol}, i}}=P_{j, i} \frac{f_{\mathrm{shp}} 8 K_{\mathrm{sat}, i}\left(h_{\mathrm{mp}, j, i}-h_{\mathrm{mt}, i}\right)}{d_{\mathrm{pol}, i}^{2}}
$$


and in case of a seepage face $\left(h_{\mathrm{mp}, j, I}=0\right)$ according to Eq. (6.36):

$$
\begin{aligned}
& q_{\mathrm{ls}, \mathrm{seep}, j, i}=-\frac{h_{\mathrm{mt}, i}}{\gamma_{\mathrm{ver}, i}+\gamma_{\mathrm{hor}, i}+\gamma_{\mathrm{rad}, i}} \\
& \gamma_{\mathrm{ver}, i}=\frac{\Delta z_{i}}{K_{\mathrm{sat}, i}} \\
& \gamma_{\mathrm{hor}, i}=\frac{d_{\mathrm{pol}, i}^{2}}{8 K_{\mathrm{sat}, i} \Delta z_{i}} \\
& \left.\gamma_{\mathrm{rad}, i}=\frac{d_{\mathrm{pol}, i} \ln \left(\frac{\Delta z_{i}}{\pi K_{\mathrm{sat}, i}}\right)}{u_{\mathrm{seep}, i}}\right)
\end{aligned}
$$

where $u_{\text {seep }, i}$ is set to $10 \%$ of $\Delta z_{i}$.

Lateral exfiltration out of the saturated matrix as interflow $q_{l i}$

Lateral exfiltration rate $q \mathrm{li}, \mathrm{j}, \mathrm{i}(\mathrm{cm} \mathrm{d}-1)$ out of compartment i with perched groundwater into macropore domain $j$ by interflow is calculated according to Eq. (6.58) and Eq. (6.59) with an opposite sign.

\section{Rapid drainage $q_{r d}$}

The actual drainage resistance $Y_{\text {act }}\left(\mathrm{d}^{-1}\right)$ for calculating rapid drainage flux $q_{\mathrm{rd}}\left(\mathrm{cm} \mathrm{d}^{-1}\right)$ according to Eq. (6.30) is obtained by:

$$
\gamma_{\mathrm{act}}=\frac{\sum_{i=n l_{\mathrm{act}}}^{n b_{\mathrm{act}}} \frac{w_{\mathrm{cr}, \mathrm{at}, i}^{r_{\mathrm{rd}}}}{d_{\mathrm{pol}, i}} \Delta z_{i}}{\sum_{i=n l_{\mathrm{ref}}}^{n b_{\mathrm{ref}}} \frac{w_{\mathrm{cr}, \mathrm{ref}, i}^{r_{\mathrm{r}}}}{d_{\mathrm{pol}, i}} \Delta z_{i}} \gamma_{\mathrm{ref}}
$$

where $n l$ and $n b$ are the numbers of the compartments with the water level and the bottom of the MB domain, respectively, for actual and reference situation.

For use in solute models, the rapid drainage flux $q_{\text {rd }}$ is distributed over the compartments $n l_{\text {act }}$ to $n b_{\text {act }}$ according to their relative KD values:

$$
q_{\mathrm{rd}, i}=\frac{\frac{w_{\mathrm{cr}, \mathrm{act}, i}^{r_{\mathrm{rd}}}}{d_{\mathrm{pol}, i}} \Delta z_{i}}{\sum_{i=n l_{\mathrm{act}}}^{n b_{\mathrm{act}}} \frac{w_{\mathrm{cr}, \mathrm{cct}, i}^{r_{\mathrm{rd}}}}{d_{\mathrm{pol}, i}} \Delta z_{i}} q_{\mathrm{rd}}
$$

Numerical solution

For the numerical solution of Richards equation (Par. 2.7.2) the partial derivative of the exchange between macropores and matrix to the pressure head must be added to the total partial derivative to the pressure head. For each compartment $i$ the macropore contribution to this derivative is the sum of the derivatives of all $n_{\mathrm{dm}, i}$ macropore domains $j$ :

$$
\frac{\partial S_{m, i}^{l+1, p}}{\partial h_{i}^{l+1, p}}=\sum_{j=1}^{n_{\mathrm{dm}, i}}\left(\frac{\partial S_{\mathrm{m}, j, i}^{l+1, p}}{\partial h_{i}^{l+1, p}}\right)
$$

where $/$ refers to the time level and $p$ to the iteration round. For the Darcy flow and seepage face fluxes $q_{\mathrm{lu}, \mathrm{D}}, q_{\mathrm{ls}}$ and $q_{\mathrm{li}}$ the derivative to the pressure head is calculated as:

$$
\frac{\partial S_{\mathrm{m}, j, i}^{l+1, p}}{\partial h_{i}^{l+1, p}}=\left(-\frac{q_{\mathrm{lu}, \mathrm{D} \_ \text {or_ls }, j, i}^{l+1, p}}{h_{\mathrm{mp}, j, i}^{l}-h_{\mathrm{mt}, i}^{l+1, p}}\right) \text { and } \frac{\partial S_{\mathrm{m}, j, i}^{l+1, p}}{\partial h_{i}^{l+1, p}}=\left(-\frac{q_{\mathrm{li}, j, i}^{l+1, p}}{h_{\mathrm{mp}, j, i}^{l}-h_{\mathrm{mt}, i}^{l+1, p}}\right)
$$


For the absorption flux $q_{\mathrm{lu}, \mathrm{ab}}$ the derivative to the pressure head is obtained by:

$$
\frac{\partial S_{\mathrm{m}, j, i}^{l+1, p}}{\partial h_{i}^{l+1, p}}=\left(-\alpha_{\mathrm{S}, i} \frac{q_{\mathrm{lu}, \mathrm{ab}, j, i}}{\left(\theta_{\mathrm{s}, i}+\theta_{\mathrm{thr}, j, i}-\theta_{0, j, i}-\theta_{i}\right)} \frac{\partial \theta_{i}}{\partial h_{i}}\right)^{l+1, p}
$$

where $\frac{\partial \theta_{i}}{\partial h_{i}}$ is the differential water capacity (Section 2.2).

\subsection{User instructions}

\subsubsection{General input parameters}

The most important aspect of macropore flow is that precipitation water is routed into macropores at the soil surface. A relatively small part of precipitation enters the macropore volume directly. Inflow of precipitation excess via overland flow in case of precipitation intensity exceeding matrix infiltration rate is the dominant source of macropore inflow at soil surface. In order to describe these inflow processes accurately, realistic precipitation intensities and matrix infiltration rates should be simulated. The consequences of this for the SWAP parameterisation other than the macropore parameters, are discussed below.

\section{Rainfall option}

For realistic rainfall intensities, rainfall option SWRAIN 3 is preferred (Section 3.7.1). Less preferable are options 1 and 2. Option 0, daily rainfall sum, is not recommended. This option may seriously underestimate macropore inflow at soil surface because of far too small rainfall intensities.

\section{Vertical discretisation}

Realistic simulation of matrix infiltration at the soil surface requires thin compartments in the top of the profile in the order of $1 \mathrm{~cm}$ thick (Van Dam and Feddes, 2000). A typical vertical discretisation for a macroporous field soil could be: for the first $10-20 \mathrm{~cm}$ compartments of $1 \mathrm{~cm}$ thick, for the next 20-30 cm $2.5 \mathrm{~cm}$ thick, until the depth of the bottom of the IC domain maximal $5 \mathrm{~cm}$ thick, until the depth of the bottom of the static macropores 5 to maximal $10 \mathrm{~cm}$ thick and below this depth compartments of $10-25 \mathrm{~cm}$ thick.

\section{Soil hydraulic functions}

The hydraulic functions of the soil matrix should be used. This implies that the saturated volumetric moisture content is without the static macropore volume. And that the saturated hydraulic conductivity concerns a soil physical conductivity of the matrix rather than a hydrological conductivity of the soil. The air-entry-value option should be switched off, thus he $=0$ (see Sections 2.3 and 2.8.1).

Time step

It is recommended to take $10^{-5}$ or $10^{-6}(\mathrm{~d})$ for the minimum time step and $10^{-1}$ (d) or less for the maximum time-step.

Output

Macropore simulation provides the option of output of a macropore water balance in the file *.BMA. For this option, switch SWBMA should have value 1 (see Box 1.4). Automatically generated are output files MacroGeom.csv and SoilShrinkChar.csv, which contain a tabular representation of the macropore geometry and the shrinkage characteristics as computed by the model on basis of the user's input.

\subsubsection{Macropore input parameters}

The typical macropore input parameters are discussed in this Section. They are listed in Boxes 6.1 (Section 6.3.2.1: Macropore geometry) and 6.2 (Section 6.3.2.2: Water flow). The presented values concern a field experiment on water, bromide tracer and pesticide transport in a tile-drained field on 
clay soil (Scorza Júnior et al., 2004). The field was located in the riverine area in the central part of the Netherlands. The soil concerned light to moderate clay (30-55 mass-\% clay) and the crop was winter wheat. At $320 \mathrm{~cm}$ depth the clay soil was underlain by a coarse sand aquifer. The presented values are the first results of a calibration of SWAP against the dataset.

\subsubsection{Macropore geometry}

The input parameters of the macropore geometry are listed in Box 6.1. They are discussed below.

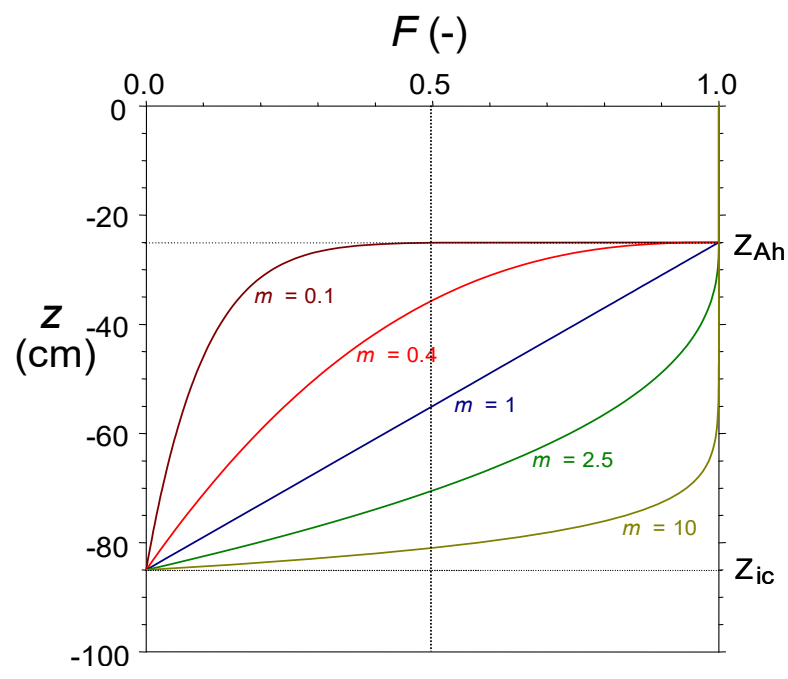

Figure 6.6 Fraction of functional IC macropores $F$ as a function of depth is described by a power law function with power $m . m=1$ describes a linear decline, while $m<1$ represents shallow IC systems and $m>1$ deep IC systems.

Illustration of the effect of parameters on macropore geometry Curve $F$ in Figure 6.6 illustrates the effect of shape-factor $m$ in combination with other macropore geometry parameters on the fraction of IC macropores that is functional at depth $z$ (in case that $R_{\text {ZAH }}=0$ ). For $m=0.1,0.4,1.0,2.5$ and 10, respectively, depth $z$ at which fraction $F$ of functional IC macropores has declined to 0.5 equals $-25.1,-35.6,-55,-70.5$ and $-81 \mathrm{~cm}$, respectively. In general, $m<1$ describes shallow IC systems, while $m>1$ represents deep IC systems; $m=1$ describes an intermediate system with linear decline of functional IC macropores with depth. Optionally, two more shape parameters can be used to describe IC macropores in more detail. The symmetry-point-parameter SPOINT allows for 'standing' $(m<1)$ and 'laying' $(m>1)$ S-shaped F-curves. In combination with switch SWPOWM turned on, these curves can be modified into double convex $(m<1)$ or double concave $(m>1)$ curves (see Appendix 3 for examples). This allows for a functional description of macropore volume for a wide range of macropore geometries.

Default value of $R_{\mathrm{ZAH}}$ is 0.0 . In Figure $6.2 R_{\mathrm{ZAH}}=0.2$, implying that at the bottom of the A-horizon $\left(z=-25 \mathrm{~cm}\right.$ ) $20 \%$ of the IC macropores has ended. If $R_{\mathrm{ZAH}}=0$, no IC macropores end above the bottom of the A-horizon. This option may be used to describe effects of tillage of the A-horizon. Data of a dye tracer experiment from Booltink (1993) for an A-horizon in a clay soil in Flevoland, the Netherlands, point out that this option may be relevant. 


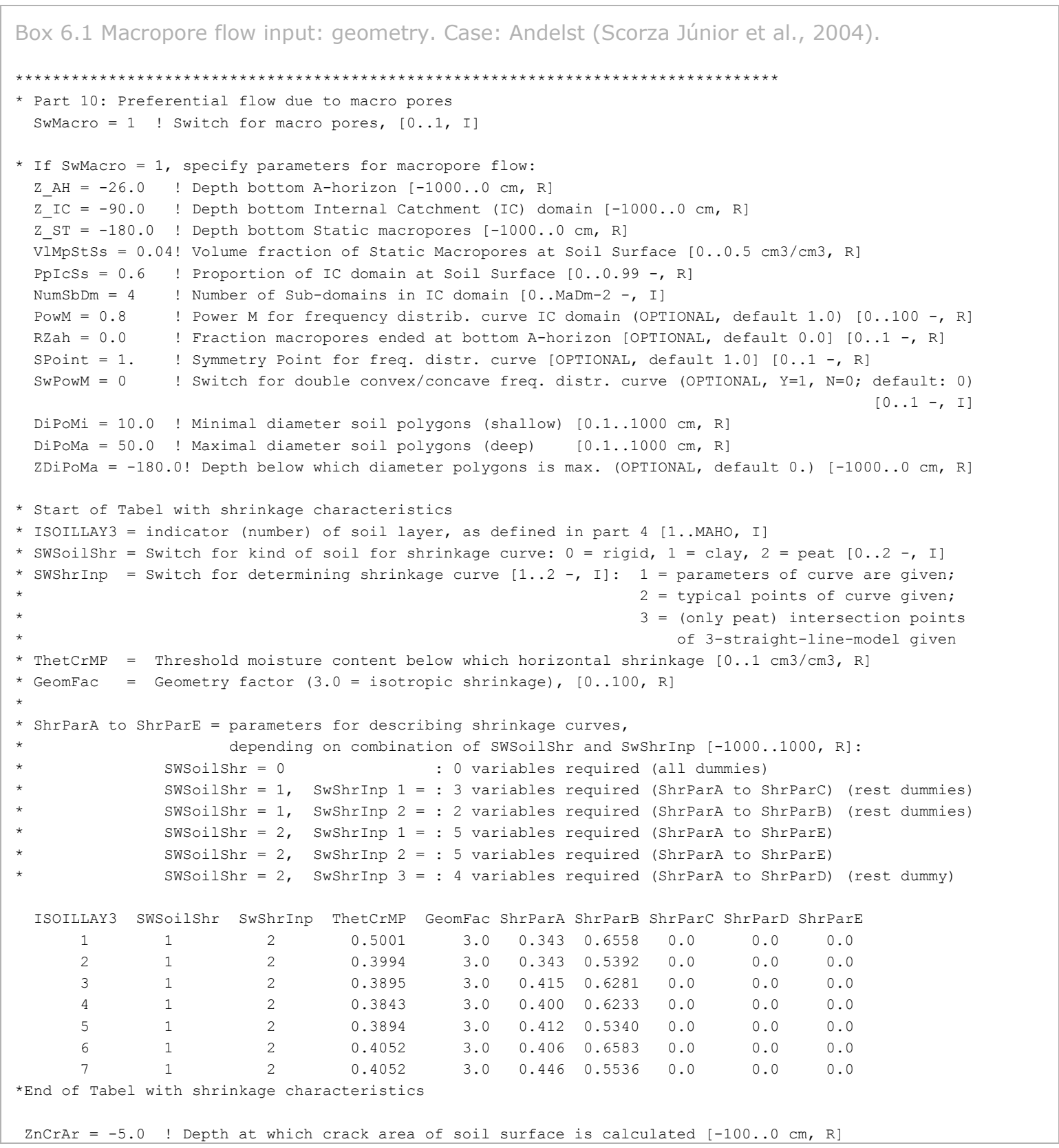

Figure 6.7 illustrates a macropore geometry with six domains: MB-domain, four IC sub-domains and Ah-sub-domain. In this example $V_{\mathrm{st}}$ at soil surface $=0.04 \mathrm{~cm}^{3} \mathrm{~cm}^{-3}, P_{\mathrm{ic}, 0}=0.6, m=0.4, n_{\mathrm{dm}}=6$, $n_{\mathrm{sd}}=4, R_{\mathrm{ZAH}}=0.2, Z_{\mathrm{AH}}=-25 \mathrm{~cm}$ and $Z_{\mathrm{ic}}=-85 \mathrm{~cm}$. $V_{\mathrm{st}, \mathrm{ic}}$ at soil surface equals $0.6 \times 0.04=$ $0.024 \mathrm{~cm}^{3} \mathrm{~cm}^{-3}$. This volume is equally divided over the $n_{\mathrm{sd}}+1$ sub-domains, including the Ah-subdomain, because at soil surface $P_{\mathrm{sd}, j}$ is equal for all five sub-domains and amounts to $0.6 / 5=0.12$. Depth $z_{\mathrm{sd}, j}$ of bottom of domains 2 to 6 , equals $-85,-54.2,-35.6,-26.9$ and $-25 \mathrm{~cm}$, respectively. Figure 6.8 presents an example of lumped sub-domains. 
A

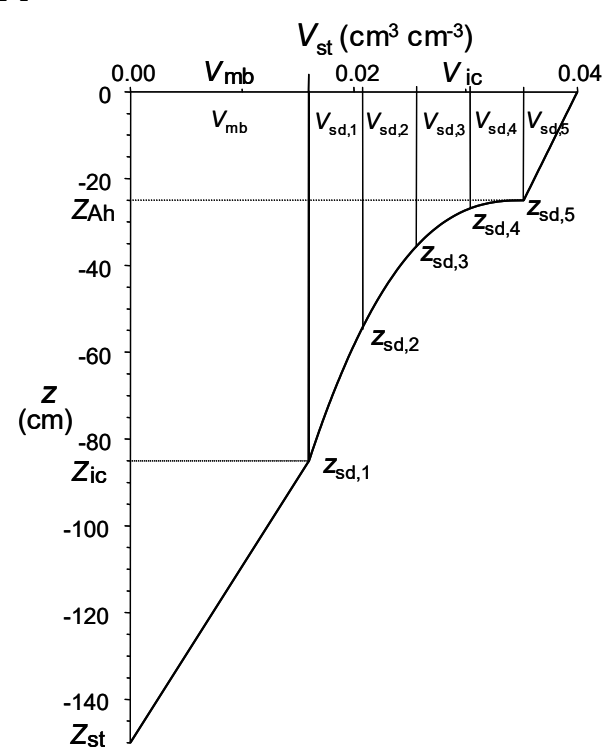

B

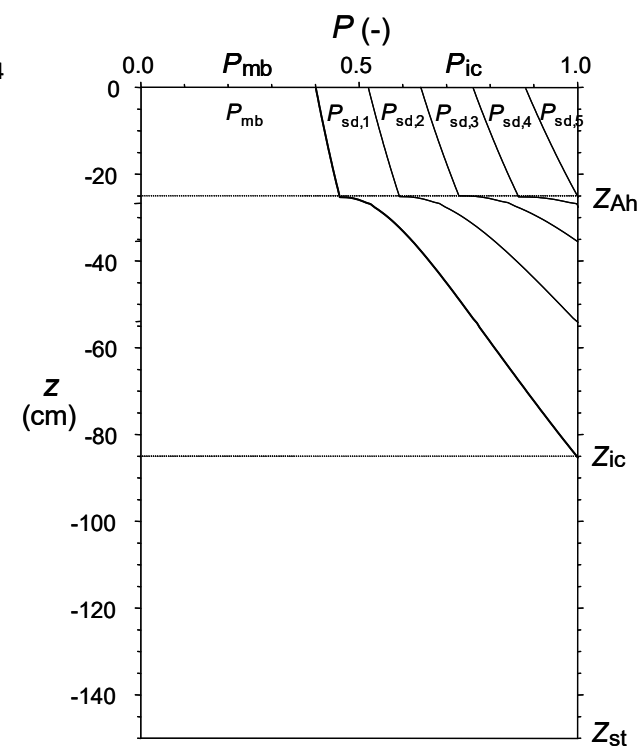

Figure 6.7 Example of macropore geometry with the IC domain partitioned in four sub-domains and an Ah-sub-domain. Static macropore volume $V_{\text {st }}$ (left) and volumetric proportion $P$ (right) for MB, IC and IC-sub-domains. $V_{s t, 0}=0.04 \mathrm{~cm}^{3} \mathrm{~cm}^{-3}, P_{i c, 0}=0.6, m=0.4, n_{d m}=6, n_{s d}=4, R_{Z A H}=0.2$, $Z_{A H}=-25 \mathrm{~cm}$ and $Z_{i c}=-85 \mathrm{~cm} . Z_{s d, j}$ is bottom of sub-domain $j$.

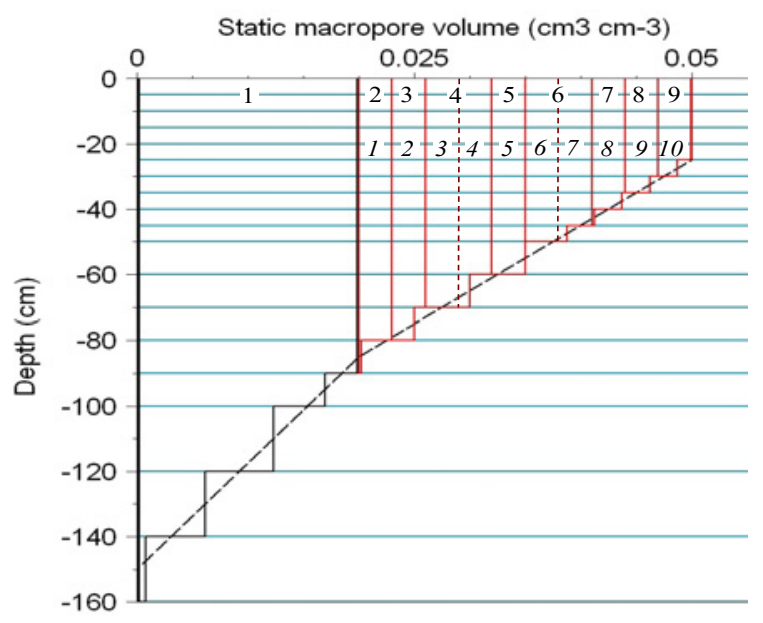

Figure 6.8 Example of a combination of horizontal and vertical discretisation: number of IC subdomains $n_{s d}=10$ (italic figures) while resulting total number of domains $n_{d m}=9$ (regular figures). Former sub-domains 3 and 4 are lumped to obtain present domain 4, and former sub-domains 6 and 7 are lumped to obtain present domain 6.

$V_{\mathrm{st}, 0}=0.1 \mathrm{~cm} 3 \mathrm{~cm}-3, P_{\mathrm{ic}, 0}=0.6, m=1, R_{\mathrm{ZAH}}=0, Z_{\mathrm{AH}}=-20 \mathrm{~cm}, Z_{\mathrm{ic}}=-80 \mathrm{~cm}, Z_{\mathrm{st}}=-120 \mathrm{~cm}$.

Obtaining parameter values for macropore continuity and distribution

Most macropore input parameters are functional parameters with a physical relevancy. Information on their values can be derived from field and lab research. This especially counts for the depths $Z_{\text {Ah, }}, Z_{\text {ic }}$ and $Z_{\text {st. }}$. Depth of A-horizon $Z_{\text {Ah }}$ may be known from soil mapping or field investigation. $Z_{\text {st }}$ could be taken at or some decimetres above the mean annual lowest groundwater table. Processes leading to the presence of static macropores like ripening of clay and peat soils, and biological activities like soil penetration by plant roots, worms, insects and small mammals, will very likely be limited to this depth. $Z_{\text {ic }}$ might be found at the depth of a clear shift in macropore density by investigation of a vertical soil profile in a pit or by taking relatively large soil samples (e.g. $20 \mathrm{~cm}$ diameter and 10-20 cm height). 
Information about macropore volume to obtain a value for static macropore volume fraction at soil surface $V_{\text {st }, 0}$ (VIMpStSs in Box 6.1) and the distribution of macropore volume with depth, can be obtained by comparing pore volume of large samples with fitted values for $\theta_{\text {sat }}$ of the original, unmodified Mualem-Van Genuchten functions. The latter expresses the pore volume of the soil matrix, while the first may comprise macropore volume as well.

Parameters which are relevant for the distribution of macropore volume with depth, PpIcSs, NumSbDm, PowM, DiPoMi, DiPoMa, and optional RZah, SPoint, SwPowM, ZDiPoMa, may be derived from inverse modelling of field experiments on tracer transport, with dye, conservative solutes or isotope tracers.

To illustrate the concept of the effective polygon diameter in case of combinations of cracks and holeshaped macropores in the field, we consider the following equation:

$$
d_{\mathrm{pol}}=\frac{1}{\frac{1}{d_{\mathrm{pf}}}+\pi \frac{\left(N_{\mathrm{h}, 1} d_{\mathrm{hf}, 1}+N_{\mathrm{h}, 2} d_{\mathrm{hf}, 2}+\ldots \ldots .\right)}{4 A_{\mathrm{h}}}}
$$

where $d_{\mathrm{pol}}(\mathrm{cm})$ is the effective polygon diameter, $d_{\mathrm{pf}}(\mathrm{cm})$ is the actual average polygon diameter in the field, $d_{\mathrm{hf}, 1}$ and $d_{\mathrm{hf}, 2}(\mathrm{~cm})$ are the diameters of two classes of hole-shaped macropores in the field and $N_{\mathrm{h}, 1}$ and $N_{\mathrm{h}, 2}$ are their numbers per area $A_{\mathrm{hf}}\left(\mathrm{cm}^{2}\right)$. If we assume that $d_{\mathrm{pf}}=15 \mathrm{~cm}$, that there are two classes of hole-shaped parameters with an average diameter of 0.4 and $1.0 \mathrm{~cm}$ and with numbers per $\mathrm{dm}^{2}$ of 3 and 1 , then the effective polygon diameter will be $11.9 \mathrm{~cm}$.

\section{Shrinkage characteristics}

The SWAP user needs to specify either the parameters of Kim's or Hendriks' relationship (see Section 6.1.1.2), or the values of typical points of the shrinkage characteristic curve. The different options and required parameters are listed in Table 6.1. The option to specify the original parameters of both relationships is especially relevant for the development of pedotransfer functions for shrinkage characteristics. Alterra is working on pedotransfer functions for shrinkage characteristics of clay and peat soils. The options to use typical points of the shrinkage characteristic curves are useful when limited information about the exact curves is available. When just a (rough) sketch of a curve is available it may be possible to recognize these typical points.

Table 6.1 Overview of required shrinkage parameters for clay and peat soils (Figure 6.4.A and 6.9).

\begin{tabular}{|c|c|c|c|c|c|c|}
\hline Soil & Input option & \multicolumn{5}{|c|}{ Shrinkage parameters } \\
\hline Clay & 1 & $\mathrm{a}_{\mathrm{K}}\left(e_{0}\right)$ & $\beta_{\mathrm{K}}$ & $Y_{K}$ & - & - \\
\hline \multirow[t]{2}{*}{ Peat } & 1 & $e_{0}$ & $\vartheta_{a}$ & $a_{H}$ & $\beta_{H}$ & $P_{\mathrm{H}}$ \\
\hline & 2 & $e_{0}$ & $\vartheta_{a}$ & $\vartheta_{r}$ & $\vartheta_{p}$ & $P_{\mathrm{H}}$ \\
\hline
\end{tabular}

For clay soils, the typical points are the void ratio $e_{0}$ at $\vartheta=0\left(\alpha_{k}\right)$ and the moisture ratio $\vartheta_{a}$ at transition of normal to residual shrinkage (Figure 6.4.A). With these two input data, SWAP generates the parameters of Kim's relationship. For peat soils, there are two options to use typical points of the shrinkage curve. The first, option 2 in Table 6.1, requires typical points with which the parameters of Hendriks' curve can be generated. The second, option 3 in Table 6.1, enables to describe the shrinkage with three straight line-pieces. 

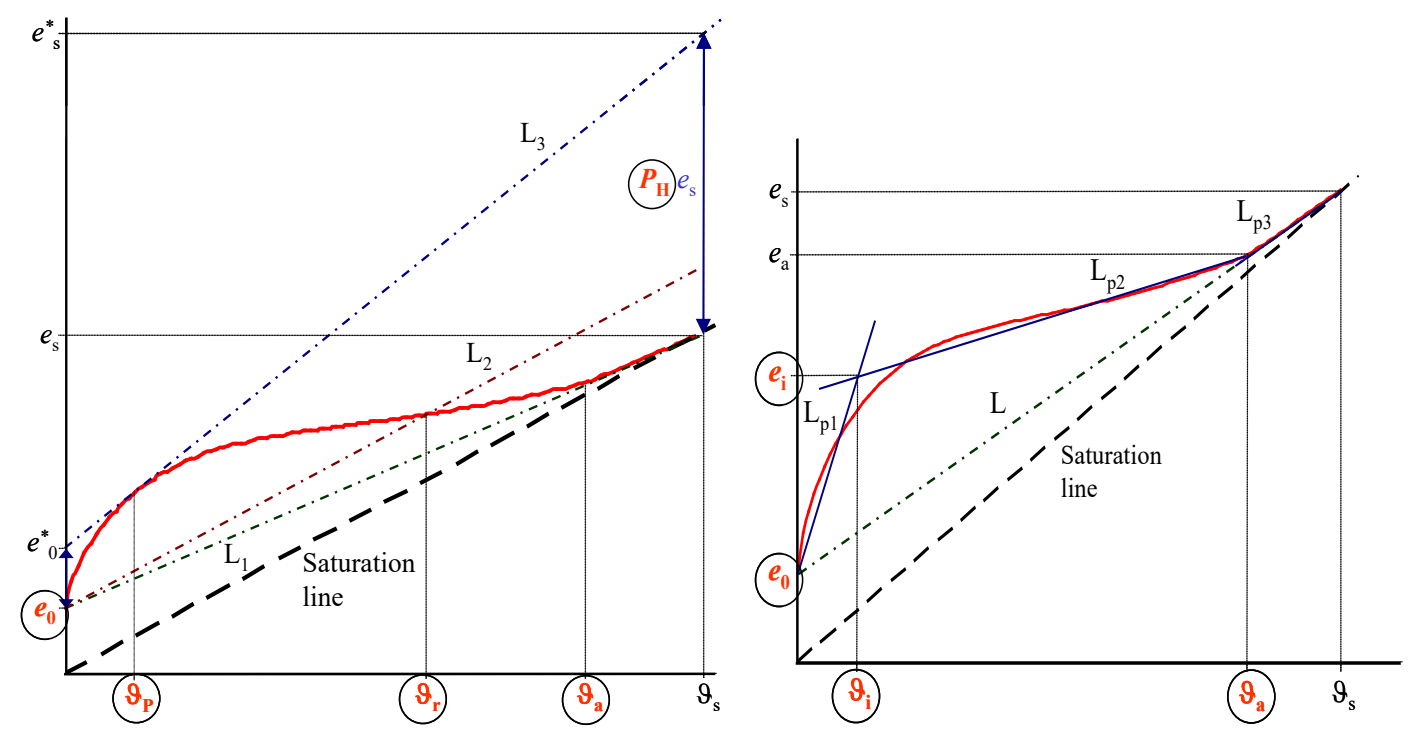

Figure 6.9 Construction of support-lines and line-pieces in the graph of the peat-shrinkage curve to find values of input parameters for Option 2 of Table 6.1: 'typical points' (left) and for Option 3 of Table 6.1: '3 straight line-pieces' (right). Symbols in circles represent input parameters.

Option 2 requires the construction of three support-lines, $L_{1}, L_{2}$ and $L_{3}$, in the graph of the shrinkage curve (Figure 6.9). $L_{1}$ connects the points $\left(0, e_{0}\right)$ and $\left(\vartheta_{s}, e_{s}\right) . L_{2}$ is parallel to the saturation line and starts at point $\left(0, e_{0}\right) . L_{3}$ connects the points $\left(0, e_{0}^{*}\right)$ and $\left(\vartheta_{s}, e_{s}^{*}\right)$ and is tangent to the shrinkage curve. In order to construct this line, parameter $P_{\mathrm{H}}$ should be found so that $\mathrm{e}_{0}^{*}=\left(1+P_{\mathrm{H}}\right) \mathrm{e}_{0}$ and $\mathrm{e}_{\mathrm{s}}^{*}=(1+$ $\left.P_{H}\right) e_{s}$. This can easily be done by trial-and-error in a spreadsheet or on paper. When $P_{H}<0, L_{2}$ must start at point $\left(0,1 / 2 \mathrm{e}_{0}\right)$ instead of point $\left(0, \mathrm{e}_{0}\right)$ (e.g. samples $\mathrm{A}-15$ and $\mathrm{V}-10$ in Appendix 11$)$. For values of $\left|P_{H}\right|<0.1$, option 3 is recommended (e.g. sample A-25 in Appendix 11). Input parameters are (Table 6.1): $\mathrm{e}_{0}, P_{\mathrm{H}}, \vartheta_{\mathrm{a}}$ (moisture ratio at transition of 'near-normal' to 'subnormal' shrinkage on $\mathrm{L}_{1}$, Figure 6.4.B), $\vartheta_{r}$ (moisture ratio at intersection point of $L_{2}$ and curve) and $\vartheta_{p}$ (moisture ratio at tangency point of $L_{3}$ to curve). Values must be given with an accuracy of at least $1 \%$ of saturated moisture ratio $\left(\vartheta_{s}\right)$.

Option 3 requires the construction of three line-pieces $L_{p 1}, L_{p 2}$ and $L_{p 3}$ and one support-line $L$ in the graph of the shrinkage curve (Figure 6.9). $L_{p 1}$ connects the points $\left(0, e_{0}\right)$ and $\left(\vartheta_{i}, e_{i}\right), L_{p 2}$ the points $\left(\vartheta_{i}, e_{i}\right)$ and $\left(\vartheta_{a}, e_{a}\right)$, and $L_{p 3}$ the points $\left(\vartheta_{a}, e_{a}\right)$ and $\left(\vartheta_{s}, e_{s}\right)$. L connects the points $\left(0, e_{0}\right)$ and $\left(\vartheta_{s}, e_{s}\right)$. Point $\left(\vartheta_{a}, e_{a}\right)$ is situated on this line and represents the point of transition of 'near-normal' to 'subnormal' shrinkage. Point $\left(\vartheta_{i}, e_{i}\right)$ should be chosen in such a way that the three line-pieces describe the shrinkage curve as accurate as possible. For use in the model, $L_{p 2}$ and $L_{p 3}$ are much more important than $L_{p 1}$. So emphasis should be put on these two line-pieces. Input parameters are (Table 6.1): $e_{0}$, $\vartheta_{a}, e_{i}$ and $\vartheta_{i}$. 


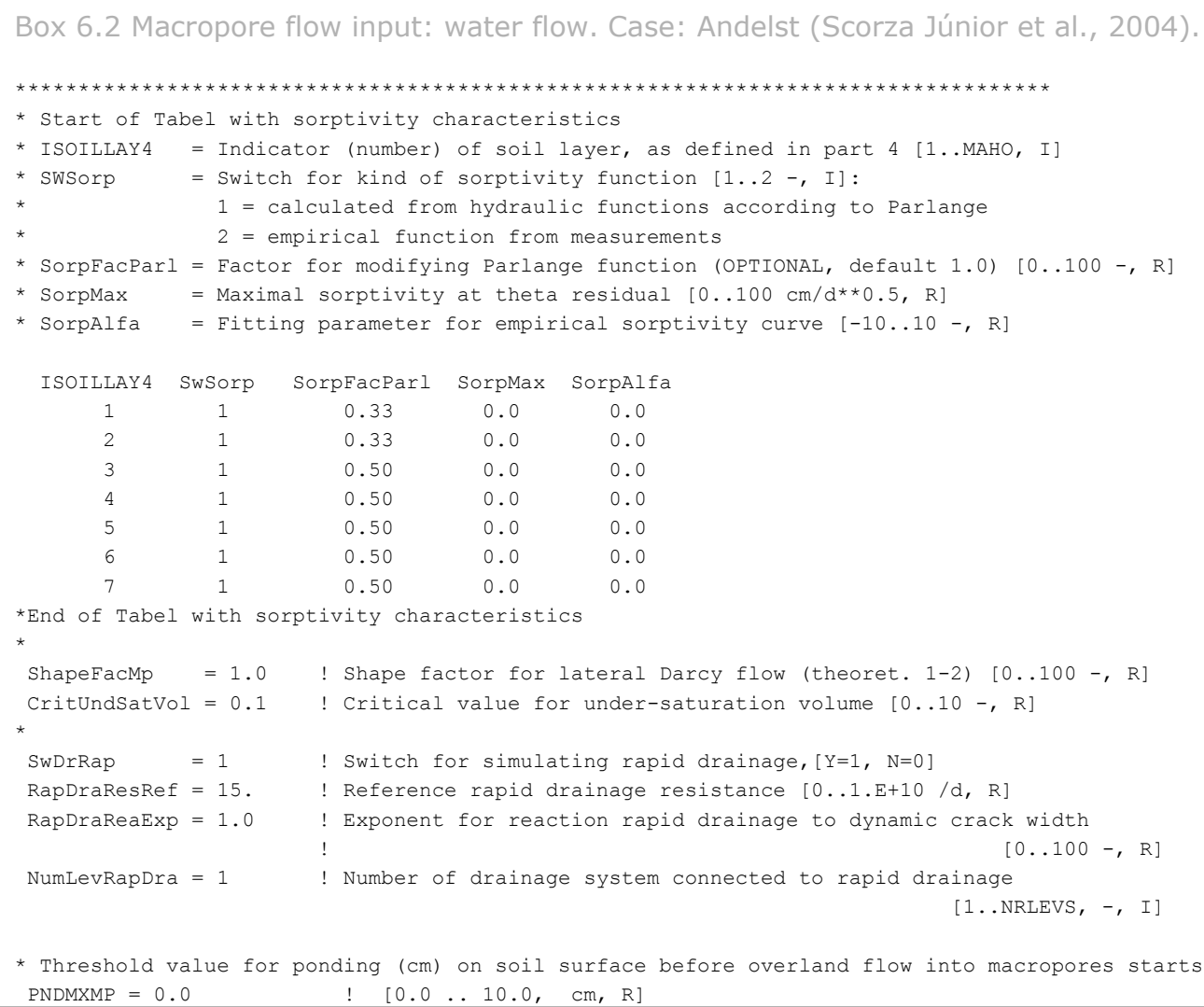

If there is no information available to decide otherwise, ThetCrMP could be taken at $90-100 \% \theta_{\text {sat, }}$ GeomFac as 3.0 and $\mathrm{ZnCrAr}$ around $-5.0 \mathrm{~cm}$.

Measured shrinkage characteristics of seven clay profiles in the Netherlands, as described by Bronswijk and Evers-Vermeer (1990), are listed in Appendix 10. Yule and Ritchie (1980a, 1980b) described shrinkage characteristics of eight Texas Vertisols, using small and large cores. Garnier et al. (1997) propose a simple evaporation experiment to determine simultaneously the moisture retention curve, hydraulic conductivity function and shrinkage characteristic. Measured shrinkage characteristics of four peat soil profiles in the Netherlands, as described by Hendriks (2004), are listed in Appendix 11.

\subsubsection{Water flow}

The input parameters of the water flow concept are listed in Box 6.2. They are discussed below.

The sorptivity parameters can be obtained by fitting Eq. (6.30) against measured values to derive a relationship between sorptivity and initial moisture content. The advantage of measured sorptivities is that they may reflect the influence of water-repellent coatings on the surface of clay aggregates which often hamper infiltration into these aggregates (Thoma et al., 1992; Dekker and Ritsema, 1996). If measured sorptivities are not available, sorptivity as a function of moisture content is derived from the soil hydraulic characteristics (Parlange, 1975). To account for water-repellent coatings a correction factor SorpFacParl can be entered. Greco et al. (1996) found values for this factor of 0.33 for the topsoil and 0.5 for the sub-soil of a Dutch clay soil similar to the Andelst soil. They describe a simple way of measuring sorptivity as a function of moisture content.

ShapeFacMp can be used to decrease or increase exchange fluxes between macropores and soil matrix. Theoretically, its value lies between 1 and 2 (see Section 6.1.2); default value is 1.0. RapDraResRef depends on the system of macropores and their connection to drains or ditches. In case of a network of structural cracks, RapDraResRef will be smaller than in case of mainly hole shaped macropores. The opposite applies to RapDraReaExp. 


\section{$7 \quad$ Crop growth}

\subsection{Introduction}

SWAP contains three crop growth routines: a simple module for a static crop and two detailed modules for dynamic crops: one for arable crops (WOFOST, WOrld FOod STudies), and a detailed module for grass (re)growth. A static crop has a fixed development of leaf area index and rooting depth, independent of climatic conditions, whereas development of a dynamic crop changes with the climatic conditions.

The main function of the simple module for static crops is to provide proper upper boundary conditions for soil water movement. The simple model is useful when crop growth doesn't need to be simulated or when crop growth input data are insufficient. Section 7.2 provides a description of the simple module.

In the footsteps of De Wit and co-workers (De Wit et al., 1978; Goudriaan, 1977; Penning de Vries and Van Laar, 1982), in the 1980s a wide range of scientists in Wageningen became involved in the development and application of dynamic crop growth models. The generic crop model SUCROS for the potential production situation was developed (Spitters et al., 1989). SUCROS formed the basis of a range of Wageningen crop models, as reviewed by Bouman et al. (1996) and Van Ittersum et al. (2003). One of the developed models is the WOFOST model, which simulates in detail photosynthesis and crop development, taking into account growth reductions due to water and/or salt stress.

WOFOST has been implemented in SWAP and is described in Section 7.3.

The detailed module for dynamic growth of grass is a modified version of WOFOST. The only species occuring in the sward is supposed to be perennial ryegrass (Lolium perenne L.). The sward is regular mowed or grazed and remains vegetative during the year. The settings for regrowth after grass cutting have a large effect on the leaf area development; therefore the application of this module requires expert judgement. The detailed module for growth of grassland is described in Section 7.4.

Section 7.5 describes the germination of crop growth, and Section 7.6 the option to simulate the impact of atmospheric $\mathrm{CO}_{2}$ changes. Section 7.7 describes the option to activate soil and crop nitrogen modules which interact with WOFOST. 


\subsection{Simple module for static crop growth}

The simple crop growth model represents a green canopy that intercepts precipitation, transpires water vapour and shades the ground. The user specifies crop variables as a function of development stage. The variables to be specified are crop height, rooting depth and either leaf area index or soil cover fraction. The development stage is controlled either by the temperature sum or can be linear in time.

The simple model does not calculate the crop potential or actual yield. However, users may define yield response factors (Doorenbos and Kassam, 1979; Smith, 1992) for various growing stages as a function of development stage. For each growing stage $k$ the actual yield $Y_{\mathrm{a}, \mathrm{k}}\left(\mathrm{kg} \mathrm{ha}^{-1}\right)$ relative to the potential yield $Y_{\mathrm{p}, \mathrm{k}}\left(\mathrm{kg} \mathrm{ha}^{-1}\right)$ during this growing stage is calculated by:

$$
1-\frac{Y_{\mathrm{a}, k}}{Y_{\mathrm{p}, k}}=K_{\mathrm{y}, k}\left(1-\frac{T_{\mathrm{a}, k}}{T_{\mathrm{p}, k}}\right)
$$

where $K_{y, k}(-)$ is the yield response factor of growing stage $k$, and $T_{\mathrm{p}, \mathrm{k}}(\mathrm{cm})$ and $T_{\mathrm{a}, \mathrm{k}}(\mathrm{cm})$ are the potential and actual transpiration, respectively, during growing stage $k$.

The relative yield of the whole growing season is calculated as the product of the relative yields of each growing stage:

$$
\frac{Y_{\mathrm{a}}}{Y_{\mathrm{p}}}=\prod_{k=1}^{n}\left(\frac{Y_{\mathrm{a}, k}}{Y_{\mathrm{p}, k}}\right)
$$

where $Y_{a}$ is the cumulative actual yield $\left(\mathrm{kg} \mathrm{ha}^{-1}\right)$ of the whole growing season, $Y_{\mathrm{p}}$ is the cumulative potential yield $\left(\mathrm{kg} \mathrm{ha}^{-1}\right)$ of the whole growing season, index $k$ is the growing stage and $n$ is the number of defined growing stages. 


\subsection{Detailed module for dynamic growth of arable crops}

Three groups of growth factors (Figure 7.1) can be distinguished to obtain a hierarchy of production levels in crop production (Van Ittersum et al., 2003). Growth defining factors, such as radiation intensity, carbon dioxide concentration, temperature and crop characteristics determine the potential production that can be achieved in a given physical environment and for a given plant species. To achieve the potential production the crop must be optimally supplied with water and nutrients and completely protected against weeds, pests, diseases and other factors that may reduce growth.

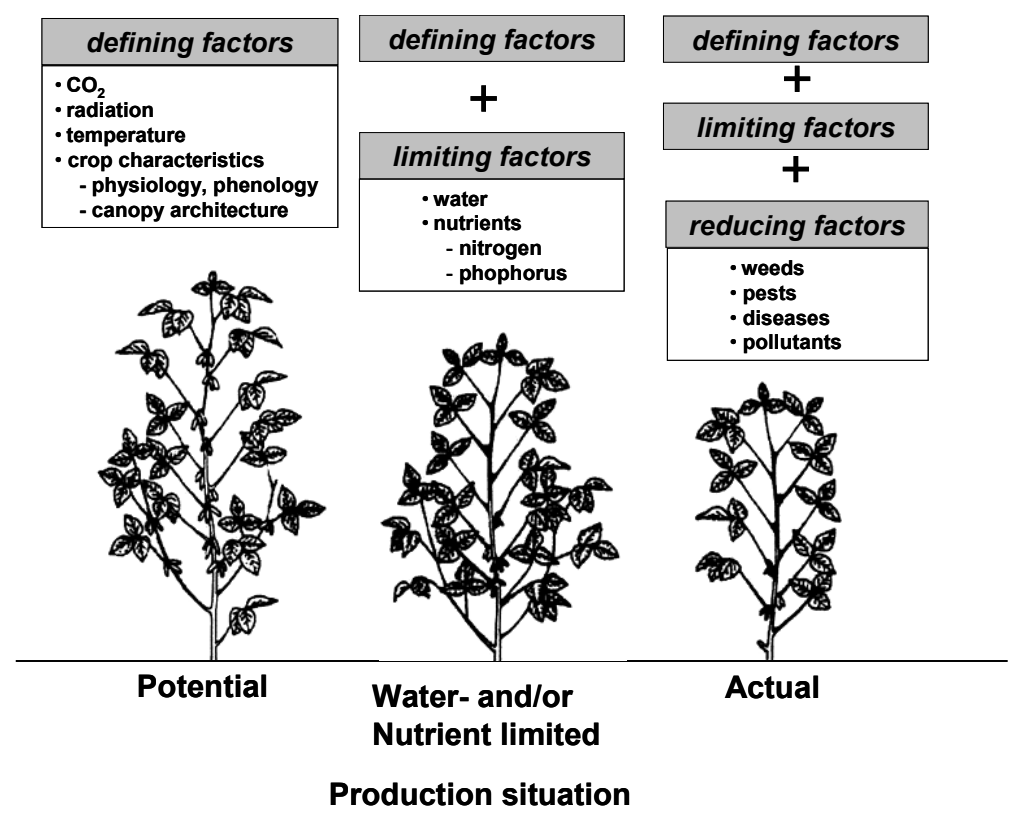

Figure 7.1 A hierarchy of growth factors, production situations and associated production levels (Van Ittersum et al., 2003).

Growth-limiting factors such as water and nutrients determine the water- or nutrient-limited production level in a given physical environment. Through management of the water and nutrients availability, crop production may increase towards potential levels. Growth reducing factors that reduce or hamper growth comprise biotic factors such as weeds, pests and diseases, and abiotoc factors such as pollutants. Crop protection aims at an effective management of these factors. In the actual production situation crop productivity is usually the results of a combination of growth-limiting and -reducing factors (Van Ittersum et al., 2003). 


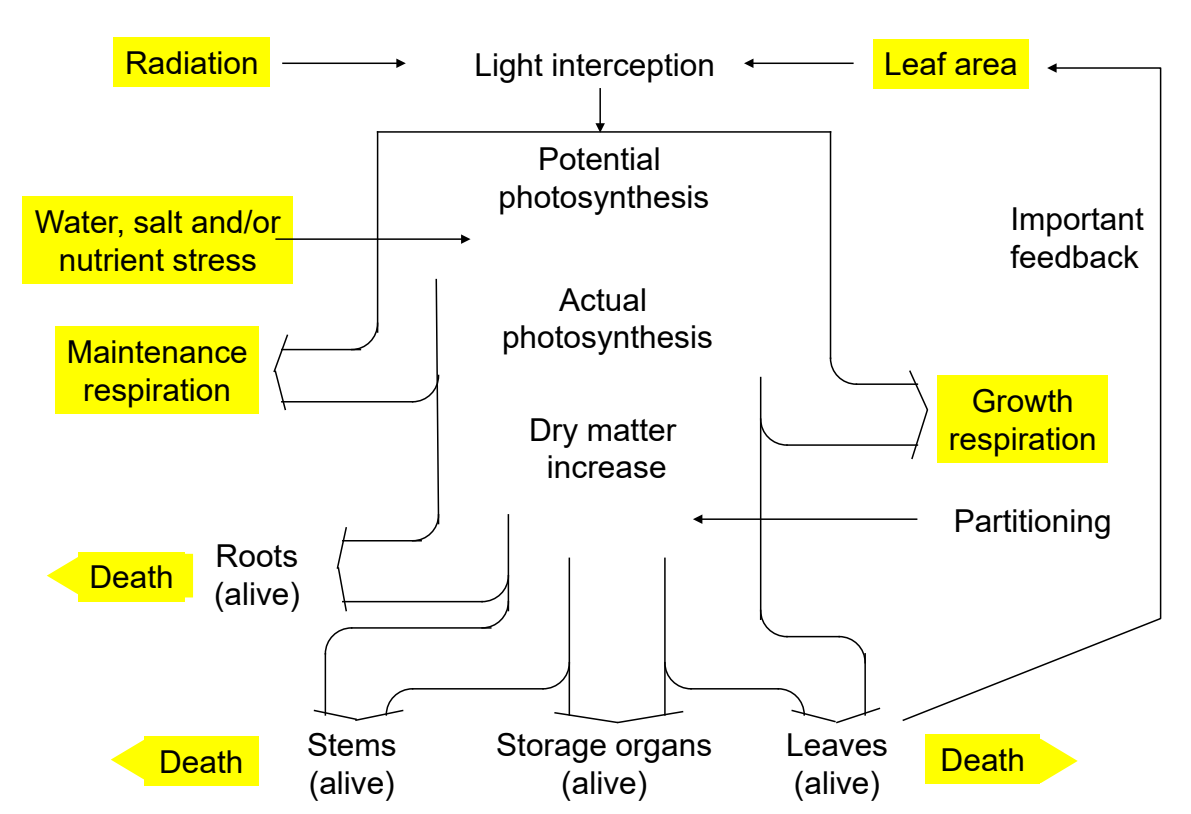

Figure 7.2 Overview of crop growth processes incorporated in WOFOST.

WOFOST (Van Keulen and Wolf, 1986; Spitters et al., 1989; Supit et al., 1994; Hijmans et al., 1994; Boogaard et al., 1998) simulates potential and limited production due to water and/or salinity and/or nutrient stress. Figure 7.2 shows the processes and relations incorporated in WOFOST. The energy that is absorbed by the canopy depends on the incoming radiation, the crop leaf area and the photosynthetic leaf characteristics. With the absorbed radiation the potential gross photosynthesis is calculated, which is subsequently reduced due to water/salinity/nutrient stress yielding the actual gross photosynthesis.

Part of the produced carbohydrates $\left(\mathrm{CH}_{2} \mathrm{O}\right)$ are used for maintenance of the living biomass (maintenance respiration). The remaining carbohydrates are converted into structural matter. This conversion process costs energy and some weight is lost (growth respiration). The remaining dry matter is partitioned among roots, leaves, stems and storage organs, using partitioning factors that are a function of the phenological development stage (Spitters et al., 1989). The fraction partitioned to the leaves, determines leaf area development and hence the dynamics of light interception. Integrating the growth rates over time yields the dry weight of the crop organs. During the seasons, part of living biomass dies due to senescence.

Light interception and $\mathrm{CO}_{2}$ assimilation are the main growth driving processes. Some of these crop growth processes are influenced by temperature, like for example the maximum photosynthesis and the maintenance respiration rate. Other processes, like the assimilate partitioning and tissue decay, are a function of the phenological development stage (which is a function of the thermal time).

\subsubsection{Phenological development stage}

As many physiological and morphological processes change with the phenological stage, quantification of the phenological development is essential in any crop growth simulation model. For many annual crops, the phenological development can be expressed as a development stage $D_{\mathrm{s}}(-)$, having the value 0 at seedling emergence, 1 at flowering and 2 at maturity (Van Heemst, 1986a; 1986b). The most important phenological change is the one from vegetative $\left(0<D_{s}<1\right)$ to reproductive stage $\left(1<D_{\mathrm{s}}<2\right)$, which changes drastically the dry matter allocation to organs. 
Crop growth simulation starts either at sowing or emergence. The sowing or emergence date should be specified by the user. A crop passes through successive phenological development stages, of which the length depends on the development rate. Development rates before floral initiation or anthesis $\left(D_{\mathrm{s}}=1\right)$ are controlled by temperature and sometimes depend on day length as well. After anthesis the dvelopment rate only depends on the temperature. Higher temperatures accelerate the development rate, leading to shorter growing periods. It has often been demonstrated, that the development rate increases more or less linearly with temperature

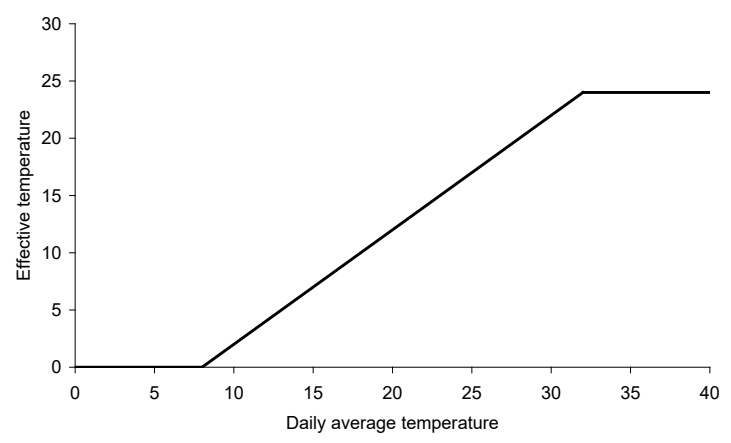

Figure 7.3 Example of effective temperature for temperature sum as function of daily average temperature.

(Van Dobben, 1962; Van Keulen and Seligman, 1987). Therefore WOFOST uses the temperature sum to describe the development stage. The temperature sum is calculated using the effective temperature $T_{\text {eff }}\left({ }^{\circ} \mathrm{C}\right)$, which is a tabular function of the daily average temperature $T_{\text {day }}\left({ }^{\circ} \mathrm{C}\right)$ (Figure 7.3). Generally for species originating from temperate regions $T_{\text {day }}-T_{\text {eff }}=0-3{ }^{\circ} \mathrm{C}$, while for species of subtropical and tropical origins $T_{\text {day }}-T_{\text {eff }}=9-14{ }^{\circ} \mathrm{C}$ (Angus et al., 1981). Note that $T_{\text {eff }}$ cannot be negative. The user should specify $T_{\text {eff }}\left(T_{\text {day }}\right)$ and the relation between crop development stage $D_{\mathrm{s}}$ and $T_{\text {eff }}$ :

$$
D_{\mathrm{s}}^{j+1}=D_{\mathrm{s}}^{j}+\frac{T_{\mathrm{eff}}}{T_{\mathrm{sum}, i}}
$$

where superscript $j$ is the day number and $T_{\text {sum,i }}$ is the temperature sum required to complete either the vegetative or the reproductive stage. Within a species, cultivars may vary substantially in their temperature requirements.

For some species or cultivars the day length during the vegetative stage should be accounted for. Approaches that describe such effects quantitatively are given, amongst others, by Weir et al. (1984), Hadley et al. (1984) and Reinink et al. (1986). In Wofost a reduction factor for the development rate as function of day length $f_{\text {lday }}(-)$ is computed:

$$
f_{\text {lday }}=\frac{L_{\text {day }}-L_{\text {cday }}}{L_{\text {oday }}-L_{\text {cday }}} \quad \text { with } \quad 0<f_{\text {lday }}<1
$$

with $L_{\text {day }}$ the actual day length (d), $L_{\text {cday }}$ the critical day length for development (d), and $L_{\text {oday }}$ the optimum day length for development $(d)$. The critical and the optimum day length should be provided by the user. Note that in modern cultivars, photosensitivity is much less pronounced than in traditional cultivars and the day length influence can be ignored by choosing an appropriate temperature sum, which leads to an equivalent crop life cycle.

Crop growth simulation stops when the development stage reaches ripeness $\left(D_{\mathrm{s}}=2\right)$ or when the crop will be harvested (i.e. before ripeness). In this later case the user should specify the harvest date. 


\subsubsection{Radiation fluxes above the canopy}

Measured or estimated daily global radiation (wave length range $300-3000 \mathrm{~nm}$ ) is input for the model. Incoming radiation is partly direct, with the angle of incidence equal to the angle of the sun, and partly diffuse, with incidence under various angles. The sine of solar elevation as a function of the day hour, can be calculated with:

$$
\sin \beta_{\text {sun }}=\sin L_{\mathrm{g}} \sin \sigma_{\text {sun }}+\cos L_{\mathrm{g}} \cos \sigma_{\text {sun }} \cos \left(\frac{2 \pi\left(t_{\mathrm{h}}+12\right)}{24}\right)
$$

with $\beta_{\text {sun }}$ is the solar elevation (degrees), $\sigma_{\text {sun }}$ is solar declination (degrees), $L_{g}$ is geographic latitude (degrees) and $t_{\mathrm{h}}$ is hour of the day. Integration of $\sin \beta_{\text {sun }}$ over the day and mulitplication with the solar constant yields the daily extra-terrestrial radiation which is also known as the Angot radiation $\left(\mathrm{J} \mathrm{m}^{-2} \mathrm{~d}^{-1}\right)$ :

$$
R_{o}=S_{c} \int \sin \beta_{\text {sun }} d t
$$

The solar constant $\mathrm{S}_{\mathrm{c}}\left(\mathrm{J} \mathrm{m}^{-2} \mathrm{~d}^{-1}\right)$ can be calculated as:

$$
S_{c}=1370\left(1+0.033 \cos \left(\frac{2 j \pi}{365}\right)\right)
$$

where $j$ is the day number in the year (DOY). Only about 50 percent of the global radiation is photosynthetically active (PAR, Photosynthetically Active Radiation, wavelength band 400-700 nm). The daily incoming PAR $\left(\mathrm{J} \mathrm{m}^{-2} \mathrm{~d}^{-1}\right)$ is calculated by multiplying half of the daily global radiation with the ratio of the actual effective solar elevation and the integral of the effective solar height, taking into account reduced atmospheric transmission at low solar elevations:

$$
P A R=0.5 R_{g} \frac{\sin \beta_{\text {sun }}\left(1+0.4 \sin \beta_{\text {sun }}\right)}{\int \sin \beta_{\text {mod, sun }}}
$$

where $R_{\mathrm{g}}$ is daily global radiation $\left(\mathrm{J} \mathrm{m}^{-2} \mathrm{~d}^{-1}\right)$ and $\int \sin \beta_{\mathrm{mod}, \text { sun }}$ the integral of $\sin \beta_{\text {sun }}$ over the day (-) which is corrected for reduced atmospheric transmission at low solar elevations. See Spitters et al. (1986).

The diffuse radiation flux results from scattering of sun rays by clouds, gases and dust in the atmosphere. To quantify the diffuse radiation first the atmospheric transmission is calculated as:

$$
T_{a t m}=\frac{R_{g}}{R_{o}}
$$

The relation between the proportion of the diffuse flux in the global irradiance $\left(R_{d f} / R_{g}\right)$ and the atmospheric transmission $T_{\text {atm }}$ is characterized by an approximately linear trend for transmissions ranging between 0.35 and 0.75 . At low transmissions, nearly all of the incoming radiation is diffuse so that the curve bends off. There is some variation among published relations, arising from differences in atmospheric conditions, especially relative sunshine duration, water content of the atmosphere, and cloud type, but also lack of fit of the presented regression equation from the data and differences in the method of measuring the diffuse radiation. The relation used in WOFOST has been derived by de Jong (1980) and has been recommended by Spitters et al. (1986):

$$
\begin{array}{ll}
\frac{R_{d f}}{R_{g}}=1 & \text { for } T_{a t m} \leq 0.07 \\
\frac{R_{d f}}{R_{g}}=1-2.3\left(T_{\dot{a} t m}-0.07\right)^{2} & \text { for } 0.07<T_{a t m} \leq 0.35 \\
\frac{R_{d f}}{R_{g}}=1.33-1.46 T_{a t m} & \text { for } 0.35<T_{a t m} \leq 0.75 \\
\frac{R_{d f}}{R_{g}}=0.23 & \text { for } T_{a t m}>0.75
\end{array}
$$


The diffuse photosynthetically active radiation $P A R_{\text {dif }}\left(\mathrm{J} \mathrm{m}^{-2} \mathrm{~d}^{-1}\right)$ can be calculated by:

$$
P A R_{\mathrm{dif}}=0.5 \frac{R_{d f}}{R_{g}} T_{a t m} R_{o}
$$

The direct radiation flux, $P A R_{\text {dir }}\left(\mathrm{J} \mathrm{m}^{-2} \mathrm{~d}^{-1}\right)$, is obtained by subtracting the diffuse part from the photosynthetically active radiation flux:

$$
P A R_{\mathrm{dir}}=P A R-P A R_{\mathrm{dif}}
$$

\subsubsection{Radiation profiles within the canopy}

The incoming PAR is partly reflected by the canopy. The reflection coefficient is defined as the fraction of the downward radiation flux that is reflected by the entire canopy. The reflection coefficient $\rho_{\text {rad }}(-)$ of a green leaf canopy with a random spherical leaf angle distribution equals (Goudriaan, 1977):

$$
\rho_{\text {rad }}=\left(\frac{1-\sqrt{1-\sigma_{\text {leaf }}}}{1+\sqrt{1-\sigma_{\text {leaf }}}}\right)\left(\frac{2}{1+1.6 \sin \beta_{\text {sun }}}\right)
$$

with $\sigma_{\text {leaf }}$ the scattering coefficient of single leaves for visible radiation $(-)$, which is set to 0.2 (Goudriaan, 1977). The first right-hand-side term of Eq. (7.13) denotes the reflection of horizontal leaves and the second term is the approximate correction factor for a spherical leaf angle distribution. The fraction $\left(1-\rho_{\mathrm{rad}}\right)$ of the incoming visible radiation is available for absorption by the canopy.

Light intensity, adjusted for crop reflection, decreases approximately exponentially with leaf area index when going deeper into the canopy:

$$
P A R_{\mathrm{L}}=\left(1-\rho_{\mathrm{rad}}\right) P A R \mathrm{e}^{-\kappa L}
$$

where $L$ is the cumulative leaf area index, $\Sigma L A I\left(\mathrm{~m}^{2}\right.$ leaf $\mathrm{m}^{-2}$ ground), counted from the top of the canopy downwards, $P A R_{\mathrm{L}}$ is the net light intensity $\left(\mathrm{J} \mathrm{m}^{-2} \mathrm{~d}^{-1}\right)$ at depth $L$, and $\kappa$ is the radiation extinction coefficient (-).

The profiles of the net diffuse flux and the net flux caused by direct irradiance can be characterized analogously (Goudriaan, 1982). Diffuse and direct fluxes each attenuate at a different rate, and both extinction coefficients are input in SWAP. For a random spherical leaf angle distribution the extinction coefficient of the direct flux component, $\kappa_{\text {dir }}(-)$, might be approximated by (Goudriaan, 1977, 1982):

$$
\kappa_{\text {dir }}=\frac{0.5}{\sin \beta_{\text {sun }}}
$$

and the extinction coefficient of the diffuse flux component, $\kappa_{\text {dif }}(-)$, might be calculated as:

$$
\kappa_{\text {dif }}=\kappa_{\text {dir }} \sqrt{1-\sigma_{\text {leaf }}}
$$

In Eq. (7.15), the factor 0.5 represents the average projection on the ground surface of leaves showing a spherical angle distribution. Averaging $0.5 / \sin \beta$ during a day with an overcast sky, gives a value of $\kappa_{\text {dir }}=0.8(-)$. The value of $\kappa_{\text {dif }}$ can be measured directly under completely overcast sky conditions, when only diffuse radation reaches the canopy. The average value is about $0.72(-)$ (Goudriaan, 1977).

In many situations, the leaf angle distribution is not spherical. Therefore, in WOFOST the actual leaf angle distribution is accounted for by using a so called cluster factor which is the measured extinction coefficient for diffuse radiation, relative to the theoretical one for a spherical leaf area distribution.

On its way through the canopy, part of the direct flux is intercepted and scattered by the leaves. Hence, the direct flux segregates into a diffuse, scattered component and another component which remains direct. Attenuation of the remaining direct component proceeds like in a hypothetical canopy of black, non scattering leaves. The diffuse component is obtained as the difference between the total direct flux and its direct component. 
The light absorption rate at depth $L$ in the canopy, $P A R_{\mathrm{L}, \mathrm{a}}\left(\mathrm{J} \mathrm{m}^{-2}\right.$ leaf $\left.\mathrm{d}^{-1}\right)$, is the derivative of Eq. (7.14) with respect to $L$ :

$$
P A R_{L, \mathrm{a}}=\kappa\left(1-\rho_{\text {rad }}\right) P A R \mathrm{e}^{-\kappa L}
$$

Similar expressions can be derived for the separate light components: the diffuse flux, the total direct radiation flux and the direct component of the direct radiation flux. The absorbed diffuse component of the direct flux is obtained by subtracting the direct component from the total direct flux.

\subsubsection{Instantaneous assimilation rates per leaf layer}

The canopy $\mathrm{CO}_{2}$ assimilation rate layer is obtained by substituting the absorbed amount of light energy into the assimilation-light response of single leaves (Peat, 1970):

$$
A_{\mathrm{L}}=A_{\max }\left(1-\mathrm{e}^{\frac{-\varepsilon_{\mathrm{PAR}} P A R_{L, \mathrm{a}}}{A_{\max }}}\right)
$$

where $A_{\mathrm{L}}$ is the gross assimilation rate at relative depth $\mathrm{L}\left(\mathrm{kg} \mathrm{CO}_{2} \mathrm{~m}^{-2}\right.$ leaf $\left.\mathrm{d}^{-1}\right), A_{\max }$ the gross assimilation rate at light saturation $\left(\mathrm{kg} \mathrm{CO}_{2} \mathrm{~m}^{-2}\right.$ leaf $\left.\mathrm{d}^{-1}\right)$, and $\varepsilon_{\text {PAR }}$ the initial slope or light use efficiency (kg $\mathrm{CO}_{2} \mathrm{~J}^{-1}$ absorbed).

Two leaf classes are distinguished: shaded leaves and sunlit leaves. The shaded leaf area absorbs the diffuse flux and the diffuse component of the direct flux. The sunlit leaf area receives diffuse and direct radiation. At every horizon within the canopy, the intensity of the unobstructed direct beam equals its intensity above the crop. llumination intensity of sunlit leaves varies strongly with leaf angle. In the model, the assimilation rate of the sunlit leaf area is therefore integrated over the leaf angle distribution.

The assimilation rate per unit leaf area in a canopy, is the sum of the assimilation rates of sunlit and shaded leaves, taking into account their proportion in each layer. The proportion of sunlit leaf area at depth $L$ in the canopy equals the proportion of the direct component of the direct flux reaching that depth. This proportion is calculated analogous to Eq. (7.17), using the extinction coefficient of the direct radiation component.

Figure 7.4 shows the $\mathrm{CO}_{2}$ assimilation rate at different sunlight intensities as measured for various crops. Striking are the higher assimilation rates of so-called $\mathrm{C}_{4}$ crops in comparison to $\mathrm{C}_{3}$ crops. The reason is that $\mathrm{C}_{4}$ plants are more effective in $\mathrm{CO}_{2}$ fixation within a leaf (Moene and Van Dam, 2014).

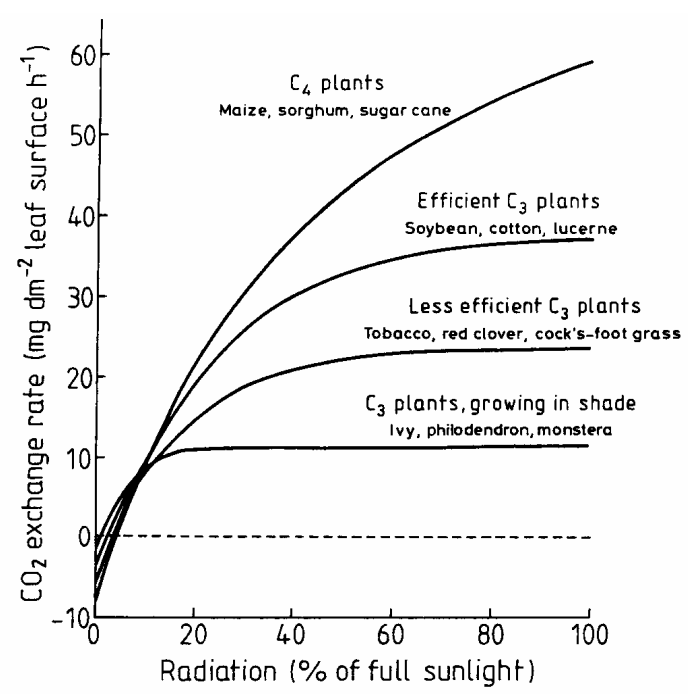

Figure 7.4 $\mathrm{CO}_{2}$ exchange rate as function of radiation amount for $C_{3}$ and $C_{4}$ plants.

At a certain light intensity, the $\mathrm{CO} 2$ uptake rate and the photosynthesis are much higher in case of $\mathrm{C} 4$ plants. 


\subsubsection{Daily gross assimilation rate of the canopy}

The maximum instantaneous $\mathrm{CO}_{2}$ assimilation rate $A_{\max }\left(\mathrm{kg} \mathrm{CO}_{2} \mathrm{ha}^{-1} \mathrm{~d}^{-1}\right)$ is crop and development stage dependent and should be provided by the user as function of development stage. $A_{\max }$ has to be corrected for the atmospherical $\mathrm{CO}_{2}$ concentration and for the average daytime temperature $T_{\text {day }}\left({ }^{\circ} \mathrm{C}\right)$ and according to:

$$
A_{\text {pgross }}^{1}=f_{\mathrm{CO}_{2}} f_{\text {tday }} A_{\text {max }}
$$

where $f_{\mathrm{CO} 2}$ is the $\left[\mathrm{CO}_{2}\right]$ dependent correction factor and $f_{\text {tday }}$ is the average daytime temperature that corrects $A_{\max }$ for sub-optimum temperatures. Both correction factors are crop specific and should be provided by the user as tables. The average daytime temperature $T_{\text {day }}\left({ }^{\circ} \mathrm{C}\right)$ is calculated as:

$$
T_{\text {day }}=0.75 T_{\max }+0.25 T_{\min }
$$

Figure 7.5 shows a typical occurence of the daytime dependent correction factor.

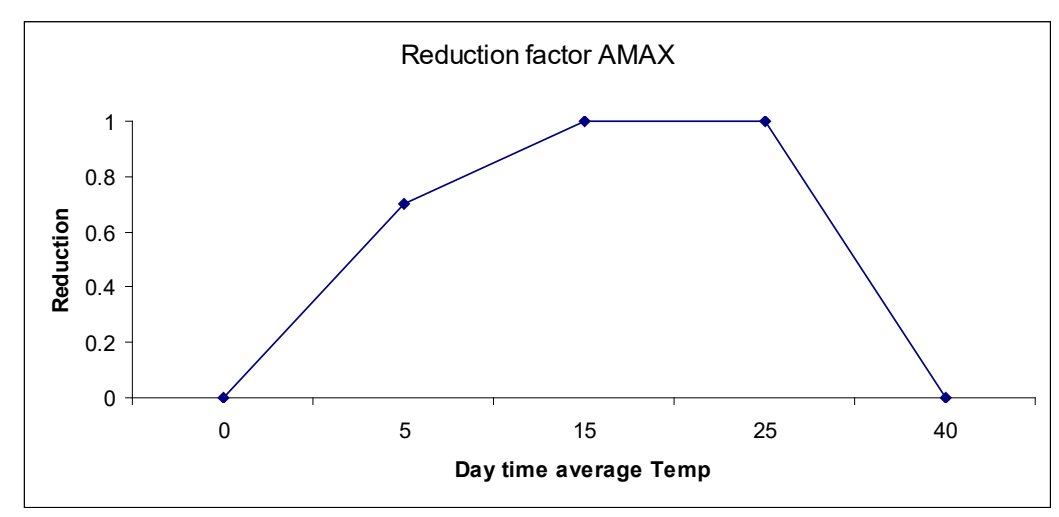

Figure 7.5 Reduction factor for the influence of day time average temperature on AMAX.

In a next step, the instantaneous rates per leaf layer are integrated over the canopy leaf area index and over the day using the Gaussian integration method (Press et al., 1989). This method specifies the discrete points at which function values have to be calculated, and the weighting factors with which the function values have to be multiplied in order to minimize deviation from analytical integration. A three-point algorithm evaluates the function at $0.1127 a, 0.5 a$ and $0.8873 a$ of the interval $(0, a)$, with weighting coefficients $1.0,1.6$ and 1.0, respectively. The Gaussian integration method is remarkably accurate in case of trigonometric (radiation) and exponential (light absorption) functions. WOFOST computes at three selected moments of the day incoming PAR just above the canopy. Using this radiation, assimilation is computed at three selected depths in the canopy (Spitters et al., 1989). Gaussian integration of these values results in the daily gross $\mathrm{CO}_{2}$ assimilation rate, $A_{\text {pgross }}\left(\mathrm{kg} \mathrm{CO}_{2} \mathrm{ha}^{-1} \mathrm{~d}^{-1}\right)$.

For each $\mathrm{kg}$ of absorbed $\mathrm{CO}_{2} 30 / 44 \mathrm{~kg}$ biomass $\left(\mathrm{CH}_{2} \mathrm{O}\right)$ is formed. Factors that reduce the daily assimilation rate are unfavourable temperatures, water shortage or excess, salinity and nutrient stress:

$$
A_{\text {gross }}=\frac{30}{44} f_{7 \min }\left(\min \left(\frac{T_{\mathrm{a}}}{T_{\mathrm{p}}}, f_{\text {nstress }}\right)\right) A_{\text {pgross }}^{1}
$$

where $A_{\text {gross }}$ is the daily gross assimilation rate $\left(\mathrm{kg} \mathrm{ha}^{-1} \mathrm{~d}^{-1}\right), T_{\mathrm{a}}$ is de actual transpiration reduced by, water, oxygen, salinity and frost stress, see equation (3.18 and 3.19), $f_{\text {nstress }}$ is the nutrient stress and $f_{7 \min }$ is stress caused by low temperatures. At night, assimilates produced during daytime, are transformed into structural biomass. This process is hampered by low temperature. If these low temperatures prevail for several days, the assimilation rate diminishes and ultimately halts. In WOFOST $f_{7 \mathrm{~min}}$ is a function of the minimum temperature during the previous seven days. $T_{p}$ is the potential transpiration and $T_{a}$ is de actual transpiration reduced by, water, oxygen, salinity and frost stress, see 
Section 3.5. Factor $f_{\text {nstress }}$ accounts for nutrient stress that is calculated as function of the crop nutrient status and supply by either artificial fertilizer, slurry or compost. The crop nutrient status is based on Shibu et al. (2010). Soil nitrogen fate is described by Groenendijk et al. (2016).

The nitrogen stress factor depends on the nitrogen application and the crop nitrogen uptake. Not all applied fertilizer will be consumed by the crop. In SWAP the reduction factor is input as function of the applied Nitrogen amount $(\mathrm{kg})$ (Figure 7.6).

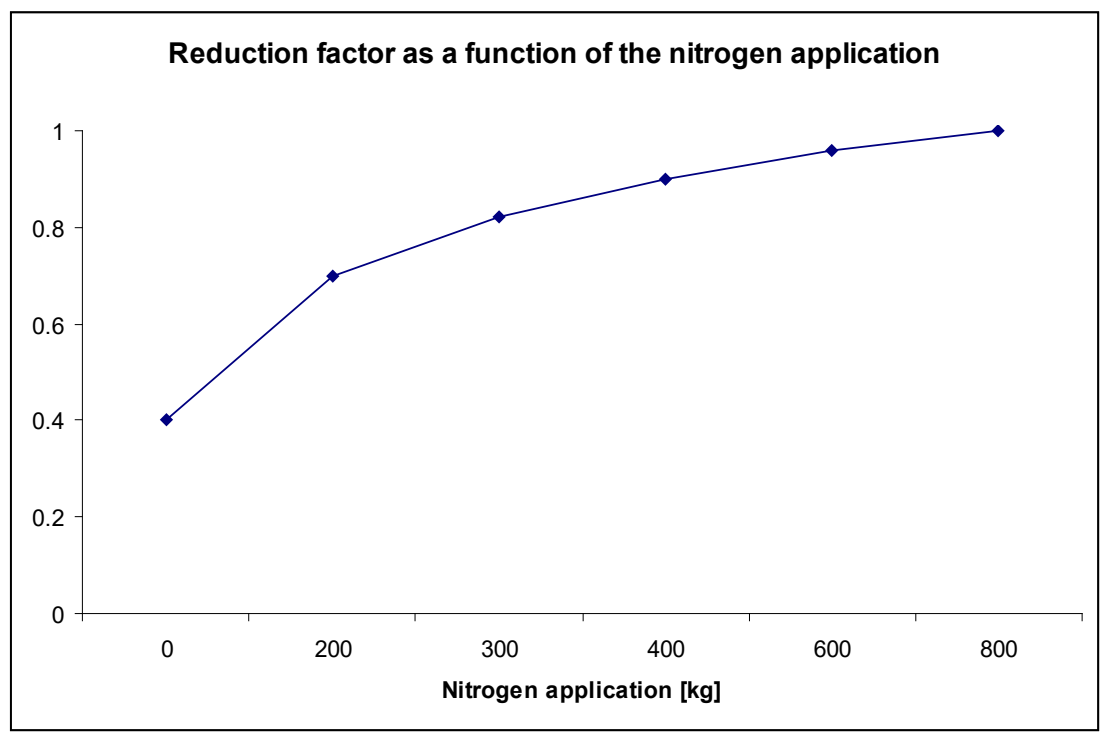

Figure 7.6 Reduction factor for influence of nitrogen supply on on AMAX (Source: Nitriogen fluxes in intensive grassland systems. Van der Meer et al. (1986).

\subsubsection{Maintenance respiration}

Some of the carbohydrates formed are respired to provide energy for maintaining the existing bio structures. This maintenance respiration consumes roughly $15-30 \%$ of the carbohydrates produced by a crop in a growing season (Penning de Vries et al., 1979). This underlines the importance of accurate quantification of this process in the model.

WOFOST estimates the maintenance costs using the approach proposed by Penning de Vries and Van Laar (1982), assuming that the reference maintenance requirements $R_{\mathrm{mref}}\left(\mathrm{kg} \mathrm{ha}^{-1} \mathrm{~d}^{-1}\right)$ are proportional to the dry weights of the plant organs to be maintained:

$$
R_{\text {mref }}=c_{\text {m,leaf }} W_{\text {leaf }}+c_{\text {m,stem }} W_{\text {stem }}+c_{\text {m,stor }} W_{\text {stor }}+c_{\text {m,root }} W_{\text {root }}
$$

where $c_{\mathrm{m}, \mathrm{i}}$ denotes the maintenance coefficient of organ $i\left(\mathrm{~kg} \mathrm{~kg}^{-1} \mathrm{~d}^{-1}\right)$ and $W_{\mathrm{i}}$ the organ dry weight $\left(\mathrm{kg} \mathrm{ha}^{-1}\right)$. The maintenance coefficients should be specified by the user.

The maintenance respiration rate still has to be corrected for senescence and temperature. The reduction factor for senescence $f_{\text {senes }}(-)$ is crop-specific and is defined as a function of development stage. Higher temperatures accelerate the turnover rates in plant tissue and hence the costs of maintenance. An increase in temperature of $10^{\circ} \mathrm{C}$ increases maintenance respiration by a factor of about 2 (Kase and Catsky, 1984; Penning de Vries and Van Laar, 1982). However, to be more flexible, the user may specify the increase factor of the respiration rate per $10^{\circ} \mathrm{C}$ temperature increase, $\mathrm{Q}_{10}(-)$ :

$$
R_{\mathrm{m}}=f_{\text {senes }} R_{\mathrm{mref}} Q_{10}^{\frac{T_{\text {avg }}-25}{10}}
$$

where $R_{\mathrm{m}}$ is the actual maintenance respiration rate $\left(\mathrm{kg} \mathrm{ha}^{-1} \mathrm{~d}^{-1}\right)$. 
It may be assumed that the vegetation will not be 'self-consuming' in terms of carbohydrates. Therefore the maintenance respiration rate cannot exceed the gross assimilation rate. The net assimilation rate $A_{\text {net }}\left(\mathrm{kg} \mathrm{ha}^{-1} \mathrm{~d}^{-1}\right)$ is the amount of carbohydrates available for conversion into structural material:

$$
A_{\text {net }}=A_{\text {gross }}-R_{\mathrm{m}} \quad \text { with } \quad A_{\text {net }} \geq 0
$$

\subsubsection{Dry matter partitioning and growth respiration}

The primary assimilates in excess of the maintenance costs, are available for conversion into structural plant material. In this conversion process $\mathrm{CO}_{2}$ and $\mathrm{H}_{2} \mathrm{O}$ are released. The magnitude of growth respiration is determined by the composition of the end product formed (Penning de Vries et al., 1974). Thus the weight efficiency of conversion of primary photosynthates into structural plant material varies with the composition of that material. Fats and lignin are produced at high costs; structural carbohydrates and organic acids are relatively cheap. Proteins and nucleic acids form an intermediate group.

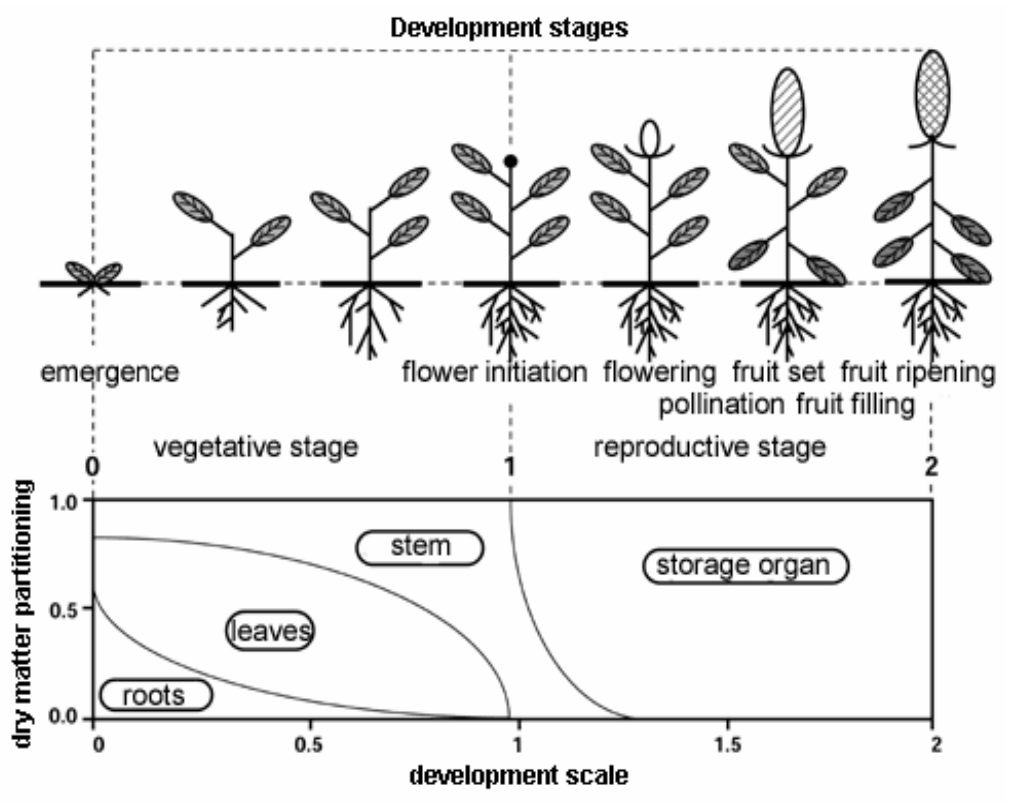

Figure 7.7 Typical partitioning of assimilated dry matter among leaves, stem, roots and storage organs as function of development stage.

At higher temperatures the conversion processes are accelerated, but the pathways are identical (Spitters et al. 1989). Hence, the assimilate requirements do not vary with temperature.

In the vegetative stage, the increase in total dry weight of the crop is partitioned over the plant organs: roots, leaves, stems and storage organs. Storage organs, however, may not only be formed from current photosyntheses but also from carbohydrates and proteins that have been stored temporarily in vegetative parts and that are redistributed during the reproductive stage. In WOFOST this redistribution process is not incorporated.

The partitioning factor depends on the crop development stage. Figure 7.7 gives a typical example. The partitioning factors are input to the model. WOFOST first divides the gross dry matter among roots and shoots (leafs, stems and storage organs together), using the partitioning factor for roots, $\xi_{\text {root }}(-)$. Next WOFOST divides the gross dry matter directed to the shoots among leafs, stems and storage organs, using the partitioning factors for these plant organs. At any development stage the sum $\xi_{\text {leaf }}+\xi_{\text {stem }}+\xi_{\text {stor }}$ must equal one. 
For lossess during conversion of general carbohydrates to specific plant tissues, average (crop specific) conversion factors $C_{e, i}\left(\mathrm{~kg} \mathrm{~kg}^{-1}\right)$ should be specified for leaf, storage organ, stem and root biomass. WOFOST calculates a weighted average, $C_{e}\left(\mathrm{~kg} \mathrm{~kg}^{-1}\right)$, of these organ specific conversion factors by multiplying the organ specific values with the partitioning factors:

$$
C_{\mathrm{e}}=\frac{1}{\left(\frac{\xi_{\text {leaf }}}{C_{\mathrm{e} \text { leaf }}}+\frac{\xi_{\text {stor }}}{C_{\mathrm{e}, \mathrm{stor}}}+\frac{\xi_{\text {stem }}}{C_{\mathrm{e}, \mathrm{stem}}}\right)\left(1-\xi_{\text {root }}\right)+\frac{\xi_{\text {root }}}{C_{\mathrm{e}, \text { root }}}}
$$

where $\xi_{i}$ is the partitioning factor for organ $i$.

The gross dry matter growth rate $w_{\text {gross }}\left(\mathrm{kg} \mathrm{ha}^{-1} \mathrm{~d}^{-1}\right)$ is related to the net assimilation rate $A_{\text {net }}$ by:

$$
w_{\text {gross }}=C_{\mathrm{e}} A_{\text {net }}
$$

Gross dry matter growth is first partitioned between shoots (leafs, stems and storage organs together) and roots:

$$
w_{\text {gross,root }}=\xi_{\text {root }} w_{\text {gross }} \quad \text { and } \quad w_{\text {gross,sh }}=\left(1-\xi_{\text {root }}\right) w_{\text {gross }}
$$

where $\xi_{\text {root }}$ is the partitioning factor for roots (-) and $w_{\text {gross, root }}$ and $w_{\text {gross,sh }}$ are the gross growing rates $\left(\mathrm{kg} \mathrm{ha}^{-1} \mathrm{~d}^{-1}\right)$ of the roots and the shoots, respectively. The gross growth rate of leaves, stems and storage organs is simply the product of the gross dry matter growth rate of the shoots and the fraction allocated to these organs.

\subsubsection{Carbon balance check}

The actual gross $\mathrm{CO}_{2}$ assimilation rate has to be identical to the amount of structural plant material produced plus the amounts used for maintenance respiration and conversion. The carbon balance has to be zero and is implemented as:

$$
0=\frac{R_{d}-R_{m, T}-R_{g} *\left(\xi_{\text {root }}+\left(\xi_{\text {leaf }}+\xi_{\text {stem }}+\xi_{\text {stor }}\right) *\left(1-\xi_{\text {root }}\right) *\left(D_{m i} / C_{e}\right)\right.}{R_{d}}
$$

where $R_{g}$ is the growth respiration rate $\left(\mathrm{kg} \mathrm{ha}^{-1} \mathrm{~d}^{-1}\right), R_{d}$ is the actual daily $\mathrm{CH}_{2} \mathrm{O}$ assimilation rate $\left(\mathrm{kg} \mathrm{ha}^{-1} \mathrm{~d}^{-1}\right) R_{m, T}$ is the maintenance respiration rate $\left(\mathrm{kg} \mathrm{ha}^{-1} \mathrm{~d}^{-1}\right)$ and $\xi_{i}$ are partitioning factors (-) of organ $i$ (i.e. leaves, stems and roots). $D_{m i}$ is the dry matter increase.

WOFOST assumes that maintenance respiration cannot exceed the actual gross assimilation rate. However, in case the daily $\mathrm{CH}_{2} \mathrm{O}$ assimilation rate comes close to zero, the maintenance respiration rate and the growth respiration rate also becomes small and a situation may occur that the numerator of the above mentioned equation becomes very small and negative. Introducing a division by $R_{d}$ in the carbon check will identify such an event.

\subsubsection{Senescence}

The death rate of storage organs is assumed to be zero. The death rate of stem and roots is crop specific and is defined as the daily amount of the living biomass that no longer participates in the plant processes. The death rate of stems and roots is considered to be a function of development stage as specified by the user.

The death rate of leaves is more complicated. Leaf senescence occurs due to water stress, shading (high LAI), and also due to exceedance of the life span.

The potential death rate of leaves due to water stress $\zeta_{\text {leaf,water }}\left(\mathrm{kg} \mathrm{ha}^{-1} \mathrm{~d}^{-1}\right)$ is calculated as:

$$
\zeta_{\text {leaf,water }}=W_{\text {leaf }}\left(1-\frac{T_{\mathrm{a}}}{T_{\mathrm{p}}}\right) \zeta_{\text {leaf, } \mathrm{p}}
$$


where $W_{\text {leaf }}$ is the leaf dry matter weight $\left(\mathrm{kg} \mathrm{ha}^{-1}\right), T_{\mathrm{a}}$ and $T_{\mathrm{p}}$ are the actual and potential transpiration rates $\left(\mathrm{cm} \mathrm{d}^{-1}\right)$, respectively, and $\zeta_{\text {leaf,p }}$ is the maximum relative death rate of leaves due to water stress $\left(\mathrm{kg} \mathrm{kg}^{-1} \mathrm{~d}^{-1}\right)$. The latter is crop specific and should be provided by the user.

A potential death rate due to self-shading, $\zeta_{\text {leaf,shade }}\left(\mathrm{kg} \mathrm{ha}^{-1} \mathrm{~d}^{-1}\right)$, is defined which increases linearly from zero at a critical leaf area index $\operatorname{LAI}_{\mathrm{c}}(-)$, to its maximum value at $2 \mathrm{LAI}_{\mathrm{c}}$ :

$$
\zeta_{\text {leaf,shade }}=0.03 W_{\text {leaf }}\left(\frac{L A I-L A I_{\mathrm{c}}}{L A I_{\mathrm{c}}}\right) \quad \text { with } \quad 0<\left(\frac{L A I-L A I_{\mathrm{c}}}{L A I_{\mathrm{c}}}\right)<1
$$

with $L A I_{\mathrm{c}}=3.2 / \kappa_{\mathrm{df}}$. Typical values for $\zeta_{\text {leaf,p }}$ and $L A I_{\mathrm{c}}$ are $0.03 \mathrm{~d}^{-1}$ and $4 \mathrm{~m}^{2} \mathrm{~m}^{-2}$, respectively (Spitters et al., 1989).

WOFOST uses the highest value of $\zeta_{\text {leaf,water }}$ and $\zeta_{\text {leaf,shade }}$ for the combined effect of water stress and mutual shading.

Leaves that have escaped from premature death due to water stress or mutual shading, inevitably die due to exceedance of the life span for leaves. Life span is defined as the maximum time a leaf can live at a constant temperature of $35^{\circ} \mathrm{C}$. A physiologic ageing factor, $f_{\text {age }}(-)$, is calculated each day:

$$
f_{\text {age }}=\frac{T-T_{\text {b,age }}}{35-T_{\mathrm{b}, \mathrm{age}}} \quad \text { with } \quad f_{\mathrm{age}} \geq 0
$$

with $T_{\mathrm{b} \text {,age }}$ the lower threshold temperature for physiologic ageing $\left({ }^{\circ} \mathrm{C}\right)$, which is crop specific and should be provided by the user.

The integral of the physiologic ageing factor over time yields the physiologic age, $P_{\text {age }}(\mathrm{d})$ :

$$
P_{\mathrm{age}}^{j+1}=P_{\mathrm{age}}^{j}+f_{\mathrm{age}} \Delta t
$$

To correct for leaf senescence, the specific leaf area of each day, $S_{\mathrm{la}}{ }^{\mathrm{j}}$ (ha kg${ }^{-1}$ ), the growth of the dry matter weight of leaves per day, $w_{\text {leaf, }}$ and the physiological age, $P_{\text {age, }}$ are stored in 3 corresponding arrays. The first element of the arrays represents the most recent day and the last element of the arrays represents the oldest day.

The leave weight that died during a day due to water stress or mutual shading is subtracted from the weight of the oldest leaf class. When senescence is larger than the amount available in the oldest leaf class, the remaining senescence is subtracted from the next oldest leaf class. Emptying of the leaf classes continues, until the amount of senescence is dissipated completely or the remaining amount of leaves becomes zero.

Leaves may maximally attain the age defined by the crop specific life span. WOFOST checks the leaf classes ages. The first class younger than the defined life span becomes the oldest class.

The stem death rate due to water stress is calculated in a similar way as the leaf death rate due to water stress using the same factor for the maximum relative death rate:

$$
\zeta_{\text {stem,water }}=W_{\text {stem }}\left(1-\frac{T_{\mathrm{a}}}{T_{\mathrm{p}}}\right) \zeta_{\text {stem }, \mathrm{p}}
$$

where $\zeta_{\text {stem, water }}$ is the stem death rate due to water stress $\left(\mathrm{kg} \mathrm{ha}^{-1} \mathrm{~d}^{-1}\right), \zeta_{\text {stem,p }}$ is the maximum relative leaf death rate due to water stress $\left(\mathrm{kg} \mathrm{ha}^{-1} \mathrm{~d}^{-1}\right), W_{\text {stem }}$ is the stem dry matter weight $\left(\mathrm{kg} \mathrm{ha}^{-1}\right)$ and $T_{a}$ and $T_{p}$ are the actual and potential transpiration respectively $\left(\mathrm{cm} \mathrm{d}^{-1}\right)$. 


\subsubsection{Net growth}

The initial amount of total dry crop weight should be provided by the user. This amount is multiplied by the partitioning factors, $\xi_{i}$, to yield the dry weight values at emergence.

The net growth rates of the plant organs, $w_{\text {net,i }}\left(\mathrm{kg} \mathrm{ha}^{-1} \mathrm{~d}^{-1)}\right.$ result from the gross growth rates (Section 7.8) and the senescence rates, $\zeta_{i}\left(\mathrm{~kg} \mathrm{~kg}^{-1} \mathrm{~d}^{-1}\right)$ :

$$
w_{\text {net }, i}=w_{\text {gross }, i}-\zeta_{i} W_{i}
$$

By integrating $w_{\text {net,i }}$ over time, the dry matter weight of organ $i, W_{\mathrm{i}}\left(\mathrm{kg} \mathrm{ha}^{-1}\right)$, is calculated.

An exception has to be made for the growth of leaves. In the initial stage, the rate of leaf appearance and final leaf size are constrained by temperature through its effect on cell division and extension, rather than by the supply of assimilates. For a relative wide range of temperatures the growth rate responds more or less linearly to temperature (Hunt et al., 1985; Causton and Venus, 1981; Van Dobben, 1962). The growth rate of the leaf area index, $w_{\text {LAI }}$ (ha ha ${ }^{-1} \mathrm{~d}^{-1}$ ), in this so-called exponential stage, is described by:

$$
w_{\mathrm{LAI}}=L A I w_{\mathrm{LAI}, \max } T_{\text {eff }}
$$

where $W_{\text {LAI, max }}$ is the maximum relative increase of leaf area index $\left({ }^{\circ} \mathrm{C}^{-1} \mathrm{~d}^{-1}\right)$.

WOFOST assumes that the exponential growth rate of leaf area index will continue until it equals the assimilation limited growth rate of the leaf area index. During this second, source limited growth stage, $w_{\text {LAI }}$ is described by:

$$
w_{\text {LAI }}=w_{\text {net,leaf }} S_{\text {la }}
$$

where $S_{\mathrm{la}}$ is the specific leaf area $\left(\mathrm{ha} \mathrm{kg}^{-1}\right)$.

The green parts of stems and storage organs, may absorb a substantial amount of radiation. Therefore the so-called green area index $G A I_{\mathrm{i}}\left(\mathrm{ha} \mathrm{ha}^{-1}\right.$ ) should be added to the leaf area index. The green area index of the stems and storage organs, are calculated from the dry matter weights of the organs:

$$
G A I_{i}=S_{\mathrm{ga}, i} W_{i}
$$

with $S_{\mathrm{ga}, \mathrm{i}}$ the specific green area (ha $\mathrm{kg}^{-1}$ ) of either stems or storage organ. $S_{\mathrm{ga}, \mathrm{i}}$ are crop specific and should be provided by the user.

\subsubsection{Root growth}

Root extension is computed in a straightforward way. The user needs to specify the initial rooting depth, the maximum rooting depth as determined by the crop and by the soil, and the maximum daily increase in rooting depth, $d_{\text {root, } \max }(\mathrm{cm})$. SWAP offers 2 methods to calculate actual rooting depth:

1. Daily increase in rooting depth is equal to the maximum daily increase, unless maximum rooting depth is reached or no assimilates are available for root growth:

$$
D_{\text {root }}^{j}=D_{\text {root }}^{j-1}+d_{\text {root, } \max } \quad \text { with } \quad D_{\text {root }}^{j} \leq D_{\text {root, } \max } \quad \text { and } \quad w_{\text {net, root }} \geq 0
$$

where $D_{\text {root }}{ }^{j}$ is the rooting depth $(\mathrm{cm})$ at day $j$.

2. Daily increase in rooting depth depends on relative dry matter increase:

$$
D_{\text {root }}^{j}=D_{\text {root }}^{j-1}+\frac{\mathrm{DMI}^{j}}{\mathrm{DMI}_{\text {pot }}^{j}} d_{\text {root, max }} \quad \text { with } \quad D_{\text {root }}^{j} \leq D_{\text {root, max }}
$$

where DMIj is the dry matter increase of the actual crop at day $j$ and DMIpotj is the dry matter increase of the potential crop. 


\subsection{Detailed module for dynamic growth of grass}

The detailed module for dynamic growth of grass is a modified version of WOFOST. Unlike common agricultural crops, grass is perennial and stays in the vegetative stage during most of it's growing season. Therefore the grass growth module deviates from the standard WOFOST. For instance the forcing functions are a function of Julian day number instead of crop development stage. Another example is grass rooting depth, which is directly related to simulated root dry matter.

Also cultivation of grass is quite different from common crops. Grass can be regularly mowed or consumed by cattle. In SWAP mowing is triggered by either a minimum amount of above ground dry matter or by a fixed date. The dry matter threshold can be defined as a constant or may vary during the season (Figure 7.8). Alternatively, the timing of a mowing event can be overruled by a maximum duration of grass growth. Note that mowing should occur within 6 weeks after the previous event in order to conserve the nutritional value of the grass. The total weight above ground biomass remaining on the field after mowing is specified by the user.

In a similar way cattle is allowed to enter the field after a specified minimum amount of above ground dry matter is exceeded. This threshold remains constant during the season. Cattle grazing can also be initiated at specific dates. In latter case still the minimum threshold of above ground dry matter should be reached before grazing starts. Grazing differs from mowing in this respect that the consumed biomass depends on the grazing days and the lifestock density. In a table the user should specify the relation between lifestock density, biomass consumption as well as dry matter losses due to manure droppings and treading.

After harvest, the leave and stem weights are reset according to the partioning factors. The leaf area index is reset based on the leave weight. Experiments show that mowing does impede growth for a number of days. This regrowth delay is a function of the dry matter harvest and is part of the input. An example is given in Figure 7.9.

Note that the grass module is experimental. The supplied input values are calibrated for Dutch conditions and may not be appropiate for other climatic zones.

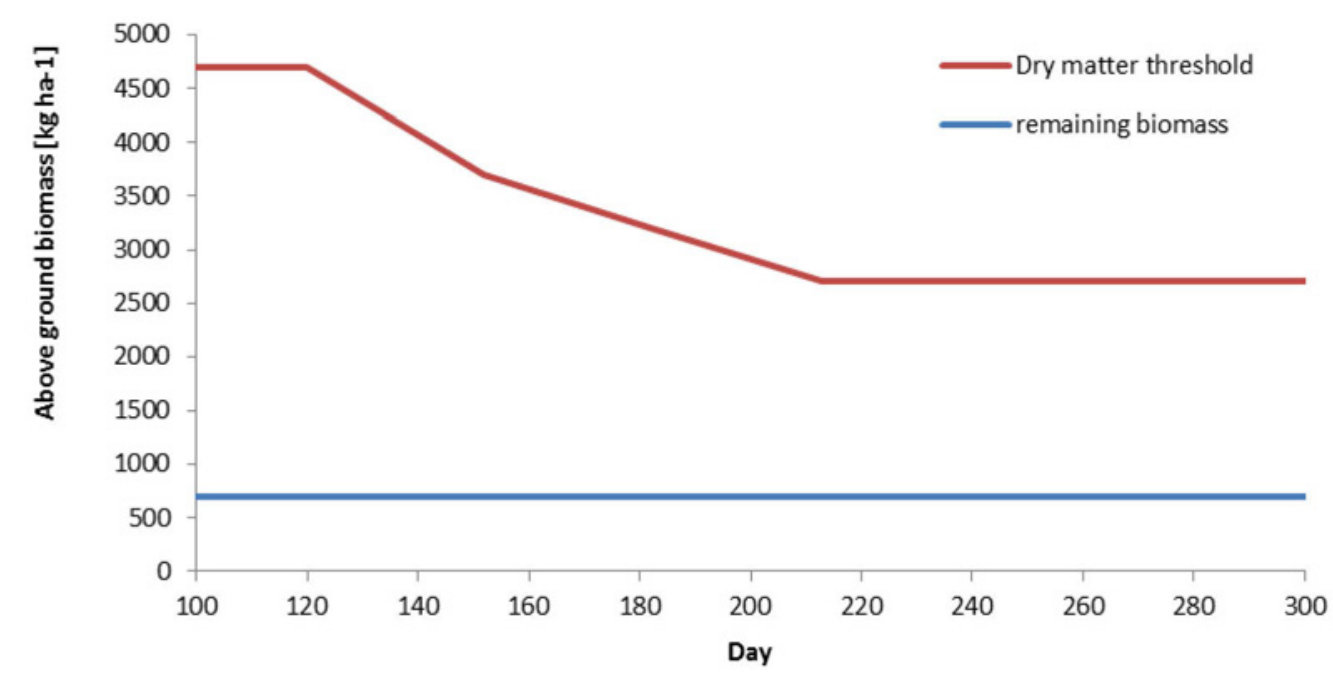

Figure 7.8 Example of mowing regime. 


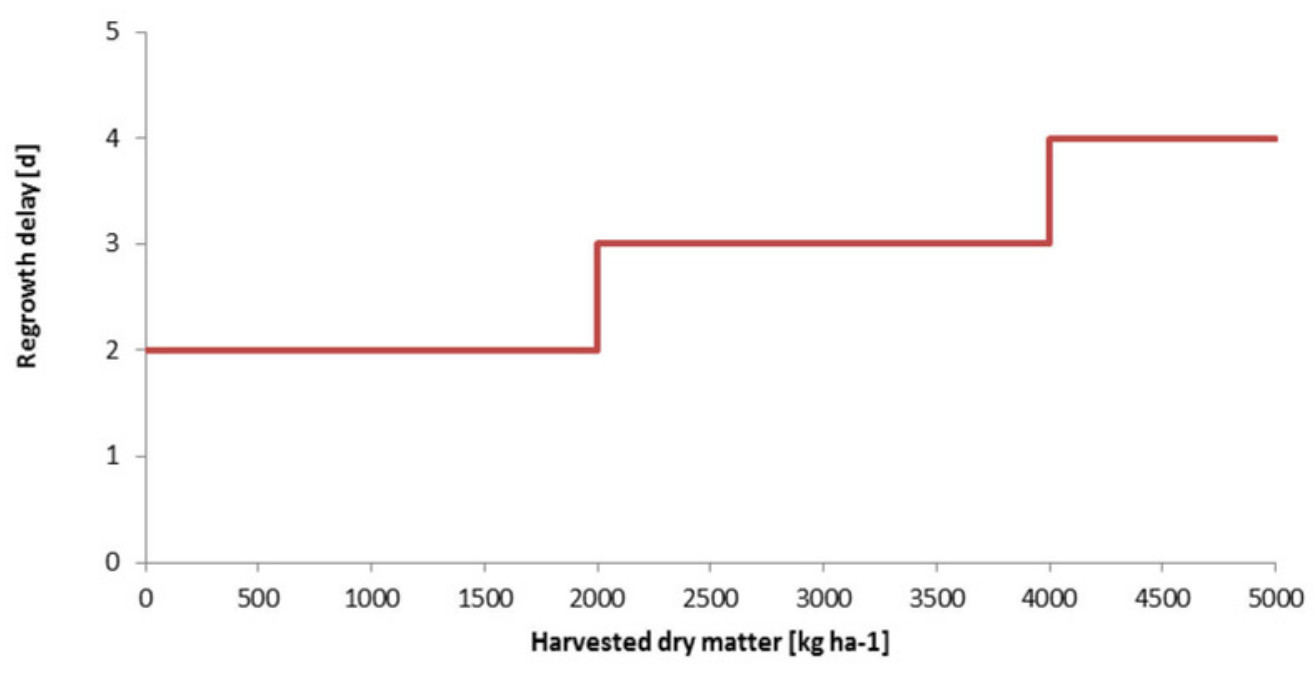

Figure 7.9 Example of regrowth delay after mowing.

\subsection{Initialisation of crop growth (Germination)}

\subsubsection{Arable crops}

For germination the method of Van Wijk et al. (1988) was implemented similar to Van Walsum and Van der Bolt (2013). Germination depends on temperature:
a. $T_{a v}<$ Tmin $_{\text {germ }}$
: Tsum $_{\text {germ }}=$ Tsum $_{\text {germ }}$
b. Tmin $_{\text {germ }}>T_{a v}<$ Tmax $_{\text {germ }}$
$:$ Tsum $_{\text {germ }}=$ Tsum $_{\text {germ }}+\frac{\text { Tsum }_{\text {eme,opt }}}{\text { Tsum }_{\text {eme }, \text { sub }}} *\left(T_{\text {av }}-\right.$ Tmin $\left._{\text {germ }}\right)$
C. $T_{a v}>$ Tmax $_{\text {germ }}$
$:$ Tsum $_{\text {germ }}=$ Tsum $_{\text {germ }}+\frac{\text { Tsum }_{\text {eme,opt }}}{\text { Tsum }_{\text {eme }, \text { sub }}} *\left(\right.$ Tmax $_{\text {germ }}-$ Tmin $\left._{\text {germ }}\right)$

where $T_{a v}$ is the mean daily air temperature $\left({ }^{\circ} \mathrm{C}\right)\left(=0.5^{*}(\mathrm{Tmin}+\mathrm{Tmax})\right), \mathrm{Tmin}_{\text {germ }}$ is the minimum temperature for Tsum calculation $\left({ }^{\circ} \mathrm{C} * \mathrm{~d}\right), \operatorname{Tmax}_{\text {germ }}$ is the maximum temperature for Tsum calculation $\left({ }^{\circ} \mathrm{C} * \mathrm{~d}\right)$, Tsum $_{\text {germ }}$ is the Tsum during the germination period $\left({ }^{\circ} \mathrm{C}^{*} \mathrm{~d}\right)$, Tsum eme, opt is the Tsum for crop emergence under optimal soil moisture and temperature conditions $\left({ }^{\circ} \mathrm{C} * \mathrm{~d}\right)$, and $T$ sum eme,sub is the Tsum for crop emergence under sub-optimal soil moisture and temperature conditions $\left({ }^{\circ} \mathrm{C}^{*} \mathrm{~d}\right)$.

Tsum for crop emergence under sub-optimal soil moisture and temperature conditions is calculated for:

a. too dry conditions as:

TSUM $_{\text {eme }, \text { sub }}=a * \log 10\left(-h_{r z}\right)+c$

b. too wet conditions as:

TSUM $_{\text {eme }, \text { sub }}=-a * \log 10\left(-h_{r z}\right)+b$

C. optimal conditions as:

TSUM $_{\text {eme,sub }}=$ TSUM $_{\text {eme, opt }}$

where $a, b$, and $c$ are input parameters, and $h_{r z}$ is the average pressure head of the upper $10 \mathrm{~cm}$ of the soil.

Emergence occurs when: Tsum $_{\text {germ }}>$ Tsumeme,opt . 


\subsubsection{Grasland}

The initialisation of grass growth has 2 options with different thresholds to start growth:

a. sum air temperatures or

b. Soil temperature at particular depth

ad a) Select sum air temperatures $>200^{\circ} \mathrm{C}$. This requires a switch which will add positive daily air temperatures until sum $>200^{\circ} \mathrm{C}$.

ad b) Select soil temperature at particular depth. This requires a temperature threshold at a given depth and a time interval (days) during which the threshold should be exceeded.

\section{6 $\quad \mathrm{CO}_{2}$ changes}

The effect of increasing atmospheric $\mathrm{CO}_{2}$ concentrations can be simulated using input of $\mathrm{CO}_{2}$ concentrations and tables defining the relation between $\mathrm{CO}_{2}$ concentration and maximum assimilation rate $A_{\max }$, light use efficiency $\varepsilon_{\mathrm{PAR}}$ and potential transpiration rate $T_{\mathrm{p}}$, respectively. These relations vary per crop and also allow distinction between $\mathrm{C}_{3}$ and $\mathrm{C}_{4}$ crops.

This option is activated when 2 conditions are met: $\mathrm{i})$ switch FLCO2 in the crop input file is present and set to true: FLCO2 = .TRUE. and ii) a file named "Atmosferic.co2" is present in the same directory/folder as the crop input file. An example and more detailed explanation are given by Groenendijk et al. (2016).

\subsection{Nitrogen limited crop growth of arable crops}

The core of the Soil-N module is a description of the nitrogen cycle, which is coupled to the organic matter cycle based upon the RothC-26.3 model.

This option also simulates nitrogen uptake and distribution in arable crops. The nitrogen routines, implemented in SWAP-WOFOST are based on Shibu et al. (2010). The carbon and nitrogen extensions for arable crops are described in more detail in a separate report (Groenendijk et al., 2016).

This option is activated with a switch (FLCROPNUT) in the .swp-file. An example and more detailed explanantion are given by Groenendijk et al. (2016).

\subsection{User instructions}

\subsubsection{Crop rotation scheme}

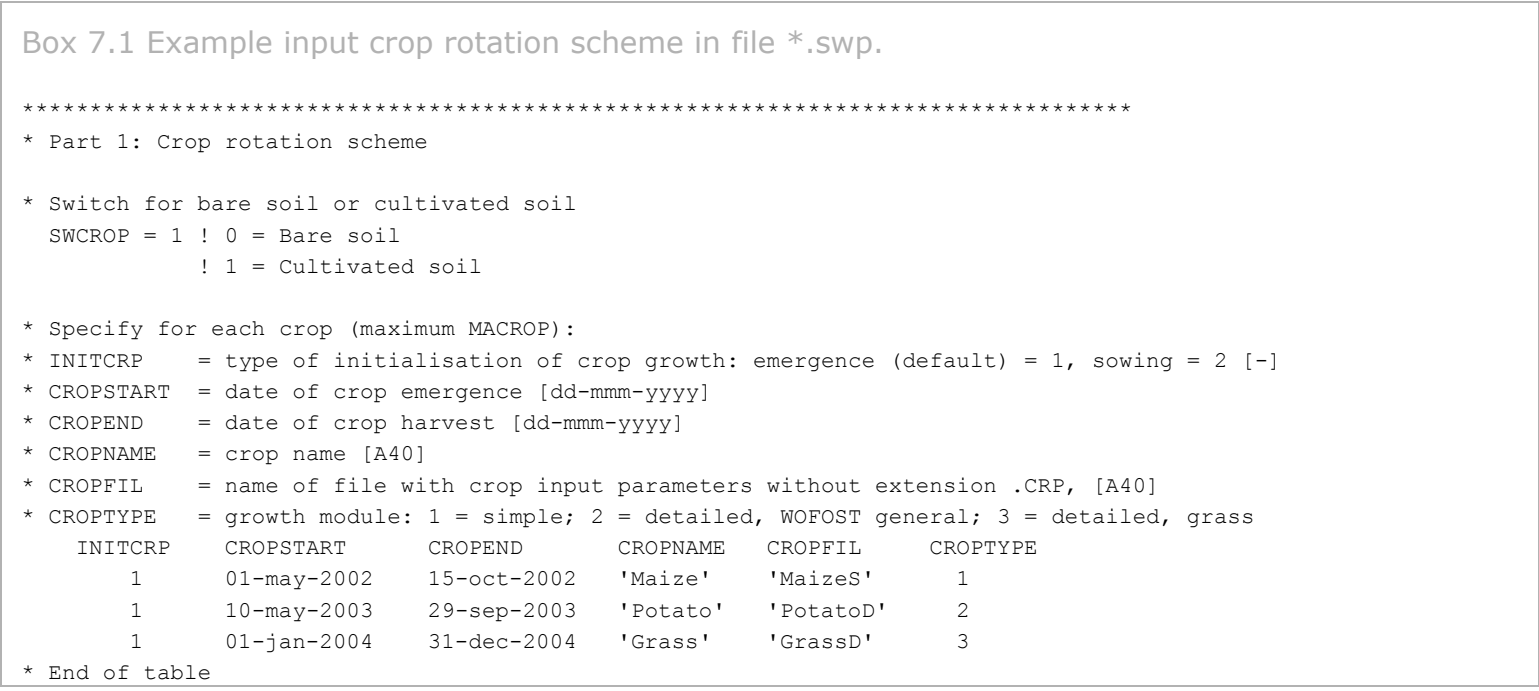


Box 7.1 shows an example of the input for the crop rotation scheme. Switch SWCROP determines whether a bare soil is simulated or a cultivated soil. For each crop the user should specify the type of initialisation, the cropping period, the crop name, the file name with crop input data and the type of growth module that should be used.

The output of crop results are written to an output file with the file name of the project (defined in *.swp-file as PROJECT=) and an extension ".crp". Since crop rotation schemes with different combinations of CROPTYPE are allowed (see Box 7.1) we standardized the header of the output file and give an explanation in Appendix 18.

\subsubsection{Simple crop module}

An example of the input file is given in Box 7.2. Most data are specified as function of crop development stage, which ranges from 0 to 2 . In part 1 , the development stage can be defined either linear in time (specify only duration of crop growth) or based on the temperature sums in the vegetative and reproductive stage.

In part 2, light extinction coefficients are used to quantify the decrease of solar radiation within a canopy (Chapter 3 ). Default values of $\kappa_{\text {dir }}=0.8$ and $\kappa_{\text {dif }}=0.72$ will suffice in most cases.

In part 3, either leaf area or soil cover during crop development should be specified, in order to distribute evapotranspiration fluxes over evaporation and transpiration, as discussed in Chapter 3.

In part 4 a choice should be made between input of crop factors or crop heights. Crop factors should be used when $E T_{\text {ref }}$ values are used as input, or when the Penman-Monteith method is used to calculate $E T_{\text {ref. }}$ Crop heights should be specified if the potential evapotranspiration fluxes are derived directly for the actual crop (see Table 3.2). In that case also the reflection coefficient and stomatal resistance of the crop should be defined.

Rooting depth during crop development (part 5) in combination with a dimensionless root length density distribution (part 10) will be used by SWAP to determine the distribution of rootwater extraction rates.

In part 6, yield response factors as function of development stage should be specified. In case of a linear relation between $Y_{\mathrm{a}} / Y_{\mathrm{p}}$ and $T_{\mathrm{a}} / T_{\mathrm{p}}$ during the whole growing period, or when no information is available of the yield response factors as function of development stage $D_{\mathrm{s}}$ for the particular crop, specify $K_{\mathrm{y}, \mathrm{k}}=1$ for $0<D_{\mathrm{s}}<2$ and specify one growing stage $k$. Please, note that increasing the number of growing stages reduces the relative yield as calculated by Eq. (7.1).

Part 7 describes the reduction function of root water uptake for oxygen and drought stress. For oxygen stress options are available for the simple reduction function according to Feddes et al. (1978), or the more process based reduction of Bartholomeus et al. (2008). For drought stress the user should choose between the simple reduction function of Feddes et al. (1978), or the more process based reduction function of De Jong van Lier et al. (2008). Appendix 8 contains critical pressure head values of drought stress for Feddes et al. (1978) based on Taylor and Ashcroft (1972). The user may extend the original Feddes reduction function with compensation according to Jarvis (1989) by specifying a value for ALPHACRIT. This parameter denotes the value of relative transpiration until which reduction of root water uptake is compensated.

Part 8 specifies the parameters which describe the reduction of root water uptake as function of salinity concentrations. Options are available for the Maas and Hoffman reduction function, and reductions based on the osmotic head. Parameters for the Maas and Hoffman reduction function are available for many crops (Maas, 1990). Appendix 9 gives input parameters SALTMAX and SALTSLOPE for a number of crops.

Interception input data are specified in Part 9. For agricultural crops, just one interception coefficient $(C O F A B)$ for the Von Hoyningen-Hune and Braden concept is required. The default value of COFAB = $0.25 \mathrm{~cm}$ will suffice for most agricultural crops. In case of forests (Gash concept) SWAP requires 
coefficients for free throughfall and stem flow, canopy storage capacity and average rainfall and evaporation fluxes as function of crop development. The specified average rainfall and evaporation fluxes values are independent of other input weather data.

Part 10 requires input of root density $\left(\mathrm{cm} / \mathrm{cm}^{3}\right)$ as function of relative root depth. In case of drought stress according to Feddes et al. (1978), relative root density (-) is sufficient.

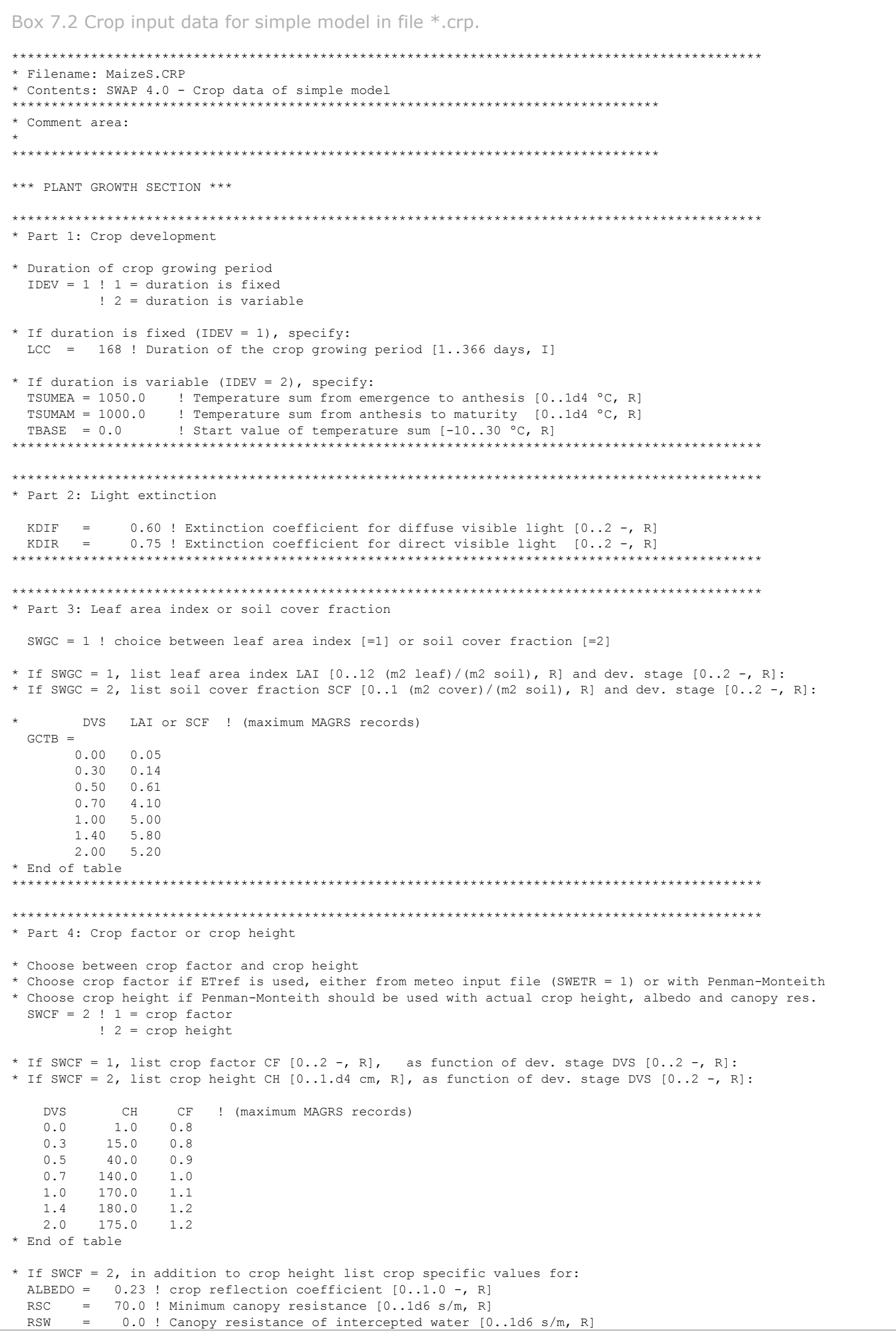




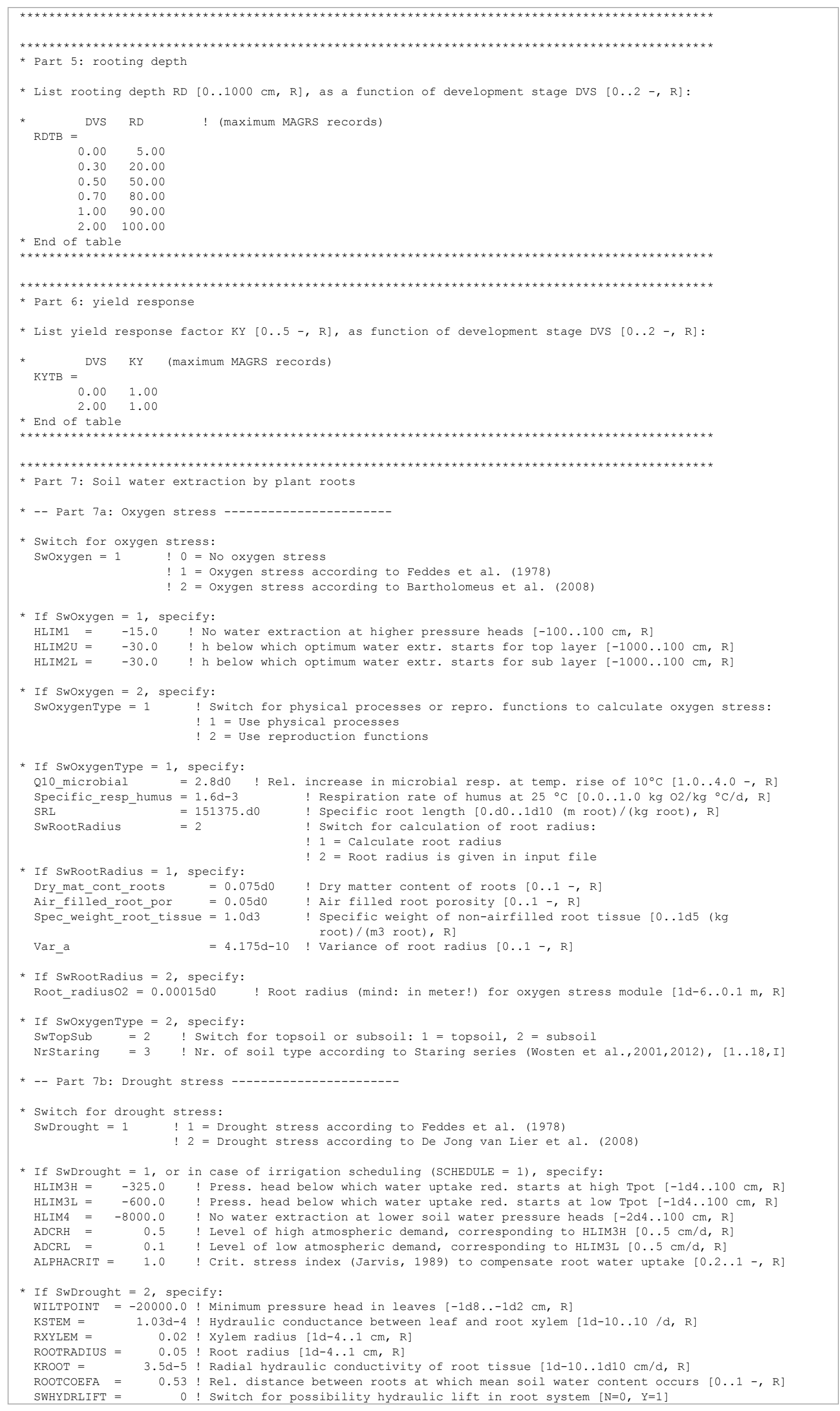




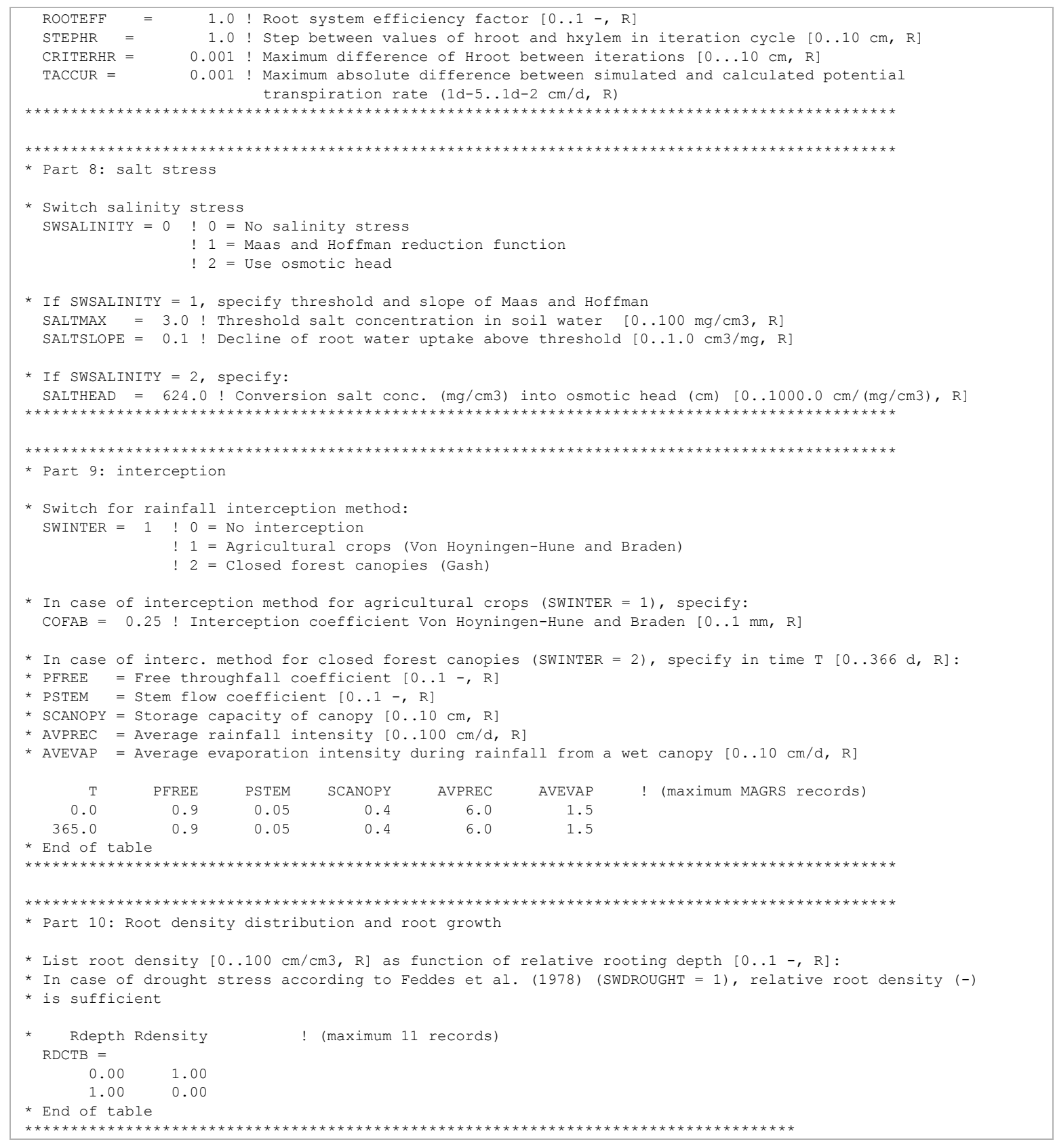




\subsubsection{Detailed crop module}

Input of the detailed crop module has been divided in 13 parts:

1. Crop factor of crop height

2. Crop development

3. Initial values

4. Green surface area

5. Assimilation

6. Conversion of assimilates into biomass

7. Maintenance respiration

8. Partitioning

9. Death rates

10. Crop water use

11. Salt stress

12. Interception

13. Root growth and root density profile

An example of the input file is given in Box 7.3. In general the theorie description in Section 7.3 in combination with the descriptions in the input file will be sufficient to guide the model user. However a few additional remarks should be made here.

In part 1 a choice should be made between input of crop factors or crop heights. Crop factors should be used when $E T_{\text {ref }}$ values are used as input, or when the Penman-Monteith method is used to calculate $E T_{\text {ref. }}$ Crop heights should be specified if the potential evapotranspiration fluxes are derived directly for the actual crop (see Table 3.2). In that case also the reflection coefficient and stomatal resistance of the crop should be defined.

In part 8 the user should specify the partitioning factors as function of crop development stage. As explained in Section 7.3.7, WOFOST first divides the gross dry matter among roots and shoots (leafs, stems and storage organs together), using the partitioning factor for roots. Next WOFOST divides the gross dry matter directed to the shoots among leafs, stems and storage organs, using the partitioning factors for these plant organs. At any development stage the sum $\xi_{\text {leaf }}+\xi_{\text {stem }}+\xi_{\text {stor }}$ must equal one.

The theoretical background of Parts 10-12 (Crop water use, Salt stress and Interception) applies to both the simple and detailed crop model and has been explained earlier.

Boons-Prins et al. (1993) documented specific parameters for the crops winter wheat, grain maize, spring barley, rice, sugar beet, potato, field bean, soy bean, winter oilseed rape and sunflower. WOFOST input files for some crops will be provided with the SWAP program.

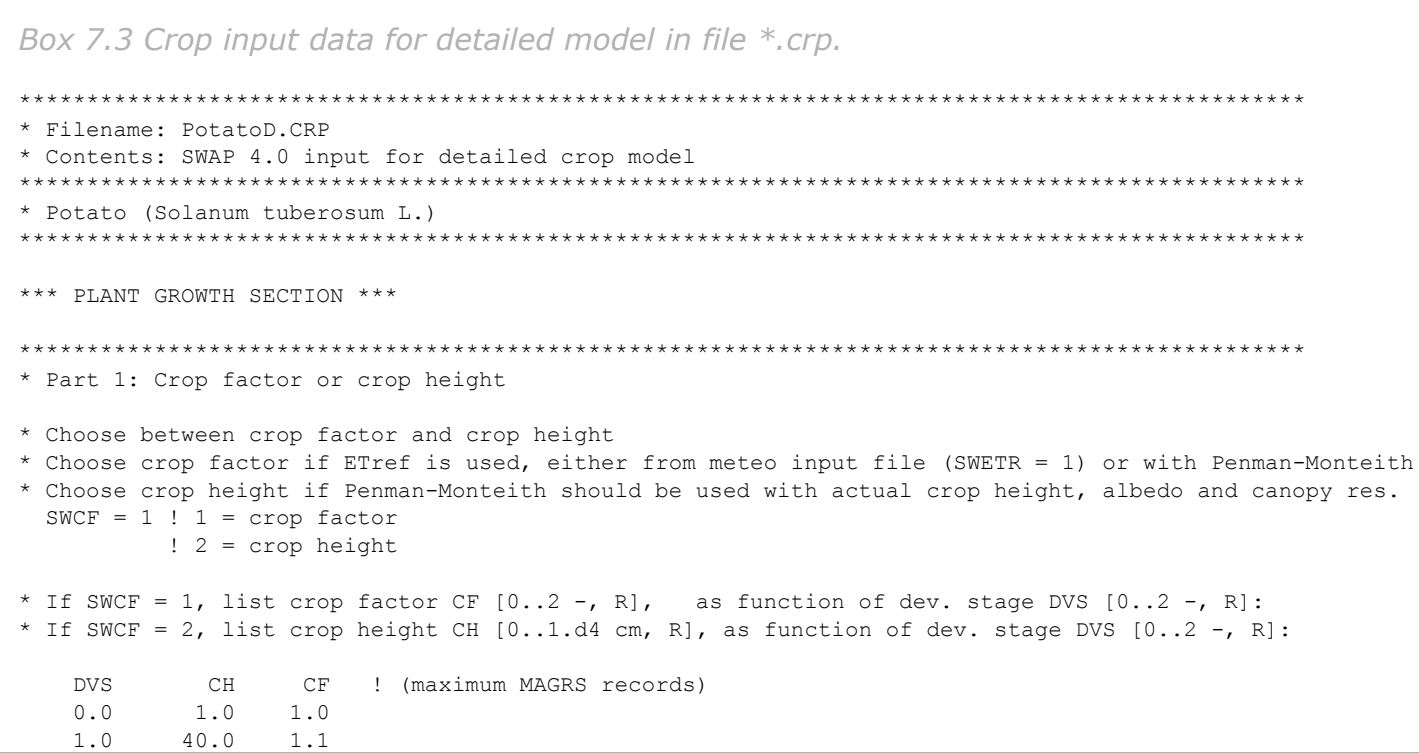




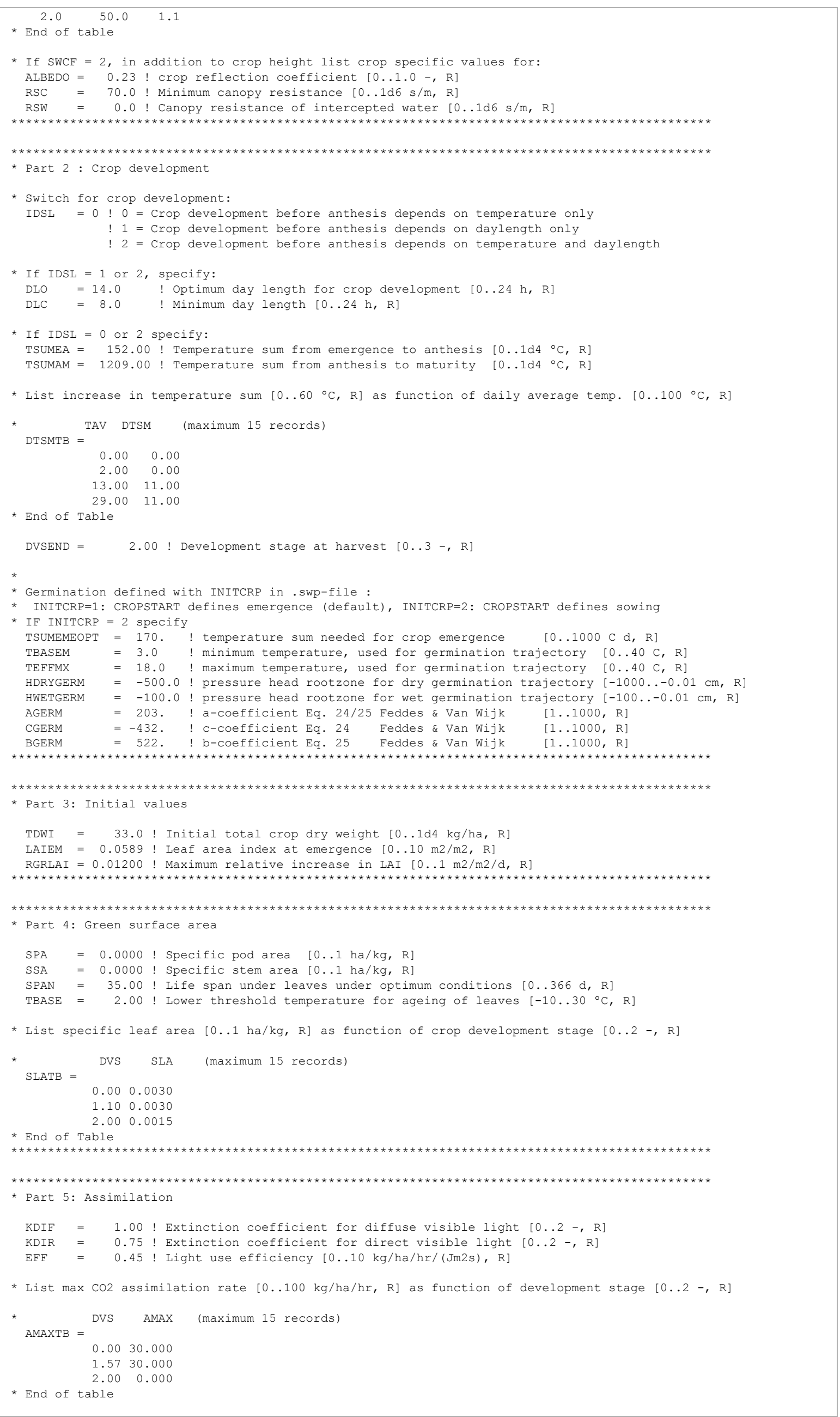




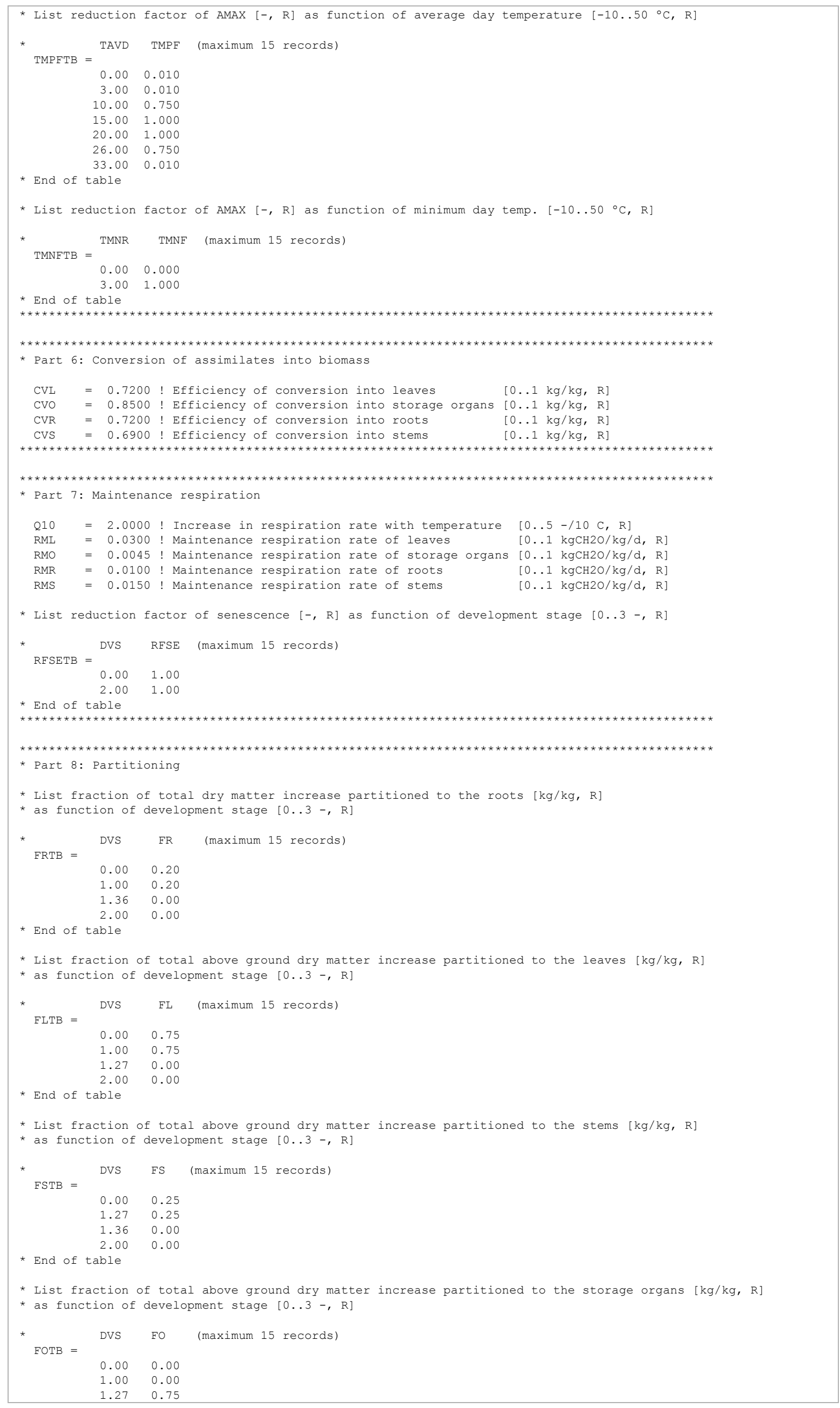




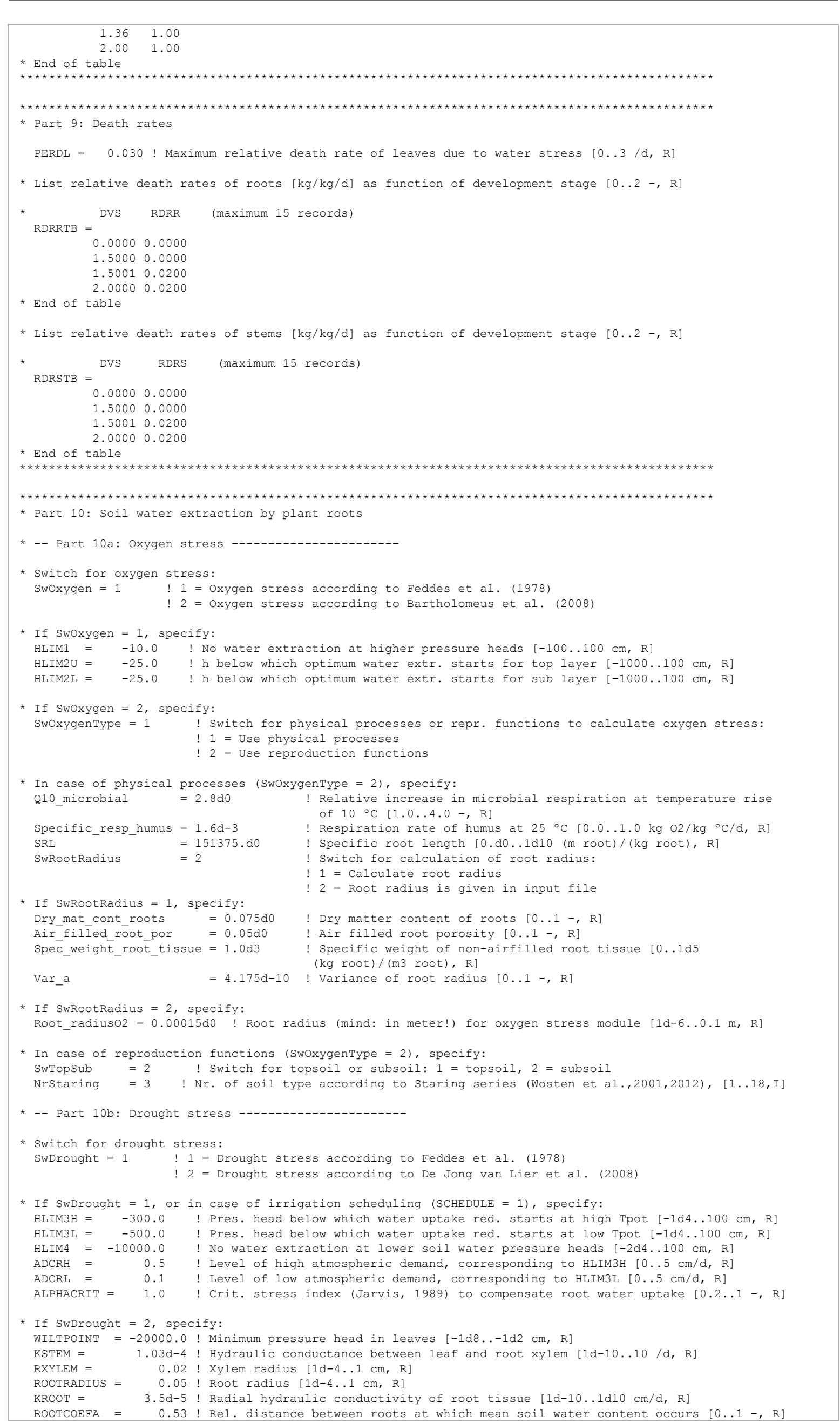




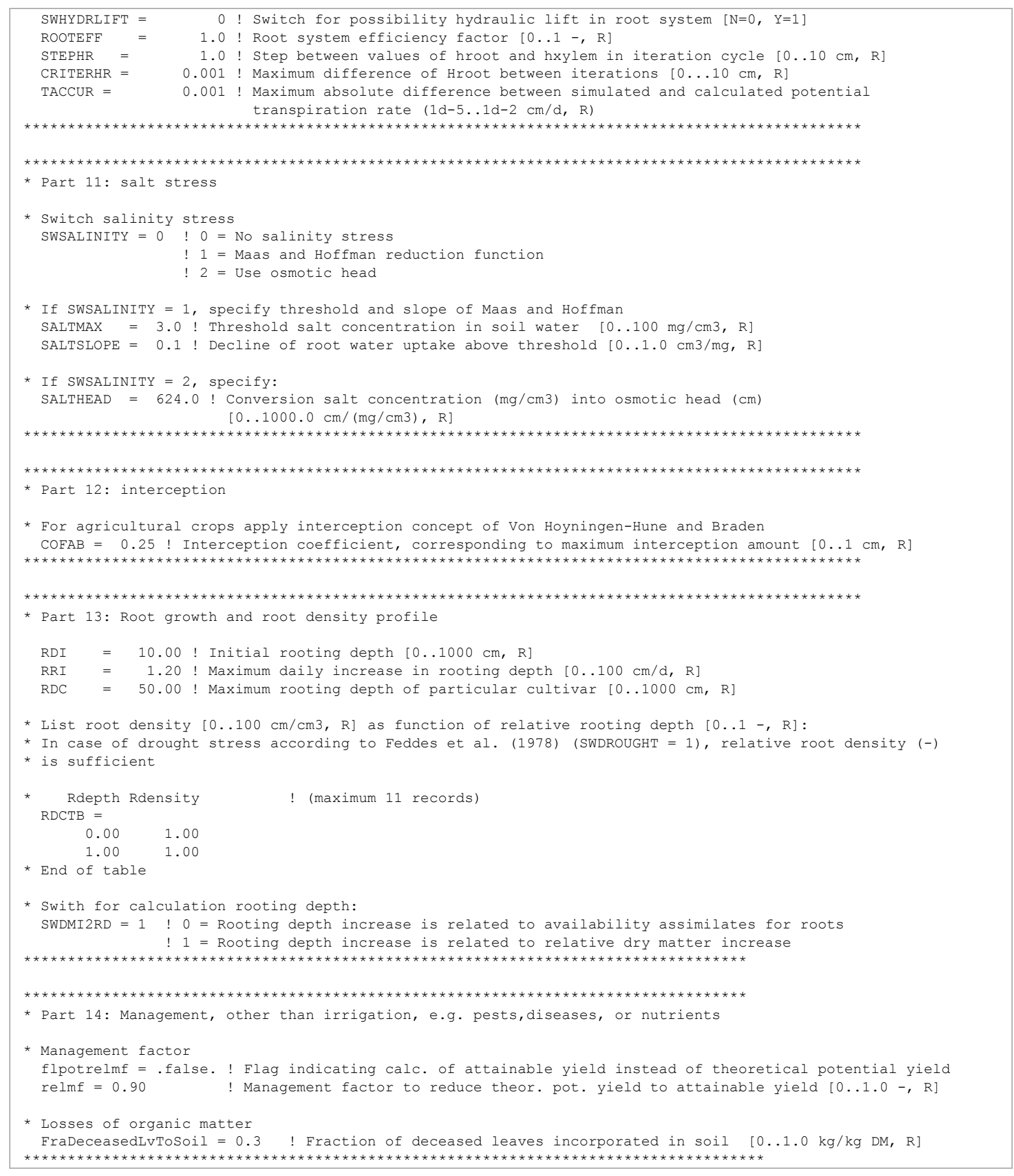




\subsubsection{Detailed grass module}

Differences of the detailed grass module input with repect to common agricultural crops are described in Par. 7.4.

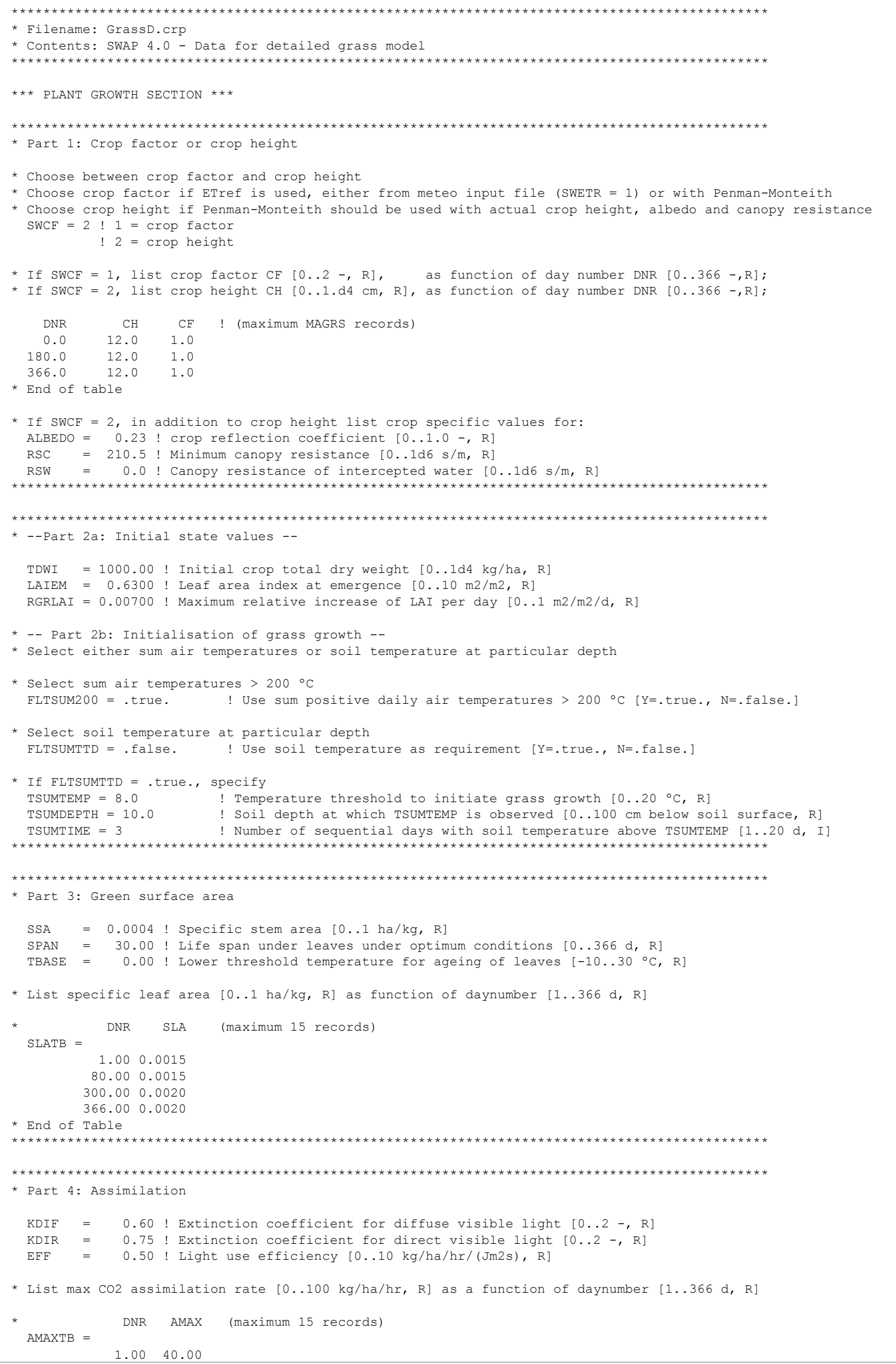




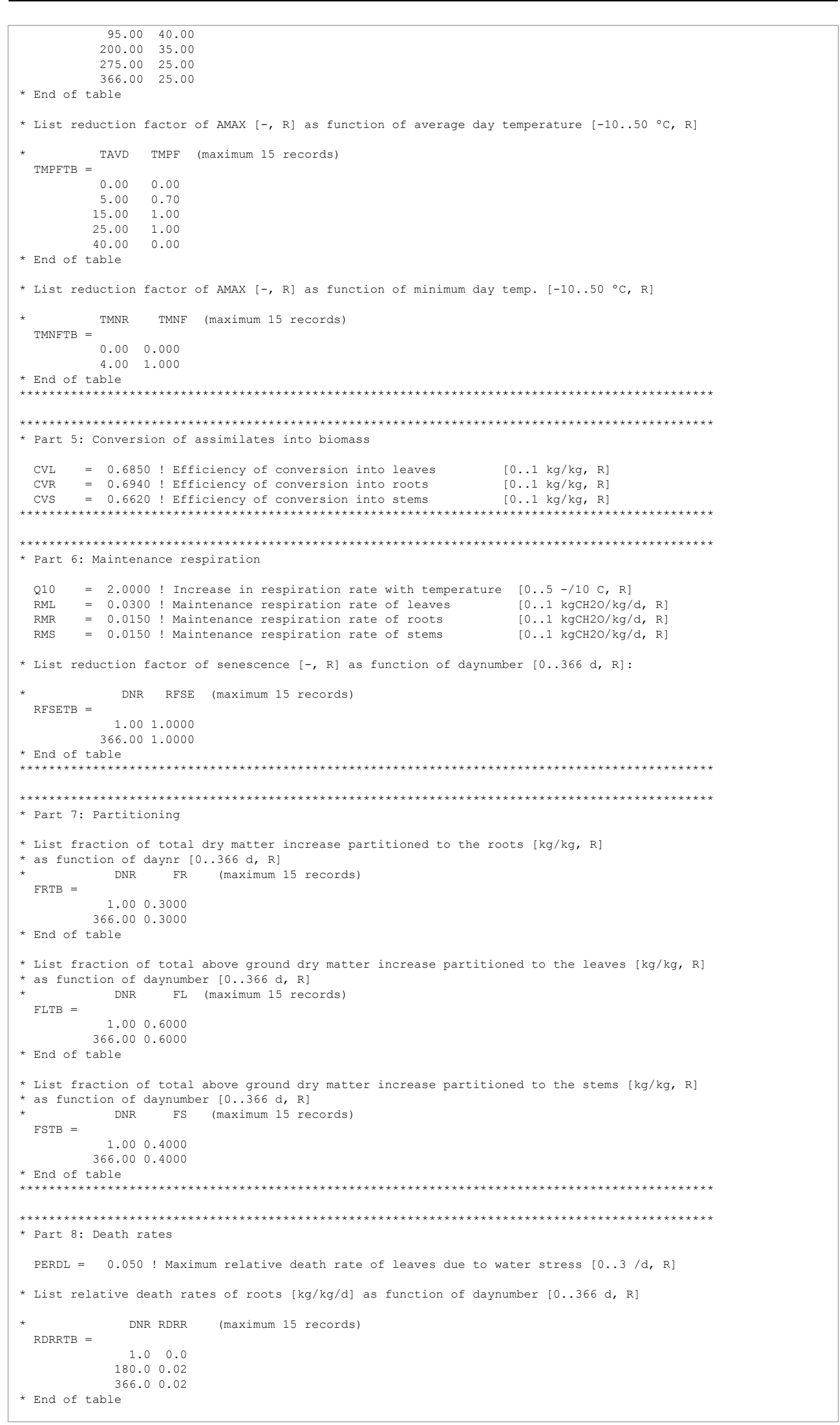




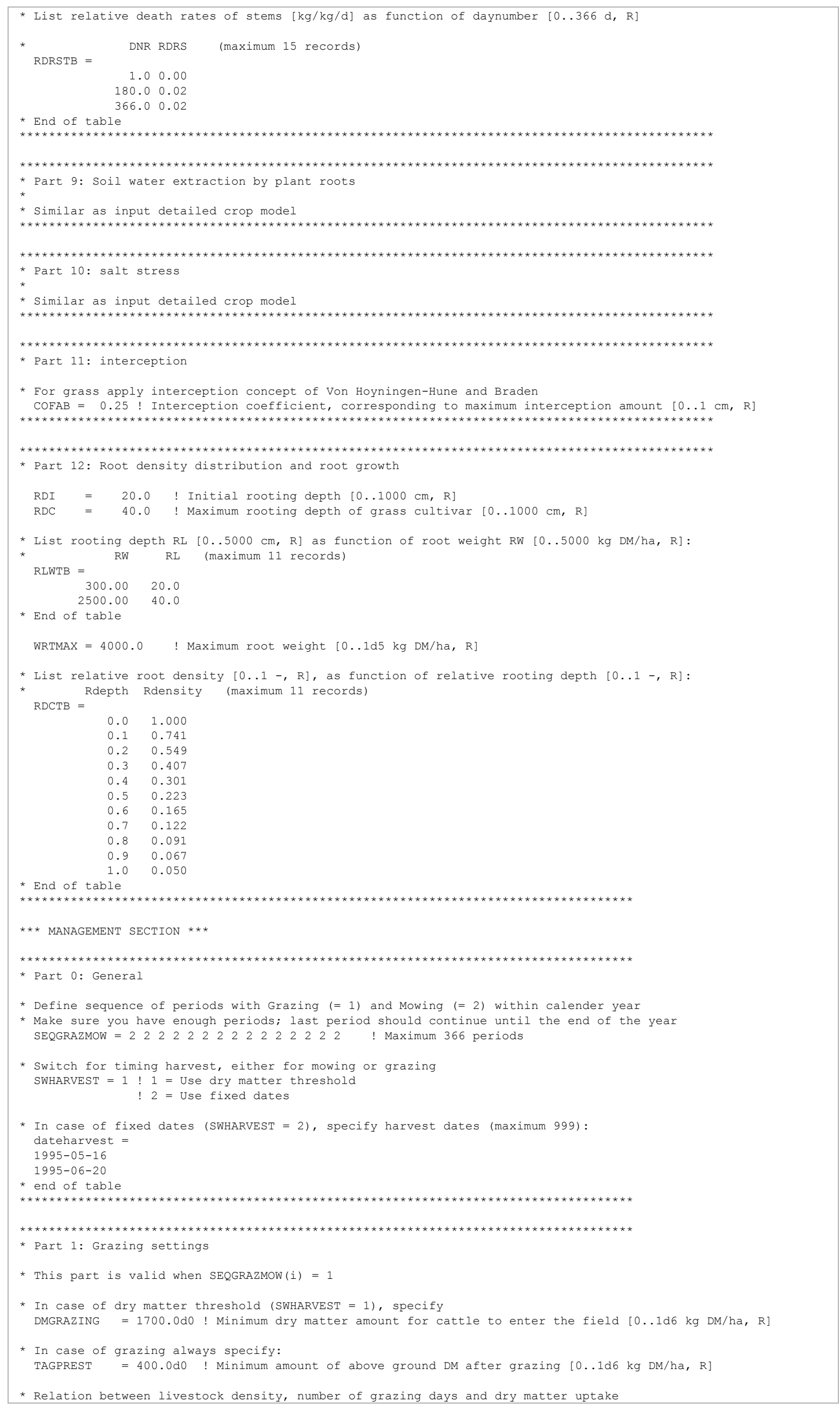


* LSDB = Basic Live Stock Density $[0.0 \ldots 1000.0 \mathrm{LS} / \mathrm{ha}, \mathrm{R}]$

* DAYSGRAZING $=$ Maximum days of grazing $[0.0 \ldots 366.0 \mathrm{~d}, \mathrm{R}]$

* UPTGRAZING = Dry matter uptake by grazing $[0.0 .1000 .0 \mathrm{~kg} / \mathrm{ha}, \mathrm{R}](\mathrm{kg} / \mathrm{ha} \mathrm{DM})$

* LOSSGRAZING = Dry matter loss during grazing due to droppings and treading $[0.0 .1000 .0 \mathrm{~kg} / \mathrm{ha}, \mathrm{R}]$ ( $\mathrm{kg} / \mathrm{ha} \mathrm{DM})$

$\begin{array}{cclc}\text { LSDb } & \text { DAYSGRAZING } & \text { UPTGRAZING } & \text { LOSSGRAZING } \\ 20.0 & 5.5 & 16.0 & 1.00 \\ 22.2 & 5.0 & 15.8 & 0.96 \\ 25.0 & 4.0 & 15.7 & 0.92 \\ 28.6 & 3.5 & 15.4 & 0.88 \\ 33.3 & 3.0 & 14.9 & 0.84 \\ 50.0 & 2.0 & 13.4 & 0.80\end{array}$

* end of table

* Actual livestock density of each grazing period

* SEQNR $=$ number of the sequence period with mowing/grazing $[0 . .366 \mathrm{~d}, \mathrm{I}]$

* LSDA = actual Live Stock Density of the grazing period [0.0.1000.0 LS/ha, R]

* Note: total number of periods should be equal to number of periods in SEQGRAZMOW

$\begin{array}{cc}\text { SEQNR } & \text { LSDA } \\ 1 & 20.0 \\ 2 & 20.0 \\ 3 & 30.0 \\ 4 & 20.0 \\ 5 & 20.0 \\ 6 & 20.0 \\ 7 & 20.0 \\ 8 & 20.0 \\ 9 & 20.0 \\ 10 & 20.0\end{array}$

* end of

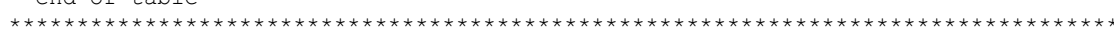

* Part 2: Mowing settings

* this part is valid when SEQGRAzMOW $(i)=2$

* In case of dry matter threshold (SWHARVEST = 1), specify

DMHARVEST $=4200.0 \mathrm{~d} 0$ ! Threshold of above ground dry matter to trigger mowing [0..1d6 kg DM/ha, R]

DAYLASTHARVEST $=289$ ! Last calendar day on which mowing may occur $[1 \ldots 366-$, I $]$

DMLASTHARVEST $=2700.0 \mathrm{do}$ ! Minimum above ground dry matter for mowing on last date [0..1d6 kg DM/ha, R]

* Optional: use of mowing table with variable threshold (in case DMMOWTB exists, DMHARVEST will be overruled)

* List threshold of above ground dry matter [0..1d6 kg DM/ha, R] to trigger mowing with daynr. [1..366 d, R]:

* DNR DMMOW (maximum 20 records)

DMMOWTB $=$

$120.0 \quad 4700.0$

$152.0 \quad 3700.0$

$182.0 \quad 3200.0$

213.02700 .0

366.02700 .0

* end of table

MAXDAYMOW $=42$ ! Maximum growing period after harvest $[1 \ldots 366-$, I]

MOWREST $=700 . \mathrm{d} 0 \quad$ ! Remaining yield above ground after mowing event $[0 \ldots 1 \mathrm{~d} 6 \mathrm{~kg} \mathrm{DM} / \mathrm{ha}, \mathrm{R}]$

* Relation between dry matter harvest [0..1d6 kg/ha, R] and delay in regrowth [0..366 d, I] after mowing DMMOWDELAY DAYDELAY

$\begin{array}{rr}0.0 & 2 \\ 2000.0 & 3\end{array}$

4000.0

* end of table

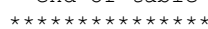

* Part 3: Management, other than mowing, grazing, irrigation, for instance pests, diseases or nutrients

FLPOTRELMF $=$.true. ! Flag indicating calculation of attainable yield instead of theoretical potential yield [Y=.true., $\mathrm{N}=$.false. $]$

RELMF $=0.90 \quad$ ! Relative management factor to reduce theoretical potential yield to attainable yield $[0 \ldots 1-, R]$ 


\section{$8 \quad$ Solute transport}

\subsection{Introduction}

Water is a great dissolver and many solutes enter the natural soil system at the Earth surface. The solute residence time in the unsaturated zone is important for soil- and groundwater pollution management. For instance organic compounds are mainly decomposed in the unsaturated zone, where the biological activity is concentrated. Most plants are able to extract water and nutrients from the soil only in the unsaturated zone. In irrigated areas, the long term salinity in the root zone will depend on the amount of percolation from the unsaturated zone. Whereas in the unsaturated zone the transport of solutes is predominantly vertical, once being in the groundwater solutes may diverge in any direction, threatening surface waters, nature reserves and drinking wells. Using an analytical model, Beltman et al. (1995) show the importance of the transport processes in the unsaturated zone as compared to the transport processes in the saturated zone. It is clear that a thorough understanding is needed of the processes that govern the transport, adsorption, root uptake and decomposition of the solutes in the unsaturated zone, in order to analyse and manage soil and water related environmental problems.

SWAP is designed to simulate basic transport processes at field scale level. Although for management purposes most farmers try to have more or less the same soil and drainage condition per field, still the existing soil spatial heterogeneity within a field may cause a large variation of solute fluxes (Biggar and Nielsen, 1976; Van de Pol et al., 1977; Van der Zee and Van Riemsdijk, 1987). Most of this variation is caused by spatial variation of the soil hydraulic functions, preferential flow due to macropores in structured soils or unstable wetting fronts in unstructured soils. In many cases it will not be possible to determine the variation (including the correlations) of all the physical parameters (Hopmans and Stricker, 1989). SWAP confines to the physical processes in order to be flexible in parameter input and allow the simulation of all kind of design and management scenarios. The spatial variability can be taken into account by inverse modelling or Monte Carlo simulation. Inverse modelling has been applied by Groen (1997). He measured for a period of time the solute concentrations in the soil profile and drainage water and determined 'field effective' transport parameters by inverse modelling. In case of Monte Carlo simulations the model is run a large number of times, while the input parameters and boundary conditions are varied according to the variation at comparable fields (Boesten and Van der Linden, 1991).

SWAP focuses on the transport of salts, pesticides and other solutes that can be described with relatively simple physical relations: convection, diffusion, dispersion, root uptake, Freundlich adsorption and first order decomposition. Transport related processes that are not considered in SWAP are:

- volatilization and gas transport

- transport of non-mixing or immiscible fluids (e.g. oil and water)

- chemical equilibria of various solutes (e.g. between $\mathrm{Na}^{+}, \mathrm{Ca}^{2+}$ and $\mathrm{Mg}^{2+}$ )

- chemical and biological chain reactions (e.g. mineralization, nitrification)

In case of advanced pesticide transport, including volatilization and kinetic adsorption, SWAP can be used in combination with the model PESTLA (Van den Berg and Boesten, 1998) and PEARL (Leistra et al., 2000; Tiktak et al., 2000). For nutrient transport (nitrogen and phosphorus), SWAP can be used in combination with the model ANIMO (Rijtema et al., 1997; Kroes and Roelsma, 1998) or Soil-N (Groenendijk et al., 2017).

In this chapter, we first describe the solute transport processes that are considered in SWAP. Next, we discuss the boundary conditions applied. Also, we consider how SWAP deals with solute transport in water repellent soils and in cracked clay soils. Salinization is a special case of solute transport. We describe the salinity stress options in SWAP. Finally we provide an overview of solute transport input data. 


\subsection{Basic equations}

\subsubsection{Transport processes}

The three main solute transport mechanisms in soil water are diffusion, convection and dispersion. Diffusion is solute transport caused by the solute gradient. Thermal motion of the solute molecules within the soil solution causes a net transport of molecules from high to low concentrations. The solute flux $J_{\text {dif }}\left(\mathrm{mg} \mathrm{cm}^{-2} \mathrm{~d}^{-1}\right)$ is generally described by Fick's first law:

$$
J_{\text {dif }}=-\theta D_{\text {dif }} \frac{\partial c}{\partial z}
$$

with $D_{\text {dif }}$ the diffusion coefficient $\left(\mathrm{cm}^{2} \mathrm{~d}^{-1}\right)$ and $c$ the solute concentration in soil water $\left.(\mathrm{mg} \mathrm{cm})^{-3}\right) . D_{\text {dif }}$ is very sensitive to the actual water content, as it strongly affects the solute transport path and the effective cross-sectional transport area. In SWAP we employ the relation proposed by Millington and Quirk (1961):

$$
D_{\text {dif }}=D_{\mathrm{w}} \frac{\theta^{7 / 3}}{\phi_{\text {por }}^{2}}
$$

with $D_{\mathrm{w}}$ the solute diffusion coefficient in free water $\left(\mathrm{cm}^{2} \mathrm{~d}^{-1}\right)$ and $\phi_{\text {por }}$ the soil porosity $\left(\mathrm{cm}^{3} \mathrm{~cm}^{-3}\right)$.
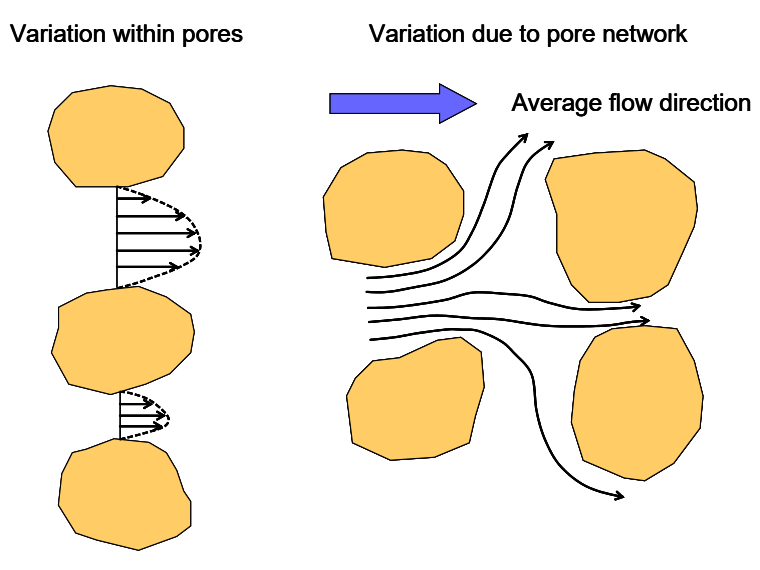

Figure 8.1 Flow velocity variation within pores and within the pore network.

The bulk transport of solutes occurs when solutes are carried along with the moving soil water. The mean flux of this transport is called the convective flux, $J_{\text {con }}\left(\mathrm{mg} \mathrm{cm}^{-2} \mathrm{~d}^{-1}\right)$, and can be calculated from the average soil water flux:

$$
J_{\text {con }}=q c
$$

When describing water flow, we usually consider the Darcy flux $q\left(\mathrm{~cm} \mathrm{~d}^{-1}\right)$, which is averaged over a certain cross section. In case of solute transport, we have to account for the water velocity variation between pores of different size and geometry and also the water velocity variation inside a pore itself (Figure 8.1). The variety of water velocities cause some solutes to advance faster than the average solute front, and other solutes to advance slower. The overall effect will be that steep solute fronts tends to smoothen or to disperse. Solutes seem to flow from high to low concentrations. If the time required for solutes to mix in the transverse direction is small, compared to the time required for solutes to move in the flow direction by mean convection, the dispersion flux $J_{\text {dis }}\left(\mathrm{mg} \mathrm{cm}^{-2} \mathrm{~d}^{-1}\right)$ is proportional to the solute gradient (Bear, 1972):

$$
J_{\text {dis }}=-\theta D_{\text {dis }} \frac{\partial c}{\partial z}
$$


with $D_{\text {dis }}$ the dispersion coefficient $\left(\mathrm{cm}^{2} \mathrm{~d}^{-1}\right)$. Under laminar flow conditions $D_{\text {dis }}$ itself is proportional to the pore water velocity $v=q / \theta$ (Bolt, 1979):

$$
D_{\text {dis }}=L_{\text {dis }}|v|
$$

with $L_{\text {dis }}$ the dispersion length $(\mathrm{cm})$. Unless water is flowing very slowly through repacked soil, the dispersion flux is usually much larger than the diffusion flux.

The total solute flux $\mathrm{J}\left(\mathrm{mg} \mathrm{cm}^{-2} \mathrm{~d}^{-1}\right)$ is therefore described by:

$$
J=J_{\text {con }}+J_{\text {dif }}+J_{\text {dis }}=q c-\theta\left(D_{\text {dif }}+D_{\text {dis }}\right) \frac{\partial c}{\partial z}
$$

\subsubsection{Continuity and transport equation}

By considering conservation of mass in an elementary volume, we may derive the continuity equation for solute transport:

$$
\frac{\partial X}{\partial t}=-\frac{\partial J}{\partial z}-S_{\mathrm{s}}
$$

with $X$ being the total solute concentration system $\left(\mathrm{mg} \mathrm{cm}^{-3}\right.$ soil) and $S_{\mathrm{s}}$ the solute sink term $\left(\mathrm{mg} \mathrm{cm}^{-3}\right.$ $\mathrm{d}^{-1}$ ) accounting for decomposition and uptake by roots.

The solutes may be dissolved in the soil water or may be adsorbed to organic matter or to clay minerals:

$$
X=\theta c+\rho_{\mathrm{b}} Q
$$

with $\rho_{\mathrm{b}}$ being the dry soil bulk density $\left(\mathrm{g} \mathrm{cm}^{-3}\right)$ and $Q$ the amount adsorbed $\left(\mathrm{mg} \mathrm{g}^{-1}\right)$. The adsorption isotherm describes the amount of solutes adsorbed in equilibrium with the dissolved concentration. At this stage we will assume instantaneous equilibrium between $c$ and $Q$ and use the non-linear Freundlich equation, which is a flexible function for many organic and inorganic solutes. Freundlich adsorption can be written as:

$$
Q=K_{\mathrm{f}} c_{\text {ref }}\left(\frac{c}{c_{\text {ref }}}\right)^{N_{\mathrm{f}}}
$$

with $K_{\mathrm{f}}$ the Freundlich coefficient $\left(\mathrm{cm}^{3} \mathrm{mg}^{-1}\right), N_{\mathrm{f}}$ is the Freundlich exponent $(-)$ and $c_{\text {ref }}$ is a reference value of the solute concentration $\left(\mathrm{mg} \mathrm{cm}^{-3}\right.$ ) which is used to make $N_{\mathrm{f}}$ dimensionless.

The solute sink term $S_{\mathrm{s}}$ can be written as:

$$
S_{\mathrm{s}}=\mu\left(\theta c+\rho_{\mathrm{b}} Q\right)+K_{\mathrm{r}} S c
$$

where $\mu$ is the first order rate coefficient of transformation $\left(d^{-1}\right), K_{r}$ is the root uptake preference factor $(-)$ and $S$ the root water extraction rate $\left(d^{-1}\right)$. At the right hand side of Eq. (8.10), the first term accounts for linear decomposition and the second term for root uptake proportional to water uptake. $K_{\mathrm{r}}$ accounts for positive or negative selection of solute ions relative to the amount of soil water that is extracted.

The coefficient $\mu$ is affected by soil temperature, water content and depth. Analogous to Boesten and Van der Linden (1991), SWAP calculates $\mu$ from:

$$
\mu=f_{\mathrm{T}} f_{\theta} f_{\mathrm{z}} \mu_{\mathrm{ref}}
$$

in which $f_{\mathrm{T}}$ is a soil temperature factor $(-), f_{\theta}$ and $f_{z}$ are reduction factors $(-)$ accounting for the effect of soil water content and soil depth, and $\mu_{\text {ref }}\left(d^{-1}\right)$ is $\mu$ at reference conditions (e.g. soil from the plough layer at $20^{\circ} \mathrm{C}$ and at soil water pressure head $h=-100 \mathrm{~cm}$ ). 
The factor $f_{\mathrm{T}}$ is described according to Boesten (1986) as:

$$
f_{\mathrm{T}}=\mathrm{e}^{\gamma_{\mathrm{T}}(T-20)}
$$

where $\gamma_{T}$ is a parameter $\left({ }^{\circ} \mathrm{C}^{-1}\right)$, and $T$ is the soil temperature in ${ }^{\circ} \mathrm{C}$.

Wolfe et al. (1990) describe the importance of the water content in transformation processes.

Realizing that it is a large simplification, in SWAP we adopt the relation as proposed by Walker (1974):

$$
f_{\theta}=\left(\frac{\theta}{\theta_{\text {ref }}}\right)^{B} \quad \text { with } \quad f_{\theta} \leq 1.0
$$

where $\theta_{\text {ref }}$ is $\theta$ at $h=-100 \mathrm{~cm}$ and $B$ is a constant $(-)$.

The transformation reduction factor for soil depth, $f_{z}$, should be derived from in situ measurements. The user may specify $f_{z}$ as function of soil depth in the input file.

Combination of Eq. (8.6), (8.7), (8.8), and (8.10), yields the transport equation applied in SWAP which is valid for dynamic, one-dimensional, convective-dispersive mass transport, including nonlinear adsorption, linear decay and proportional root uptake in unsaturated/saturated soil (Van Genuchten and Cleary, 1979; Nielsen et al., 1986; Boesten and Van der Linden, 1991):

$$
\frac{\partial\left(\theta c+\rho_{\mathrm{b}} Q\right)}{\partial t}=-\frac{\partial(q c)}{\partial z}+\frac{\partial\left[\theta\left(D_{\text {dif }}+D_{\text {dis }}\right) \frac{\partial c}{\partial z}\right]}{\partial z}-\mu\left(\theta c+\rho_{\mathrm{b}} Q\right)-K_{\mathrm{r}} S c
$$

An explicit, central finite difference scheme is used to solve Eq. (8.14):

$$
\begin{aligned}
& \frac{\theta_{i}^{j+1} c_{i}^{j+1}+\rho_{\mathrm{b}} Q_{i}^{j+1}-\theta_{i}^{j} c_{i}^{j}-\rho_{\mathrm{b}} Q_{i}^{j}}{\Delta t^{j}}= \\
& \frac{q_{i-1 / 2}^{j} c_{i-1 / 2}^{j}-q_{i+1 / 2}^{j} c_{i+1 / 2}^{j}}{\Delta z_{i}}+\frac{1}{\Delta z_{i}}\left[\frac{\theta_{i-1 / 2}^{j} D_{i-1 / 2}^{j}\left(c_{i-1}^{j}-c_{i}^{j}\right)}{1 / 2\left(\Delta z_{i-1}+\Delta z_{i}\right)}-\frac{\theta_{i+1 / 2}^{j} D_{i+1 / 2}^{j}\left(c_{i}^{j}-c_{i+1}^{j}\right)}{1 / 2\left(\Delta z_{i}+\Delta z_{i+1}\right)}\right]- \\
& \mu_{i}^{j}\left(\theta_{i}^{j} c_{i}^{j}+\rho_{\mathrm{b}} Q_{i}^{j}\right)-K_{r} S_{i}^{j} c_{i}^{j}
\end{aligned}
$$

where $D\left(=D_{\text {dif }}+D_{\text {dis }}\right)$ is the overall dispersion coefficient $\left(\mathrm{cm}^{2} \mathrm{~d}^{-1}\right)$; the superscript $j$ denotes the time level, subscript $i$ the node number and subscripts $i-1 / 2$ and $i+1 / 2$ refer to linearly interpolated values at the upper and lower compartment boundary, respectively. Compared to an implicit, iterative scheme, above explicit scheme has the advantage that incorporation of non-linear adsorption, mobile/immobile concepts, and other non-linear processes is relatively easy. In order to ensure stability of the explicit scheme, the time step $\Delta t^{\mathrm{j}}$ should meet the criterium (Van Genuchten and Wierenga, 1974; corresponding to the Fourier number being $<0.5$ ):

$$
\Delta t^{j} \leq \frac{\Delta z_{i}^{2} \theta_{i}^{j}}{2 D_{i}^{j}}
$$

This stability criterium applies to non-sorbing substances and is therefore also safe for sorbing substances.

\subsection{Boundary conditions}

As initial condition, the user needs to specify the solute concentrations $c_{\mathrm{i}}\left(\mathrm{mg} \mathrm{cm}^{-3}\right.$ soil water) at different soil depths.

For the top boundary condition, the solute concentrations in irrigation and rain water, $c_{\text {irr }}$ and $c_{\text {prec }}$ $\left(\mathrm{mg} \mathrm{cm}{ }^{-3}\right.$ ), need to be specified. During evaporation no solutes leave the soil profile at the surface. During infiltration, the solute concentration of water that enters the soil profile at the top, $c_{\text {pond }}$ 
( $\mathrm{mg} \mathrm{cm}^{-3}$ ), is affected by the ponding layer and its concentration at the former time step, the solute amounts coming in by rain and irrigation, and the solute amounts transported laterally to cracks:

$$
c_{\text {pond }}^{j}=\frac{\left(P_{\text {net }}^{j} c_{\text {prec }}+I_{\text {net }}^{j} c_{\text {irr }}\right) \Delta t^{j}+h_{\text {pond }}^{j-1} c_{\text {pond }}^{j-1}}{h_{\text {pond }}^{j}-\left(q_{\text {top }}+q_{\text {lat }}\right) \Delta t^{j}}
$$

where $P_{\text {net }}$ is the net precipitation rate $\left(\mathrm{cm} \mathrm{d}^{-1}, I_{\text {net }}\right.$ is the net irrigation rate $\left(\mathrm{cm} \mathrm{d}^{-1}\right), h_{\text {pond }}$ is the height of water ponding on the soil surface, $q_{\text {top }}$ is the water flux at the soil surface $\left(\mathrm{cm} \mathrm{d}^{-1}\right.$, positive upward) and $q_{\text {lat }}$ is the water flux flowing to cracks ( $\mathrm{cm} \mathrm{d}^{-1}$, see Section 8.4). The solute flux $J_{\text {top }}\left(\mathrm{mg} \mathrm{cm}^{-2}\right)$ entering the soil at the surface, equals:

$$
J_{\text {top }}=q_{\text {top }} c_{\text {pond }}\left(1.0-A_{\mathrm{c}}\right)
$$

where $A_{\mathrm{c}}$ is the relative crack area $\left(\mathrm{cm}^{2} \mathrm{~cm}^{-2}\right)$.

For the drainage boundary condition, during drainage $\left(q_{\text {drain }}>0\right)$ the solute flux $J_{\text {drain }}\left(\mathrm{mg} \mathrm{cm}^{-2}\right)$ that leaves the one-dimensional soil profile is accumulated for each compartment below groundwater level:

$$
J_{\text {drain }}=\sum_{i=\mathrm{n}_{\mathrm{gwl}}}^{\mathrm{n}} q_{\mathrm{drann}, i} c_{i}
$$

where $n_{\text {gwl }}$ is the compartment with the groundwater level and $q_{\text {drain, }, i}$ is the lateral drainage flux $\left(\mathrm{cm} \mathrm{d}^{-1}\right)$ of compartment $i$. During infiltration $\left(q_{\text {drain }}<0\right), J_{\text {drain }}$ follows from:

$$
J_{\text {drain }}=\sum_{i=\mathrm{n}_{\mathrm{gul}}}^{\mathrm{n}} q_{\text {drain, }, i} c_{\text {surf }}
$$

where $c_{\text {surf }}$ is the solute concentration in surface water $\left(\mathrm{mg} \mathrm{cm}^{-3}\right)$, specified as input.

For the bottom boundary condition, SWAP uses the flux through the bottom of the soil profile $q_{\text {bot }}$ $\left(\mathrm{cm} \mathrm{d}^{-1}\right)$. In case of upward flow $\left(q_{\mathrm{bot}}>0\right)$, the solute flux $J_{\mathrm{bot}}\left(\mathrm{mg} \mathrm{cm}^{-2}\right.$, positive is upwards) equals:

$$
J_{\text {bot }}=q_{\text {bot }} c_{\text {seep }}
$$

where $c_{\text {seep }}$ is the solute concentration in groundwater $\left(\mathrm{mg} \mathrm{cm}^{-3}\right)$ specified as input. This concentration can be specified as constant or variable in time. Also the user may select $c_{\text {surf }}$ in stead of $c_{\text {seep }}$.

If $q_{\text {bot }}$ is directed downwards $\left(q_{\text {bot }}<0\right)$, the solute flux $J_{\text {bot }}\left(\mathrm{mg} \mathrm{cm}^{-2}\right)$ equals:

$$
J_{\text {bot }}=q_{\text {bot }} c_{\mathrm{n}}
$$

\subsection{Crack solute transport}

In order to calculate solute transport in combination with macropore flow, SWAP may generate soil water fluxes which are input to the pesticide model PEARL or the nutrient model ANIMO.

\subsection{Residence time in the saturated zone}

In the case of heterogeneous groundwater flow or multi-level drainage, the residence time approach described in Chapter 4 is used. This section describes an alternative concept assuming a homogeneous aquifer and field drainage at one level.

Ernst (1973) and Van Ommen (1985) showed that the breakthrough curve of a field with fully penetrating drainage canals, is identical to the breakthrough curve of a reservoir with complete mixing. This is also valid if adsorption can be described by a linear isotherm and transformation occurs proportional to the existing concentration (Van Ommen, 1985). 
Linear adsorption might be described by:

$$
Q=k_{\mathrm{ads}} c_{\mathrm{gr}}
$$

where $k_{\text {ads }}$ is the linear adsorption coefficient in the saturated zone $\left(\mathrm{cm}^{3} \mathrm{mg}^{-1}\right)$. Numerical analysis by Duffy and Lee (1992) showed that dispersion in the saturated zone has only a minor effect for $L_{\text {drain }} / d_{\text {aquif }}$ $\geq 10$, where $L_{\text {drain }}$ is the distance between the drainage canals $(\mathrm{cm})$ and $d_{\text {aquif }}$ the thickness of the aquifer $(\mathrm{cm})$. Generally $L_{\text {drain }} / d_{\text {aquif }}$ will be around 10 or larger, therefore SWAP ignores dispersion.

In order to derive the breakthrough curve, the similarity is used between breakthrough curves of drained fields and mixed reservoirs. Starting point is the solute transport equation of the unsaturated zone, Eq. (8.14). Replacement of non-linear adsorption by linear adsorption, and removal of dispersion and root water uptake, results in the mass balance equation of the saturated zone:

$$
\frac{\partial\left(\theta_{\mathrm{s}} c_{\mathrm{gr}}+\rho_{\mathrm{b}} k_{\mathrm{ads}} c_{\mathrm{gr}}\right)}{\partial t}=\frac{q_{\mathrm{drain}}}{d_{\text {aquif }}}\left(c_{\mathrm{in}}-c_{\mathrm{gr}}\right)-\mu_{\mathrm{gr}}\left(\theta_{\mathrm{s}} c_{\mathrm{gr}}+\rho_{\mathrm{b}} k_{\mathrm{ads}} c_{\mathrm{gr}}\right)
$$

where $\theta_{\mathrm{s}}$ is the saturated water content $\left(\mathrm{cm}^{3} \mathrm{~cm}^{-3}\right), q_{\text {drain }}$ is the drainage flux $\left(\mathrm{cm} \mathrm{d}^{-1}\right), c_{\text {in }}$ is the solute concentration of water percolating from the unsaturated zone $\left(\mathrm{mg} \mathrm{cm}^{-3}\right)$ and $\mu_{\mathrm{gr}}$ is the first order rate coefficient for transformation in the saturated zone $\left(\mathrm{d}^{-1}\right)$. Eq. (8.24) applies to a drainage situation $\left(q_{\text {drain }}>0\right)$. In case of infiltration $\left(q_{\text {drain }}<0\right)$, SWAP assumes the infiltrating water from the drainage system to be solute free, and Eq. (8.24) transforms into:

$$
\frac{\partial\left(\theta_{\mathrm{s}} c_{\mathrm{gr}}+\rho_{\mathrm{b}} k_{\mathrm{ads}} c_{\mathrm{gr}}\right)}{\partial t}=\frac{q_{\mathrm{drain}}}{d_{\text {aquif }}} c_{\mathrm{gr}}-\mu_{\mathrm{gr}}\left(\theta_{\mathrm{s}} c_{\mathrm{gr}}+\rho_{\mathrm{b}} k_{\mathrm{ads}} c_{\mathrm{gr}}\right)
$$

Eq. (8.24) and (8.25) are discretized as an explicit, forward difference scheme. The boundary conditions that apply to the saturated zone, are included in Eq. (8.24) and (8.25).

\subsection{Salinity stress}

Salinity stress reduces the root water uptake and is related to the solute concentration in soil water. Two options are offered in SWAP:

1. Linear reduction function (Maas and Hoffman, 1977)

2. Osmotic head (De Jong van Lier et al., 2009)

Figure 8.2 shows the reduction function of Maas and Hoffman (1977). The reduction of root water uptake $\left(a_{r s}\right)$ when the salt concentration exceeds a threshold value is linearly related to the salinity concentration:

$$
\alpha_{\mathrm{rs}}=1.0-\left(c-S_{\max }\right) S_{\text {slope }}
$$

where $c$ is the salinity concentration $\left(\mathrm{mg} \mathrm{cm}^{-3}\right), S_{\max }$ is the salinity threshold value $\left(\mathrm{mg} \mathrm{cm}^{-3}\right)$ and $S_{\text {slope }}$ is the decline of the root water uptake factor per unit increase of salinity concentration $\left(\mathrm{cm}^{3} \mathrm{mg}^{-1}\right)$.

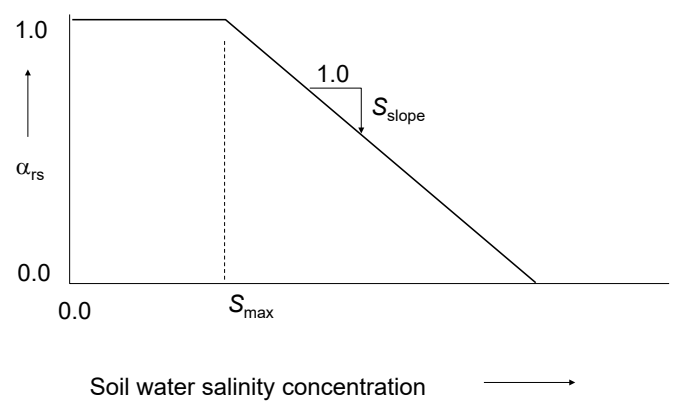

Figure 8.2 Reduction coefficient for root water uptake, $\alpha_{r s,}$ as function of soil water salinity concentration (after Maas and Hoffman, 1977). 
De Jong van Lier et al. (2009) demonstrated how the osmotic head due to salts in soil water can be included in microscopic root water uptake. To apply this concept for salinity stress, the root water uptake under dry conditions should be based on the microscopic approach (see Par. 3.5.4). Mind that if salinity stress is based on osmotic head, toxic effects of salts are not included. The application of the osmotic head requires tuning of the drought input parameters of the crop input file. Currently this option is advised for specialists only.

\subsection{Groundwater age}

Soil moisture and groundwater age can be calculated as the time elapsed after entering the soil water domain described by the SWAP model. Following the method used by Goode (1996) and Lemieux and Sudicky (2010), the soil moisture age is governed by a convection-dispersion equation for an inert solute with an internal source of unit (1) strength:

$$
\frac{\partial \theta A}{\partial t}=-\frac{\partial}{\partial z} q_{z} A+\frac{\partial}{\partial z}\left(\theta D \frac{\partial A}{\partial z}\right)-S A+\theta
$$

where $A$ is the average age of water particles in a package of water (d).

The age of water entering the model boundaries is set to 0 , irrespective of the boundary type. In soil profiles exposed to a constant upward flux at the bottom boundary, the age at the bottom equals zero and the distribution with depth shows a curved course.

The capabilities of the age module implemented in the SWAP model is illustrated in Figure 8.3. It shows the long term averaged age distribution with depth for four classes of hydrological plots in the Dutch Nationwide STONE model (Groenendijk et al, 2013).

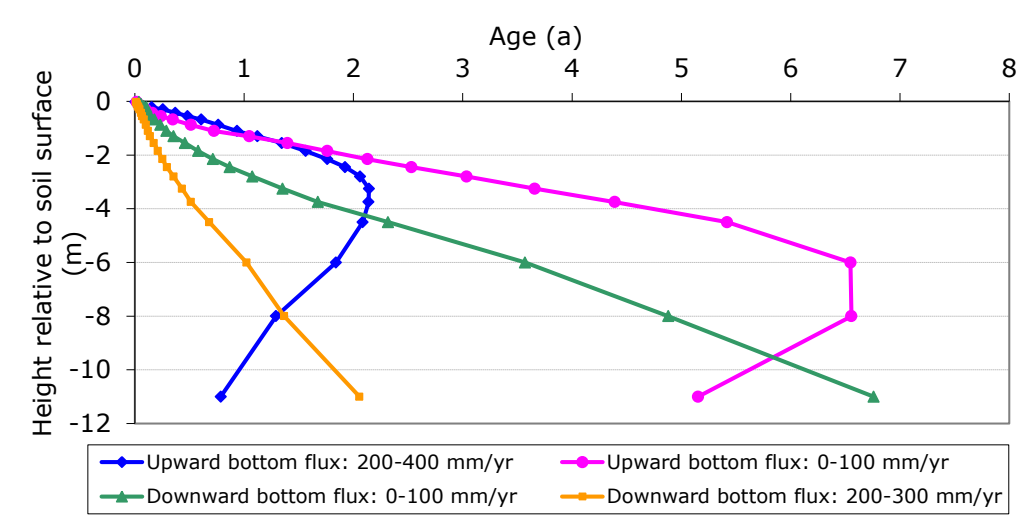

Figure 8.3 Age is a function of depth for different classes of hydrological plots in the Dutch nationwide STONE model (Groenendijk et al, 2013).

The age of soil water in the profiles with a relative low leaching rate $\left(0-100 \mathrm{~mm} \mathrm{yr}^{-1}\right)$ is higher than in the profiles with a relative high leaching rate $\left(200-300 \mathrm{~mm} \mathrm{yr}^{-1}\right)$ and the age in the profiles with a relative low upwarde seepage $\left(0-100 \mathrm{~mm} \mathrm{yr}^{-1}\right)$ is higher than in the profiles with a relative high upwarde seepage rate rate $\left(200-400 \mathrm{~mm} \mathrm{yr}^{-1}\right)$. The position of the maximum age in the profiles with upward seepage depends on the seepage flow rate and the lay-out of the drainage systems. 


\subsection{User instructions}

Box 8.1 lists the input data for solute transport, which are divided over the parts:

1. Main switch

2. Top and initial boundary condition

3. Miscellaneous parameters as function of soil depth

4. Diffusion coefficient and solute uptake by roots

5. Adsorption

6. Decomposition

7. Solute residence in the saturated zone

8. Soil moisture and groundwater age

In general the theorie description in Sections $8.2-8.6$ in combination with the descriptions in the input file will be sufficient to guide the model user. A few additional remarks are appropriate at this place.

In case conservative solute are simulated, like salts are non-reactive tracers, we need only to consider the transport processes convection, diffusion, dispersion and passive uptake by plant roots.

At most field conditions we may neglect the effect of diffusion with respect to dispersion and therefore may specify $D_{\text {dif }}=0$. The parameter dispersion length, $L_{\text {dis }}(\mathrm{cm})$, depends on the scale over which the water flux and solute convection are averaged. Typical values of $L_{\text {dis }}$ are $0.5-2.0 \mathrm{~cm}$ in packed laboratory columns and 5-20 cm in the field (Jury et al., 1991; Vanderborght and Vereecken, 2007).

SWAP supports two methods to account for the residence time of solutes in the saturated zone. The first one by proper distribution of the lateral drainage flux over the saturated compartments (Chapter 4). In that case we may set SWBR $=0$ and specify the solute concentration in the groundwater as boundary condition for upward flow (Box 8.1, Part 7). The second method has been described in this chapter and views the saturated zone as one mixed reservoir (Section 8.5). In that case we should set SWBR $=1$ and provide the effective transport properties of the saturated zone (Box 8.1, Part 7).

The input data for salinity stress depend on the plant specie and are listed in the crop input file. SWAP input data for the Maas and Hoffman reduction function are listed in Appendix 9.

To simulate soil moisture and groundwater age the user should add a flag to the section for solute transport (Box 8.1, Part 8). When this flag (flAgeTracer) is set with the value .TRUE. the model will automatically produce 2 output files: i) result.ageeffluent.csv and ii) Result.ageProfile.csv with groundwater age $(d)$ of respectively effluent and soil water.

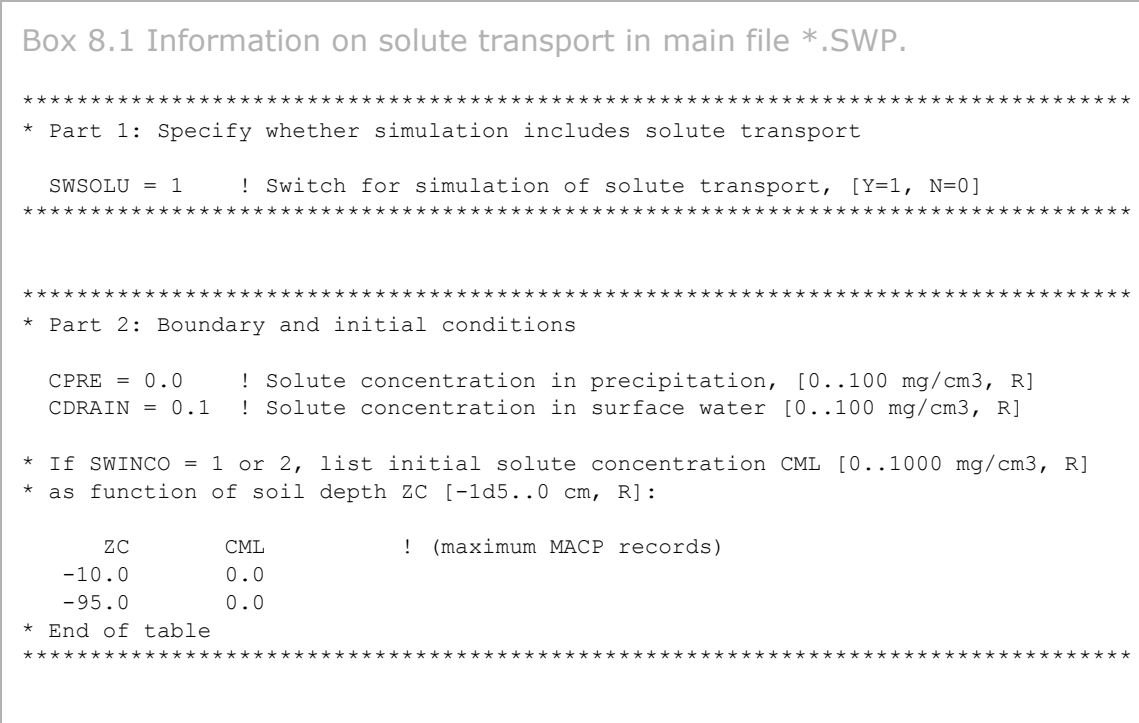




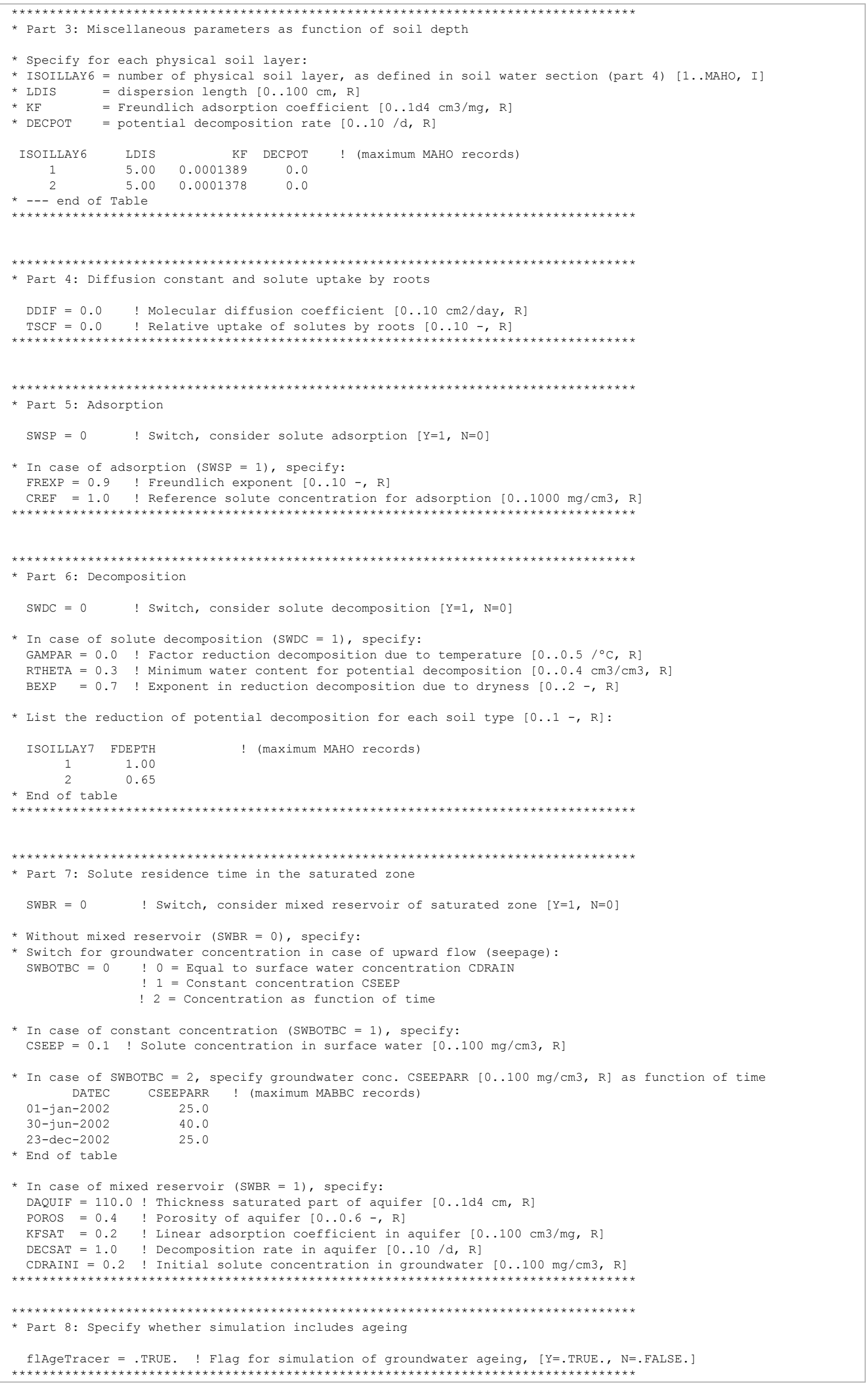




\section{Soil temperature}

Soil temperature affects many physical, chemical and biological processes in the top soil, for instance the surface energy balance, soil hydraulic properties, decomposition rate of solutes and growth rate of roots. Currently, SWAP uses soil temperature in the following processes:

- suppression of grass growth due to low temperatures

- reduction of hydraulic conductivity and root water uptake at low temperatures

- mineralization rate

- respiration rate (and some other oxygen related processes)

- solute decomposition rate

Other temperature relations can be easily included, if needed. SWAP calculates the soil temperatures either analytically or numerically. In the following sections the heat flow equations and the applied analytical and numerical solutions are discussed.

\subsection{Temperature conductance equation}

If we consider heat transport only by convection, the one-dimensional soil heat flux, $q_{\text {heat }}\left(\mathrm{J} \mathrm{cm}^{-2} \mathrm{~d}^{-1}\right)$, can be described as:

$$
q_{\text {heat }}=-\lambda_{\text {heat }} \frac{\partial T}{\partial z}
$$

where $\lambda_{\text {heat }}$ is the thermal conductivity $\left(\mathrm{J} \mathrm{cm}^{-1}{ }^{\circ} \mathrm{C}^{-1} \mathrm{~d}^{-1}\right)$ and $T$ is the soil temperature $\left({ }^{\circ} \mathrm{C}\right)$.

Conservation of energy results in:

$$
C_{\text {heat }} \frac{\partial T}{\partial t}=\frac{-\partial q_{\text {heat }}}{\partial z}
$$

where $C_{\text {heat }}$ is the soil heat capacity $\left(\mathrm{J} \mathrm{cm}^{-3}{ }^{\circ} \mathrm{C}^{-1}\right)$.

Combination of Eq. (9.1) and (9.2) yields the differential equation for soil heat flow:

$$
C_{\text {heat }} \frac{\partial T}{\partial t}=\frac{\partial\left(\lambda_{\text {heat }} \frac{\partial T}{\partial z}\right)}{\partial z}
$$

In general in the liquid phase, radiation and convection will also transport heat. As the contribution of radiation and convection to soil heat transport in general is small compared to conductance (e.g. Moene and van Dam, 2014), SWAP only considers conductance. In the vapour phase, diffusion may contribute to soil heat transport. The rate of heat transfer by water vapour diffusion is small and proportional to the temperature gradient (De Vries, 1975). Therefore, such diffusion can be taken into account by slightly increasing the soil thermal diffusivity. This approach is followed in SWAP as well. Apparent thermal properties rather than real thermal properties are assumed to account for both conductive and non-conductive heat flow. 


\subsection{Numerical solution}

The parameters $\lambda_{\text {heat }}$ and $C_{\text {heat }}$ strongly depend on the soil moisture content. Therefore in general Eq. (9.3) can only be solved with a numerical solution. SWAP employs a fully implicit finite difference numerical scheme to solve Eq. (9.3):

$$
C_{i}^{j+1}\left(T_{i}^{j+1}-T_{i}^{j}\right)=\frac{\Delta t^{j}}{\Delta z_{i}}\left[\lambda_{i-1 / 2}^{j+1 / 2} \frac{T_{i-1}^{j+1}-T_{i}^{j+1}}{\Delta z_{\mathrm{u}}}-\lambda_{i+1 / 2}^{j+1 / 2} \frac{T_{i}^{j+1}-T_{i+1}^{j+1}}{\Delta z_{\ell}}\right]
$$

where superscript $j$ denotes the time level, subscript $i$ is the node number, $\Delta z_{\mathrm{u}}=z_{\mathrm{i}-1}-z_{\mathrm{i}}$ and $\Delta z_{\mathrm{I}}=z_{\mathrm{i}}-$ $z_{i+1}$. As the coefficients $C_{\text {heat }}$ and $\lambda_{\text {heat }}$ are not affected by the soil temperature itself, Eq. (9.4) is a linear equation.

Both $C_{\text {heat }}$ and $\lambda_{\text {heat }}$ depend on the soil composition. The volumetric heat capacity is calculated as weighted mean of the heat capacities of the individual components (De Vries, 1963):

$$
C_{\text {heat }}=f_{\text {sand }} C_{\text {sand }}+f_{\text {clay }} C_{\text {clay }}+f_{\text {organic }} C_{\text {organic }}+\theta C_{\text {water }}+f_{\text {air }} C_{\text {air }}
$$

where $f$ and $C$ on the right hand side of Eq. (9.4) are the volume fraction $\left(\mathrm{cm}^{3} \mathrm{~cm}^{-3}\right)$ and volumetric heat capacity $\left(\mathrm{J} \mathrm{cm}^{-3}{ }^{\circ} \mathrm{C}^{-1}\right)$ of each component, respectively, and the components are indicated in the subscripts. Table 9.1 gives values of $C$ for the different soil components.

Table 9.1 Volumetric heat capacity and thermal conductivity of the soil components.

\begin{tabular}{llc} 
Component & Volumetric heat capacity, & Thermal conductivity, \\
& $C_{\text {heat }}\left(\mathrm{J} \mathrm{cm}^{-3}{ }^{\circ} \mathrm{C}^{-1}\right)$ & $\lambda_{\text {heat }}\left(\mathrm{J} \mathrm{cm}^{-1} \mathrm{C}^{-1} \mathrm{~d}^{-1}\right)$ \\
Sand & 2.128 & 7603 \\
\hline Clay & 2.385 & 2523 \\
\hline Organic & 2.496 & 216 \\
\hline Water & 4.180 & 492 \\
\hline Air $\left(20^{\circ} \mathrm{C}\right)$ & 0.001212 & variable \\
\hline
\end{tabular}

In order to calculate $C_{\text {heat }}$ from (9.5), the percentage (by volume) of sand and clay, denoted $V P_{\text {sand }}$ and $V P_{\text {clay, }}$ respectively, must be specified by the SWAP user. $V P_{\text {sand }}$ and $V P_{\text {clay }}$ should be provided as percentages of the total solid soil matter and may differ for each soil layer. The total volume fraction of solid matter is given by:

$$
\theta_{\text {solid }}=1-\theta_{\text {sat }}
$$

where $\theta_{\text {sat }}$ is the saturated volumetric water content. The volume fraction of air is equal to the saturated minus the actual water content:

$$
f_{\text {air }}=\theta_{\text {sat }}-\theta
$$

$f_{\text {sand, }} f_{\text {clay }}$ and $f_{\text {organic }}$ are then calculated by:

$$
\begin{aligned}
& f_{\text {sand }}=\frac{V P_{\text {sand }}}{100} \theta_{\text {solid }} \\
& f_{\text {clay }}=\frac{V P_{\text {clay }}}{100} \theta_{\text {solid }} \\
& f_{\text {organic }}=\theta_{\text {solid }}-f_{\text {sand }}-f_{\text {clay }}
\end{aligned}
$$

where it has been assumed that solid matter that is not sand or clay, is organic.

Table 9.1 also lists the thermal conductivities, which are largest for sand and clay, an order smaller for organic material and water, and again an order smaller for air. Hence the space-average thermal 
conductivity of a soil depends upon its mineral composition and organic matter content, as well as the volume fractions of water and air. Since the thermal conductivity of air is much smaller than that of water or solid matter, a high air content (or low water content) corresponds to a low thermal conductivity.

The components that affect $\lambda_{\text {heat }}$ are the same as those affecting $C_{\text {heat }}$. However, the variation in $\lambda_{\text {heat }}$ is much greater than the variation of $C_{\text {heat }}$. In the range of soil wetness normally experienced in the field, $C_{\text {heat }}$ may undergo a threefold or fourfold change, whereas the corresponding change in $\lambda_{\text {heat }}$ may be hundredfold or more. Unlike heat capacity, thermal conductivity is also sensitive to the sizes, shapes, and spatial arrangements of the soil particles (Hillel, 1980).

The thermal conductivity is found by considering the soil as a continuous liquid or gaseous phase in which soil and respectively gas or liquid 'particles' are dispersed. In the case of a 'wet' soil $\left(\theta>\theta_{\text {wet }}\right)$ liquid water is assumed to be the continuous phase and the thermal conductivity is given by:

$$
\lambda_{\text {heat }}=\frac{x_{\text {sand-water }} f_{\text {sand }} \lambda_{\text {sand }}+x_{\text {clay-water }} f_{\text {clay }} \lambda_{\text {clay }}+x_{\text {organic-water }} f_{\text {organic }} \lambda_{\text {organic }}+x_{\text {water-water }} \theta \lambda_{\text {water }}+x_{\text {air-water }} f_{\text {air }} \lambda_{\text {air }}}{x_{\text {sand-water }} f_{\text {sand }}+x_{\text {clay-water }} f_{\text {clay }}+x_{\text {organic-water }} f_{\text {organic }}+x_{\text {wate-water }} \theta+x_{\text {air-water }} f_{\text {air }}}
$$

The $\lambda$-values on the right hand side of Eq. (9.11) refer to the thermal conductivities $\left(\mathrm{J} \mathrm{cm}^{-1}{ }^{\circ} \mathrm{C}^{-1} \mathrm{~d}^{-1}\right)$ of each individual component, as listed in Table 9.1. The weighting factors $x_{\mathrm{mn}}$ for component $m$ particles suspended in the continuous phase $n$ phase depend on the ratio of the specific thermal conductivities of component $m$ and $n$ and on the shape of $m$ particles in the direction of the temperature gradient. When we assume the particles to be spheroids whose axes are randomly oriented in the soil (Ten Berge, 1986), the weighting factors can be calculated by:

$$
x_{\mathrm{mn}}=\frac{0.66}{1+g_{\mathrm{m}}\left(\lambda_{\mathrm{m}} / \lambda_{\mathrm{n}}-1\right)}+\frac{0.33}{1+\left(\lambda_{\mathrm{m}} / \lambda_{\mathrm{n}}-1\right)\left(1-2 g_{\mathrm{m}}\right)}
$$

The shape factors and weights calculated using Eq. (9.12) are given in Table 9.2. The thermal conductivity is quite sensitive to the air shape factor $\left(g_{\mathrm{a}}\right)$, which appears to depend on the air content itself and is calculated as:

in wet soils $\left(\theta>\theta_{\text {wet }}\right): \quad g_{\mathrm{a}}=0.333-\frac{\theta_{\text {sat }}-\theta}{\theta_{\text {sat }}}(0.333-0.035)$

in dry soils $\left(\theta<\theta_{d r y}\right): \quad g_{\mathrm{a}}=0.013+\frac{\theta}{\theta_{\mathrm{dry}}}\left(g_{\mathrm{a}, \mathrm{dry}}-0.013\right)$

where $g_{a, d r y}$ is the value of Eq. (9.13) at $\theta=\theta_{d r y}$. In this way $g_{a}$ varies between $0.013(\theta=0)$ and $0.333\left(\theta=\theta_{\text {sat }}\right)$.

For 'dry' soil $\left(\theta<\theta_{\text {dry }}\right)$ air is considered as the continuous phase and the conductivity is given by:

$$
\lambda_{\text {heat }}=1.25 \frac{x_{\text {sand-air }} f_{\text {sand }} \lambda_{\text {sand }}+x_{\text {clay-air }} f_{\text {clay }} \lambda_{\text {clay }}+x_{\text {organic-air }} f_{\text {organic }} \lambda_{\text {organic }}+x_{\text {water-air }} \theta \lambda_{\text {water }}+x_{\text {air-air }} f_{\text {air }} \lambda_{\text {air }}}{x_{\text {sand-air }} f_{\text {sand }}+x_{\text {clay-air }} f_{\text {clay }}+x_{\text {organic-air }} f_{\text {organic }}+x_{\text {water-air }} \theta+x_{\text {air-air }} f_{\text {air }}}
$$

which is similar to Eq. (9.11) with an empirical correction factor (Ten Berge, 1986).

In the case that neither water nor air can be considered as the continuous phase $\left(\theta_{\mathrm{dry}}<\theta<\theta_{\text {wet }}\right) \lambda_{\text {heat }}$ is found by interpolation between values at the wet and dry limits:

$$
\lambda_{\text {heat }}(\theta)=\lambda_{\text {heat }}\left(\theta_{\text {dry }}\right)+\frac{\lambda_{\text {heat }}\left(\theta_{\text {wet }}\right)-\lambda_{\text {heat }}\left(\theta_{\text {dry }}\right)}{\theta_{\text {wet }}-\theta_{\text {dry }}}\left(\theta-\theta_{\text {dry }}\right)
$$

The values of $\theta_{\text {dry }}$ and $\theta_{\text {wet }}$ are taken as 0.02 and $0.05 \mathrm{~cm}^{3} \mathrm{~cm}^{-3}$, respectively. We refer to De Vries (1975), Ten Berge (1986) and Moene and Van Dam (2014) for more information on the calculation of $\lambda_{\text {heat. }}$ 
Table 9.2 Shape and weight factors for different components in water and air phases, as used for thermal conductivity calculations (Ashby et al., 1996).

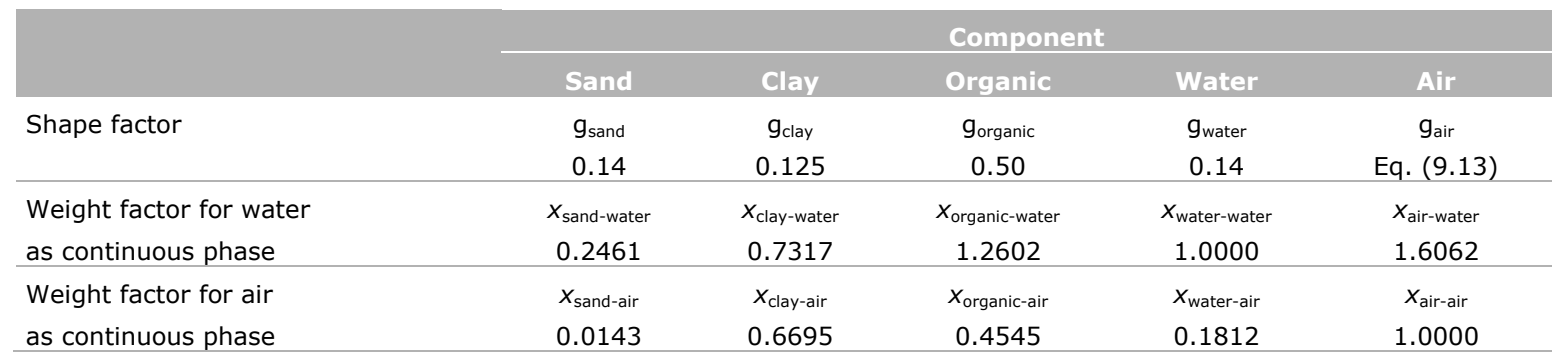

At the soil surface either the daily average air temperature $T_{\text {avg }}$ or measured soil surface temperatures can be used as a boundary condition. In case of a snow layer and the use of $T_{\text {avg, }}$ SWAP will adjust $T_{\text {avg }}$ as described in Chapter 10. At the bottom of the soil profile either soil temperatures can be specified or $q_{\text {heat }}=0.0$ can be selected. The latter option is valid for large soil columns.

Application of Eq. (9.4) to each node and including the boundary conditions at the top and bottom of the soil profile, results in a tri-diagonal system of equations, as shown in Appendix 6. SWAP solves the equations with $L U$-decomposition for tridiagonal systems (Press et al., 1989).

\subsection{Analytical solution}

If the values of $\lambda_{\text {heat }}$ and $C_{\text {heat }}$ are considered to be constant within the discretized soil layer and time interval, the soil thermal diffusivity $D_{\text {heat }}\left(\mathrm{cm}^{2} \mathrm{~d}^{-1}\right)$ can be defined as:

$$
D_{\text {heat }}=\frac{\lambda_{\text {heat }}}{\mathrm{C}_{\text {heat }}}
$$

and Eq. (9.3) simplifies to:

$$
\frac{\partial T}{\partial t}=D_{\text {heat }} \frac{\partial^{2} T}{\partial z^{2}}
$$

This partial differential equation can be solved for simple boundary conditions, assuming $D_{\text {heat }}$ constant or very simple functions for $D_{\text {heat }}$ (Van Wijk, 1966; Feddes, 1971; Wesseling, 1987). A commonly used top boundary condition is a sinusoidally varying soil surface temperature:

$$
T(0, t)=T_{\text {mean }}+T_{\text {ampl }} \sin \left(1 / 2 \pi+\omega\left(t-t_{\text {max }}\right)\right)
$$

where $T_{\text {mean }}$ is the mean yearly temperature $\left({ }^{\circ} \mathrm{C}\right), T_{\text {ampl }}$ is the wave amplitude $\left({ }^{\circ} \mathrm{C}\right), \omega=2 \pi / \tau$ is the angular frequency, where $\tau$ is the period of the wave (d), $t$ is time (d) starting January $1^{\text {st }}$ and $t_{\max }$ equals $t$ when the temperature reaches its maximum. In case of a semi-infinite soil profile with constant $D_{\text {heat }}$ and using Eq. (9.19), the solution to Eq. (9.18) is:

$$
T(z, t)=T_{\text {mean }}+T_{\text {ampl }} \mathrm{e}^{\frac{z}{d_{\text {temp }}}} \sin \left(1 / 2 \pi+\omega\left(t-t_{\text {max }}\right)+\frac{z}{d_{\text {temp }}}\right)
$$

where $d_{\text {temp }}$ is the damping depth $(\mathrm{cm})$, which equals:

$$
d_{\text {temp }}=\sqrt{\frac{2 D_{\text {heat }}}{\omega}}
$$

Equation (9.20) can be used for daily or yearly fluctuations. Measured values of $D_{\text {heat }}$ for various dry and wet soils are given in Table 9.3. Figure 9.1 gives an example of calculated soil temperatures for a dry and wet sand soil. The sinusoidal temperature fluctuations at each depth are reduced in amplitude and delayed in time with respect to the top boundary condition. Although the heat capacity of wet sand is higher than of dry sand, the temperature wave in the wet sand is less attenuated due to the higher thermal conductivity. 
Table 9.3 Thermal diffusivity $D_{\text {heat }}\left(\mathrm{cm}^{2} \mathrm{~d}^{-1}\right)$ for various dry and wet soils (Jury et al., 1991).

\begin{tabular}{llllllll} 
& \multicolumn{1}{c}{ Sand } & \multicolumn{3}{c}{ Loam } & \multicolumn{3}{c}{ Clay } \\
Dry & Wet & Dry & Wet & Dry & Wet & Dry & Wet \\
\hline 147 & 380 & 156 & 518 & 156 & 320 & 112 & 104 \\
\hline
\end{tabular}

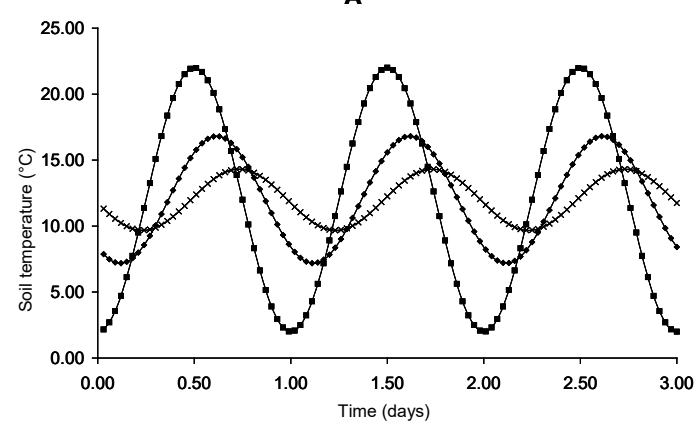

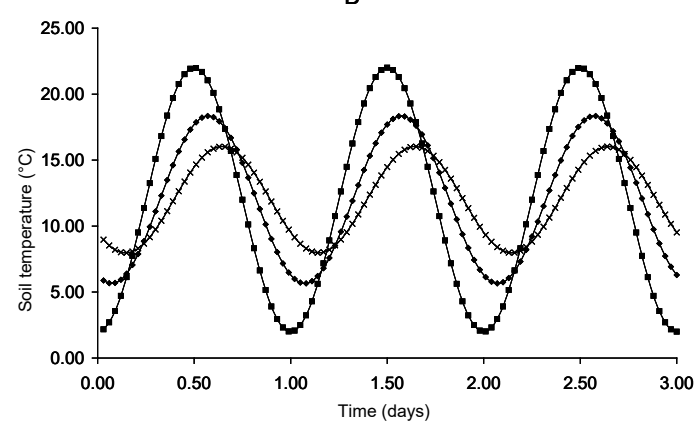

Figure 9.1 Calculated soil temperatures at depths $z=0, z=-5$ en $z=-10 \mathrm{~cm}$ for a dry $(A)$ and a wet $(B)$ sand soil. The following input date were used: $T_{\text {mean }}=12^{\circ} \mathrm{C}, T_{\text {ampli }}=10{ }^{\circ} \mathrm{C}, \tau=1 \mathrm{~d}$, $t_{\max }=0.5 \mathrm{~d}$ en $D_{\text {heat }}=147$ (droog) en 380 (nat) $\mathrm{cm}^{2} \mathrm{~d}^{-1}$.

\subsection{User instructions}

Box 9.1 lists the input data for heat transport. When the analytical method is used, the parameters describing the soil surface temperature wave and the demping depth should be specified. The damping depth might be derived from Eq. (9.21) and the thermal diffusivity values from Table 9.3. When the numerical method is used, information should be given of the soil texture, initial soil temperatures and type of bottom boundary condition.

In case top soil temperatures are provided in a separate file (SwTopHea $=2$ ), the file TSOILFILE must have the extension .TSS. The files should contain two data columns: the first column (with header DATET) contains the date (dd-mm-yyyy), and the second column (header TTOP) contains the soil surface temperature $\left({ }^{\circ} \mathrm{C}\right)$. 


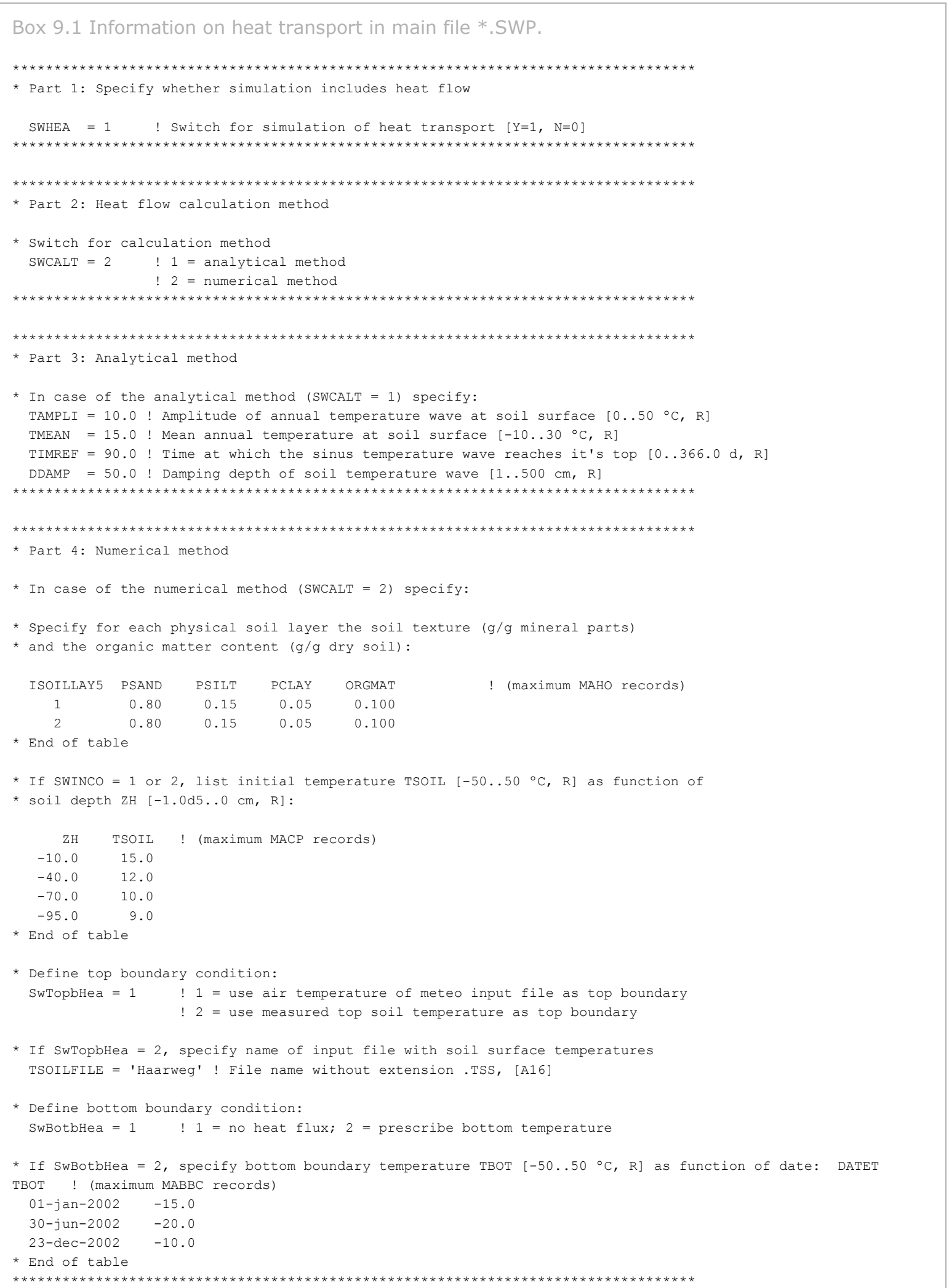




\section{Snow and frost}

SWAP contains separate switches for simulating snow and frost conditions. When both switches are turned off, in the simulations precipitation and soil water remain unfrozen at temperatures below zero ${ }^{\circ} \mathrm{C}$. Snow is described in Section 10.1 and frost in Section 10.2.

\subsection{Snow}

When the snow option is switched on, SWAP simulates snowfall, accumulation of snow in a snowpack and the water balance of the snowpack. The present approach is quite simple and consists of the most basic processes, including the insulating effect of snow on energy transport. Simulation of snowfall and water balance of the snowpack is performed on a daily basis. Snowfall and snowpack are described in the next two Sections.

\subsubsection{Snowfall}

Snowfall occurs when air temperature drops below a threshold value. In that case precipitation falls partly or completely as snow. The division of total precipitation $P\left(\mathrm{~cm} \mathrm{~d}^{-1}\right)$ into snow $P_{\mathrm{s}}\left(\mathrm{cm} \mathrm{d}^{-1}\right)$ and rain $P_{\mathrm{r}}\left(\mathrm{cm} \mathrm{d}^{-1}\right)$ depends on the daily average air temperature. For air temperatures $T_{\mathrm{av}}\left({ }^{\circ} \mathrm{C}\right)$ below the threshold temperature $T_{\text {snow }}\left({ }^{\circ} \mathrm{C}\right)$ all precipitation is snow, while for air temperatures above the threshold temperature $T_{\text {rain }}\left({ }^{\circ} \mathrm{C}\right)$ all precipitation is rain. Between both threshold temperatures the snow fraction $f_{\text {snow }}(-)$ and rain fraction $f_{\text {rain }}(-)$ of the precipitation are obtained by linear interpolation:

$$
\begin{array}{ll}
f_{\text {snow }}=1 & \text { for } T_{\mathrm{av}} \leq T_{\text {snow }} \\
f_{\text {snow }}=\frac{T_{\text {rain }}-T_{\mathrm{av}}}{T_{\text {rain }}-T_{\text {snow }}} \quad \text { for } T_{\text {snow }}<T_{\text {av }}<T_{\text {rain }} \\
f_{\text {snow }}=0 & \text { for } T_{\text {av }} \geq T_{\text {rain }} \\
f_{\text {rain }}=1-f_{\text {snow }} & \\
P_{\mathrm{s}}=f_{\text {snow }} P \text { and } P_{\mathrm{r}}=f_{\text {rain }} P
\end{array}
$$

\subsubsection{Snowpack}

Snow that falls on the soil surface is accumulated in a snowpack, provided that the soil surface temperature is below $0.5^{\circ} \mathrm{C}$. The water balance of the snowpack includes storage, the incoming fluxes snow and rain and the outgoing fluxes melt and sublimation (Figure 10.1) and reads:

$$
S_{\text {snow }}^{t}-S_{\text {snow }}^{t-1}=\left(P_{r}+P_{s}-q_{\text {melt }}-q_{\text {melt }, r}-E_{s}\right) \Delta t
$$

in which $S_{\text {snow }}$ is snow storage at day $t$ or the previous day $t-1$ in $\mathrm{cm}$ water equivalent (cm w.e.), $P_{\mathrm{s}}$ and $P_{\mathrm{r}}$ are the two precipitation terms (cm w.e. $\left.\mathrm{d}^{-1}\right), q_{\text {melt }}$ and $q_{\text {melt,r }}$ are two snow melt terms (cm w.e. $\left.\mathrm{d}^{-1}\right), E_{\mathrm{s}}$ is snow sublimation ( $\mathrm{cm}$ w.e. $\mathrm{d}^{-1}$ ) and $\Delta t$ is the time step of one day. 


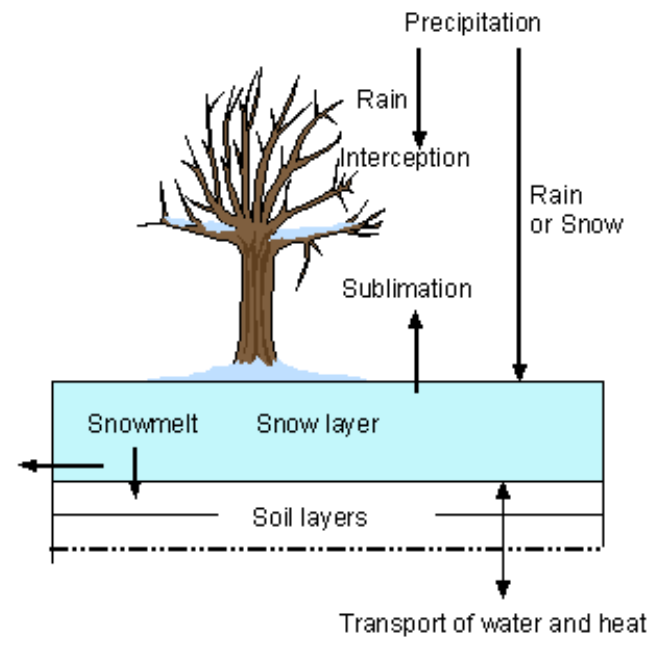

Figure 10.1 Water fluxes to and from the snow layer.

Two forms of snowmelt are included in the model:

1. air temperature rise above a threshold value, according to the 'degree-day model' (Kustas \& Rango, 1994):

$$
\begin{array}{ll}
q_{\text {melt }}=a\left(T_{\mathrm{av}}-T_{\mathrm{b}}\right) & \text { for } T_{\mathrm{av}}>T_{\mathrm{b}} \\
q_{\text {melt }}=0 & \text { for } T_{\mathrm{av}} \leq T_{\mathrm{b}}
\end{array}
$$

where $a$ is the 'degree-day factor' $\left(\mathrm{cm}^{\circ} \mathrm{C}^{-1} \mathrm{~d}^{-1}\right), T_{\mathrm{av}}$ is the daily average air temperature $\left({ }^{\circ} \mathrm{C}\right)$ and $T_{\mathrm{b}}$ is the base temperature $\left({ }^{\circ} \mathrm{C}\right)$ which is set to $0{ }^{\circ} \mathrm{C}$ according to Kustas \& Rango (1994). The value of $a$ can be specified by the user, and ranges usually between 0.35 and $0.60 \mathrm{~cm}^{\circ} \mathrm{C}^{-1} \mathrm{~d}^{-1}$.

2. heat release from rainfall $P_{\mathrm{r}}$ on the snowpack: additional melt will occur due to heat released by splashing raindrops. This snowmelt rate $q_{\text {melt, } r}$ is calculated with (Fernández, 1998; Singh et al., 1997):

$$
\begin{array}{ll}
q_{\text {melt }, \mathrm{r}}=\frac{P_{\mathrm{r}} C_{\mathrm{m}}\left(T_{\mathrm{av}}-T_{\text {snow }}\right)}{L_{\mathrm{f}}} & \text { for } T_{\mathrm{av}}>T_{\text {snow }} \\
q_{\text {melt }, \mathrm{r}}=0 & \text { for } T_{\mathrm{av}} \leq T_{\text {snow }}
\end{array}
$$

where $C_{\mathrm{m}}$ is the heat capacity of water $\left(4180 \mathrm{~J} \mathrm{~kg}^{-1}{ }^{\circ} \mathrm{C}^{-1}\right), L_{\mathrm{f}}$ is the latent heat of fusion (333580 $\mathrm{J} \mathrm{kg}^{-1}$ ) and $T_{\text {snow }}$ is the temperature of the snowpack, which is set to $0{ }^{\circ} \mathrm{C}$.

The melt fluxes leave the snow pack as infiltration into the soil and/or runoff when infiltration capacity of the soil is exceeded.

Snow can evaporate directly into the air, a process called sublimation. The sublimation rate $E_{\mathrm{s}}$ is taken equal to the potential evaporation rate $E_{\mathrm{p}}$ (see Chapter 3 ). When a snow pack exists, the evapotranspiration from the soil and vegetation is set to zero.

A snowpack on top of the soil surface has a large impact on soil temperatures. Because of the low thermal conductivity of snow (0.1-0.4 times thermal conductivity of water), a snowpack can form a perfect insulating layer that will considerably dampen the effects of air temperature on soil temperature. The insulating effect of a snowpack on soil temperature is accounted for by calculating the temperature at the soil surface, the driving force for soil temperature calculations (see Chap. 9), taking into account the thermal conductivity and thickness of the snowpack. Therefore, the surface temperature $T_{\mathrm{ss}}\left({ }^{\circ} \mathrm{C}\right)$ is calculated as a weighted average derived from the distances from the top of the snow cover and the first soil temperature node to the surface and the respective temperatures of air and soil, and thermal conductivities of snow and soil (Granberg et al., 1999): 


$$
T_{\mathrm{ss}}=\frac{T_{1}+a T_{\mathrm{av}}}{1+a}
$$

where:

$$
a=\frac{\lambda_{\text {snow }}\left(\frac{\Delta z_{1}}{2}\right)}{\lambda_{1} \Delta z_{\text {snow }}}
$$

with $\lambda_{1}\left(\mathrm{~J} \mathrm{~cm}^{-1}{ }^{\circ} \mathrm{C}^{-1} \mathrm{~d}^{-1}\right)$ and $\Delta z_{1}(\mathrm{~cm})$ are thermal conductivity and thickness of the first soil compartment. Thermal conductivity of the snowpack $\lambda_{\text {snow }}\left(\mathrm{J} \mathrm{cm}^{-1}{ }^{\circ} \mathrm{C}^{-1} \mathrm{~d}^{-1}\right)$ depends on density $\rho_{\text {snow }}$ $\left(\mathrm{kg} \mathrm{cm}^{-3}\right.$ ) of the snowpack (Granberg et al., 1999):

$$
\lambda_{\text {snow }}=k_{\text {snow }} \rho_{\text {snow }}^{2}
$$

where $k_{\text {snow }}$ is a thermal conductivity parameter for snow $\left(24.71 \cdot 10^{8} \mathrm{~J} \mathrm{~cm}^{5} \mathrm{~kg}^{-2}{ }^{\circ} \mathrm{C}^{-1} \mathrm{~d}^{-1}\right)$. For $\rho_{\text {snow }}$ the average value of $170 \cdot 10^{-6} \mathrm{~kg} \mathrm{~cm}^{-3}$ is taken (Granberg et al., 1999), so that $\lambda_{\text {snow }}$ equals $71.4 \mathrm{~J} \mathrm{~cm}^{-1}$ ${ }^{\circ} \mathrm{C}^{-1} \mathrm{~d}^{-1}\left(15 \%\right.$ of $\left.\lambda_{\text {water }}\right)$.

Thickness of the snowpack $\Delta z_{\text {snow }}(\mathrm{cm})$ is calculated from storage and density of snow, and density of water $\left(1000 \cdot 10^{-6} \mathrm{~kg} \mathrm{~cm}^{-3}\right)$ :

$$
\Delta z_{\text {snow }}=\frac{\rho_{\text {water }}}{\rho_{\text {snow }}} S_{\text {snow }}=\frac{1000}{170} S_{\text {snow }}
$$

\subsection{Frost}

If the option for frost is activated, SWAP simulates freezing of soil water when soil temperature drops below a threshold value $T_{\mathrm{frz}}\left({ }^{\circ} \mathrm{C}\right)$. Soil ice has a sharp impact on water flow and storage in the soil. To simulate this impact in situations where soil ice occurs, some transport parameters are adjusted. This is achieved by using a factor $f_{\mathrm{T}}(z)$, which introduces a correction when soil temperatures are below $T_{\text {frz }}$ at depth $z$. This correction factor is assumed to be linear related to the fraction of soil ice $f_{\text {ice }}(z)(-)$ at depth $z$ :

$$
f_{T}(z)=1-f_{\text {ice }}(z)
$$

where $f_{\text {ice }}(z)$ is the fraction of the free volumetric soil water content (actual water content minus residual water content) at depth $(z)$.

According to measured data from Kujala (1991), $f_{\text {ice }}(z)$ can reasonably well be described by a linear function of soil temperature $T(z)\left({ }^{\circ} \mathrm{C}\right)$ between two threshold temperatures:

$$
\begin{array}{ll}
f_{\text {ice }}(z)=0 & \text { for } T(z) \geq T_{\mathrm{frz}} \\
f_{\text {ice }}(z)=\frac{T_{\mathrm{frz}}-T(z)}{T_{\mathrm{frz}}-T_{\mathrm{mlt}}} & \text { for } T_{\mathrm{mlt}}<T(z)<T_{\mathrm{frz}} \\
f_{\text {ice }}(z)=1 & \text { for } T(z) \leq T_{\mathrm{mlt}}
\end{array}
$$

where $T_{\text {frz }}$ is the temperature below which soil water starts freezing, and $T_{\mathrm{mlt}}$ is the temperature above which soil ice starts melting and below which all soil water except $\theta_{\text {res }}$ is frozen. A value of $T_{\text {frz }}<0{ }^{\circ} \mathrm{C}$ expresses freezing point depression. $T_{\text {frz }}$ and $T_{\text {mlt }}$ are model input with default values of 0 and $-1{ }^{\circ} \mathrm{C}$.

The following parameters are adjusted in case of soil ice:

1. hydraulic conductivity $K$ :

$$
K^{*}(z)=f_{T}(z)\left(K(z)-K_{\min }\right)+K_{\min }
$$


where $K^{*}(z)$ is the adjusted hydraulic conductivity at depth $z\left(\mathrm{~cm} \mathrm{~d}^{-1}\right)$ and $K_{\min }$ is a very small hydraulic conductivity $\left(\mathrm{cm} \mathrm{d}^{-1}\right)$. For $K_{\min }$ a default value is taken of $10^{-10} \mathrm{~cm} \mathrm{~d}^{-1}$;

2. actual crop uptake is reduced as:

$$
S_{\mathrm{a}}(z)=\alpha_{\mathrm{rf}} S_{\mathrm{a}}(z) \quad \text { with } \alpha_{\mathrm{rf}}=0 \quad \text { for } \quad T(z)<0{ }^{0} \mathrm{C}
$$

where $\alpha_{\mathrm{rf}}$ is a multiplication factor for soil temperatures (-);

3. drainage fluxes of all drainage levels:

$$
q_{\text {drain }, i}(z)=f_{T}(z) q_{\text {drain }, i}(z)
$$

where $q_{\text {drain }, i}(z)$ is the drainage flux at depth $z$ from drainage level $i\left(\mathrm{~cm} \mathrm{~d}^{-1}\right)$;

4. bottom flux:

$$
q_{b o t}=f_{T}(z) q_{b o t}
$$

where $q_{b o t}$ is the flux across the bottom of the modelled soil profile and $z$ the bottom depth;

5. boundary fluxes (drainage and bottom) when the available air volume is very low:

When drainage does not occur and the available air volume is very low $\left(<0.01 \mathrm{~cm} \mathrm{~cm}^{-3}\right)$, the bottom flux is reduced to zero.

When drainage occurs and the available air volume is very low $\left(<0.01 \mathrm{~cm} \mathrm{~cm}^{-3}\right)$, the drainage fluxes of frozen soil compartments above the drainage level are reduced to zero.

The (available) air volume in the soil $V_{\text {air }}(\mathrm{cm})$ for a soil profile that becomes saturated equals:

$$
V_{\text {air }}=\sum_{i=n}^{m}\left\{\left(\theta_{s, i}-\theta_{i}\right) \Delta z_{i}\right\}
$$

where $\theta_{s, i}$ is the saturated water content $\left(\mathrm{cm} \mathrm{cm}^{-3}\right), \theta_{i}$ is the actual water content $\left(\mathrm{cm}^{3} \mathrm{~cm}^{-3}\right), i$ is a particular node number, $n$ is the node number of the bottom compartment, $m$ is the node number of the highest soil compartment with a temperature below $T_{\text {mlt }}$ starting to count from the bottom compartment, and $\Delta z_{1}$ is the nodal distance $\left(z_{i}-z_{i+1}\right)$

When a soil compartment is frozen $\left(T(z)<T_{\mathrm{mlt}}\right)$ the pore volume of the total soil profile becomes smaller, because only the compartments below this layer are used in the calculation.

An example is a soil in spring that is melting (Figure 10.2). The lower compartments were never frozen and the melting starts at the soil surface. It is possible that the first 4 compartments have melted and only the compartments 5 - 8 are frozen. Now the air volume is only calculated for compartments $n$ (bottom) to $m=5$ (frozen). The following is then valid:

- when drainage does not occur and the available air volume is very low $\left(<0.01 \mathrm{~cm} \mathrm{~cm}^{-3}\right)$, the bottom flux is reduced to zero;

- when drainage does occur and the available air volume is very low $\left(<0.01 \mathrm{~cm} \mathrm{~cm}^{-3}\right)$, the drainage fluxes of all drainage systems, that have a drainage level above the lowest frozen soil compartment, are reduced to zero.

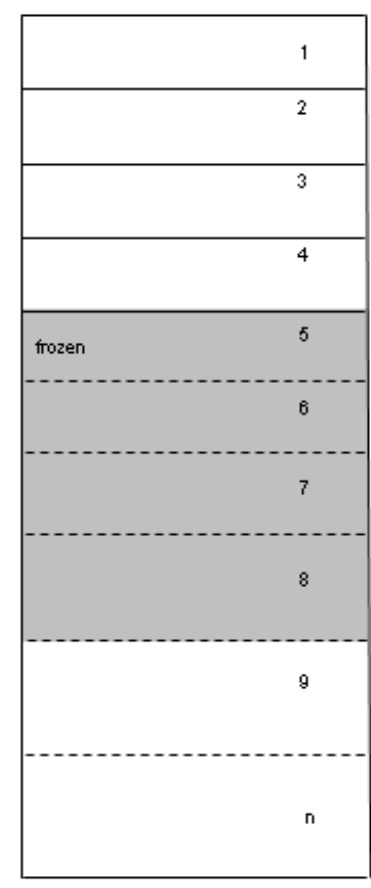

Figure 10.2 Partly frozen soil profile. 


\subsection{User instructions}

Obviously the options for snow and frost can only be used when the soil temperature simulation is activated.

For the snow option, the two threshold temperatures $T_{\text {rain }}$ and $T_{\text {snow, }}$ the initial storage of snow at the beginning of the simulations $S_{\text {snow }}$ and the 'degree-day factor' a are required as model input (Box 10.1).

The frost option requires input for the two threshold temperatures $T_{\mathrm{frz}}$ and $T_{\mathrm{mlt}}$ (Box 10.1).

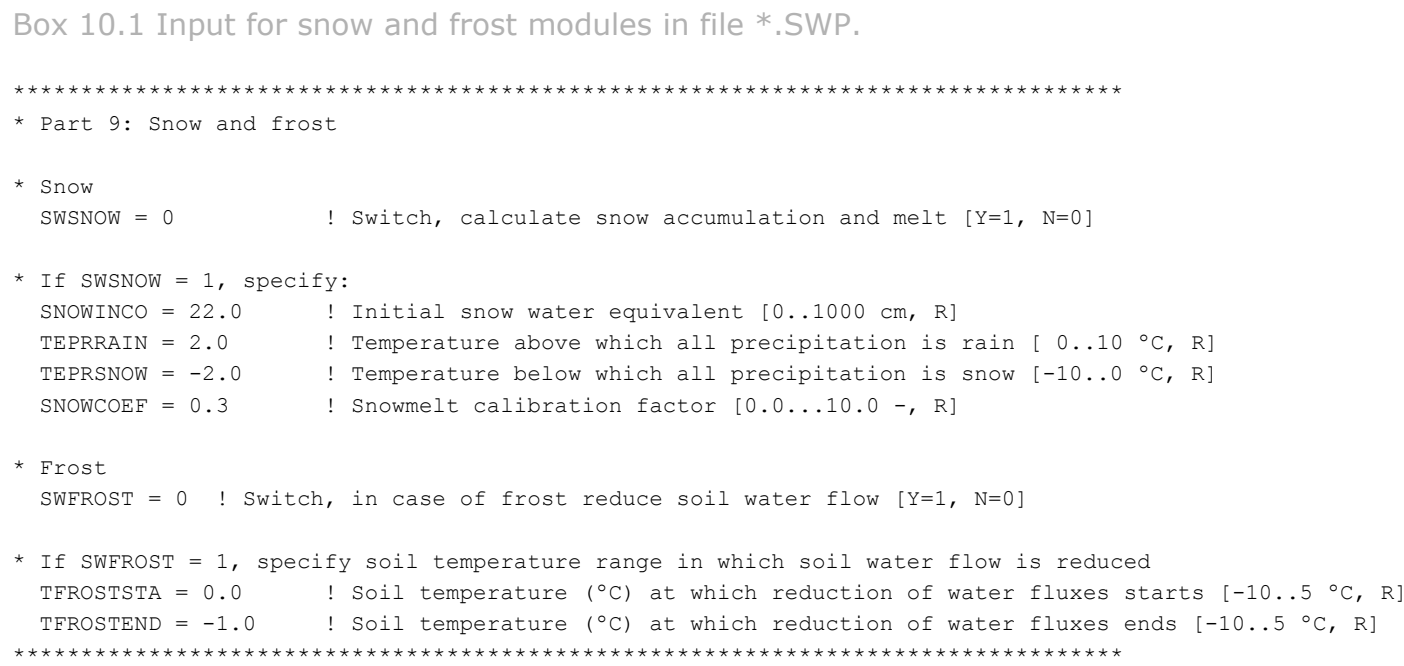




\section{Irrigation}

The SWAP agrohydrological simulations are useful to develop optimal irrigation schedules by evaluating various application strategies.

Irrigation strategies may be applied with a fixed or a scheduled regime. The fixed regime is defined by the time and depth of irrigation application (Par. 11.1).

The scheduled regime is defined by different criteria for time and depth of an irrigation application (Par. 11.2). A combination of a fixed and a scheduled regime is also possible. This regime allows the evaluation of water productivity in relation to several degrees of water stress.

Irrigation or flooding may also be simulated using supply of water from a nearby field or from surface waters (Par. 11.3).

This chapter ends with a description of input requirements (Par. 11.4).

\subsection{Fixed irrigation regime}

At user-defined dates a fixed application depth may be applied as an observed gross irrigation dose $\left(I_{g}\right)$. Interception of irrigation water may occur, dependent on the type of application (surface irrigation or sprinkling):

$$
I_{n}=I_{g}-E_{i}
$$

where $I_{n}$ is the net amount of irrigation water $(\mathrm{cm} / \mathrm{d}), I_{g}$ is the gross given amount of fixed irrigation water $(\mathrm{cm} / \mathrm{d}), E_{i}$ is the amount of intercepted irrigation water $(\mathrm{cm} / \mathrm{d})$. The interception irrigation water $\left(E_{i}\right)$ is assumed to evaporate at the same day as the irrigation occurs.

\subsection{Scheduled irrigation regime}

A specific combination of timing and depth criteria is valid from a user-defined date in the growing season until the end of crop growth. Both timing and depth criteria may be defined as a function of crop development stage. Scheduled irrigation only occurs when a crop is present.

\subsubsection{Timing criteria}

For the timing of the irrigation schedule one out of five different criteria must be selected:

- Allowable daily stress

- Allowable depletion of readily available water in the root zone

- Allowable depletion of totally available water in the root zone

- Allowable depletion amount of water in the root zone

- Critical pressure head or moisture content at sensor depth

At the start of each day the selected criterion is evaluated based on state variables at the start of the day. The outcome of this evaluation may generate an irrigation event on that same day.

The five different evaluation criteria are explained in Par. 11.2.1.1 - 11.2.1.5.

In addition the user may prescribe a fixed or a minimum interval between irrigated applications

(Par. 11.2.1.6 and 11.2.1.7).

\subsubsection{Allowable daily stress}

The level of soil water shortage by drought and salinity stress may be diagnosed from a threshold defined by the ratio of reduced transpiration $T_{\mathrm{r}}$ to potential transpiration $T_{\mathrm{p}}$. Irrigation is applied whenever reduced transpiration becomes lower than a limit defined by this threshold: 


$$
T_{\mathrm{r}} \leq f_{1} T_{\mathrm{p}}
$$

where $T_{\mathrm{r}}$ is the transpiration reduced by drought and salinity stress $\left(\mathrm{cm} \mathrm{d}^{-1}\right), T_{\mathrm{p}}$ is the potential transpiration $\left(\mathrm{cm} \mathrm{d}^{-1}\right), f_{1}(-)$ is a user defined factor for allowable daily stress.

\subsubsection{Allowable depletion of readily available water}

In order to optimize irrigation scheduling where irrigation is always secured before conditions of soil moisture stress occur, the maximum amount of depletion of readily available water in the root zone can be specified. Irrigation is then applied whenever the water depletion exceeds fraction $f_{2}$ of the readily available water amount:

$$
\left(U_{\text {field }}-U_{\mathrm{a}}\right) \geq f_{2}\left(U_{\text {field }}-U_{\mathrm{h} 3}\right)
$$

where $U_{\mathrm{a}}(\mathrm{cm})$ is the actual water storage in the root zone, $U_{\text {field }}(\mathrm{cm})$ is the root zone water storage at $h=$ given value for field capacity, and $U_{\mathrm{h} 3}(\mathrm{~cm})$ is the root zone water storage at $h=h_{3}$, the pressure head from where root water extraction starts being reduced due to drought stress, $f_{2}(-)$ is a userdefined depletion fraction.

$U_{\mathrm{a}}$ is calculated by integrating numerically the water content in the rooting layer. For deficit irrigation purposes, stress can be allowed by specifying $f_{2}>1$.

\subsubsection{Allowable depletion of totally available water}

Depletion of water in the root zone can also be evaluated relative to the total amount of water available in the root zone as given by the difference between the field capacity and the wilting point. Irrigation is then applied whenever the depletion of water in the root zone exceeds fraction $f_{3}(-)$ of the available water:

$$
\left(U_{\text {field }}-U_{\mathrm{a}}\right) \geq f_{3}\left(U_{\text {field }}-U_{\mathrm{h} 4}\right)
$$

where $U_{\mathrm{h} 4}(\mathrm{~cm})$ is the root zone water storage at $h=h_{4}$, the pressure head at which root water extraction is reduced to zero, $f_{3}(-)$ is a user-defined factor depletion fraction.

\subsubsection{Allowable depletion of field capacity water}

In case of high-frequency irrigation systems (drip) it may be useful to specify the maximum amount of water that may be extracted below field capacity, $\Delta U \max (\mathrm{cm})$. Irrigation is then applied if:

$$
U_{\mathrm{a}} \leq U_{\text {field }}-\Delta U_{\max }
$$

\subsubsection{Critical pressure head or moisture content}

The user may specify a soil moisture threshold value $\theta_{\min }\left(\mathrm{cm}^{3} \mathrm{~cm}^{-3}\right)$ or pressure head threshold value $h_{\min }(\mathrm{cm})$ and a corresponding depth for which the threshold values are valid.

This option may be used to simulate irrigation with automated systems relying on soil moisture measurements. Irrigation is then applied whenever a threshold is exceeded:

$$
\theta_{\text {sensor }} \leq \theta_{\text {min }} \quad \text { or } \quad h_{\text {sensor }} \leq h_{\text {min }}
$$

where $\theta_{\text {sensor }}$ and $h_{\text {sensor }}$ are the sensor values for soil moisture and pressure head, which are simulated by SWAP.

In addition the user may specify a salinity threshold value above which over-irrigation is applied to decrease the high salinity concentrations.

\subsubsection{Fixed interval}

By default an irrigation interval has a minimum of one day and the length of the interval is variable and determined by the moment when one of the previously mentioned timing criteria becomes valid. The user may optionally choose a fixed interval of one week between possible irrigation events. Irrigation events occur weekly during crop growth when the required amount of water to bring the rootzone to field capacity exceeds a given threshold value. This threshold value is input to the model. 


\subsubsection{Minimum interval}

The length of the interval between irrigation events may also be variable and be determined by the moment when one of the timing criteria becomes valid.

The user may select this option in addition to one of the previous five criteria (Par. 11.2.1.1 11.2.1.5) to have a minimum time interval between irrigation applications.

\subsubsection{Depth criteria}

Scheduled irrigation results in gross irrigation depths. Interception of irrigation water may occur in case of sprinkling irrigation:

$$
I_{n}=I_{g}-E_{i}
$$

Two option are available for the amount of irrigation:

- An amount which increases root zone soil moisture to field capacity

- A fixed amount

The actual amount of the application may be limited to a minimum and a maximum amount. Both criteria are explained in more detail hereafter.

\subsubsection{Back to Field Capacity}

The soil water content in the root zone is brought back to field capacity. This option may be useful in case of sprinkler and micro irrigation systems, which allow variation of irrigation application depth. An additional irrigation amount $(\mathrm{d} I)$ can be defined to leach salts, while the user may define a smaller irrigation amount when rainfall is expected. This amount is added to field capacity when the rain amount is larger than a specified threshold value (see Box 11.4).

\subsubsection{Fixed irrigation depth}

A specified amount of water is applied. This option applies to most gravity systems, which allow little variation in irrigation application depth.

\subsubsection{Limited depth}

With this option enabled the calculated irrigation depth $I_{g}$ will be limited to the range:

$$
I_{g, \min } \leq I_{g} \leq I_{g \text { max }}
$$

where $I_{g, \min }$ and $I_{g, \max }$ are the specified minimum and maximum irrigation amount values $(\mathrm{mm})$, respectively.

\section{$11.3 \quad$ Flooding}

Both fixed and scheduled irrigation may result in flooding conditions. Flooding may also be generated by increasing surface water levels or by adding water from a nearby field (runon). The option for input of surface water levels is described in Chapter 5 (Surface water management). The option for runon for nearby fields as input to the model is described in Par. 4.5.1 (Surface Runoff).

\subsection{User instructions}

\subsubsection{Fixed irrigation regime}

Fixed irrigation depths must be entered in the *.SWP file (Box 11.1). 


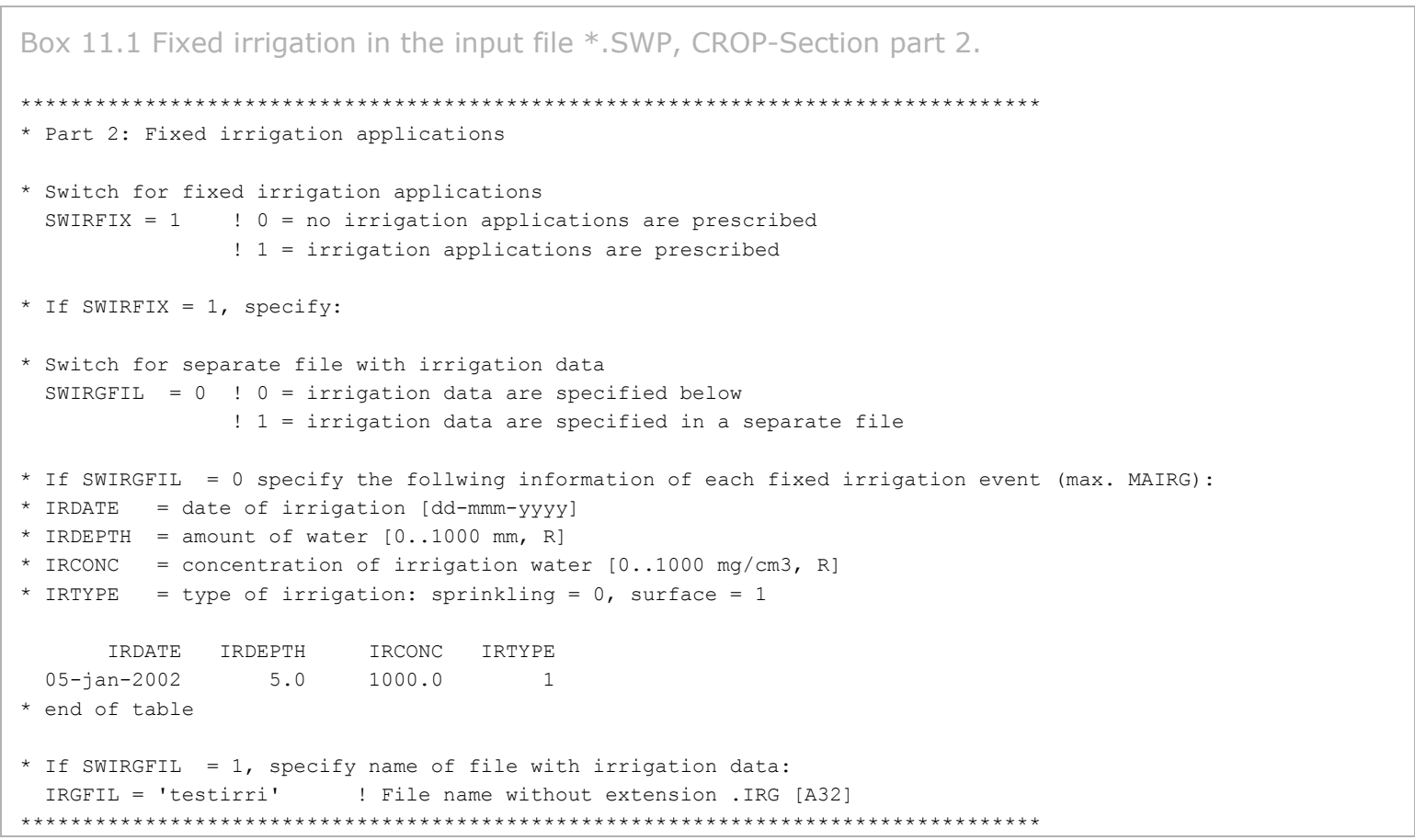

\subsubsection{Scheduled irrigation}

Scheduled irrigation enters the model by means of general, timing and depth criteria in the .CRP-file, see respectively Boxes 11.2, 11.3 and 11.4.

\section{Box 11.2 Scheduled irrigation in the input file *.CRP, IRRIGATION SCHEDULING part 1.}

$* * *$ IRRIGATION SCHEDULING SECTION ***

* Part 1: General

SCHEDULE $=0$ ! Switch for application irrigation scheduling $[\mathrm{Y}=1, \mathrm{~N}=0$ ]

* If SCHEDULE $=0$, no more information is required in this input file!

* If SChedule $=1$, continue ....

STARTIRR $=303$ ! Specify day and month at which irrigation scheduling starts [dd $\mathrm{mm}$ ]

ENDIRR = 3112 ! Specify day and month at which irrigation scheduling stops [dd $\mathrm{mm}$ ]

CIRRS $=0.0 \quad$ ! Solute concentration of irrigation water $[0 \ldots 100 \mathrm{mg} / \mathrm{cm} 3, \mathrm{R}]$

ISUAS $=1 \quad$ ! Switch for type of irrigation method:

! $0=$ sprinkling irrigation

! 1 = surface irrigation

* Specify pressure head at field capacity which will be used for irrigation timing options phFieldCapacity $=-100.0$ ! Soil water pressure head at field capacity $[-1000 \ldots 0 \mathrm{~cm}, \mathrm{R}]$

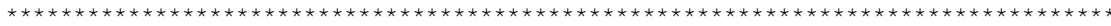


Box 11.3 Scheduled irrigation - timing criteria: in the .CRP-file IRRIGATION SCHEDULING, part 2.

$\star * \star$ IRRIGATION SCHEDULING SECTION $* * *$

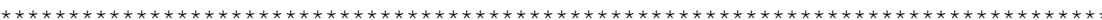

* Part 2: Irrigation time criteria

*** Choose one of the following 5 timing options:

TCS $=1$ ! Switch for timing criterion $[1 \ldots 6-$, I $]$

$! 1$ = Ratio actual/potential transpiration

! 2 = Depletion of Readily Available Water

! 3 = Depletion of Totally Available Water

! 4 = Depletion of absolute Water Amount

! 5 = Pressure head or moisture content

! 6 = Fixed weekly irrigation, bring root zone back to field capacity

* Ratio actual/potential transpiration $($ TCS $=1)$

* If TCS $=1$, specify mimimum of ratio actual/potential transpiration Trel $\left[0 \ldots 1-, R^{2}\right]$

* as function of crop development stage

DVS tc1 Trel ! (maximum 7 records)

$0.0 \quad 0.95$

$2.0 \quad 0.95$

* End of table

* Depletion of Readily Available Water (TCS $=2$ )

* If TCS $=2$, specify minimum fraction of readily available water RAW [0..1 -, R]

* as function of crop development stage

DVS tc2 RAW ! (maximum 7 records)

$0.0 \quad 0.95$

$2.0 \quad 0.95$

* End of table

* Depletion of Totally Available Water (TCS = 3)

* If TCS $=3$, specify minimal fraction of totally available water TAW [0..1 -, R]

* as function of crop development stage

DVS_tc3 TAW ! (maximum 7 records)

$0.0 \quad 0.50$

$2.0 \quad 0.50$

* End of table

* Depletion of absolute Water Amount (TCS $=4$ )

* If TCS $=4$, specify maximum amount of water depleted below field capacity DWA [0..500 mm, R]

* as function of crop development stage

DVS tc4 DWA ! (maximum 7 records)

$0.0 \quad 40.0$

$2.0 \quad 40.0$

* End of table

* Pressure head or Moisture content (TCS = 5), specify

PHORMC $=0$ ! Switch, use either pressure head $($ PHORMC $=0)$ or water content $($ PHORMC $=1)$

DCRIT $=-30.0 !$ Depth of the sensor $[-100 \ldots 0 \mathrm{~cm}, \mathrm{R}]$

* Also specify critical pressure head $[-1 \mathrm{~d} 6 \ldots-100 \mathrm{~cm}, \mathrm{R}]$ or moisture content $[0 \ldots 1 \mathrm{~cm} 3 / \mathrm{cm} 3, \mathrm{R}]$

* as function of crop development stage

DVS tc5 Value tc5

$0.0-1000.0$

$2.0-1000.0$

* End of table

* In case $\operatorname{TCS}=5$, over-irrigation can be applied if the salinity concentration exceeds a threshold salinity

* Switch for over-irrigation:

SWCIRRTHRES $=0 \quad ! \quad 0=$ No over-irrigation

! 1 = Apply over-irrigation

* If SWCIRRTHRES $=1$, specify:

CIRRTHRES $=8.0 \quad$ ! Threshold salinity conc above which over-irrigation occurs $[0 . .100 \mathrm{mg} / \mathrm{cm} 3, \mathrm{R}]$

PERIRRSURP $=10.0$ ! Over-irrigation as percentage of the usually scheduled irrigation depth [0...100 $\%$ R]

* Fixed weekly irrigation, root zone back to field capacity (TCS $=6$ ), specify

* Threshold value for weekly irrigation; only irrigate when soil water deficit is larger than threshold

IRGTHRESHOLD $=1.0 \quad$ ! threshold value $[0.20 \mathrm{~mm}, \mathrm{R}]$

* Switch for minimum time interval between irrigation applications

TCSFIX $=0 \quad$ ! $0=$ no minimum time interval

$1 \quad 1=$ define minimum time interval

* If TCSFIX $=1$, specify:

IRGDAYFIX $=7$ ! Minimum number of days between irrigation applications $\left[\begin{array}{llll}1.366 & d & \text { I }\end{array}\right]$ 
Box 11.4 Scheduled irrigation - depth criteria: in the .CRP-file IRRIGATION SCHEDULING, part 3.

* Part 3: Irrigation depth criteria

* Choose one of the following two options for irrigation depth:

DCS $=1 \quad ! 1=$ Back to Field Capacity

! 2 = Fixed Irrigation Depth

* Back to Field Capacity (DCS $=1$ )

* If DCS $=1$, specify amount of under $(-)$ or over $(+)$ irrigation dI $[-100 \ldots 100 \mathrm{~mm}, \mathrm{R}]$,

* as function of crop development stage $[0 . .2, \mathrm{R}]$ DVS_dc1 dI (maximum 7 records)

$0.0 \quad 10.0$

$2.0 \quad 10.0$

* End of table

RAITHRESHOLD $=10.0$ ! When rainfall exceeds RAITHRESHOLD, irr. is reduced with rainfall [0..1000 cm, R]

* Fixed Irrigation Depth (DCS $=2$ )

* If DCS $=2$, specify fixed irrigation depth FID $[0.400 \mathrm{~mm}, \mathrm{R}]$,

* as function of crop development stage [0..2, R]

DVS_dc2 FID ! (maximum 7 records)

$0.0 \quad 60.0$

2.060 .0

* End of table

* Select minimum and maximum of irrigation depths:

dcslim $=0 \quad$ ! Switch, limit range irrigation depth $[Y=1, N=0]$

* If deslim $=1$, specify:

irgdepmin $=10.0 \quad$ ! Minimum irrigation depth $[0 . .100 \mathrm{~mm}, \mathrm{I}]$

irgdepmax $=80.0 \quad !$ Maximum irrigation depth $[$ irgdepmin..1d7 mm, I] 


\section{Case studies}

\subsection{Hupsel Brook}

The Hupsel Brook catchment has been a well-known field site for hydrological studies since the 1960 s. It has been used for studies on evapotranspiration (Stricker and Brutsaert, 1978), soil physical properties (Hopmans and Stricker, 1989), relations between flow routes and water quality (Van den Eertwegh, 2002; Rozemeijer et al., 2010; Van der Velde et al., 2012) and rainfall-runoff modelling (Bierkens and Puente, 1990; Brauer et al., 2014). The catchment of $6.5 \mathrm{~km}^{2}$ is slightly sloping (0.8\%). Its soil consists of a loamy sand layer (with some clay, peat and gravel) of 0.2 to $10 \mathrm{~m}$ thickness on an impermeable clay layer of more than 20 m thickness (Brauer et al., 2014).

Period 1 Jan 2002 - 31 Dec 2004, weather data meteorological station Hupsel Brook Land use: maize (1 May 2002 - 15 Oct 2002), potato (10 May 2003 - 29 Sep 2003), grass (1 Jan 2004 - 31 Dec 2004)

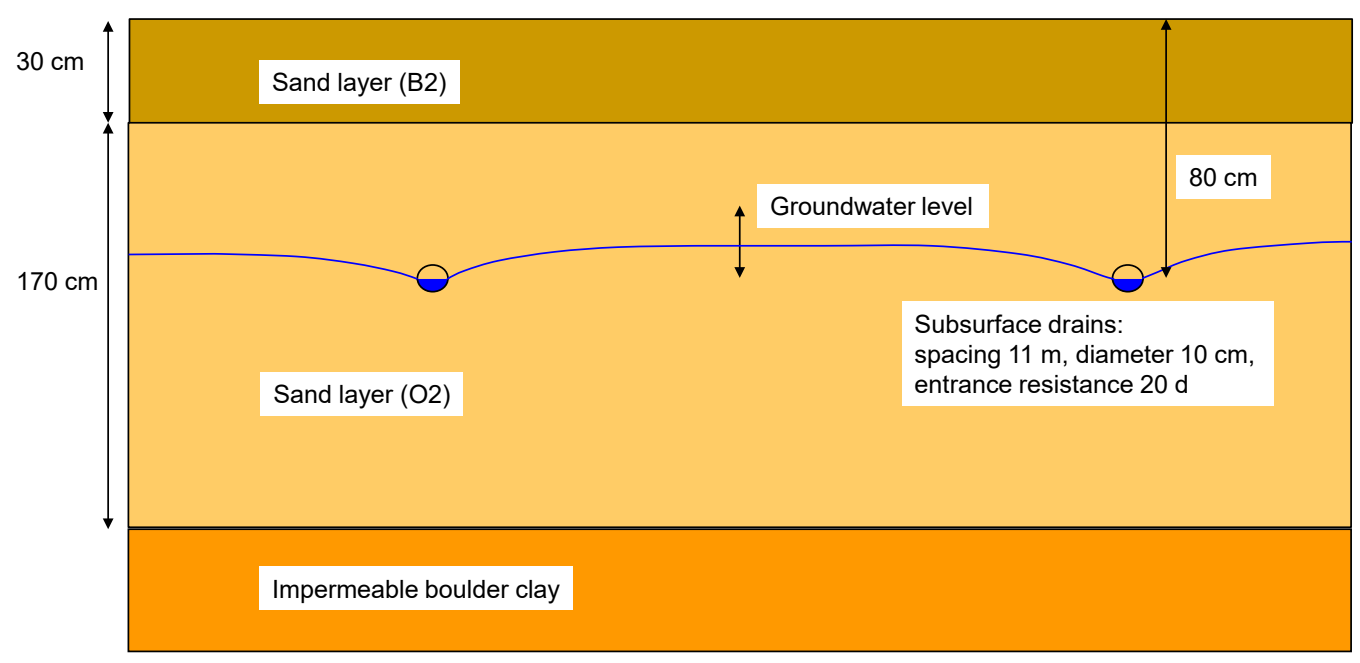

Figure 12.1 Environmental input data of case 1: typical field in Hupsel Brook catchment.

We selected a typical field in the Hupsel Brook catchment to demonstrate SWAP in- and output files. Figure 12.1 shows the environmental settings. The simulation run covers the period 2002-2004 with 3 crops: maize, potato and grass. Each crop is simulated with a different crop module in SWAP: maize with the simple module for a static crop, potato with the WOFOST module for a dynamic crop, and the specialized WOFOST module for grass growth.

Potential evapotranspiration fluxes are calculated with daily meteorological observations and the Penman-Monteith equation. Also daily values for rainfall are used, as in the catchment with its mild slopes and sandy soils no runoff is expected.

The sandy soil profile consists of a top- and sublayer with thicknesses of 30 and $170 \mathrm{~cm}$, respectively. The soil hydraulic functions are derived from the Staring series (Appendix 7): texture B2 for the toplayer and texture $\mathrm{O} 2$ for the sublayer.

At the bottom of the soil profile a layer of boulder clay with low permeability prevents vertical soil water movement. Therefore at the bottom a zero flux condition is specified. Drainage fluxes are calculated for subsurface drains at $80 \mathrm{~cm}$ depth and with a lateral distance of $11 \mathrm{~m}$. 
Initial soil water contents are assumed to be in hydrostatic equilibrium with a groundwater level at 75 $\mathrm{cm}$ depth. On 5 January 2002 a tracer is applied, which leaches in the subsequent years towards the drains.

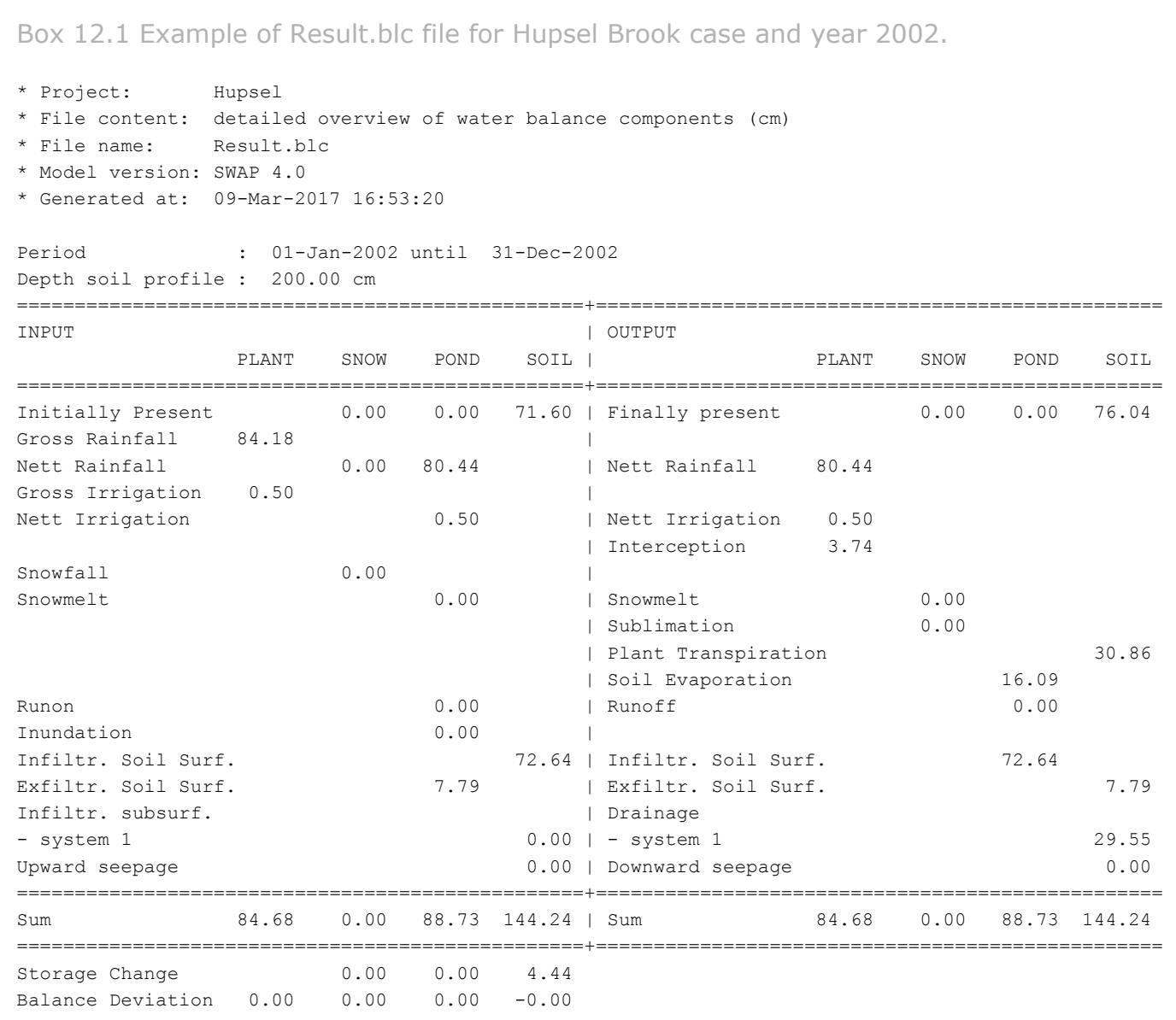

SWAP prints two yearly water balances: a summary (*.BAL) and an extensive (*.BLC). Box 12.1 shows an example of the extensive water balance for the year 2002. In this output file the fluxes are presented between the subdomains plant, snow, pond layer, soil, and their environment, as depicted in Figure 12.2. In addition to the in- and outgoing fluxes, of each subdomain also the water storage and balance is depicted. For instance the ponding layer received $80.44 \mathrm{~cm}$ rain and $7.79 \mathrm{~cm}$ soil water from the first soil compartment. Of this amount $16.09 \mathrm{~cm}$ evaporated towards the atmosphere, and $72.64 \mathrm{~cm}$ infiltrated into the first soil compartment. As both the initial and final storage of the ponding layer are zero, the storage change is also zero. 


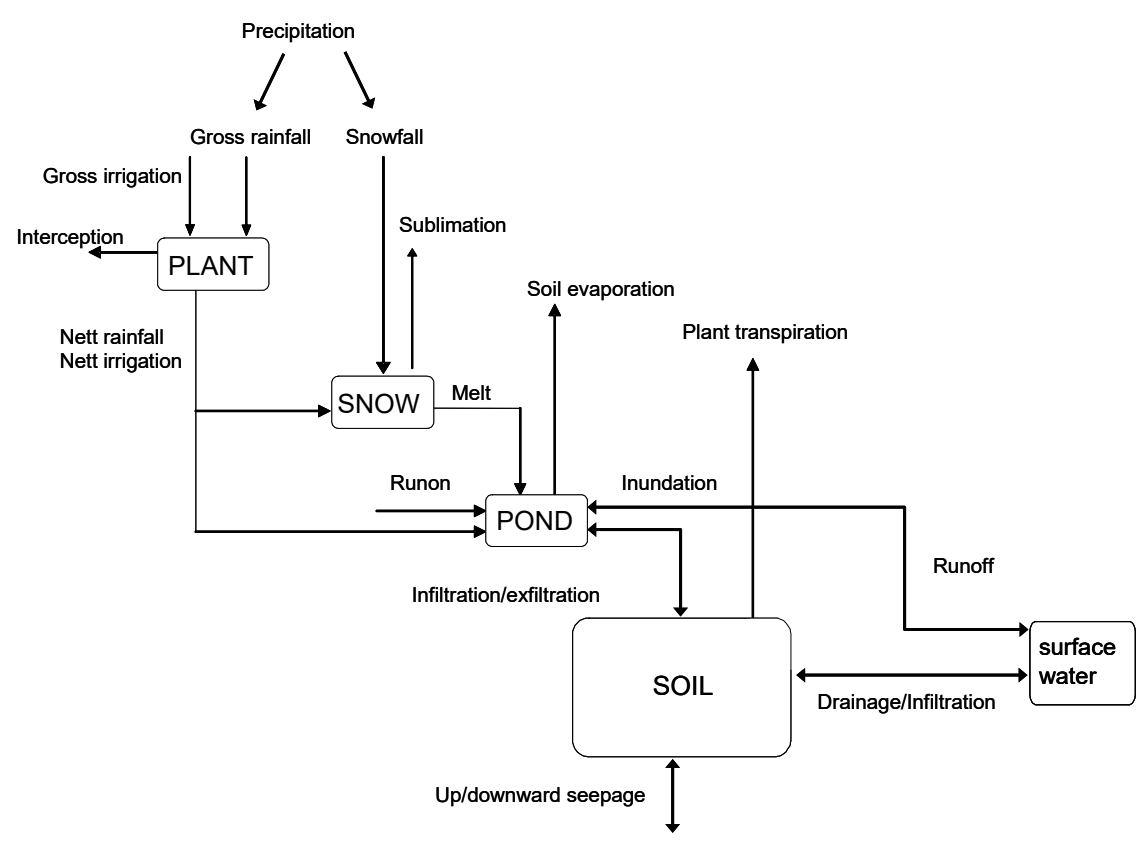

Figure 12.2 Scheme of water fluxes between the subdomains plant, snow, ponding layer, soil and surface water.

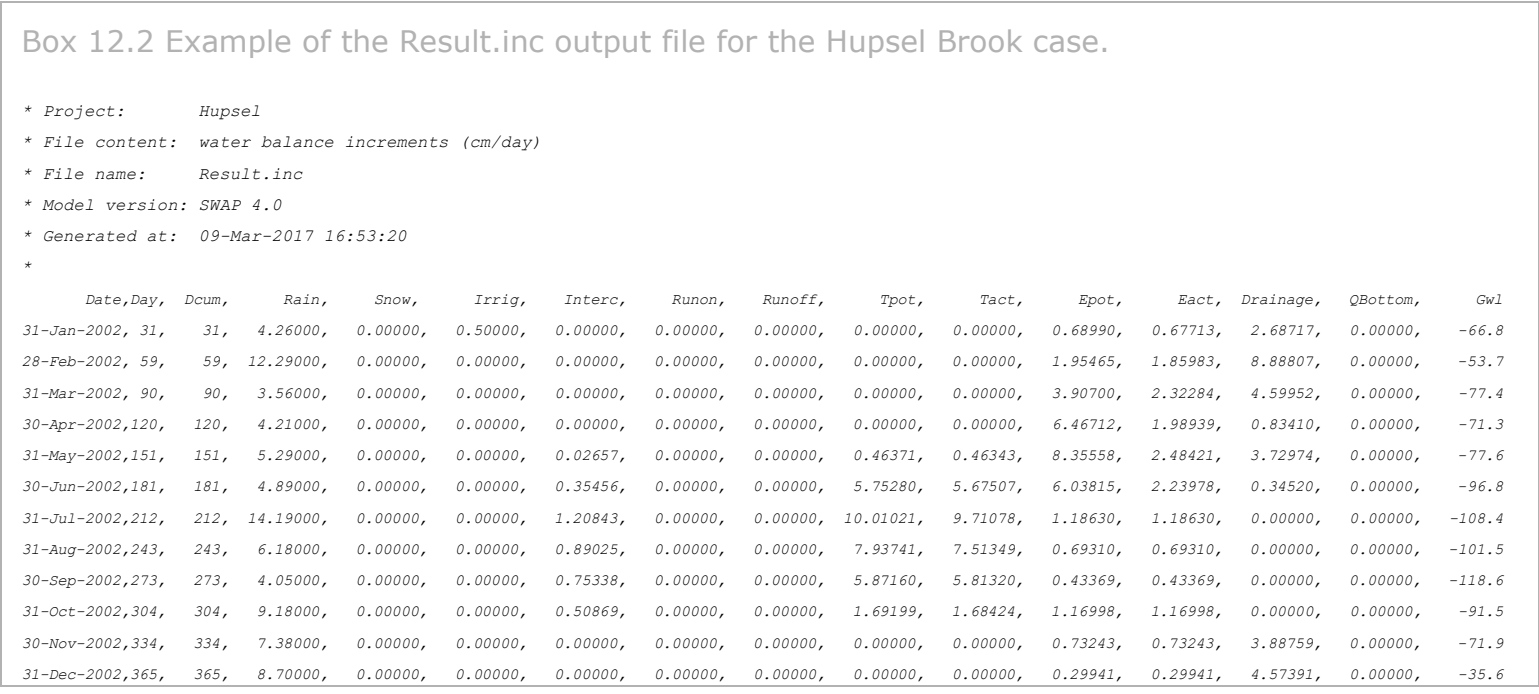

Output intervals may range from 0.001 day to 1 year. In this case output is requested at the end of each month (SWMONTH = 1). SWAP prints both incremental and cumulative water balance fluxes.

Box 12.2 shows the incremental water fluxes for year 2002. The actual transpiration rates are close to the potential transpiration rates due to the high rain amounts in the summer season and the shallow groundwater levels. In May the maize is not yet covering the soil and the actual soil evaporation rate is substantial: $2.48 \mathrm{~cm} / \mathrm{month}$. Drainage mainly occurs in the winter months Jan - Mar and Oct - Dec. In the summer months May and June the soil water storage declines with 1.41 and $3.72 \mathrm{~cm}$, respectively. Due to large rainfall amounts in July, during this month the soil water storage increases with $2.08 \mathrm{~cm}$. At the monthly resolution the simulated groundwater levels fluctuate between $-35.6 \mathrm{~cm}$ (31 Dec) and $-118.64 \mathrm{~cm}$ (30 Sep) with respect to soil surface. 


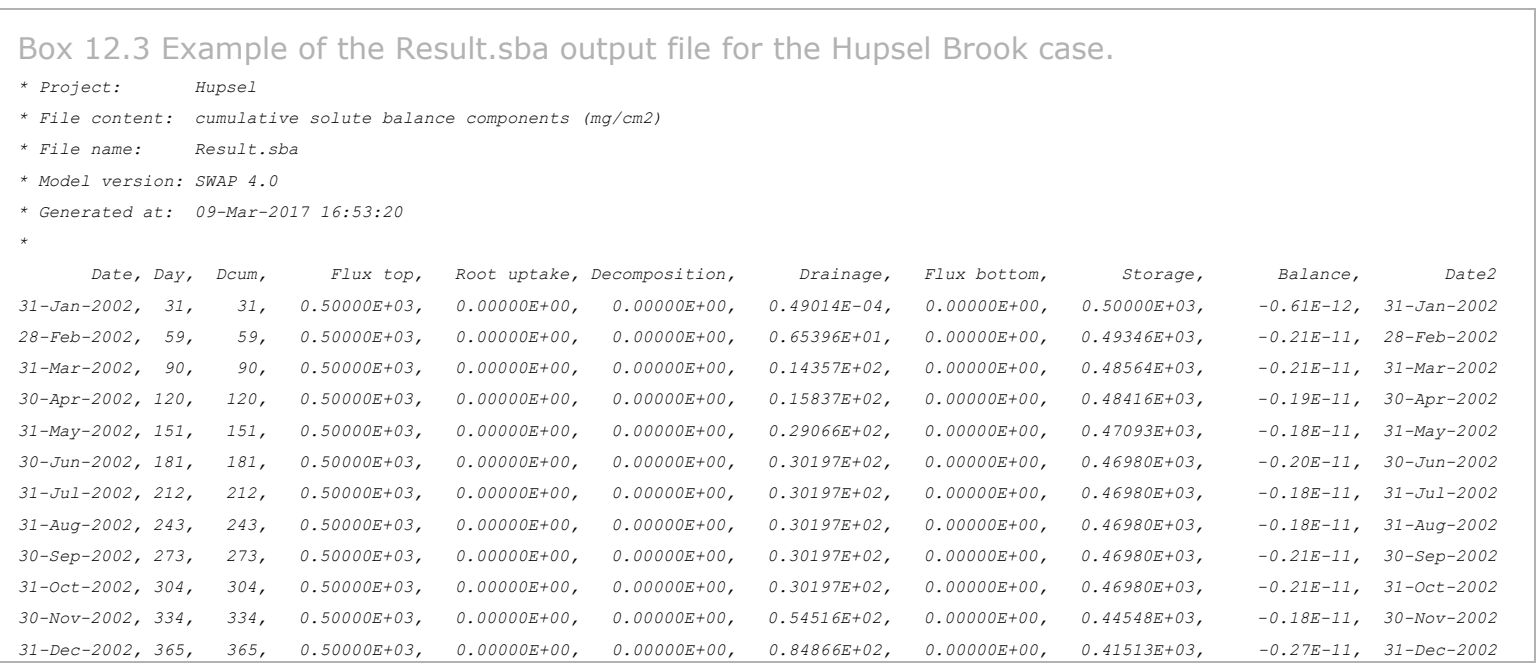

On 5 January 2002 a tracer is applied $\left(500 \mathrm{mg} / \mathrm{cm}^{2}\right)$. Box 12.3 lists the simulated cumulative solute balance fluxes for the year 2002. After one year, $84.87 \mathrm{mg} / \mathrm{cm}^{2}$ solutes have leached towards the drains. The remaining amount, $415.1 \mathrm{mg} / \mathrm{cm}^{2}$ or $83.0 \%$ of the applied dose, is still in the soil profile and groundwater on 31 December 2002.

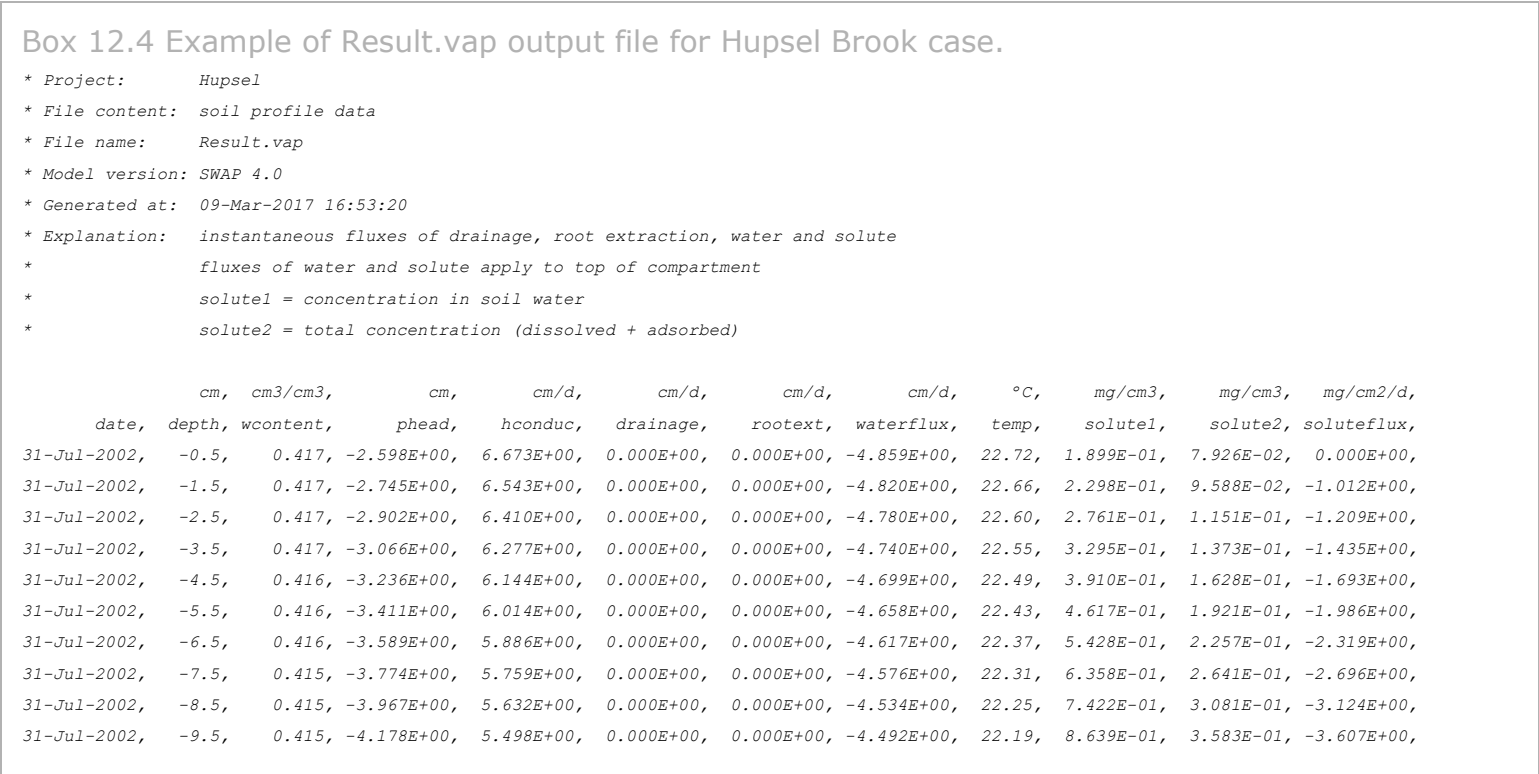

Box 12.4 shows a part of the *.VAP output file with soil profile data on water content, pressure head, hydraulic conductivity, lateral drainage, root water extraction, vertical water fluxes, soil temperature, solute concentrations in soil water and in soil compartment, and solute fluxes. When output intervals are larger than 1 day, as the case here, the data pertain to the situation at the end of the day. Mind that the specified flux rates are valid at the particular date and time; they are not averages of the entire day. 


\subsection{Grass growth}

This case describes growth of grassland and was used by Kroes and Supit (2011) to study the effects of increasing salinity of groundwater, drought and water excess on grass production in The Netherlands. One field of Ruurlo was selected to show the method and some of the options to simulate growth of grassland. It is a field with high fertilization of $600 \mathrm{~kg} / \mathrm{ha} \mathrm{N}$.

Meteorological data are a combination of rainfall from a local weather station and other data (radiation, temperature, windspeed and humidity) from an automatic Dutch national weather station De Bilt (www.knmi.nl).

Grass growth is initiated when a temperature sum of $200^{\circ} \mathrm{C}$ is exceeded.

Growth parameters are similar to arable and grassland crops.

Management has a separate section in the input file (Box 12.5).

This management section in the input file has 4 parts:

- Part 13: General : in this part the sequence of grass growth is defined: mowing and/or grazing has to be choosen.

- Part 14: Grazing settings : this part defines the grazing amount, timing and uptake of lifestock.

- Part 15: Mowing settings : this part defines timing and thresholds for mowing.

- Part 16: Management not modeled (like impact of pests, diseases, nutrients).

The remainder of the input file for grassland crops (Part 17-19) is about irrigation and is explained in Chapter 11.

The ouput files of crop-simulation results have a file extension "crp". These files have a uniform header which is described in Appendix 18.

A simulation can be carried out and a graph made with the R-procedure "PlotResult.R" should look like Figure 12.3.

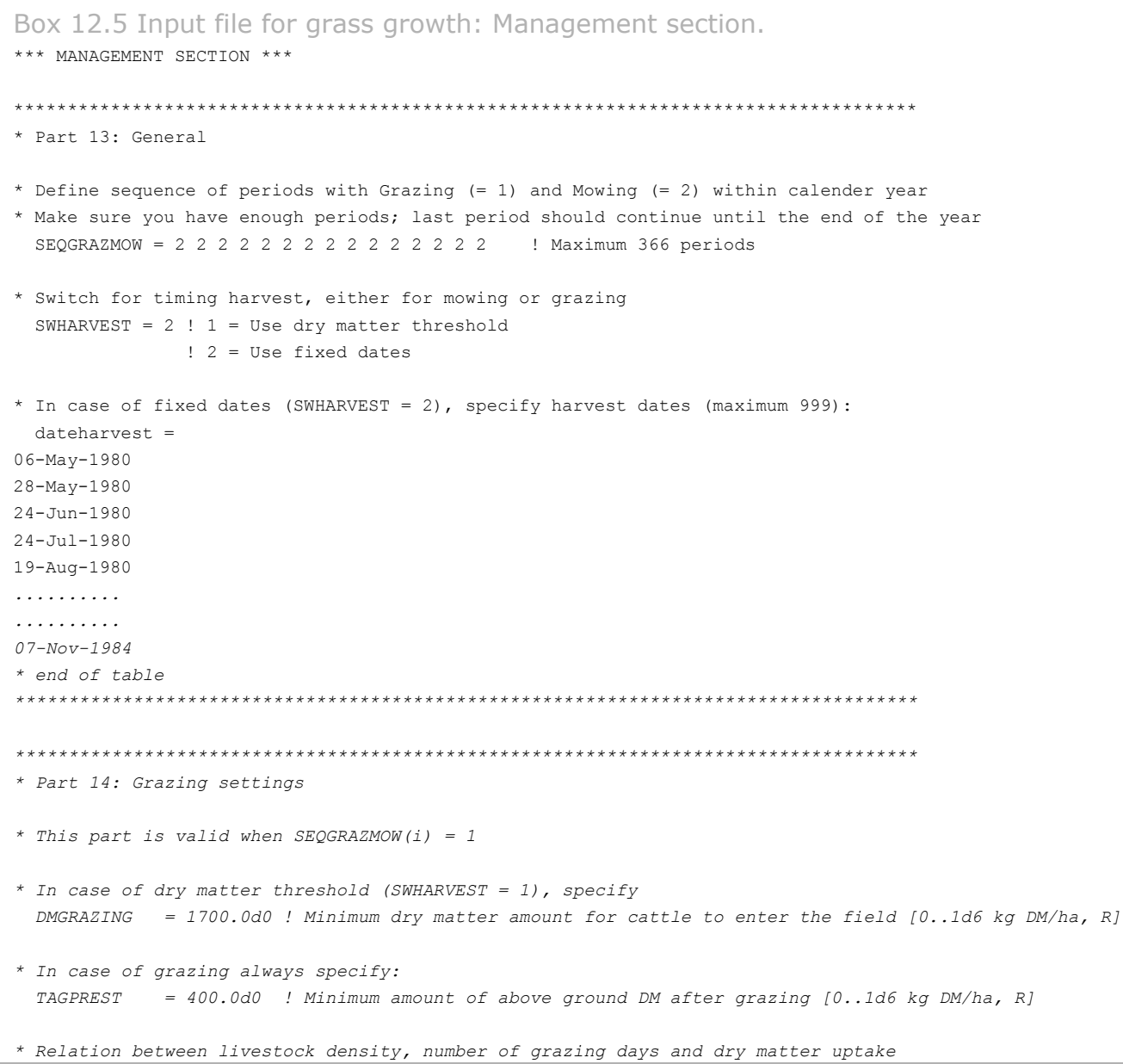


* LSDB = Basic Live Stock Density [0.0.1000.0 LS/ha, R]

* DAYSGRAZING = Maximum days of grazing $[0.0 .366 .0 \mathrm{~d}, \mathrm{R}]$

* UPTGRAZING = Dry matter uptake by grazing $[0.0 .1000 .0 \mathrm{~kg} / \mathrm{ha}, \mathrm{R}](\mathrm{kg} / \mathrm{ha} \mathrm{DM})$

* LOSSGRAZING = Dry matter loss during grazing due to droppings and treading [0.0.1000.0 kg/ha, R] (kg/ha DM)

LSDD DAYSGRAZING UPTGRAZING LOSSGRAZING

$\begin{array}{llll}20.0 & 5.5 & 16.0 & 1.00\end{array}$

$\begin{array}{llll}22.2 & 5.0 & 15.8 & 0.96\end{array}$

$\begin{array}{llll}25.0 & 4.0 & 15.7 & 0.92\end{array}$

$\begin{array}{llll}28.6 & 3.5 & 15.4 & 0.88\end{array}$

$\begin{array}{llll}33.3 & 3.0 & 14.9 & 0.84\end{array}$

$\begin{array}{llll}50.0 & 2.0 & 13.4 & 0.80\end{array}$

* end of table

* Actual livestock density of each grazing period

* SEQNR $=$ number of the sequence period with mowing/grazing $[0.366 \mathrm{~d}$, I]

* LSDA = actual Live Stock Density of the grazing period [0.0.1000.0 LS/ha, R]

* Note: total number of periods should be equal to number of periods in SEQGRAZMOW

$\begin{array}{cc}S E Q N R & \text { LSDA } \\ 1 & 20.0 \\ 2 & 20.0 \\ 3 & 30.0 \\ 4 & 20.0 \\ 5 & 20.0 \\ 6 & 20.0 \\ 7 & 20.0 \\ 8 & 20.0 \\ 9 & 20.0 \\ 10 & 20.0 \\ * \text { end of table }\end{array}$

end of table

* Part 15: Mowing settings

* this part is valid when SEQGRAZMOW(i) $=2$

* In case of dry matter threshold (SWHARVEST = 1), specify

DMHARVEST $\quad=4200.0 \mathrm{do} !$ Threshold of above ground dry matter to trigger mowing [0..1d6 $\mathrm{kg} D M / \mathrm{ha}, R]$

DAYLASTHARVEST $=289$ ! Last calendar day on which mowing may occur [1.366 -, I]

DMLASTHARVEST $=2700.0 \mathrm{d0} !$ Minimum above ground dry matter for mowing on last date $[0 . .1 d 6 \mathrm{~kg} D M / \mathrm{ha}, \mathrm{R}]$

* Optional: use of mowing table with variable threshold (in case DMMOWTB exists, DMHARVEST will be overruled)

* List threshold of above ground dry matter [0..1d6 kg DM/ha, R] to trigger mowing as function of daynumber [1..366 d, $R$ ]:

* DNR DMMOW (maximum 20 records)

DMMOWTB $=$

$120.0 \quad 4700.0$

$152.0 \quad 3700.0$

$182.0 \quad 3200.0$

$213.0 \quad 2700.0$

$366.0 \quad 2700.0$

* end of table

MAXDAYMOW $=42$ ! Maximum growing period after harvest [1..366 -, I]

MOWREST $=700 . d 0$ ! Remaining yield above ground after mowing event [0..1d6 $\mathrm{kg} \mathrm{DM/ha,} \mathrm{R]}$

* Relation between dry matter harvest [0..1d6 kg/ha, R] and days of delay in regrowth [0..366 d, I] after mowing DMMOWDELAY DAYDELAY

0.02

$2000.0 \quad 3$

4000.0

* end of table

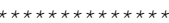

* Part 16: Management, other than mowing, grazing, irrigation, for instance pests, diseases or nutrients

FLPOTRELMF $=$.false. ! Flag indicating calculation of attainable yield instead of theoretical potential yield [Y=.true., $N=$. false.]

RELMF $=0.90$

I Relative management factor to reduce theoretical potential vield to attainable vield $[0,1$-, $R]$ 
Grassland, Yield (kg.ha DM)

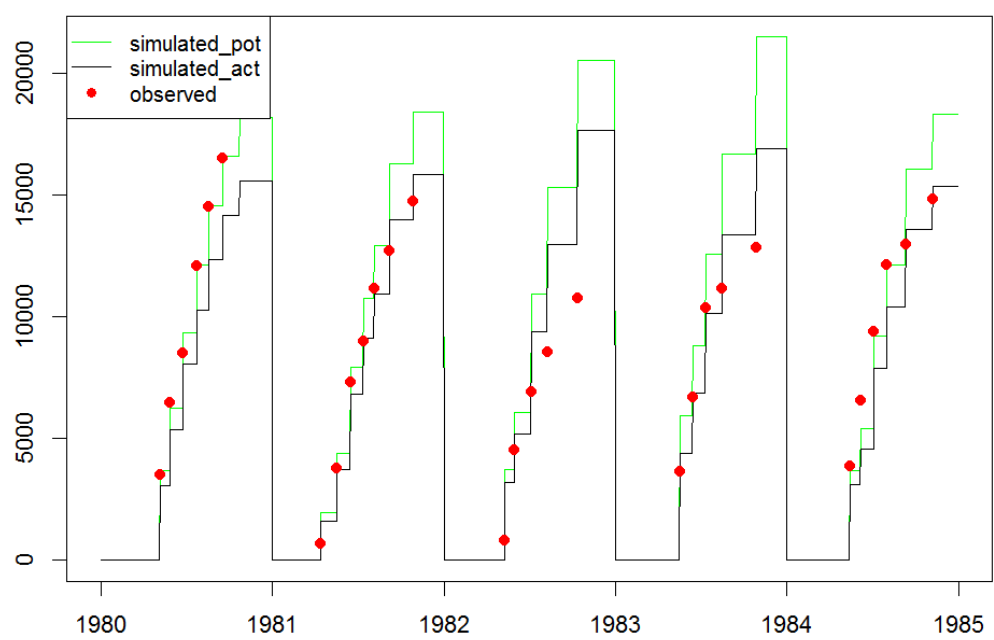

Figure 12.3 Observed, potential simulated and actual simulated yields ( $\mathrm{kg} \mathrm{ha}^{-1}$ ) of a grassland field experiments in Ruurlo (600 kg N, field16-600n-2k-80).

\subsection{Macropore flow}

This case describes a field experiment where macro pore flow is evident; a detailed description of the field experiment has been given by Smelt et al., (2003).

The precipitation excess is discharged from a field with a shallow groundwater level between 0.5 and 1.5 meter below the soil surface. Dischage occurs towards drains was simulated in the framework of pesticide leaching studies (Tiktak et al., 2012a) who also applied the same model tools at a national level (Tiktak et al., 2012b).
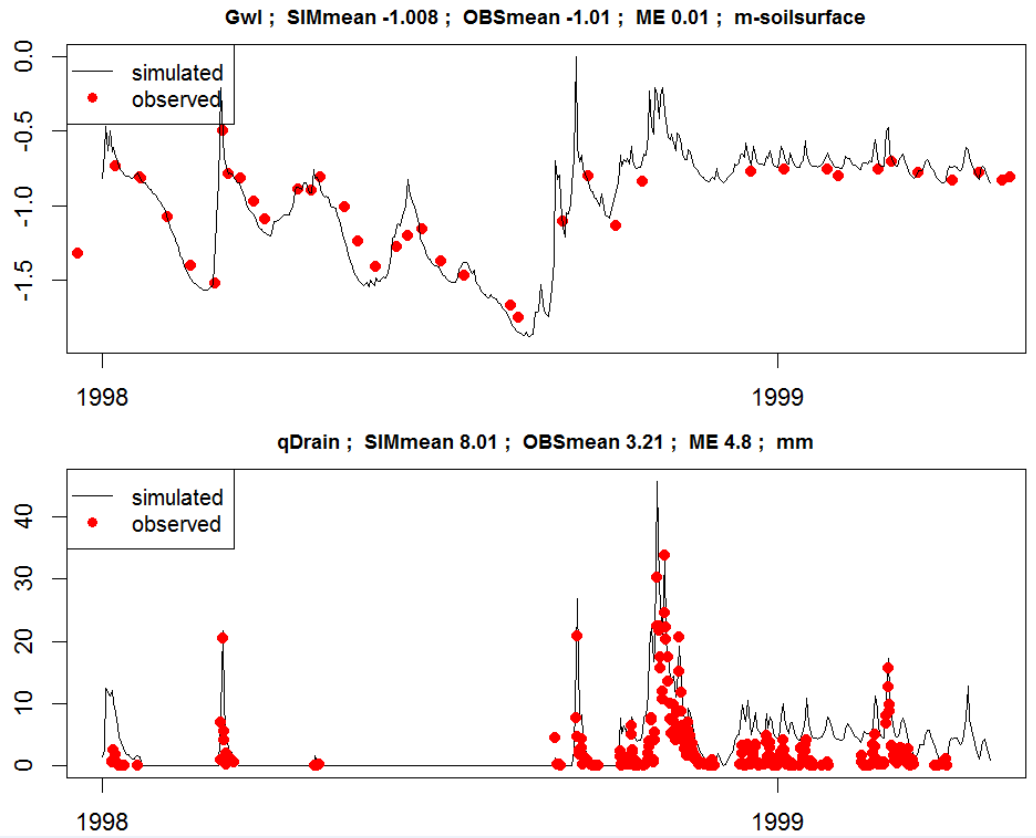

Figure 12.4 Upper figure: Observed, and simulated groundwater level ( $m$-soil surface) of a field experiments in Andelst; Lower figure: Observed, and simulated drainage discharge ( $\mathrm{mm} /$ day) of a field experiment in Andelst. 


\subsection{Oxygen stress}

This case describes a field experiment where oxygen stress occurs. It is a grassland field with a shallow groundwater level between 0.0 and 0.5 meter below the soil surface. The input parameters are described in paragraph 3.5.3.

Results of the simulation are displayed in Figure 12.5, showing oxygen stress as transpiration reduction occuring especially in the wet year 1998.
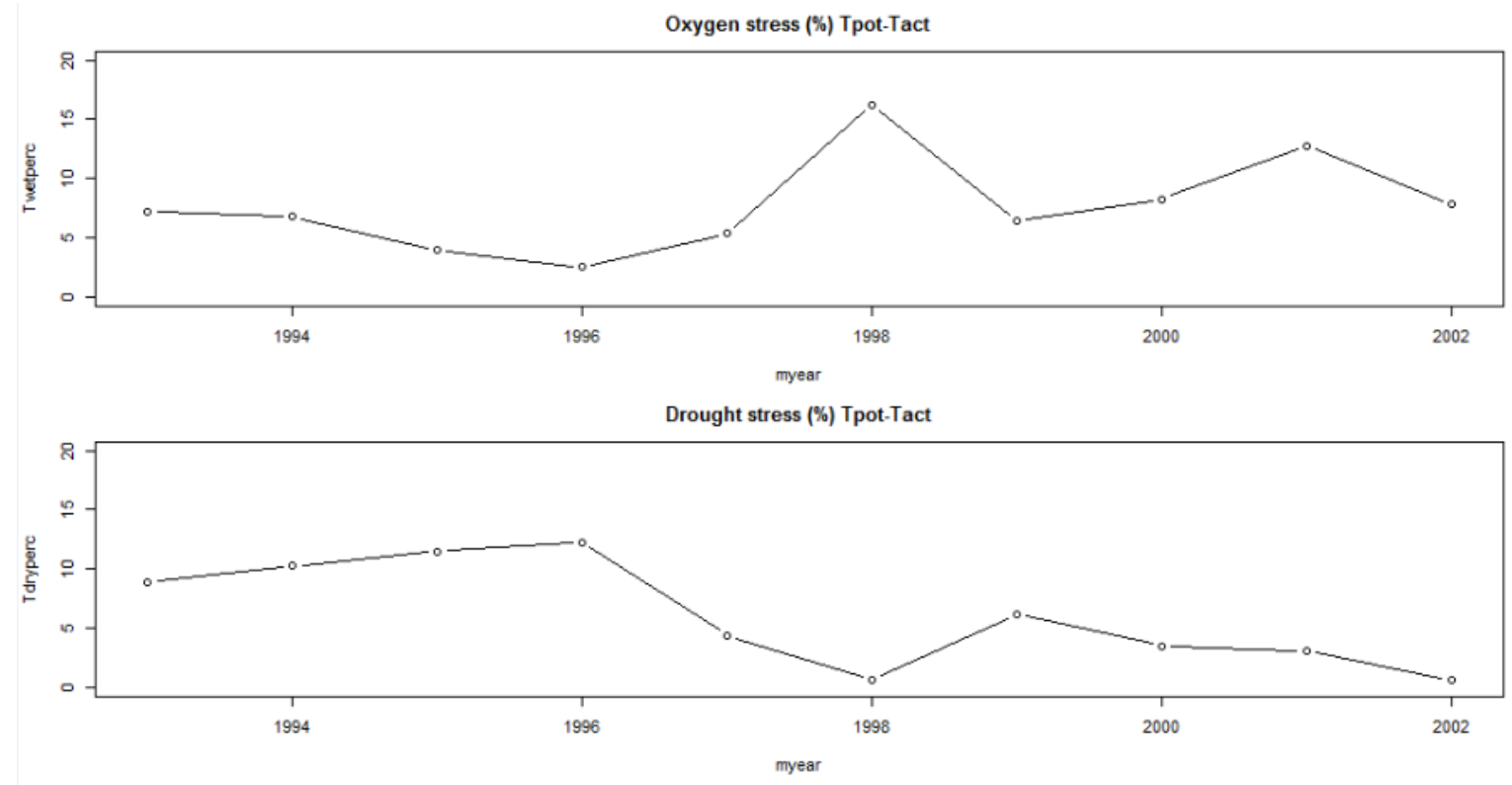

Figure 12.5 Transpiration reduction (Tact/(Tpot-Tact) due to oxygen stress (upper figure) and drought (bottom figure) below a grassland field in The Netherlands.

For more information and a description of oxygen stress simulation in case of climate-adaptive water management, we refer to Bartholomeus et al. (2015). 


\subsection{Salinity stress}

Salinization is one of the major threats to agriculture worldwide, and is an increasing problem. In the Netherlands it is expected that salinization of arable land will increase up to 125.000 hectares (Kempenear et al., 2007).

Since 2012 field trials have been performed at the open-air laboratory of Salt Farm Texel in the Netherlands in order to investigate salt tolerance of different crops (www.SaltFarmTexel.com). Between 2012 and 2015 varieties of potatoes were daily irrigated (drip irrigation) with seven different salt concentrations located in 56 fields (maximum of eight replica's for each salt concentration). During the experiment, soil moisture of the top layer, soil water salinity at different depths and the crop yields were monitored (De Vos et al., 2016).

For this example simulation results of one field with one potato variety are presented. The salinity of the irrigation water during the growing seasons of 2012 until 2015 was set to respectively 1.7, 16, 20 and $12 \mathrm{dS} \mathrm{m}^{-1}$. The threshold of salt concentration in soil water after which the root water uptake is declining was set to $0.732 \mathrm{mg} \mathrm{cm}^{-3}$ and the decline of root water uptake above the threshold was set to $0.0868 \mathrm{~cm}^{3} \mathrm{mg}^{-1}$. Box 12.6 shows the salt stress settings which is part of the *.CRP input file.

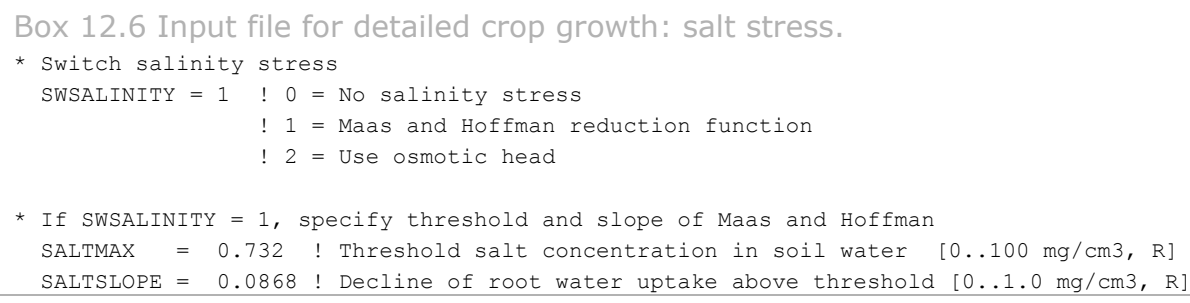

Meteorological data are gained from an nearby automatic Dutch national weather station De Kooy (www.knmi.nl). Potato growth parameters are based on standard potatoes for this region (Solanum Tuberosum L.) and set similar to the HupselBrook case with a management factor set to 0.8 . The settings of the growing season is shown in Table 12.1.

Table 12.1 Crop rotation scheme of the potato salinity example.

\begin{tabular}{llll} 
Year & Start & End & Days \\
\hline 2012 & 17 April & 19 July & 94 \\
\hline 2013 & 1 May & 29 August & 121 \\
\hline 2014 & 29 April & 12 August & 106 \\
\hline 2015 & 7 May & 18 August & 104 \\
\hline
\end{tabular}

Figure 12.6 shows the simulated salinity of the soil water. 
Solute Concentration $[\mathrm{mg} / \mathrm{I}]$

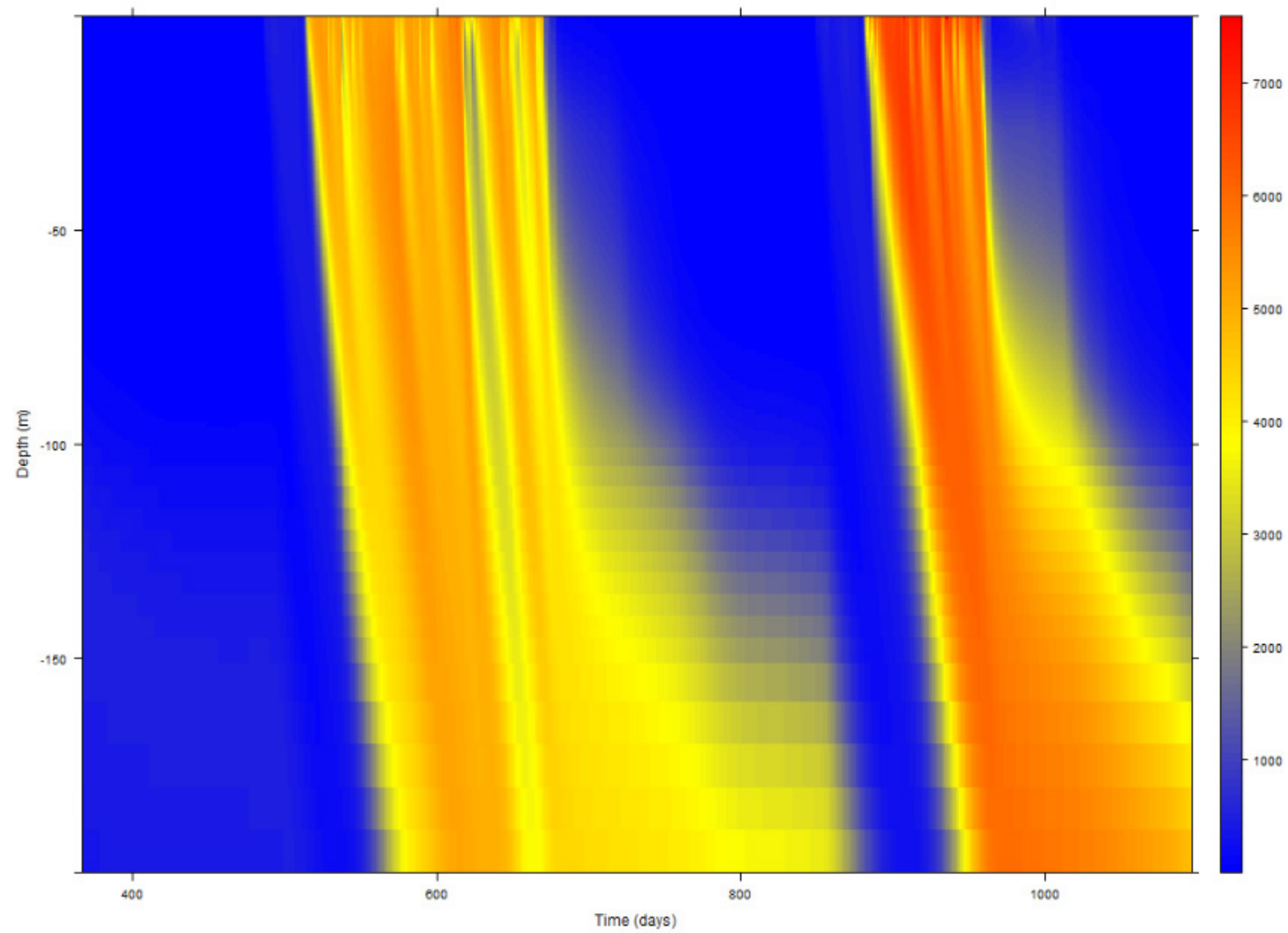

Figure 12.6 Simulated salinity concentrations in soil water ( $\mathrm{mg} / \mathrm{l})$. 


\subsection{Surface water}

The surface water case is given to explain the capabilities of the SWAP model to simulate the interaction between groundwater and surface water. Many applications of the SWAP model have a focus on the impact of measures in the surface water system on the groundwater and indirectly on the vadose zone. This is driven by the fact that water managers have control over the surface water system. This case illustrates model use to quantify the impact of elevated surface water levels on water and nutrient management of neighboring farmer fields. Input data were derived from the results of a monitoring program, which was carried out in several fields surrounding the "Wildenborch" estate in the eastern part of The Netherlands (Massop et al., 1994). Data series were collected on meteorology, soil, groundwater and surface water during several years. Here we focus on a field with a groundwater observation point (GWL in Figure 12.7) and measurements in the adjacent surface water (SWL and weir in Figure 12.7).

The SWAP model was applied assuming a seepage flux at the lower boundary described as a Cauchy condition due to regional flow and local flow to surface water systems at the lateral boundary. The surface water system was schematized in two systems. The first system has a weir with a movable crest and a depth of 1.0 meter below the soil surface. The second system has a depth of 0.6 meter below the soil surface. The drainage resistances of the two systems were calibrated with PEST (Doherty et al., 1995). The crest level was specified according to monitored data.
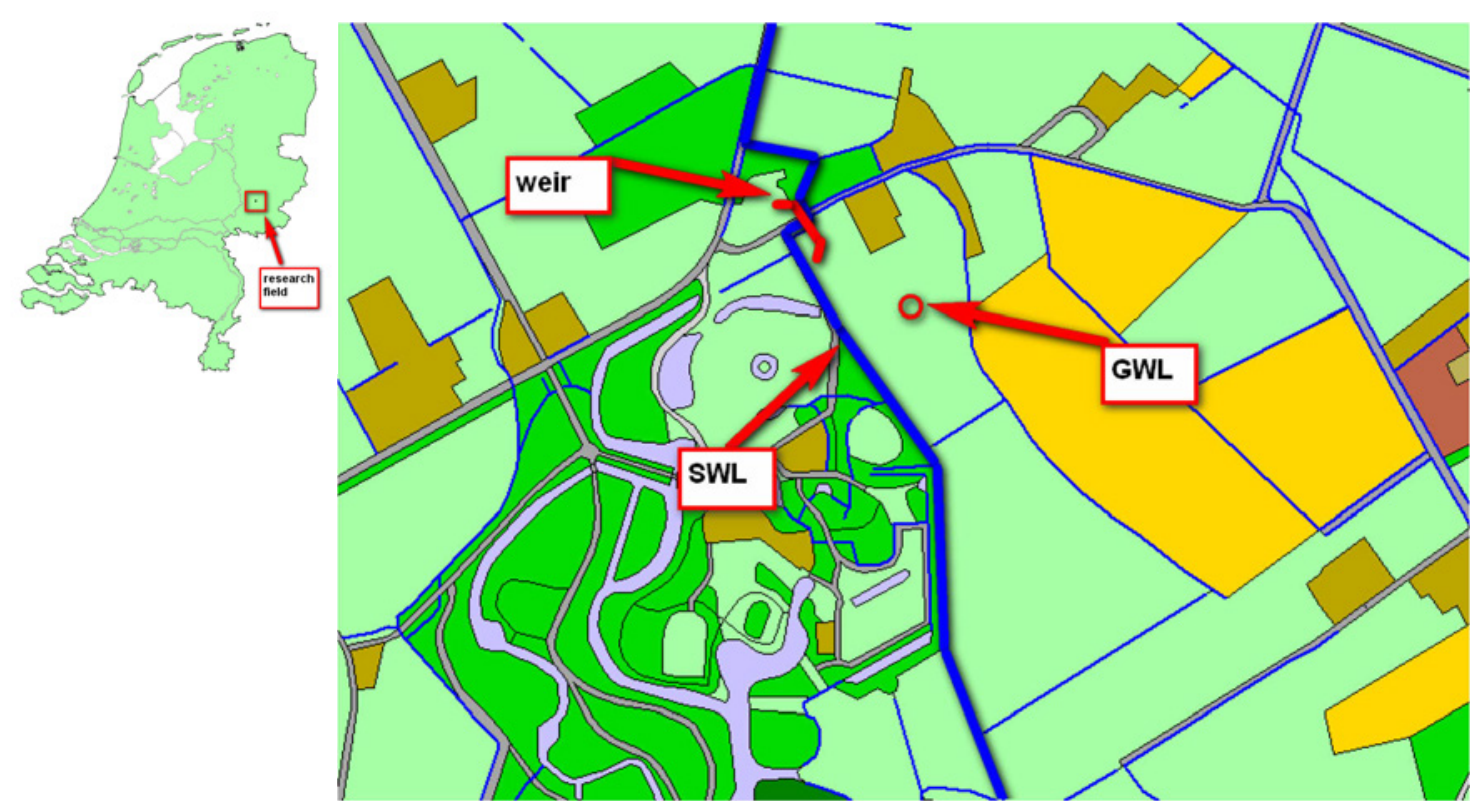

Figure 12.7 Location of monitoring site at the Wildenborch estate where groundwater levels (GWL) and surface water levels (SWL) were measured and controlled by a weir.

The flexible crest level resulted in a dynamic surface water level (Figure 12.8), which follows the monitored levels of the weir crest. Periods of discharge mainly occur in the winter period. Surface water levels drop below the weir crest in dry summer periods, during which groundwater levels also decrease. Groundwater levels, using the calibrated drainage resistances, showed a good agreement between simulated and measures levels (Figure 12.8). The hydrological results were used as input for nutrient studies (Kroes et al., 2002). 


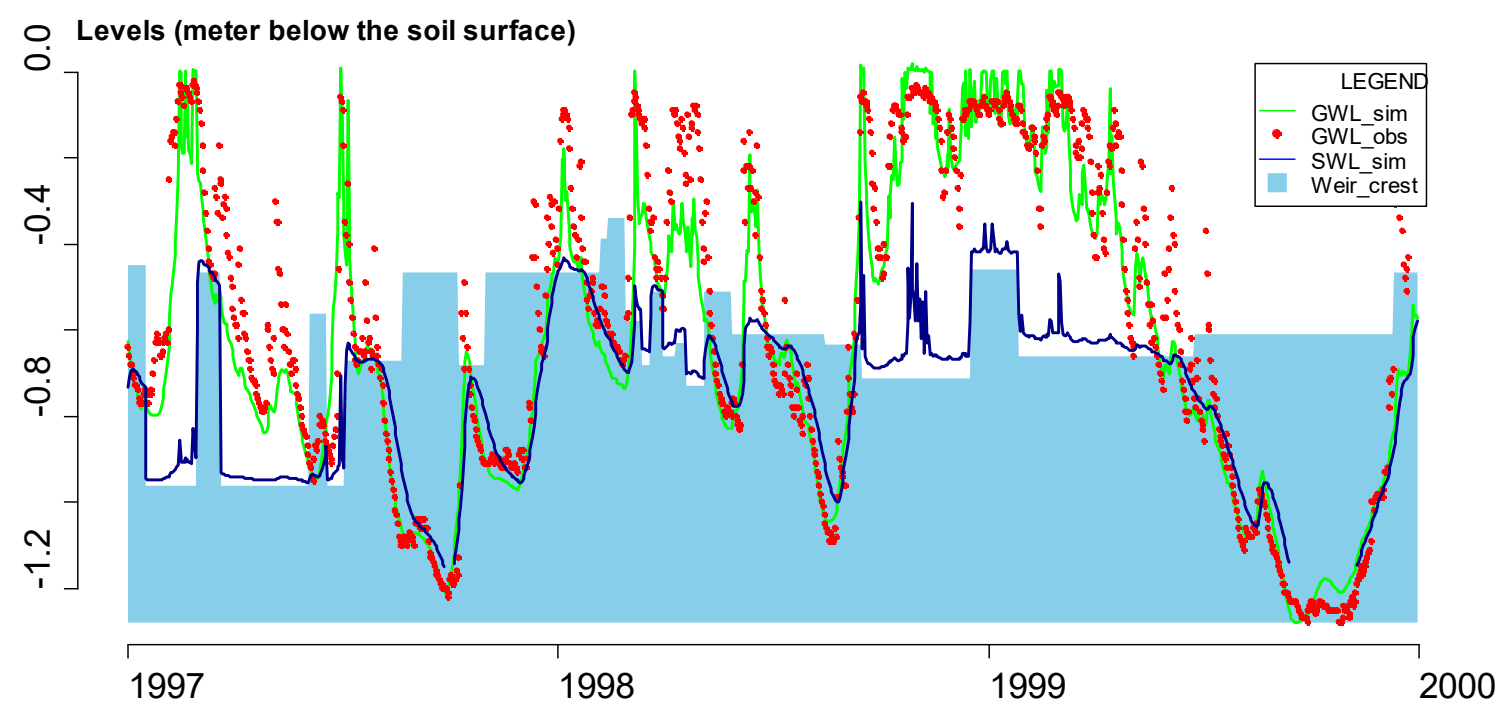

Figure 12.8 Calculated and measured groundwater levels (GWL simulated and GWL observed) and calculated surface water levels (SWL) and controlled by a weir with a variable crest level. 


\section{References}

Abenney-Mickson, S., A. Yomota, and T. Miura, 1997. Water balance of field plots planted with soybean and pumpkin. Trans. ASAE, 40: 899-909.

Allen, R.G., M.E. Wright and R.D. Burman, 1989. Operational estimates of evapotranspiration. Agron. J., 81, 650-662.

Allen, R.G., 1991. REF-ET Reference evapotranspiration calculator, version 2.1. Utah State University, Logan, $39 \mathrm{pp}$.

Allen, R.G., L.S. Pereira, D. Raes, and M. Smith, 1998. Crop evapotranspiration. Guidelines for computing crop water requirements. Irrigation and Drainage Paper 56, FAO, Rome, Italy, 300 p.

Amthor, J.F., 2000. The McCree-de Wit-Penning de Vries-Thornley respiration paradigms: 30 Years Later. Ann. Bot., 86, 1-20.

Angus, J.F., R.B. Cunningham, M.W. Moncur and D.H. Mackenzie, 1981. Phasic development in field crops. I. Thermal response in seedling phase. Field Crops Research 3, 365-378.

Ashby, M., A.J. Dolman, P. Kabat, E.J. Moors and M.J. Ogink-Hendriks, 1996. SWAPS version 1.0. Technical reference manual. Technical document 42, Winand Staring Centre, Wageningen.

Bartholomeus, R.P., J.P.M. Witte, P.M. van Bodegom, J.C. van Dam, and R. Aerts, 2008. Critical soil conditions for oxygen stress to plant roots: substituting the Feddes-function by a process-based model. J. Hydrol., 360, 147-165.

Bartholomeus, R.P., J.P.M. Witte, P.M. van Bodegom, J.C. van Dam, and R. Aerts, 2011. Climate change threatens endangered plant species by stronger and interacting water-related stresses. J. Geophys. Res., 116.

Bartholomeus, R.P., G.W.H. Simons, and G.A.P.H. van den Eertwegh, 2015. Anticipating on amplifying water stress: Optimal crop production supported by climate-adaptive water management. KWR report 2015.062, KWR, Nieuwegein.

Bastiaanssen, W.G.M., M.J.M. Cheema, W.W. Immerzeel, I.J. Miltenburg, and H. Pelgrum, 2012. Surface energy balance and actual evapotranspiration of the transboundary Indus Basin estimated from satellite measurements and the ETLook model. Water Res. Res., 48, W11512, doi: 10.1029/2011WR010482.

Bear, J., 1972. Dynamics of fluids in porous media. Elsevier, Amsterdam.

Belmans, C., J.G. Wesseling and R.A. Feddes, 1983. Simulation of the water balance of a cropped soil: SWATRE. J. Hydrol., 63, 271-286.

Beltman, W.H.J., J.J.T.I. Boesten and S.E.A.T.M. van der Zee, 1995. Analytical modelling of pesticide transport from the soil surface to a drinking water well. J. Hydrol., 169, 209-228.

Beven, K. and P. Germann, 1982. Macropores and water flow in soils. Water Resour. Res., 18: 1311-1325.

Bierkens, M.F.P., C.E. Puente, 1990. Analytically derived runoff models based on rainfall point processes. Water Resour. Res., 26, 2653-2659.

Biggar, J.W. and D.R. Nielsen, 1976. The spatial varability of the leaching characteristics of a field soil. Water Resour. Res., 12, 78-84.

Black, T.A., W.R. Gardner and G.W. Thurtell, 1969. The prediction of evaporation, drainage, and soil water storage for a bare soil. Soil Sci. Soc. Am. J., 33, 655-660.

Boesten, J.J.T.I., 1986. Behaviour of herbicides in soil : Simulation and experimental assessment. Ph.D. thesis Winand Staring Centre, Wageningen.

Boesten, J.J.T.I. and L. Stroosnijder, 1986. Simple model for daily evaporation from fallow tilled soil under spring conditions in a temperate climate. Neth. J. Agric. Sci., 34, 75-90.

Boesten, J.J.T.I. and A.M.A. van der Linden, 1991. Modeling the influence of sorption and transformation on pesticide leaching and persistence. J. Environ. Qual., 20, 425-435.

Bolt, G.H., 1979. Movement of solutes in soils: principles of adsorption/exchange chromatography. In: G.H. Bolt (Ed.), Soil Chemistry B, Physico-Chemical Models. Elsevier, Amsterdam. p. 285-348.

Boogaard, H.L., C.A. van Diepen, R.P. Rötter, J.C.M.A. Cabrera, and H.H. van Laar, 1998. WOFOST 7.1. User guide for the WOFOST 7.1 crop growth simulation model and WOFOST Control Center 5.1. Techn. Doc. 52, Alterra, WUR, Wageningen, The Netherlands, pp. 144. 
Booltink, H.W.G., 1993. Morphometric methods for simulation of water flow. PhD-thesis, Wageningen University, $169 \mathrm{p}$.

Booltink, H.W.G. and J. Bouma, 1993. Sensitivity analysis on processes affecting bypass flow. Hydrol. Process., 7: 33-43.

Boons-Prins, E.R., G.H.J. de Koning, C.A. van Diepen and F.W.T. Penning de Vries, 1993. Crop specific simulation parameters for yield forecasting across the European Community. Simulation Rep. 32, CABO-DLO and SC-DLO, Wageningen, The Netherlands.

Bos, M.G., J. Vos and R.A. Feddes, 1996. CRIWAR 2.0. A simulation model on crop irrigation water requirements. ILRI publ. 46, Wageningen, The Netherlands.

Bouma, J. and J.L. Anderson, 1973. Relationships between soil structure characteristics and hydraulic conductivity. p. 77-105. In: R.R. Bruce (ed.). Field soil moisture regime. SSSA Special Publ. no. 5. Am. Soc. of Agron. Madison, Wis.

Bouma, J. and L.W. Dekker, 1978. A case study on infiltration into a dry clay soil. I. Morphological observations. Geoderma, 20: 27-40.

Bouma, J., C. Belmans, L.W. Dekker and W.J.M. Jeurissen, 1983. Assessing the suitability of soils with macropores for subsurface liquid waste disposal. J. Environ. Qual. 12, 305-311.

Bouma, J., 1990. Using morphometric expressions for macropores to improve soil physical analyses of fields soils. Geoderma 46, 3-11.

Bouman, B.A.M., H. van Keulen, H.H. van Laar and R. Rabbinge, 1996. The 'School of de Wit' crop growth simulation models: a pedigree and historical overview. Agric. Systems 56, 171-198.

Bouten, W., 1992. Monitoring and modelling forest hydrological processes in support of acidification research. Diss. Univ. A'dam. 218 pp.

Braden, H., 1985. Ein Energiehaushalts- und Verdunstungsmodell for Wasser und Stoffhaushaltsuntersuchungen landwirtschaftlich genutzer Einzugsgebiete. Mittelungen Deutsche Bodenkundliche Geselschaft, 42, 294-299.

Brauer, C.C., 2014. Modelling rainfall-runoff processes in lowland catchments. PhD thesis, Wageningen University.

Bresler, E., and G. Dagan, 1983. Unsaturated flow in spatially variable fields. 2. Application of water flow models to various fields. Water Resour. Res., 19: 421-428.

Bronswijk, J.J.B. 1988. Modeling of water balance, cracking and subsidence of clay soils. J. Hydrol., 97: $199-212$.

Bronswijk, J.J.B. and J.J. Evers-Vermeer, 1990. Shrinkage of Dutch clay soil aggregates. Neth. J. of Agric. Sci., 38, 175-194.

Bronswijk, J.J.B., W. Hamminga and K. Oostindie, 1995. Field-scale solute transport in a heavy clay soil. Water Resour. Res., 31, 517-526.

Brooks, R.H. and A.T. Corey, 1964. Hydraulic properties of porous media. Colorado State Univ., Hydrology paper no. 3, p. 27.

Brooks, R.H., and A.T. Corey. 1966. Properties of porous media affecting fluid flow. J. Irrig. Drain. Div. 92(IR2):61-88.

Bruin, R.A., 1998. Micrometeorology. Lecture notes 06252207, Wageningen University, 156 p.

Brunt, D., 1952. Physical and dynamical meteorology. Second edition, University Press, Cambridge, $428 \mathrm{pp}$.

Burman, R.D., M.E. Jensen and R.G. Allen, 1987. Thermodynamic factors in evapotranspiration. In 'Proc. Irrig. and Drain. Spec. Conf.', L.G. James and M.J. English (Eds.), ASCE, Portland, Ore., July, p. 28-30.

Carrera, J. and S.P. Neuman, 1986. Estimation of aquifer parameters under transient and steady state conditions. 2. Uniqueness, stability, and solution algorithms. Water Resour. Res. 22, 211-27.

Carsel, R.F. and R.S. Parrish, 1988. Developing joint probability distributions of soil water characteristics. Water Resour. Res., 24, 755-769.

Causton, D.R. and J.C. Venus, 1981. The biometry of plant growth. Edward Arnold, London. 307 pp.

Celia, M.A., E.T. Bouloutas and R.L. Zarba, 1990. A general mass-conservative numerical solution for the unsaturated flow equation. Water Resour. Res., 26, 1483-1496.

Clausnitzer, V., J.W. Hopmans and D.R. Nielsen, 1992. Simultaneous scaling of soil water retention and hydraulic conductivity curves. Water Resour. Res., 28, 19-31.

Clothier, B.E., M.B. Kirkham and J.E. McLean, 1992. In situ measurement of the effective transport volume for solute moving through soil. Soil Sci. Soc. Am. J., 56, 733-736. 
Dane, J.H., and G.C. Topp, 2002. Methods of soil analysis. Part 4. Physical methods. SSSA Book series, number 5, Madison, Wisconsin, $1692 \mathrm{p}$.

De Jong van Lier, Q., J.C. van Dam, K. Metselaar, R. de Jong, and W.H.M. Duijnisveld, 2008. Macroscopic root water uptake distribution using a matric flux potential approach. Vadose Zone $\mathrm{J}$. 7:1065-1078, doi:10.2136.

De Jong van Lier, Q., J.C. van Dam and K. Metselaar, 2009. Root water extraction under combined water and osmotic stress. Soil Sci. Soc. Am. J., 73, 862-875.

De Jong van Lier, Q., J.C. van Dam, A. Durigon, M.A. dos Santos and K. Metselaar, 2013. Modeling water potentials and flows in the soil-plant system comparing hydraulic resistances and transpiration reduction functions. Vadose Zone J., doi:10.2136/vzj2013.02.0039.

Kempenaer, J.G. de, Brandenburg, W.A., \& Hoof, L.J.W. van. (2007). Het zout en de pap Een verkenning bij marktexperts naar lange termijnmogelijkheden voor zilte landbouw. Report from project "Markttrends Zilte Productie Retrieved from http://edepot.wur.nl/25020.

Dekker, L.W. and P.D. Jungerius, 1990. Water repellency in the dunes with special reference to the Netherlands. In 'Dunes of the European Coasts', Catena Suppl., 18, 173-183.

Dekker, L.W. and C.J. Ritsema, 1994. How water moves in a water repellent sandy soil. 1. Potential and actual water repellency. Water Resour. Res., 30, 2507-2517.

Dekker, L.W. and C.J. Ritsema, 1996. Preferential flow paths in a water repellent clay soil with grass cover. Water. Resour. Res. 32: 1239-1249.

Dekker, L.W., 1998. Moisture variability resulting from water repellency in Dutch soils. PhD-thesis Wageningen University, 240 p.

De Rooij, G.H., 1996. Preferential flow in water-repellent sandy soils. Model development and lysimeter experiments. Ph.D. thesis, Wageningen Agricultural University, The Netherlands, 229 p.

Desbarats, A.J., 1995. An interblock conductivity scheme for finite difference models of steady unsaturated flow in heterogeneous media. Water Resour. Res., 31, 2883-2889.

De Smedt, F. and P.J. Wierenga, 1979. A generalized solution for solute flow in soils with mobile and immobile water. Water Resour. Res., 1137-1141.

De Vries, D.A., 1975. Heat transfer in soils. In 'Heat and mass transfer in the biosphere. I. Transfer processes in plant environment', De Vries, D.A. and N.H. Afgan (eds.), Scripts Book Company, Washington D.C., p. 5-28.

De Willigen, P., and W. van Noordwijk, 1987. Roots, plant production and nutrient use efficiency. PhD thesis, Agricultural University Wageningen, $282 \mathrm{pp}$.

Deru, J., N.V. Eekeren, and H.D. Boer, 2010. Beworteling van grasland-een literatuurstudie: nutriëntenopname in relatie tot bewortelingsdiepte en-intensiteit; factoren en potentiële maatregelen die de beworteling beïnvloeden.

De Wit, C.T. et al., 1978. Simulation of assimilation and transpiration of crops. Simulation Monographs, Pudoc, Wageningen, The Netherlands. $100 \mathrm{pp}$.

Dirksen, C., 1979. Flux-controlled sorptivity measurements to determine soil hydraulic property functions. Soil Sci. Soc. Am. J., 43, 827-834.

Dirksen, C., 1987. Water and salt transport in daily irrigated root zone. Neth. J. Agric. Sci., 35: 395-406.

Dirksen, C., 1991. Unsaturated hydraulic conductivity. In 'Soil analysis, physical methods', K.A. Smith and C.E. Mullins (eds), Marcel Dekker, New York, p. 209-269.

Dirksen, C., J.B. Kool, P. Koorevaar and M.Th. van Genuchten, 1993. Hyswasor: simulation model of hysteretic water and solute transport in the root zone. In: D. Russo and G. Dagan (Eds.), Water flow and solute transport in soils. Springer-Verlag, Adv. Series in Agric. Sci., 20, 99-122.

Dirksen, C. and S. Matula, 1994. Automatic atomized water spray system for soil hydraulic conductivity measurements. Soil Sci. Soc. Am. J., 58, 319-325.

Doherty, J., 2010. PEST: Model independent parameter estimation. Watermark Numer, Comput., Brisbane, Queensland, Australia.

Doorenbos, J. and W.O. Pruitt, 1977. Guidelines for predicting crop water requirements. Irrigation and Drainage Paper 24, FAO, Rome, Italy.

Doorenbos, J. and A.H. Kassam, 1979. Yield response to water. FAO Irrigation and Drainage Paper 33, FAO, Rome, Italy.

Ehlert, P., H. Pasterkamp, and G. Brouwer, 2004. Fosfaatbehoefte van bloembollen: onderbouwing van de fosfaatbemestingsadviezen. Alterra, Wageningen. 
Elrick, D.E. and W.D. Reynolds, 1992. Infiltration from constant-head well permeameters and infiltrometers. In 'Advances in measurement of soil physical properties: bringing theory into practice', G.C. Topp, W.D. Reynolds and R.E. Green (eds.), SSSA special publication no. 30, p. 1-24.

Ernst, L.F., 1956. Calculation of the steady flow of groundwater in vertical cross-sections. Netherlands Journal of Agricultural Science 4, 126-131.

Ernst, L.F. 1962. Groundwater flow in the saturated zone and its calculation when parallel open conduits are present. Thesis (Dutch with English summary), University of Utrecht, $189 \mathrm{pp}$.

Ernst, L.F., 1973. The determination of residence times in case of groundwater flow. Nota 755 I.C.W., now Winand Staring Centre, Wageningen (in Dutch).

Ernst, L.F. 1978. Drainage of undulating sandy soils with high groundwater tables. I en II. Journal of Hydrology 39, 1-50.

Ernst, L.F. and R.A. Feddes, 1979. Invloed van grondwateronttrekking voor beregening en drinkwater op de grondwaterstand. Report 1116, ICW (currently Winand Staring Centre), Wageningen, The Netherlands.

Farahani, H.J., T.A. Howell, W.J. Shuttleworth, and W.C. Bausch, 2007. Evapotranspiration: progress in measurement and modelling in agriculture. Transactions of the ASABE 50, 1627-1638.

Feddes, R.A., 1971. Water, heat and crop growth. Ph.D. thesis, Wageningen Agricultural University, The Netherlands.

Feddes, R.A., P.J. Kowalik and H. Zaradny, 1978. Simulation of field water use and crop yield. Simulation Monographs. Pudoc. Wageningen. 189 pp.

Feddes, R.A., 1987. Crop factors in relation to Makking reference crop evapotranspiration. In 'Evaporation and weather', TNO Committee on Hydrological Research, Proceedings and information no 39, p. 33-46.

Feddes, R.A., P. Kabat, P.J.T. van Bakel, J.J.B. Bronswijk and J. Halbertsma, 1988. Modelling soil water dynamics in the unsaturated zone - state of the art. J. Hydrol., 100, 69-111.

Feddes, R.A., G.H. de Rooij, J.C. van Dam, P. Kabat, P. Droogers, and J.N.M. Stricker, 1993 a. Estimation of regional effective soil hydraulic parameters by inverse modelling. In 'Water flow and solute transport in soils: modelling and application', D. Russo and G. Dagan (Eds.), Springer Verlag, Berlin, p. 211-231.

Feddes, R.A., M. Menenti, P. Kabat, and W.G.M. Bastiaanssen, 1993b. Is large scale inverse modelling of unsaturated flow with areal average evaporation and surface soil moisture as estimated from remote sensing feasible? J. Hydrol., 143: 125-152.

Feddes, R.A. and K.J. Lenselink, 1994. Evapotranspiration. In 'Drainage priciples and applications', H.P. Ritzema (ed.), ILRI publication 16, second ed., Wageningen, p. 145-174.

Fernández, A., 1998. An Energy Balance Model of Seasonal Snow Evolution. Physical Chemistry of the Earth 23, 5-6: 661-666.

Fierer, N., B.P. Colman, J.P. Schimel, and R.B. Jackson, 2006. Predicting the temperature dependence of microbial respiration in soil: A continental-scale analysis. Global Biogeochemical Cycles, 20.

Flury, M. and H. Flühler, 1995. Tracer characteristics of Brilliant Blue FCF. Soil Sci. Soc. Am. J., 59, 22-27.

Fukusako, S. 1990. Thermophysical Properties of Ice, Snow, and Sea Ice. Int. J. Thermophysics, 11(2): 353-372.

Garnier, P., Rieu, M., Boivin, P., Vauclin, M., and Baveye, P. 1997. Determining the hydraulic properties of a swelling soil from a transient evaporation experiment. Soil Sci. Soc. Am. J., 61: 1555-1563.

Gash, J.H.C., 1979. An analytical model of rainfall interception by forests. Q. J. R. Meteor. Soc. 105, 43-55.

Gash, J.H.C., C.R. Lloyd and G. Lachaud, 1995. Estimating sparse forest rainfall interception with an analytical model. Journal of Hydrology 170 (1995) 79-86.

Gelhar, L.W. and J.L. Wilson, 1974. Groundwater quality modeling. Ground Water, 12, 399-408.

Gerke, H.H. and M.Th van Genuchten, 1993. A dual-porosity model for preferential movement of water and solutes in structured porous media. Water Resour. Res., 29, 305-319.

Fierer, N., B.P. Colman, J.P. Schimel, and R.B. Jackson, 2006. Predicting the temperature dependence of microbial respiration in soil: A continental-scale analysis. Global Biogeochemical Cycles, 20.

Goode, D.J. (1996). Direct simulation of groundwater age. Water Resources Research, 32(2), 289-296.

Goudriaan, J., 1977. Crop meteorology: a simulation study. Simulation monographs, Pudoc, Wageningen. 
Goudriaan, J., 1982. Some techniques in dynamic simulation. In 'Simulation of plant growth and crop production', F.W.T. Penning de Vries and H.H. van Laar (Eds.), Simulation Monographs, Pudoc, Wageningen, p. 66-84.

Granberg, G., H. Grip, M. Ottoson Lofvenius, I. Sundh, B.H. Svensson and M. Nilsson, 1999. A simple model for simulation of water content, soil frost, and soil temperatures in boreal mixed mires. Water Resources Research 35: 3771-3782.

Greco, R., R.F.A. Hendriks and W. Hamminga, 1997. Clay soil aggregate sorptivity measurements under different water contents. Proceedings of the National Hydraulics Conference, Turin, Italy, November 1996.

Groen, K.P., 1997. Pesticide leaching in polders. Field and model studies on cracked clays and loamy sand. PhD thesis, Wageningen Agricultural University, Wageningen, The Netherlands, 296 pp.

Groenendijk, P., L.V. Renaud, and J. Roelsma, 2005. Prediction of Nitrogen and Phosphorus leaching to groundwater and surface waters; Process descriptions of the ANIMO 4.0 model. Alterra-Report 983. Wageningen.

Groenendijk, P., \& Renaud, L.V. (2013). Voorbereiding STONE2.4 op berekeningen voor de Evaluatie Meststoffenwet 2012, Alterra rapport 2462, Wageningen

Groenendijk, P., Boogaard, H., Heinen, M., Kroes, J., Supit, I., \& Wit, A. De. (2016). Simulation of nitrogen-limited crop growth with SWAP / WOFOST. Report 2721. Alterra Rapport, 2721.

Hack-ten Broeke, M.J.D., J.G. Kroes, R.P Bartholomeus, J.C. van Dam, A.J.W. de Wit, I. Supit, D.J.J. Walvoort, P.J.T. van Bakel and R. Ruijtenberg, 2016. Quantification of the impact of hydrology on agricultural production as a result of too dry, too wet or too saline conditions. Soil 2 , 391-402.

Hadley, P., E.H. Roberts, R.J. Summerfield and F.R. Minchin, 1984. Effects of temperature and photoperiod on flowering in soya bean: a quantitative model. Annals of Botany, 53, 669-681.

Hargreaves, G.L., and Z.A. Samani, 1985. Reference crop evapotranspiration from temperature. Applied Engineer. In Agric., 1, 2, 96-99.

Harrison, L.P., 1963. Fundamental concepts and definitions relating to humidity. In 'Humidity and moisture', A. Wexler (Ed.), Vol. 3, Reinhold Publishing Company, New York.

Haverkamp, R., M. Vauclin, J. Touma, P.J. Wierenga and G. Vachaud, 1977. A comparison of numerical simulation models for one-dimensional infiltration. Soil Sci. Soc. Am. J., 41, 285-294.

Haverkamp, R., and M. Vauclin, 1979. A note on estimating finite difference interblock hydraulic conductivity values for transient unsaturated flow problems. Water Resour. Res., 15, 181-187.

Hendriks, R.F.A., K. Oostindie, and P. Hamminga, 1999. Simulation of bromide tracer and nitrogen transport in a cracked clay soil with the FLOCR/ANIMO model combination. J. Hydrol., 215: 94-115.

Hendriks, R.F.A. (2004). An analytical equation for describing the shrinkage characteristics of peat soils. In J. Päivänen (Ed.) Wise use of Peatlands. Proceedings of the 12th International Peat Congress. Tampere, Finland.

Hijmans, R.J., I.M. Guiking-Lens and C.A. van Diepen, 1994. User's guide for the WOFOST 6.0 crop growth simulation model. Technical Document 12, Winand Staring Centre, Wageningen, The Netherlands, $144 \mathrm{p}$.

Hillel, D., 1980. Fundamentals of soil physics. Academic Press, San Diego, CA, 412 p.

Hooghoudt, S.B., 1940. Algemene beschouwing van het probleem van de detailontwatering en de infiltratie door middel van parallel lopende drains, greppels, sloten en kanalen. Versl. Landbouwk. Onderz., 46, B, $193 \mathrm{p}$.

Hoogmoed, W.B., and Bouma, J. 1980. A simulation model for predicting infiltration into a cracked clay soil. Soil Sci. Soc. Am. J., 44: 458-461.

Homaee, M., 1999. Root water uptake under non-uniform transient salinity and water stress. PhDthesis, Wageningen University, $173 \mathrm{p}$.

Hopmans, J.W., and J.N.M. Stricker, 1989. Stochastic analysis of soil water regime in a watershed. J. Hydrol., 105, 57-84.

Hopmans, J.W., K.C. Roy, and W.W. Wallender, 1991. Irrigation water management and soil-water hysteresis - a computer modeling study with stochastic soil hydraulic properties. Transactions of the ASAE, 34: 449-459.

Hopmans, J.W., J.C. van Dam, S.O. Eching and J.N.M. Stricker, 1994. Parameter estimation of soil hydraulic functions using inverse modeling of transient outflow experiments. Trends in Hydrology, 1, 217-242. 
Hornung, U., and W. Messing, 1983. Truncation errors in the numerical solution of horizontal diffusion in saturated/unsaturated media. Adv. Water Resour., 6, 165-168.

Huang, K., B.P. Mohanty and M.Th. van Genuchten, 1996. A new convergence criterion for the modified Picard iteration method to solve the variably saturated flow equation. J. Hydrol., 178, 69-91.

Hunt, E.R., J.A. Weber and D.M. Gates, 1985. Effects of nitrate application on Amaranthuspowellii Wats. I. Changes in photosynthesis, growth rates, and leaf area. Plant Physiology 79, 609-613.

Ippisch, O., H.-J. Vogel and P. Bastian, 2006. Validity limits for the van Genuchten-Mualem model and implications for parameter estimation and numerical simulation. Adv. Water Resour., 29, 1780-1789.

Iwama, K., 1998. Development of nodal and lateral roots in potato under field conditions. Journal of the Faculty of Agriculture, 68, 33-44.

Jackson, R.B., J. Canadell, J.R. Ehleringer, H.A. Mooney, O.E. Sala, and E.D. Schulze, 1996. A global analysis of root distributions for terrestrial biomes. Oecologia, 108, 389-411.

Jacucci, G., P. Kabat, L. Pereira, P. Verrier, P. Steduto, C. Uhrik, G. Bertanzon, J.Huygen, B. van den Broek, J. Teixeira, R. Fernando, G. Giannerini, F. Carboni, M. Todorovic, G. Toller, G. Tziallas, E. Fragaki, J. Vera Munoz, D. Carreira, P. Yovchev, D. Calza, E. Valle and M. Douroukis, 1994. The Hydra Project: a Decision Support System for Irrigation Water Management. Proceedings of the International Conference on Land and Water Resources Management in the Mediterranean Region. 4-8 September 1994, Valenzano (Bari), Italy.

Jarvis, N.J., 1989. A simple empirical model of root water uptake. J. Hydrol., 107, 57-72.

Jarvis, N.J., 1989. CRACK - A model of water and solute movement in cracking clay soils. Technical description and user notes. Report 159, Dept. Soil Sci., Swedish Univ. Agric. Sci., Uppsala, Sweden, $37 \mathrm{pp}$.

Jarvis, N.J., 2011. Simple physics-based models of compensatory plant water uptake: concepts and eco-hydrological consequences. Hydrol. Earth Syst. Sci., 15, 3431-3446.

Jaynes, D.B., 1984. Comparison of soil water hysteresis models. J. Hydrol., 75, 287-299.

Jensen, M.E., R.D. Burman and R.G. Allen, 1990. Evapotranspiration and irrigation water requirements. ASCE manuals and reports on enigineering practice 70, ASCE, New York. 332 pp.

Jury, W.A., 1982. Simulation of solute transport using a transfer function mode. Water Resour. Res., $18,363-368$.

Jury, W.A., D. Russo and G. Sposito, 1987. The spatial variability of water and solute transport properties in unsaturated soil, II Scaling of water transport. Hilgardia, 55, 33-56.

Jury, W.A., W.R. Gardner and W.H. Gardner, 1991. Soil Physics. Fifth edition. Wiley, New York. 330 pp.

Kabat, P., B.J. Broek, van den and R.A. Feddes, 1992. SWACROP: A water management and crop production simulation model. ICID Bulletin 92, vol. 41 No. 2, 61-84.

Kaluarachchi, J.J., and J.C. Parker, 1987. Effects of hysteresis with air entrapment on water flow in the unsaturated zone. Water Resour. Res., 23: 1967-1976.

Kamaluddin, M., and J.J. Zwiazek, 2001. Metabolic inhibition of root water flow in red-osier dogwood (Cornus stolonifera) seedlings. J. Exp. Bot., 52, 739-745.

Kase, M. and J. Catský, 1984. Maintenance and growth components of dark respiration rate in leaves of $C_{3}$ and $C_{4}$ plants as affected by leaf temperature. Biologia Plantarum 26, 461-470.

Kim, D.J., 1992. Characterization of swelling and shrinkage behaviour, hydraulic properties and modelling of water movement in a physically ripening marine clay soil. PhD thesis, Catholic University Leuven.

Kim, R., 1995. The water budget of heterogeneous areas. Doctoral thesis. Wageningen Agricultural University, Wageningen, The Netherlands, $182 \mathrm{pp}$.

Kool, J.B., J.C. Parker and M.Th. van Genuchten, 1985. Determining soil hydraulic properties from One-step outflow experiments by parameter estimation: I. Theory and numerical studies. Soil Sci. Soc. Am. J.,49, 1348-1354.

Kool, J.B., and J.C. Parker, 1987. Development and evaluation of closed form expressions for hysteretic soil hydraulic properties. Water Resour. Res., 23, 105-114.

Kool, J.B., J.C. Parker and M.Th. van Genuchten, 1987. Parameter estimation for unsaturated flow and transport models - a review. J. Hydrol., 91, 255-293.

Kool, J.B., and M.Th. van Genuchten, 1991. HYDRUS, One-dimensional variably saturated flow and transport model including hysteresis and root water uptake. Research Report 124, U.S. Salinity Laboratory, USDA, ARS, Riverside, CA.

Koorevaar, P., G. Menelik and C. Dirksen, 1983. Elements of soil physics. Developments in Soil Science 13, Elsevier, Amsterdam, p. 223. 
Kosugi, K., J.W. Hopmans, and J.H. Dane. 2002. Parametric models. In: J.H. Dane and G.C. Topp, editors, Methods of soil analysis. Part 4. Physical methods. SSSA Book Ser. 5. SSSA, Madison, WI. p. 728-757. doi:10.2136/sssabookser5.4.c27

Klute, A., 1986. Water retention: laboratory methods. In 'Methods of soil analysis; Part 1; Physical and Mineralogical methods', A. Klute (Ed.), Agronomy series n. 9, ASA and SSSA, Madison, Wisconsin, p. 635-662.

Klute, A., and C. Dirksen, 1986. Hydraulic conductivity and diffusivity: laboratory methods. In 'Methods of soil analysis; Part 1; Physical and Mineralogical methods', A. Klute (Ed.), Agronomy series n. 9, ASA and SSSA, Madison, Wisconsin, p. 687-734.

Kraalingen, D.W.G, and C. Rappoldt, 2000. Reference manual of the FORTRAN utility library TTUTIL v. 4. Report 5. Plant Research International, Wageningen.

Kramer, P.J., 1951. Causes of injury to plants resulting from flooding of the soil. Plant Physiology, 722-736.

Krammes, J.S., and L.F. DeBano, 1965. Soil wettability: a neglected factor in watershed management. Water Resour. Res., 1, 283-288.

Kraijenhoff van de Leur, D.A., 1958. A study of non-steady groundwater flow with special references to a reservoir coefficient. Ingenieur, 70: B87-B94.

Kroes, J.G., and J. Roelsma, 1997. User's Guide ANIMO 3.5; input instructions and technical programme description. Technical Document 46, DLO Winand Staring Centre, Wageningen.

Kroes, J.G., P.J.T. van Bakel, J. Huygen, T. Kroon en R. Pastoors, 2001. Actualisatie van de hydrologie voor STONE 2.0. Rapport 298, Alterra, Wageningen, 68 p.

Kroes, J.G., J. Beldman, H. te Beest, D. Boland \& T. Vellinga, 2002. Conflicting interests in a Dutch agricultural dairy farming system. In: J.[H.A.M.] Steenvoorden, F. Claessen \& J. Willems (eds.), Agricultural effects on ground and surface waters: research at the edge of science and society. Wallingford (UK), IAHS, 2002. IAHS Publ. 273, pp. 41-48.

Kroes, J.G. and J.C. van Dam (Eds), 2003. Reference manual SWAP version 3.03. Alterra report 773. Wageningen. $211 \mathrm{p}$.

Kroes, J.G., J.C. van Dam, P. Groenendijk, R.F.A. Hendriks and C.M.J. Jacobs, 2008. SWAP version 3.2. Theory description and user manual. Alterra report 1649, Wageningen University and Research centre, Wageningen.

Kroes, J.G., J.C. van Dam, P. Groenendijk, R.F.A. Hendriks and C.M.J. Jacobs, 2009. SWAP version 3.2. Theory description and user manual. Alterra report 1649 update 02, Wageningen University and Research centre, Wageningen.

Kroes, J.G., \& Supit, I., 2011. Impact analysis of drought, water excess and salinity on grass production in The Netherlands using historical and future climate data. Agriculture, Ecosystems \& Environment, 144(1), 370-381. http://doi.org/10.1016/j.agee.2011.09.008

Kropff, M.J., H.H. van Laar and H.F.M. ten Berge (Eds.), 1993. ORYZA1 A basic model for irrigated lowland rice production. IRRI, Los Banos, The Philippines.

Kujala, K., 1991. Factors affecting frost susceptibility and heaving pressure in soils. Acta Univ. Oul. C 58. Department of Civil Engineering, University of Oulu, Finland, 104 pag.

Kustas, W., A. Rango, 1994. A simple energy algorithm for the snowmelt runoff model. Water Resources Research 30: 1515-1527.

Leij, F.J., W.B. Russell, and S.M. Lesch, 1997. Closed-form expressions for water retention and conductivity data. Ground Water 35:848-858. doi:10.1111/j.1745-6584.1997.tb00153.x

Leij, F.J., W.J. Alves, M.Th. van Genuchten and J.R. Williams, 1996. The UNSODA Unsaturated Soil Hydraulic Database. User's manual Version 1.0, Soil Salinity Laboratory, Riverside, California.

Leistra, M., A.M.A. van der Linden, J.J.T.I. Boesten, A. Tiktak and F. van den Berg. 2001. PEARL model for pesticide behaviour and emissions in soil-plant systems. Description of processes. Alterra report 13, RIVM report 711401009, Alterra, Wageningen, $107 \mathrm{pp}$.

Lemieux, J-M. and E.A. Sudicky, 2010. Simulation of groundwater age evolution during the Wisconsinian glaciation over the Canadian landscape. Environ. Fluid Mech 10, 91-102.

Leong, E.C., and H. Rahardjo, 1997. Review of soil water characteristic curve equations. J. Geotech. Geoenviron. Eng. 123:1106-1117. doi:10.1061/(ASCE)1090-0241(1997)123:12(1106).

Lin, H.C., Richards, D.R., Yeh, G.T., Cheng, J.R., Cheng, H.P., Jones, N.L., 1997. FEMWATER: A ThreeDimensional Finite Element Computer Model for Simulating Density-Dependent Flow and Transport in Variably Saturated Media. Technical Report CHL-97-12. Waterways Experiment Station, U.S. Army Corps of Engineers, Vicksburg, MS 39180-6199. 
Maas, E.V., and G.J. Hoffman, 1977. Crop salt tolerance-current assessment. J. Irrig. and Drainage Div., ASCE 103, 115-134.

Maas, E.V., 1990. Crop salt tolerance. In 'Agricultural salinity assessment and management', K.K. Tanji (Ed.), ASCE Manuals and Reports on Engineering practice, No 71, New York.

Makkink, G.F., 1957. Testing the Penman formule by means of lysimeters. J. Int. Water Eng., 11: 277-288.

Massop, H.Th.L., J.M.P.M. Peerboom, and H.C. van Vessem, 1994. Effects of measures to alleviate desiccation at rural estate 'De Wildenborch'. Report 342, Alterra Green World Research, Wageningen, 128 p. (in Dutch)

Massop, H.Th.L., and P.A.J.W. de Wit, 1994. Hydrologisch onderzoek naar de gewasweerstanden van het tertiair ontwateringsstelsel in Oost-Gelderland. Report 373, Winand Staring Centre, Wageningen, The Netherlands, $132 \mathrm{p}$.

Miller, E.E., and R.D. Miller, 1956. Physical theory for capillary flow phenomena. J. Appl. Phys., 27, 324-332.

Millington, R.J., and J.P. Quirk., 1961. Permeability of porous solids. Trans. Faraday Soc., 57, 1200-1207.

Milly, P.C.D., 1985. A mass conservative procedure for time-stepping in models of unsaturated flow. Adv. Water Resour., 8, 32-36.

Moene, A.F., \& Van Dam, J.C. (2014). Transport in the Atmosphere-Vegetation-Soil Continuum (Vol. 87). Cambridge: Cambridge University Press. http://doi.org/10.1016/j.apradiso.2013.12.019

Monteith, J.L., 1965. Evaporation and the Environment. In: G.E. Fogg (ed.), The state and movement of water in living organisms. Cambridge University Press, p. 205-234.

Monteith, J.L., 1981. Evaporation and surface temperature. Quarterly J. Royal Soc., 107, 1-27.

Mualem, Y., 1976. A new model for predicting the hydraulic conductivity of unsaturated porous media. Water Resour. Res., 12, 513-522.

Murray, F.W., 1967. On the computation of saturation vapor pressure. J. Appl. Meteor., 6, 203-204.

Nieber, John, L., Tammo S. Steenhuis, Todd Walter \& Mark Bakker, 2006. Enhancement of seepage and lateral preferential flow by biopores on hillslopes, in Biologia.

Nielsen, D.R., M.Th. van Genuchten and J.W. Biggar, 1986. Water flow and solute transport in the unsaturated zone. Water Resour. Res., 22, supplement, 89S-108S.

Nimmo, J.R., J. Rubin and D.P. Hammermeister, 1987. Unsaturated flow in a centrifugal field: Measurement of hydraulic conductivity and testing of Darcy's law. Water Resour. Res., 32, 124-134.

Parlange, J.Y., 1975. On solving the flow equation in unsaturated soils by optimization: horizontal infiltration. Soil Sci. Soc. Am. Proc. 39, 415-418.

Peat, W.E., 1970. Relationships between photosynthesis and light intensity in the tomato. Annal Bot., 34, 319-328.

Peck, A.J., R.J. Luxmoore and J.L. Stolzy, 1977. Effects of spatial variability of soil hydraulic properties in water budget modeling. Water Resour. Res., 13, 348-354.

Penning de Vries, F.W.T., A.H.M. Brunsting and H.H. van Laar, 1974. Products requirements and efficiency of biosynthesis: a quantitative approach. Journal of Theoretical Biology 45, 339-377.

Penning de Vries, F.W.T., J.M. Witlage and D. Kremer, 1979. Rates of respiration and of increase in structural dry matter in young wheat, ryegrass and maize plants in relation to temperature, to water stress and to their sugar content. Annals of Botany (London) 44, 595-609.

Penning de Vries, F.W.T. and H.H. van Laar, 1982. Simulation of growth processes and the model BACROS. In Penning de Vries, F.W.T. and H.H. van Laar (Eds.) Simulation of plant growth and crop production. Simulation Monographs, Pudoc, Wageningen, The Netherlands. p. 114-135.

Penning de Vries, F.W.T., D.M. Jansen, H.F.M. ten Berge and A. Bakema, 1989. Simulation of ecophysiological processes of growth in several annual crops. Pudoc, Wageningen, the Netherlands, $271 \mathrm{pp}$.

Penman, H.L., 1948. Natural evaporation from open water, bare soil, and grass. Proc. Royal Society, London 193, 120-146.

Philip, J.R., 1957. The theory of infiltration: 4. Sorptivity and algebraic infiltration equations. Soil Science 84, 264-275.

Press, W.H., B.P. Flannery, S.A. Teukolsky and W.T. Vetterling, 1989. Numerical Recipes. The art of scientific computing. Cambridge University Press. 702 pp.

Priestley, C.H.B., and R.J. Taylor, 1972. On the assessment of surface heat flux and evaporation using large scale parameters. Mon. Weath. Rev., 100, 81-92. 
Qin, R., P. Stamp, and W. Richner, 2006. Impact of tillage on maize rooting in a Cambisol and Luvisol in Switzerland. Soil and Tillage Research, 85, 50-61.

$\mathrm{R}, 2013$. A language and environment for statistical computing. R Foundation for Statistical Computing. Vienna, Austria. URL http://www.R-project.org/

Raats, P.A.C., 1973. Unstable wetting fronts in uniform and nonuniform soils. Soil Sci. Soc. Am. J., 37, 681-685.

Raes, D., H. Lemmens, P. van Aelst, M. van der Bulcke and M. Smith, 1988. IRSIS (Irrigation Scheduling Information System), reference manual. Laboratory of Land Management, K.U. Leuven, Belgium.

Rawsthorne, D., and B. Brodie, 1986. Relationship between root growth of potato, root diffusate production, and hatching of Globodera rostochiensis. J. Nematol., 18, 379.

Reinink, K., I. Jorritsma and A. Darwinkel, 1986. Adaption of the AFRC wheat phenology model for Dutch conditions. Neth. J. Agric. Science, 34, 1-13.

Rhoades, J.D., Kandiah, A., Mashali, A.M., 1992. The use of saline waters for crop production - FAO irrigation and drainage paper 48.

Rijniersce, K., 1983. A simulation model for physical ripening in the IJsselmeerpolders. Rijksdienst voor IJsselmeerpolders. Lelystad, The Netherlands, $216 \mathrm{pp}$.

Rijtema, P.E., P. Groenendijk and J.G. Kroes, 1997. ANIMO, a dynamic simulation model for transport and transformation of nutrients and organic materials in soils. Report 30, DLO Winand Staring Centre, Wageningen, in press.

Ritchie, J.T., 1972. A model for predicting evaporation from a row crop with incomplete cover. Water Resour. Res., 8, 1204-1213.

Ritsema, C.J., L.W. Dekker, J.M.H. Hendrickx and W. Hamminga, 1993. Preferential flow mechanism in a water repellent sandy soil. Water Resour. Res., 29, 2183-2193.

Ritsema, C.J., and L.W. Dekker, 1994. How water moves in a water repellent sandy soil. 2. Dynamics of fingered flow. Water Resour. Res., 30, 2519-2531.

Ritsema, C.J., 1998. Flow and transport in water repellent sandy soils. PhD-thesis Wageningen University, 213 p.

Ritzema, H.P., 1994. Subsurface flow to drains. In 'Drainage principles and applications', H.P. Ritzema (Ed. in Chief), ILRI publication 16, second edition, Wageningen, p. 263-304.

Ross, P.J., 1990. Efficient numerical methods for infiltration using Richards' equation. Water Resour. Res., 26, 279-290.

Rozemeijer, J.C., Y. van der Velde, F.C. van Geer, G.H. de Rooij, P.J.J.F. Torfs, H.P. Broers, 2010. Improving load estimates for NO3 and $P$ in surface waters by characterizing the concentration response to rainfall events. Environ. Sci. Technol., 44 (16), 6305-6312.

Russo, D., E. Bresler, U. Shani and J. Parker, 1991. Analysis of infiltration events in relation to determining soil hydraulic properties by inverse problem methodology. Water Resour. Res., 27, 1361-1373.

Rutter, A.J., Kershaw, K.A., Robins, P.C., and Morton, A.J., 1971. A predictive model of rainfall interception in forests, 1 . Derivation of the model from observations in a plantation of Corsican Pine, Agr. Meteorol., 9, 367-384.

Saxena, R.K., N.J. Jarvis and L. Bergström, 1994. Interpreting non-steady state tracer breakthrough experiments in sand and clay soils using a dual-porosity model. J. Hydrol., 162, 279-298.

Schaap, Marcel G. and Martinus Th. Van Genuchten, 2006. A Modified Mualem-van Genuchten Formulation for Improved Description of the Hydraulic Conductivity Near Saturation. In: Vadose Zone Journal 5:27-34.

Scorza Júnior, R.P., J.H. Smelt, J.T.I. Boesten, R.F.A. Hendriks and S.E.A.T.M. van der Zee (2004). Preferential flow of bromide, bentazon and imidacloprid in a Dutch clay soil. J. Environ. Qual. 33:1473-1486.

Scott, P.S., G.J. Farquhar and N. Kouwen, 1983. Hysteretic effects on net infiltration. In 'Advances in infiltration', American Society of Agricultural Engineers, St. Joseph, Mich, p. 163-170.

Shalhevet, J., 1994. Using water of marginal quality for crop production: major issues. Agric. Water Man., 25, 233-269.

Sidle, R.C., S. Noguchi, Y. Tsuboyama and K. Laursen, 2001. A conceptual model of preferential flow systems in forested hillslopes: evidence of self-organization. Hydrol. Process., 15: 1675-1692.

Singh, P., G. Spitzbart, H. Hübl, H.W. Weinmeister, 1997. Hydrological response of snowpack under rain-on-snow events: a field study. Journal of Hydrology 202: 1-20. 
Šimůnek, J., T. Vogel and M.Th. van Genuchten, 1992. The SWMS-2D code for simulating water flow and solute transport in two-dimensional variably saturated media. Version 1.1. Res. Rep. 126, U.S. Salinity Lab., Agric. Res. Ser., U.S. Dept. of Agric., Riverside, Calif.

Šimůnek, J., K. Huang, M. Šejna, and M.Th. van Genuchten. 1998. The HYDRUS-1D Software Package for Simulating the One-Dimensional Movement of Water, Heat and Multiple Solutes in VariablySaturated Media, Version 1.0. IGWMC-TPS-70, Int. Ground Water Modeling Center, Colorado School of Mines, Golden, CO., 186 p.

Šimůnek, J., R. Kodesova, M.M. Gribb and M.T. van Genuchten, 1999. Estimating hysteresis in the soil water retention function from cone permeameter experiments. Water Resour. Res., 35: 1329-1345.

Šimůnek, J., M. Šejna and M.Th. van Genuchten, 2007. The HYDRUS Software Package for Simulating the Two- and Three-Dimensional Movement of Water, Heat, and Multiple Solutes in VariablySaturated Media. User Manual Version 1.0. PC-Progress, Prague, Czech Republic.

Skaggs, T.H., M.Th van Genuchten, P.J. Shouse, and J.A. Poss, 2006. Macroscopic approaches to root water uptake as a function of water and salinity stress. Agric. Water Man., 86, 140-149.

Smelt, J.H., Hendriks, R.F.A., Pas, L.J.T. van der, Matser, A.M., Toorn, A. van den, K. Oostindie, O.M. van Dijk-Hooijer J.J.T.I Boesten, R.P. Scorza (2003). Transport of water, bromide ion, nutrients and the pesticides bentazone and imidacloprid in a cracking, tile drained clay soil at Andelst, The Netherlands, rapport 289. Wageningen.

Smith, M., 1992. CROPWAT, a computer program for irrigation planning and management. FAO Irrigation and Drainage Paper 46. Rome, Italy.

Spitters, C.J.T., H. van Keulen and D.W.G. van Kraalingen, 1989. A simple and universal crop growth simulator: SUCROS87. In: R. Rabbinge, S.A. Ward and H.H. van Laar (Eds.) Simulation and systems management in crop protection. Simulation Monographs, Pudoc, Wageningen, The Netherlands. p. 147-181.

Stolte, J., J.I. Freijer, W. Bouten, C. Dirksen, J.M. Halbertsma, J.C. van Dam, J.A. van den Berg, G.J. Veerman and J.H.M. Wösten, 1994. Comparison of six methods to determine unsaturated soil hydraulic conductivity. Soil Sci. Soc. Am. J., 58, 1596-1603.

Stricker, J.N.M., W. Brutsaert, 1978. Actual evapotranspiration over a summer in the Hupsel Catchment. J. Hydrol., 39, 139-157.

Spitters, C.J.T., Toussaint, H.A.J.M., Goudriaan, J., 1986. Separating the diffuse and direct component of global radiation and its implications for modelling canopy photosynthesis. Part I: Components of incoming radiation. Agricultural and Forest Meteorology, 38:217-229.

Spitters, C.J.T., Toussaint, H.A.J.M., Goudriaan, J., 1986. Separating the diffuse and direct component of global radiation and its implications for modelling canopy photosynthesis. Part II: Calculation of canopy photosynthesis/ Agricultural and Forest Meteorology, 38:231-242.

Stroosnijder, L., 1975. Infiltration and redistribution of water in the soil. PhD-thesis, Wageningen University, 213 p. (in Dutch)

Supit, I., A.A. Hooyer and C.A. van Diepen (Eds.), 1994. System description of the WOFOST 6.0 crop simulation model implemented in CGMS. Vol. 1: Theory and algorithms. EUR publication 15956, Agricultural series, Luxembourg, $146 \mathrm{p}$.

Taylor, S.A., and G.M. Ashcroft, 1972. Physical Edaphology. Freeman and Co., San Francisco, California, p. 434-435.

Ten Berge, H.F.M., 1986. Heat and water transfer at the bare soil surface: aspects affecting thermal images. PhD thesis, Wageningen Agricultural University, Wageningen, The Netherlands.

Tetens, O., 1930. Uber einige meteorologische Begriffe. Z. Geophys, 6, 297-309.

Thoma, S.G., D.P. Gallegos and D.M. Smith, 1992. Impact of fracture coatings on fracture/matrix flow interactions in unsaturated porous media. Water Resources Res., 28, 1357-1367.

Thoms, R.B., R.L. Johnson, and R.W. Healy, 2006. User's guide to the variability saturated flow (VSF) process for MODFLOW. USGS Techniques and Methods 6-A18. Reston, Virginia: U.S. Geological Survey.

Tiktak, A., F. van den Berg, J.J.T.I. Boesten, M. Leistra, A.M.A. van der Linden, and D. van Kraalingen, 2000. Pesticide Emission at Regional and Local scales: Pearl version 1.1 User Manual. RIVM report 711401008, report 29, Alterra Green World Research, Wageningen.

Tiktak, A., Hendriks, R.F.A., \& Boesten, J.J.T.I. (2012a). Simulation of movement of pesticides towards drains with a preferential flow version of PEARL. Pest Management Science, 68(2), 290-302. http://doi.org/10.1002/ps.2262 
Tiktak, A., Hendriks, R.F.A., Boesten, J.J.T.I., \& van der Linden, A.M.A. (2012b). A spatially distributed model of pesticide movement in Dutch macroporous soils. Journal of Hydrology, 470-471, 316-327. http://doi.org/10.1016/j.jhydrol.2012.09.025

Van Bakel, P.J.T., 1986. Planning, design and operation of surface water management systems; a case study. PhD thesis, Wageningen Agricultural University.

Van Dam, J.C., J.M.H. Hendrickx, H.C. van Ommen, M.H. Bannink, M.Th. van Genuchten and L.W. Dekker, 1990. Water and solute movement in a coarse-textured water-repellent field soil. J. Hydrol., 120, 359-379.

Van Dam, J.C., J.N.M. Stricker and P. Droogers, 1994. Inverse method to determine soil hydraulic functions from multi-step outflow experiments. Soil Sci. Soc. Am. J., 58, 647-652.

Van Dam, J.C., J.H.M. Wösten and A. Nemes, 1996. Unsaturated soil water movement in hysteretic and water repellent soils. J. Hydrol., 184, 153-173.

Van Dam, J.C., J. Huygen, J.G. Wesseling, R.A. Feddes, P. Kabat, P.E.V. van Walsum, P. Groenendijk and C.A. van Diepen, 1997. Theory of SWAP version 2.0. Simulation of water flow, solute transport and plant growth in the Soil-Water-Atmosphere-Plant environment. Wageningen University and Alterra. Technical Document 45.

Van Dam, J.C., 2000. Field scale water flow and solute transport. SWAP model concepts, parameter estimation and case studies. PhD thesis, Wageningen Universiteit, $167 \mathrm{p}$.

Van Dam, J.C., and R.A. Feddes, 2000. Simulation of infiltration, evaporation and shallow groundwater levels with the Richards' equation. J. of Hydrol., 233, 72-85.

Van Dam, J.C., Groenendijk, P., Hendriks, R.F.A., \& Kroes, J.G., 2008. Advances of Modeling Water Flow in Variably Saturated Soils with SWAP. Vadose Zone Journal, 7(2), 640-653.

Van de Pol, R.M., P.J. Wierenga and D.R. Nielsen, 1977. Solute movement in a field soil. Soil Sci. Soc. Am. J., 41, 10-13.

Van den Berg, F., and J.J.T.I. Boesten, 1998. PEsticide Leaching and Accumulation model (PESTLA) version 3.4; description and user's guide. Technical Document 43, Alterra Green World Research, Wageningen, $150 \mathrm{p}$.

Van den Broek, B.J., J.C. van Dam, J.A. Elbers, R.A. Feddes, J. Huygen, P. Kabat and J.G. Wesseling, 1994. SWAP 1993, input instructions manual. Report 45, Dep. Water Resources, Wageningen Agricultural University.

Van den Eertwegh, G.A.P.H., 2002. Water and nutrient budgets at field and regional scale: travel times of drainage water and nutrient loads to surface water. PhD thesis, Wageningen University.

Vanderborght, J., \& Vereecken, H., 2007. Review of Dispersivities for Transport Modeling in Soils. Vadose Zone Journal, 6(1), 29. http://doi.org/10.2136/vzj2006.0096

Van der Molen, W.H., and J. Wesseling, 1991. A solution in closed form and a series solution to replace the tables for the thickness of the equivalent layer in Hooghoudt's drain spacing formula. Agricultural Water Management 19, p. 1-16.

Van der Velde, Y., P.J.J.F. Torfs, S.E.A.T.M. van der Zee, R. Uijlenhoet, 2012. Quantifying catchmentscale mixing and its effect on time varying travel time distributions. Water Resour. Res., 48, W06536.

Van der Zee, S.E.A.T.M., and W.H. van Riemsdijk, 1987. Transport of reactive solute in spatially variable soil systems. Water Resour. Res., 23, 2059-2069.

Van Dobben, W.H., 1962. Influence of temperature and light conditions on dry matter distribution, development rate and yield in arable crops. Netherlands Journal of Agricultural Science 10, 377-389.

Van Genuchten, M.Th., and P.J. Wieringa, 1974. Simulation of one-dimensional solute transfer in porous media. New Mexico State University Agric. Exp. Stn. Bull. 628, New Mexico.

Van Genuchten, M.Th., and R.W. Cleary, 1979. Movement of solutes in soil : computer simulated and laboratory results. In: G.H. Bolt (Ed.), Soil Chemistry B, Physico-Chemical Models. Elsevier, Amsterdam, pp. 349-386.

Van Genuchten, M.Th., 1980. A closed form equation for predicting the hydraulic conductivity of unsaturated soils. Soil Sci. Soc. Am. J., 44, 892-898.

Van Genuchten, M.Th., 1982. A comparison of numerical solutions of the one-dimensional unsaturated-saturated flow and transport equations. Adv. Water Resour., 5, 47-55.

Van Genuchten, M.Th., 1987. A numerical model for water and solute movement in and below the root zone. Res. Report, US Salinity Laboratory, Riverside, CA. 
Van Genuchten, M.Th., and R.J. Wagenet, 1989. Two-site/two-region models for pesticide transport and degradation: Theoretical development and analytical solutions. Soil Sci. Soc. Am. J., 53, 1303-1310.

Van Genuchten, M.Th., F.J. Leij and S.R. Yates, 1991. The RETC code for quantifying the hydraulic functions for unsaturated soils. U.S. Salinity Laboratory, Riverside, California.

Van Genuchten, M.Th., and F.J. Leij, 1992. On estimating the hydraulic properties of unsaturated soils. In 'Indirect methods for estimating hydraulic properties of unsaturated soils', M.Th. van Genuchten and F.J. Leij (eds.), Proc. Int. Workshop, Riverside, California, p. 1-14.

Van Grinsven, J.J.M., C. Dirksen and W. Bouten, 1985. Evaluation of hot air method for measuring soil water diffusivity. Soil Sci. Soc. Am. J., 49, 1093-1099.

Van Heemst, H.D.J., 1986a. The distribution of dry matter during growth of a potato crop. Potato Research 29, 55-66.

Van Heemst, H.D.J., 1986b. Crop phenology and dry matter distribution. In: H. van Keulen and J. Wolf (Eds.). Modelling of agricultural production: soil, weather and crops. p. 13-60.

Van Ittersum, M.K., P.A. Leffelaar, H. van Keulen, M.J. Kropff, L. Bastiaans and J. Goudriaan, 2003. On approaches and applications of the Wageningen crop models. Europ. J. Agronomy 18, 201-234.

Van Keulen, H., 1975. Simulation of water use and herbage growth in arid regions. Simulation Monographs. Pudoc, Wageningen, the Netherlands. $184 \mathrm{pp}$.

Van Keulen, H., N.G. Seligman and R.W. Benjamin, 1981. Simulation of water use and herbage growth in arid regions - A re-evaluation and further development of the model 'Arid Crop'. Agricultural systems 6, 159-193.

Van Keulen, H., and J. Wolf, 1986. Modelling of agricultural production: weather, soil and crops. Simulation Monographs. Pudoc, Wageningen, The Netherlands, pp. 479.

Van Keulen, H., and N.G. Seligman, 1987. Simulation of water use, nitrogen nutrition and growth of a spring wheat crop. Simulation Monographs. Pudoc, Wageningen, The Netherlands. 310 pp.

Van Laar, H.H., J. Goudriaan and H. van Keulen (Eds.), 1992. Simulation of crop growth for potential and water-limited production situations (as applied to spring wheat). Simulation reports CABO-TT 27. CABO-DLO, WAU-TPE, Wageningen. 72 pp.

Van Ommen, H.C., M.Th. van Genuchten, W.H. van der Molen, R. Dijksma and J. Hulshof, 1989. Experimental assessment of preferential flow paths in a field soil. J. Hydrol., 105, 253-262.

Van Stiphout, T.P.J., H.A.J. van Lanen, O.H. Boersma, and J. Bouma, 1987. The effect of bypass flow and internal catchment of rain on the water regime in a clay loam grassland soil. J. Hydrol. 95, 1-11.

Van Walsum, P.E.V., \& Supit, I., 2012. Influence of ecohydrologic feedbacks from simulated crop growth on integrated regional hydrologic simulations under climate scenarios. Hydrology and Earth System Sciences, 16(6), 1577-1593. http://doi.org/10.5194/hess-16-1577-2012

Van Walsum, P.E.V., and F.J.E. van der Bolt, 2013. "Sensitivity of the Delta Model to Evapotranspiration Exploring Structural Uncertainties due to Evapotranspiration Concepts. Alterra Report 2481."

Van Wijk, A.L.M., R.A. Feddes, J.G. Wesseling, and J. Buitendijk, 1988. "Effecten van Grondsoort En Ontwatering Op de Opbrengst van Akkerbouwgewassen". ICW Rapporten nieuwe serie 31. Wageningen.

Van Wijk, W.R. (Ed.), 1966. Physics of plant environment. North Holland Publ. Comp., Amsterdam, The Netherlands, 2nd edition. $382 \mathrm{pp}$.

Vereecken, H., M. Weynants, M. Javaux, Y. Pachepsky, M.G. Schaap and M.Th. van Genuchten, 2010. Using pedotransferfunctions to estimate the van Genuchten-Mualem soil hydraulic properties: a review. Vadose Zone J., 9, 795-820.

Vogel, T., M.Th. van Genuchten, M. Cislerova, 2001. Effect of the shape of the soil hydraulic functions near saturation on variably-saturated flow predictions. Advances in Water Resources, 24, $133-144$.

Von Hoyningen-Hüne, J., 1983. Die Interception des Niederschlags in landwirtschaftlichen Beständen. Schriftenreihe des DVWK 57, 1-53.

Vos, A. de, B. Bruning, G. van Straten, R. Oosterbaan, J. Rozema and P. van Bodegom (2016). Crop salt tolerance under controlled field conditions in The Netherlands, based on trials conducted at Salt Farm Texel. SaltFarm Texel. Texel. The Netherlands. Retrieved from www.saltfarmtexel.com as http://www.saltfarmtexel.com/application/files/5714/8776/5471/saltfarmtexel_brochure_A4.pdf, dd 24-April-2017

Vos, J., and J. Groenwold, 1986. Root growth of potato crops on a marine-clay soil. Plant Soil, 94, 17-33. 
Voss, C.I., and A.M. Provost, 2002. SUTRA, A model for saturated-unsaturated variable-density ground-water flow with solute or energy transport. U.S. Geological Survey Water-Resources Investigations Report 02-4231, $250 \mathrm{p}$.

Walker, A., 1974. A simulation model for prediction of herbicide persistence. J. Environ. Qual., 3, 396-401.

Warrick, A.W., 1991. Numerical approximations of darcian flow through unsaturated soil. Water Resour. Res., 27, 1215-1222.

Weir, A.H., P.L. Bragg, J.R. Porter and J.H. Rayner, 1984. A winter wheat crop simulation model without water and nutrient limitations. Journal of Agricultural Science 102, 371-382.

Wendroth, O., W. Ehlers, J.W. Hopmans, H. Kage, J. Halbertsma and J.H.M. Wösten, 1993. Reevaluation of the evaporation method for determining hydraulic functions in unsaturated soils. Soil Sci. Soc. Am. J., 57, 1436-1443.

Wesseling, J.G.; Wesseling, J., 1984. Influence of seepage on the depth of water tables in drainage. J. Hydrol., 73, 289-297

Wesseling, J.G., 1987. Invloed van bodemsoort en vochtgehalte op de bodemtemperatuur. Een theoretische beschouwing. Cultuurtechnisch tijdschrift, 27(2), 117-128.

Wesseling, J.G., J.A. Elbers, P. Kabat and B.J. van den Broek, 1991. SWATRE; instructions for input. Internal note, Winand Staring Centre, Wageningen.

Wesseling, J.G., J.G. Kroes, and K. Metselaar, 1998. Global sensitivity analysis of the Soil-WaterAtmosphere-Plant (SWAP) model. Report 160, Alterra, Wageningen, $67 \mathrm{p}$.

Wolfe, N.L., U. Mingelgrin, G.C. Miller, 1990. Abiotic transformations in water, sediments and soil. In: H.H. Cheng (Ed.), Pesticides in the soil environment: processes, impacts and modeling. SSSA Book Series no. 2, Madison, Wisconsin, USA.

Wopereis, F.A., 1994. Invloed van bodemverdichting op de wortelontwikkeling van grasland op zandgrond. Rapport DLO-Staring Centrum 260, Wageningen.

Wösten, J.H.M., G.J. Veerman and J. Stolte, 1994. Water retention and hydraulic conductivity functions of top- and subsoils in The Netherlands: The Staring series. Technical Document 18, Winand Staring Centre, Wageningen, The Netherlands, 66 p. (in Dutch).

Wösten, J.H.M., A. Lilly, A. Nemes and C. Le Bas, 1998. Using existing soil data to derive hydraulic properties for simulation models in environmental studies and in land use planning. Report 156, Winand Staring Centre, The Netherlands.

Wösten, J.H.M., G.J. Veerman, W.J.M. de Groot, and J. Stolte, 2001. Water retention and hydraulic conductivity functions of top- and subsoils in The Netherlands: The Staring series. Alterra report 153, Wageningen, The Netherlands, 86 p. (in Dutch).

Wösten, H., Vries, F. De, Hoogland, T., Massop, H., Veldhuizen, A., Vroon, H., Wesseling, J., Heijkers, J., Bolman, A., 2013. BOFEK2012, de nieuwe, bodemfysische schematisatie van Nederland. Alterra-rapport 2387. Wageningen, The Netherlands, 88 p. (in Dutch). See also http://www.wur.nl/nl/show/Bodemfysische-Eenhedenkaart-BOFEK2012.htm

Yates, S.R., M.Th. van Genuchten, A.W. Warrick and F.J. Leij, 1992. Analysis of measured, predicted and estimated hydraulic conductivity using the RETC computer program. Soil Sci. Soc. Am. J., 56, 347-354.

Youngs, E.G., and R.I. Price, 1981. Scaling of infiltration behaviour in dissimilar porous materials. Water Resour. Res., 17, 1065-1070.

Yule, D.F., and J.T. Ritchie, 1980a. Soil shrinkage relationships of Texas Vertisols: I. Small cores. Soil Sci. Soc. Am. J., 44: 1285-1291.

Yule, D.F., and J.T. Ritchie, 1980b. Soil shrinkage relationships of Texas Vertisols: II. Large cores. Soil Sci. Soc. Am. J., 44: 1291-1295.

Zaidel, J., and D. Russo, 1992. Estimation of finite difference interblock conductivities for simulation of infiltration into initially dry soils. Water Resour. Res., 28, 2285-2295. 


\section{Appendix 1 Application Penman Monteith method}

Description of Penman-Monteith method after Allen et al. (1998).

The original form of the Penman-Monteith equation can be written as (Monteith, 1965, 1981):

$$
E T_{p}=\frac{\frac{\Delta_{v}}{\lambda_{w}}\left(R_{n}-G\right)+\frac{p_{1} \rho_{\text {air }} C_{\text {air }} \frac{e_{\text {sat }}-e_{a}}{\lambda_{w}}}{r_{\text {air }}}}{\Delta_{v}+\gamma_{\text {air }}\left(1+\frac{r_{\text {crop }}}{r_{\text {air }}}\right)}
$$

where $E T_{\mathrm{p}}$ is the potential transpiration rate of the canopy $\left(\mathrm{mm} \mathrm{d}^{-1}\right), \Delta_{\mathrm{V}}$ is the slope of the vapour pressure curve $\left(\mathrm{kPa}{ }^{\circ} \mathrm{C}^{-1}\right), \lambda_{w}$ is the latent heat of vaporization $\left(\mathrm{J} \mathrm{kg}^{-1}\right), R_{\mathrm{n}}$ is the net radiation flux at the canopy surface $\left(\mathrm{J} \mathrm{m}^{-2} \mathrm{~d}^{-1}\right), G$ is the soil heat flux $\left(\mathrm{J} \mathrm{m}^{-2} \mathrm{~d}^{-1}\right), p_{1}$ accounts for unit conversion $\left(=86400 \mathrm{~s} \mathrm{~d}^{-1}\right), \rho_{\text {air }}$ is the air density $\left(\mathrm{kg} \mathrm{m}^{-3}\right), C_{\text {air }}$ is the heat capacity of moist air $\left(\mathrm{J} \mathrm{kg}^{-1}{ }^{\circ} \mathrm{C}^{-1}\right), e_{\text {sat }}$ is the saturation vapour pressure $(\mathrm{kPa}), e_{\mathrm{a}}$ is the actual vapour pressure $(\mathrm{kPa}), \gamma_{\text {air }}$ is the psychrometric constant $\left(\mathrm{kPa}{ }^{\circ} \mathrm{C}^{-1}\right), r_{\text {crop }}$ is the crop resistance $\left(\mathrm{s} \mathrm{m}^{-1}\right)$ and $r_{\text {air }}$ is the aerodynamic resistance $\left(\mathrm{s} \mathrm{m}^{-1}\right)$.

To facilitate analysis of the combination equation, an aerodynamic and radiation term are defined:

$$
E T_{p}=E T_{\text {rad }}+E T_{\text {aero }}
$$

where $E T_{\mathrm{p}}$ is potential transpiration rate of crop canopy $\left(\mathrm{cm} \mathrm{d}^{-1}\right), E T_{\text {rad }}$ is the radiation term ( $\left.\mathrm{cm} \mathrm{d}^{-1}\right)$ and $E T_{\text {aero }}$ is the aerodynamic term $\left(\mathrm{cm} \mathrm{d}^{-1}\right)$.

The radiation term equals:

$$
E T_{\text {rad }}=\frac{\Delta_{v}\left(R_{n}-G\right)}{\lambda_{w}\left(\Delta_{v}+\gamma_{a i r}^{*}\right)}
$$

where the modified psychrometric constant $\left(\mathrm{kPa}{ }^{\circ} \mathrm{C}^{-1}\right)$ is:

$$
\gamma_{\text {air }}^{*}=\gamma_{\text {air }}\left(1+\frac{r_{\text {crop }}}{r_{\text {air }}}\right)
$$

The aerodynamic term equals:

$$
E T_{\text {aero }}=\frac{p_{1} \rho_{\text {air }} C_{\text {air }}\left(e_{\text {sat }}-e_{a}\right)}{\lambda_{w}\left(\Delta_{v}+\gamma_{\text {air }}^{*}\right) r_{\text {air }}}
$$

Many meteorological stations provide mean daily values of air temperature $T_{\text {air }}\left({ }^{\circ} \mathrm{C}\right)$, global solar radiation $R_{\mathrm{s}}\left(\mathrm{J} \mathrm{m}^{-2} \mathrm{~d}^{-1}\right)$, wind speed $u_{0}\left(\mathrm{~m} \mathrm{~s}^{-1}\right)$ and air humidity $e_{\text {act }}(\mathrm{kPa})$. These basic meteorological data are used to apply the Penman Monteith equation. 


\section{Radiation term}

The net radiation $R_{\mathrm{n}}\left(\mathrm{J} \mathrm{m}^{-2} \mathrm{~d}^{-1}\right)$ is the difference between incoming and outgoing radiation of both short and long wavelengths. It is the balance between the energy adsorbed, reflected and emitted by the earth's surface:

$$
R_{\mathrm{n}}=\left(1-\alpha_{\mathrm{r}}\right) R_{\mathrm{s}}-R_{\mathrm{nl}}
$$

where $\alpha_{r}$ is the reflection coefficient or albedo $(-)$ and $R_{\mathrm{nl}}$ is the net longwave radiation $\left(\mathrm{J} \mathrm{m}^{-2} \mathrm{~d}^{-1}\right)$. The albedo is highly variable for different surfaces and for the angle of incidence or slope of the ground surface. It may be as large as 0.95 for freshly fallen snow and as small as 0.05 for a wet bare soil. A green vegetation cover has an albedo of about 0.20-0.25 (De Bruin, 1998). SWAP will assume in case of a crop $\alpha_{r}=0.23$, in case of bare soil $\alpha_{r}=0.15$.

The earth emits longwave radiation, which increases with temperature and which is adsorbed by the atmosphere or lost into space. The longwave radiation received by the atmosphere increases its temperature and, as a consequence, the atmosphere radiates energy of its own. Part of this radiation finds its way back to the earth's surface. As the outgoing longwave radiation is almost always greater than the incoming longwave radiation, the net longwave radiation $R_{\mathrm{n}}$ represents an energy loss. Allen et al. (1998) recommend the following formula for the net longwave radiation:

$$
R_{\mathrm{nl}}=\sigma_{\mathrm{sb}}\left[\frac{T_{\max }^{4}+T_{\min }^{4}}{2}\right]\left(0.34-0.14 \sqrt{e_{\mathrm{act}}}\right)\left(0.1+0.9 N_{\text {rel }}\right)
$$

where $\sigma_{\mathrm{sb}}$ is the Stefan-Boltzmann constant $\left(4.90310^{-3} \mathrm{~J} \mathrm{~K}^{-4} \mathrm{~m}^{-2} \mathrm{~d}^{-1}\right), T_{\min }$ and $T_{\max }$ are the minimum and maximum absolute temperatures during the day $(\mathrm{K})$, respectively, $e_{\text {act }}$ is the actual vapour pressure $(\mathrm{kPa})$, and $N_{\text {rel }}$ is the relative sunshine duration. The latter can be derived from the measured global solar radiation $R_{\mathrm{s}}$ and the extraterrestrial radiation $R_{\mathrm{a}}\left(\mathrm{J} \mathrm{m}^{-2} \mathrm{~d}^{-1}\right)$, which is received at the top of the Earth's atmosphere on a horizontal surface:

$$
N_{\text {rel }}=\left(\frac{R_{\mathrm{s}}}{R_{\mathrm{a}}}-a\right) \frac{1}{b}
$$

where $a$ and $b$ are so-called Angstrom coefficients which depend on the local climate. For international use Allen et al. (1998) recommend $a=0.25$ and $b=0.50$.

The extraterrestrial radiation $R_{\mathrm{a}}$ depends on the latitude and the day of the year. $R_{\mathrm{a}}$ is calculated with:

$$
R_{\mathrm{a}}=\frac{G_{\mathrm{sc}}}{\pi} d_{\mathrm{r}}\left[\omega_{\mathrm{s}} \sin (\varphi) \sin (\delta)+\cos (\varphi) \cos (\delta) \sin \left(\omega_{\mathrm{s}}\right)\right]
$$

where $G_{s c}$ is the amount of solar radiation striking a surface perpendicular to the sun's rays at the top of the Earth's atmosphere, called the solar constant $\left(\mathrm{J} \mathrm{m}^{-2} \mathrm{~d}^{-1}\right), d_{\mathrm{r}}$ is the inverse relative distance Earth-Sun ( -$), \omega_{\mathrm{s}}$ is the sunset hour angle (rad), $\varphi$ is the latitude (rad) and $\delta$ is the solar declination (rad). The inverse relative distance Earth-Sun and the solar declination are given by:

$$
\begin{aligned}
& d_{\mathrm{r}}=1+0.033 \cos \left(\frac{2 \pi}{365} J\right) \\
& \delta=0.409 \sin \left(\frac{2 \pi}{365} J-1.39\right)
\end{aligned}
$$

where $J$ is the number of the day in the year (1-365 or 366, starting January 1$)$. The sunset hour angle expresses the day length and is given by:

$$
\omega_{\mathrm{s}}=\arccos [-\tan (\varphi) \tan (\delta)]
$$




\section{Aerodynamic term}

Latent heat of vaporization, $\lambda_{w}\left(\mathrm{~J} \mathrm{~g}^{-1}\right)$, depends on the air temperature $T_{\text {air }}\left({ }^{\circ} \mathrm{C}\right)$ (Harrison, 1963):

$$
\lambda_{w}=2501-2.361 T_{\text {air }}
$$

Saturation vapour pressure, $e_{\text {sat }}(\mathrm{kPa})$, also can be calculated from air temperature (Tetens, 1930):

$$
e_{\text {sat }}=0.611 \exp \left(\frac{17.27 T_{\text {air }}}{T_{\text {air }}+237.3}\right)
$$

The slope of the vapour pressure curve, $\Delta_{v}\left(\mathrm{kPa}{ }^{\circ} \mathrm{C}^{-1}\right)$, is calculated as (Murray, 1967):

$$
\Delta_{v}=\frac{4098 e_{\text {sat }}}{\left(T_{\text {air }}+237.3\right)^{2}}
$$

The psychrometric constant, $\gamma_{\text {air }}\left(\mathrm{kPa}{ }^{\circ} \mathrm{C}^{-1}\right)$, follows from (Brunt, 1952):

$$
\gamma_{\text {air }}=1.63 \frac{p_{\text {air }}}{\lambda_{\mathrm{w}}}
$$

with $p_{\text {air }}$ the atmospheric pressure $(\mathrm{kPa})$ at elevation $z_{0}(\mathrm{~m})$, which is calculated from (Burman et al., 1987):

$$
p_{\text {air }}=101.3\left(\frac{T_{\mathrm{air}, \mathrm{K}}-0.0065 z_{0}}{T_{\mathrm{air}, \mathrm{K}}}\right)^{5.256}
$$

Employing the ideal gas law, the atmospheric density, $\rho_{a}\left(\mathrm{~g} \mathrm{~cm}^{-3}\right)$, can be shown to depend on $p$ and the virtual temperature $T_{\text {vir }}(\mathrm{K})$ :

$$
\rho_{\text {air }}=3.48610^{-3} \frac{p_{\text {air }}}{T_{\text {vir }}}
$$

where the virtual temperature is derived from:

$$
T_{\mathrm{vir}}=\frac{T_{\mathrm{air}, \mathrm{K}}}{1-0.378 \frac{e_{\mathrm{act}}}{p_{\mathrm{air}}}}
$$

The heat capacity of moist air, $C_{\text {air }}\left(\mathrm{J} \mathrm{g}^{-1}{ }^{\circ} \mathrm{C}^{-1}\right)$, follows from:

$$
C_{\text {air }}=0.622 \frac{\gamma_{\text {air }} \lambda_{\mathrm{w}}}{p_{\text {air }}}
$$

\section{Aerodynamic resistance}

The aerodynamic resistance $r_{\text {air }}$ depends on the wind speed profile and the roughness of the canopy and is calculated as (Allen et al., 1998):

$$
r_{\mathrm{air}}=\frac{\ln \left(\frac{z_{\mathrm{m}}-d}{z_{\mathrm{om}}}\right) \cdot \ln \left(\frac{z_{\mathrm{h}}-d}{z_{\mathrm{oh}}}\right)}{\kappa_{\mathrm{vk}}^{2} \cdot u}
$$

where $z_{\mathrm{m}}$ is height of wind speed measurements $(\mathrm{m}), z_{\mathrm{h}}$ is height of temperature and humidity measurements $(\mathrm{m}), d$ is zero plane displacement of wind profile $(\mathrm{m}), z_{\mathrm{om}}$ is roughness parameter for momentum $(\mathrm{m})$ and $z_{\mathrm{oh}}$ is roughness parameter for heat and vapour $(\mathrm{m}), \mathrm{k}_{\mathrm{vk}}$ is von Karman constant $=0.41(-), u$ is wind speed measurement at height $z_{\mathrm{m}}\left(\mathrm{m} \mathrm{s}^{-1}\right)$.

The parameters $d, z_{\mathrm{om}}$ and $z_{\mathrm{oh}}$ are defined as:

$$
\begin{aligned}
& d=\frac{2}{3} h_{\text {crop }} \\
& z_{\text {om }}=0.123 h_{\text {crop }}
\end{aligned}
$$




$$
z_{\text {oh }}=0.1 z_{\text {om }}
$$

with $h_{\text {crop }}$ the crop height $(\mathrm{cm})$

A default height of $2 \mathrm{~m}$ is assumed for wind speed measurements $\left(z_{\mathrm{m}}\right)$ and height of temperature and humidity measurements $\left(z_{\mathrm{h}}\right)$.

Meteorological stations generally provide 24 hour averages of wind speed measurements, according to international standards, at an altitude of 10 meter.

To calculate $r_{\text {air, }}$ the average daytime wind $(7.00-19.00 \mathrm{~h})$ should be used. For ordinary conditions we assume (Smith, 1991) for the average daytime windspeed $\left(u_{0, \text { day }}\right)$ :

$$
u_{0, \text { day }}=1.33 u_{0}
$$

where $u_{0}$ is the measured average wind speed over 24 hours $\left(\mathrm{m} \mathrm{s}^{-1}\right)$.

When crop height $\left(h_{\text {crop }}\right)$ reaches below or above measurement height $\left(z_{\mathrm{m}, \text { meas }}\right)$, the wind speed is corrected with the following assumptions:

- a uniform wind pattern at an altitude of 100 meter;

- wind speed measurements are carried out above grassland;

- a logarithmic wind profile is assumed;

- below 2 meter wind speed is assumed to be unchanged with respect to a value at an altitude of 2 meter; applying a logarithmic wind profile at low altitudes is not carried out due to the high variation below 2 meter.

These assumptions result in the following equation for wind speed correction:

$$
u=\frac{\ln \left(\frac{z_{\text {act }}-d_{\text {act }}}{z_{\text {om,act }}}\right)}{\ln \left(\frac{z_{100}-d_{\text {act }}}{z_{\text {om,act }}}\right)} \frac{\ln \left(\frac{z_{100}-d_{\text {grass }}}{z_{\text {om,grass }}}\right)}{\ln \left(\frac{z_{\text {m,meas }}-d_{\text {grass }}}{z_{\text {om,grass }}}\right)} u_{0, \text { day }}
$$

where: $u$ wind speed at crop height $\left(\mathrm{m} \mathrm{s}^{-1}\right), z_{\text {act }}$ is the actual crop height with a minimum value of $2 \mathrm{~m}$, $d_{\text {act }}$ and $d_{\text {grass }}$ are zero plane displacement of actual crop and grass $(\mathrm{m}), z_{\mathrm{om}, \text { act }}$ and $z_{\mathrm{om}, \text { grass }}$ are roughness parameter for momentum actual crop and grass $(m)$. 


\section{Appendix 2 Derivation of some macropore geometry equations}

Basis of the determination of the effective vertical macropore wall area and the effective crack width is the assumption that the natural variety of soil matrix polygons can be described in terms of one effective regular soil matrix polygon. Crucial condition for this polygon is that many of it should fit together without any gaps to 'tile the plane'. From the regular polygons, only equilateral triangles, squares and regular hexagons have this quality. Empirical experience points out that squares and hexagons in particular are the most likely candidates for these polygons. Which of the two should be chosen, is irrelevant. They are both regular polygons with an even number of sides. All of these evensided regular polygons, from square to circle, have two relevant, special qualities: the quotient of their perimeter divided by their area is independent of the number of sides and their area is a function of the squared diameter.

\section{Effective vertical macropore wall area}

All even-sided regular polygons with $n$ sides are built up of $n$ equal isosceles triangles with base of length $x(\mathrm{~cm})$ and height $1 / 2 d_{\mathrm{pol}}(\mathrm{cm})$ (Figure A2.1). The perimeter of the polygon equals $n$ times $x$ and the area equals $n$ times the area of the triangle. The latter equals $1 / 4 d_{\mathrm{pol}} x\left(\mathrm{~cm}^{2}\right)$, so that:

$$
\frac{\text { perimeter }_{\mathrm{pol}}}{\text { area }_{\mathrm{pol}}}=\frac{n x}{n-d_{\mathrm{pol}} x}=\frac{4}{d_{\mathrm{pol}}}
$$

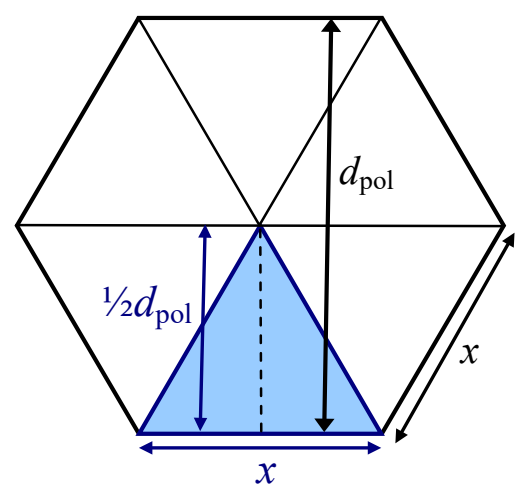

Figure A2.1 Hexagon: 6-sided regular polygon, consists of 6 isosceles triangles with area $1 / 2 d p o l \cdot 1 / 2 x=1 / 4$ dpolx. Area of polygon $=6 \cdot 1 / 4$ dpol $x$ and perimeter $=6 x$. Vertical wall area per unit of volume $A^{*}$ wall $=6 \times /(6 \cdot 1 / 4 d p o l x)=4 /$ dpol.

The vertical area of the wall of the polygon of Figure A2.1 per unit of depth is equal to the perymeter of the polygon. In order to express this area per unit of horizontal area it is divided by the area of the polygon. Thus, the effective vertical area of the wall of the matrix polygons $A^{*}$ wall per unit of depth and horizontal area, which implies per unit of volume, equals the quotient of the polygons' perimeter divided by their area, which equals $4 / d_{\text {pol }}\left(\mathrm{cm}^{-1}\right)$. 


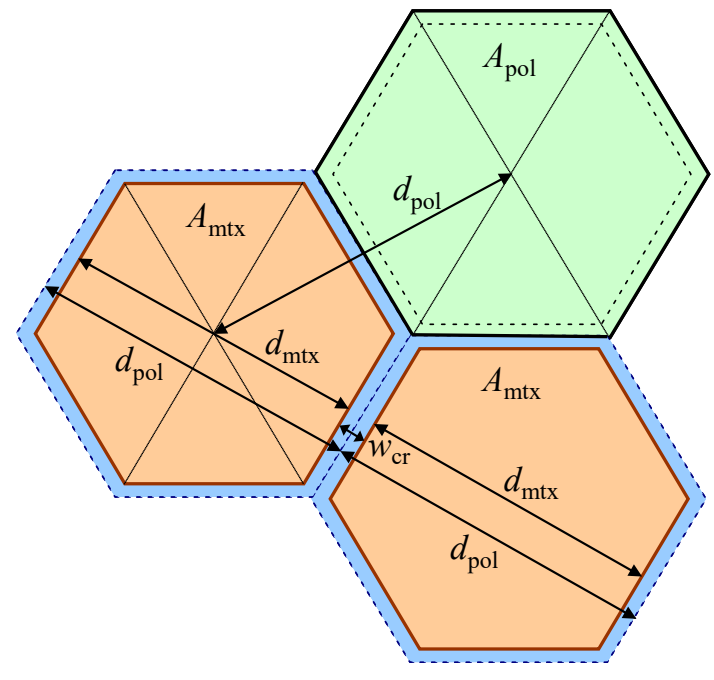

Figure A2.2 Area $A$ and diameter $d$ of basis polygon (pol) and matrix polygon (mtx). Crack width $w_{c r}$ is equal to the difference between $d_{p o l}$ and $d_{m t x}$.

\section{Effective crack width}

For even-sided regular polygons it can be derived that their sides $x$ (Figure A2.1) can be expressed as $\left(C_{n} / n\right) d_{\text {pol, }}$, where $C_{n}(-)$ is a constant that depends on $n$. For squares, hexagons and circles, $C_{n}$ equals, $4,2 \sqrt{ } 3$ and $n$, respectively. With $C_{n}$ a general equation for the area $A_{\text {pol }}\left(\mathrm{cm}^{2}\right)$ of an even-sided regular polygon as function of $d^{2}$ pol can be derived:

$$
A_{\mathrm{pol}}=n \frac{1}{4} d_{\mathrm{pol}} x=n \frac{1}{4} d_{\mathrm{pol}} \frac{\mathrm{C}_{n}}{n} d_{\mathrm{pol}}=\frac{1}{4} \mathrm{C}_{n} d_{\mathrm{pol}}^{2}
$$

For this equation, we define $d_{\mathrm{pol}}$ as the distance between the centres of two adjacent basis polygons (Figure A2.2). The value of this diameter is fixed. The value of the actual soil matrix polygon diameter $d_{\mathrm{mtx}}$ depends on the crack width, which is not fixed in case of a shrinking matrix. Thus, the crack width $w_{\mathrm{cr}}$ can be calculated as (Figure A2.2):

$$
w_{c r}=d_{\mathrm{pol}}-d_{\mathrm{mtx}}
$$

The horizontal area of the cracks $A_{\mathrm{cr}}\left(\mathrm{cm}^{2}\right)$ as fraction of $A_{\text {pol }}$ depends on the macropore volume fraction $V_{\mathrm{mp}}\left(\mathrm{cm}^{3} \mathrm{~cm}^{-3}\right)$ as:

$$
A_{\mathrm{cr}}=V_{\mathrm{mp}} A_{\mathrm{pol}}
$$

The horizontal area of the matrix polygon $A_{\mathrm{mtx}}\left(\mathrm{cm}^{2}\right)$ is a function of $d^{2}{ }_{\mathrm{mtx}}$ according to Eq. (A2.2):

$$
A_{\mathrm{mtx}}=\frac{1}{4} \mathrm{C}_{n} d_{\mathrm{mtx}}^{2}
$$

$A_{\mathrm{mtx}}$ is also equal to the difference between basis polygon area $A_{\mathrm{pol}}$ and crack area $A_{\mathrm{cr}}$ :

$$
A_{\mathrm{mtx}}=A_{\mathrm{pol}}-A_{\mathrm{cr}}=A_{\mathrm{pol}}-V_{\mathrm{mp}} A_{\mathrm{pol}}=\left(1-V_{\mathrm{mp}}\right) A_{\mathrm{pol}}=\left(1-V_{\mathrm{mp}}\right) \frac{1}{4} \mathrm{C}_{n} d_{\mathrm{pol}}^{2}
$$

Combining the right hands terms of Eq. (A2.5) and Eq. (A2.6) yields:

$$
\frac{1}{4} \mathrm{C}_{n} d_{\mathrm{mtx}}^{2}=\left(1-V_{\mathrm{mp}}\right) \underset{4}{-1} \mathrm{C}_{n} d_{\mathrm{pol}}^{2}
$$

and:

$$
d_{\mathrm{mtx}}^{2}=\left(1-V_{\mathrm{mp}}\right) d_{\mathrm{pol}}^{2}
$$

so that:

$$
d_{\mathrm{mtx}}=d_{\mathrm{pol}} \sqrt{1-V_{\mathrm{mp}}}
$$


And finally, the crack width is expressed as:

$$
w_{\mathrm{cr}}=d_{\mathrm{pol}}-d_{\mathrm{mtx}}=d_{\mathrm{pol}}\left(1-\sqrt{1-V_{\mathrm{mp}}}\right)
$$

Figure A2.3 shows the crack width $w_{\mathrm{cr}}$ as function of the macropore volume fraction $V_{\mathrm{mp}}$ for different polygon diameters $d_{\text {pol }}$.

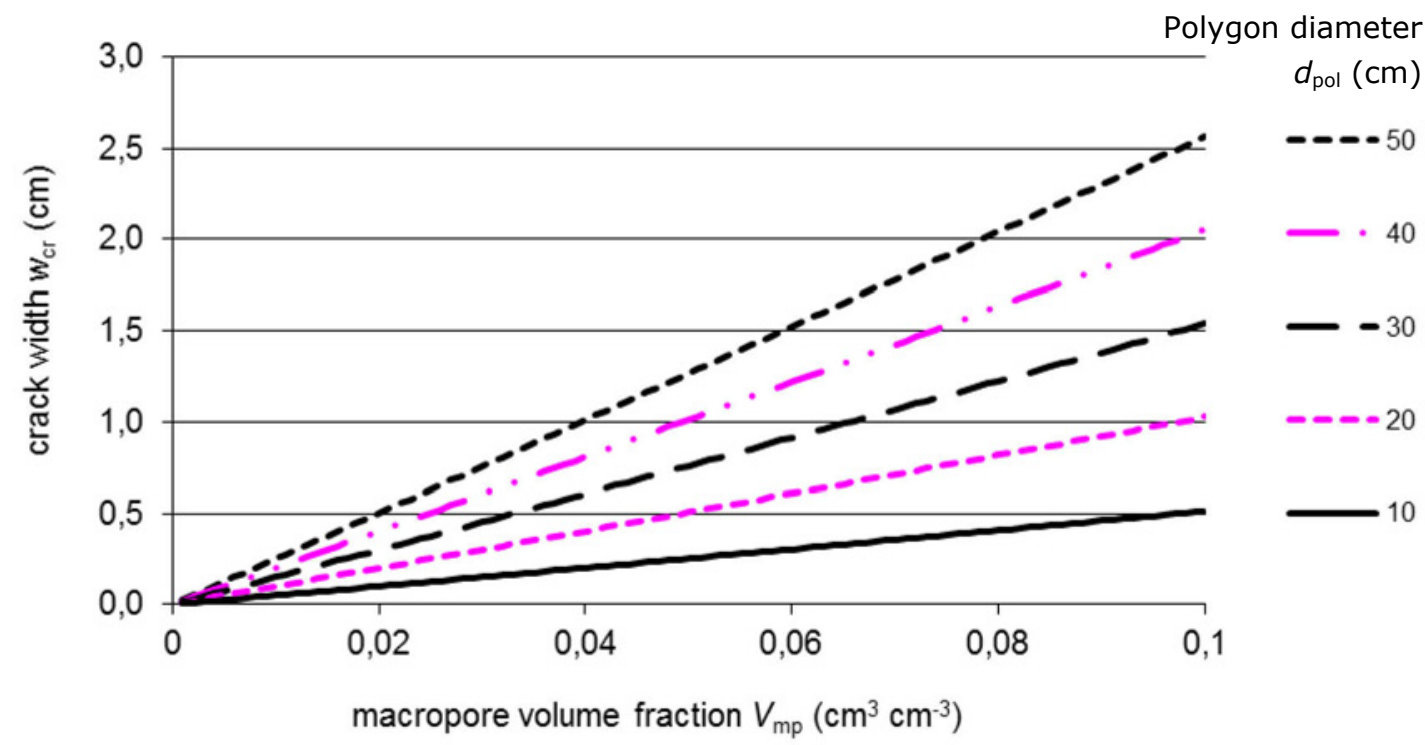

Figure A2.3 Crack width $w_{c r}$ as function of the macropore volume fraction $V_{m p}$ for different polygon diameters $d_{p o l}$

\section{Effect of crack width $\mathbf{w}_{\mathrm{cr}}$ on calculation of area of vertical wall $A *$ wall and distance $\mathbf{x}_{\mathrm{po}}$}

Strictly speaking, the vertical macropore wall area $A^{*}$ wall and the horizontal distance $x_{\text {pol }}$ should be calculated on the basis of $d_{\mathrm{mtx}}$ instead of $d_{\mathrm{pol}}$. However, $x_{\mathrm{pol}}$ is always used in combination with $A^{*}$ wall as: $A^{*}{ }_{\text {wall }} / x_{\mathrm{pol}}$ (Eq. (6.32), Eq. (6.35) and Eq. (6.36)). This quotient is similar for using $d_{\mathrm{mtx}}$ and $d_{\mathrm{pol}}$ :

$$
\frac{A_{\mathrm{wall}, \mathrm{mtx}}^{*}}{x_{\mathrm{pol}, \mathrm{mtx}}}=\frac{\frac{4}{d_{\mathrm{mtx}}} \frac{A_{\mathrm{mtx}}}{A_{\mathrm{pol}}}}{\frac{1}{2} d_{\mathrm{mtx}}}=\frac{\frac{4}{d_{\mathrm{mtx}}} \frac{d_{\mathrm{mtx}}^{2}}{d_{\mathrm{pol}}^{2}}}{\frac{1}{2} d_{\mathrm{mtx}}}=\frac{4}{\frac{1}{2} d_{\mathrm{pol}}^{2}}=\frac{A_{\mathrm{wall}}^{*}}{x_{\mathrm{pol}}}
$$

Only in Eq. (6.29), the calculation of the absorption, $A^{*}$ wall is used without dividing by $x_{\text {pol }}$. In that case, $A *_{\text {wall, mtx }}$ is used for $A{ }^{*}{ }_{\text {wall }}$. Therefore, $A^{*}{ }_{\text {wall }}$ is corrected with Eq. (A2.9) according to:

$$
A_{\mathrm{wall}, \mathrm{mtx}}^{*}=\frac{d_{\mathrm{mtx}}}{d_{\mathrm{pol}}} A_{\mathrm{wall}}^{*}=\frac{d_{\mathrm{pol}} \sqrt{1-V_{\mathrm{mp}}}}{d_{\mathrm{pol}}} A_{\mathrm{wall}}^{*}=\sqrt{1-V_{\mathrm{mp}}} A_{\mathrm{wall}}^{*}
$$




\section{Appendix 3 Examples of description of macropore geometry}

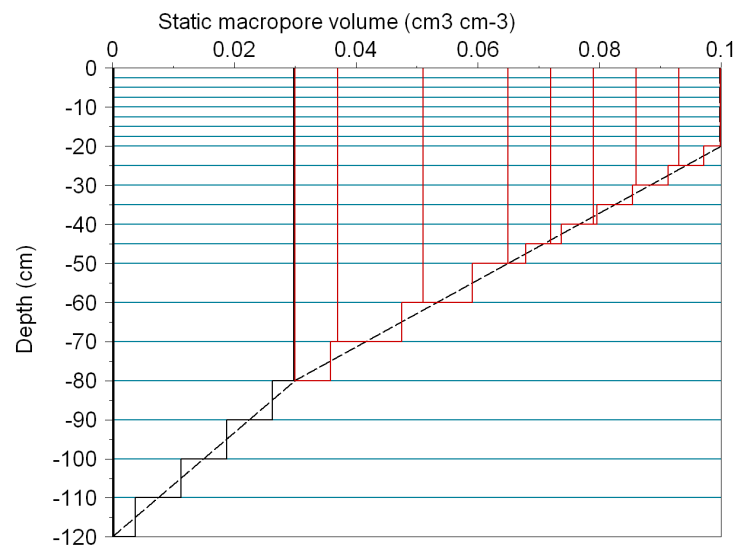

$\begin{array}{ll}\text { VLMPSTSS } & =v_{\text {St }, 0} \\ \text { PPICSS } & =P_{\text {ic }, 0} \\ \text { NUMSBDM } & =n_{\text {sd }} \\ \text { POWM } & =m \\ \text { RZAH } & =R_{\text {ZAh }} \\ \text { SPOINT } & =S_{p} \\ \text { SWPOWM } & =S w_{\text {powm }}\end{array}$

$Z_{\text {ah }}=-20 \mathrm{~cm} ; Z_{\mathrm{ic}}=-80 \mathrm{~cm} ; Z_{\text {st }}=-120 \mathrm{~cm}$;

$v_{\mathrm{st}, 0}=0.1 \mathrm{~cm}^{3} \mathrm{~cm}^{-3} ; P_{\mathrm{ic}, 0}=0.7 ; m=1.0 ; n_{\mathrm{sd}}=10$

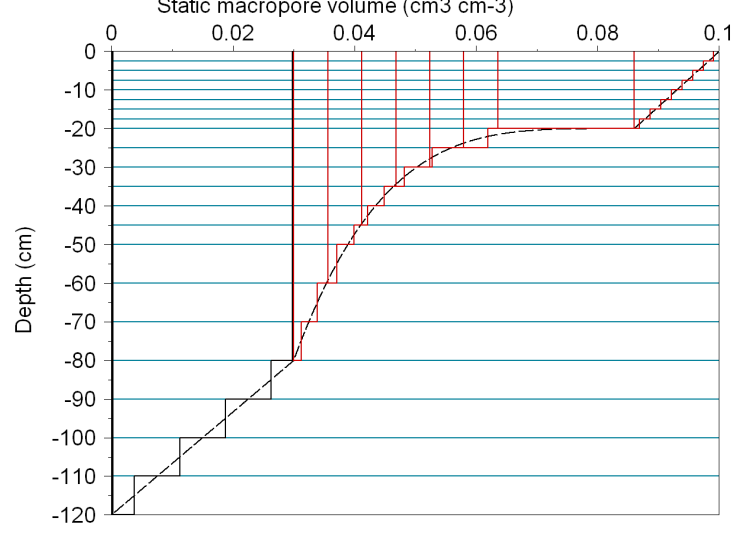

$m=0.25 ; R_{\mathrm{ZAh}}=0.2$

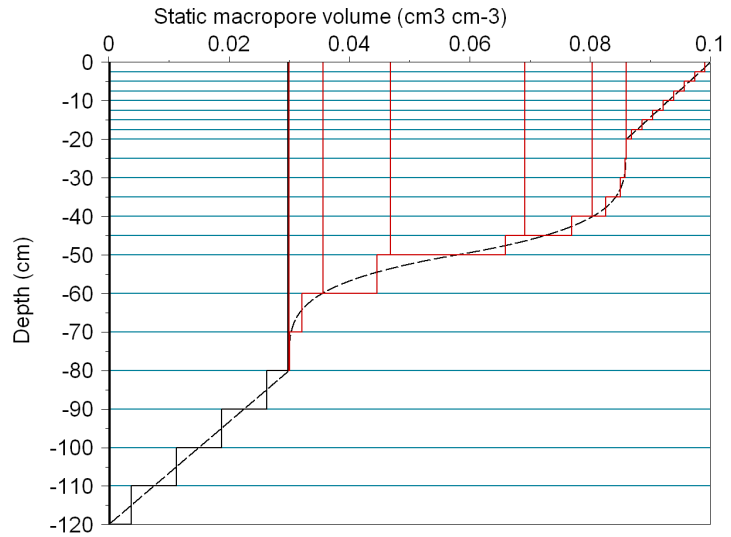

$m=4.0 ; S_{p}=0.5$

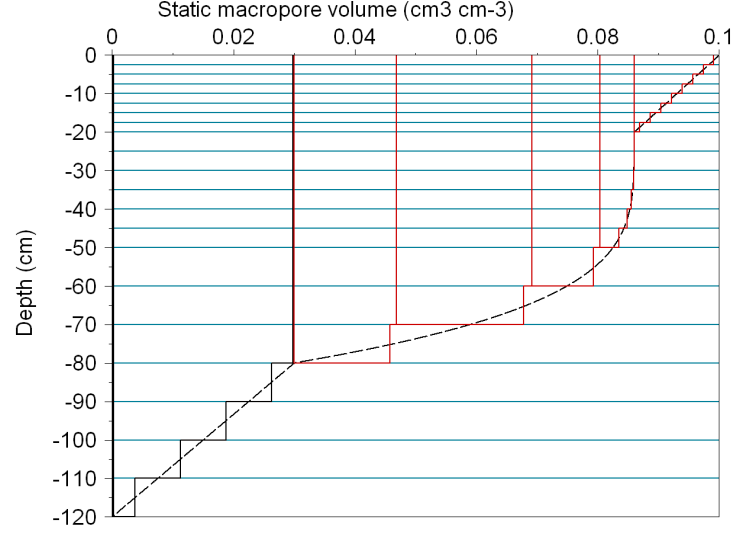

$m=4.0$

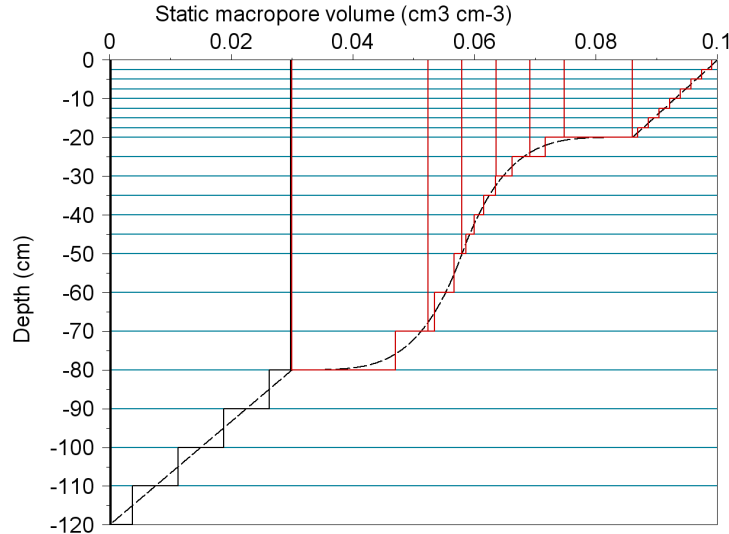

$m=0.25 ; S_{\mathrm{p}}=0.5$ 


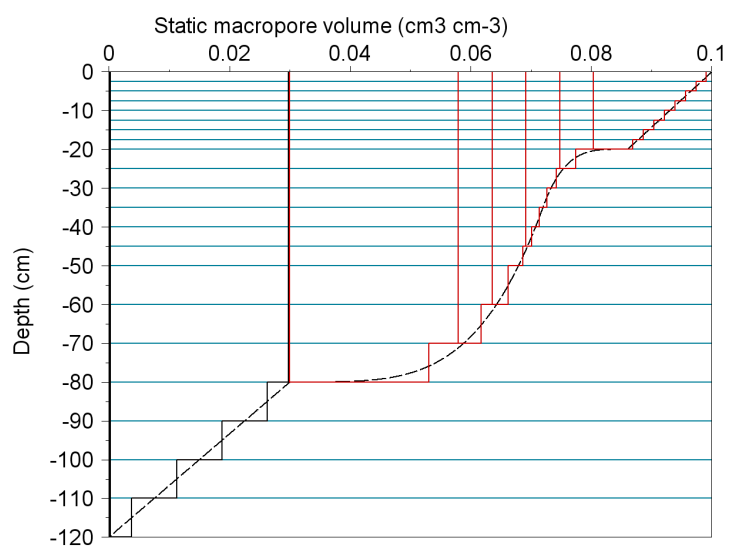

$m=0.25 ; S_{\mathrm{p}}=0.25$

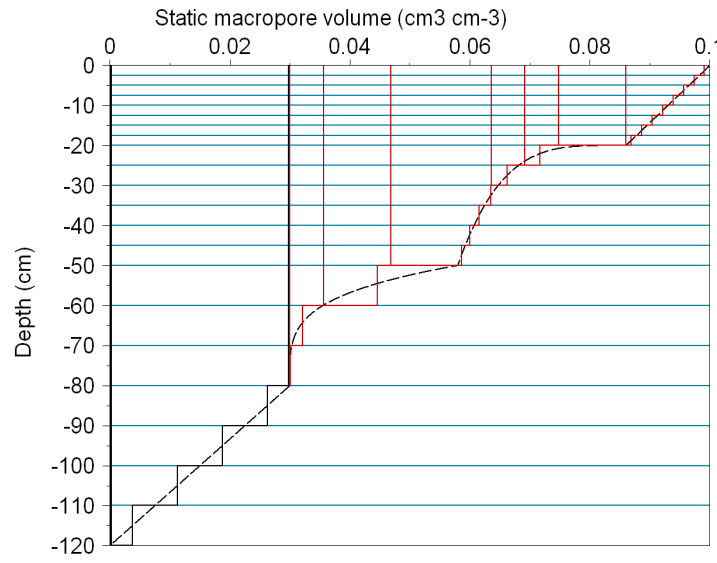

$S_{\mathrm{p}}=0.5 ; S w_{\text {powm }}=1$

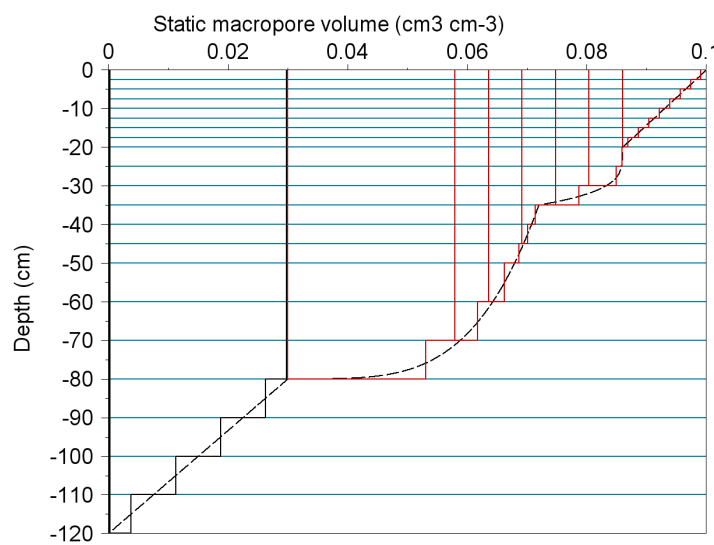

$m=4.0 ; S_{p}=0.25 ; S w_{\text {powm }}=1$
Static macropore volume $(\mathrm{cm} 3 \mathrm{~cm}-3)$

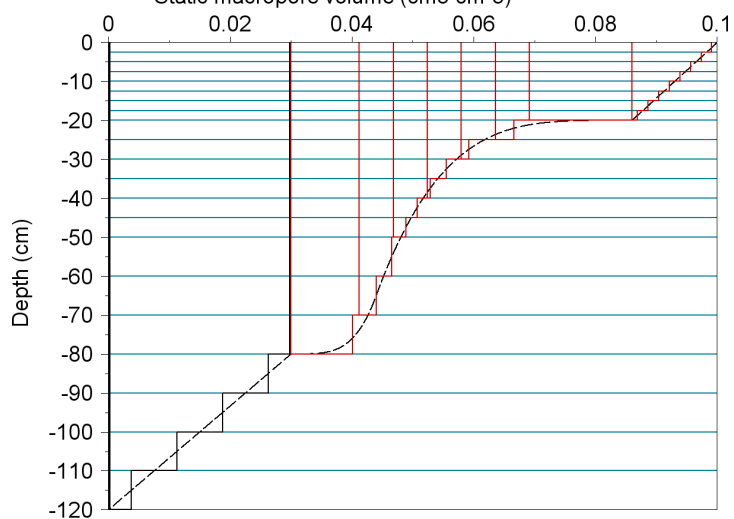

$m=0.25 ; S_{p}=0.75$

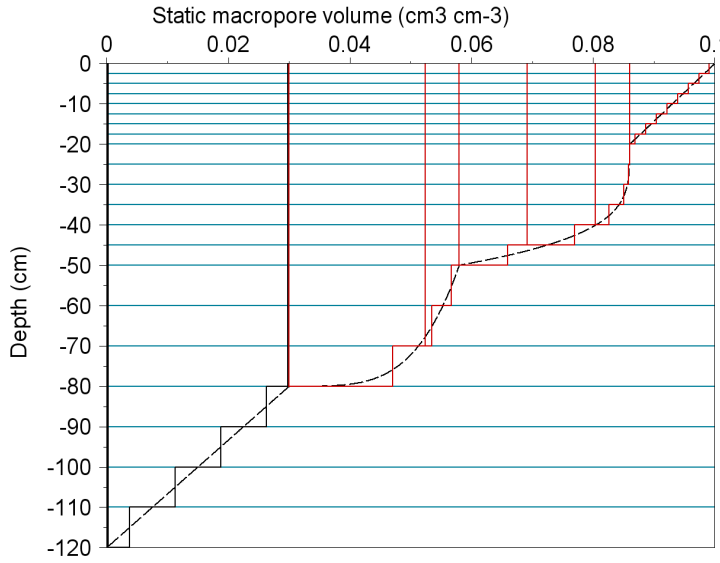

$m=4.0 ; S_{p}=0.5 ; S w_{\text {powm }}=1$

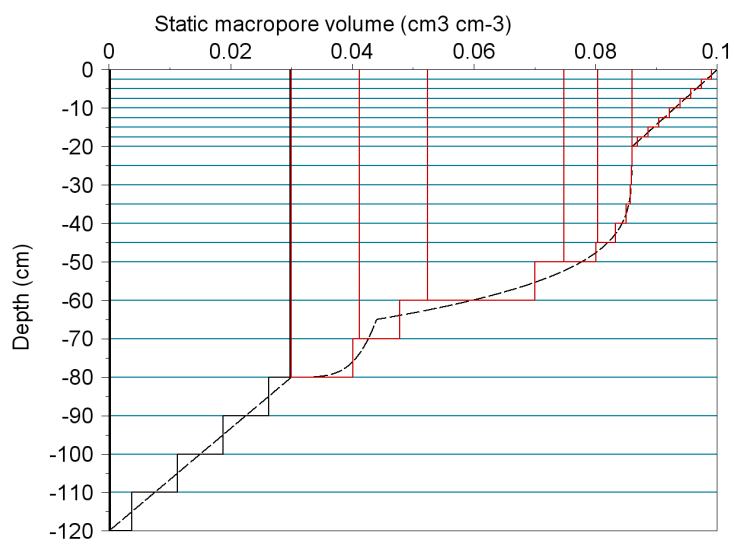

$m=4.0 ; S_{\mathrm{p}}=0.75 ; S w_{\text {powm }}=1$ 


\section{Appendix 4 Partial derivatives of $F_{i}$ to pressure heads}

The coefficients of the Jacobian are given by:

$$
\begin{aligned}
\frac{\partial F_{i}}{\partial h_{i-1}^{j+1, p}} & =-\frac{K_{i-1 / 2}^{j+\kappa \kappa p}}{1 / 2\left(\Delta z_{i-1}+\Delta z_{i}\right)}-\kappa \frac{\partial K_{i-1 / 2}^{j+\kappa, \kappa p}}{\partial h_{i-1}^{j+1, p}}\left(\frac{h_{i-1}^{j+1, p}-h_{i}^{j+1, p}}{1 / 2\left(\Delta z_{i-1}+\Delta z_{i}\right)}+1\right) \\
\frac{\partial F_{i}}{\partial h_{i}^{j+1, p}} & =\frac{\Delta z_{i}}{\Delta t^{j}} C_{i}^{j+1, p}+\Delta z_{i} \frac{\partial S_{m, i}^{j+1, p}}{\partial h_{i}^{j+1, p}}+\frac{K_{i-1 / 2}^{j+\kappa, \kappa p}}{1 / 2\left(\Delta z_{i-1}+\Delta z_{i}\right)}+\frac{K_{i+1 / 2}^{j+\kappa, \kappa p}}{1 / 2\left(\Delta z_{i}+\Delta z_{i+1}\right)} \\
& -\kappa \frac{\partial K_{i-1 / 2}^{j+\kappa, \kappa p}}{\partial h_{i}^{j+1, p}}\left(\frac{h_{i-1}^{j+1, p}-h_{i}^{j+1, p}}{1 / 2\left(\Delta z_{i-1}+\Delta z_{i}\right)}+1\right)+\kappa \frac{\partial K_{i+1 / 2}^{j+\kappa}}{\partial h_{i}^{j+1, p}}\left(\frac{h_{i}^{j+1, p}-h_{i+1}^{j+1, p}}{1 / 2\left(\Delta z_{i}+\Delta z_{i+1}\right)}+1\right) \\
\frac{\partial F_{i}}{\partial h_{i+1}^{j+1, p}} & =-\frac{K_{i+1 / 2}^{j+\kappa, \kappa p}}{1 / 2\left(\Delta z_{i}+\Delta z_{i+1}\right)}+\kappa \frac{\partial K_{i+1 / 2}^{j+\kappa, \kappa} p}{\partial h_{i+1}^{j+1, p}}\left(\frac{h_{i}^{j+1, p}-h_{i+1}^{j+1, p}}{1 / 2\left(\Delta z_{i}+\Delta z_{i+1}\right)}+1\right)
\end{aligned}
$$

Where $C_{i}^{j+1, p}$ is the differential moisture capacity $\left(\mathrm{cm}^{-1}\right) \cdot \frac{\partial K_{i-1 / 2}^{j+\kappa, \kappa}}{\partial h_{i}^{j+1, p}}$ and $\frac{\partial K_{i+1 / 2}^{j+\kappa, \kappa}}{\partial h_{i}^{j+1, p}}$ are the partial derivatives of the internodal conductivity to the pressure head. The calculation of the partial derivatives for the top and bottom compartment requires special attention.

The Jacobian coefficient for the first compartment reads as:

Flux controlled top boundary condition

$$
\frac{\partial F_{1}}{\partial h_{1}^{j+1}}=\frac{\Delta z_{1}}{\Delta t^{j}} C_{1}^{j+1}+\Delta z_{1} \frac{\partial S_{m, 1}^{j+1}}{\partial h_{1}^{j+1}}+\frac{K_{1 / 2}^{j+\kappa}}{1 / 2\left(\Delta z_{i}+\Delta z_{i+1}\right)}+\kappa \frac{\partial K_{1 / 2}^{j+\kappa}}{\partial h_{1}^{j+1}}\left(\frac{h_{1}^{j+1}-h_{2}^{j+1}}{1 / 2\left(\Delta z_{1}+\Delta z_{2}\right)}+1\right)
$$

Head controlled top boundary condition

$$
\begin{aligned}
\frac{\partial F_{1}}{\partial h_{1}^{j+1}=} & \frac{\Delta z_{1}}{\Delta t^{j}} C_{1}^{j+1}+\Delta z_{1} \frac{\partial S_{m, 1}^{j+1}}{\partial h_{1}^{j+1}}+\frac{K_{1 / 2}^{j+1}}{1 / 2 \Delta z_{i}}+\frac{K_{1 / 2}^{j+\kappa}}{1 / 2\left(\Delta z_{i}+\Delta z_{i+1}\right)} \\
& -\frac{\partial K_{1 / 2}^{j+1}}{\partial h_{1}^{j+1}}\left(\frac{h_{0}^{j+1}-h_{1}^{j+1}}{1 / 2 \Delta z_{1}}+1\right)+\kappa \frac{\partial K_{1 / 2}^{j+\kappa}}{\partial h_{1}^{j+1}}\left(\frac{h_{1}^{j+1}-h_{2}^{j+1}}{1 / 2\left(\Delta z_{1}+\Delta z_{2}\right)}+1\right)
\end{aligned}
$$

The internodal conductivity $K_{1 / 2}^{j+1}$ is irrespective the value of $\kappa$ always treated implicitly.

The Jacobian coefficient for the last compartment reads as:

Flux controlled bottom boundary

$$
\frac{\partial F_{1}}{\partial h_{n}^{j+1}}=\frac{\Delta z_{n}}{\Delta t^{j}} C_{n}^{j+1}+\Delta z_{n} \frac{\partial S_{m, n}^{j+1}}{\partial h_{n}^{j+1}}+\frac{K_{n-1 / 2}^{j+\kappa}}{1 / 2\left(\Delta z_{n-1}+\Delta z_{n}\right)}-\kappa \frac{\partial K_{n-1 / 2}^{j+\kappa}}{\partial h_{n}^{j+1}}\left(\frac{h_{n-1}^{j+1}-h_{n}^{j+1}}{1 / 2\left(\Delta z_{n-1}+\Delta z_{n}\right)}+1\right)
$$


Head controlled bottom boundary

$$
\begin{aligned}
\frac{\partial F_{n}}{\partial h_{n}^{j+1, p}} & =\frac{\Delta z_{n}}{\Delta t^{j}} C_{n}^{j+1}+\Delta z_{n} \frac{\partial S_{m, n}^{j+1}}{\partial h_{n}^{j+1}}+\frac{K_{n-1 / 2}^{j+\kappa}}{1 / 2\left(\Delta z_{n-1}+\Delta z_{n}\right)}+\frac{K_{n+1 / 2}^{j+\kappa}}{1 / 2 \Delta z_{n}} \\
& -\kappa \frac{\partial K_{n-1 / 2}^{j+\kappa}}{\partial h_{n}^{j+1}}\left(\frac{h_{n-1}^{j+1}-h_{n}^{j+1}}{1 / 2\left(\Delta z_{n-1}+\Delta z_{n}\right)}+1\right)+\kappa \frac{\partial K_{n+1 / 2}^{j+\kappa}}{\partial h_{n}^{j+1}}\left(\frac{h_{n}^{j+1}-h_{b o t}}{1 / 2 \Delta z_{n}}+1\right)
\end{aligned}
$$

Predefined groundwater levels

$$
\begin{aligned}
\frac{\partial F_{i}}{\partial h_{n^{*}}^{j+1}} & =\frac{\Delta z_{i}}{\Delta t^{j}} C_{n^{*}}^{j+1}+\Delta z_{n^{*}} \frac{\partial S_{m, n^{*}}^{j+1}}{\partial h_{n^{*}}^{j+1}}+\frac{K_{n^{*}-1 / 2}^{j+\kappa}}{1 / 2\left(\Delta z_{n^{*}-1}+\Delta z_{n^{*}}\right)}-\kappa \frac{\partial K_{n^{*}-1 / 2}^{j+\kappa}}{\partial h_{n^{*}}^{j+1}}\left(\frac{h_{n^{*}-1}^{j+1}-h_{n^{*}}^{j+1}}{1 / 2\left(\Delta z_{n^{*}-1}+\Delta z_{n^{*}}\right)}+1\right) \\
& +\left(\frac{z_{n^{*}}-z_{n^{*}+1}}{z_{n^{*}}-g w l}\right) \frac{K_{n^{*}+1 / 2}^{j+\kappa}}{1 / 2\left(\Delta z_{n^{*}}+\Delta z_{n^{*}+1}\right)}+\kappa \frac{\partial K_{n^{*}+1 / 2}^{j+\kappa}}{\partial h_{n^{*}}^{j+1}}\left(\left(\frac{z_{n^{*}}-z_{n^{*}+1}}{z_{n^{*}}-g w l}\right) \frac{h_{n^{*}}^{j+1}}{1 / 2\left(\Delta z_{n^{*}}+\Delta z_{n^{*}+1}\right)}+1\right)
\end{aligned}
$$

\section{Cauchy relation for the bottom boundary}

$$
\begin{aligned}
\frac{\partial F_{n}}{\partial h_{n}^{j+1, p}} & =\frac{\Delta z_{n}}{\Delta t^{j}} C_{n}^{j+1}+\Delta z_{n} \frac{\partial S_{m, n}^{j+1}}{\partial h_{n}^{j+1}}+\frac{K_{n-1 / 2}^{j+\kappa}}{1 / 2\left(\Delta z_{n-1}+\Delta z_{n}\right)}+\frac{K_{n+1 / 2}^{j+\kappa}}{1 / 2 \Delta z_{n}+c K_{n+1 / 2}^{j+\kappa}} \\
& -\kappa \frac{\partial K_{n-1 / 2}^{j+\kappa}}{\partial h_{n}^{j+1}}\left(\frac{h_{n-1}^{j+1}-h_{n}^{j+1}}{1 / 2\left(\Delta z_{n-1}+\Delta z_{n}\right)}+1\right)+\kappa \frac{\partial K_{n+1 / 2}^{j+\kappa} \Delta z_{n}}{\partial h_{n}^{j+1}}\left(\frac{h_{n}^{j+1}-\left(\phi-z_{n}\right)}{\left(1 / 2 \Delta z_{n}+c K_{n+1 / 2}^{j+\kappa}\right)^{2}}\right)
\end{aligned}
$$

Seepage face

$$
\begin{aligned}
& h_{n}^{j+1}+1 / 2 \Delta z_{n}<0 \rightarrow \frac{\partial F_{1}}{\partial h_{1}^{j+1}}=\frac{\Delta z_{1}}{\Delta t^{j}} C_{1}^{j+1}+\Delta z_{1} \frac{\partial S_{m, 1}^{j+1}}{\partial h_{1}^{j+1}}+\frac{K_{1 / 2}^{j+\kappa}}{1 / 2\left(\Delta z_{i}+\Delta z_{i+1}\right)}+\kappa \frac{\partial K_{1 / 2}^{j+\kappa}}{\partial h_{1}^{j+1}}\left(\frac{h_{1}^{j+1}-h_{2}^{j+1}}{1 / 2\left(\Delta z_{1}+\Delta z_{2}\right)}+1\right)
\end{aligned}
$$

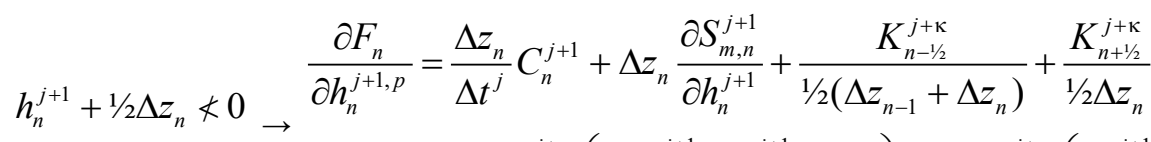

$$
\begin{aligned}
& -\kappa \frac{\partial K_{n-1 / 2}^{j+\kappa}}{\partial h_{n}^{j+1}}\left(\frac{h_{n-1}^{j+1}-h_{n}^{j+1}}{1 / 2\left(\Delta z_{n-1}+\Delta z_{n}\right)}+1\right)+\kappa \frac{\partial K_{n+1 / 2}^{j+\kappa}}{\partial h_{n}^{j+1}}\left(\frac{h_{n}^{j+1}}{1 / 2 \Delta z_{n}}+1\right)
\end{aligned}
$$

Free drainage

$$
\begin{aligned}
\frac{\partial F_{n}}{\partial h_{n}^{j+1, p}} & =\frac{\Delta z_{n}}{\Delta t^{j}} C_{n}^{j+1}+\Delta z_{n} \frac{\partial S_{m, n}^{j+1}}{\partial h_{n}^{j+1}}+\frac{K_{n-1 / 2}^{j+\kappa}}{1 / 2\left(\Delta z_{n-1}+\Delta z_{n}\right)}+\frac{K_{n+1 / 2}^{j+1}}{1 / 2 \Delta z_{n}} \\
& -\kappa \frac{\partial K_{n-1 / 2}^{j+\kappa}}{\partial h_{n}^{j+1}}\left(\frac{h_{n-1}^{j+1}-h_{n}^{j+1}}{1 / 2\left(\Delta z_{n-1}+\Delta z_{n}\right)}+1\right)+\kappa \frac{\partial K_{n+1 / 2}^{j+1}}{\partial h_{n}^{j+1}}
\end{aligned}
$$

The internodal conductivity $K_{n+1 / 2}^{j+1}$ is in the case of free drainage irrespective the value of $\kappa$ always treated implicitly 


\section{Appendix 5 Implicit linearization of hydraulic conductivities}

An implicit linearization of hydraulic conductivities in the numerical elaboration of the Richards equation (Appendix 4) requires expressions for the derivative of the conductivity to the pressure head:

Arithmetic mean:

$$
\frac{\partial K_{i-1 / 2}^{j+\kappa, \kappa}}{\partial h_{i-1}^{j+1, p}}=1 / 2 \frac{\partial K_{i-1}^{j+1, p}}{\partial h_{i-1}^{j+1, p}}
$$

$$
\frac{\partial K_{i-1 / 2}^{j+\kappa, \kappa}}{\partial h_{i}^{j+1, p}}=1 / 2 \frac{\partial K_{i}^{j+1, p}}{\partial h_{i}^{j+1, p}}
$$

$$
\frac{\partial K_{i-1 / 2}^{j+\kappa, \kappa p}}{\partial h_{i-1}^{j+1, p}}=\frac{\Delta z_{i-1}}{\Delta z_{i-1}+\Delta z_{i}} \frac{\partial K_{i-1}^{j+1, p}}{\partial h_{i-1}^{j+1, p}}
$$

Weighted arithmetic mean:

$$
\frac{\partial K_{i-1 / 2}^{j+\kappa, \kappa p}}{\partial h_{i}^{j+1, p}}=\frac{\Delta z_{i}}{\Delta z_{i-1}+\Delta z_{i}} \frac{\partial K_{i}^{j+1, p}}{\partial h_{i}^{j+1, p}}
$$

$$
\frac{\partial K_{i-1 / 2}^{j+\kappa, \kappa p}}{\partial h_{i-1}^{j+1, p}}=1 / 2\left(\frac{K_{i}^{j+1, p}}{K_{i-1}^{j+1, p}}\right)^{1 / 2} \frac{\partial K_{i-1}^{j+1, p}}{\partial h_{i-1}^{j+1, p}}
$$

Geometric mean:

$$
\frac{\partial K_{i-1 / 2}^{j+\kappa p}}{\partial h_{i}^{j+1, p}}=1 / 2\left(\frac{K_{i-1}^{j+1, p}}{K_{i}^{j+1, p}}\right)^{1 / 2} \frac{\partial K_{i}^{j+1, p}}{\partial h_{i}^{j+1, p}}
$$

$$
\frac{\partial K_{i-1 / 2}^{j+\kappa, \kappa p}}{\partial h_{i-1}^{j+1, p}}=\frac{\Delta z_{i-1}}{\Delta z_{i-1}+\Delta z_{i}}\left(\frac{K_{i}^{j+1, p}}{K_{i-1}^{j+1, p}}\right)^{\frac{\Delta z_{i}}{\Delta z_{i-1}+\Delta z_{i}}} \frac{\partial K_{i-1}^{j+1, p}}{\partial h_{i-1}^{j+1, p}}
$$

Weighted geometric mean:

$$
\frac{\partial K_{i-1 / 2}^{j+\kappa p}}{\partial h_{i}^{j+1, p}}=\frac{\Delta z_{i}}{\Delta z_{i-1}+\Delta z_{i}}\left(\frac{K_{i-1}^{j+1, p}}{K_{i}^{j+1, p}}\right)^{\frac{\Delta z_{i-1}}{\Delta z_{i-1}+\Delta z_{i}}} \frac{\partial K_{i}^{j+1, p}}{\partial h_{i}^{j+1, p}}
$$

A relation for the conductivity derivative to the pressure head $\partial K / \partial h$ is given by:

$$
\frac{\partial K}{\partial h}=\frac{\partial K}{\partial S_{e}} \frac{\partial S_{e}}{\partial h}
$$

Where for the Mualem (1976) $K\left(S_{\mathrm{e}}\right)$ relationship:

$$
\begin{aligned}
& \frac{\partial K}{\partial S_{e}}=K_{s a t} S_{e}^{\lambda-1}\left[1-\left(1-S_{e}^{\frac{1}{m}}\right)^{m}\right]\left[\lambda+\left(1-S_{e}^{\frac{1}{m}}\right)^{m-1}\left((2+\lambda) S_{e}^{\frac{1}{m}}-\lambda\right)\right] \\
& \frac{\partial S_{e}}{\partial h}=\frac{C}{\theta_{\text {sat }}-\theta_{\text {res }}}
\end{aligned}
$$


The coefficients of the Jacobian are given by:

$$
\begin{aligned}
\frac{\partial F_{i}}{\partial h_{i-1}^{j+1, p}} & =-\frac{K_{i-1 / 2}^{j+\kappa, \kappa p}}{1 / 2\left(\Delta z_{i-1}+\Delta z_{i}\right)}-\kappa \frac{\partial K_{i-1 / 2}^{j+\kappa, \kappa p}}{\partial h_{i-1}^{j+1, p}}\left(\frac{h_{i-1}^{j+1, p}-h_{i}^{j+1, p}}{1 / 2\left(\Delta z_{i-1}+\Delta z_{i}\right)}+1\right) \\
\frac{\partial F_{i}}{\partial h_{i}^{j+1, p}} & =\frac{\Delta z_{i}}{\Delta t^{j}} C_{i}^{j+1, p}+\Delta z_{i} \frac{\partial S_{m, i}^{j+1, p}}{\partial h_{i}^{j+1, p}}+\frac{K_{i-1 / 2}^{j+\kappa, \kappa p}}{1 / 2\left(\Delta z_{i-1}+\Delta z_{i}\right)}+\frac{K_{i+1 / 2}^{j+\kappa, \kappa p}}{1 / 2\left(\Delta z_{i}+\Delta z_{i+1}\right)} \\
& -\kappa \frac{\partial K_{i-1 / 2}^{j+\kappa, \kappa}}{\partial h_{i}^{j+1, p}}\left(\frac{h_{i-1}^{j+1, p}-h_{i}^{j+1, p}}{1 / 2\left(\Delta z_{i-1}+\Delta z_{i}\right)}+1\right)+\kappa \frac{\partial K_{i+1 / 2}^{j+\kappa} p}{\partial h_{i}^{j+1, p}}\left(\frac{h_{i}^{j+1, p}-h_{i+1}^{j+1, p}}{1 / 2\left(\Delta z_{i}+\Delta z_{i+1}\right)}+1\right) \\
\frac{\partial F_{i}}{\partial h_{i+1}^{j+1, p}} & =-\frac{K_{i+1 / 2}^{j+\kappa, \kappa p}}{1 / 2\left(\Delta z_{i}+\Delta z_{i+1}\right)}+\kappa \frac{\partial K_{i+1 / 2}^{j+\kappa, p}}{\partial h_{i+1}^{j+1, p}}\left(\frac{h_{i}^{j+1, p}-h_{i+1}^{j+1, p}}{1 / 2\left(\Delta z_{i}+\Delta z_{i+1}\right)}+1\right)
\end{aligned}
$$




\section{Appendix 6 Numerical solution heat flow equation}

The discretized form of the heat flow equation as described in Chapter 9, is:

$$
C_{i}^{j+1 / 2}\left(T_{i}^{j+1}-T_{i}^{j}\right)=\frac{\Delta t^{j}}{\Delta z_{i}}\left[\lambda_{i-1 / 2}^{j+1 / 2} \frac{T_{i-1}^{j+1}-T_{i}^{j+1}}{\Delta z_{\mathrm{u}}}-\lambda_{i+1 / 2}^{j+1 / 2} \frac{T_{i}^{j+1}-T_{i+1}^{j+1}}{\Delta z_{\ell}}\right]
$$

where for notational convencience the subscript heat of thermal conductivity $\lambda$ and soil heat capacity $C$ is omitted. Equation [A6.1] can be rewritten as:

$$
-\frac{\Delta t^{j}}{\Delta z_{i} \Delta z_{\mathrm{u}}} \lambda_{i-1 / 2}^{j+1 / 2} T_{i-1}^{j+1}+\left[C_{i}^{j+1 / 2}+\frac{\Delta t^{j}}{\Delta z_{i} \Delta z_{\mathrm{u}}} \lambda_{i-1 / 2}^{j+1 / 2}+\frac{\Delta t^{j}}{\Delta z_{i} \Delta z_{\ell}} \lambda_{i+1 / 2}^{j+1 / 2}\right] T_{i}^{j+1}-\frac{\Delta t^{j}}{\Delta z_{i} \Delta z_{\ell}} \lambda_{i+1 / 2}^{j+1 / 2} T_{i+1}^{j+1}=C_{i}^{j+1 / 2} T_{i}^{j}
$$

Application of Eq. (A6.2) to each node results in a tri-diagonal matrix:

$$
\left[\begin{array}{ccccccc}
\beta_{1} & \gamma_{1} & & & & & \\
\alpha_{2} & \beta_{2} & \gamma_{2} & & & & \\
& \alpha_{3} & \beta_{3} & \gamma_{3} & & & \\
& & & & & & \\
& & & & \alpha_{n-1} & \beta_{n-1} & \gamma_{n-1} \\
& & & & & \alpha_{n} & \beta_{n}
\end{array}\right]\left[\begin{array}{c}
T_{1}^{j+1} \\
T_{2}^{j+1} \\
T_{3}^{j+1} \\
T_{n-1}^{j+1} \\
T_{n}^{j+1}
\end{array}\right]=\left[\begin{array}{c}
f_{1} \\
f_{2} \\
f_{3} \\
\\
f_{n-1} \\
f_{n}
\end{array}\right]
$$

where $n$ is the number of nodal points. Next the coefficients $\alpha_{i}, \beta_{i}, \gamma_{i}$, and $f_{i}$ are explained for the intermediate nodes and for the top and bottom node.

\section{Intermediate nodes}

From Eq. (A6.2) and Eq. (A6.3) we may derive the coefficients:

$$
\begin{aligned}
& \alpha_{i}=-\frac{\Delta t^{j}}{\Delta z_{i} \Delta z_{\mathrm{u}}} \lambda_{i-1 / 2}^{j+1 / 2} \\
& \beta_{i}=C_{i}^{j+1 / 2}+\frac{\Delta t^{j}}{\Delta z_{i} \Delta z_{\mathrm{u}}} \lambda_{i-1 / 2}^{j+1 / 2}+\frac{\Delta t^{j}}{\Delta z_{i} \Delta z_{\ell}} \lambda_{i+1 / 2}^{j+1 / 2} \\
& \gamma_{i}=-\frac{\Delta t^{j}}{\Delta z_{i} \Delta z_{\ell}} \lambda_{i+1 / 2}^{j+1 / 2} \\
& f_{i}=C_{i}^{j+1 / 2} T_{i}^{j}
\end{aligned}
$$




\section{Top node}

The temperature at soil surface is set equal to the daily average air temperature, $T_{\text {avg. }}$. Therefore, in case of the top node, Eq. (A6.1) transforms to:

$$
C_{1}^{j+1 / 2}\left(T_{1}^{j+1}-T_{1}^{j}\right)=\frac{\Delta t^{j}}{\Delta z_{1}}\left[\lambda_{1 / 2}^{j+1 / 2} \frac{T_{\text {avg }}-T_{1}^{j+1}}{\Delta z_{\mathrm{u}}}-\lambda_{1 / 2}^{j+1 / 2} \frac{T_{1}^{j+1}-T_{2}^{j+1}}{\Delta z_{\ell}}\right]
$$

which can be written as:

$$
\left[C_{1}^{j+1 / 2}+\frac{\Delta t^{j}}{\Delta z_{1} \Delta z_{\mathrm{u}}} \lambda_{1 / 2}^{j+1 / 2}+\frac{\Delta t^{j}}{\Delta z_{1} \Delta z_{\ell}} \lambda_{11 / 2}^{j+1 / 2}\right] T_{1}^{j+1}-\frac{\Delta t^{j}}{\Delta z_{1} \Delta z_{\ell}} \lambda_{11 / 2}^{j+1 / 2} T_{2}^{j+1}=C_{1}^{j+1 / 2} T_{1}^{j}+\frac{\Delta t^{j}}{\Delta z_{1} \Delta z_{\mathrm{u}}} \lambda_{1 / 2}^{j+1 / 2} T_{\text {avg }}
$$

Combination of Eq. (A6.9) and (A6.3) gives the following coefficients:

$$
\begin{aligned}
& \beta_{1}=C_{1}^{j+1 / 2}+\frac{\Delta t^{j}}{\Delta z_{1} \Delta z_{\mathrm{u}}} \lambda_{1 / 2}^{j+1 / 2}+\frac{\Delta t^{j}}{\Delta z_{1} \Delta z_{\ell}} \lambda_{1 / 2}^{j+1 / 2} \\
& \gamma_{1}=-\frac{\Delta t^{j}}{\Delta z_{1} \Delta z_{\ell}} \lambda_{11 / 2}^{j+1 / 2} \\
& f_{1}=C_{1}^{j+1 / 2} T_{1}^{j}+\frac{\Delta t^{j}}{\Delta z_{1} \Delta z_{\mathrm{u}}} \lambda_{1 / 2}^{j+1 / 2} T_{\text {avg }}
\end{aligned}
$$

In case of a snow cover at the soil surface, the average air temperature can no longer be used as a top boundary condition, because of the insulating properties of snow. The corrected top boundary temperature, $T_{\text {top }}\left({ }^{0} \mathrm{C}\right)$, is calculated as follows.

$$
\begin{aligned}
& T_{\text {top }}=\frac{T_{\text {soil }}(1)+a T_{\text {avg }}}{1+a} \\
& a=0.5 \frac{\lambda_{\text {snow }}}{\lambda(1)} \frac{\Delta z(1)}{d z_{\text {snow }}} \\
& \lambda_{\text {snow }}=2.86 \cdot 10^{-6} \times 864 \times R_{\text {snow }}^{2} \\
& d z_{\text {snow }}=\frac{S_{\text {snow }}}{0.17}
\end{aligned}
$$

where $T_{\text {soil }}(1)$ is the current soil temperature of the first soil layer $\left({ }^{0} \mathrm{C}\right), T_{\text {avg }}$ is the average air temperature $\left({ }^{0} \mathrm{C}\right), \Delta z(1)$ is the thickness of the first soil layer $(\mathrm{cm}), d z_{\text {snow }}$ is the snow layer thickness (cm; see Chapter 10), $s_{\text {snow }}$ is the amount of snow (equivalent liters of water), $R_{\text {snow }}$ is the density of snow ( $\mathrm{kg} \mathrm{m}^{-3}$; Granberg et al., 1999), and $\lambda_{\text {snow }}$ is the thermal conductivity of snow $\left(\mathrm{J} \mathrm{cm}^{-1} \mathrm{~d}^{-1}{ }^{0} \mathrm{C}^{-1}\right)$. The expression for $\lambda_{\text {snow }}$ was taken as one of the possibilities listed in Fukusako (1990; see also Chapter 10$)$. In case $\lambda(1)<10^{-10}, \lambda(1)$ is set equal to 100 . 


\section{Bottom node}

SWAP adopts a heat flow rate $q_{\text {heat,bot }}\left(\mathrm{J} \mathrm{cm}^{-2} \mathrm{~d}^{-1}\right)$ at the bottom of the soil profile. At the bottom node, the general heat flow equation, Eq. (A6.1), transforms to:

$$
C_{n}^{j+1 / 2}\left(T_{n}^{j+1}-T_{n}^{j}\right)=\frac{\Delta t^{j}}{\Delta z_{n}}\left[\lambda_{n-1 / 2}^{j+1 / 2} \frac{T_{n-1}^{j+1}-T_{n}^{j+1}}{\Delta z_{\mathrm{u}}}-q_{\text {heat,bot }}\right]
$$

which can be written as:

$$
-\frac{\Delta t^{j}}{\Delta z_{n} \Delta z_{\mathrm{u}}} \lambda_{n-1 / 2}^{j+1 / 2} T_{n-1}^{j+1}+\left[C_{n}^{j+1 / 2}+\frac{\Delta t^{j}}{\Delta z_{n} \Delta z_{\mathrm{u}}} \lambda_{n-1 / 2}^{j+1 / 2}\right] T_{n}^{j+1}=C_{n}^{j+1 / 2} T_{n}^{j}+\frac{\Delta t^{j}}{\Delta z_{n}} q_{\text {heat,bot }}
$$

Combination of Eq. (A6.15) and (A6.3) gives the following coefficients:

$$
\begin{aligned}
& \alpha_{n}=-\frac{\Delta t^{j}}{\Delta z_{n} \Delta z_{\mathrm{u}}} \lambda_{n-1 / 2}^{j+1 / 2} \\
& \beta_{n}=C_{n}^{j+1 / 2}+\frac{\Delta t^{j}}{\Delta z_{n} \Delta z_{\mathrm{u}}} \lambda_{n-1 / 2}^{j+1 / 2} \\
& f_{n}=C_{n}^{j+1 / 2} T_{n}^{j}-\frac{\Delta t^{j}}{\Delta z_{n}} q_{\text {heat,bot }}
\end{aligned}
$$

In case of prescribed temperature $T_{\text {bot }}$ at the soil profile bottom, Eq. (A6.1), transforms to:

$$
C_{n}^{j+1 / 2}\left(T_{n}^{j+1}-T_{n}^{j}\right)=\frac{\Delta t^{j}}{\Delta z_{n}}\left[\lambda_{n-1 / 2}^{j+1 / 2} \frac{T_{n-1}^{j+1}-T_{n}^{j+1}}{\Delta z_{\mathrm{u}}}-\lambda_{n+1 / 2}^{j+1 / 2} \frac{T_{n}^{j+1}-T_{b o t}}{\Delta z_{\ell}}\right]
$$

which can be written as:

$$
-\frac{\Delta t^{j}}{\Delta z_{n} \Delta z_{\mathrm{u}}} \lambda_{n-1 / 2}^{j+1 / 2} T_{n-1}^{j+1}+\left[C_{n}^{j+1 / 2}+\frac{\Delta t^{j}}{\Delta z_{n} \Delta z_{\mathrm{u}}} \lambda_{n-1 / 2}^{j+1 / 2}+\frac{\Delta t^{j}}{\Delta z_{n} \Delta z_{\ell}} \lambda_{n+1 / 2}^{j+1 / 2}\right] T_{n}^{j+1}=C_{n}^{j+1 / 2} T_{n}^{j}+\frac{\Delta t^{j}}{\Delta z_{n} \Delta z_{\ell}} \lambda_{n+1 / 2}^{j+1 / 2} T_{\text {bot }}
$$

Combination of Eq. (A6.20) and (A6.3) gives the following coefficients:

$$
\begin{aligned}
& \alpha_{n}=-\frac{\Delta t^{j}}{\Delta z_{n} \Delta z_{\mathrm{u}}} \lambda_{n-1 / 2}^{j+1 / 2} \\
& \beta_{n}=C_{n}^{j+1 / 2}+\frac{\Delta t^{j}}{\Delta z_{n} \Delta z_{\mathrm{u}}} \lambda_{n-1 / 2}^{j+1 / 2}+\frac{\Delta t^{j}}{\Delta z_{n} \Delta z_{\ell}} \lambda_{n+1 / 2}^{j+1 / 2} \\
& f_{n}=C_{n}^{j+1 / 2} T_{n}^{j}+\frac{\Delta t^{j}}{\Delta z_{n} \Delta z_{\ell}} \lambda_{n+1 / 2}^{j+1 / 2} T_{\text {bot }}
\end{aligned}
$$




\section{Appendix 7 Parameters of soil hydraulic functions: Staring series}

Topsoils according to Wösten et al. (2013)

\begin{tabular}{|c|c|c|c|c|c|c|c|c|c|}
\hline Soil & Top & Dutch Nomenclature of Texture & $\theta_{r}$ & $\theta_{s}$ & a & $\mathbf{n}$ & $\mathbf{K}_{\mathrm{s}}$ & $\mathrm{I}$ & Staring series \\
\hline Type & Soils & & $\left(\mathrm{cm}^{3} / \mathrm{cm}^{3}\right)$ & $\left(\mathrm{cm}^{3} / \mathrm{cm}^{3}\right)$ & $(1 / \mathrm{cm})$ & $(-)$ & $(\mathrm{cm} / \mathrm{d})$ & $(-)$ & version* \\
\hline Sand & B1 & Leemarm, zeer fijn tot matig fijn zand & 0.00 & 0.37 & 0.0208 & 1.646 & 33.34 & 0.571 & 1987 \\
\hline Sand & B2 & Zwak lemig, zeer fijn tot matig fijn zand & 0.00 & 0.43 & 0.0224 & 1.436 & 32.21 & -0.304 & 1987 \\
\hline Sand & B3 & Sterk lemig, zeer fijn tot matig fijn zand & 0.00 & 0.45 & 0.0152 & 1.412 & 17.81 & -0.213 & 1987 \\
\hline Sand & B4 & $\begin{array}{l}\text { Zeer sterk lemig, zeer fijn tot matig fijn } \\
\text { zand }\end{array}$ & 0.00 & 0.42 & 0.0163 & 1.559 & 54.80 & 0.177 & 1987 \\
\hline Sand & B5 & Grof zand & 0.01 & 0.36 & 0.0452 & 1.933 & 52.91 & -0.359 & 2001 \\
\hline Sand & B6 & Keileem & 0.01 & 0.38 & 0.0222 & 1.238 & 100.69 & -1.747 & 2001 \\
\hline Loam & B7 & Zeer lichte zavel & 0.00 & 0.40 & 0.0158 & 1.287 & 25.10 & 0.248 & 1987 \\
\hline Loam & B8 & Matig lichte zavel & 0.00 & 0.40 & 0.0313 & 1.200 & 22.90 & -3.578 & 1987 \\
\hline Loam & B9 & Zware zavel & 0.00 & 0.43 & 0.0065 & 1.325 & 1.54 & -2.161 & 1994 \\
\hline Clay & B10 & Lichte klei & 0.00 & 0.44 & 0.0519 & 1.216 & 31.10 & -6.552 & 1987 \\
\hline Clay & B11 & Matig zware klei & 0.00 & 0.51 & 0.1562 & 1.099 & 63.60 & -8.067 & 1987 \\
\hline Clay & B12 & Zeer zware klei & 0.00 & 0.57 & 0.1689 & 1.068 & 98.20 & -10.286 & 1987 \\
\hline Silt & B13 & Zandige leem & 0.01 & 0.42 & 0.0084 & 1.441 & 12.98 & -1.497 & 2001 \\
\hline Silt & B14 & Siltige leem & 0.01 & 0.42 & 0.0051 & 1.305 & 0.80 & 0.000 & 1994 \\
\hline Peat & B15 & Venig zand & 0.01 & 0.53 & 0.0242 & 1.280 & 81.28 & -1.476 & 2001 \\
\hline Peat & B16 & Zandig veen en veen & 0.00 & 0.73 & 0.0134 & 1.320 & 13.44 & 0.534 & 1987 \\
\hline Peat & B17 & Venige klei & 0.00 & 0.72 & 0.0180 & 1.140 & 4.46 & -0.350 & 1994 \\
\hline Peat & B18 & Kleiig veen & 0.00 & 0.71 & 0.0284 & 1.141 & 34.80 & 1.086 & 1987 \\
\hline
\end{tabular}

Subsoils according to Wösten et al. (2013)

\begin{tabular}{|c|c|c|c|c|c|c|c|c|c|}
\hline Soil & Sub & Dutch Nomenclature of Texture & $\theta_{\mathrm{r}}$ & $\theta_{s}$ & a & n & $\mathbf{K}_{\mathrm{s}}$ & 1 & Staring series \\
\hline Type & Soils & & $\left(\mathrm{cm}^{3} / \mathrm{cm}^{3}\right)$ & $\left(\mathrm{cm}^{3} / \mathrm{cm}^{3}\right)$ & $(1 / \mathrm{cm})$ & $(-)$ & $(\mathrm{cm} / \mathrm{d})$ & $(-)$ & version* \\
\hline Sand & 01 & Leemarm, zeer fijn tot matig fijn zand & 0.00 & 0.35 & 0.0220 & 2.186 & 99.70 & 0.796 & 1987 \\
\hline Sand & $\mathrm{O} 2$ & Zwak lemig, zeer fijn tot matig fijn zand & 0.00 & 0.38 & 0.0182 & 1.870 & 63.90 & 0.911 & 1987 \\
\hline Sand & $\mathrm{O} 3$ & Sterk lemig, zeer fijn tot matig fijn zand & 0.00 & 0.34 & 0.0265 & 1.543 & 44.60 & -0.333 & 1987 \\
\hline Sand & 04 & Zeer sterk lemig, zeer fijn tot matig fijn zand & 0.00 & 0.36 & 0.0216 & 1.540 & 53.10 & -0.520 & 1987 \\
\hline Sand & 05 & Grof zand & 0.00 & 0.33 & 0.0524 & 1.912 & 223.00 & 0.873 & 1987 \\
\hline Loam & 06 & Keileem & 0.00 & 0.41 & 0.0291 & 1.152 & 5.48 & -6.864 & 1987 \\
\hline Loam & 07 & Beekleem & 0.01 & 0.51 & 0.0123 & 1.152 & 39.10 & -2.023 & 2001 \\
\hline Loam & 08 & Zeer lichte zavel & 0.00 & 0.42 & 0.0248 & 1.321 & 26.40 & -0.622 & 1987 \\
\hline Loam & 09 & Matig lichte zavel & 0.00 & 0.41 & 0.0280 & 1.283 & 24.00 & -1.559 & 1987 \\
\hline Clay & 010 & Zware zavel & 0.00 & 0.44 & 0.0231 & 1.212 & 25.60 & -2.220 & 1987 \\
\hline Clay & 011 & Lichte klei & 0.00 & 0.42 & 0.0420 & 1.125 & 61.00 & -3.706 & 1987 \\
\hline Clay & 012 & Matig zware klei & 0.00 & 0.49 & 0.0384 & 1.113 & 10.80 & -6.743 & 1987 \\
\hline Silt & 013 & Zeer zware klei & 0.00 & 0.58 & 0.1122 & 1.063 & 38.00 & -12.538 & 1987 \\
\hline Silt & 014 & Zandige leem & 0.00 & 0.38 & 0.0025 & 1.686 & 0.36 & 0.057 & 1994 \\
\hline Peat & 015 & Siltige leem & 0.00 & 0.43 & 0.0207 & 1.224 & 57.42 & -2.077 & 1987 \\
\hline Peat & 016 & Oligotroof veen & 0.00 & 0.87 & 0.0179 & 1.275 & 14.66 & 0.539 & 1987 \\
\hline Peat & 017 & Mesotroof en eutroof veen & 0.00 & 0.89 & 0.0145 & 1.252 & 30.45 & 1.019 & 1987 \\
\hline Peat & 018 & Moerige tussenlaag & 0.01 & 0.57 & 0.0138 & 1.323 & 34.45 & -1.204 & 2001 \\
\hline
\end{tabular}

*References to Staring Series versions:

Wösten, J. H. M. (1987). Beschrijving van de Waterretentie- en doorlatendheidskarakteristieken uit de Staringreeks met analytische functies. Stiboka rapport nr 2019.

Wösten, J. H. ., Veerman, G. J., \& Stolte, J. (1994). Waterretentie- en doorlatend- heidskarakteristieken van boven- en ondergronden in Nederland: de Staringreeks 1994. Technisch Document 18.

Wösten, J., Veerman, G., DeGroot, W., \& Stolte, J. (2001). Waterretentie- en doorlatendheids-karakteristieken van boven- en ondergronden in Nederland: de Staringreeks. Alterra Rapport, 153, 86. 
Soil texture according to Wösten et al. (2001)

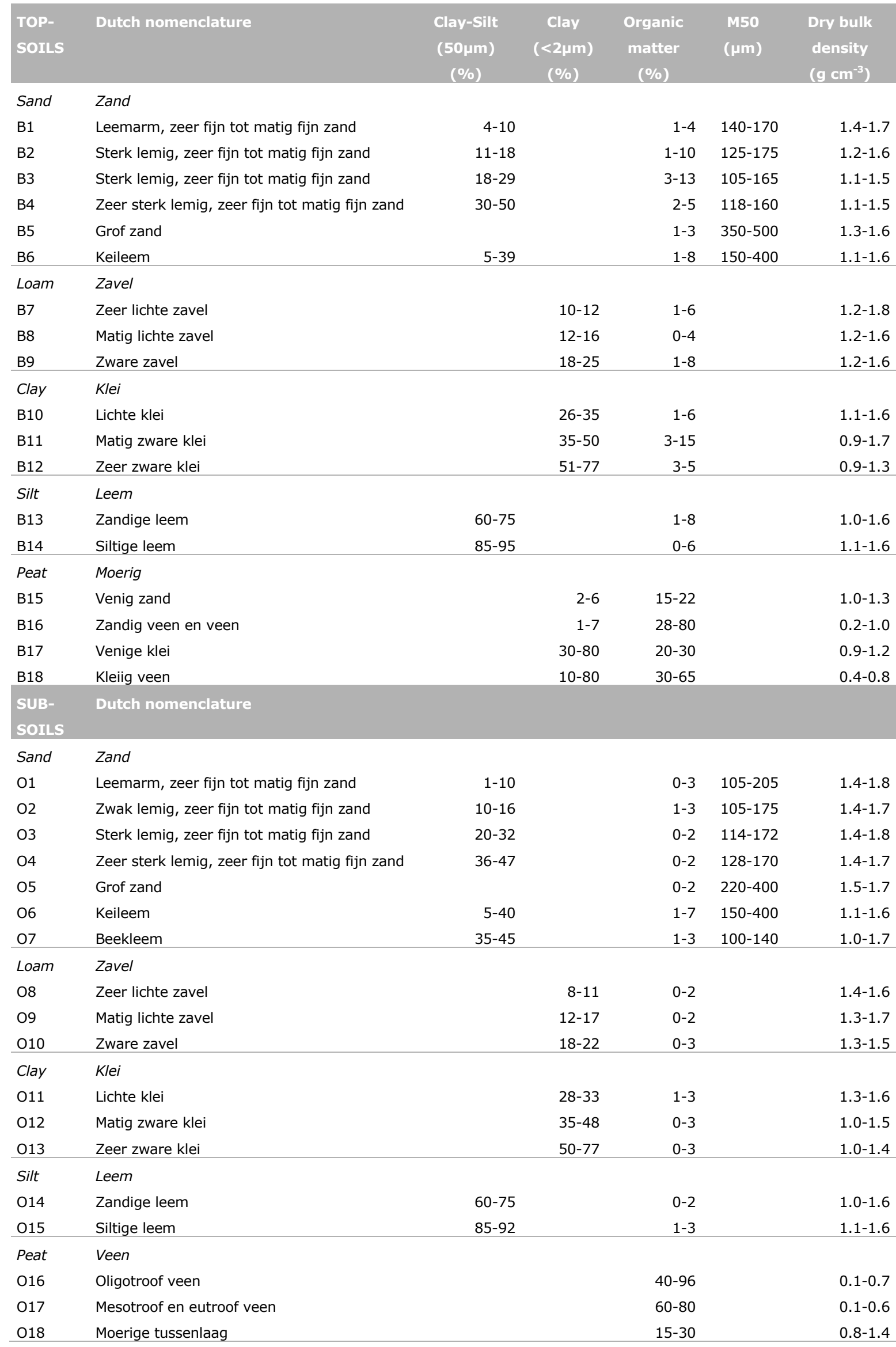




\section{Appendix 8 Critical pressure head values of root water extraction}

After Taylor and Ashcroft (1972)

\begin{tabular}{|c|c|c|c|c|c|}
\hline Crop & $h_{3 h}$ & $h_{31}$ & Crop & $h_{3 h}$ & $h_{31}$ \\
\hline Vegetative crops & & & Deciduous fruit & -500 & -800 \\
\hline Alfalfa & -1500 & -1500 & Avocadoes & -500 & -500 \\
\hline Cabbage & -600 & -700 & early season & -400 & -500 \\
\hline Canning peas & -300 & -500 & during maturity & -1000 & -1000 \\
\hline Grass & -300 & -1000 & Cantaloupe & -350 & -450 \\
\hline Lettuce & -400 & -600 & Tomatoes & -800 & -1500 \\
\hline Tobacco & -300 & -800 & Bananas & -300 & -1500 \\
\hline \multicolumn{6}{|l|}{ Sugar cane } \\
\hline \multirow[t]{2}{*}{ Turfgrass } & -240 & -360 & during ripening & -8000 & -12000 \\
\hline & & & Small grains & & \\
\hline Root crops & & & vegetative period & -400 & -500 \\
\hline Onions & & & during ripening & -8000 & -12000 \\
\hline early growth & -450 & -550 & & & \\
\hline bulbing time & -550 & -650 & Seed crops & & \\
\hline Sugar beets & -400 & -600 & Alfalfa & & \\
\hline Potatoes & -300 & -500 & prior to bloom & -2000 & -2000 \\
\hline Carrots & -550 & -650 & during bloom & -4000 & -8000 \\
\hline Fruit crops & & & at $15 \mathrm{~cm}$ depth & -1500 & -1500 \\
\hline Lemons & -400 & -400 & Lettuce & & \\
\hline Oranges & -200 & -1000 & during productive phase & -3000 & -3000 \\
\hline
\end{tabular}




\section{Appendix 9 Salt tolerance data}

Salt tolerance data of Maas $(1990)^{2}$ translated into SWAP salt input parameters

\begin{tabular}{|c|c|c|c|c|c|c|c|}
\hline Crop common name & Crop botanical name & $\begin{array}{l}\text { ECmax }^{3} \\
\left(\mathrm{dS} \mathrm{m}^{-1}\right)\end{array}$ & $\begin{array}{l}\text { ECslope } \\
\left(\% \text { per dS } \mathrm{m}^{-1}\right)\end{array}$ & $\begin{array}{l}\text { SALTMAX } \\
\left(\mathrm{mg} \mathrm{cm}^{-3}\right)\end{array}$ & $\begin{array}{l}\text { SALTSLOPE }^{3} \\
\left(\mathrm{~cm}^{-3} \mathrm{mg}\right)\end{array}$ & Rating ${ }^{5}$ & Ref. $^{6}$ \\
\hline \multicolumn{8}{|l|}{ Fiber and grain crops } \\
\hline Barley $^{7}$ & Hordeum vulgare & 8.0 & 5.0 & 10.24 & 0.039 & $\mathrm{~T}$ & 1 \\
\hline Bean & Phaseolus vulgaris & 1.0 & 19.0 & 1.28 & 0.148 & $\mathrm{~S}$ & 1 \\
\hline Corn & Zea mays & 1.7 & 12.0 & 2.18 & 0.094 & MS & 1 \\
\hline Cotton & Gossypium hirsutum & 7.7 & 5.2 & 9.86 & 0.041 & $\mathrm{~T}$ & 1 \\
\hline Peanut & Arachis hypogaea & 3.2 & 29.0 & 4.10 & 0.227 & MS & 1 \\
\hline Rice (paddy) & Oryza sativa & 3.0 & 12.0 & 3.84 & 0.094 & $\mathrm{~S}$ & 1 \\
\hline Rye & Secale cereale & 11.4 & 10.8 & 14.59 & 0.084 & $\mathrm{~T}$ & 2 \\
\hline Sorghum & Sorghum bicolor & 6.8 & 16.0 & 8.70 & 0.125 & MT & 2 \\
\hline Soybean & Glycine max & 5.0 & 20.0 & 6.40 & 0.156 & MT & 1 \\
\hline Sugar beet ${ }^{8}$ & Beta vulgaris & 7.0 & 5.9 & 8.96 & 0.046 & $\mathrm{~T}$ & 1 \\
\hline Sugar cane & Sacharum officinarum & 1.7 & 5.9 & 2.18 & 0.046 & MS & 1 \\
\hline Wheat & Triticum aestivum & 6.0 & 7.1 & 7.68 & 0.055 & MT & 1 \\
\hline Wheat, durum & Triticum turgidum & 5.9 & 3.8 & 7.55 & 0.030 & $\mathrm{~T}$ & 2 \\
\hline \multicolumn{8}{|c|}{ Grasses and forage crops } \\
\hline Alfalfa & Medicago sativa & 2.0 & 7.3 & 2.56 & 0.057 & MS & 1 \\
\hline Barley (forage) ${ }^{\mathrm{e}}$ & Hordeum vulgare & 6.0 & 7.1 & 7.68 & 0.055 & MT & 1 \\
\hline 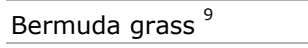 & Cynodon dactylon & 6.9 & 6.4 & 8.83 & 0.050 & $\mathrm{~T}$ & 1 \\
\hline Clover, ladino & Trifolium repens & 1.5 & 12.0 & 1.92 & 0.094 & MS & 1 \\
\hline Corn (forage) & Zea mays & 1.8 & 7.4 & 2.30 & 0.058 & MS & 1 \\
\hline Cowpea (forage) & Vigna unguiculata & 2.5 & 11.0 & 3.20 & 0.086 & MS & 3 \\
\hline Ryegrass, perennial & Lolium perenne & 5.6 & 7.6 & 7.17 & 0.059 & MT & 1 \\
\hline Sundan grass & Sorghum sudanese & 2.8 & 4.3 & 3.58 & 0.036 & MT & 1 \\
\hline Wheat (forage) ${ }^{10}$ & Triticum aestivum & 4.5 & 2.6 & 5.76 & 0.020 & MT & 2 \\
\hline Wheat, durum (forage) & Triticum turgidum & 2.1 & 2.5 & 2.69 & 0.020 & MT & 2 \\
\hline \multicolumn{8}{|c|}{ Vegetables and fruit crops } \\
\hline Bean & Phaseolus vulgaris & 1.0 & 19.0 & 1.28 & 0.148 & $\mathrm{~s}$ & 1 \\
\hline Beet, red ${ }^{f}$ & Beta vulgaris & 4.0 & 9.0 & 5.12 & 0.070 & MT & 1 \\
\hline Broccoli & Brassica oleracea botrytis & 2.8 & 9.2 & 3.58 & 0.072 & MS & 1 \\
\hline Cabbage & Brassica oleracea capitata & 1.8 & 9.7 & 2.30 & 0.076 & MS & 1 \\
\hline Carrot & Daucus carota & 1.0 & 14.0 & 1.28 & 0.109 & $\mathrm{~s}$ & 1 \\
\hline Corn, sweet & Zea mays & 1.7 & 12.0 & 2.18 & 0.094 & MS & 1 \\
\hline Cucumber & Cucumis sativus & 2.5 & 13.0 & 3.20 & 0.102 & MS & 1 \\
\hline Lettuce & Lactuca sativa & 1.3 & 13.0 & 1.66 & 0.102 & MS & 1 \\
\hline Onion & Allium cepa & 1.2 & 16.0 & 1.54 & 0.125 & $\mathrm{~S}$ & 1 \\
\hline Potato & Solanum tuberosum & 1.7 & 12.0 & 2.18 & 0.094 & MS & 1 \\
\hline Spinach & Spinacia oleracea & 2.0 & 7.6 & 2.56 & 0.059 & MS & 1 \\
\hline Tomato & Lycopersicon lycopersicum & & & 3.20 & 0.077 & MS & 1 \\
\hline
\end{tabular}

2 These data serve only as a guideline to relative tolerances among crops. Absolute tolerances vary, depending on climate, soil conditions and cultural practices

3 In gypsiferous soils, plants will tolerate ECe values about $2 \mathrm{dS} / \mathrm{m}$ higher than indicated

4 SALTMAX and SALTSLOPE are values for total salinity in soil water assuming total salinity $(\mathrm{mg} / \mathrm{cm} 3)=0.64 \times \mathrm{EC}(\mathrm{dS} / \mathrm{m})$

5 (Rhoades et al., (1992)) and EC soil water $\approx 2 \mathrm{EC}_{\mathrm{e}}$

5 Ratings according to Maas (1990): S sensitive, MS moderately sensitive, MT moderately tolerant, and T tolerant

6 References: 1 Maas and Hoffman (1977), 2 Francois et al. (1986), 3 West and Francois (1982)

7 Less tolerant during seedling stage, ECe at this stage should not exceed $4 \mathrm{dS} / \mathrm{m}$ or $5 \mathrm{dS} / \mathrm{m}$

8 Sensitive during germination and emergence, ECe should not exceed $3 \mathrm{dS} / \mathrm{m}$

9 Average of several varieties. Suwannee and Coastal are about $20 \%$ more tolerant, and common and Greenfield are about

$1020 \%$ less tolerant than the average

10 Data from one cultivar, 'Pobred'. 


\section{Appendix 10 Shrinkage characteristic data}

After Bronswijk and Vermeer (1990)

\begin{tabular}{|c|c|c|c|c|c|c|c|c|c|c|c|c|}
\hline \multirow{3}{*}{$\begin{array}{l}\text { Place } \\
\text { (1) }\end{array}$} & \multirow{3}{*}{$\begin{array}{l}\text { Depth } \\
\mathrm{cm}\end{array}$} & \multirow{3}{*}{$\begin{array}{l}\text { Horizon } \\
\text { - }\end{array}$} & \multirow{3}{*}{$\begin{array}{l}\rho_{\mathrm{s}}^{(2)} \\
\mathrm{g} \mathrm{cm}^{-3}\end{array}$} & \multicolumn{6}{|c|}{ Composition } & \multicolumn{3}{|c|}{ Shrinkage par. } \\
\hline & & & & \multicolumn{2}{|c|}{ weight $\%$ of soil } & \multicolumn{4}{|c|}{ weight $\%$ of mineral parts } & \multirow{2}{*}{$\begin{array}{l}e_{0} \\
-\end{array}$} & \multirow{2}{*}{$\begin{array}{l}v_{1} \\
-\end{array}$} & \multirow{2}{*}{$\begin{array}{l}v_{\mathrm{s}} \\
-\end{array}$} \\
\hline & & & & $\mathrm{CaCO}_{3}$ & $H^{(3)}$ & $<2$ & $2-16$ & $16-50$ & $>50 \mu \mathrm{m}$ & & & \\
\hline \multirow[t]{4}{*}{1} & $0-22$ & A11 & 2.52 & 0.0 & 10.3 & 39.9 & 20.9 & 33.4 & 5.8 & 0.45 & 1.0 & 0.0 \\
\hline & $22-42$ & $\mathrm{ACg}$ & 2.60 & 0.0 & 6.9 & 40.7 & 25.9 & 28.3 & 5.1 & 0.37 & 0.6 & 0.0 \\
\hline & $42-78$ & $\mathrm{C} 1 \mathrm{~g}$ & 2.66 & 2.5 & 4.5 & 58.1 & 24.7 & 16.2 & 1.1 & 0.43 & 0.7 & 0.0 \\
\hline & $78-120$ & $\mathrm{C} 2 \mathrm{~g}$ & 2.68 & 6.9 & 2.2 & 24.1 & 14.3 & 53.5 & 8.1 & 0.56 & 0.7 & 0.0 \\
\hline \multirow[t]{5}{*}{2} & $0-26$ & Ap & 2.64 & 1.4 & 4.8 & 45.4 & 27.8 & 16.6 & 10.2 & 0.52 & 0.8 & 0.2 \\
\hline & $26-34$ & $\mathrm{~A} 12$ & 2.61 & 0.8 & 3.9 & 45.9 & 27.4 & 18.9 & 6.8 & 0.46 & 0.9 & 0.0 \\
\hline & $34-56$ & $\mathrm{C} 11 \mathrm{~g}$ & 2.62 & 1.7 & 2.2 & 51.6 & 29.2 & 15.4 & 3.8 & 0.48 & 0.9 & 0.1 \\
\hline & $56-75$ & $\mathrm{C} 12 \mathrm{~g}$ & 2.68 & 3.3 & 1.9 & 39.1 & 24.1 & 32.8 & 4.0 & 0.50 & 0.9 & 0.1 \\
\hline & $75-107$ & $\mathrm{C} 13 \mathrm{~g}$ & 2.69 & 0.3 & 3.0 & 59.3 & 31.7 & 6.9 & 2.1 & 0.50 & 0.9 & 0.05 \\
\hline \multirow[t]{5}{*}{3} & $0-29$ & Ap & 2.65 & 9.0 & 3.3 & 52.0 & 24.2 & 20.4 & 3.4 & 0.49 & 0.7 & 0.2 \\
\hline & $29-40$ & $A C$ & 2.67 & 10.6 & 2.9 & 62.9 & 17.0 & 17.7 & 2.4 & 0.50 & 0.8 & 0.2 \\
\hline & $40-63$ & $\mathrm{C} 21$ & 2.69 & 11.3 & 2.7 & 52.4 & 25.3 & 18.3 & 4.0 & 0.55 & 0.8 & 0.1 \\
\hline & $63-80$ & $\mathrm{C} 22 \mathrm{~g}$ & 2.66 & 9.8 & 2.8 & 55.8 & 24.1 & 16.7 & 3.4 & 0.58 & 1.0 & 0.1 \\
\hline & $80-100$ & $\mathrm{C} 23 \mathrm{~g}$ & 2.69 & 11.6 & 2.2 & 59.6 & 26.4 & 12.2 & 1.8 & 0.57 & 1.0 & 0.1 \\
\hline \multirow[t]{4}{*}{4} & $0-21$ & A11 & 2.59 & 11.7 & 5.9 & 34.8 & 17.9 & 27.9 & 19.5 & 0.52 & 1.0 & 0.0 \\
\hline & $21-52$ & A12 & 2.61 & 11.1 & 6.2 & 42.9 & 22.1 & 26.5 & 8.5 & 0.53 & 0.9 & 0.0 \\
\hline & $52-77$ & $\mathrm{C} 21 \mathrm{~g}$ & 2.62 & 17.6 & 3.7 & 32.1 & 20.4 & 33.2 & 14.2 & 0.82 & 1.2 & 0.0 \\
\hline & $77-100$ & $\mathrm{C} 22 \mathrm{~g}$ & 2.63 & 18.8 & 3.1 & 16.2 & 10.1 & 37.8 & 36.0 & 0.79 & 1.0 & 0.0 \\
\hline \multirow[t]{5}{*}{5} & $0-22$ & Ap1 & 2.66 & 9.9 & 2.6 & 36.8 & 22.2 & 27.5 & 13.5 & 0.48 & 0.8 & 0.0 \\
\hline & $22-38$ & A12 & 2.66 & 8.1 & 2.2 & 45.6 & 27.2 & 22.9 & 4.3 & 0.56 & 0.8 & 0.0 \\
\hline & $38-60$ & $\mathrm{C} 22 \mathrm{~g}$ & 2.63 & 6.6 & 7.6 & 35.3 & 43.9 & 19.7 & 1.1 & 0.68 & 1.2 & 0.1 \\
\hline & $60-90$ & $\mathrm{C} 23 \mathrm{~g}$ & 2.59 & 5.8 & 7.0 & 15.9 & 23.9 & 58.2 & 2.0 & 1.10 & 2.0 & 0.0 \\
\hline & $90-110$ & $\mathrm{C} 24 \mathrm{~g}$ & 2.57 & 4.6 & 10.5 & 20.2 & 27.2 & 51.2 & 1.4 & 1.10 & 2.1 & 0.0 \\
\hline \multirow[t]{4}{*}{6} & $0-18$ & A11 & 2.52 & 0.0 & 9.9 & 58.1 & 30.7 & 10.2 & 1.0 & 0.30 & 0.9 & 0.0 \\
\hline & $18-30$ & $\mathrm{~A} 12$ & 2.60 & 0.0 & 7.5 & 55.8 & 35.5 & 8.1 & 0.6 & 0.34 & 0.9 & 0.0 \\
\hline & $30-58$ & $\mathrm{C} 11 \mathrm{~g}$ & 2.64 & 0.0 & 3.7 & 59.6 & 29.5 & 10.1 & 0.8 & 0.37 & 0.5 & 0.0 \\
\hline & $58-85$ & $\mathrm{C} 12 \mathrm{~g}$ & 2.59 & 0.0 & 3.8 & 51.7 & 37.0 & 9.6 & 1.7 & 0.40 & 0.8 & 0.05 \\
\hline \multirow[t]{4}{*}{7} & $0-35$ & Ap & 2.67 & 10.2 & 2.1 & 30.8 & 15.7 & 30.2 & 23.3 & 0.43 & 1.0 & 0.0 \\
\hline & $35-60$ & $\mathrm{C} 21 \mathrm{~g}$ & 2.67 & 13.6 & 1.6 & 46.4 & 20.5 & 21.2 & 11.9 & 0.45 & 0.8 & 0.0 \\
\hline & $60-80$ & $\mathrm{C} 22 \mathrm{~g}$ & 2.70 & 15.7 & 1.3 & 41.9 & 18.3 & 23.3 & 15.5 & 0.40 & 1.3 & 0.0 \\
\hline & $80-95$ & $\mathrm{C} 23 \mathrm{~g}$ & 2.69 & 9.5 & 0.3 & 16.2 & 6.7 & 21.0 & 56.1 & 0.40 & 1.3 & 0.0 \\
\hline
\end{tabular}

(1) Locations: 1-Oosterend, 2-Nieuw Beerta, 3-Nieuw Statenzijl, 4-Schermerhorn, 5-Dronten, 6-Bruchem and 7-Kats.

(2) Density of the solid phase

(3) Organic matter 


\section{Appendix 11 Examples of shrinkage characteristics of peat}

Shrinkage characteristics of peat and peaty soils (after Hendriks, 2004). Black dots are measurements and lines are fits with Eq. (6.19). Parameter values concern parameters of Eq. (6.19).

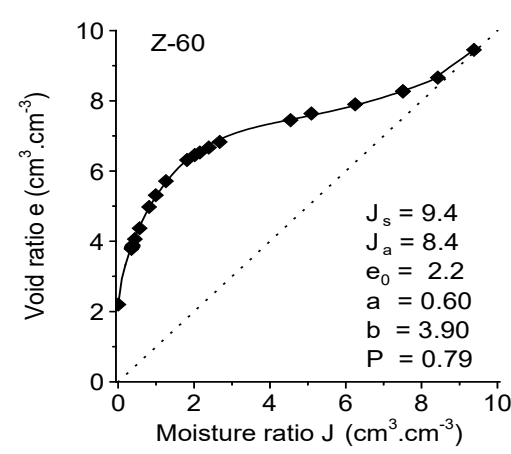

Description, organic matter and clay content (mass-\%) of the peat soils. Figures in sample codes refer to sample depths in $\mathrm{cm}$.

\begin{tabular}{lllc} 
Sample & Soil & Org. & Clay \\
code & description & matter & \\
A-15 & peaty clay & 33 & 40 \\
A-25 & clayey peat & 62 & 21 \\
\hline A-45 & $\begin{array}{l}\text { sphagnum } \\
\text { peat }\end{array}$ & 93 & 1 \\
& wood peat & 81 & 5 \\
\hline D-80 & sphagnum & 91 & 2 \\
p-80 & peat & & \\
\hline V-10 & $\begin{array}{l}\text { peaty, sandy } \\
\text { clay }\end{array}$ & 18 & 17 \\
\hline Z-10 & clayey peat & 48 & 36 \\
\hline Z-60 & wood peat & 82 & 4 \\
\hline Z-80 & wood peat & 83 & 4 \\
\hline
\end{tabular}
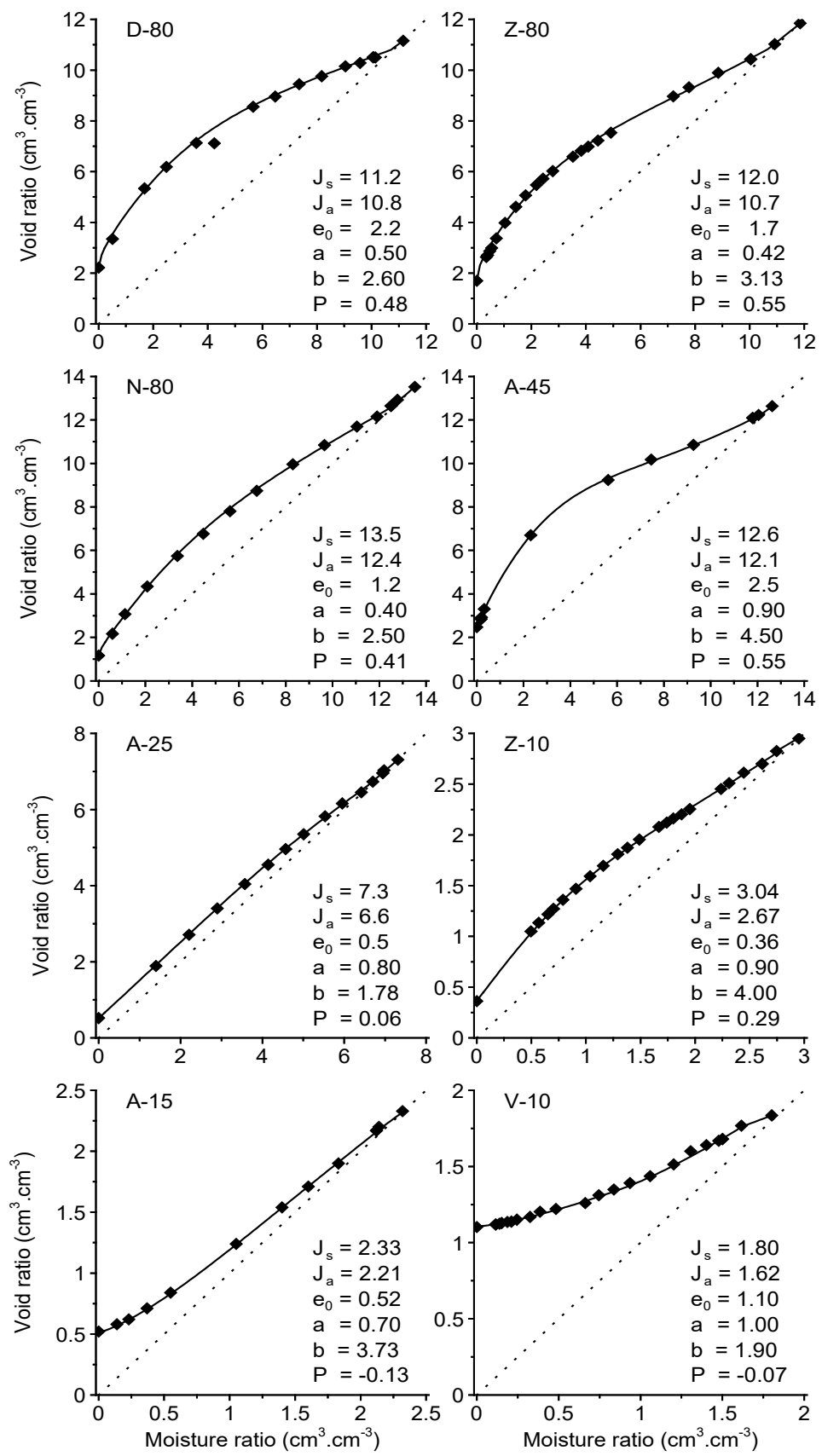


\section{Appendix 12 List of input array lengths}

The array lengths of input data are defined as parameters in the Fortran subroutine 'param.fi'. The array lengths can be enlarged by adjusting the values in 'param. fi' en recompilation of the Fortran code. In the internet version SWAP 4 we did define the array lengths as listed in Box 12.1.

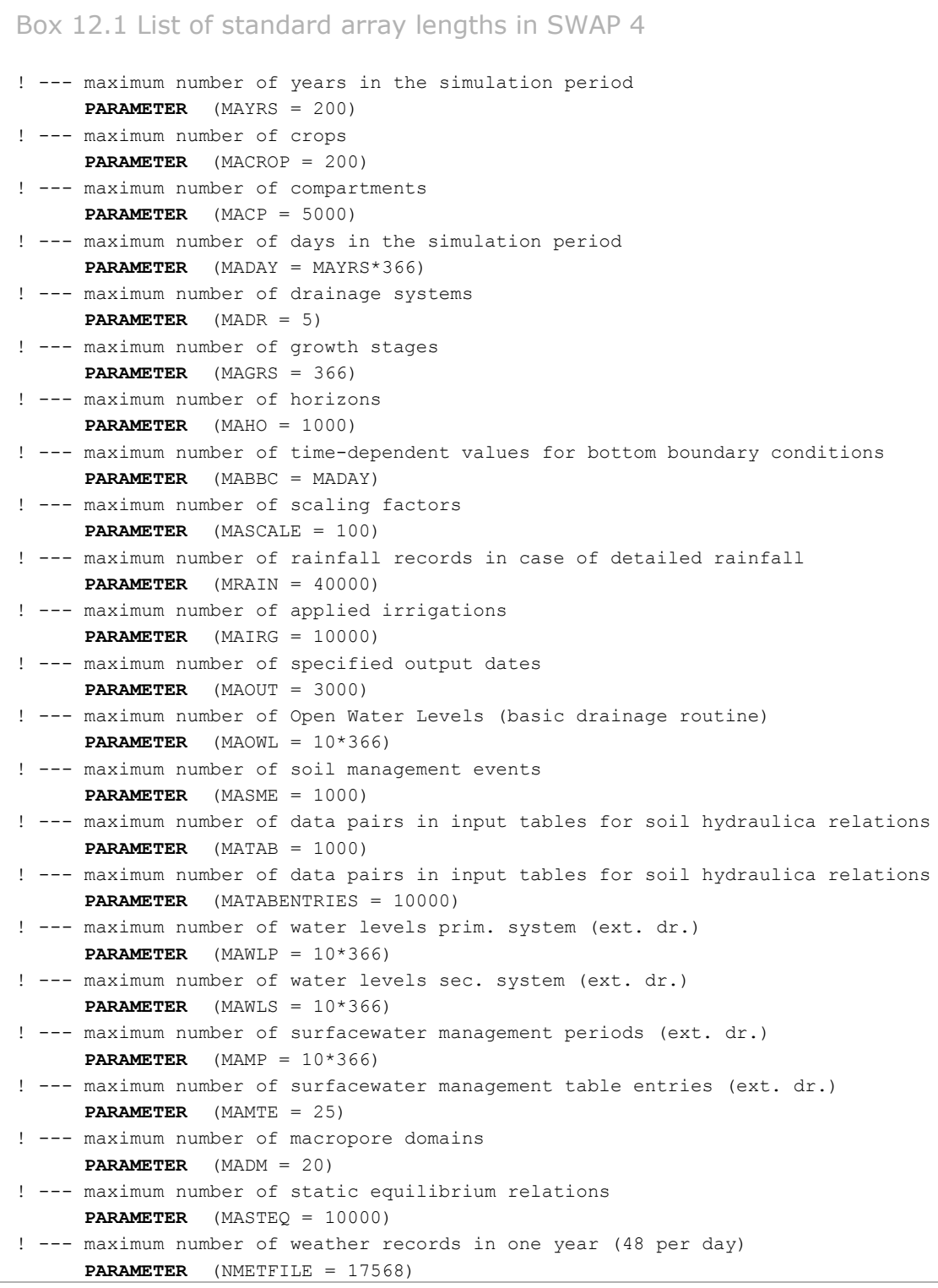




\title{
Appendix 13 List of main SWAP subroutines
}

\author{
Assim \\ Bocodrb \\ gross $\mathrm{CO} 2$ assimilation rate of the crop \\ Bocodre \\ Boundbottom \\ calculate lateral drainage rate and state variables \\ Boundtop \\ CalcGwl \\ CalcGrid \\ CropFixed \\ Drainage \\ DeVries \\ DivDra \\ Fluxes \\ Grass \\ HConduc \\ HeadCalc \\ Hysteresis \\ Integral \\ Irrigation \\ MacroPore \\ MeteoInput \\ MoisCap \\ NoCrop \\ OutAfo \\ OutAun \\ OutBal \\ OutBlc \\ OutDrf \\ OutEnd \\ OutInc \\ OutSba \\ OutSwb \\ OutTem \\ OutVap \\ OutWba \\ Penmon \\ Ponding \\ PrHead \\ Radiat \\ ReadSwap \\ ReducEva \\ RootExtraction \\ Snow \\ SoilWater \\ Solute \\ SurfaceWater \\ Temperature \\ TimeControl \\ Totass \\ Warn \\ WatCon \\ WatStor \\ Wofost \\ WriteHead \\ calculate lateral drainage rate and state variables, including surface water system \\ calculate lower boundary conditions \\ calculate top boundary conditions \\ search for groundwater levels \\ converts vertical discretization \\ prescribed crop growth \\ lateral drainage \\ calculate soil thermal properties \\ divide drainage flux to compartments \\ calculate bottom and compartment fluxes \\ detailed grass growth routine \\ calculate hydraulic conductivity from water content \\ calculate pressure heads at next time \\ check hysteretic reversal and update scanning curve \\ integrate intermediate and cumulative water fluxes \\ initialize and calculate irrigation \\ calculate crack shrinkage and swelling, including fluxes \\ read meteorological data and return values of actual day \\ calculate moisture capacity from pressure head \\ specify crop characteristics for bare soil \\ formatted hydrological output for ANIMO/PESTLA (*.AFO) \\ unformatted hydrological output for ANIMO/PESTLA (*.AUN) \\ write overview balances (*.BAL) \\ write total water balances (*.BLC) \\ write drainage fluxes, runoff, etc. (*.DRF) \\ write final soil state variables for next initial condition \\ write water balance increments (*.INC) \\ write solute balance (*.SBA) \\ write surface water balance (*.SWB) \\ write soil temperatures (*.TEM) \\ write water and solute profile data (*.VAP) \\ write water balance (*.WBA) \\ calculate potential evaporation and transpiration rates \\ calculation of runoff \\ calculate pressure head from water content \\ calculate fluxes of diffuse and PAR radiation \\ read SWAP main input file \\ calculate actual soil evaporation \\ calculate potential and actual water extraction by roots \\ snow submodel \\ calculate soil water rate/state variables \\ calculate solute transport \\ calculate rate/state variables of surface water system \\ calculate soil temperatures \\ handles time variables, switches and flags \\ calculate daily total gross assimilation \\ output of warnings to screen and log file \\ calculate water content from pressure head \\ calculate water storage in soil profile and cracks \\ detailed crop growth routine \\ write header with model version, project name, etc.
}




\section{Appendix 14 Description of output files $*$.afo and *aun}

This annex describes the content of the output files with extension *.afo and *.aun. The content of both files is identic; they only differ in format: one file is binary and unformatted (*.aun) and the other file is formatted (*.afo). The description given in this annex uses the following symbols:

- Unit = units as applied in these output files; units differ from those applied in Swap !

- $\mathrm{R}=$ data are written to a new record;

- DT = data type; R means Real*4, I means Integer*2;

- Mnemonic = the name of the variable as applied in the source code of Swap

\begin{tabular}{|c|c|c|c|c|c|}
\hline Description of variable & Unit & Range & $\mathbf{R}$ & DT & Mnemonic \\
\hline \multicolumn{6}{|l|}{ Time domain } \\
\hline Year when hydrological simulation started & - & {$[1 . . \infty>$} & * & I & bruny \\
\hline $\begin{array}{l}\text { Time (Julian daynumber) when hydrological simulation } \\
\text { started (Minimum); will be } 0.0 \text { when simulation started at } \\
\text { 1st of January, } 00.00 \text { hour. }\end{array}$ & - & {$[0.0 . .366]$} & - & $\mathrm{R}$ & brund-1 \\
\hline $\begin{array}{l}\text { Time (Julian daynumber) when hydrological simulation } \\
\text { ended (Maximum) }\end{array}$ & - & {$[0.0 . .366]$} & - & $\mathrm{R}$ & erund \\
\hline Step size of time-interval for dynamic hydrological data & d & {$[1.0 . .30 .0]$} & - & $\mathrm{R}$ & period \\
\hline \multicolumn{6}{|l|}{ Geometry of model system } \\
\hline \multicolumn{6}{|c|}{ The following 4 variables (botcom - thetawp) are given for the horizons 1 - numlay: } \\
\hline $\begin{array}{l}\text { Compartment number of the deepest compartment (bottom) } \\
\text { of each horizon/layer }\end{array}$ & - & {$[1 .$. numnod] } & * & I & botcom(numlay) \\
\hline Volume fraction moisture at Saturation & $m^{3} m^{-3}$ & {$[0.0 \ldots 1.0]$} & $*$ & $\mathrm{R}$ & thetas (numlay) \\
\hline Volume fraction moisture at Field Capacity & $m^{3} m^{-3}$ & {$[0.0 \ldots 1.0]$} & $*$ & $\mathrm{R}$ & thetafc(numlay) \\
\hline Volume fraction moisture at Wilting point & $\mathrm{m}^{3} \mathrm{~m}^{-3}$ & {$[0.0 \ldots 1.0]$} & $*$ & $\mathrm{R}$ & thetawp(numlay) \\
\hline \multicolumn{6}{|c|}{ The following variable $d z$ is given for the compartments 1 - numnod } \\
\hline Thickness of compartments & $\mathrm{m}$ & {$[0.001 . .100]$} & * & $\mathrm{R}$ & $\mathrm{dz}$ (numnod) \\
\hline
\end{tabular}




\section{Initial conditions}

The following variable theta is given for the compartments 1 - numnod

Volume fraction moisture inItially present in compartments $\mathrm{m}^{3} \mathrm{~m}^{-3}$

$[0.0 \ldots 1.0] * \mathrm{R} \quad$ theta(numnod)

1 - NUMNOD

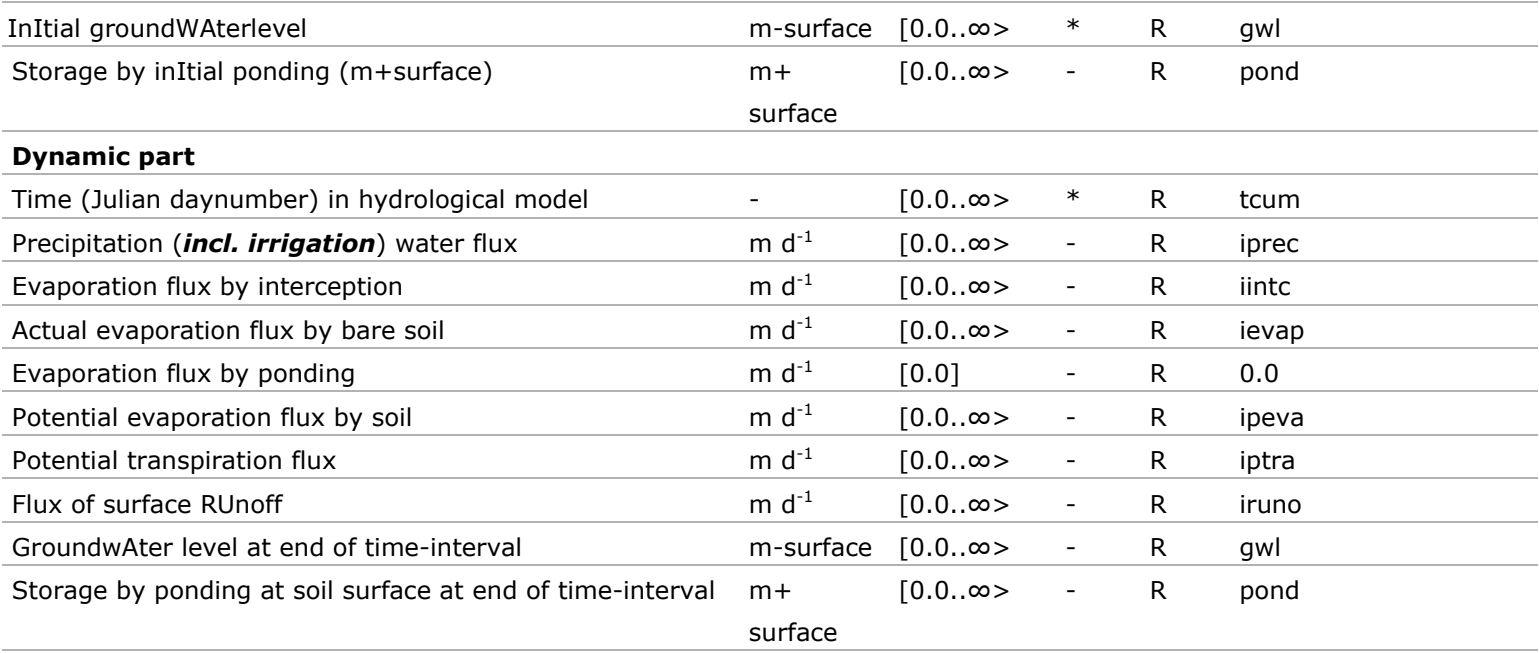

The variables $h$ - inqdra are given for the compartments 1 - numnod, with one exception for inq, which is given for the compartments 1 - numnod +1

Suction (pressure head) of soil moisture (negative when cm unsaturated)

Volume fraction of moisture at end of time-interval

$\mathrm{cm}$

$$
<-\infty . .+\infty>\quad * \quad \mathrm{R} \quad \mathrm{h} \text { (numnod) }
$$

Actual transpiration flux

$\mathrm{m}^{3} \mathrm{~m}^{-3}$

$[0.0 \ldots 1.0]$

$\mathrm{R}$

theta(numnod)

Flux incoming from above (compartments 1 - numnod $+1-\mathrm{m} \mathrm{d}^{-1}$ $[0.0 . . \infty>$ inqrot(numnod)

downward=positive)

$[0.0 . . \infty>\quad * \quad \mathrm{R} \quad$ inq(numnod +1$)$

The presence of values for variables inqdra1-inqdra5 is determined by the variable nrlevs. The value of nrlevs determines the number of drainage systems for which flux densities must be given.

Flux of drainage system of 1 st order (e.g. canal) $\quad \mathrm{m} \mathrm{d}^{-1} \quad[0.0 . \infty>* * \quad \mathrm{R} \quad$ inqdra(1,numnod)

Flux of drainage system of 2 nd order (e.g. ditch) $\quad \mathrm{m} \mathrm{d}^{-1} \quad[0.0 . . \infty>\quad * \quad \mathrm{R} \quad$ inqdra(2,numnod)

Flux of drainage system of 3rd order (e.g. trench) $\quad \mathrm{m} \mathrm{d}^{-1} \quad[0.0 . \infty>\quad * \quad \mathrm{R} \quad$ inqdra(3,numnod)

Flux of drainage system of 4th order (e.g. tube drain) $\quad \mathrm{m} \mathrm{d}^{-1} \quad[0.0 . \infty>\quad * \quad \mathrm{R} \quad$ inqdra(4,numnod)

Flux of drainage system of 5 th order (e.g. rapid drainage) $\mathrm{m} \mathrm{d}^{-1} \quad[0.0 . \infty>\quad * \quad \mathrm{R} \quad$ inqdra(5,numnod) 


\section{Appendix 15 Description of output files $*$.bfo and *.bun}

This annex describes the content of the output files with extension *.bfo and *.bun. The content of both files is identical; they only differ in format: one file is binary and unformatted (*.bun) and the other file is ascii and formatted (*.bfo). Differences between the (*.bfo, *.bun) and (*.aun, *.afo, 0) are indicated with a vertical line next to the text.

Part of the content of this file is optional and indicated with grey shading of the corresponding rows. The optional content is indiced with the switch SWOP (see section File Options).

The temperature parameter (Tsoil) has a value of "-99.9" when temperature processes were not simulated.

The snow-parameters (Ssnow, Igsnow, Isubl) have a value of "0", when snow processes were not simulated. This 0 -value instead of -99.9 -value is applied to facilitate uniformity of water balance calculations.

The description given in these pages uses the following symbols:

- Unit = units as applied in these output files; units mostly differ from those applied in Swap

- Range = upper and lower boundary of given data

- $\mathrm{R}=$ an asterisk $(*)$ indicates that data are written to a new record;

- DT = data type; R means Real*4, I means Integer*2, C means CharacterString;

- Mnemonic = the name of the variable as applied in the source code of Swap

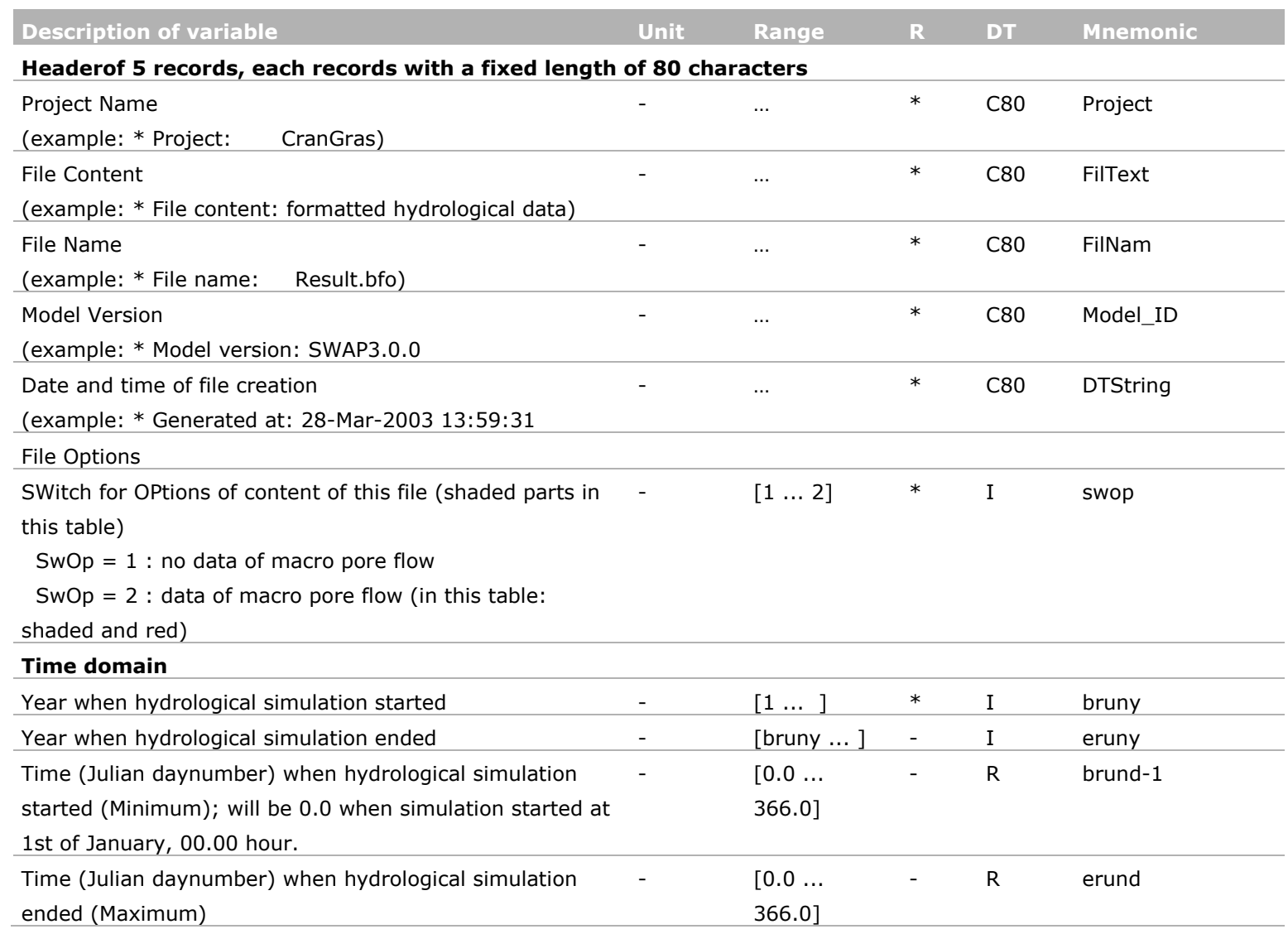


Geometry of model system

Number of model compartments

[1

$*$

I

numnod

Number of horizons

numnod]

Number of drainage systems

$[1 \ldots$

numlay]

(value must be $0,1,2,3,4$ or 5 )

$[0 \ldots 5] \quad-\quad$ I $\quad$ nrlevs

The following 4 variables (botcom ... thetawp) are given for the horizons 1 ... numlay:

Compartment number of the deepest compartment

$[1 \ldots$

* I

botcom(numlay)

(bottom) of each horizon/layer

numnod]

Volume fraction moisture at Saturation

$\mathrm{m}^{3} \mathrm{~m}^{-3} \quad[0.0 \ldots 1.0] \quad * \quad \mathrm{R} \quad$ thetas (numlay)

Volume fraction moisture at Field Capacity

$\mathrm{m}^{3} \mathrm{~m}^{-3} \quad[0.0 \ldots 1.0] * \mathrm{R} \quad$ thetafc(numlay)

Volume fraction moisture at Wilting point

$\mathrm{m}^{3} \mathrm{~m}^{-3} \quad[0.0 \ldots 1.0] * \quad \mathrm{R} \quad$ thetawp(numlay)

The following variable $d z$ is given for the compartments 1 ... numnod

Thickness of compartments

$[0.001 \ldots$

$* \quad \mathrm{R}$

R dz(numnod)

100.0]

\section{Geometry of macropore system}

Areic volume of static macropores in domain 1 (Main

$m^{3} m^{-2}$

$[0.0 \ldots]$

R

VIMpStDm1(numnod)

Bypass Flow domain) per compartment 1 ... NUMNOD

\begin{tabular}{|c|c|c|c|c|c|}
\hline $\begin{array}{l}\text { Areic volume of static macropores in domain } 2 \text { (Internal } \\
\text { Catchment domain) per compartment } 1 \ldots \text { NUMNOD }\end{array}$ & $\mathrm{m}^{3} \mathrm{~m}^{-2}$ & {$[0.0 \ldots]$} & $*$ & $\mathrm{R}$ & VIMpStDm2(numnod) \\
\hline $\begin{array}{l}\text { Diameter of soil matrix polygones per compartment } \\
1 \ldots \text { NUMNOD }\end{array}$ & $\mathrm{m}$ & $\begin{array}{l}{[0.001 \ldots} \\
10.01\end{array}$ & $*$ & $\mathrm{R}$ & DiPoCp(numnod) \\
\hline
\end{tabular}

\section{Initial conditions}

The following variable theta and tempi are given for the compartments 1 ... numnod

Volume fraction moisture initially present in

$\mathrm{m}^{3} \mathrm{~m}^{-3} \quad[0.0 \ldots 1.0] \quad * \quad \mathrm{R} \quad$ Theta(numnod)

compartments 1 ... NUMNOD

Initial groundwaterlevel

(negative below soil surface, when positive use Pond)

Storage by initial ponding

Storage by snow

Soil temperature of compartments 1 ... NUMNOD

m-surf. $[0.0 \ldots] \quad * \quad \mathrm{R} \quad \mathrm{GW}$

$\begin{array}{lllll}\mathrm{m} & {[0.0 \ldots]} & - & \mathrm{R} & \text { Pond } \\ \mathrm{m} & {[0.0 \ldots]} & * & \mathrm{R} & \text { Ssnow } \\ { }^{\circ} \mathrm{C} & {[-50.0 \ldots} & * & \mathrm{R} & \text { Tsoil(numnod) } \\ & 50.0] & & & \end{array}$

Initial conditions for macropores, domain 1 (Main Bypass Flow domain)

\begin{tabular}{|c|c|c|c|c|c|}
\hline Water level & m-surf. & {$[0.0 \ldots]$} & $*$ & $\mathrm{R}$ & WaLevDm1 \\
\hline Areic volume & $m^{3} m^{-2}$ & {$[0.0 \ldots]$} & - & $\mathrm{R}$ & VIMpDm1 \\
\hline Areic volume of water stored & $m^{3} m^{-2}$ & {$[0.0 \ldots]$} & - & $\mathrm{R}$ & WaSrDm1 \\
\hline Areic volume & $m^{3} m^{-2}$ & {$[0.0 \ldots]$} & - & $\mathrm{R}$ & VIMpDm2 \\
\hline
\end{tabular}




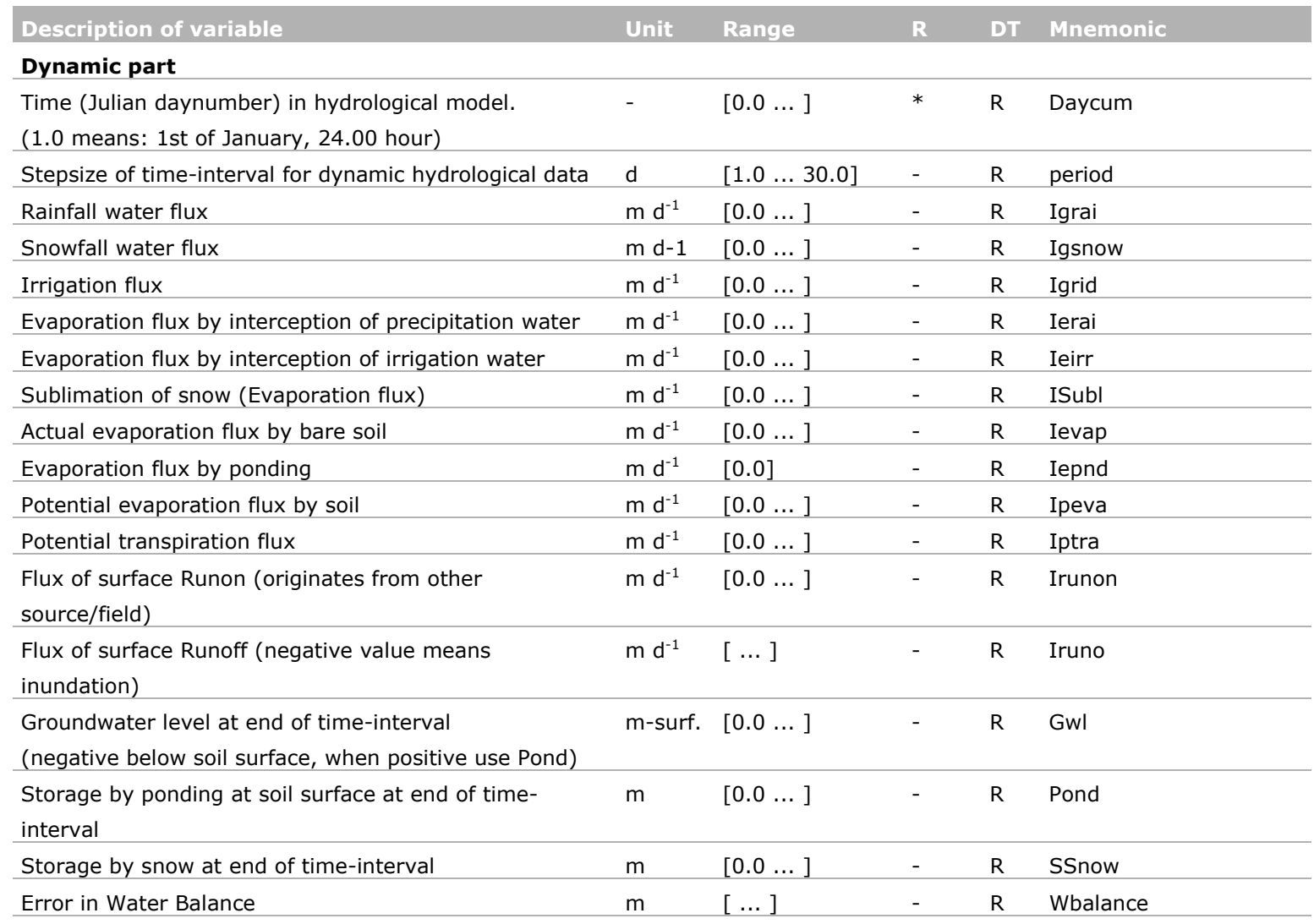

The variables $h$... inqdra are given for the compartments 1 ... numnod, with one exception for inq, which is given for the compartments 1 ... numnod +1

\begin{tabular}{|c|c|c|c|c|c|}
\hline $\begin{array}{l}\text { Suction (pressure head) of soil moisture } \\
\text { (negative = unsaturated) }\end{array}$ & $\mathrm{cm}$ & {$[\cdots]$} & * & $\mathrm{R}$ & h(numnod) \\
\hline Volume fraction of moisture at end of time-interval & $\mathrm{m}^{3} \mathrm{~m}^{-3}$ & {$[0.0 \ldots 1.0]$} & * & $\mathrm{R}$ & theta(numnod) \\
\hline Flux incoming from above (compartments $1 \ldots$ & $m d^{-1}$ & {$[\ldots]$} & $*$ & $\mathrm{R}$ & inq(numnod +1$)$ \\
\hline
\end{tabular}

numnod +1 , positive = downward)

The presence of values for variables inqdra1...inqdra5 is determined by the variable nrlevs. The value of nrlevs determines the number of drainage systems for which flux densities must be given (postive: from soil to drainage system)

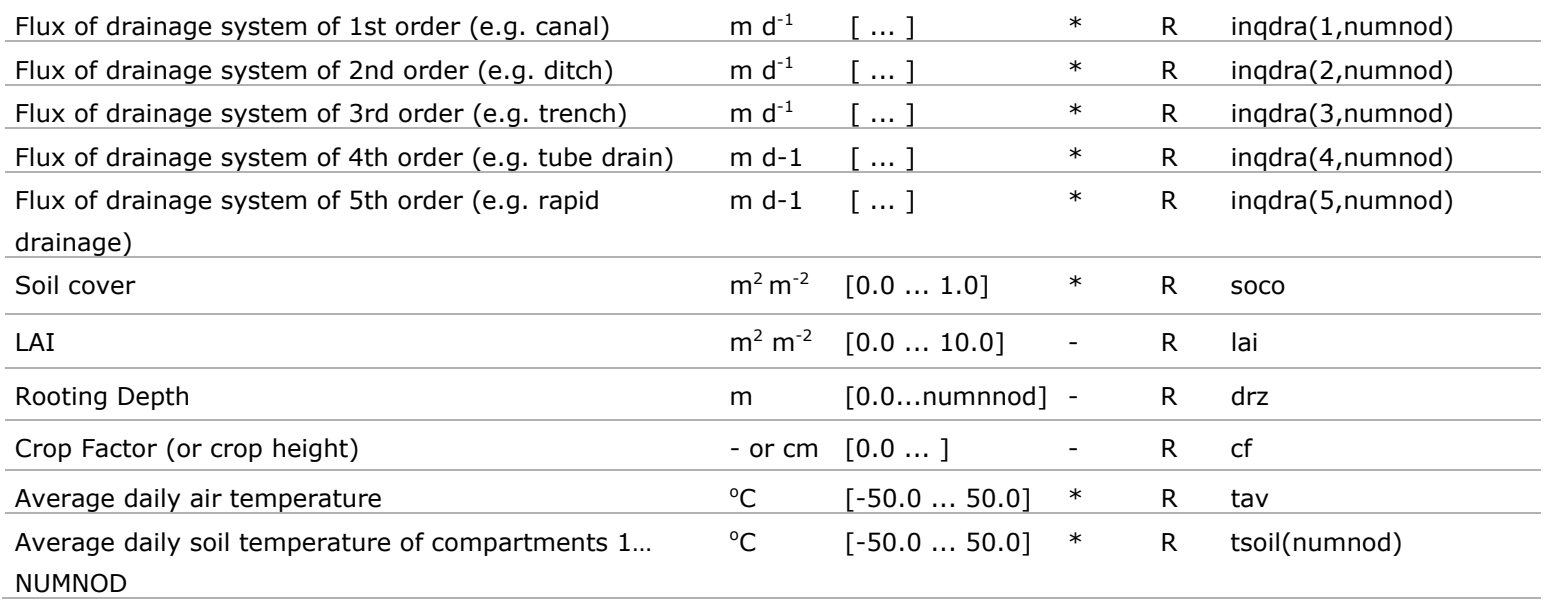


Dynamic part for macropores, domain 1 (Main Bypass Flow domain

Water level at end of time-interval

m-surf. $\quad[0.0 \ldots]$

$\mathrm{R}$

WaLevDm1

Areic volume at end of time-interval

$\mathrm{m}^{3} \mathrm{~m}^{-2} \quad[0.0 \ldots]$

R VIMpDm1

Areic volume of water stored at end of time-interval $\mathrm{m}^{3} \mathrm{~m}^{-2} \quad[0.0 \ldots]$

R WaSrDm1

Infiltration flux at soil surface directly by precipitation

$\mathrm{m} \mathrm{d}^{-1} \quad[0.0 \ldots]$

R IQInTopPreDm1

Infiltration flux at soil surface indirectly by lateral

$\mathrm{m} \mathrm{d}^{-1} \quad[0.0 \ldots]$

R IQInTopLatDm1

overland flow (runoff)

Exchange flux with soil matrix per compartment 1-

$\mathrm{m} \mathrm{d}-1 \quad[\ldots]$

$\mathrm{R}$

InQExcMtxDm1Cp(numnod)

numnod (positive: from macropores into matrix)

Rapid drainage flux towards drain tube per

$\mathrm{m} \mathrm{d}^{-1} \quad[0.0 \ldots]$

R InQOutDrRapCp(numnod)

compartment 1-numnod

Average fraction of macropore wall in contact with

$\mathrm{m} \mathrm{d}^{-1} \quad[0.0 \ldots]$

R FrMpWalWetDm1(numnod)

macropore water during timestep per comp. 1-numnod

Dynamic part for macropores, domain 2 (Internal Catchment domain)

Areic volume at end of time-interval $\mathrm{m}^{3} \mathrm{~m}^{-2}[0.0 \ldots]$

Areic volume of water stored at end of time-interval

$m^{3} m^{-2}$

$[0.0 \ldots]$

VIMpDm2

Infiltration flux at soil surface directly by precipitation

$\mathrm{m} \mathrm{d}^{-1}$

$[0.0 \ldots]$

R WaSrDm2

Infiltration flux at soil surface indirectly by lateral

$m d^{-1}$

$[0.0 \ldots]$

$\mathrm{R}$

IQInTopPreDm2

overland flow (runoff)

Exchange flux with soil matrix per compartment 1-

$\mathrm{m} \mathrm{d}^{-1} \quad[\ldots]$

numnod (positive: from macropores into matrix)

Average fraction of macropore wall in contact with

$\mathrm{m} \mathrm{d}^{-1} \quad[0.0 \ldots]$

R InQExcMtxDm2Cp(numnod)

macropore water during timestep per comp. 1-numnod 


\section{Appendix 16 Crop growth of grassland: acronyms}

\begin{tabular}{|c|c|c|}
\hline admi(pot) & above ground dry matter increase & {$\left[\mathrm{kg} \mathrm{ha}^{-1} \mathrm{~d}^{-1}\right]$} \\
\hline amax & instantaneous gross assimilation at light saturation & {$\left[\mathrm{kg} \mathrm{ha}^{-1} \mathrm{hr}^{-1}\right]$} \\
\hline amaxtb & list max. $\mathrm{CO}_{2}$ assimilation rate as a function of day number & {$\left[\mathrm{kg} \mathrm{ha}^{-1} \mathrm{hr}^{-1}\right]$} \\
\hline $\operatorname{asrc}($ pot $)$ & assimilates available for growth & {$\left[\mathrm{kg} \mathrm{ha}^{-1} \mathrm{~d}^{-1}\right]$} \\
\hline cptro & accumulated potential transpiration & {$[\mathrm{cm}]$} \\
\hline ctro & accumulated actual transpiration & {$[\mathrm{cm}]$} \\
\hline crto & relative transpiration & {$[-]$} \\
\hline $\mathrm{cvl}$ & efficiency of conversion into leaves & {$\left[\mathrm{kg} \mathrm{kg}^{-1}\right]$} \\
\hline cvs & efficiency of conversion into stems & {$\left[\mathrm{kg} \mathrm{kg}^{-1}\right]$} \\
\hline $\mathrm{cvr}$ & efficiency of conversion into roots & {$\left[\mathrm{kg} \mathrm{kg}^{-1}\right]$} \\
\hline dmi(pot) & dry matter increase & {$\left[\mathrm{kg} \mathrm{ha} \mathrm{a}^{-1} \mathrm{~d}^{-1}\right]$} \\
\hline dalv(pot) & leaf death rate due to ageing & {$\left[\mathrm{kg} \mathrm{d}^{-1}\right]$} \\
\hline $\operatorname{drrt}($ pot $)$ & root death rate roots & {$\left[\mathrm{kg} \mathrm{ha}^{-1} \mathrm{~d}^{-1}\right]$} \\
\hline $\operatorname{drst}($ pot) & stem death rate & {$\left[\mathrm{kg} \mathrm{ha}^{-1} \mathrm{~d}^{-1}\right]$} \\
\hline drst1 & stem death rate due to water stress & {$\left[\mathrm{kg} \mathrm{ha}^{-1} \mathrm{~d}^{-1}\right]$} \\
\hline drst2 & stem death rate due to ageing & {$\left[\mathrm{kg} \mathrm{ha}^{-1} \mathrm{~d}^{-1}\right]$} \\
\hline $\mathrm{ds} \operatorname{lv}(\mathrm{pot})$ & leaf death rate due to stress (shading or water) & {$\left[\mathrm{kg} \mathrm{d}^{-1}\right]$} \\
\hline dslv1 & leaf death rate due to water stress & {$\left[\mathrm{kg} \mathrm{d}^{-1}\right]$} \\
\hline dslv2 & leaf death rate due to mutual shading & {$\left[\mathrm{kg} \mathrm{d}^{-1}\right]$} \\
\hline $\operatorname{drlv}($ pot $)$ & total death rate leaves & {$\left[\mathrm{kg} \mathrm{d}^{-1}\right]$} \\
\hline dtga(pot) & gross daily $\mathrm{CO}_{2}$ assimilation rate & {$\left[\mathrm{kg} \mathrm{ha}^{-1} \mathrm{~d}^{-1}\right]$} \\
\hline dwlv(pot) & dry weight death leaves & {$\left[\mathrm{kg} \mathrm{ha}^{-1}\right]$} \\
\hline dwrt(pot) & dry weight death roots & {$\left[\mathrm{kg} \mathrm{ha}^{-1}\right]$} \\
\hline dwst(pot) & dry weight death stems & {$\left[\mathrm{kg} \mathrm{ha}^{-1}\right]$} \\
\hline dteff & daily effective temperature & {$\left[{ }^{\circ} \mathrm{C}\right]$} \\
\hline eff & light use efficiency of single leaf & {$\left[\mathrm{kgha}^{-1} \mathrm{hr}^{-1}\left(\mathrm{Jm}^{2} \mathrm{~s}\right)^{-1}\right]$} \\
\hline $\mathrm{fr}$ & root partitioning factor & {$[-]$} \\
\hline fs & stem partitioning factor & {$[-]$} \\
\hline $\mathrm{fl}$ & leaves partitioning factor & {$[-]$} \\
\hline fltb & $\begin{array}{l}\text { list fraction of total dry matter increase partitioned to the leaves as function of } \\
\text { day number }\end{array}$ & {$\left[\mathrm{kg} \mathrm{kg}^{-1}\right]$} \\
\hline frtb & $\begin{array}{l}\text { list fraction of total dry matter increase partitioned to the roots as function of } \\
\text { day number }\end{array}$ & {$\left[\mathrm{kg} \mathrm{kg}^{-1}\right]$} \\
\hline fstb & $\begin{array}{l}\text { list fraction of total dry matter increase partitioned to the stems as function of } \\
\text { day number }\end{array}$ & {$\left[\mathrm{kg} \mathrm{kg}^{-1}\right]$} \\
\hline gass(pot) & available assimilates after correction for drought stress & {$\left[k g h a^{-1} d^{-1}\right]$} \\
\hline glaiexp(pot) & leaf area index growing rate during the exponential growing phase & {$\left[\right.$ ha ha- $\left.{ }^{-1} d^{-1}\right]$} \\
\hline gla(pot) & leaf area index growth rate & \\
\hline glasol(pot) & source limited leaf area index growing rate & {$\left[\right.$ ha ha- $\left.{ }^{-1} d^{-1}\right]$} \\
\hline grlv(pot) & leaf growth rate & {$\left[k g h a^{-1} d^{-1}\right]$} \\
\hline grrt(pot) & roots growth rate & {$\left[\mathrm{kg} \mathrm{ha}^{-1} \mathrm{~d}^{-1}\right]$} \\
\hline grst(pot) & stem growth rate & {$\left[\mathrm{kg} \mathrm{ha}^{-1} \mathrm{~d}^{-1}\right]$} \\
\hline gwrt(pot) & net growth rate roots & {$\left[\mathrm{kg} \mathrm{ha}^{-1} \mathrm{~d}^{-1}\right]$} \\
\hline gwst(pot) & net stem growth rate & {$\left[\mathrm{kg} \mathrm{ha}^{-1} \mathrm{~d}^{-1}\right]$} \\
\hline idregr(pot) & starting day re-growth after mowing & [d] \\
\hline il & counter leaf age classes & {$[-]$} \\
\hline Ilvold(pot) & counter leaf age classes & {$[-]$} \\
\hline kdif & extinction coefficient for diffuse visible light & {$[-]$} \\
\hline kdir & extinction coefficient for direct visible light & {$[-]$} \\
\hline lai(pot) & total green area index (leaves + stems) & {$\left[\right.$ ha ha $\left.{ }^{-1}\right]$} \\
\hline laicr & critical leaf area index & {$[$ ha ha-1] } \\
\hline laiem & leaf area index at emergence & {$[$ ha ha-1] } \\
\hline
\end{tabular}




\begin{tabular}{|c|c|c|}
\hline laiexp(pot) & leaf area index during exponential growing phase & {$[$ ha ha-1] } \\
\hline laisum & total leaf area of all leaf classes & {$\left[\right.$ ha ha- $\left.{ }^{-1}\right]$} \\
\hline $\operatorname{lv}(\mathrm{pot})$ & array that contains the leaf weight classes & {$[\mathrm{kg}]$} \\
\hline Ivage(pot) & array that contains the leaf age classes & [d] \\
\hline perdl & maximum rel. leaf death rate due to water stress & {$\left[d^{-1}\right]$} \\
\hline pgass(pot) & gross daily $\mathrm{CH}_{2} \mathrm{O}$ assimilation rate & {$\left[\mathrm{kg} \mathrm{ha}^{-1} \mathrm{~d}^{-1}\right]$} \\
\hline ptra & potential transpiration rate & {$\left[\mathrm{cm} \mathrm{d}^{-1}\right]$} \\
\hline $\mathrm{q} 10$ & relative increase of the respiration rate per $10^{\circ} \mathrm{C}$ temperature increase & {$[-]$} \\
\hline rdc & maximum rooting depth determined by the crop & {$[\mathrm{cm}]$} \\
\hline rd(pot) & rooting dept & {$[\mathrm{cm}]$} \\
\hline rdi & Initial rooting depth & {$[\mathrm{cm}]$} \\
\hline rds & maximum rooting depth determined by the soil & {$[\mathrm{cm}]$} \\
\hline relni & reduction factor of the gross daily assimilation rate as & {$[-]$} \\
\hline relnitab & $\begin{array}{l}\text { table containing the gross daily assimilation factors as a function of the } \\
\text { nitrogen application }\end{array}$ & \\
\hline reltr & ratio actual transpiration over potential transpiration & {$[-]$} \\
\hline rest(pot) & death plant material in current time step & {$\left[\mathrm{kg} \mathrm{ha}^{-1}\right]$} \\
\hline rfsetb & list senescence reduction factor as function of day number & {$[-]$} \\
\hline rgrlai & maximum relative increase of LAI per day & {$\left[\right.$ ha ha $\left.{ }^{-1}\right]$} \\
\hline rid & day number & [d] \\
\hline rml & rel. maintenance respiration rate of leaves & {$\left[\mathrm{kg} \mathrm{kg}^{-1} \mathrm{~d}^{-1}\right]$} \\
\hline rmres(pot) & maintenance respiration at reference temperature of $25^{\circ} \mathrm{C}$ & {$\left[\mathrm{kg} \mathrm{ha}^{-1} \mathrm{~d}^{-1}\right]$} \\
\hline rms & rel. maintenance respiration rate of stems & {$\left[\mathrm{kg} \mathrm{kg}^{-1} \mathrm{~d}^{-1}\right]$} \\
\hline rmr & rel. maintenance respiration rate of roots & {$\left[\mathrm{kg} \mathrm{kg}^{-1} \mathrm{~d}^{-1}\right]$} \\
\hline $\operatorname{rr}(\mathrm{pot})$ & growth rate roots & {$\left[\mathrm{cm} \mathrm{d}^{-1}\right]$} \\
\hline rri & maximum daily growth rate roots & {$\left[\mathrm{cm} \mathrm{d}^{-1}\right]$} \\
\hline rdrstb & list relative stem death rates as function of day number & {$\left[\mathrm{kg} \mathrm{kg}^{-1} \mathrm{~d}^{-1}\right]$} \\
\hline sla(pot) & array that contains the specific leaf area classes & {$[$ ha kg-1] } \\
\hline slat(pot) & specific leaf area at time step $t$ & {$\left[\mathrm{ha} \mathrm{kg}{ }^{-1}\right]$} \\
\hline slatb & list specific leaf area as function of day number & {$\left[\right.$ ha $\left.\mathrm{kg}^{-1}\right]$} \\
\hline span & life span of leaves under optimum conditions & [d] \\
\hline ssa & specific stem area & {$\left[\right.$ ha $\left.\mathrm{kg}^{-1}\right]$} \\
\hline $\operatorname{tagp}(\mathrm{pot})$ & total above ground production (stems +leaves) & {$\left[\mathrm{kg} \mathrm{ha}^{-1}\right]$} \\
\hline tagps(pot) & total above ground death plant material & {$\left[\mathrm{kg} \mathrm{ha}^{-1}\right]$} \\
\hline tagptpt(pot) & accumulated total above ground death plant material & {$\left[\mathrm{kg} \mathrm{ha}^{-1}\right]$} \\
\hline $\operatorname{tav}$ & average temperature & {$\left[{ }^{\circ} \mathrm{C}\right]$} \\
\hline tbase & lower threshold temperature for ageing of leaves & {$\left[{ }^{\circ} \mathrm{C}\right]$} \\
\hline tdwi & initial total crop dry weight & {$\left[\mathrm{kg} \mathrm{ha}^{-1}\right]$} \\
\hline teff & temperature factor for the maintenance respiration & {$[-]$} \\
\hline tmnftb & list AMAX reduction factor as function of minimum day temperature & {$[-]$} \\
\hline tmpftb & list AMAX reduction factor as function of average day temperature & {$[-]$} \\
\hline tra & actual transpiration rate & {$\left[\mathrm{cm} \mathrm{d}^{-1}\right]$} \\
\hline twlv(pot) & total weight leaves (living + death) & {$\left[\mathrm{kg} \mathrm{ha}^{-1}\right]$} \\
\hline twst(pot) & total stems leaves (living + death) & {$\left[\mathrm{kg} \mathrm{ha}^{-1}\right]$} \\
\hline wrt(pot) & dry matter weight roots & {$\left[\mathrm{kg} \mathrm{ha}^{-1}\right]$} \\
\hline$w \operatorname{lv}(p o t)$ & dry matter weight leaves & {$\left[\mathrm{kg} \mathrm{ha}^{-1}\right]$} \\
\hline wst(pot) & dry matter weight stems & {$\left[\mathrm{kg} \mathrm{ha}^{-1}\right]$} \\
\hline
\end{tabular}




\section{Appendix 17 Numerical scheme soil water boundary conditions}

\section{Top boundary condition}

Appropriate criteria for the procedure with respect to the top boundary condition are important for accurate simulation of rapidly changing soil water fluxes near the soil surface. This is for instance the case with infiltration/runoff events during intensive rain showers or when the soil occasionally gets flooded in areas with shallow groundwater tables. At moderate weather and soil wetness conditions the soil top boundary condition will be flux-controlled. In either very wet or very dry conditions the prevailing water pressure head at the soil surface starts to govern the boundary condition.

In case of a Flux controlled top boundary the term $-K_{i-1 / 2}^{j+\kappa, \kappa p}\left(\frac{h_{i-1}^{j+1, p}-h_{i}^{j+1, p}}{1 / 2\left(\Delta z_{i-1}+\Delta z_{i}\right)}+1\right)$ is replaced by the flux through the soil surface qtop $\left(\mathrm{cm} \mathrm{d}^{-1}\right)$ which yields the following expression:

$$
F_{1}=\frac{\Delta z_{1}}{\Delta t^{j}}\left(\theta_{1}^{j+1}-\theta_{1}^{j}\right)+q_{\text {top }}+K_{1 / 2}^{j+\kappa} \frac{h_{1}^{j+1}-h_{2}^{j+1}}{1 / 2\left(\Delta z_{1}+\Delta z_{2}\right)}+K_{1 / 2}^{j+\kappa}+\Delta z_{1} S_{a, 1}^{j+\kappa}+\Delta z_{1} S_{d, 1}^{j}+\Delta z_{1} S_{m, 1}^{j+1}
$$

where $q_{\text {top }}$ is calculated from external driving factors as net precipitation $\left(q_{\text {prec }}\right)$, irrigation $\left(q_{\text {irri }}\right)$, melt of a snow pack $\left(q_{\text {melt }}\right)$ runon originating from adjacent fields $\left(q_{\text {runon }}\right)$ and inundation from adjacent water courses $\left(q_{\text {inun }}\right)$

$$
q_{\text {top }}=-q_{\text {prec }}-q_{\text {irri }}-q_{\text {melt }}-q_{\text {runon }}-q_{\text {inun }}
$$

In case of a Head controlled top boundary the term $-K_{i-1 / 2}^{j+\kappa, \kappa p}\left(\frac{h_{i-1}^{j+1, p}-h_{i}^{j+1, p}}{1 / 2\left(\Delta z_{i-1}+\Delta z_{i}\right)}+1\right)$ is replaced by $-K_{1 / 2}^{j+1}\left(\frac{h_{0}^{j+1}-h_{1}^{j+1}}{1 / 2\left(\Delta z_{1}\right)}+1\right)$, where $h_{0}^{j+1}$ is the pressure head at the soil surface at the new time level.

The internodal conductivity $K_{1 / 2}^{j+1}$ is always treated implicitly.

Within each iteration round and also within each backtracking sub-cycle it is tested whether the combination of $q_{\text {top }}$ and $h_{1}^{j+1}$ would lead to $h_{1}^{j+1}-1 / 2\left(\Delta z_{1}\right)\left(\frac{q_{\text {top }}}{K_{1 / 2}^{j+1}}+1\right)>0$. In such a case it is decided that the head boundary condition holds and the water balance of the so-called ponding layer is calculated which includes the surface runoff flux and the ponding depth at time level $j+1$. The value of $h_{0}^{j+1}$ is set to the ponding depth at time level $j+1$. The water balance of the ponding layer reads as:

$$
\frac{h_{0}^{j+1}-h_{0}^{j}}{\Delta t}=-K_{1 / 2}^{j+1}\left(\frac{h_{0}^{j+1}-h_{1}^{j+1}}{1 / 2\left(\Delta z_{1}\right)}+1\right)+q_{\text {prec }}+q_{\text {irri }}+q_{\text {melt }}+q_{\text {runon }}+q_{\text {inun }}-q_{e, \text { pond }}-q_{\text {runoff }}-I_{r u}
$$

Where $I_{r u}$ is the runoff into the macropores (Section 6.1.2).

The surface runoff flux $q_{\text {runoff }}$ is defined as a function of the ponding height:

$$
\begin{array}{ll}
h_{0}^{j+1} \leq h_{0, \text { threshold }} & q_{\text {runoff }}=0 \\
h_{0}^{j+1}>h_{0, \text { threshold }} & q_{\text {runoff }}=\alpha\left(h_{0}^{j+1}-h_{0, \text { threshold }}\right)^{\beta}
\end{array}
$$


Where $\alpha$ and $\beta$ are coefficients of the surface runoff equation employed by the SWAP model (see

Section 4.1). Substitution of surface runoff expression into water balance equation for the ponding layer yields the relation which is solved in the iteration procedure:

$$
\begin{aligned}
& h_{0}^{j+1}\left(\frac{1}{\Delta t}+\frac{K_{1 / 2}^{j+1}}{1 / 2\left(\Delta z_{1}\right)}\right)+\alpha\left(h_{0}^{j+1}-h_{0, \text { threshold }}\right)^{\beta}= \\
& \frac{h_{0}^{j}}{\Delta t}+\frac{K_{1 / 2}^{j+1}}{1 / 2\left(\Delta z_{1}\right)} h_{1}^{j+1}-K_{1 / 2}^{j+1}+q_{\text {prec }}+q_{\text {melt }}+q_{\text {runon }}-q_{e, \text { pond }}-I_{r u}
\end{aligned}
$$

which is solved in the iteration procedure.

\section{Bottom boundary condition}

The SWAP model provides a number of options to describe the relation between saturated shallow soil layers with deep groundwater (see Chapter 5). Beside handling the flux controlled boundary condition and head controlled boundary condition, the model has additional capabilities to combine these basic types of conditions. Additional options comprise the handling of:

- Predefined groundwater levels

- Cauchy relation for the bottom boundary

- Free drainage

- Free outflow

Flux controlled bottom boundary

For $\mathrm{i}=\mathrm{n}$, the $K_{i+1 / 2}^{j+\kappa, \kappa p}\left(\frac{h_{i}^{j+1, p}-h_{i+1}^{j+1, p}}{1 / 2\left(\Delta z_{i}+\Delta z_{i+1}\right)}+1\right)$-term in Eq. (2.29) is replaced by $-q_{b o t}$, which yields:

$$
F_{n}=\frac{\Delta z_{n}}{\Delta t^{j}}\left(\theta_{n}^{j+1}-\theta_{n}^{j}\right)-K_{n-1 / 2}^{j+\kappa}\left(\frac{h_{n-1}^{j+1}-h_{n}^{j+1}}{1 / 2\left(\Delta z_{n-1}+\Delta z_{n}\right)}+1\right)-q_{b o t}+\Delta z_{n}\left(S_{a, n}^{j+\kappa}+S_{d, n}^{j}+S_{m, n}^{j+1}\right)
$$

Beside the flux boundary condition, the SWAP model has options to handle groundwater level dependent bottom fluxes. The flux can be formulated as an exponential function of groundwater level, or as the difference between groundwater level and hydraulic head in deep groundwater outside the flow domain divided by a flow resistance. Such a flux is calculated explicitly at the start of the current time step and is treated as a flux condition in the numerical scheme.

Head controlled bottom boundary

For $i=n, \Delta z_{i+1}$ is set to zero and $h_{i+1}^{j+1, p}=h_{b o t}$, which leads to the following expression:

$$
\begin{aligned}
F_{n}= & \frac{\Delta z_{n}}{\Delta t^{j}}\left(\theta_{n}^{j+1}-\theta_{n}^{j}\right)-K_{n-1 / 2}^{j+\kappa}\left(\frac{h_{n-1}^{j+1}-h_{n}^{j+1}}{1 / 2\left(\Delta z_{n-1}+\Delta z_{n}\right)}+1\right)+K_{n+1 / 2}^{j+\kappa}\left(\frac{h_{n}^{j+1}-h_{b o t}}{1 / 2 \Delta z_{n}}+1\right) \\
& +\Delta z_{n}\left(S_{a, n}^{j+\kappa}+S_{d, n}^{j}+S_{m, n}^{j+1}\right)
\end{aligned}
$$

\section{Predefined groundwater levels}

First, the lowest partially unsaturated compartment is searched for and is called $n^{*}$. The set of $n^{*}$ nonlinear equations for $F(h)$ is then solved for the unsaturated compartments. The bottom boundary condition for this set of equations is defined by:

$$
\begin{aligned}
F_{n^{*}} & =\frac{\Delta z_{n^{*}}}{\Delta t^{j}}\left(\theta_{n^{*}}^{j+1}-\theta_{n^{*}}^{j}\right)-K_{n^{*}-1 / 2}^{j+\kappa}\left(\frac{h_{n^{*}-1}^{j+1}-h_{n^{*}}^{j+1}}{1 / 2\left(\Delta z_{n^{*}-1}+\Delta z_{n^{*}}\right)}+1\right)+K_{n^{*+1 / 2}}^{j+\kappa}\left(\frac{h_{n^{*}}^{j+1}-h_{n^{*}+1}^{j+1}}{1 / 2\left(\Delta z_{n^{*}}+\Delta z_{n^{*}+1}\right)}+1\right) \\
& +\Delta z_{n}\left(S_{a, n^{*}}^{j+\kappa}+S_{d, n^{*}}^{j}+S_{m, n^{*}}^{j+1}\right)
\end{aligned}
$$


The groundwater level is situated between the nodal points $n^{*}$ and $n^{*}+1$. The pressure head of nodal point $n^{*}+1$ is approximated $h_{n^{*}}^{j+1}$ by:

$$
h_{n^{*}+1}^{j+1}=-h_{n^{*}}^{j+1} \frac{g w l-z_{n^{*}+1}}{z_{n^{*}}-g w l}
$$

where $z_{n^{*}}$ is the height of the node in compartment $n^{*}$ and $g w /$ is the groundwater level. Substitution into Eq. (2.30) yields:

$$
\begin{aligned}
F_{n^{*}} & =\frac{\Delta z_{n^{*}}}{\Delta t^{j}}\left(\theta_{n^{*}}^{j+1}-\theta_{n^{*}}^{j}\right)-K_{n^{*}-1 / 2}^{j+\kappa}\left(\frac{h_{n^{*}-1}^{j+1}-h_{n^{*}}^{j+1}}{1 / 2\left(\Delta z_{n^{*}-1}+\Delta z_{n^{*}}\right)}+1\right)+K_{n^{*}+1 / 2}^{j+\kappa}\left(\frac{h_{n^{*}}^{j+1}\left(\frac{z_{n^{*}}-z_{n^{*}+1}}{z_{n^{*}}-g w l}\right)}{1 / 2\left(\Delta z_{n^{*}}+\Delta z_{n^{*}+1}\right)}+1\right) \\
& +\Delta z_{n}\left(S_{a, n^{*}}^{j+\kappa}+S_{d, n^{*}}^{j}+S_{m, n^{*}}^{j+1}\right)
\end{aligned}
$$

After iteratively solving the set of equations for $i \leq n^{*}$, the pressure head profile of the compartments $i>n^{*}$ can be calculated from the pressure head of the two adjacent upward nodes.

$$
\begin{aligned}
h_{i+1}^{j+1}= & h_{i}^{j+1}\left(1+\frac{K_{i-1 / 2}^{j+\kappa}\left(\Delta z_{i}+\Delta z_{i+1}\right)}{K_{i+1 / 2}^{j+\kappa}\left(\Delta z_{i-1}+\Delta z_{i}\right)}\right)-h_{i-1}^{j+1} \frac{K_{i-1 / 2}^{j+\kappa}\left(\Delta z_{i}+\Delta z_{i+1}\right)}{K_{i+1 / 2}^{j+\kappa}\left(\Delta z_{i-1}+\Delta z_{i}\right)}+1 / 2\left(\Delta z_{i}+\Delta z_{i+1}\right)\left(1-\frac{K_{i-1 / 2}^{j+\kappa}}{K_{i+1 / 2}^{j+\kappa}}\right) \\
& +\frac{1 / 2\left(\Delta z_{i}+\Delta z_{i+1}\right)}{K_{i+1 / 2}^{j+\kappa}}\left(\frac{\Delta z_{i}}{\Delta t^{j}}\left(\theta_{s a t, i}-\theta_{i}^{j}\right)+\Delta z_{i}\left(S_{a, i}^{j+\kappa}+S_{d, i}^{j}+S_{m, i}^{j+1}\right)\right)
\end{aligned}
$$

Cauchy relation for the bottom boundary

The flux through the bottom boundary is defined by the difference the hydraulic head at the lower boundary and the hydraulic head $\phi(\mathrm{cm})$ of the regional groundwater specified by the user, divided by a flow resistance $c(d)$. The hydraulic head at the lower boundary is approximated by the pressure head of the lowest nodal point plus the elevation head of node $n$.

$$
q_{b o t}=\frac{h_{n}^{j+1}+z_{n}-\phi}{\frac{1 / 2 \Delta z_{n}}{K_{n+1 / 2}^{j+\kappa}}+c}
$$

Substitution into Eq. (2.30) yields:

$$
\begin{aligned}
F_{n} & =\frac{\Delta z_{n}}{\Delta t^{j}}\left(\theta_{n}^{j+1}-\theta_{n}^{j}\right)-K_{n-1 / 2}^{j+\kappa}\left(\frac{h_{n-1}^{j+1}-h_{n}^{j+1}}{1 / 2\left(\Delta z_{n-1}+\Delta z_{n}\right)}+1\right)+K_{n+1 / 2}^{j+\kappa} \frac{h_{n}^{j+1}-\left(\phi-z_{n}\right)}{1 / 2 \Delta z_{n}+c K_{n+1 / 2}^{j+\kappa}} \\
& +\Delta z_{n}\left(S_{a, n}^{j+\kappa}+S_{d, n}^{j}+S_{m, n}^{j+1}\right)
\end{aligned}
$$

\section{Seepage face}

The seepage face option is used to simulate the soil moisture flow in a lysimeter with an open outlet at the bottom. No outflow occurs when the bottom soil layer is still unsaturated. Since the flow resistance of the outlet is negligible small, no positive pressure head values will be build up at the bottom when the soil water percolates at the bottom. Within the iteration cycle for solving the numerical expression of the Richards equation, it is checked whether the flux or the head controlled boundary condition prevails. When $h_{n}^{j+1}+1 / 2 \Delta z_{n}<0$ the bottom flux $q_{b o t}$ is set to zero, but when $h_{n}^{j+1}+1 / 2 \Delta z_{n}$ tends to take values larger than zero, the pressure at the bottom is set to zero $\left(h_{b o t}=0\right)$. The numerical implementation is as follows:

$$
\begin{aligned}
& h_{n}^{j+1}+1 / 2 \Delta z_{n}<0 \underset{n}{\rightarrow} F_{n}=\frac{\Delta z_{n}}{\Delta t^{j}}\left(\theta_{n}^{j+1}-\theta_{n}^{j}\right)-K_{n-1 / 2}^{j+\kappa}\left(\frac{h_{n-1}^{j+1}-h_{n}^{j+1}}{1 / 2\left(\Delta z_{n-1}+\Delta z_{n}\right)}+1\right) \\
& +\Delta z_{n}\left(S_{a, n}^{j+\kappa}+S_{d, n}^{j}+S_{m, n}^{j+1}\right)
\end{aligned}
$$




$$
\begin{aligned}
& h_{n}^{j+1}+1 / 2 \Delta z_{n} \nless 0 \rightarrow{ }_{n}=\frac{\Delta z_{n}}{\Delta t^{j}}\left(\theta_{n}^{j+1}-\theta_{n}^{j}\right)-K_{n-1 / 2}^{j+\kappa}\left(\frac{h_{n-1}^{j+1}-h_{n}^{j+1}}{1 / 2\left(\Delta z_{n-1}+\Delta z_{n}\right)}+1\right) \\
& +K_{n+1 / 2}^{j+\kappa}\left(\frac{h_{n}^{j+1}}{1 / 2 \Delta z_{n}}+1\right)+\Delta z_{n}\left(S_{a, n}^{j+\kappa}+S_{d, n}^{j}+S_{m, n}^{j+1}\right)
\end{aligned}
$$

Free drainage

The free drainage option is applied for soil profiles with deep groundwater levels. The bottom flux is only provoked by gravity flow and the head pressure gradient equals zero:

$$
q_{b o t}=-K_{n+1 / 2}^{j+1}\left(\left(\frac{\partial h}{\partial z}\right)_{n+1 / 2}+1\right) \rightarrow q_{b o t}=-K_{n+1 / 2}^{j+1}
$$

Substitution into Eq. (2.30) yields:

$$
\begin{aligned}
F_{n}= & \frac{\Delta z_{n}}{\Delta t^{j}}\left(\theta_{n}^{j+1}-\theta_{n}^{j}\right)-K_{n-1 / 2}^{j+\kappa}\left(\frac{h_{n-1}^{j+1}-h_{n}^{j+1}}{1 / 2\left(\Delta z_{n-1}+\Delta z_{n}\right)}+1\right)+K_{n+1 / 2}^{j+1} \\
& +\Delta z_{n}\left(S_{a, n}^{j+\kappa}+S_{d, n}^{j}+S_{m, n}^{j+1}\right)
\end{aligned}
$$




\section{Appendix 18 Explanation of header of crop output file}

\begin{tabular}{|c|c|c|}
\hline Name & Unit & Description \\
\hline Date & - & Julian Date \\
\hline Daynr & day & Day number within a calender year starting with $1=$ January 1 \\
\hline Daycrp & day & Day number starting at crop emergence \\
\hline DVS & - & DeVelopment Stage \\
\hline TSUM & $\operatorname{grC}$ & Temperature SUM from cropstart \\
\hline LAIpot & - & Leaf Area Index of crop under potential growth conditions \\
\hline LAI & - & Leaf Area Index of crop under actual growth conditions \\
\hline Height & $\mathrm{cm}$ & Height of crop under actual growth conditions \\
\hline CrpFac & - & Crop factor \\
\hline RootdPot & $\mathrm{cm}$ & Rooting depth under potential growth conditions \\
\hline Rootd & $\mathrm{cm}$ & Rooting depth under actual growth conditions \\
\hline PWLV & $\mathrm{kg} / \mathrm{ha}$ & Potential dry weight of living plant leaves \\
\hline WLV & $\mathrm{kg} / \mathrm{ha}$ & Actual dry weight of living plant leaves \\
\hline PWST & $\mathrm{kg} / \mathrm{ha}$ & Potential dry weight of living plant stem \\
\hline WST & $\mathrm{kg} / \mathrm{ha}$ & Actual dry weight of living plant stem \\
\hline PWRT & $\mathrm{kg} / \mathrm{ha}$ & Potential dry weight of living plant root \\
\hline WRT & $\mathrm{kg} / \mathrm{ha}$ & Actual dry weight of living plant root \\
\hline CPWDM & $\mathrm{kg} / \mathrm{ha}$ & Cumulative from cropstart potential dry weight of dead and living above ground plant organs \\
\hline CWDM & $\mathrm{kg} / \mathrm{ha}$ & Cumulative from cropstart actual dry weight of dead and living above ground plant organs \\
\hline CPWSO & $\mathrm{kg} / \mathrm{ha}$ & Cumulative from flowering potential dry weight of living storage organs \\
\hline CWSO & $\mathrm{kg} / \mathrm{ha}$ & Cumulative from flowering actual dry weight of s living torage organs (wso) \\
\hline PGRASSDM & $\mathrm{kg} / \mathrm{ha}$ & Potential dry weight of dead and living grass \\
\hline GRASSDM & $\mathrm{kg} / \mathrm{ha}$ & Actual dry weight of dead and living grass (tagp $=$ wlv + dwlv + wst $+d w s t)$ \\
\hline PMOWDM & $\mathrm{kg} / \mathrm{ha}$ & Potential amount of harvested grass by mowing \\
\hline MOWDM & $\mathrm{kg} / \mathrm{ha}$ & Actual amount of harvested grass by mowing (tagpt $=$ tagpt $+($ tagp $-(w l v+d w l v+w s t+d w s t)))$ \\
\hline PGRAZDM & $\mathrm{kg} / \mathrm{ha}$ & Potential amount of grazed grass by lifestock \\
\hline GRAZDM & $\mathrm{kg} / \mathrm{ha}$ & Actual amount of consumed grass by lifestock (cuptgraz) \\
\hline DWLVCROP & $\mathrm{kg} / \mathrm{ha}$ & Cumulative from cropstart actual dry weight of deceased leafs allocated to soil \\
\hline DWLVSOIL & $\mathrm{kg} / \mathrm{ha}$ & Cumulative from cropstart actual dry weight of deceased leafs allocated to soil \\
\hline DWST & $\mathrm{kg} / \mathrm{ha}$ & Cumulative from cropstart actual dry weight of deceased plant stems \\
\hline DWRT & $\mathrm{kg} / \mathrm{ha}$ & Cumulative from cropstart actual dry weight of deceased plant roots \\
\hline DWSO & $\mathrm{kg} / \mathrm{ha}$ & Cumulative from cropstart actual dry weight of deceased plant storage organs \\
\hline HarLosOrm & $\mathrm{kg} / \mathrm{ha}$ & Harvest losses added to the soil (roots+fr_shoots_fr_stems+fr_stor.organs) at harvest \\
\hline
\end{tabular}


Wageningen Environmental Research P.O. Box 47

6700 AA Wageningen

The Netherlands

T +31 (0)317480700

www.wur.nl/environmental-research

Wageningen Environmental Research Report 2780

ISSN 1566-7197
The mission of Wageningen University and Research is "To explore the potential of nature to improve the quality of life". Under the banner Wageningen University \& Research, Wageningen University and the specialised research institutes of the Wageningen Research Foundation have joined forces in contributing to finding solutions to important questions in the domain of healthy food and living environment. With its roughly 30 branches, 5,000 employees and 10,000 students, Wageningen University \& Research is one of the leading organisations in its domain. The unique Wageningen approach lies in its integrated approach to issues and the collaboration between different disciplines.

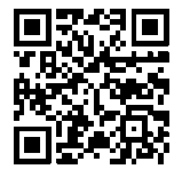




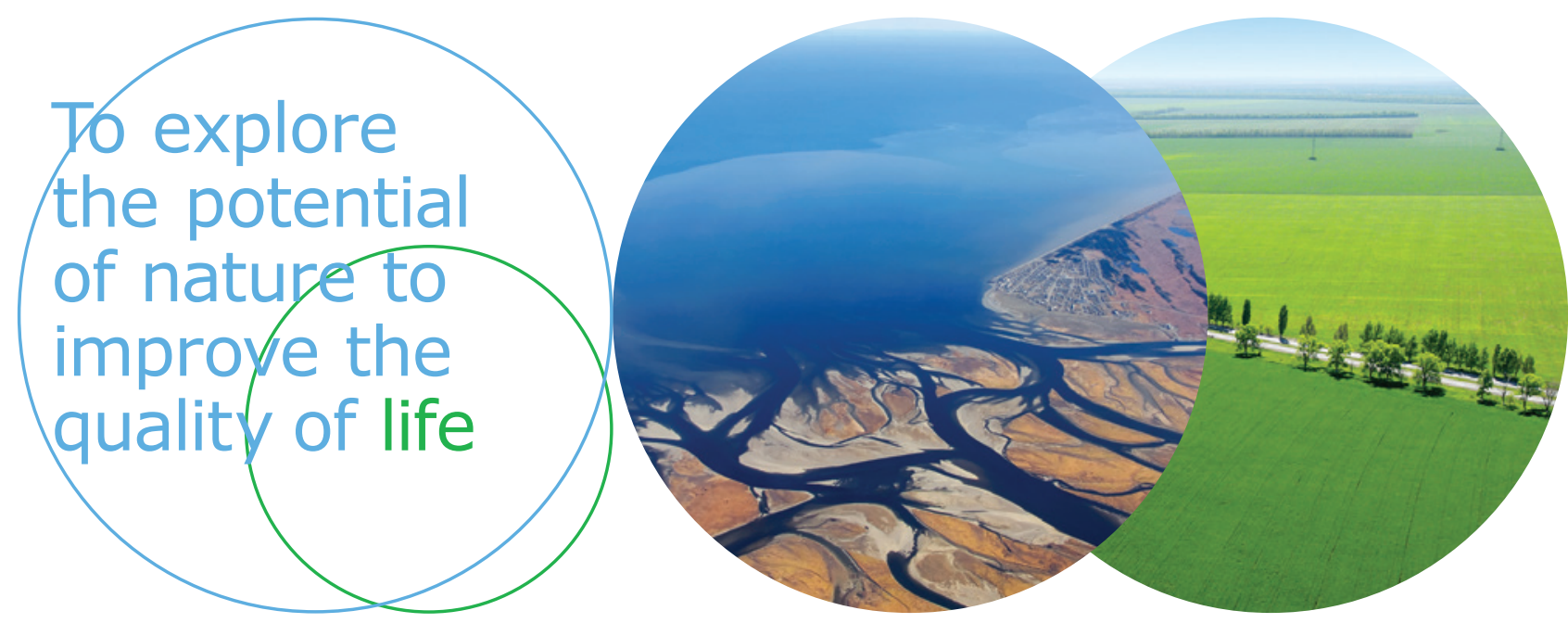

Wageningen Environmental Research P.O. Box 47

$6700 \mathrm{AB}$ Wageningen

The Netherlands

$\mathrm{T}+31(0) 317480700$

www.wur.eu/environmental-research

Report 2780

ISSN 1566-7197
The mission of Wageningen University and Research is "To explore the potential of nature to improve the quality of life". Under the banner Wageningen University \& Research, Wageningen University and the specialised research institutes of the Wageningen Research Foundation have joined forces in contributing to inding solutions to important questions in the domain of healthy food and living environment. With its roughly 30 branches, 5,000 employees and 10,000 students, Wageningen University \& Research is one of the leading organisations in its domain. The unique Wageningen approach lies in its integrated approach to issues and the collaboration between different disciplines. 